\title{
Detection of subsurface anomalies in fiber-reinforced polymer (FRP) wrapped timber bridge components using infrared thermography
}

William Edward Steele III

West Virginia University

Follow this and additional works at: https://researchrepository.wvu.edu/etd

\section{Recommended Citation}

Steele, William Edward III, "Detection of subsurface anomalies in fiber-reinforced polymer (FRP) wrapped timber bridge components using infrared thermography" (2001). Graduate Theses, Dissertations, and Problem Reports. 1209.

https://researchrepository.wvu.edu/etd/1209

This Thesis is protected by copyright and/or related rights. It has been brought to you by the The Research Repository @WVU with permission from the rights-holder(s). You are free to use this Thesis in any way that is permitted by the copyright and related rights legislation that applies to your use. For other uses you must obtain permission from the rights-holder(s) directly, unless additional rights are indicated by a Creative Commons license in the record and/ or on the work itself. This Thesis has been accepted for inclusion in WVU Graduate Theses, Dissertations, and Problem Reports collection by an authorized administrator of The Research Repository @ WVU. For more information, please contact researchrepository@mail.wvu.edu. 


\section{DETECTION OF SUBSURFACE ANOMALIES IN FIBER REINFORCED POLYMER (FRP) WRAPPED TIMBER BRIDGE COMPONENTS USING INFRARED THERMOGRAPHY}

By

William Edward Steele III

\section{A THESIS}

Submitted to the

College of Engineering and Mineral Resources

at West Virginia University

In partial fulfillment of the requirements

for the degree of

Master of Science in Engineering

Udaya Halabe, Ph.D., P.E., Chair

Hota GangaRao, Ph.D., P.E.

Powsiri Klinkhachorn, Ph.D.

Department of Civil and Environmental Engineering

Morgantown, West Virginia

2001

Keywords: Anomalies, Composite, Debonds, Defects, Delaminations, FRP, Infrared

Thermography, Nondestructive Testing, Temperature gradients, Wrap 


\author{
ABSTRACT \\ DETECTION OF SUBSURFACE ANOMALIES IN FIBER \\ REINFORCED POLYMER (FRP) WRAPPED TIMBER \\ BRIDGE COMPONENTS USING INFRARED \\ THERMOGRAPHY
}

\author{
William Edward Steele III
}

This thesis presents the results of an experimental study on the use of Infrared Thermography technique for detection of subsurface anomalies in fiber reinforced polymer (FRP) wrapped timber bridge components. An extensive literature review on the application of various nondestructive evaluation techniques to composite structures has also been presented.

Simulated subsurface delaminations were constructed in the laboratory in timber piles wrapped with FRP composite fabric. The delaminations varied in size, thickness, and severity. These delaminations were placed between the $1 / 8$ " thick FRP wrap and timber surface. The thermal images from the delaminated specimens were compared with thermal images from undamaged specimens to study the effect of subsurface anomalies. In addition, several field tests were conducted using the infrared imaging system on three timber railroad bridges located in Moorefield, West Virginia that were reinforced with FRP composite fabric. The field test data was used to detect debonds at the composite-timber interface and study the effect of environmental parameters on infrared images.

This study shows that the infrared thermography technique can be used to effectively to detect subsurface delaminations in timber components wrapped with FRP composite fabric. The study also shows the effect of different parameters (environmental conditions, heat source, etc.) on the clarity of infrared images. 


\section{ACKNOWLEDGEMENT}

The author wishes to express his sincere gratitude to his research advisor and committee chairman, Dr. Udaya B. Halabe, for all his guidance, help, and encouragement during the course of this research. The author is also grateful to Dr. Hota V. S. GangaRao and Dr. Powsiri Klinkhachorn for serving on his thesis committee and providing valuable help and support during the completion of this research.

The author would like to thank the USDOT-FRA and WVDOT-South Branch Valley Railroad for all their cooperation and help during the field study part of this research. And the author would also like to thank the USDOT-FRA and USDOT-FHWA for funding various aspects of this research through the Constructed Facilities Center at West Virginia University.

Appreciation is also extended to all the faculty and students that I have had the privilege of working with throughout my years of schooling at WVU. 


\section{Table of Contents}

$\underline{\text { Description }}$

Page No.

Chapter 1 - Introduction................................................1

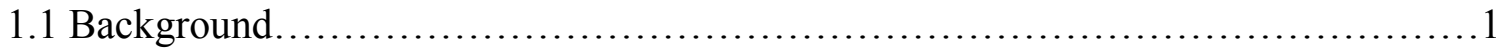

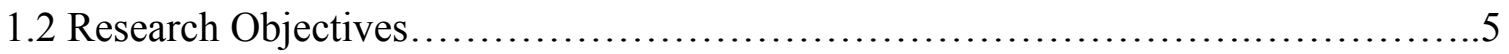

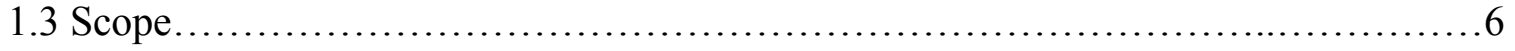

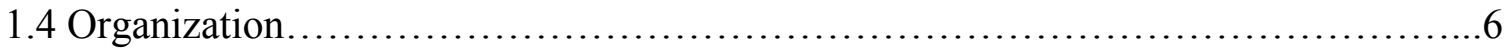

\section{Chapter 2 - Literature Review of NDE Techniques For Evaluating}

Composite Materials.........................................8

2.1 Infrared Thermography Applications $\ldots \ldots \ldots \ldots \ldots \ldots \ldots \ldots \ldots \ldots \ldots \ldots \ldots \ldots \ldots \ldots$

2.1.1 Rapid NDT of Composite Aircraft Components Using Lock-In

Ultrasonic and Halogen Lamp Thermography...........................9

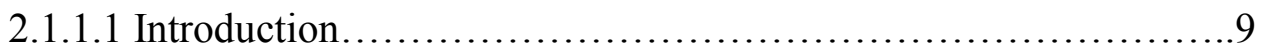

2.1.1.2 Thermal Non-Destructive Testing Techniques...................10

2.1.1.2.1 Lock-in Thermography..............................11

2.1.1.2.2 Lock-in Halogen Lamp Heating........................12

2.1.1.2.3 Lock-in Ultrasonic Transducer Heating..................13 
2.1.1.3 Sample Preparation........................................ 15

2.1.1.4 Experimental Apparatus......................................16

2.1.1.5 Results and Discussion......................................16

2.1.1.5.1 Ultrasonic C-Scan Results............................16

2.1.1.5.2 Lock-in Halogen Lamp Results........................17

2.1.1.5.3 Lock-in Ultrasonic Transducer Results.................19

2.1.1.5.4 Comparison of the NDT Techniques..................20

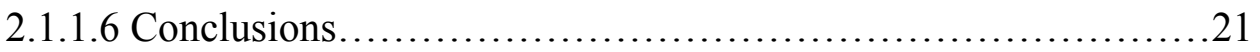

2.1.2 Feasibility of Evaluating the Performance of Fiber Reinforced Plastic (FRP) Wrapped Reinforced Concrete Columns Using Ground Penetrating Radar (GPR) and Infrared (IR) Thermography Techniques....................22

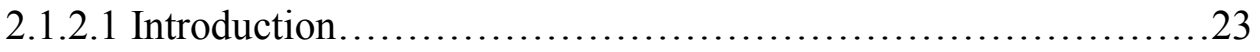

2.1.2.2 Ground Penetrating Radar (GPR).............................24

2.1.2.3 Infrared (IR) Thermography................................24

2.1.2.4 GPR Survey Results.......................................24

2.1.2.5 IR Survey Results.........................................

2.1.2.6 Concluding Remarks .....................................26

2.1.3 Thermal Imaging Technique to Detect Delaminations in CFRP Plated

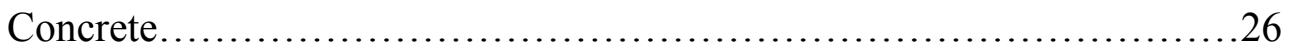

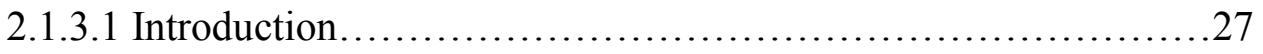

2.1.3.2 Active Infrared Thermography.............................28

2.1.3.3 Experimental Procedure......................................29

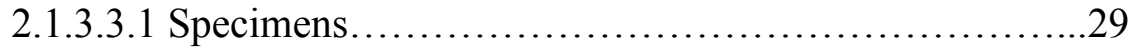


2.1.3.3.2 Equipment Setup...................................29

2.1.3.3.3 Procedure ........................................... 30

2.1.3.4 Results........................................................

2.1.3.4.1 One and Two Ply Specimen...........................31

2.1.3.4.2 Four and Eight Ply Specimen.........................31

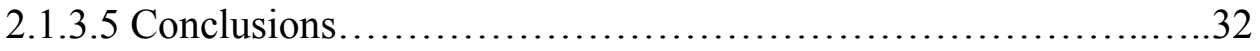

2.1.4 Detecting Manufacturing Flaws in Composite Retrofits...................32

2.1.4.1 Composite Manufacturing Techniques...........................33

2.1.4.1.1 Hand Lay-up.........................................33

2.1.4.1.2 Pre-Cured Shells......................................33

2.1.4.1.3 Machine Wrap.......................................34

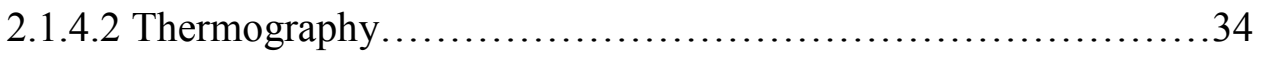

2.1.4.2.1 Thermography Background..........................34

2.1.4.2.2 Thermography System................................35

2.1.4.3 Bridge Inspection Results....................................36

2.1.4.3.1 Hand Lay-up.........................................36

2.1.4.3.2 Pre-Cured Shells......................................37

2.1.4.3.3 Machine Wrap Debonds...............................39

2.1.4.4 Advantages of Thermography................................. 39

2.1.4.4.1 Remote Inspection Capability.........................39

2.1.4.4.2 Rapid Inspection..................................40

2.1.4.4.3 Data Storage ...................................... 40

2.1.4.5 Issues Encountered and Potential Solutions.......................41 
2.1.4.5.1 Uniform Heating...................................41

2.1.4.5.2 Material Thickness Variations.........................41

2.1.4.5.3 Cleanliness........................................42

2.1.4.5.4 Image Processing...................................42

2.1.4.5.5 Inspection Speed....................................42

2.1.4.6 Summary ..................................................... 43

2.1.5 Thermal Nondestructive Testing (TNDT) of Adhesively Bonded Composite Reinforcements Applied to Concrete Civil Structures.....................43

2.1.5.1 Structures Tested...........................................44

2.1.5.1.1 Caltrans Column Confinement Qualification Test.......44

2.1.5.1.2 Navy Pier 12 San Diego...............................45

2.1.5.2 Principles of Thermographic Testing ...........................47

2.1.5.3 TNDT Testing of a Concrete Column...........................48

2.1.5.4 TNDT Testing of a Naval Pier..................................49

2.1.5.4.1 Pultruded Graphite fiber rods........................50

2.1.5.4.2 Pultruded fiberglass I-Beams........................50

2.1.5.4.3 Pultruded Graphite fiber strips........................50

2.1.5.4.4 Laminated Graphite/epoxy fabric......................51

2.1.5.4.5 Laminated Vinyl Ester/Fiberglass Shells...............53

2.1.5.5 Conclusions..............................................53

2.1.6 Conclusion on Infrared Thermography.............................54

2.2 Ultrasonic Wave Propagation.................................................55 
2.2.1 Non-Contact Ultrasound Studies of Composite Materials: New

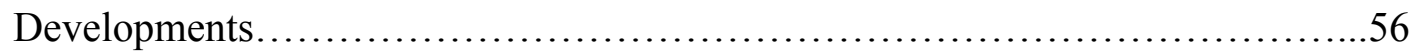

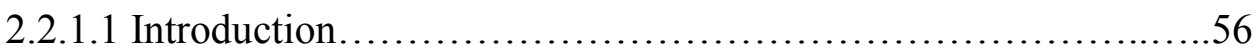

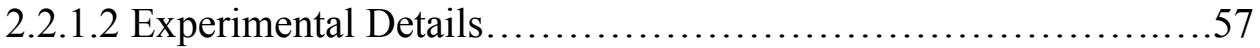

2.2.1.3 Results................................................. 58

2.2.1.3.1 Nd:YAG Laser.......................................58

2.2.1.3.2 TEA $\mathrm{CO}_{2} \ldots \ldots \ldots \ldots \ldots \ldots \ldots \ldots \ldots \ldots \ldots \ldots \ldots \ldots \ldots$

2.2.1.3.3 OPO.............................................61

2.2.1.3.4 OBD............................................ 62

2.2.1.3.5 Air Coupled Detection................................63

2.2.1.4 Discussion and Conclusion...................................65

2.2.2 Ultrasonic Evaluation of Co-Cured Composite Structures..................65

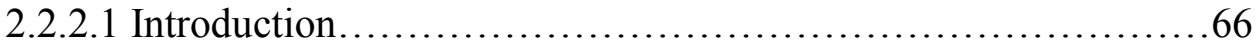

2.2.2.2 Co-Cured Non-Destructive Inspection.........................66

2.2.2.2.1 Two-Sided Inspection...............................67

2.2.2.2.2 One-Sided Inspection...............................67

2.2.2.2.3 Joint Inspection.....................................69

2.2.2.2.3(a) Potential Joint Defects......................69

2.2.2.2.3(b) Hand-Held Radius Inspection.................70

2.2.2.2.3(c) Automated Radius Scanning..................71

2.2.2.3 Conclusion and Summary...................................73

2.2.3 Nondestructive Assessment of the Post-Impact Properties of FRP Composite

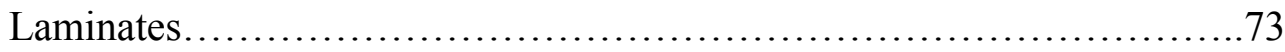


2.2.3.1 Introduction....................................................

2.2.3.2 Nondestructive Ultrasonic Experiments........................74

2.2.3.2.1 Through-Thickness Wave Speed Measurement.........75

2.2.3.2.2 Transverse Vibratory Response Measurement...........76

2.2.3.3 Experimental Results and Discussion............................77

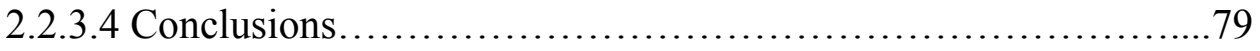

2.2.4 Nondestructive Evaluation of Critical Pultruded Composite Material Bridge

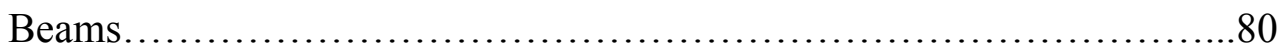

2.2.4.1 Introduction..................................................... 80

2.2.4.2 Acoustic-Ultrasonic Monitoring..............................83

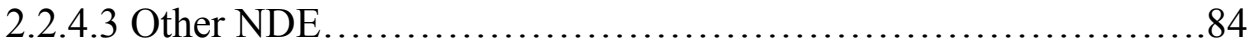

2.2.4.4 Conclusion...................................................... 84

2.2.5 Microwave and Ultrasonic NDE of Thick Glass-Fiber-Reinforced

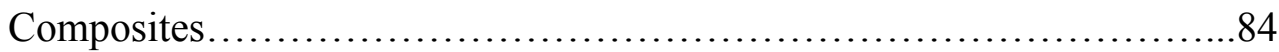

2.2.5.1 Introduction............................................... 85

2.2.5.2 Experimental.................................................. 86

2.2.5.2.1 Ultrasound.......................................... 86

2.2.5.2.2 Microwave Technique..............................90

2.2.5.3 Conclusion....................................................992

2.2.6 Composite Material Defects Characterization Using Leaky Lamb Wave Dispersion Data................................................... 93

2.2.6.1 Introduction.............................................93

2.2.6.2 Leaky Lamb Wave Phenomenon................................93 
2.2.6.3 Theory and Data Inversion..................................95

2.2.6.4 LLW Experimental Capability Enhancement....................96

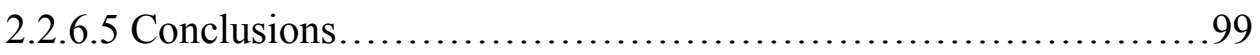

2.2.7 Conclusion on Microwave and Ultrasound Wave Propagation.............100

2.3 Acoustic Wave Propagation Technique.......................................... 101

2.3.1 Blind Deconvolution of Acoustic Emission Signals for Damage

Identification in Composites........................................ 101

2.3.1.1 Introduction.............................................. 102

2.3.1.2 Signal Processing.......................................... 104

2.3.1.3 Experimental............................................ 105

2.3.1.4 Results and Discussions...................................106

2.3.1.5 Conclusions..................................................111

2.3.2 Experimental Investigation into the Use of Vibration Data for Long Term Monitoring of an All Composite Bridge ...............................112

2.3.2.1 Introduction............................................. 113

2.3.2.2 Conceptual Design.........................................113

2.3.2.3 Fabrication................................................ 114

2.3.2.4 Modal Testing.............................................115

2.3.2.5 Equipment............................................... 116

2.3.2.5.1 Accelerometers...................................116

2.3.2.5.2 Excitation and Force Gage..........................117

2.3.2.5.3 Analyzer........................................117

2.3.2.6 Data Capture.............................................. 117 
2.3.2.7 Modal Analysis.............................................118

2.3.2.8 Final Modal Results.......................................118

2.3.2.9 Analytical Determination of Fundamental Resonant Frequency.121

2.3.2.10 Discussion of Modal Results...............................121

2.3.2.11 Data Quality............................................ 122

2.3.2.12 Conclusion............................................... 122

2.3.3 Conclusion on Acoustic Wave Propagation..............................123

2.4 Load Testing with Strain Gages..............................................124

2.4.1 Evaluating Effectiveness of FRP Composites for Bridge Rehabilitation

through Load Testing................................................ 124

2.4.1.1 Introduction............................................... 124

2.4.1.2 Service Load Stresses.......................................125

2.4.1.3 Load Testing............................................. 126

2.4.1.4 Load Test Results.........................................128

2.4.1.5 Conclusion................................................130

2.4.2 Proof Load Testing and Monitoring of an FRP Composite Bridge.........130

2.4.2.1 Introduction............................................ 130

2.4.2.2 Bridge Structure........................................... 131

2.4.2.3 Design and Fabrication...................................... 132

2.4.2.4 Sensors and Instrumentation................................ 132

2.4.2.5 Proof Load Test.............................................133

2.4.2.6 Test Results............................................. 134

2.4.2.7 Visual Inspection........................................... 135 
2.4.2.8 Conclusions.............................................. 136

2.4.3 Conclusion on Load Testing with Strain Gages.........................136

2.5 Fiber Optic Health Monitoring Systems.......................................137

2.5.1 A Composite Bridge Health Monitoring System (Thomas 1999)..........137

2.5.1.1 Introduction............................................ 137

2.5.1.2 System Description.......................................137

2.5.1.2.1 Bridge..........................................137

2.5.1.2.2 Fiber Optic Instrumentation.........................138

2.5.1.2.2(a) Bragg Grating Strain Sensors...............139

2.5.1.2.2(b) Sapphire Fiber Chemical Sensors...........142

2.5.1.3 Field Tests............................................. 146

2.5.1.3.1 Live Load Tests....................................147

2.5.1.3.1(a) Winter Tests - January 1998................147

2.5.1.3.1(b) Summer Tests - August 1998...............152

2.5.1.3.2 Long - Term Monitoring..............................159

2.5.1.3.3 Bondline Condition Monitoring......................161

2.5.1.4 Commercial System Design....................................163

2.5.1.4.1 Bondline Chemical Sensor System....................163

2.5.1.4.2 Bragg Grating Strain Sensor System...................164

2.5.1.5 Conclusion and Recommendations............................164

2.5.2 Application of Optical Fiber Sensors to the Intelligent Processing

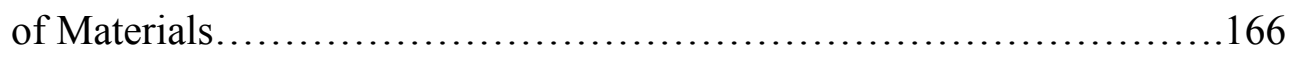

2.5.2.1 Introduction................................................ 167 
2.5.2.2 Intelligent Processing of Materials - Polymer-Matrix

Composites..................................................

2.5.2.2.1 Epoxy - Matrix Composites.........................170

2.5.2.2.2 EFPI-based Optical Fiber Sensors....................170

2.5.2.2.3 Epoxy Matrix Processing using EFPI Sensors.........173

2.5.2.2.4 Experiments..................................... 175

2.5.2.3 Intelligent Processing of Materials - Multilayer Ceramic Actuator

Elements................................................... 179

2.5.2.4 High Temperature Optical Fiber Sensors for the Intelligent

Processing of Materials -Titanium Matrix Composites............181

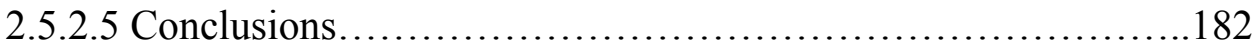

2.5.3 Fiber Optic Health Monitoring System for Composite Bridge Decks......183

2.5.3.1 Introduction................................................ 183

2.5.3.2 Evaluation and Down-Selection of Sensors and

Instrumentation.......................................... 185

2.5.3.2.1 Sensor Classification and Selection....................185

2.5.3.3 Laboratory Demonstration of the EFPI Sensor..................187

2.5.3.4 Sensor Installation and Testing............................. 188

2.5.3.4.1 Sensor Installation and Testing of the First Three-Point-

Bend Composite Bridge Deck Specimen...............188

2.5.3.4.2 Sensor Installation and Testing of the Second Three-

Point-Bend Composite Bridge Deck Test Specimen...190

2.5.3.5 Field Testing. .191 
2.5.3.6 Future Testing. 192

2.5.3.7 Conclusions. 194

2.5.4 Conclusion on Fiber Optics Health Monitoring Systems.... 195

2.6 New NDE Techniques 196

2.6.1 Characterization of Composite Microstructure and Damage Using Optical

Coherence Tomography.........................................196

2.6.1.1 Introduction................................................ 196

2.6.1.2 Experimental........................................ 198

2.6.1.2.1 Materials........................................198

2.6.1.2.2 Instrumentation.............................. 198

2.6.1.3 Results and Discussion......................................199

2.6.2 Non-contact Laser Based Computation Method of NDE of Composites and other Structures.............................................202

2.6.2.1 Introduction...............................................202

2.6.2.2 Description of the RAID NDE Technique....................203

2.6.2.2.1 System Description..............................203

2.6.2.2.2 Methodology of Defect Detection....................204

2.6.2.3 Application of RAID Technology to Composites...............206

2.6.2.4 Conclusions.................................................207

2.6.3 FOPSESPI for Non-Destructive Evaluation (NDE) of Composites........208

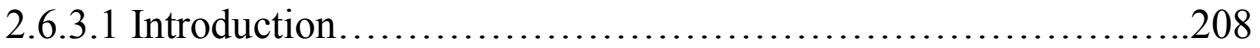

2.6.3.2 Fiber Optic Phase Shifting ESPI System.....................209

2.6.3.3 ESPI - Principle........................................210 
2.6.3.4 Specimen Preparation.......................................210

2.6.3.5 Results and Discussions..................................211

2.6.3.6 Detection of Fiber Breakage..................................212

2.6.3.7 NDT of GFRP Specimens with Programmed Delamination......213

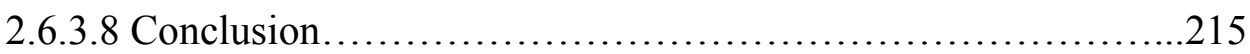

2.6.4 Cost Effective Advances in Portable Radioscopic NDT of Composite

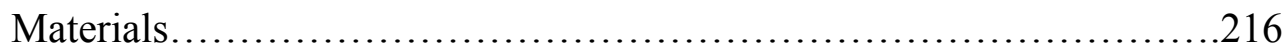

2.6.4.1 Advances in Radioscopic Imaging...........................216

2.6.4.2 NDE / NDT Training............................................

2.6.4.3 Composite Radioscopic Technique...........................217

2.6.4.4 Radioscopic Applications...................................217

2.6.4.5 Cost Consideration............................................217

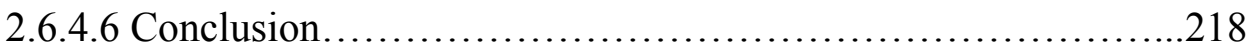

2.6.5 Nondestructive Characterization of Layered Composite Materials with a

Laser Optoacoustic Sensor.........................................218

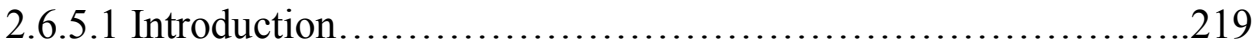

2.6.5.2 Materials and Methods........................................221

2.6.5.2.1 Principles of the Optoacoustic Evaluation..............221

2.6.5.2.2 Components of the Optoacoustic Sensor...............222

2.6.5.2.3 Epoxy-Graphite Samples..........................222

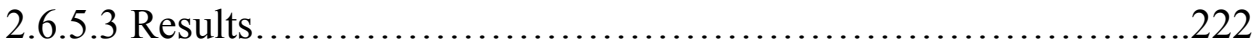

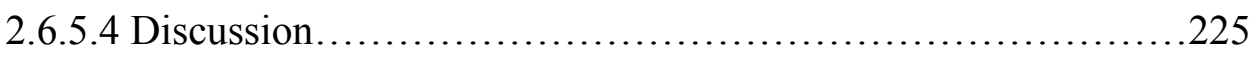

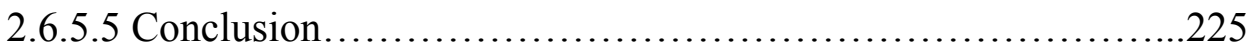


2.6.6 Nondestructive Evaluation of Fatigue Changes of Composite Structure by Laser-Excited Ultrasonic Waves..................................226

2.6.6.1 Introduction.........................................226

2.6.6.2 Experimental Setup....................................227

2.6.6.3 Investigated Samples.....................................228

2.6.6.4 Experimental Results and Discussion......................229

2.6.6.4.1 Attenuation of Ultrasound in Green-State (Before Cyclic

Loading) Composite Sample......................230

2.6.6.4.2 Attenuation of Ultrasound in Composite Samples After

Service Life Testing............................231

2.6.6.4.3 Classification of Composite Samples According to the

Aging........................................233

2.6.6.5 Conclusions..........................................233

2.6.7 Conclusion on New NDE Techniques...............................234

2.7 Final Thoughts on All NDT Methods Used for Evaluating Composite Materials and

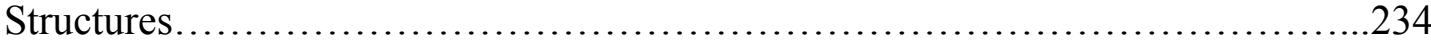

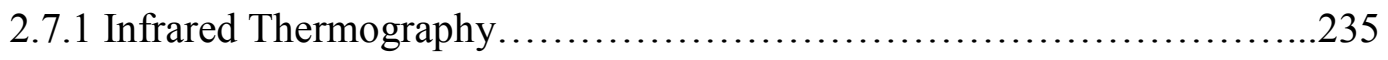

2.7.2 Wave Propagating NDE Techniques (Acoustic, Ultrasound, Microwave,

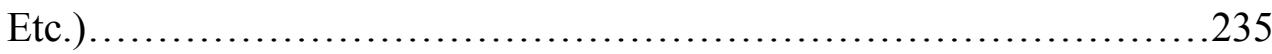

2.7.3 Load Testing with Strain Gages..................................236

2.7.4 Fiber Optic Health Monitoring Systems............................236

2.7.5 New NDE Techniques Being Developed...........................237 
Chapter 3 - Basic Theory of Infrared Thermography..................238

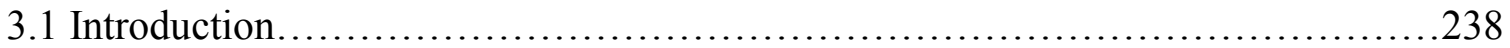

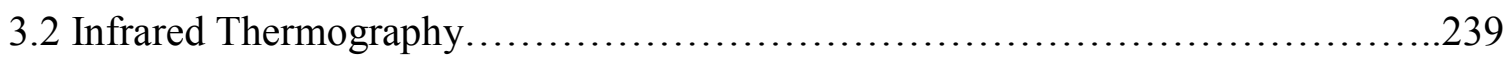

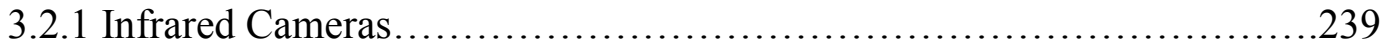

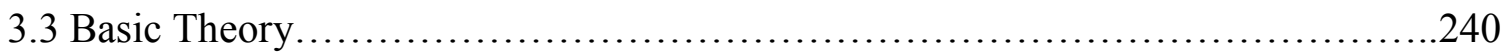

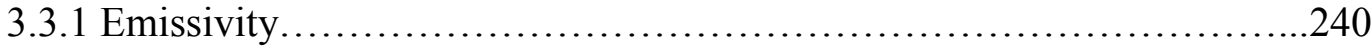

3.3.2 The Stefan-Boltzmann Law.........................................241

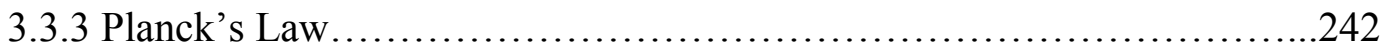

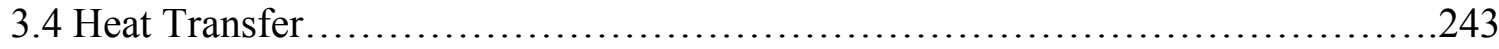

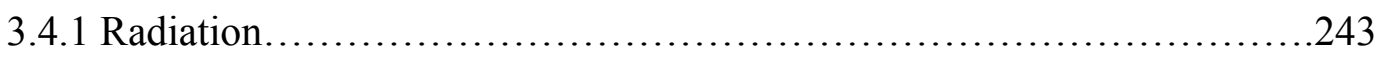

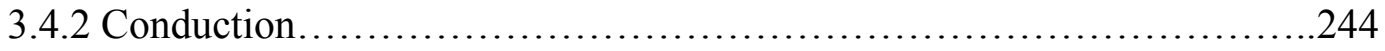

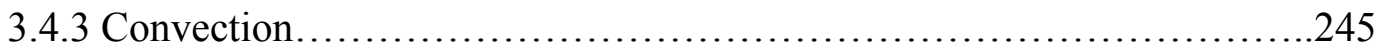

3.5 Application of Infrared Thermography to Rehabilitated Structures with Composite

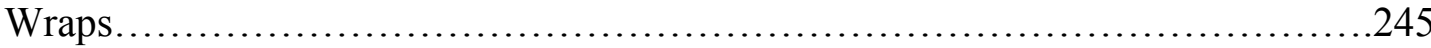

Chapter 4 - Laboratory Experiments..................................249

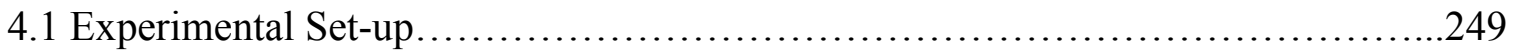

4.1.1 Heating Sources................................................... 250

4.1.1.1 Quartz Tower Heater.......................................250

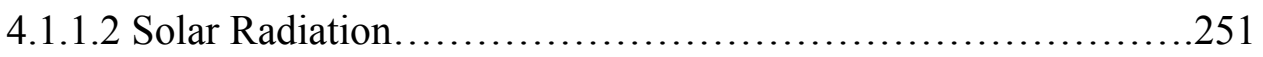




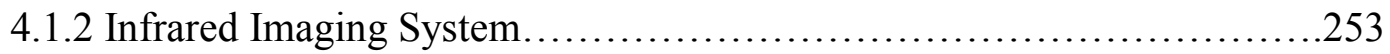

4.2 Specimen Set-up................................................. 255

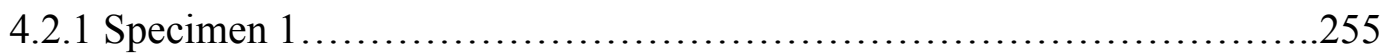

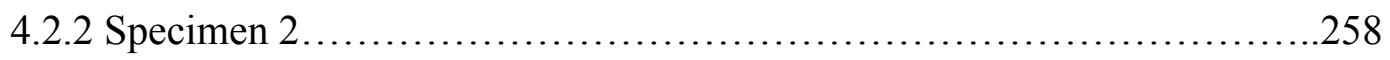

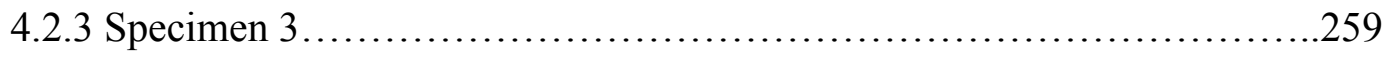

4.3 Experimental Results................................................ 260

4.3.1 Quartz Tower Heater..........................................260

4.3.2 Solar Radiation..............................................272

4.3.3 Greenhouse Effect................................................ 274

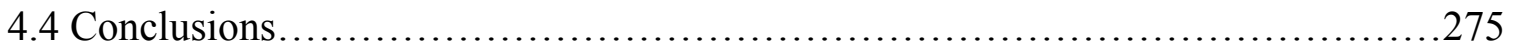

Chapter 5 - Field Study............................................277

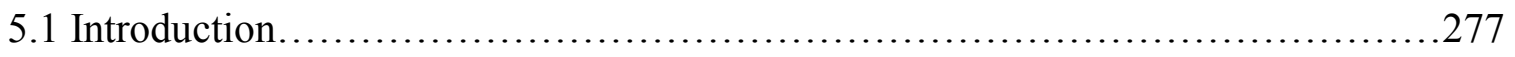

5.2 Description of Bridges...............................................280

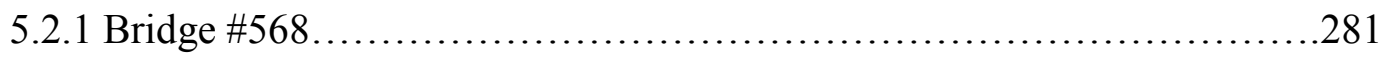

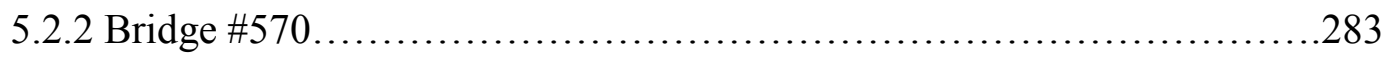

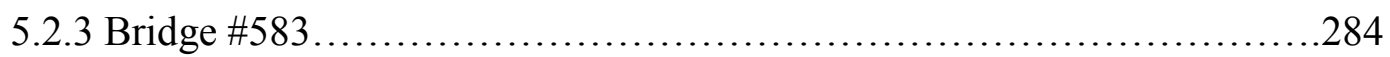

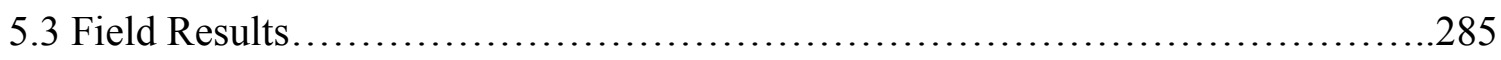

5.3.1 Equipment Set-up..........................................285

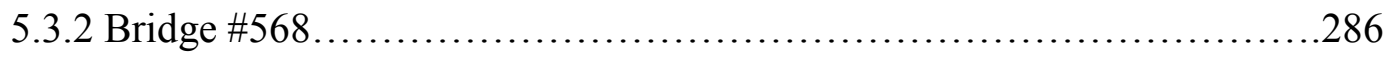

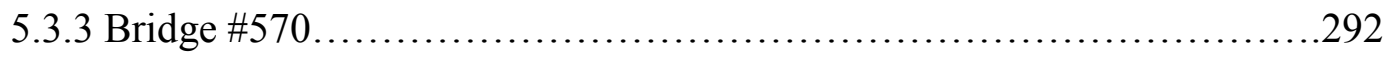

xviii 
5.4 Conclusions...

Chapter 6 - Conclusions and Recommendations......................300

6.1 Conclusions....................................................... 300

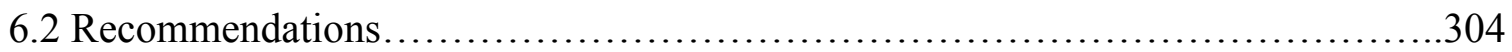

References.........................................................................306 


\section{List of Figures}

\section{Description}

Page No.

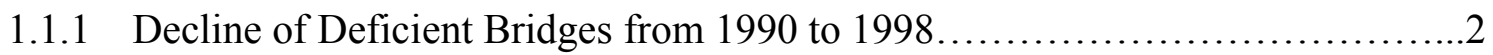

1.1.2 The Collapse of the Silver Bridge.........................................

2.1.1.1 Halogen lamp lock-in thermography test bed.............................13

2.1.1.2 Ultrasonic transducer lock-in thermography test bed........................ 14

2.1.1.3 Ultrasonic c-scan before (a) and after (b) thermal NDT $\ldots \ldots \ldots \ldots \ldots \ldots \ldots \ldots 17$

2.1.1.4 Lock-in halogen lamp phase image at $0.01 \mathrm{~Hz}$ modulation frequency and $25 \%$

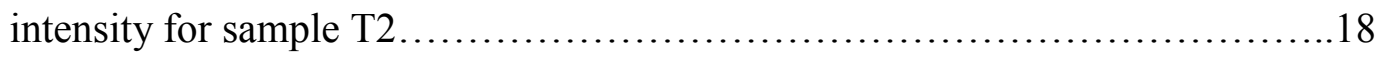

2.1.1.5 Lock-in ultrasonic transducer amplitude image at $0.01 \mathrm{~Hz}$ modulation frequency

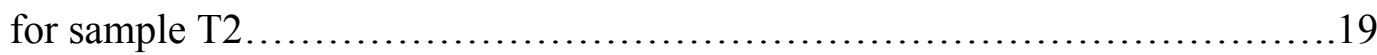

2.1.2.1 (a) Photograph of an area on one of the FRP wrapped columns, which had disbondment and voids (b) IR image of same area. Red colored areas indicates voids.

2.1.2.2 (a) Area with blisters, disbondment, and delaminations on one of the FRP wrapped columns, (b) IR thermography of the area marked with a square in (a) showing delamination, disbondment, and blistering. The scale on the left side of the image represents relative depth in inches............................26

2.1.3.1 Typical Active Thermography Setup...................................28

2.1.3.2 Test Specimen....................................................29

2.1.3.3 Experimental Setup..................................................30

2.1.3.4 1 and 2 Ply specimen after 60 s Heating................................... 31 
2.1.3.5 4 and 8 Ply Specimen after 150s Heating.

2.1.4.1 Thermographic inspection consists of 2 phases: (1) development of a temperature gradient and (2) image development.

2.1.4.2 Image of rectangular column wrapped with composite material (east face).

Extensive debond indications were seen along the corners of the overwrap.

Similar results were obtained from the tap testing.

2.1.4.3 Image of rectangular column wrapped with composite material (west face).

Extensive debond indications were noted at the edges of the wrap. Again, tap testing confirmed the results

2.1.4.4 Thermographic image of a cylindrical column retrofitted with a pre-cured shell showing small debond. .38

2.1.4.5 Thermographic images of a cylindrical column retrofitted with a pre-cured shell showing a large debond and a localized deep debond. ... .38

2.1.4.6 Core section of large debond discovered in prefabricated shell....

2.1.4.7 Sample thermographic image from an IR inspection with the imager located over $50 \mathrm{ft}$. from the column surface. Void indications with features of less than 2 inches are clearly visible in the outlined inspection area. The scan took less than one minute to perform. .40

2.1.5.1 Column Confinement Configuration. .44

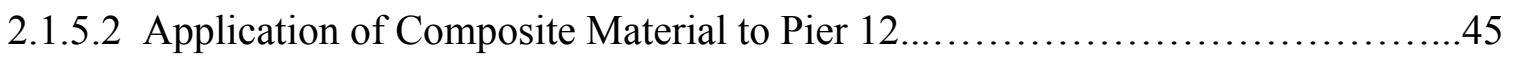

2.1.5.3 Thermal Image of a Disbond in Half-inch Thick Fiberglass Adhesively bonded to a Concrete Column. 
2.1.5.4 (a) Photograph of the area being tested, and (b) thermal image of the pultruded Graphite fiber strips shown in (a) ......................................51

2.1.5.5 Thermal Image of a 1 inch Square Simulated Defect in the Bondline of a 0.5 inch Thick Laminated Graphite/Epoxy Fabric................................52

2.1.5.6 Thermal Image of Delaminations in the Laminated Graphite/Epoxy Fabric......52

2.2.1.1 Experimental set up for measuring epicentral displacements..................58

2.2.1.2 Nd:YAG epicentral waveforms, (a) buried thermoelastic source and (b) above

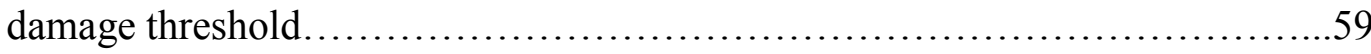

2.2.1.3 Longitudinal displacement versus laser fluence (energy of $6.2 \mathrm{~mJ}$ )............59

2.2.1.4 TEA $\mathrm{CO}_{2}$ epicentral waveforms, (a) buried thermoelastic source and (b) above damage threshold .......................................................60

2.2.1.5 Longitudinal displacement versus laser fluence for 9, 10, and $11 \mathrm{~mm}$ iris aperture

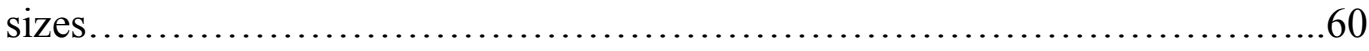

2.2.1.6 Epicentral waveforms generated at the absorption peaks for the CFRC.........61

2.2.1.7 Principle of an Optical Beam Deflection detector............................62

2.2.1.8 Off-epicentral OBD detection of bulk waves in CFRC plate..................63

2.2.1.9 $1 \mathrm{MHz}$ send receive air coupled ultrasound echoes from a CFRC plate...........64

2.2.1.10 $\mathrm{TEA} \mathrm{CO}_{2}$ generated-air coupled detected wave propagated through a $4 \mathrm{~mm}$ thick

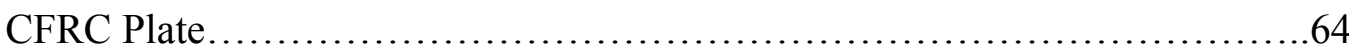

2.2.2.1 UT methods for inspecting co-cured structure............................67

2.2.2.2 Examples of the different inspection orientations that can be required to find flaws of different orientations. The images on the right are the corresponding $\mathrm{P} / \mathrm{E} \mathrm{UT}$ amplitude scans of joints containing cracks 
2.2.2.3 Examples of joint flaws found in co-cured structures.

2.2.2.4 Diagram of a co-cured joint, showing potential locations of flaws. Rotation of the transducer and identification of the flaw signal in time are necessary for locating and sizing of the flaws. .71

2.2.3.1 Configuration for through-thickness wave speed measurement test.............75

2.2.3.2 Sample through-thickness excitation and response............................76

2.2.3.3 Configuration for transverse vibration measurement test.......................77

2.2.3.4 (a) A sample transverse vibration response; (b) the corresponding frequency-

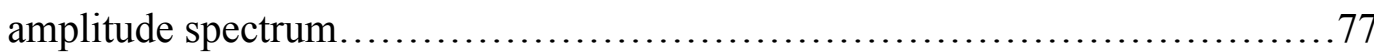

2.2.3.5 (a) Relationship between normalized through-thickness wave speed and impact energy; (b) relationship between residual strength and through-thickness wave

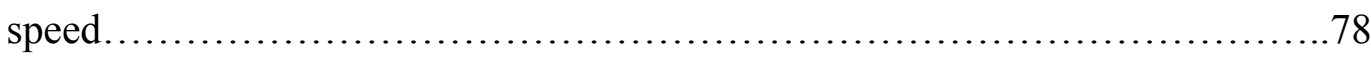

2.2.3.6 (a) Relationship between normalized mean-square amplitude and impact energy;

(b) relationship between residual strength and mean-square amplitude...........79

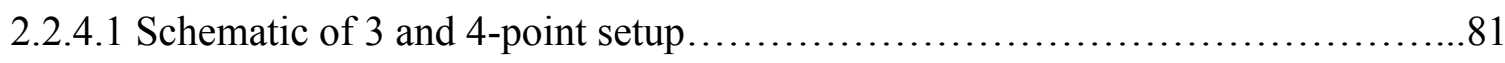

2.2.4.2 AE versus Strain (a) compression, and (b) tension..........................82

2.2.4.3 Acoustic-ultrasonic measurement on the compression flange during a four-point bend flexural fatigue test (a) parallel to the fiber direction, and (b) transverse to

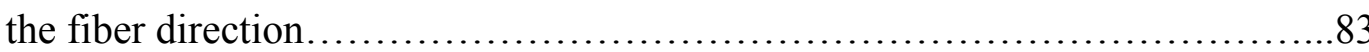

2.2.5.1 Ultrasonic pulse echo display for a 0.5 inch thick Lucite slab (top signal), a 0.5 inch thick GRP sample (middle signal), and a 0.25 inch thick GRP sample

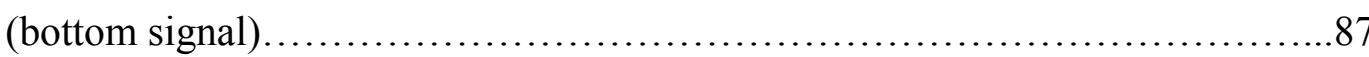


2.2.5.2 Ultrasonic pulse echo display for Lucite (bottom signal) and GRP sample (top signal). Both specimens were 1.0 inch thick................................ 88

2.2.5.3 Ultrasonic pulse echoes generated by a dual element transducer at $2.25 \mathrm{MHz}$. Uppermost trace was in Lucite, 0.25 inch thick; other traces, successively, were

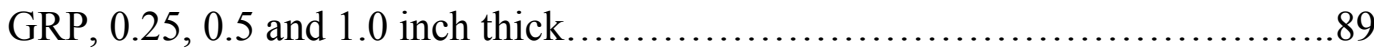

2.2.5.4 Ultrasonic pulse echo displays $(500 \mathrm{kHz})$ for a 0.375 inch diameter hole.........90

2.2.5.5 Microwave pulse echo patterns (mean frequency of $30 \mathrm{GHz}$ ) in GRP. Material thickness was 0.5 inch (top signal), 1 inch (middle signal), and 2 inches (bottom

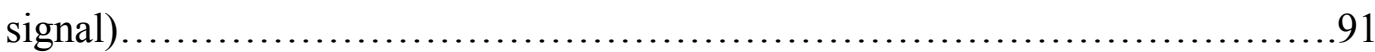

2.2.5.6 Microwave pulse echo patterns (mean frequency of $30 \mathrm{GHz}$ ) for the $0.375 \mathrm{inch}$ diameter hole. Top signal, drilled hole 0.75 inch from surface; bottom signal, drilled hole 1.25 inch from surface.......................................92

2.2.6.1 A view of the computer screen with the reflection spectra on the top and the accumulating dispersion curve on the bottom. The inverted elastic stiffness constants are shown on the left............................................

2.2.6.2 The measured dispersion curves of a unidirectional graphite-epoxy panel before

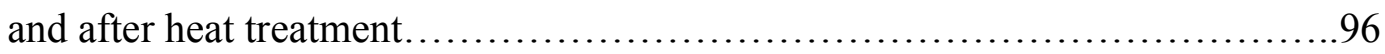

2.2.6.3 A schematic view of the rapid LLW test system............................97

2.2.6.4 Dispersion data for a defect free 16 layer unidirectional laminate...............98

2.2.6.5 The effect of porosity layer between the $8^{\text {th }}$ and $9^{\text {th }}$ layers....................98

2.2.6.6 Dispersion curves obtained at a delaminated area between the $8^{\text {th }}$ and $9^{\text {th }}$ layers.99

2.3.1.1 Sensor arrangement........................................................ 106

2.3.1.2 AE signals and their recovered wave sources...............................108

xxiv 
2.3.1.3 Signals of matrix cracking and their recovered wave sources.................109

2.3.1.4 Signals of fiber/fiber bundle breakage and recovered wave sources.............110

2.3.1.5 Signals of fiber pull-out and recovered wave sources......................111

2.3.2.1 Conceptual design: placement of bottom facesheet, wrapped cores, and top facesheet. The splice plates are adhesively attached on-site..................114

2.3.2.2 Southeast portion of grid pattern for upper deck surface $\ldots \ldots \ldots \ldots \ldots \ldots \ldots \ldots . \ldots 116$

2.3.2.3 Mode shape for full scale bridge section with (a) $18.6 \mathrm{~Hz}$ resonant frequency, (b) 45.0 Hz resonant frequency, (c) $76.6 \mathrm{~Hz}$ resonant frequency, (d) $90.5 \mathrm{~Hz}$ resonant frequency, (e) $107.51 \mathrm{~Hz}$ resonant frequency, (f) $117.3 \mathrm{~Hz}$ resonant frequency, (g) $146.83 \mathrm{~Hz}$ resonant frequency, and (h) $178.36 \mathrm{~Hz}$ resonant frequency.......120

2.4.1.1 Details of the FRP laminate strengthening system.........................126

2.4.1.2 Instrumentation Plan. (3a) Gage numbers and location, (3b) Gage locations on center beam .................................................... 127

2.4.1.3 Truck weights and configuration..................................... 128

2.4.1.4 Comparison of main rebar stresses: (a) and (b) Predicted Vs measured for a "Before" and "After" installation, (c) "Before" Vs "After"....................129

2.4.2.1 Proof load testing of the FRP superstructure..............................131

2.4.2.2 Locations of strain gages............................................. 133

2.4.2.2 Locations of strain gages.......................................... 135

2.5.1.1 "Tech-21" all composite Bridge........................................ 138

2.5.1.2 Cross-Section of the Bridge......................................... 138

2.5.1.3 Bragg grating strain sensor operation.................................139

2.5.1.4 Locations of the Bragg grating strain sensors.............................. 141 
2.5.1.5 Bragg grating sensor locations.......

2.5.1.6 Schematic of Bragg grating sensor system............................... 144

2.5.1.7 Schematic of chemical sensor operation............................... 145

2.5.1.8 Sapphire fiber sensor packaging .................................... 146

2.5.1.9 Layout of bondline sensor assemblies..............................146

2.5.1.10Bridge response - truck traveling east, right lane - January $1998 \ldots \ldots \ldots \ldots . .149$

2.5.1.11 Bridge response - truck traveling east, left lane, January $1998 \ldots \ldots \ldots \ldots \ldots . . . .150$

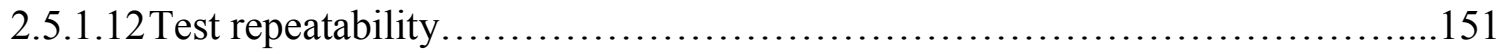

2.5.1.13 Voltage plot showing limited resolution.............................. 151

2.5.1.14(a) Bridge response - truck traveling east, right lane - August $1998 \ldots \ldots \ldots \ldots 153$

2.5.1.14(b) Bridge response - truck traveling east, right lane - August 1998

continued......................................................... 154

2.5.1.14(c) Bridge response - truck traveling east, right lane - August 1998

continued......................................................... 154

2.5.1.15(a) Bridge response - truck traveling east, left lane - August 1998.............155

2.5.1.15(b) Bridge response - truck traveling east, left lane - August 1998

continued......................................................... 155

2.5.1.15(c) Bridge response - truck traveling east, left lane - August 1998

continued........................................................ 156

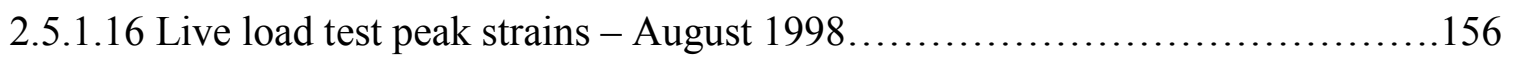

2.5.1.17(a) Comparison of conventional and fiber optic sensor results................157

2.5.1.17(b) Comparison of conventional and fiber optic sensor results continued........158

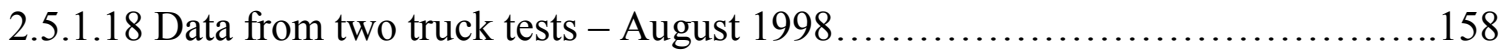

xxvi 
2.5.1.19 Comparison of single and two truck passes................................159

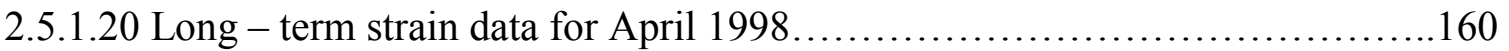

2.5.1.21 Long - term monitoring data - January to September $1998 \ldots \ldots \ldots \ldots \ldots \ldots \ldots . . . . .161$

2.5.1.22 Bondline chemical sensor data - east half...............................162

2.5.1.23 Bondline chemical sensor data - west half................................162

2.5.2.1 IPM application for optical fiber sensors.................................... 169

2.5.2.2 Geometry of conventional EFPI strain sensor..............................172

2.5.2.3 Variation of output intensity y (in arbitrary units) with changes in gap separations

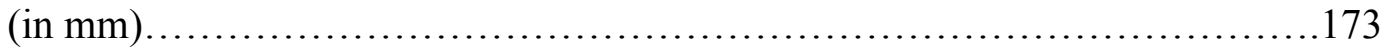

2.5.2.4 Modifications to EFPI sensor for cure state monitoring........................174

2.5.2.5 Reflectometer output as a function of refractive index.......................176

2.5.2.6 Output of reflectometer for curing 5-minute epoxy.........................177

2.5.2.7 Cure monitor sensor output (without air gap) for curing 5-minute epoxy.......177

2.5.2.8 Collimation of single mode fiber to increase sensor signal...................178

2.5.2.9 Output of collimated cure monitor for curing Devcon 5-minute $®$ epoxy........179

2.5.2.10 Schematic of the experimental arrangement used in the IPM of MCA

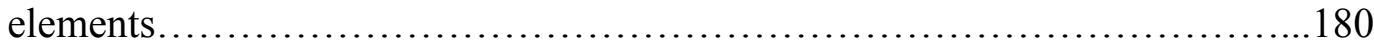

2.5.2.11 Fiber sensor data from MCA burnout tests............................... 181

2.5.2.12 Intrinsic Fabry - Perot interferometer design.............................. 182

2.5.3.1 (a) Schematic of the Luna strain, crack opening, and temperature sensor system.

(b) Modulated spectral output characteristic of absolute EFPIs.................187

2.5.3.2 Sensor response as the specimen was loaded to (a) $20,00 \mathrm{~N}$ at a rate of $2 \mathrm{~mm} / \mathrm{min}$,

(b) $30,000 \mathrm{~N}$ at a rate of $2 \mathrm{~mm} / \mathrm{min}$, and (c) $50,000 \mathrm{~N}$ at a rate of $4 \mathrm{~mm} / \mathrm{min} \ldots 188$ xxvii 
2.5.3.3 Plot of strain versus time during 3-point -bending of composite bridge deck...192

2.5.3.4 Stain vs. time data from (a) sensor 1 and (b) sensor $2 \ldots \ldots \ldots \ldots \ldots \ldots \ldots \ldots \ldots$

2.5.3.5 Stain vs. time data from (a) sensor 9 and (b) sensor $4 \ldots \ldots \ldots \ldots \ldots \ldots \ldots \ldots \ldots . \ldots \ldots$

2.5.3.6 Strain vs. time data from (a) sensor 5 and (b) sensor $6 \ldots \ldots \ldots \ldots \ldots \ldots \ldots \ldots \ldots$

2.5.3.7 Strain vs. time data from (a) sensor 7 and (b) sensor $8 \ldots \ldots \ldots \ldots \ldots \ldots \ldots \ldots . \ldots \ldots$

2.6.1.1 Schematic representation of the solid state laser and OCT system layout........199

2.6.1.2 OCT volumetric reconstruction of an epoxy/unidirectional E-glass composite..200

2.6.1.3 OCT volumetric reconstruction of an epoxy $/ 0-90^{\circ}$ woven composite...........200

2.6.1.4 OCT image of impact damaged epoxy/unidirectional E-glass composite at the surface .201

2.6.1.5 OCT image of impact damaged epoxy/unidirectional E-glass composite $337 \mu \mathrm{m}$ from surface .201

2.6.1.6 OCT image of impact damaged epoxy/unidirectional E-glass composite $652 \mu \mathrm{m}$ from surface .202

2.6.2.1 Schematic diagram of the acoustic transducer without shielding...............203

2.6.2.2 Schematic diagram of the remote acoustic Doppler NDT system................204

2.6.2.3 Velocity color map at a single frequency line..............................205

2.6.2.4 Three-dimensional representation of the analysis concept...................206

2.6.2.5 Seven defects in the parametric repair panel revealed.......................207

2.6.2.6 Detection of defect in carbon foam composite...............................207

2.6.3.1 Schematic of the optical head for the developed ESPI system.................209

2.6.3.2 Schematic of the developed ESPI System.................................211 
2.6.3.3 (a) Shear (slope) fringes for a defect free diaphragm, and (b) shear (slope) fringes obtained for the same specimen with fiber breakage.........................212

2.6.3.4 Slope fringes for circular diaphragm with defect size (a) $5 \mathrm{~mm}$, (b) $10 \mathrm{~mm}$, and (c) $15 \mathrm{~mm}$ .214

2.6.3.5 Slope profiles of defective GFRP circular diaphragm.......................215

2.6.5.1 Principle schematics of the laser optoacoustic sensors for detection in (a) "forward" signal propagation mode (LOAS-F), and (b) "backward" signal

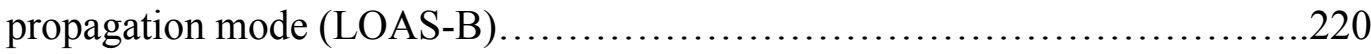

2.6.5.2 Ultrasonic frequency spectra of the acoustic attenuation coefficient measured in layered graphite-epoxy composite samples with different porosity $(<0.1 \%$ in sample \#1, $0.4 \%$ in sample \#2, and $1.2 \%$ in sample \#3).

2.6.5.3 Temporal profiles of ultrasonic pulses measured in backward detection mode for graphite composite samples with two different percentage of porosity $(<0.1 \%$ in

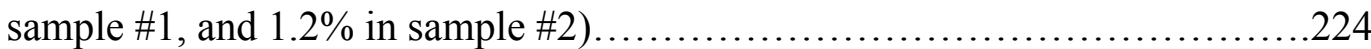

2.6.5.4 Measured and smoothed acoustic spectra of backscattered ultrasonic pulses, determined for the graphite composite sample with porosity of $1.2 \% \ldots \ldots \ldots \ldots .225$

2.6.6.1 Wide-band acoustic spectrometer with laser excitation of ultrasound (1- OA source, 2- investigated sample, 3- piezoelectric receiving transducer)...........228

2.6.6.2 Glass-fiber-reinforced composite sample. A - unloaded section, B - statically

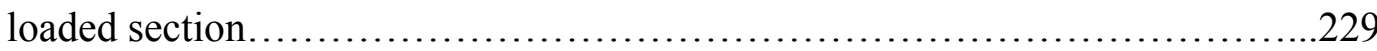

2.6.6.3 Static loading curve for composite samples..................................229 
2.6.6.4 Frequency dependencies of ultrasound attenuation coefficient in green-state composite samples. Signal A - unloaded section, and signal B - statically loaded

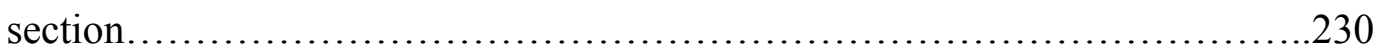

2.6.6.5 Frequency dependencies of ultrasound attenuation coefficient in composite samples after service life testing. Signal A - unloaded section, signal B statically loaded section, and signal $\mathrm{C}$ - difference of attenuation spectra in

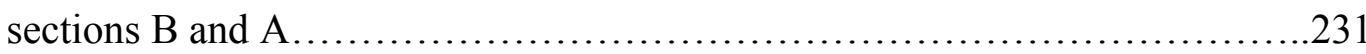

2.6.6.6 Frequency dependence of ultrasound attenuation coefficient in a composite sample after service life testing (fatigue testing). Signal A - unloaded section,

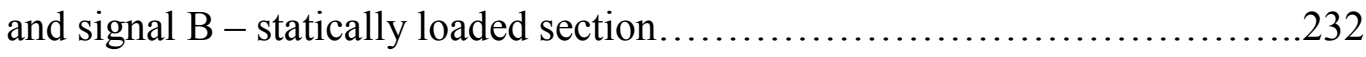

2.6.6.7 Qualitative classification of composite samples according to the aging.........233

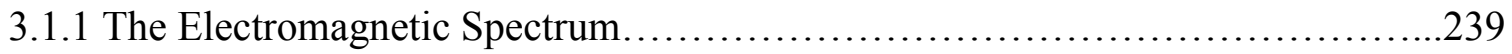

3.3.1 variation of energy per interval of wavelength at different temperatures...........243

3.5.1 Illustration of how a defect affects heat flow..................................246

3.5.2 Field data for temperature differences between solid and delaminated area in bridge deck. .247

4.1.1 The two phases of infrared thermography: (a) creating thermal gradients with a heat source, and (b) capturing the thermal gradients with an infrared imaging device..249 4.1.2 A photograph of the quartz tower heater used to heat the specimens.............250

4.1.3 Specimens heated by solar radiation.......................................251

4.1.4 Simulated greenhouse created from clear plastic............................252

4.1.5 Experimental set-up using the quartz heater to heat specimens.................254 
4.1.6 Experimental set-up using the sunlight and a simulated greenhouse to heat the

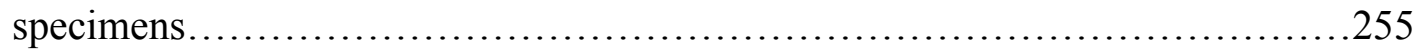

4.2.1 Cordless saw and screwdriver used to remove composite wrap................256

4.2.2 Cordless screwdriver with a 1-1/2 inch drill bit used to create delaminations......257

4.2.3 A thin plastic film was used to cover the pockets to prevent them from filling with resin .257

4.2.4 Photograph of the specimen with the composite wrap reapplied and duct tape

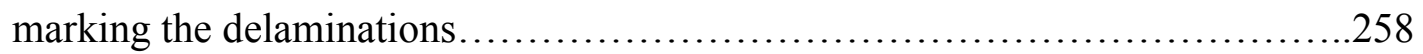

4.2.5 Creating a delamination by prying composite wrap away from timber surface....259

4.3.1 (a) Photograph of the surface where the first simulated delamination was placed on specimen 1, and (b) thermal image of the first simulated delamination detected by

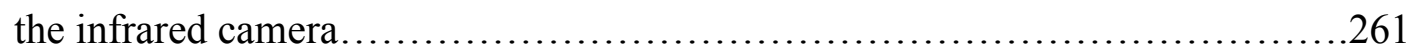

4.3.2 (a) Photograph of the surface where the second simulated delamination was placed on specimen 1, and (b) thermal image of the second simulated delamination

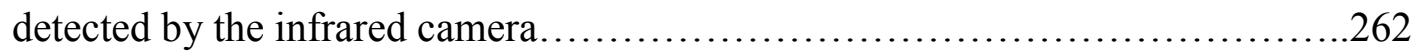

4.3.3 (a) Photograph of the surface between the first and second delamination on specimen 1, and (b) thermal image of the bonded area between the first and second delamination .263

4.3.4 First and second delamination on specimen 1 (marked with duct tape) being heated by the quartz tower heater. .264

4.3.5 (a) Photograph of the surface where the third simulated delamination was placed on specimen 1, and (b) thermal image of the third simulated delamination detected by the infrared camera. .265 
4.3.6 (a) Photograph of the surface where the fourth simulated delamination was placed on specimen 1, and (b) thermal image of the fourth simulated delamination detected

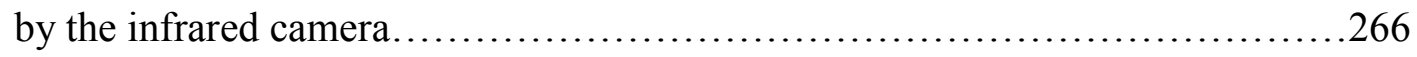

4.3.7 (a) Photograph of the surface between the third and fourth delamination on specimen 1, and (b) thermal image of the bonded area between the third and fourth delamination. .267

4.3.8 Third and fourth delamination on specimen 1 (marked with duct tape) being heated by the quartz tower heater..... .268

4.3.9 (a) Photograph of the surface where the simulated delamination was placed on specimen 2, and (b) thermal image of the simulated delamination detected by the infrared camera. .269

4.3.10 (a) Photograph of the surface where no simulated delaminations were detected (specimen 3), and (b) thermal image of the bonded area with no defects or delaminations .271

4.3.11 (a) Photograph of specimen 1 and 2, and (b) thermal image of specimen 1 and 2 using solar radiation as the heat source. .273

4.3.12 Thermal image of specimen 1 and specimen 2 using the greenhouse effect and solar radiation as the heat source. .275

5.1.1 (a) A pile cap from bridge \#568 wrapped with GFRP composite wrap, (b) a timber pile from bridge \#570 wrapped with GFRP composite wrap, (c) a stringer from bridge \#583 wrapped with GFRP composite wrap ...........................279

5.2.1 Location of Moorefield, West Virginia......................................281

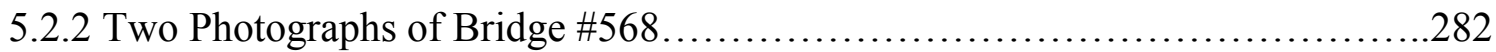




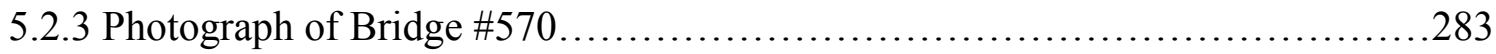

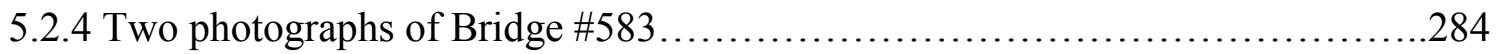

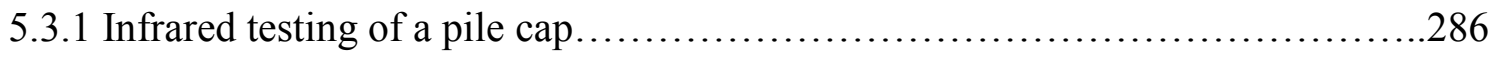

5.3.2 (a) Photograph of the pile cap section before being wrapped in plastic wrap, and (b) thermal image of the pile cap section after the plastic wrap was removed........288

5.3.3 The section of the pile cap wrapped in plastic wrap to create the greenhouse

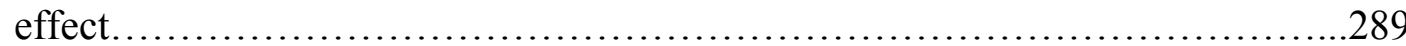

5.3.4 (a) Photograph of the pile cap section with the observed black spot, and (b) thermal image of the pile cap section that was wrapped in plastic wrap. The black spot could be caused by a metal nail or bolt under the GFRP wrap........................290

5.3.5 (a) Photograph of a stringer with a bolt going through it, (b) thermal image of the stringer. The dark area by the cross hairs represents the bolt.... .291

5.3.6 Different sections of the stringer heated by plastic wrap and a quartz tower heater. .293

5.3.7 (a) Photograph the stringer section heated with the quartz tower heater, (b) thermal image of the stringer with the quartz tower heater placed 2 inches away, (c) 4 inches away, and (d) 8 inches away from the stringer surface. .295

5.3.8 (a) A photograph of the stringer's surface, and (b) thermal image of the stringer's surface detecting significant surface temperature differentials. .296

5.3.9 (a) Photograph of the stringer's surface, and (b) thermal image of the stringer's surface that was non-uniformly heated .298 


\section{List of Tables}

$\underline{\text { Description }}$

Page No.

2.1.1.1 Impact damage sample data........................................... 16

2.3.2.1 Natural Frequencies and Corrected Viscous Damping Ratios for Full Composite

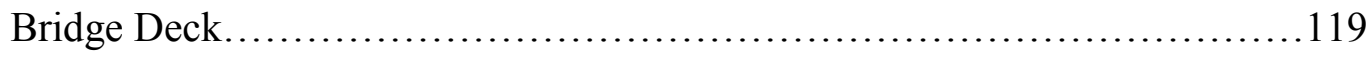

2.4.1.1 Before and After live load distribution factors for Beam 11_.................129

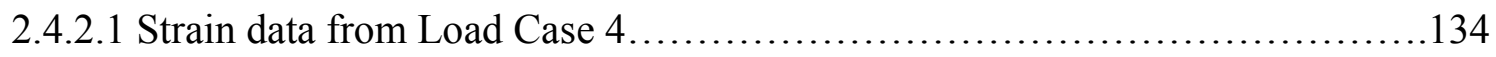

2.5.11 Bragg grating strain sensor system.................................... 164

2.6.5.1 Parameters of the evaluated samples of graphite-epoxy composite material....222 


\section{CHAPTER 1}

\section{INTRODUCTION}

\subsection{Background}

The nation's transportation system, which includes highways and bridges in many forms, touches our lives every day. The continued economic strength and growth of the United States is mainly dependent on the strength and reliability of our highways and bridges. The nation spends at least $\$ 5$ billion per year for bridge design, construction, replacement, and rehabilitation (USDOT FHWA Annual editions 1999). Of the 583,000 bridges in operation in 1997, about 30 percent were either structurally deficient or functionally obsolete. Although the percent of deficient bridges has declined from over 40 percent in 1990 to 30 percent in 1997 (illustrated in Figure 1.1.1), the amount of expenditures continues to rise (USDOT FHWA 1998b). Therefore, it is up to the engineers to develop new high performance and innovative materials to be used for the construction and rehabilitation of bridges. One such promising new material is fiber reinforced polymer (FRP) composite. With these new materials, comes the need to develop new nondestructive testing (NDT) methods for evaluating the service-life performance and quality of FRP composite based structures. 
Bridge Conditions: 1992-1998

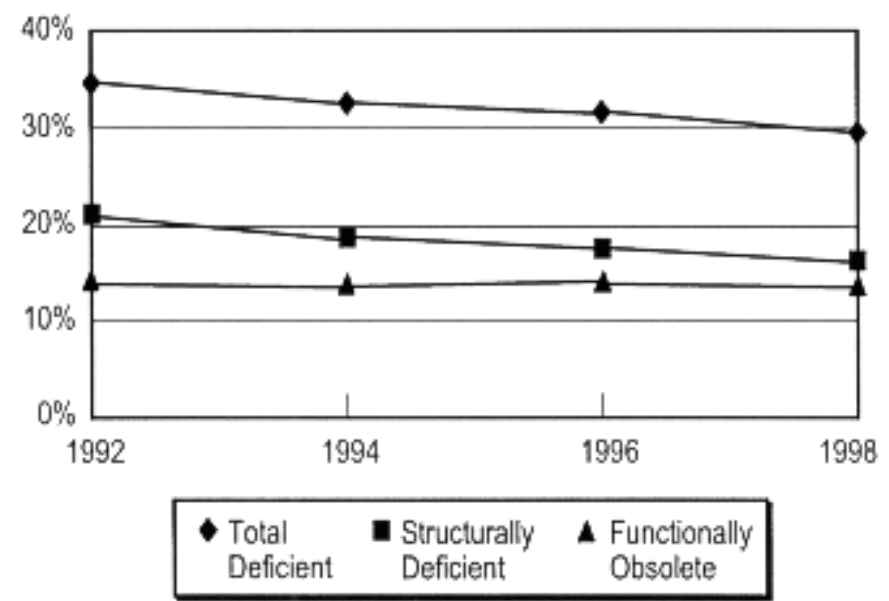

Figure 1.1.1 Decline of Deficient Bridges from 1990 to 1998 (USDOT FHWA 1998b)

The need to carefully monitor the condition of bridges became apparent after the collapse of the Silver Bridge between Point Pleasant, WV and Gallipolis, OH in 1967 (Figure 1.1.2). The loss of 46 lives due to a stress corrosion failure of an eyebar link caused great concern about the safety of bridges. The cost of this disaster was 175 million dollars, in 1967 dollars. In this case and many other cases, improved inspection technology such as infrared thermographies, ultrasonic, fiber optics, etc. would have allowed the problems to be identified before the disaster occurrence. Although the consequences of ineffective bridge inspection are usually not as severe as the Silver Bridge, repair and retrofit costs on bridges represent a very significant portion of the transportation budget. Timely maintenance and rehabilitation utilizing improved inspection technologies can go a long way in reducing repair and retrofit costs and at the same time improve safety and serviceability of the structure. 


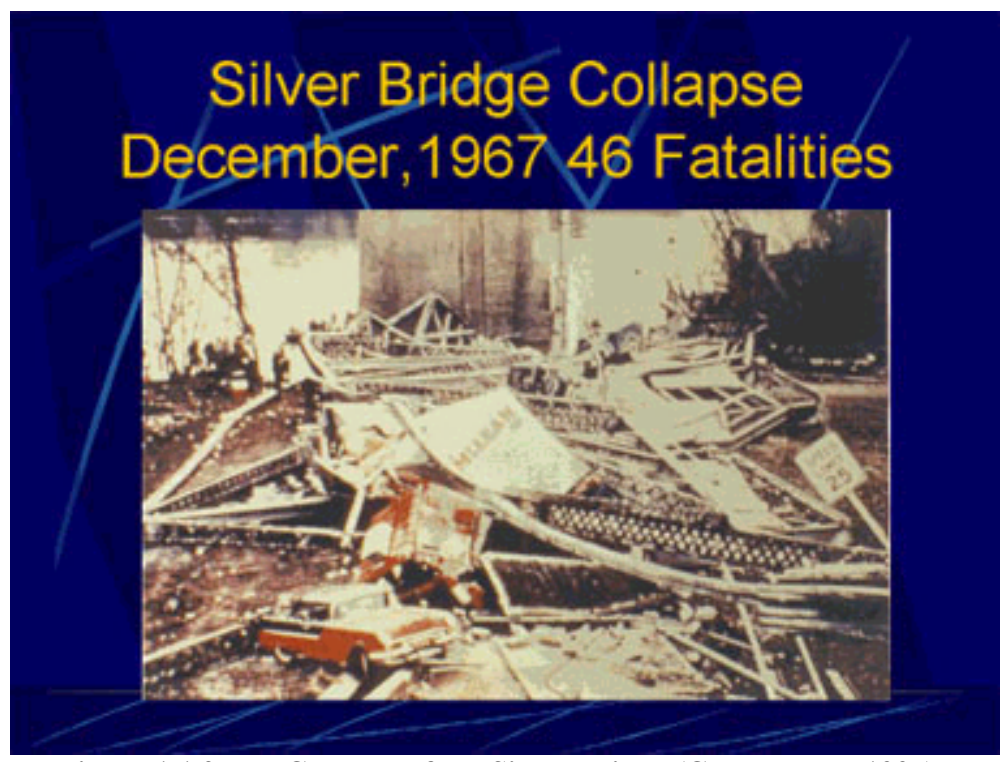

Figure 1.1.2 The Collapse of the Silver Bridge (Cooper et al. 1995)

Recent studies show that the average life span of a bridge is about 70 years and typically, significant rehabilitation is needed when a bridge reaches mid-life. As the Country's bridges age, deteriorated structures pose safety as well as potential liability concerns. In the future, replacement of a bridge will become an increasingly unattractive alternative. Construction cost growth, and disruption of traffic during a total bridge replacement will force life extension to be the only viable alternative for many of our aging bridges. Composite materials have proven to be very useful in the rehabilitation of old structures. Some of the advantages in using fiber reinforced polymer (FRP) composites for bridge rehabilitation include higher strength and stiffness to weight ratio, better fatigue resistance, and slower-rate of deterioration, than conventional materials. Because of these advantages, FRP composites are now being used in the construction of several bridges in West Virginia (CFC 2001) as well as other states. 
The FRPs are being used also by the U.S railroad industry to rehabilitate timber bridges. Nationwide, nearly 50 percent of existing timber bridges are classified as structurally deficient (USDOT-BTS 1998). Because of the increased train axle load capacity, speed and aging of creosote-treated timber components, FRP composite wraps are being used to reinforce the structural elements.

The use of this new high performance alternative composite material for highway and railroad structures requires that new types of field testing and evaluation methods be developed. Major research is being conducted on nondestructive evaluation (NDE) techniques for condition assessment of structural members reinforced with composite wraps and all-composite structural members. Effective application of improved NDE techniques will more reliably determine the condition of these structures and eliminate the need for frequent re-inspection of the structures. Also, more timely repairs can favorably reduce the overall repairs and/or rehabilitation costs. Infrared thermography has been emerging as the more promising NDE technique for field evaluation of structures wrapped in composite materials. This is because the infrared equipment is portable and easy to use, and the data interpretation is relatively easy compared to many other NDE techniques.

The infrared thermography technique consists of two phases. First, one needs to generate a thermal gradient through the thickness of the structure being tested. Then, the surface temperature of the structure is monitored as it returns to thermal equilibrium. The thermal gradient can be established through the use of a heating source (heat gun, heating 
blanket, solar heating, etc.) or a cooling method can be used. The thermal images are then created using an infrared camera to generate a temperature profile of the surface being tested. Subsurface defects affect the surface temperature because of their thermal conductivity differences; therefore, they can be observed in the thermal images.

This research focuses on detecting defects (debonds, delaminations, etc.) below the composite surface (FRP wraps used for retrofits) using infrared thermography.

\subsection{Research Objectives}

The objectives of this research are to:

1. Review the literature on the various NDE techniques used for testing composite materials and structures. The review deals with NDE techniques such as infrared thermography, ultrasonic wave propagation, acoustic wave propagation, microwave wave propagation, use of fiber optics, and other methods being experimented by the aerospace industry. Parameters, advantages, disadvantages, and reliability will all be considered in the review.

2. Study the ability of detecting sub-surface anomalies (e.g. debonds and delaminations) using infrared thermography on timber components with composite wrap under controlled laboratory conditions. Two methods of creating thermal gradients will be experimented with. Using plastic wrap and solar heat to create a green house effect to heat timber piles wrapped with FRP composites will be demonstrated. The development of a temperature gradient on timber piles will also be performed using a 
1500 Watt heater. The various timber piles include different delamination sizes and thickness.

3. Conduct infrared field testing on three timber bridges with timber components wrapped with FRP composites to detect delaminations at the composite-timber interface and within the composite itself. The two heating methods and their application in the field will be demonstrated.

\subsection{Scope}

The scope of this research involves the study of different heating methods to be used with the infrared thermography technique to detect delaminations in timber piles with composite wrap. The scope also includes using infrared thermography for field testing of timber components of three railroad bridges to detect delaminations between the composite-timber interface and within the composite wrap itself. The three bridges are part of WVDOT - South Branch Valley Railroad located in Moorefield, West Virginia. The field testing was conducted to demonstrate the effect of environmental conditions on infrared thermography.

\subsection{Organization}

This thesis consists of six chapters and a list of references. The first chapter presents an introduction to infrared thermography and its needed to evaluate highway and railroad infrastructures. Chapter 1 also outlines the objectives and scope of this research. Chapter 2 presents an extensive literature review on various NDE techniques used to 
evaluate composite materials with focus on the infrared thermography technique. The application, technique, and basic theory of infrared thermography are discussed in Chapter 3. The next chapter, Chapter 4, gives a detailed description of the experimental set-up and fabricated specimens. This chapter includes a description of the equipment used as well as the results obtained. Chapter 5 discusses the results obtained during field testing. This chapter includes a detailed description of the bridges tested and the equipment used. The last chapter, Chapter 6, presents conclusions on infrared thermography and also recommendations for future research on infrared thermography. 


\section{CHAPTER 2}

\section{LITERATURE REVIEW OF NDE TECHNIQUES FOR EVALUATING COMPOSITE MATERIALS}

This chapter presents a review of the different types of nondestructive evaluation (NDE) techniques associated with testing composite members (focusing on composite bridges and bridge structures rehabilitated with composite wraps). The various NDE techniques associated with evaluating composite materials (used in aerospace and civil engineering applications) will be reviewed in this chapter. The review emphasizes the NDE techniques that could be applied to civil structures. These techniques are infrared thermography (IR), ground penetrating radar (GRP), wave propagation (ultrasonic, acoustic, and microwave), and fiber optics. The advantages and disadvantages, parameters of interest, and data from previous NDE applications will be discussed in this chapter.

\subsection{Infrared Thermography Applications}

This section discusses different techniques of infrared thermography that were used to evaluate composite materials; i.e., lock-in ultrasonic thermography, halogen lamp thermography, active thermography, and passive thermography. Infrared thermography has been used to evaluate bridge structures as well as composite aircraft components. 


\subsubsection{Rapid NDT of Composite Aircraft Components Using Lock-In Ultrasonic and Halogen Lamp Thermography (Bates et al. 2000)}

The rapid NDT of composite aircraft components has directly influenced the NDT of composite infrastructures. Although, the techniques talked about in this section are used on composite aircraft components the methods may be helpful to evaluate other structures made from composite materials. This section describes two thermal techniques for the rapid in-service inspection of impact damaged composite aircraft components. The two techniques discussed are the lock-in halogen lamp technique and the newly developed lock-in ultrasonic transducer technique. These two techniques were experimented with by using samples prepared to simulate Barely Visible Impact Damage (BVID) in composite laminates to represent material flaws in the in-service environment. It was concluded that the ultrasonic lock-in thermography was a better technique for detecting BVIDs than the halogen lamp lock-in thermography technique and up to ten times quicker than underwater ultrasonic c-scanning (Bates et al. 2000).

\subsubsection{Introduction}

The speed of in-service NDT, because of time and economics, has become a major factor in determining the best method to use for the evaluation of composite materials. Some of the other competing rapid NDT systems include thermography, laser ultrasonics, shearography and visual methods. This section focuses on two thermography techniques for the rapid inspection of glass fiber thermoplastic composite aircraft components. 
The lock-in halogen lamp and lock-in ultrasonic transducer thermography techniques were investigated by preparing samples to simulate BVID (Barely Visible Impact Damage) in glass fiber reinforced thermoplastic and then inspected using these two techniques.

\subsubsection{Thermal Non-Destructive Testing Techniques}

Thermal NDT works by capturing transient thermal images from the sample under inspection, which produces thermal waves within the sample from heat energy. When a plane thermal wave entering a sample passes through a material imperfection it is disrupted and some of its energy is reflected back to the surface. This reflection may cause time delays between the emergence of heat patterns from different damaged sites on the surface of the sample, which can be observed using a thermal camera.

Thermal inspection requires a heat source to heat the sample or a cooling source to cool the sample being inspected. Many different heat sources can be used. The halogen lamp transient thermography typically heats the sample using a short pulse of $3 \mathrm{~s}$ duration whereas lock-in halogen lamp thermography reported in this section uses a continuous harmonically modulated heating source. Other methods reported in recent literature have included induction heating and ultrasonic heating which use either a water bath or a transducer to heat the sample (Bates et al. 2000). 
Halogen lamp and ultrasonic transducer lock-in thermography are presently two of the leading thermal NDT techniques (Bates et al. 2000). Applying the heat to the sample is the main difference in the two methods.

\subsection{Lock-in Thermography}

Lock-in thermography uses a harmonically modulated heating source to generate sinusoidal thermal waves in the frequency range 0.001 to $2 \mathrm{~Hz}$ to create heat inside the sample. To improve the temperature measurement and final image, a computer synchronizes the infrared image captured and external thermal source. Phase and amplitude images of the thermal wave (within the sample) are generated from computer software by acquiring one image for every quarter of the modulation period. These phase and amplitude images can be used to identify and locate material imperfections in the manufacturing process and in-service environments.

The thermal diffusion length can be derived from the formula of thermal wave propagation (Bates et al. 2000). The thermal diffusion length, which is the relevant parameter for the successful use of lock-in NDT, is given by:

$$
\mu=\sqrt{ }(2 \mathrm{k} / \omega \rho c) \text { (Bates et al. 2000) }
$$

where,

$$
\begin{aligned}
& \mathrm{k}=\text { thermal conductivity }(\mathrm{W} / \mathrm{mK}) \\
& \omega=\text { Angular modulation frequency of the heat source }(\mathrm{rad} / \mathrm{s}) \\
& \rho=\text { Density of the material }\left(\mathrm{Kg} / \mathrm{m}^{3}\right) \\
& \mathrm{c}=\text { Specific heat capacity }(\mathrm{J} / \mathrm{Kg} \cdot \mathrm{K})
\end{aligned}
$$


The thermal diffusion length provides an indication of the depth to which a thermal wave technique will be effective, making it a significant parameter in the use of lock-in TNDT. The thermal diffusion length can be adjusted by changing the modulation frequency making this and the modulation intensity the relevant experimental parameters for lock-in thermography using halogen lamp heating. Both of these parameters were investigated fully during the experiments.

Very low modulation frequencies required to penetrate deep into the sample took more than 25 minutes, which is an unacceptable time period when using rapid thermal NDT. Using higher modulation frequencies such as $0.01 \mathrm{~Hz}, 0.05 \mathrm{~Hz}, 0.10 \mathrm{~Hz}, 0.15 \mathrm{~Hz}$, $0.20 \mathrm{~Hz}, 0.25 \mathrm{~Hz}$ produced rapid thermal scans in 40 seconds.

To reduce the effect of variation in surface emissivity, which in turn reduces the risk of misidentification of material imperfections through external reflections, ultrasonic transducers or halogen lamps are used for complete heating the component.

\subsection{Lock-in Halogen Lamp Heating}

The lock-in halogen lamp testing setup that was used in these experiments is illustrated in Figure 2.1.1.1. This experimental configuration was used for one-sided investigations. The modulated thermal source used was a lock-in amplifier and signal generator, which were synchronized with the thermal image capture. The phase image, which measures the relative phase between the incident heat radiation on the samples' surface and the reflected thermal waves from a defect, was used to determine the depth of 
internal flaws. To determine flaws at greater depths lower modulation frequencies have to be used, with modulation frequencies as low as $0.007 \mathrm{~Hz}$ being used to measure defects at depths of up to $2 \mathrm{~mm}$ in carbon fiber composites.

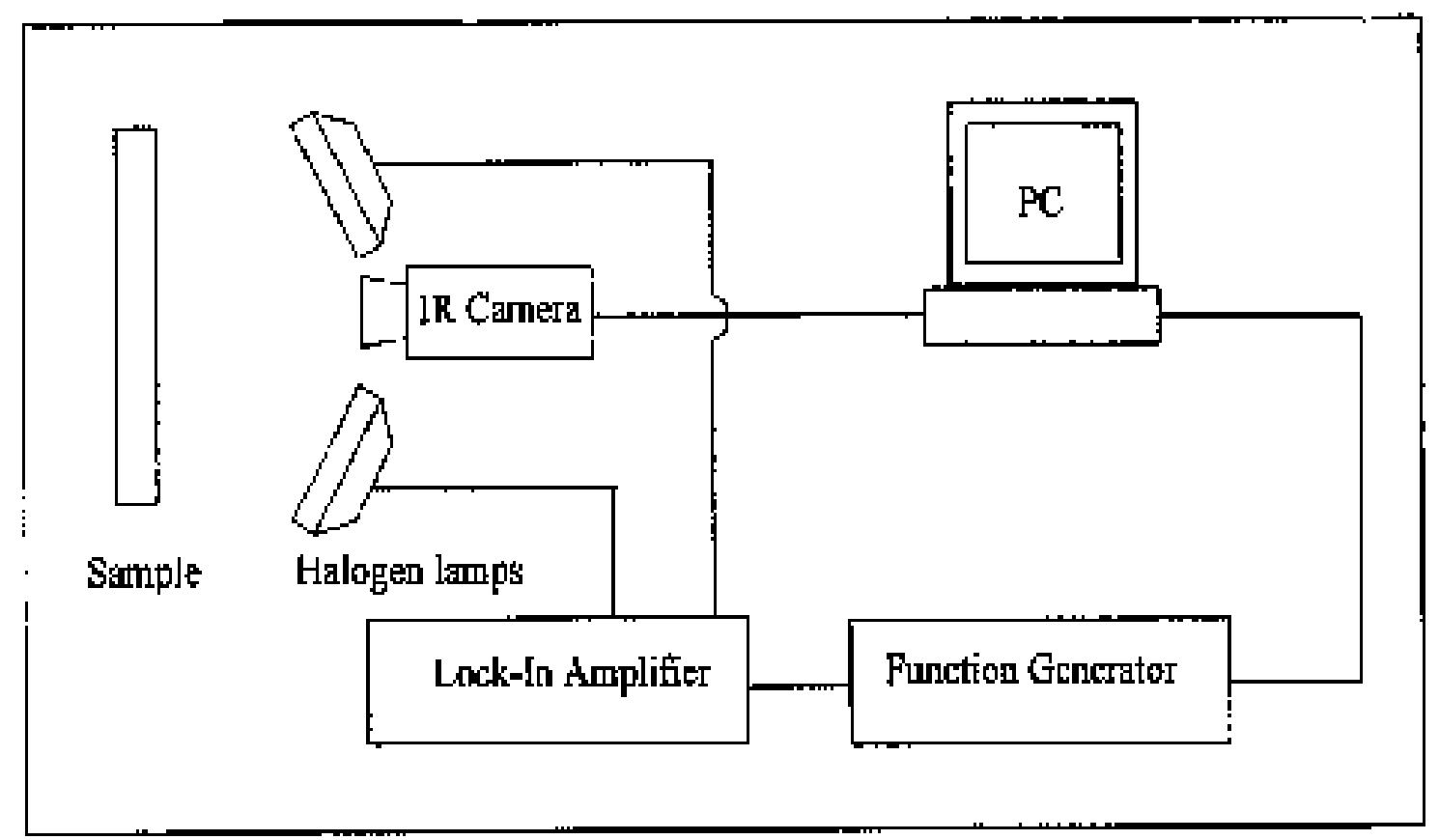

Figure 2.1.1.1 Halogen lamp lock-in thermography test bed (Bates et al. 2000)

The total intensity of the lamps was 2000 Watts; the percentage of the intensity was decreased from $100 \%$ to $25 \%$ as the distance between the lamps and the sample was increased.

\subsection{Lock-in Ultrasonic Transducer Heating}

High power ultrasonic energy can be describes as a low amplitude and high frequency mechanical vibration. The ultrasonic method has high hysteresis loss in the impact damaged regions causing an increased heating of the defective region. Therefore, the defect itself emits a thermal wave, which can be can be observed using an infrared camera. When ultrasonic heating is used over traditional mechanical vibrations, a large 
thermal signal is obtained at lower stress levels in the component ensuring that the technique is truly non-destructive. There is also a smaller signal attenuation allowing defects at greater depths to be detected.

The lock-in ultrasonic transducer test apparatus is presented in Figure 2.1.1.2. The ultrasonic transducer was attached to the sample so that it was outside the field of view of the thermal image. The transducer and amplifier operated at $40 \mathrm{kHz}$ with a maximum power output of 700 Watts. The amplitude modulated over a range of $0.01-$ $10 \mathrm{~Hz}$

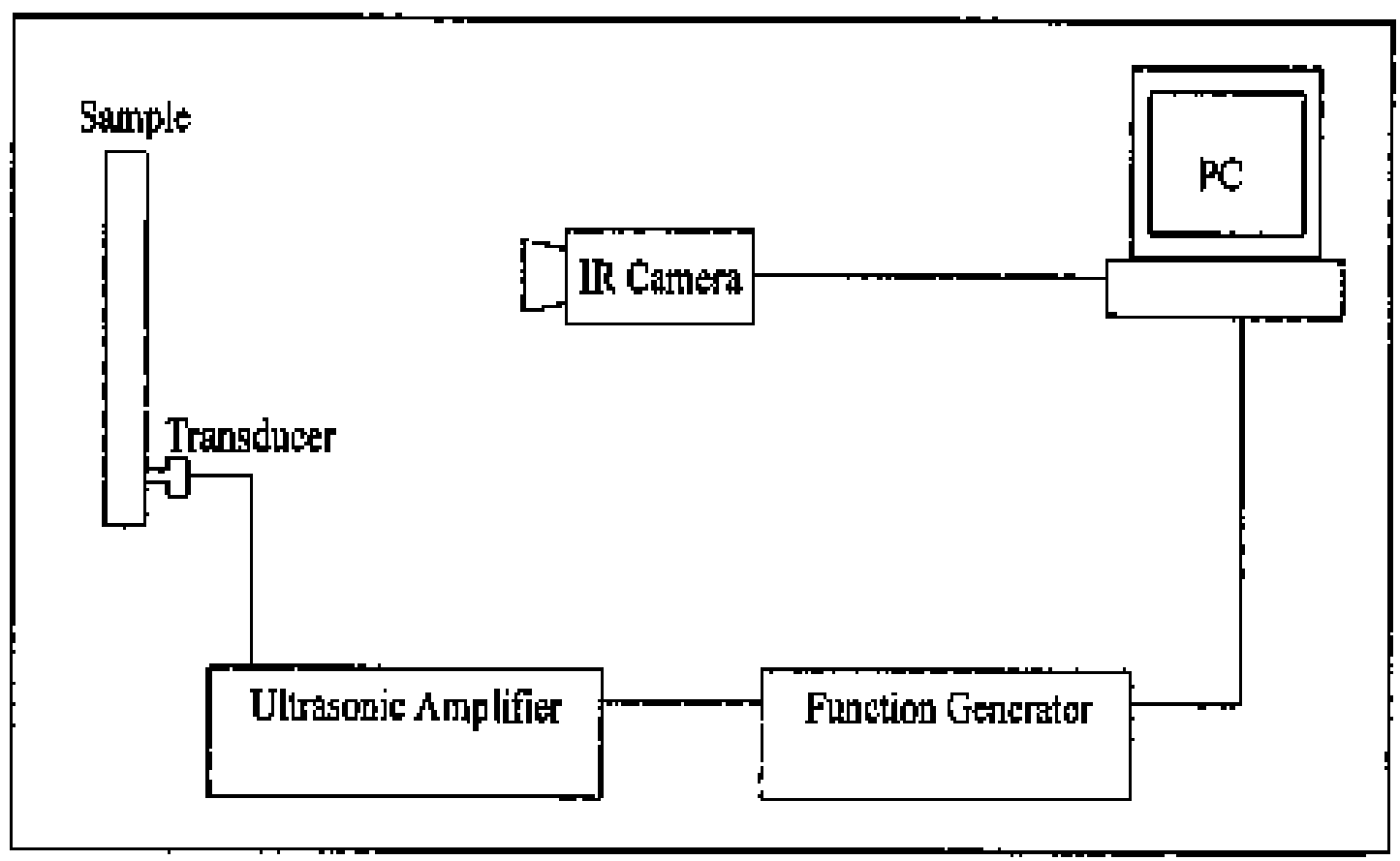

Figure 2.1.1.2 Ultrasonic transducer lock-in thermography test bed (Bates et al. 2000)

Ultrasonic energy entered the sample through mechanical coupling, which caused the entire component to start oscillating through material lattice vibrations known as phonons. The defected areas release the ultrasonic energy in the form of thermal energy 
at a higher rate than the non-damaged areas. The different temperatures are then seen using the thermal camera.

\subsubsection{Sample Preparation}

When preparing samples with faults, which occur in real life composite components, different factors need to be considered, which include surface emissivity variations, accurate fault representation and component curvature or geometry. To eliminate spurious reflections from radiant emitters such as lights or human bodies, the samples were painted with a thin matt black coating with an emissivity value of 0.92 .

To simulate the low velocity impact damage of interest, a drop weight impact machine produced impact velocities less than $3 \mathrm{~m} / \mathrm{s}$, which is usually the velocity that causes low impact damage. This produced samples for testing whether the thermal NDT techniques had the capability to detect such small damage. The Barely Visible Impact Damage (BVID) was produced from a single strike of the impact head using an instrumented impact-testing machine. Six specimens ranging in thickness from $1.133 \mathrm{~mm}$ to $1.613 \mathrm{~mm}$ with induced BVIDs ranging in dent size from $.315 \mathrm{~mm}$ to $.89 \mathrm{~mm}$ were tested. Table 2.1.1.1 gives the sample thickness with their damage parameters. The impact head of the testing machine was $16 \mathrm{~mm}$ in diameter and $15.6 \mathrm{Kg}$ in mass. All samples were simply supported to ensure consistency in the sample preparation. 
Table 2.1.1.1 Impact damage sample data (Bates et al. 2000)

\begin{tabular}{|c|c|c|c|c|c|c|}
\hline Specimen & T1 & T2 & T3 & T7 & T8 & T9 \\
\hline Thickness $(\mathbf{m m})$ & 1.133 & 1.140 & 1.123 & 1.600 & 1.653 & 1.613 \\
\hline Impact Energy $(\mathrm{J})$ & $\mathbf{1 0 . 7 2}$ & 8.27 & 5.52 & $\mathbf{8 . 6 2}$ & 7.87 & 5.56 \\
\hline Impact Velocity $\left(\mathbf{m} \mathrm{s}^{-1}\right)$ & 1.17 & 1.03 & 0.84 & 1.17 & 1.00 & 0.84 \\
\hline Dent (mm) & $\mathbf{0 . 3 8}$ & 0.315 & 0.89 & 0.455 & 0.315 & 0.330 \\
\hline
\end{tabular}

\subsubsection{Experimental Apparatus}

For the thermal NDT experiments described in section 2.4 a Cedip IRC-128MWIR infrared focal plane array camera was used. This camera captures radiation in the spectral band $3-5 \mu \mathrm{m}$. In most typical cases damage would occur where access is only available from one side, therefore a one-sided inspection method was used for all experimental trials.

The thermal images captured by the infrared camera were compared to the images captured from a USL underwater ultrasonic c-scan system. The c-scan technique was used for comparison because it was able to detect and quantify delamination damage in all thickness of composites since it is a more powerful technique.

\subsubsection{Results and Discussion}

The results presented in this section compare c-scan images of signal attenuation and thermal images from both lock-in halogen lamp and lock-in ultrasonic transducer.

\subsection{Ultrasonic C-Scan Results}

Figure 2.1.1.3 presents the ultrasonic c-scan results for the sample of thickness $1.14 \mathrm{~mm}$ and a damage of $0.315 \mathrm{~mm}$ in diameter. The two images show that the ultrasonic technique was truly non-destructive. This sample was chosen for the experiments 
because it exhibits the minimum possible size of a BVID, producing the most stringent tests for the thermal NDT techniques.

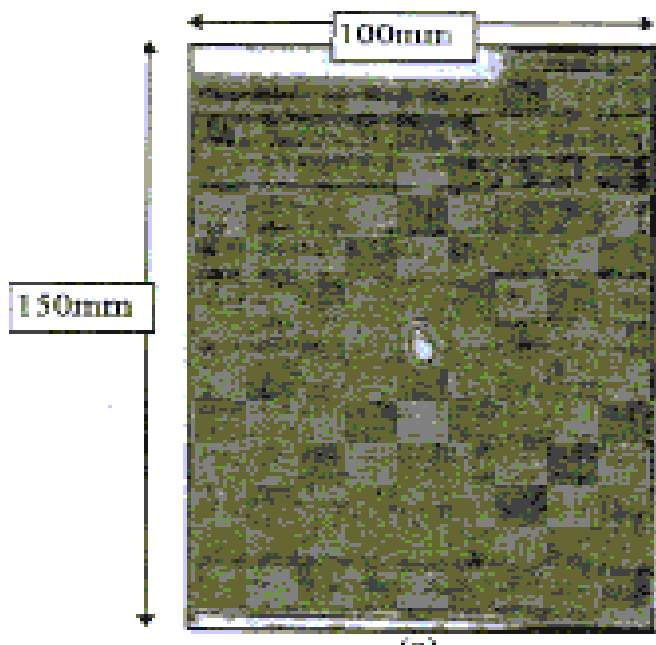

(a)

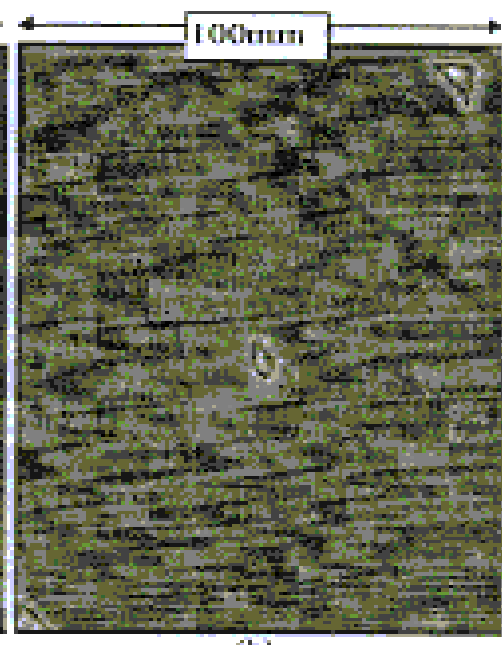

(b)

Figure 2.1.1.3 Ultrasonic c-scan before (a) and after (b) thermal NDT (Bates et al. 2000)

\subsection{Lock-in Halogen Lamp Results}

Only two of the phase images captured using the lock-in halogen lamp technique were able to locate the BVID site. The image that did clearly capture the damage was produced using a modulation frequency of $0.01 \mathrm{~Hz}$ and a lamp intensity of $25 \%$. This image is presented in Figure 2.1.1.4. The non-uniform illumination observed in Figure 2.1.1.4 was due to heat transfer from the bottom to the top of the sample. Placing the samples on a horizontal surface could have eliminated this but the vertical experimental procedure was deemed a more real life in-service inspection. This observation suggests that a certain degree of non-uniform illumination should be a necessary parameter to consider. 

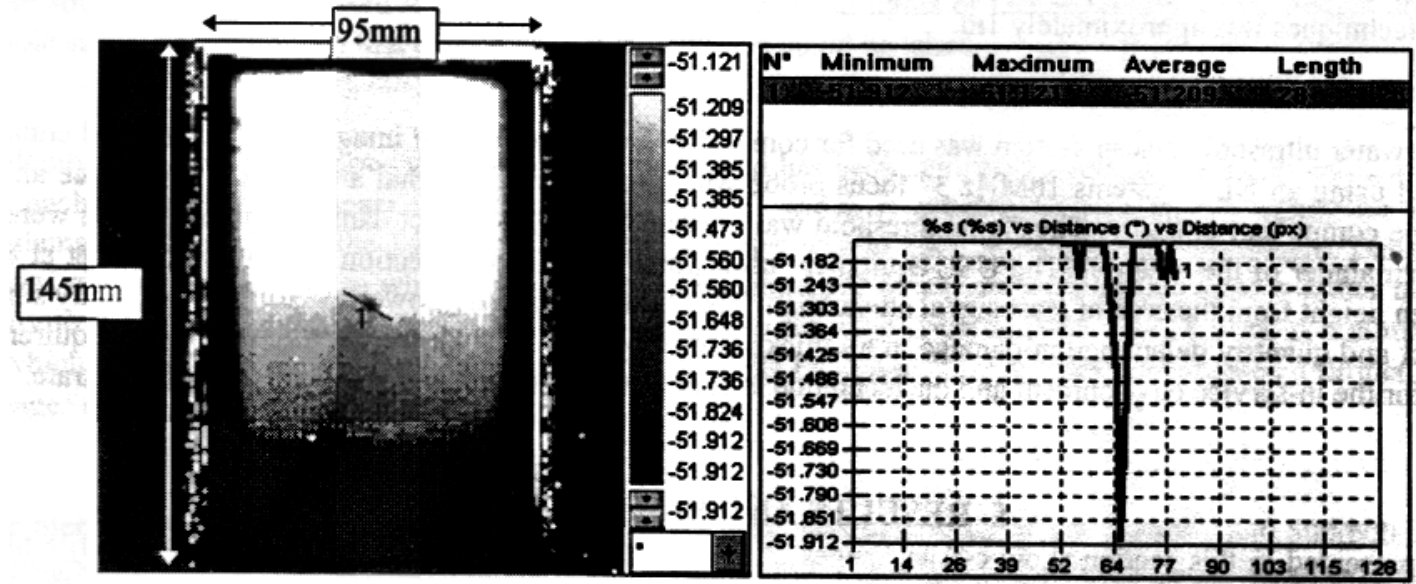

Figure 2.1.1.4 Lock-in halogen lamp phase image at $0.01 \mathrm{~Hz}$ modulation frequency and $25 \%$ intensity for sample T2 (Bates et al. 2000)

The lock-in halogen lamp thermal images demonstrated that none of the amplitude images and only two of the phase images were able to locate the BVID. Moreover, the phase images only detected the damage when $25 \%$ lamp intensity and $0.01 \mathrm{~Hz}$ modulation frequency were used. Although the phase images captured the damage, the $0.315 \mathrm{~mm}$ BVID was just detectable indicating that this is close to the detectable limit for the lock-in halogen lamp technique.

After thorough experimentation using lock-in halogen lamp thermal NDT it was demonstrated that the detection of the impact damage did indeed depend on the light intensity and modulation frequency. Since the $0.315 \mathrm{~mm}$ BVID was only just visible it was concluded that this level of BVID could be easily missed when using the lock-in halogen lamp technique to scan large areas. However, the thermal image took approximately 1.5 minutes to produce, which was 10 times faster than the approximate 15 minutes it took the c-scan to produce an image. 


\subsection{Lock-in Ultrasonic Transducer Results}

The lock-in ultrasonic transducer heating technique was able to locate the BVID sites in all of the amplitude images and two of the phase images. A modulation frequency of $0.01 \mathrm{~Hz}$ produced the clearest images. One of these images is presented in Figure 2.1.1.5. This figure shows some hot regions away from the damaged area, which was said to be areas of natural resonance of the component caused by small amplitude oscillations. However, these anomalous hot regions were approximately $80 \%$ less than the amplitude of the damaged areas.

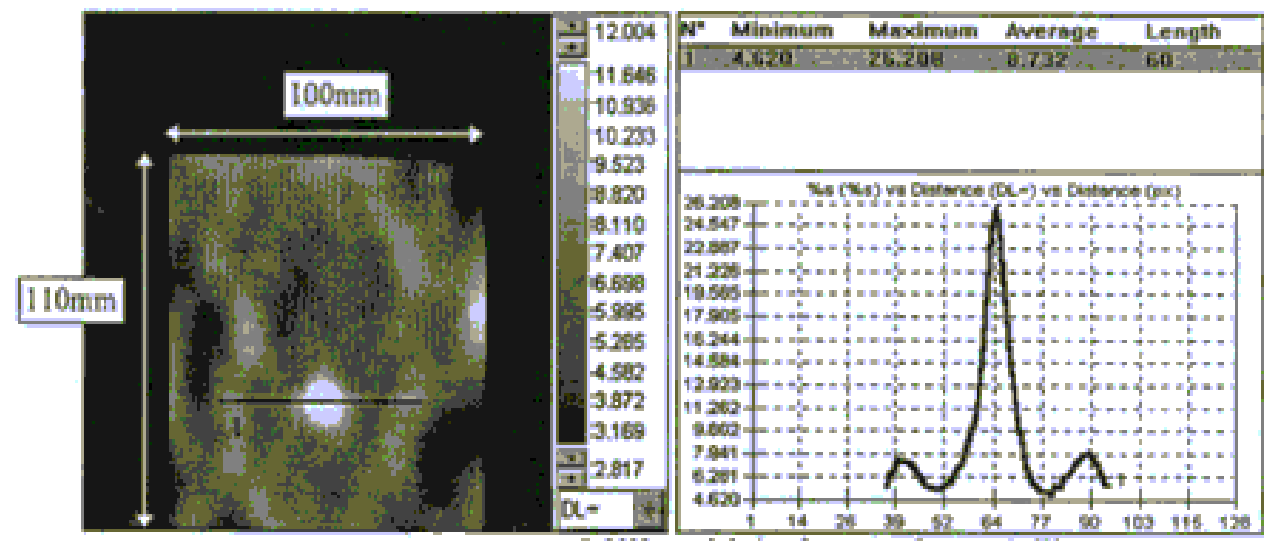

Figure 2.1.1.5 Lock-in ultrasonic transducer amplitude image at $0.01 \mathrm{~Hz}$ modulation frequency for sample $\mathrm{T} 2$ (Bates et al. 2000)

All lock-in ultrasonic thermal images were much more uniformly illuminated than that of the lock-in halogen lamp technique. The amplitude images, in general, produced clearer images than that of the phase images. It was concluded that the detection of the impact damage by ultrasonic lock-in thermal NDT depends on the modulation frequency and produces the best results with a modulation frequency of $0.01 \mathrm{~Hz}$. 
Since the $0.315 \mathrm{~mm}$ BVID was easily detected using the lock-in ultrasonic thermal NDT, this technique could be used in real life in-service inspection to scan large areas for very small damages. Again, the thermal images were produced ten times faster than the images produced using the ultrasonic c-scan method.

These results, however, ignored some parameters such as natural or resonant frequencies, which can depend on the size, mass, shape, and joint configuration. The results will still serve as a good pointer and a general experimental investigation of the technique for further works (Bates et al. 2000).

\subsection{Comparison of the NDT Techniques}

The results from the sample with the smallest BVID $(0.315 \mathrm{~mm})$ were used to compare the two techniques. It was reported that the lock-in ultrasonic technique produced a phase amplitude 28 times larger than the lock-in halogen technique when used to detect the $0.315 \mathrm{~mm}$ BVID (Bates et al. 2000). The larger the phase amplitude the better the signal to noise ratio becomes (Bates et al. 2000). Therefore, the lock-in ultrasonic technique was more accurate in locating the impact damage.

A further conclusion was that the lock-in ultrasonic thermography did not produce any "blind spot" caused by the incident and returning thermal waves being in-phase (Bates et al. 2000). There were no "blind spots" because only one thermal wave was produced within the sample at the BVID site, which was used as the thermal wave generators. This is a major advantage because when incident thermal waves and the 
returning thermal waves are in phase, the BVID is invisible using phase image data.

Producing only one thermal wave within the sample decreased the overall time since only one modulation frequency was necessary.

The last comparison made between the three NDT techniques was the relative speed of the procedure. Both lock-in thermography procedures were up to 10 times quicker than the ultrasonic c-scanning. Also, using larger thermal camera focal plane arrays can increase the area being inspected by up to four times, which would greatly decrease the amount of time it would take to inspect larger areas.

\subsubsection{Conclusions}

Section 2.1.1 explained the use of lock-in halogen lamp and lock-in ultrasonic transducer thermal NDT to detect impact damage in glass fiber composite components used in the aircraft industry. The two thermal NDT techniques were then compared to ultrasonic c-scans. It was determined that the lock-in ultrasonic thermal NDT detected the BVIDs with a greater signal to noise ratio producing more accurate results than that of the lock-in halogen lamp thermal NDT. The lock-in ultrasonic technique also produced more uniformly illuminated images and did not create "blind spots" in the images.

Both lock-in thermography techniques were demonstrated to be 10 times quicker than the ultrasonic c-scan method; therefore, larger areas could be inspected in less time. Although the lock-in thermography techniques demonstrated in this section were used for 
the inspection of composite aircraft components, the techniques could eventually be introduced to the field for the inspection of structures made from composite materials such as composite bridge decks.

\subsubsection{Feasibility of Evaluating the Performance of Fiber Reinforced Plastic (FRP) Wrapped Reinforced Concrete Columns Using Ground Penetrating Radar (GPR) and Infrared (IR) Thermography Techniques (Jackson, D.R. et al. 2000)}

To get a total non-destructive evaluation of FRP wrapped reinforced concrete columns two techniques were used together, ground penetrating radar (GPR) and infrared (IR) thermography. An embedded instrumentation system was also installed in the FRP wrapped columns to provide periodic monitoring of the columns. The tests were conducted on the columns of a bridge in Owego, NY.

Section 2.1.2 discusses the results of the IR and GPR on FRP wrapped columns. It was concluded that both methods were effective NDE techniques for detecting and assessing various types of deterioration in FRP wrapped concrete columns. The IR technique was used to find shallow defects or delaminations in the FRP wrap where GPR was more useful in finding progressive deterioration of the concrete deep beneath the FRP wrap. 


\subsubsection{Introduction}

Besides rehabilitating bridge decks, FRP has proven to be a promising and economical way to repair the columns of deteriorating structures. FRP sheets and strips have proven to be a light, easy to assemble, and durable repair systems, which can increase load carrying capacity, increase ductility capacity, and prevent brittle catastrophic structural failure.

Currently, there are several investigations trying to determine whether the application of FRP wraps is a viable alternative for concrete column repair and rehabilitation (Jackson, D.R. et al. 2000). To investigate whether the FRP wrap did strengthen and prevent further corrosion-induced deterioration of columns, the Court Street Bridge over the Susquehanna River in Owego, New York was experimented with.

The main purpose of the experiment was to determine the feasibility of utilizing IR thermography and GPR as tools for non-destructively locating potential defects in the FRP wrap (e.g., possible entrapped moisture behind the wrap, subsurface delaminations between the concrete surface and FRP wrap, etc). These techniques were chosen because

they proved to be powerful tools in monitoring the performance of the FRP wraps and the underlying concrete (Jackson, D.R. et al. 2000). Embedded instrumentation such as corrosion rate probes, moisture sensors, and strain gauges were also installed in the FRP wraps for future monitoring. 


\subsubsection{Ground Penetrating Radar (GPR)}

GPR is a very powerful non-destructive technique that can be used to detect subsurface defects or flaws. The most common GPR technique uses short pulse radar. This technique is a tradeoff between propagation depth through solid, nonmetallic materials and resolution in the medium.

The primary advantage of GPR is that echoes and reflections originating at the interface of differing mediums can be analyzed to detect delaminations.

\subsubsection{Infrared (IR) Thermography}

IR thermography can detect flaws and/or delaminations by measuring temperature differentials within the material. This technique is sensitive to ambient air temperature as well as the thermal characteristics of the material. Because of these parameters, IR thermography is a multi-disciplinary science that requires a strong understanding of the thermal dynamics of the application, but with recent enhancements in hardware and software, the technique has become more accurate.

\subsubsection{GPR Survey Results}

The GPR experiments discussed in section 2.1.2 were performed using Pulse Radar. The results indicated that GPR could easily pick up voids inside the columns (Jackson, D.R. et al. 2000). Concrete delaminations located by GPR near the surface compared reasonably well to the delaminations found by hammer soundings. However, the deeper delaminations detected by the GPR could not be detected by the hammer 
soundings. The 1 and $2 \mathrm{GHz}$ frequencies used with the GPR did not detect disbondments or other defects between the FRP wrap and the concrete columns; whereas, the hammer soundings did easily detect the flaws.

\subsubsection{IR Survey Results}

Due to ambient temperature conditions a portable propane heater was used to heat up the FRP wrapped surfaces. Under these conditions, the IR system easily detected disbondments and blistering between the FRP wrap and the concrete column. The results were confirmed with a hammer sounding. These results proved that the IR thermography could detect shallow delaminations as easily as the hammer sounding. Figure 2.1.2.1(a) shows a photograph of the area on one of the FRP wrapped columns, which had disbondment and voids while Figure 2.1.2.1(b) shows an IR thermography of the same area. The red color depicts the greater voids.
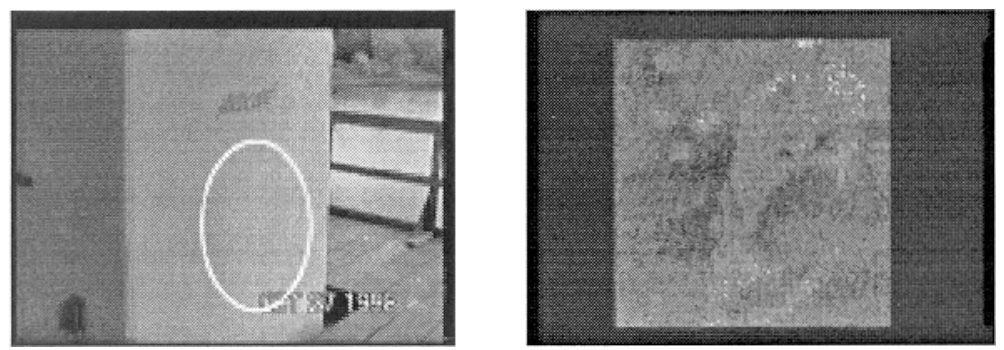

Figure 2.1.2.1 (a) Photograph of an area on one of the FRP wrapped columns, which had disbondment and voids (b) IR image of same area. Red colored areas indicates voids (Jackson, D.R. et al. 2000)

The size and depth of delaminations and debonding flaws can also be determined from IR data. This can be achieved through a computerized image processing technique. The standard two hundred and fifty six gray levels within an image can mathematically separate all signatures. Figure 2.1.2.2 shows how a computerized image of an IR thermography can be used to determine void depths. 

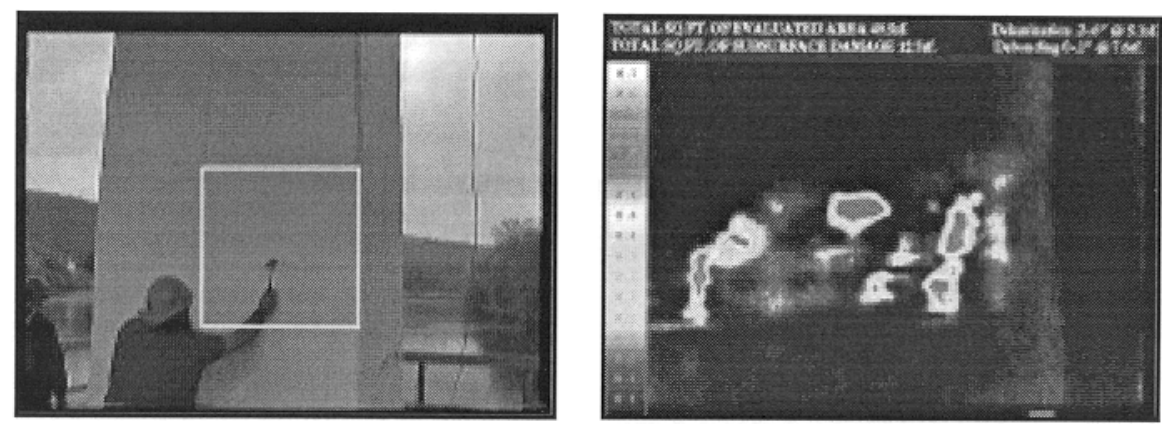

Figure 2.1.2.2 (a) Area with blisters, disbondment, and delaminations on one of the FRP wrapped columns, (b) IR thermography of the area marked with a square in (a) showing delamination, disbondment, and blistering. The scale on the left side of the image represents relative depth in inches (Jackson, D.R. et al. 2000)

\subsubsection{Concluding Remarks}

From the results obtained with the GPR and IR thermography techniques, it was concluded that the two techniques used together can be a powerful tool for detecting and assessing various types of defects in FRP wrapped concrete columns. The GPR technique can be used to detect deep defects and/or deterioration of the concrete that cannot be detected by IR thermography. The IR thermography can be used to detect very shallow defects, including FRP disbondment, and blisters, which cannot be detected easily with the GPR technique.

\subsubsection{Thermal Imaging Technique to Detect Delaminations in CFRP Plated}

\section{Concrete (Mandic et al. 1998)}

Fiber reinforced plastic (FRP) sheets applied to concrete beams has become a popular strengthening method. However, disbonds and delaminations are major defects that are of great concern. A thermographic technique for detecting these defects was experimented with and the results are presented in section 2.1.3. The technique involved heating the specimens (with seeded flaws of various thickness and depth) with a projector 
heat source and capturing thermal images with an infrared camera. Digital image processing techniques were then used to enhance the image quality. The advantages and disadvantages of this technique are discussed in this section.

\subsubsection{Introduction}

The growing number of deteriorating civil infrastructures (especially bridges) in the United States has caused great interest in cost-effective rehabilitation methods. One promising rehabilitation method that has been investigated is the use of composite sheets to repair and strengthen concrete structures. This reinforcement of concrete structures is achieved by bonding thin sheets of FRPs to the tensile side of a beam. FRP reinforcement is of great interest because it is lightweight (e.g. easily handled and installed), and it resists corrosion better than steel plating (Mandic et al. 1998).

Concrete columns are also being reinforced with FRP wraps for seismic protection. This method involves wrapping the columns with composite materials in order to provide concrete containment and increase strain resistance.

The major disadvantage of using FRP to reinforce concrete structures is its lack of standardized design, application, and inspection procedures. The concrete structure is not fully reinforced if there is poor bond quality between the FRP sheet and the concrete. This section discusses an active infrared thermography technique used to inspect the bond quality in a rapid, non-contacting, easily interpreted and archived inspection. 


\subsubsection{Active Infrared Thermography}

Infrared thermography is a truly nondestructive evaluation, which was first introduced in the aerospace industry for locating defects in thin aluminum aircraft skins (Mandic et al. 1998). This method is now proving to be an effective method for detecting delaminations and subsurface impact damage in many advanced composite materials.

Active infrared thermography uses an external uniform heat source (lamp, laser, etc.) to drive heat through or across the object of interest. The thermal gradients developed at the surface are then observed using an infrared imaging device. Cracks, inclusions, voids, or delaminations that transfer heat at different rates cause these thermal gradients. When a delamination occurs between the FRP and concrete structure it acts as an insulator causing a higher surface temperature than the areas that are securely bonded. However, the heat source needs to distribute the heat uniformly since thermal gradients induced by non-uniform heating can be mistaken for defects. A basic thermography setup is illustrated in Figure 2.1.3.1.
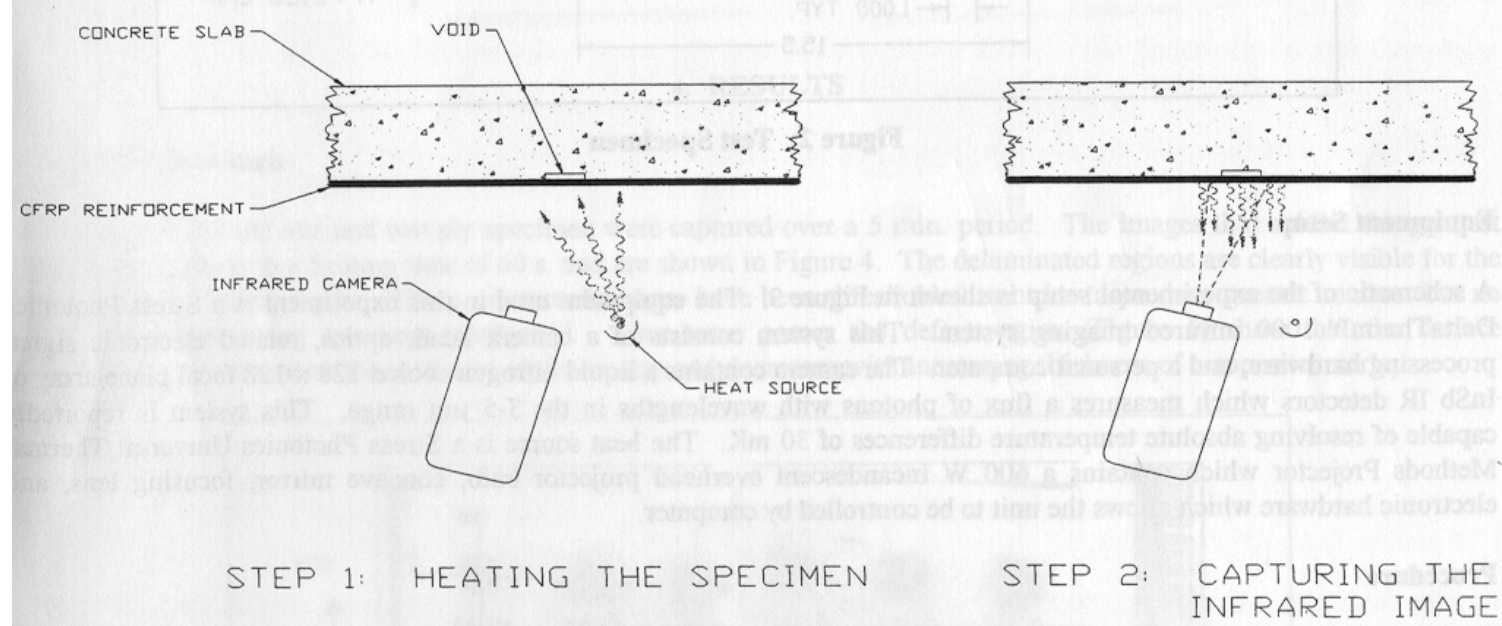

Figure 2.1.3.1 Typical Active Thermography Setup (Mandic et al. 1998) 


\subsubsection{Experimental Procedure}

\subsection{Specimens}

Test specimens were created with simulated delaminations (1 in. $\mathrm{x}$ 1in.) with depths ranging from 0.015 in. to 0.120 in. (illustrated in Figure 2.1.3.2). The FRP system used for testing was unidirectional carbon fiber Tow sheets, primer, and saturant.

Specimens were prepared with 1, 2, 4, and 8 layers of the Tow sheet to investigate the effects of composite thickness. Each layer had a thickness of 0.0065 in. To prevent the simulated delaminations from filling with saturant a thin plastic film was used to cover the pockets.

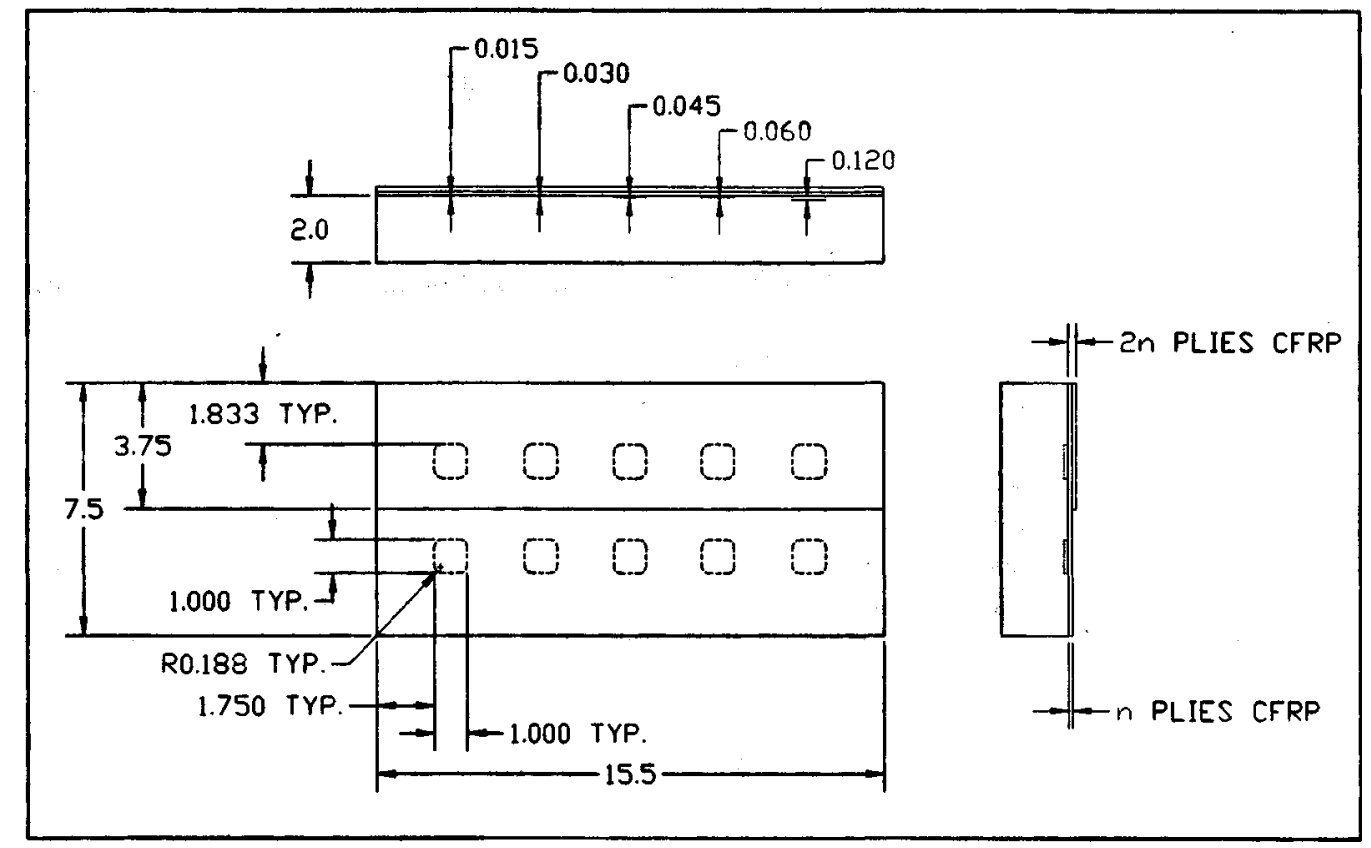

Figure 2.1.3.2 Test Specimen (Mandic et al. 1998)

\subsection{Equipment Setup}

Figure 2.1.3.3 is a schematic of the experimental setup used. The infrared imaging system used was a Stress Photonics DeltaTherm ${ }^{\mathrm{TM}} 1000$. The camera was able to measure a flux of photons with wavelengths in the $3-5 \mu \mathrm{m}$ range. The heat source 
used was a Stress Photonics Universal Thermal Methods Projector, which contained a $600 \mathrm{~W}$ incandescent overhead projector bulb, concave mirror, focusing lens, and electronic hardware allowing the unit to be controlled by a computer.

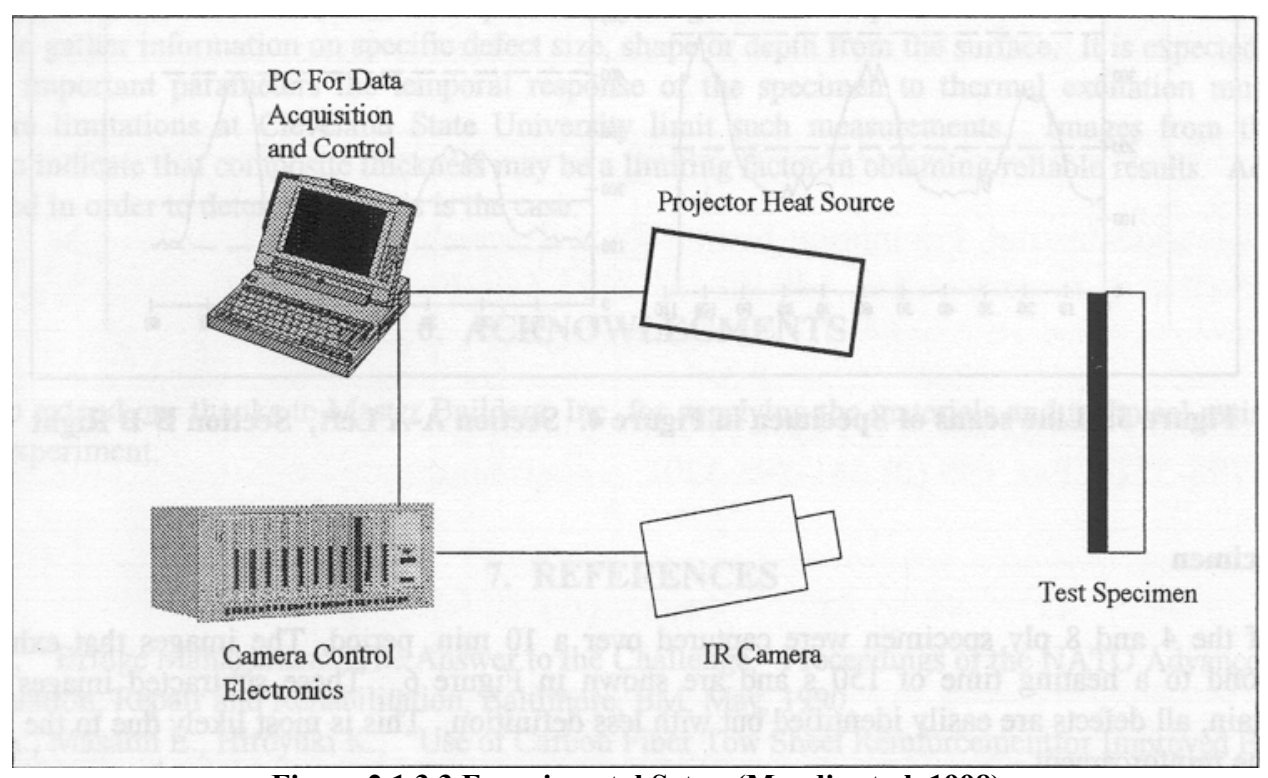

Figure 2.1.3.3 Experimental Setup (Mandic et al. 1998)

\subsection{Procedure}

The procedure used in these experiments placed the heat source and camera on the same side of the test specimens as illustrated in Figure 2.1.3.3. This setup allowed the specimens to be heated uniformly. Heating a specimen with uniform thermal properties and capturing an infrared image checked the uniform heating setup. The heat source to specimen distance used in this setup was approximately $4 \mathrm{ft}$.

To remove undesirable features in the images, the cold image (image captured prior to heating) was subtracted from the hot image (image captured during heating). This improved the quality of the image. 


\subsubsection{Results}

\subsection{One and Two Ply Specimen}

The images that captured the delaminations the best for the 1 and 2 ply specimen were heated 60s (illustrated in Figure 2.1.3.4). It was observed that the entire range of defect depths was clearly visible. It was also observed that an increase in the air gap thickness increased the surface temperature.

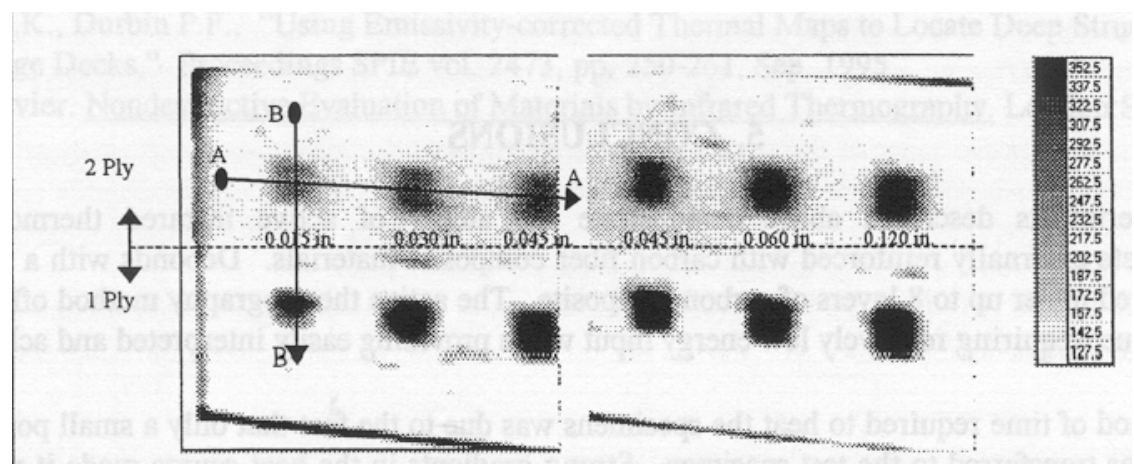

Figure 2.1.3.4 1 and 2 Ply specimen after 60s Heating (Mandic et al. 1998)

\subsection{Four and Eight Ply Specimen}

The images that captured the delaminations the best for the 4 and 8 ply specimen were heated over a 150s time period (illustrated in Figure 2.1.3.5). All of the defects were identified but with less quality. This was determined to be from lateral heat flow in the plane of the reinforcement.

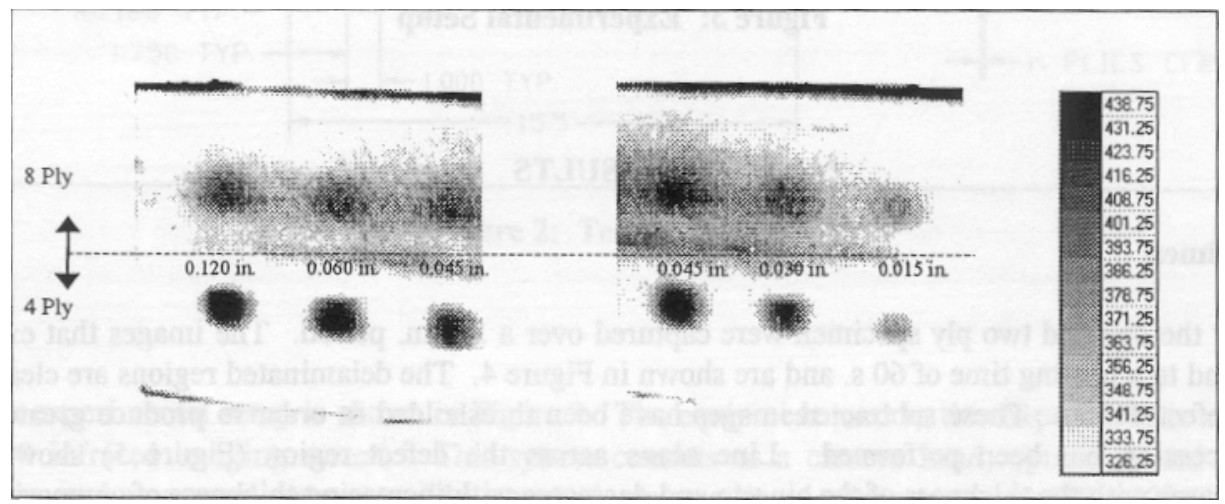

Figure 2.1.3.5 4 and 8 Ply Specimen after 150s Heating (Mandic et al. 1998) 


\subsubsection{Conclusions}

The active infrared thermography technique describe in this section has successfully detected delaminations in concrete externally reinforced with carbon fiber composite materials. Delaminations with a thickness as small as 0.015 in. were detected under as many as 8 layers of carbon composite.

Even though the testing was performed in a reasonable time period, the inspection time could be reduce by using an array of lamps to significantly heat the specimens uniformly.

\subsubsection{Detecting Manufacturing Flaws in Composite Retrofits (Hawkins et al. 1999)}

Recently many bridges and other concrete structures have been rehabilitated using composite materials. This section focuses on using composite wraps applied to concrete columns. There are three methods talked about in this section used for applying composite wraps to concrete columns. Each method has the potential for creating debonds at the composite-concrete interface and within the composite wrap itself. Infrared thermography is a nondestructive test method being experimented with composite materials to detect debonds below the surface. This section presents the results of thermographic tests of a variety of retrofit applications. 


\subsubsection{Composite Manufacturing Techniques}

The three primary methods for overwrapping concrete columns (hand lay-up, pre-cured shells, and machine wraps) were investigated using thermographic inspection. Each method had its own unique flaws caused by difficulties with manufacturing.

\subsection{Hand Lay-up}

The hand lay-up process involves manually applying the wrap to the columns and allowing it to cure. The wrap typically comes in wide rolls and cut to convenient lengths. A resin is applied to the wrap by either dipping the wrap in a bath or applying it with a brush or paint roller. The manually applied wrap needs to be smoothed once the resin is applied to release any air pockets.

\subsection{Pre-Cured Shells}

This wrapping technique uses factory manufactured shells to contain the columns. Fiberglass mats are molded and cured to form a cylindrical shell of the same diameter as the concrete column being wrapped. The column is cleaned and sprayed with an adhesive. The shell is then slit longitudinally and pried open so it can be positioned around the column (additional shells are applied to achieve proper thickness). Once the shells are in position cinching straps are tightened over the shells to squeeze out excess adhesive and tighten the shells onto the column. 


\subsection{Machine Wrap}

This method uses a machine to wrap fibers directly from a spool onto the column. The resin is either pre-impregnated into the fibers on the spool or impregnated by dipping the fibers into an epoxy bath before they are wrapped onto the column. The fibers are wrapped onto the column using a machine constructed at the job site.

\subsubsection{Thermography}

It was concluded that thermography was the best NDE technique for detecting debonds present in composite seismic retrofits. IR thermography was conducted on several full scale columns retrofitted by the three methods discussed above.

\subsection{Thermography Background}

In order for thermography inspection to be effective a thermal gradient through the thickness of the structure under inspection needs to be generated and then the surface temperature of the structure needs to be monitored as it returns to thermal equilibrium (Figure 2.1.4.1). The thermal gradient can be established using a heat gun, a warm water soak, a heat lamp, etc. It is important that the heat be uniformly distributed. Defects in the composite (debonds, porosity, etc.) conduct heat differently because the air in the voids act as insulators. These thermal differences can be captured with the use of an IR imager and, hence, pinpoint the location, shape and approximate size of a subsurface flaw. 


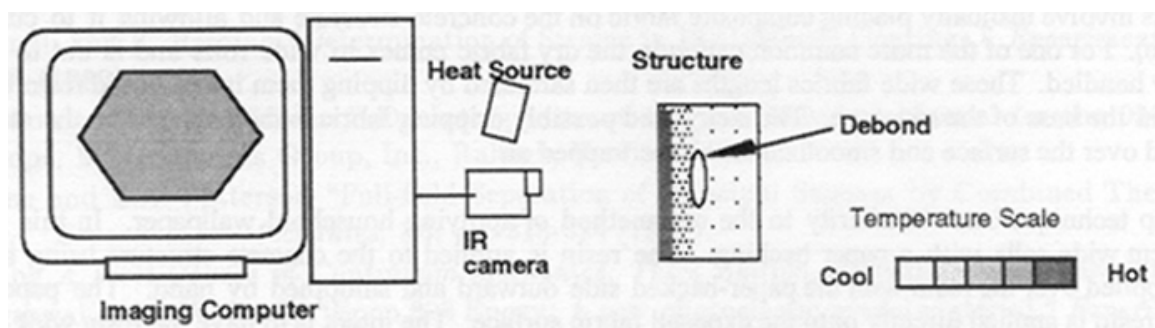

Develop thermal gradient

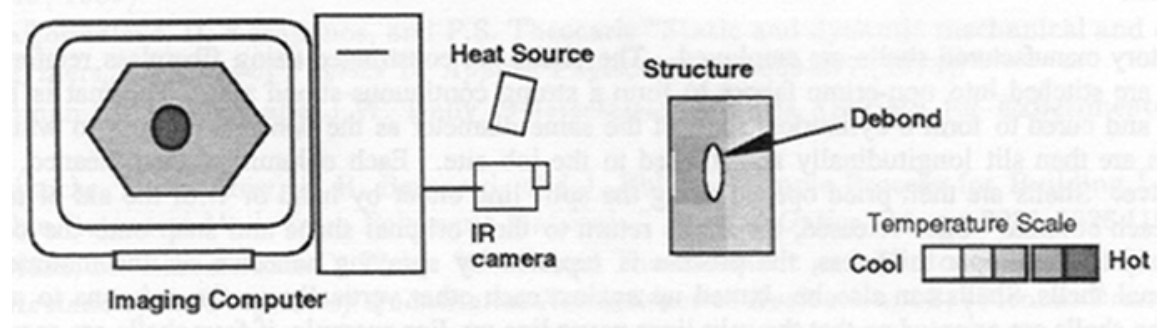

Image development

Figure 2.1.4.1 Thermographic inspection consists of 2 phases: (1) development of a temperature gradient and (2) image development (Hawkins et al. 1999)

There are many IR cameras that can be selected for developing a thermography system. This is the most expensive part of the system. The newer cameras are sensitive to IR radiation in either the $3-5 \mu \mathrm{m}$ or $8-12 \mu \mathrm{m}$ wavelength. However, the low - mid range cameras are also being used to measure thermal differences as small as $0.1{ }^{\circ} \mathrm{C}$ to 2 ${ }^{\circ} \mathrm{C}$. The selection of an IR camera mainly depends on the temperature range that the camera can view, which depends on the lens that are available for the camera.

\subsection{Thermography System}

The IR camera used for the experiments described in section 2.1.4 was a $256 \mathrm{x}$ 256 InSb FPA camera operating in the 3-5 $\mu \mathrm{m}$ band with an onboard Stirling cycle cooler. The lens that came with the camera permitted sufficient flexibility for a wide range of inspection applications. A couple different methods to produce the thermal gradient were experimented with. For defects close to the surface, photographic flash 
lamps were used. In other applications, where deeper flaws were expected, high intensity heat lamps (500 W lamps) placed $50 \mathrm{~mm}$ from the surface and scanned at $\sim 0.5 \mathrm{~m} / \mathrm{sec}$ were used. Solar heat from the sunlight was used in some situations where columns where in direct sunlight. One method that was suggested for future IR thermography was the use of high intensity spotlights or arc lamps, which would allow IR thermography to be performed by a remote.

\subsubsection{Bridge Inspection Results}

IR thermography was used to inspect several bridge columns wrapped with composite fabric manufactured by the hand lay-up method, the pre-cured shell method, and the machine wrapped method. Below are the images obtained from a thermography system.

\subsection{Hand Lay-up}

Figures 2.1.4.2 and 2.1.4.3 are the images obtained from a rectangular column, which was hand wrapped. The detection of elevated temperatures suggested debonding or porosity within the wrap. The defects detected in the thermal images where compared to the results obtained from tap testing. 


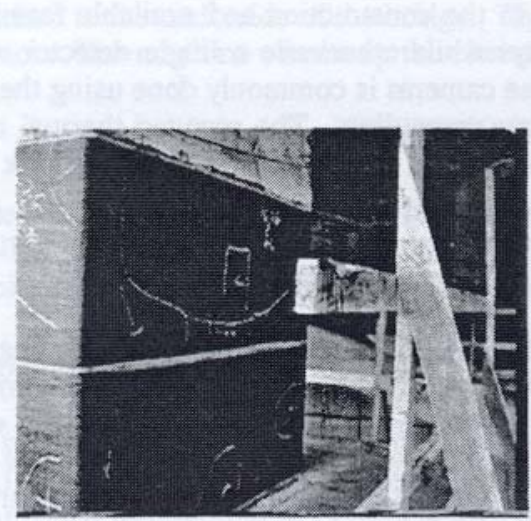

Tap test markings
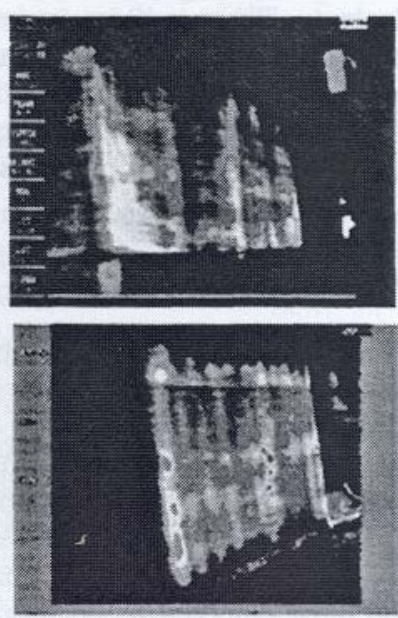

IR Image

Figure 2.1.4.2 Image of rectangular column wrapped with composite material (east face). Extensive debond indications were seen along the corners of the overwrap. Similar results were obtained from the tap testing (outlined with chalk) (Hawkins et al. 1999)

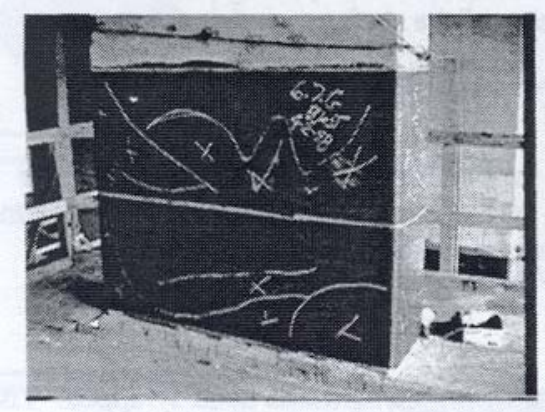

Tap Test markings

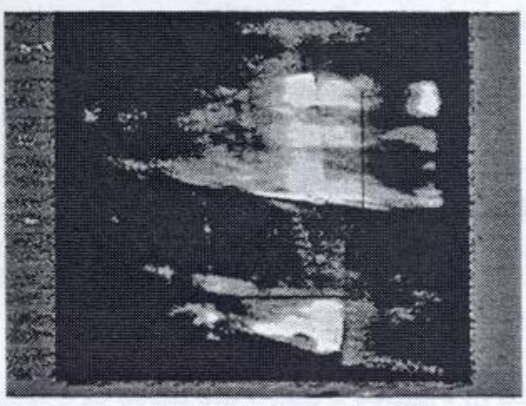

IR Images

Figure 2.1.4.3 Image of rectangular column wrapped with composite material (west face). Extensive debond indications were noted at the edges of the wrap. Again, tap testing confirmed the results (Hawkins et al. 1999)

\subsection{Pre-Cured Shells}

Figures 2.1.4.4 through 2.1.4.6 are the images obtained from cylindrical columns, which were retrofitted with pre-cured shells. The bright areas in the figures represent the debonds that were detected. It was observed that debonds tended to be close to the slits (also evident in the images). The large debond shown in Figure 2.1.4.5 was detected from both sides of the column. Figure 2.1.4.6 had a core specimen removed to represent a large defect, which is clearly visible in the IR image. 


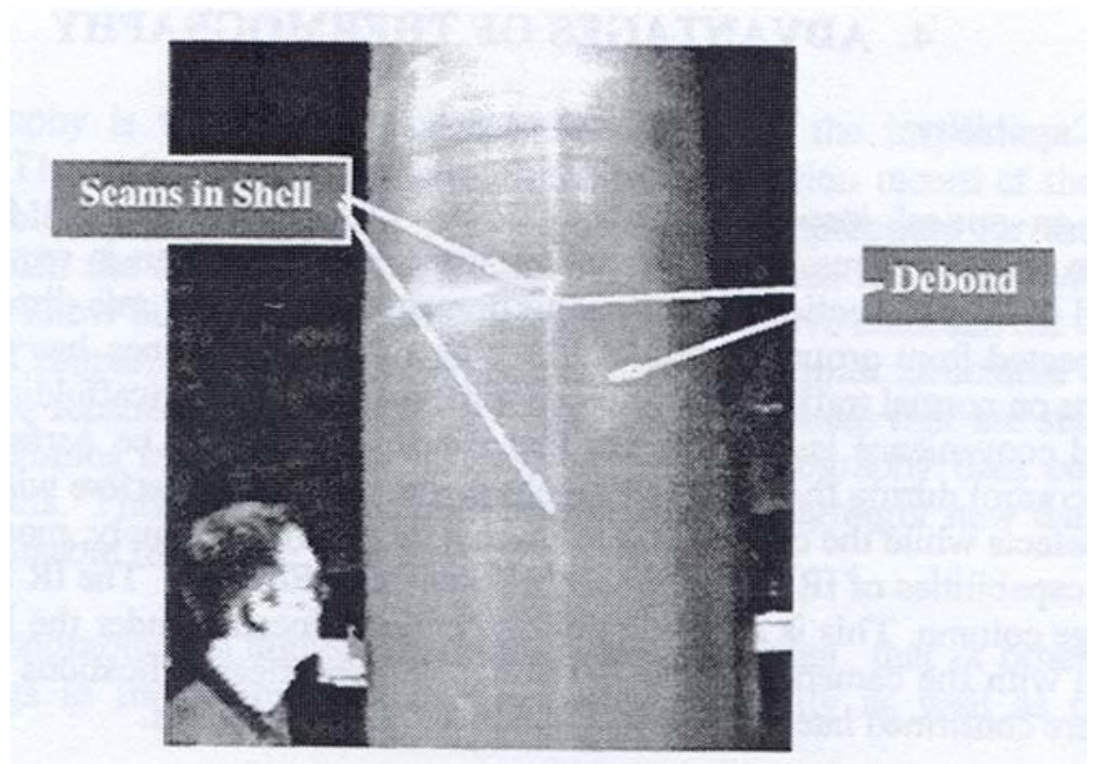

Figure 2.1.4.4 Thermographic image of a cylindrical column retrofitted with a pre-cured shell showing small debonds (Hawkins et al. 1999)

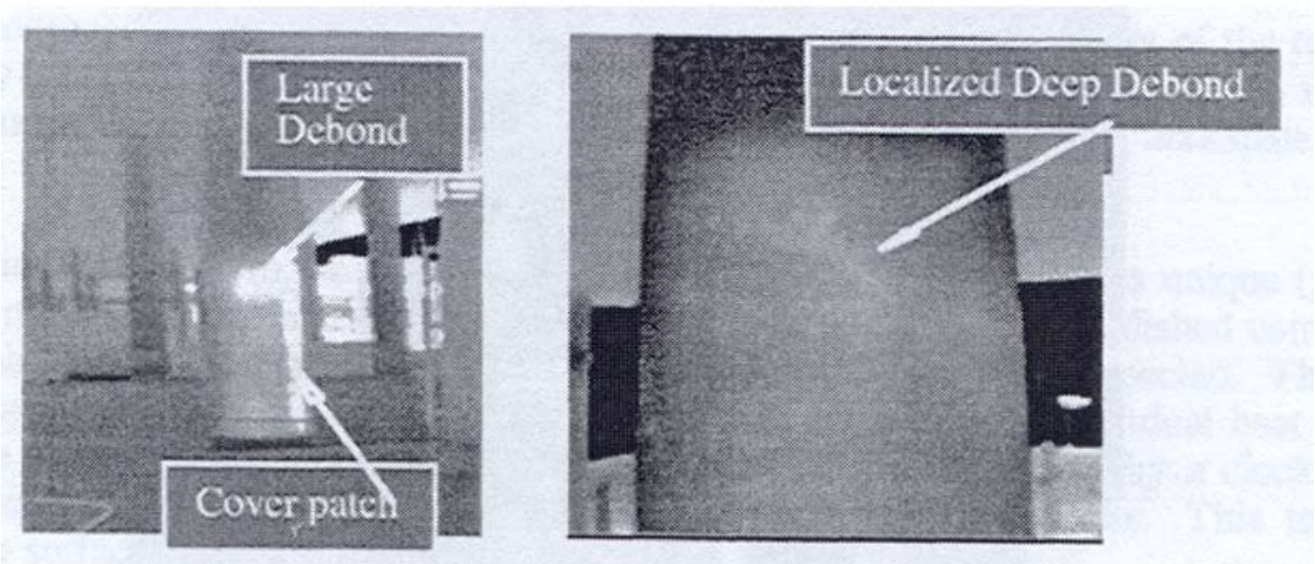

Figure 2.1.4.5 Thermographic images of a cylindrical column retrofitted with a pre-cured shell showing a large debond and a localized deep debond (Hawkins et al. 1999) 


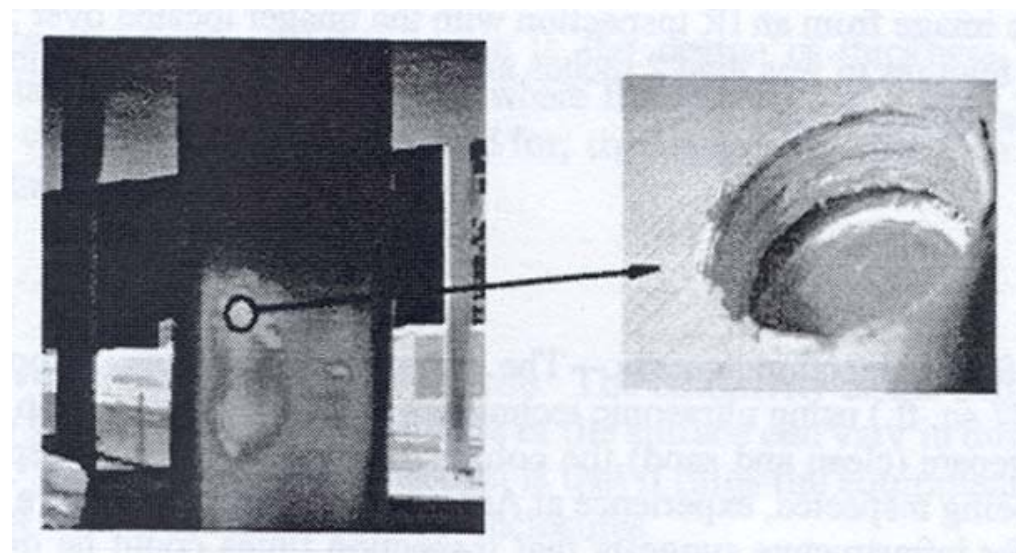

Figure 2.1.4.6 Core section of large debond discovered in prefabricated shell (Hawkins et al. 1999)

\subsection{Machine Wrap Debonds}

IR thermography was conducted on machine overwrapped circular columns and no debonds were detected. This indicates that laying down the fibers one strand at a time appears to preclude the formation of large debonds on circular columns. However, large debonds have been detected on machine wrapped rectangular columns tested in the past (Hawkins et al. 1999).

\subsubsection{Advantages of Thermography}

\subsection{Remote Inspection Capability}

Thermography is a totally nondestructive test because no physical contact with the test specimen is required (remote capability). Since no physical contact is required, personnel can work at safe distance and hard to reach areas can be inspected. Because this technique can be performed remotely, a unique means of real time quality control can be provided during the construction phase that should not interfere with manufacturing process (Hawkins et al. 1999). This would allow for a timely, cost effective repair of voids, debonds, and other defects on site. Figure 2.1.4.7 illustrates the capabilities of IR 
thermography. An IR camera positioned 50 feet from the column produced this image, which detected defects of less than 2 inches in diameter.

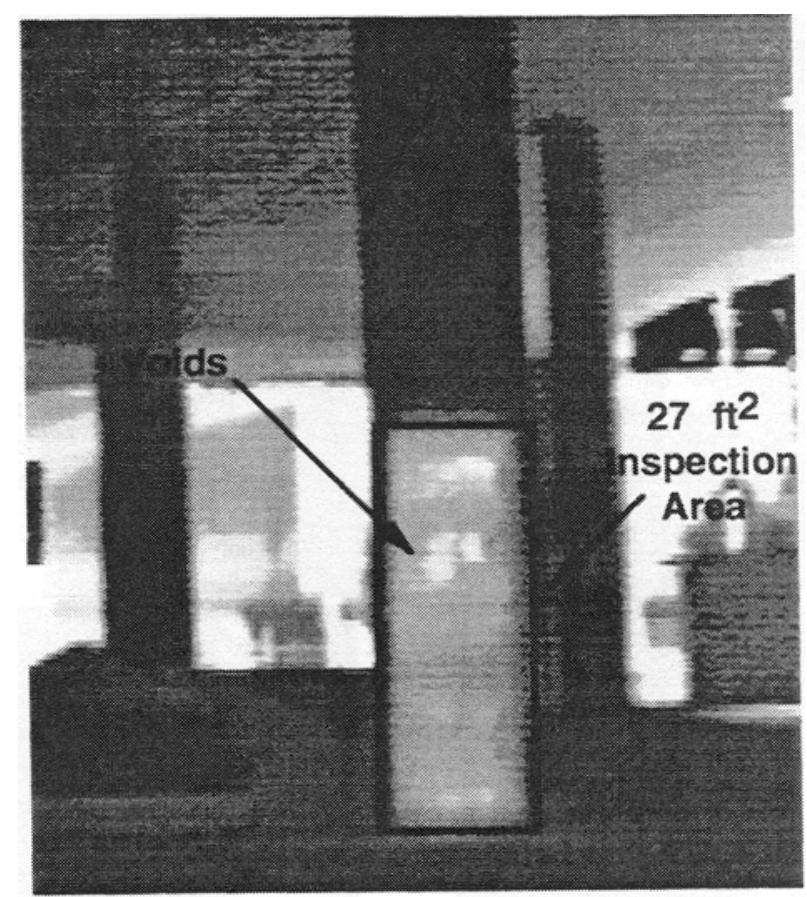

Figure 2.1.4.7 Sample thermographic image from an IR inspection with the imager located over 50 ft. from the column surface. Void indications with features of less than 2 inches are clearly visible in the outlined inspection area. The scan took less than one minute to perform (Hawkins et al. 1999)

\subsection{Rapid Inspection}

Large areas can be inspected in very timely manner. The inspection of the column illustrated in Figure 2.1.4.7 took less than one minute to perform. Ultrasonic evaluation of the same column would have taken over an hour to perform (Hawkins et al. 1999).

\subsection{Data Storage}

The inspection data of thermography can be stored in a number of formats including video tape and digital pictures. Storing data and comparing it to future data will establish a comprehensive database for the monitoring and evaluation of structures 
over their life span. This will help predict flaws in future structures and allow quick repair before safety is compromised.

\subsubsection{Issues Encountered and Potential Solutions}

\subsection{Uniform Heating}

Uneven heating can change the image of a debond, with respect to the image of a bonded area. Hawkins et al. produced a thermography system to minimize heating variations. The heating system developed used heat lamps that were held a constant distance away from the surface under inspection. A clock drive motor was used to rotate the heating system so a uniform line of heat traverses along the surface at a controlled rate. This system was able to uniformly heat a large area in a short amount of time.

Environmental variations (weather, time of day, etc.) can also affect the cooling rate of the structure, which will decrease the quality of the thermographic images. If different sections of the column undergo different cooling rates then debonds and other defects will be less distinct.

\subsection{Material Thickness Variations}

Variations in the material thickness (composite material or adhesive) can cause different thermal conductivity. If these variations are not accounted for, different thickness can be mistaken as large debonded areas. 


\subsection{Cleanliness}

If there are particles of dirt, dust, etc. left on the surface being inspected, they can be mistaken as defects in the thermal images.

\subsection{Image Processing}

Emissivity variations can also cause problems for thermography. It was suggested that images be taken at different times and subtracted from one another. This process works well, but it can become timely and costly because the images must first be digitized.

\subsection{Inspection Speed}

The inspection speed of any technique is very important because it is closely related to the expense of the inspection. If the inspection is timely and therefore more expensive a less reliable technique could be used that has the potential to miss defects.

An important parameter to the inspection speed is the development time (the time between heating the specimen and capturing thermal images). This time depends on the

thickness and thermal diffusivity of the material. For composite materials this time varies for a couple of seconds to a couple of minutes. The development time is usually not a large contributor to the overall inspection time.

The main parameter is the time it takes to set up, tear down, and move the equipment for one site to the next. It was suggested that the thermography system be 
entirely battery powered to eliminate having to move cables from site to site. It was also suggested that the images be captured on a video recorder so all of the equipment can be attached to one cart or tripod, which can be easily moved from site to site.

\subsubsection{Summary}

Advantages and disadvantages of thermographic methods for the inspection of composite retrofits to concrete structures have been discussed in section 2.1.4. It was concluded that advantages in using thermography included increased sensitivity, remote inspection capability, decreased inspection times and convenient means for data archival. The main disadvantages of this technique are the high costs of the initial equipment and uniformly heating the specimen. Other areas that would improve the technique include developing a remote heating source, and developing a thermography system that is easier to move from site to site.

\subsubsection{Thermal Nondestructive Testing (TNDT) of Adhesively Bonded Composite Reinforcements Applied to Concrete Civil Structures (Burleigh et al. 1999)}

Thermographic nondestructive testing (TNDT) was performed on a composite reinforced cement column representing a freeway support column and on a composite reinforced navy pier. The navy pier was to be upgraded from a 30 ton load capacity to a 50 ton load capacity. TNDT was used to detect both simulated and actual disbonds in several types of composite reinforcements. This section presents the results obtained 
from these tests and points out the advantages and disadvantages of using TNDT. From these results, TNDT standards were created for use in the field.

\subsubsection{Structures Tested}

\subsection{Caltrans Column Confinement Qualification Test}

The University of California at Irvine performed Caltrans (California Department of Transportation) column confinement qualification tests. It was a rectangular column having dimensions approximately 18 inches x 24 inches. Composite reinforcement was applied to the column using 8 "L" shaped shell components epoxy bonded in place around the concrete column creating an overlapping configuration (Figure 2.1.5.1). The minimum thickness of the final configuration was 0.30 inches and the maximum thickness (at the corners) was 0.60 inches.

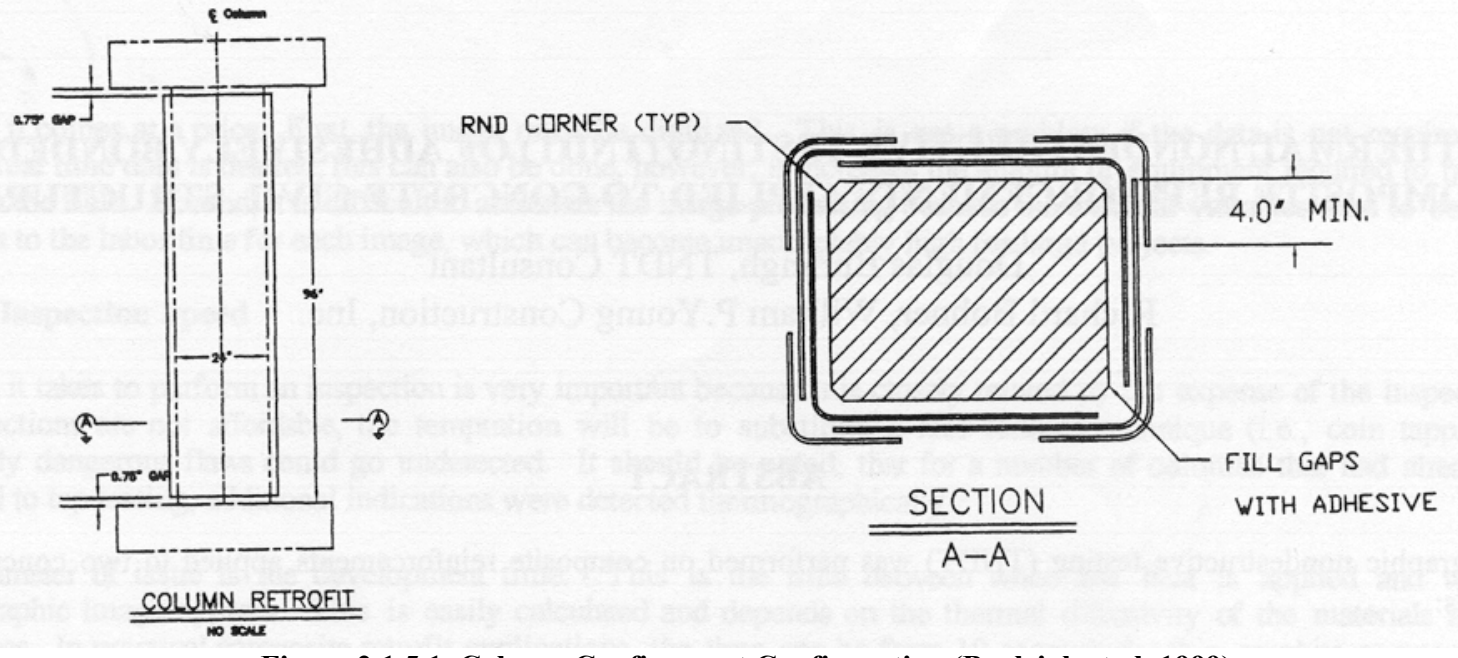

Figure 2.1.5.1 Column Confinement Configuration (Burleigh et al. 1999) 


\subsection{Navy Pier 12 San Diego}

Navy Pier 12 at San Diego Naval Station was to be upgraded from 30 to 50 tons.

This project was designed to examine several different structural design approaches using composite material systems to increase the load capacity of concrete civil structures.

Five structural reinforcing systems fabricated with five different composite materials were examined (Figure 2.1.5.2). Each system and the materials used in each system are described below.

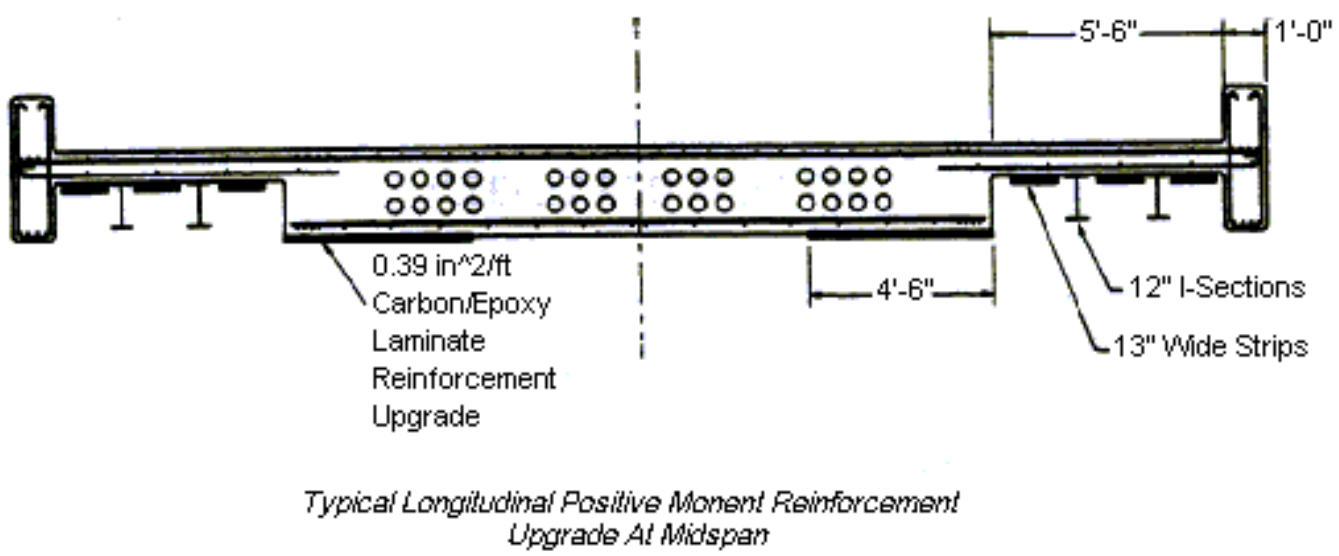

Figure 2.1.5.2 Application of Composite Material to Pier 12 (Burleigh et al. 1999)

\section{Pultruded Graphite fiber rods embedded into slots saw cut into the top deck for increased negative moment capacity.}

Ten feet long rods comprised of $65 \%$ Graphite fiber and 35\% epoxy resin where placed into $5 / 8$ " wide by $7 / 8$ " deep grooves saw cut into the top deck on 4 " centers. An $1 / 8$ " top coat of sand and epoxy was placed over the rods to prevent ultraviolet degradation of the epoxy.

\section{Pultruded fiberglass I-beams bolted and epoxy bonded to the under deck surface for increased shear strength positive moment capacity.}


Fiberglass I-beams (12"x6"x1/2”) where bonded directly to the under deck surface and anchored to the pile caps to provide increased positive moment capacity. Composite I-beams where considered for this application because of its superior corrosion resistance in salt water environments, and also its light weight makes it easy to lift the beams into place.

Pultruded Graphite fiber strips epoxy bonded to the under deck surface for increased positive moment capacity.

Pultruded Graphite fiber strips where bonded to the under deck surface using approximately $1 / 8$ " of epoxy material. Adhesive was applied to both the deck surface and the Graphite fiber strips after being cut to length. The strips where positioned into place and a roller was used to help ensure consist thickness and bonding to the deck.

Laminated Graphite/epoxy fabric adhesively bonded to the under deck surface for increased positive moment capacity.

A 4.5'x9' rectangular wet epoxy laminated Graphite fiber system was added to the underside of the 24 " thick section of the deck to strength the positive moment capacity. The system consisted of a 4 ply unidirectional Graphite fabric laminated with an epoxy resin, which had a total thickness of approximately 0.4 ". The saturated fabric was carried to the site and positioned into place using rolls. 


\section{Laminated vinyl ester/fiberglass shells installed on selected vertical piles to provide improved ductility and side load capacity.}

Several rectangular concrete piles of Pier 12 were retrofitted with the confinement composite jacket system to improve ductility and side load capacity. The confinement composite jacket system consisted of vinyl ester/fiberglass and was approximately 0.45 inches thick. Half shells were connected using composite "H" connectors. After the jackets were installed, a grout was poured into the gap between the composite shell and the pile. TNDT was used to find voids in the adhesive of the " $\mathrm{H}$ " connector.

\subsubsection{Principles of Thermographic Testing}

Thermography is a technique that measures surface temperatures to produce thermal images. The technique usually uses an infrared camera to capture the thermal images and a TV monitor to display the images. The images are made up of thousands of pixels, which represent the level of infrared energy radiating from a point on the surface.

Thermography nondestructive testing was developed primarily in the aerospace industry for evaluating composite materials (Burleigh et al. 1999). TNDT is most effective when the surface being tested is uniformly heated. Defects such as voids and delaminations will act as insulators causing the surface above the defect to be warmer than the surrounding areas. These defects can then be detected from the thermal images.

There are many parameters that effect TNDT. Many of these parameters are associated with the type of infrared camera one uses to perform the tests. The selection 
of a camera is based on requirements for spatial resolution, thermal resolution, speed, wavelength sensitivity, selection of appropriate lenses, portability, cost, and availability (Burleigh et al. 1999). Many other parameters are associated with heating the material. The selection of heating is based on the requirements for the energy and duration of the heat pulse, the surface properties of the material, portability, power consumption, cost, and availability (Burleigh et al. 1999). And many other parameters are associated with the material being evaluated such as specific heat, thermal conductivity, thermal diffusivity, thermal effusivity, thermal mass, density, thickness, etc.

TNDT cannot detect defects directly. It is the operator's ability to understand thermal images that allows defects to be detected. An appropriate thermal gradient and proper environmental conditions make identifying defects from thermal images easier. However, some thermal gradients caused by variations in the structure may be misinterpreted as defects. To avoid these misinterpretations an operator must understand the process of TNDT as well as the structure of the material being inspected.

\subsubsection{TNDT Testing of a Concrete Column}

Several methods of heating the column were performed for TNDT. An industrial heat gun was the first method of heating tried. This method did produce thermal gradients but it was questionable how uniform this heating process was. The second test used a 1000 Watt quartz lamp to heat the column. This method also produced thermal gradients, but its ability to heat the column uniformly was questioned. 
Anomalies were detected using TNDT with both heating methods. The results were compared to the results obtained from tap testing. Many of the defects located using TNDT were confirmed by the results from tap testing. However, some of the defects detected using TNDT were not detected using tap testing. It was concluded that TNDT was a more reliable technique than tap testing.

Figure 2.1.5.3 shows the thermal image of a disbond approximately six inches long under the fiberglass shell. The vertical line represents the free edge of the fiberglass shell and was not considered a disbond.
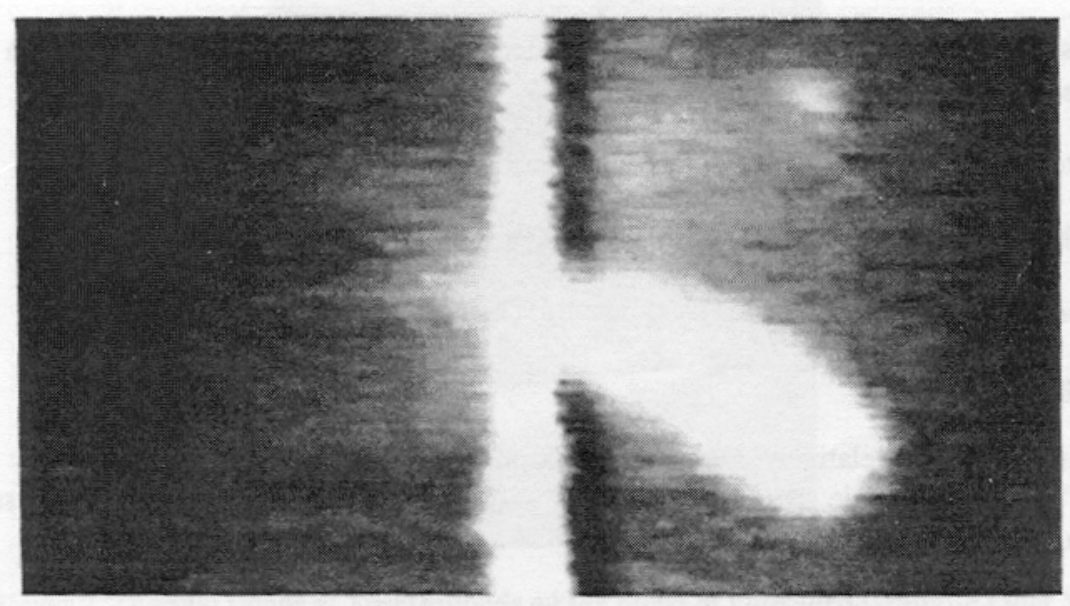

Figure 2.1.5.3 Thermal Image of a Disbond in Half-inch Thick Fiberglass Adhesively bonded to a Concrete Column (Burleigh et al. 1999)

\subsubsection{TNDT Testing of a Naval Pier}

TNDT of the pier was more challenging than the column because the pier was in an outside environment and many of the bonded composite reinforcements were overhead. Heating the surfaces was very difficult due to the cold winds $\left(\sim 50^{\circ} \mathrm{F}\right.$ and 15 $20 \mathrm{mph}$ ) and wet conditions from working on the water. 
Five different composite materials were used on the pier and defects were simulated by inserting thin, 0.05 to 0.10 inch thick, closed cell foam pieces into the bondline between the composite and cement.

\subsection{Pultruded Graphite fiber rods}

The pultruded Graphite fiber rods described in section 2.1.5.1.2 was not considered to be a reasonable candidate for TNDT, and was not tested.

\subsection{Pultruded fiberglass I-Beams}

This was a 0.50 inch thick relatively dense material made of approximately $55 \%$ glass fibers with a vinyl resin system. These composite beams were bonded to the pier as well as mechanically fastened.

Defects were constructed ranging in size from 0.25 to 1.0 square inches. However, TNDT was unsuccessful in detecting the defects.

\subsection{Pultruded Graphite fiber strips}

Strips of Graphite fiber approximately $1 \times 12$ feet in area and 0.05 inches in thickness were bonded to the pier. TNDT was very effective since the strips were so thin. Figure 2.1.5.4 shows the section that was tested and the thermal image that was obtained. 


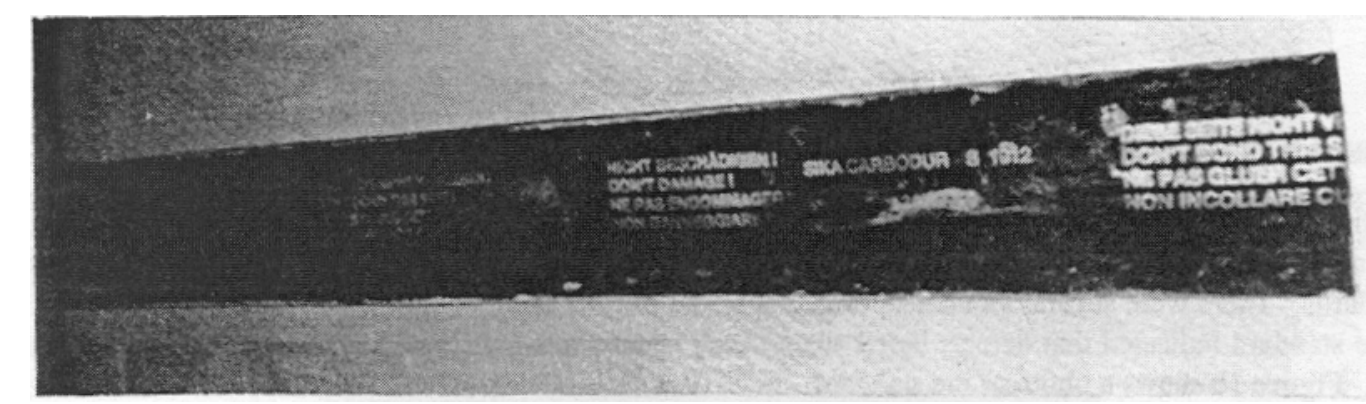

(a)

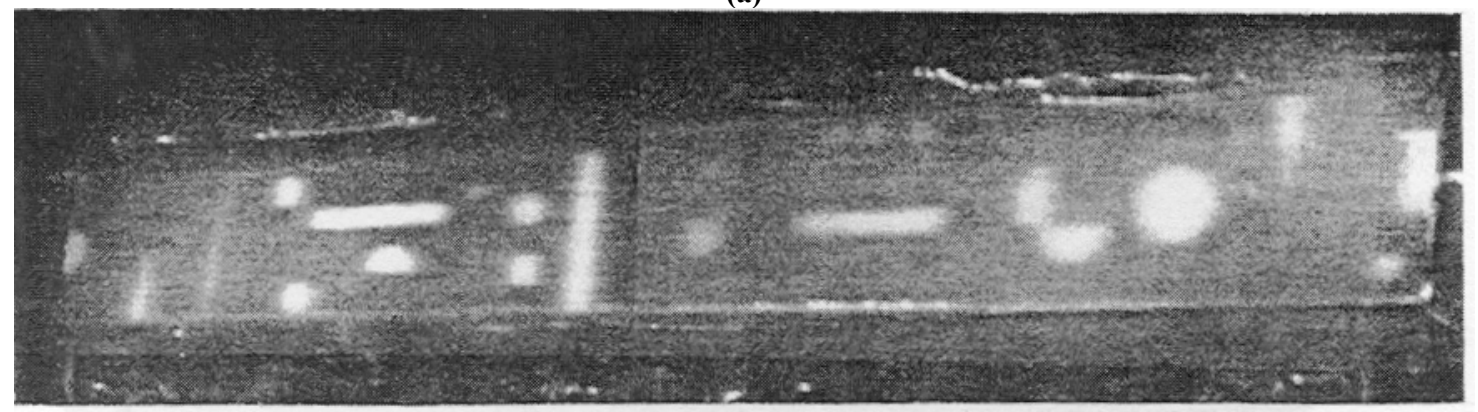

(b)

Figure 2.1.5.4 (a) Photograph of the area being tested, and (b) thermal image of the pultruded Graphite fiber strips shown in (a) (Burleigh et al. 1999)

\subsection{Laminated Graphite/epoxy fabric}

A laminated Graphite/epoxy fabric was applied to an area approximately $4.5 \times 12$ foot on the pier. The total thickness of the four ply laminated material once in place was approximately 0.4 inches. Several simulated defects were inserted in the bondline, ranging in size from 0.25 to 1.0 inch squares. Figure 2.1.5.5 shows the thermal image of a one inch square defect. Figure 2.1.5.6 shows the thermal image of an area that contained delaminations. The TNDT results were compared to the results of tap testing. The hammer tap testing detected a defect that was not detected using TNDT. When further destructive analysis was performed, a sub-surface anomaly was detected. However, there was no disbond between the composite and the concrete, which was the reason it did not show up in the thermal image. 


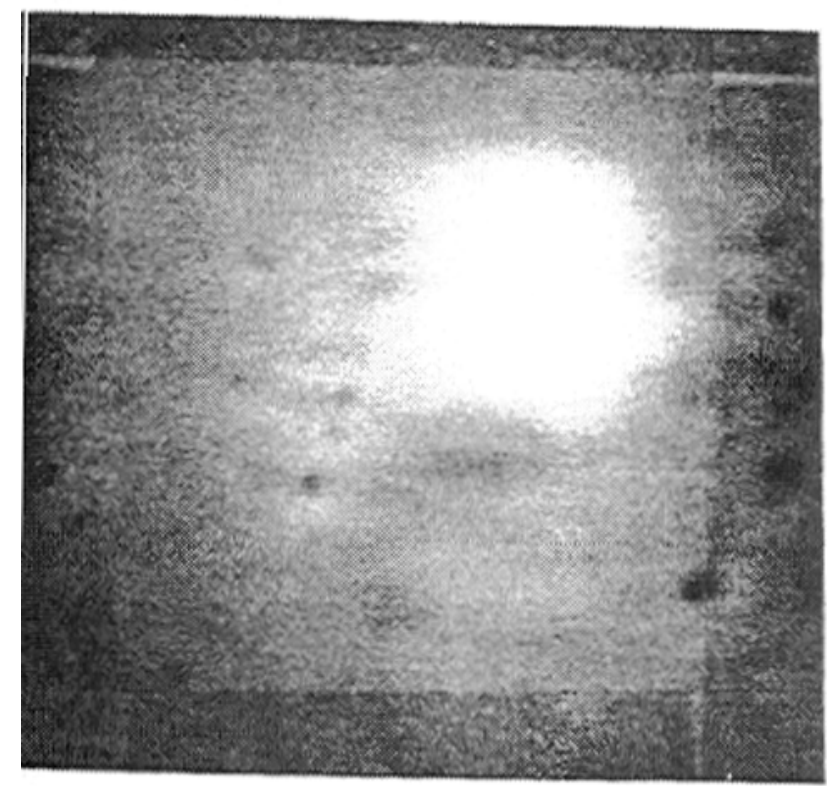

Figure 2.1.5.5 Thermal Image of a 1 inch Square Simulated Defect in the Bondline of a 0.5 inch Thick Laminated Graphite/Epoxy Fabric (Burleigh et al. 1999)

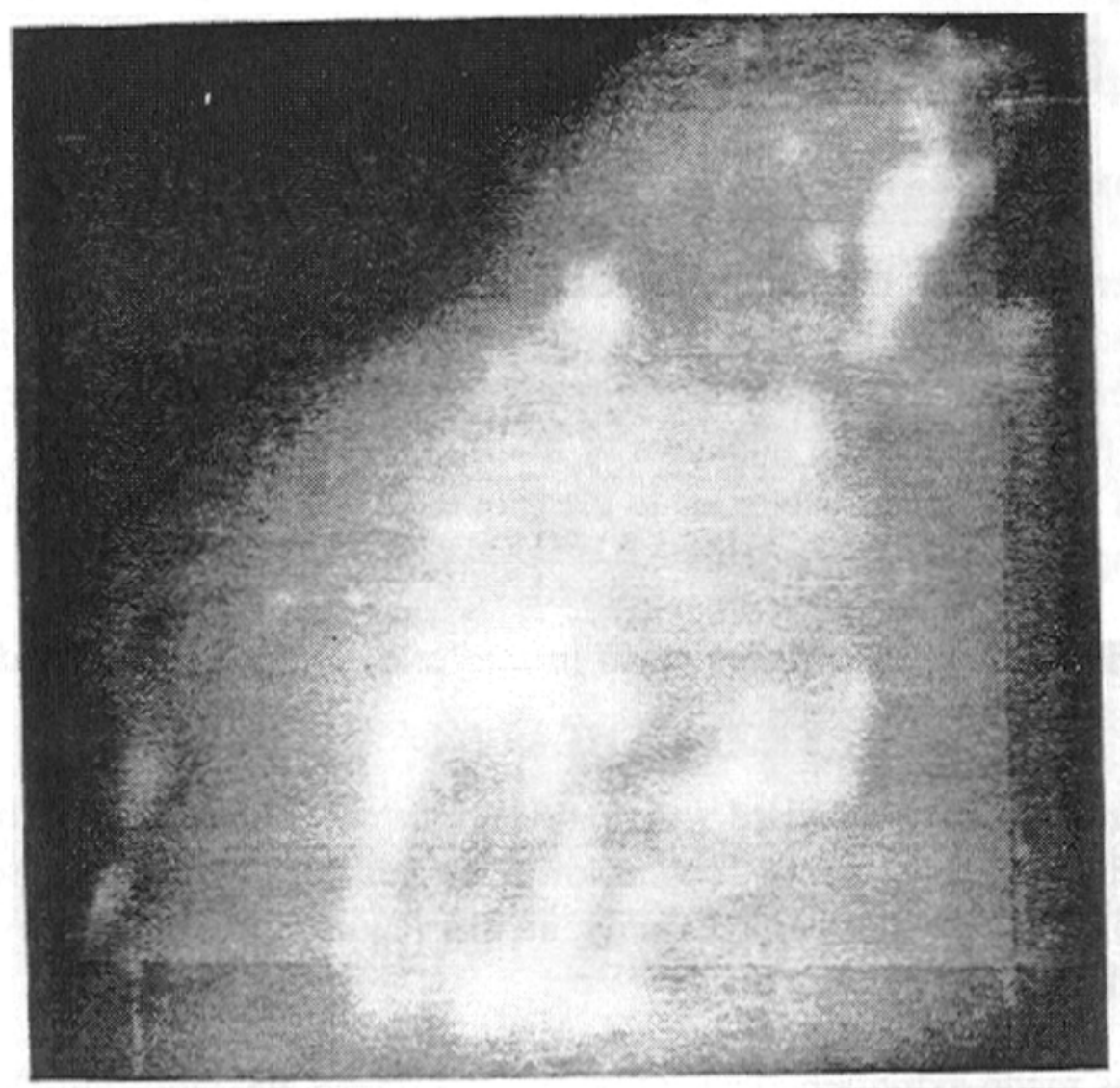

Figure 2.1.5.6 Thermal Image of Delaminations in the Laminated Graphite/Epoxy Fabric (Burleigh et al. 1999) 


\subsection{Laminated Vinyl Ester/Fiberglass Shells}

The shells were placed around the columns of Pier 12, which formed an " $\mathrm{H}$ " configuration. This configuration produced two adhesive bond layers in each joint with different thickness as illustrated in Figure 2.1.5.1. It was concluded that voids in the adhesive layer would appear in the first bondline but not as clear in the second bondline.

After thermographic inspection of several of these joints in the field, only a single one inch void was detected in adhesive bondline closest to the surface. Some disbands were detected in the second bondline, but not with good reliability.

It was concluded that TNDT was not very reliable in detecting debonds and other defects in the laminated vinyl ester/fiberglass shells because it requires thermal energy to pass through the shell, which has variable thickness and thermal properties.

\subsubsection{Conclusions}

TNDT has been experimented with for the evaluation of composite materials bonded to concrete structures, and many NDT standards have been developed for thermographic inspection.

It was demonstrated that as the thickness of the composite increased the minimum detectable disbond size increased. However, the defects found were smaller than the "critical flaw size" for column structures. 
Alternative heating methods need to be developed for future testing because environmental problems made some of the inspections very difficult.

\subsubsection{Conclusion on Infrared Thermography}

Based on the literature review, it was concluded that infrared thermography has many advantages, but also some disadvantages. However, this chapter has indicated that IR thermography has the ability to detect and characterize defects in structures made entirely of composite materials and also structures rehabilitated with FRP wraps. It was also concluded that future research needs to be conducted to ensure that the infrared thermography technique produces reliable results.

It can be concluded that IR thermography is a promising technique for evaluating composite materials and structures. This technique seems to be more effective for evaluating structures that have been wrapped with FRP composites (columns, beams, etc.). IR thermography has proved to be very successful in detecting defects such as disbonds and delaminations close to the surface. However, this technique could be used with other NDT techniques such as ground penetrating radar (GPR) to detect defects deep in the structure as well as defects close to the surface.

The main disadvantage this technique has is the expense of buying an infrared camera and other equipment. 
Other disadvantages include:

- $\quad$ The technique can only be used for short term monitoring and not for realtime monitoring.

- The detection of very small cracks or deep defects is almost impossible.

The advantages of using infrared thermography to evaluate composite materials includes:

- It is a non-contact technique and does not use external sensors and wires.

- $\quad$ The technique is relatively reliable for detecting internal defects of critical size close to the surface.

- Infrared data can be interpreted with relative ease.

\subsection{Ultrasonic Wave Propagation}

Different NDE techniques using ultrasound waves to propagate through composite materials are evaluated in section 2.2. These techniques use high frequency waves (generally greater than $20 \mathrm{kHz}$ ) to locate surface and internal defects in composite materials. The waves are generated by a source (transducer, laser, etc.) and the recorded signal is analyzed to yield information about defects along the propagating path. The parameters of interest, advantages, and disadvantages of the ultrasonic inspection technique will be evaluated and compared to other wave propagating techniques such as acoustic and microwave. 


\subsubsection{Non-Contact Ultrasound Studies of Composite Materials: New Developments}

\section{(Edwards et al. 2000)}

Non-contact ultrasonic inspection is a rapidly growing technique for the evaluation of composite materials. These techniques are being developed worldwide, which include laser generation and optical detection of ultrasound. Section 2..2.1 compares the generation efficiency and damage thresholds of a range of different types of lasers such as the Nd:YAG laser, the TEA $\mathrm{CO}_{2}$ laser, and the ND:YAG laser with an Optical Parametric Oscillator. The material absorbs the laser energy as the wavelength and pulse duration of the laser affects the temperature of the material. However, the laser energy needs to remain in the thermoelastic regime in order not to damage the material. This section talks about a modified Michelson interferometer, which is used to detect the absolute displacement of the ultrasound.

\subsubsection{Introduction}

Laser based ultrasound has become a widely used technique in the aerospace industry for the non-contact ultrasonic inspection of carbon fiber reinforced composites (CFRCs). However, it is also becoming an established technique in other engineering fields as well. Laser generated ultrasound can be produced in the thermoelastic regime or the plasma regime. In the thermoelastic regime, acoustic waves are generated by rapid thermal expansion. Ultrasound in the thermoelastic regime typically does not produce accurate results when testing metals but it is totally non-destructive. Results that are more accurate are achieved when the plasma regime is reached but is not totally nondestructive as a small damage pit typically $\sim 1 \mu \mathrm{m}$ deep is created. 
Thermoelastic regime is typically used in plastics where the laser energy is absorbed in the optical absorption depth $\alpha^{-1}$. Tailoring the laser wavelength to the material efficiency generates longitudinal waves, which have different absorption depths depending on the laser used to generate the waves. An absorption depth of about $100 \mu \mathrm{m}$ is achieved with the TEA $\mathrm{CO}_{2}$ laser, which has a wavelength of $10.6 \mu \mathrm{m}$. This is referred to as a buried thermoelastic source. The Nd:YAG laser, which has a wavelength of $1.06 \mu \mathrm{m}$, would only be weakly absorbed in a much greater depth. While burying the source reduces the maximum surface temperature and enhances the amplitude and directivity of the thermoelastic longitudinal waves, it also results in temporal broadening on the acoustic pulses. Hence, there is an optimum to be reached for a particular material in terms of damage free amplitude and frequency content.

The lasers most commonly used as generate ultrasound are Q-switched Nd:YAG and TEA $\mathrm{CO}_{2}$ lasers (Edwards et al. 2000). The $\mathrm{CO}_{2}$ laser is typically used with CFRCs because its wavelength of $10.6 \mu \mathrm{m}$ produces relatively strong longitudinal waves from a thermoelastic source, which does not damage the material. Section 2.2.1 investigates laser-generated ultrasound in CFRC samples at different laser wavelengths.

\subsubsection{Experimental Details}

Section 2.2.1 examines laser generation in CFRC samples using three different lasers, a TEA $\mathrm{CO}_{2}$ laser, a fundamental Nd:YAG, and a new Nd:YAG laser with an Optical Parametric Oscillator (OPO). Neutral density filters were used to reduce the beam energy of the Nd:YAG laser so non-destructive testing could be performed. A 24 
$\mathrm{cm}$ focal length silica lens focussed the Nd:YAG laser beam onto the sample after the laser spot sizes were measured. The laser spot sizes produced "burn marks" on photographic paper, which could then be measured with a microscope. For the $\mathrm{CO}_{2}$ laser an adjustable iris, silica wafers and a $20 \mathrm{~cm}$ focal germanium lens was used to focus the beam and the spot size was measured from "burn marks" on perspex paper coated with a thin layer of black paint. The OPO laser used silica lens to focus the beam, while the spot size and laser power were estimated from manufacture's manual. All the data was captured on a LeCroy 9310 digital storage oscilloscope. The experimental setup is shown in Figure 2.2.1.1.

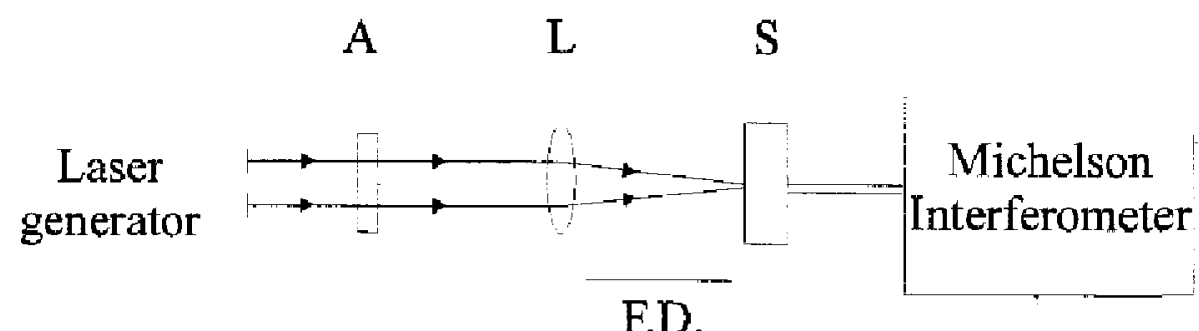

$$
\begin{aligned}
& A=\text { Attenuator and Iris } \\
& L=\text { Focusing lens } \\
& \text { F.D. = Focal Distance }
\end{aligned}
$$

F.D.

Figure 2.2.1.1 Experimental set up for measuring epicentral displacements (Edwards et al. 2000)

\subsubsection{Results}

\subsection{Nd:YAG Laser}

The YAG laser with energy of $6.2 \mathrm{~mJ}$ produced damage to the sample when the beam was progressively focused to smaller spot sizes. Figure 2.2.1.2 shows typical epicentral waveforms above and below the damage threshold. Figure 2.2.1.3 is a plot of 
the first longitudinal spike amplitude and laser fluence. The risetime of the longitudinal spikes was between $20-30 \mathrm{~ns}$.

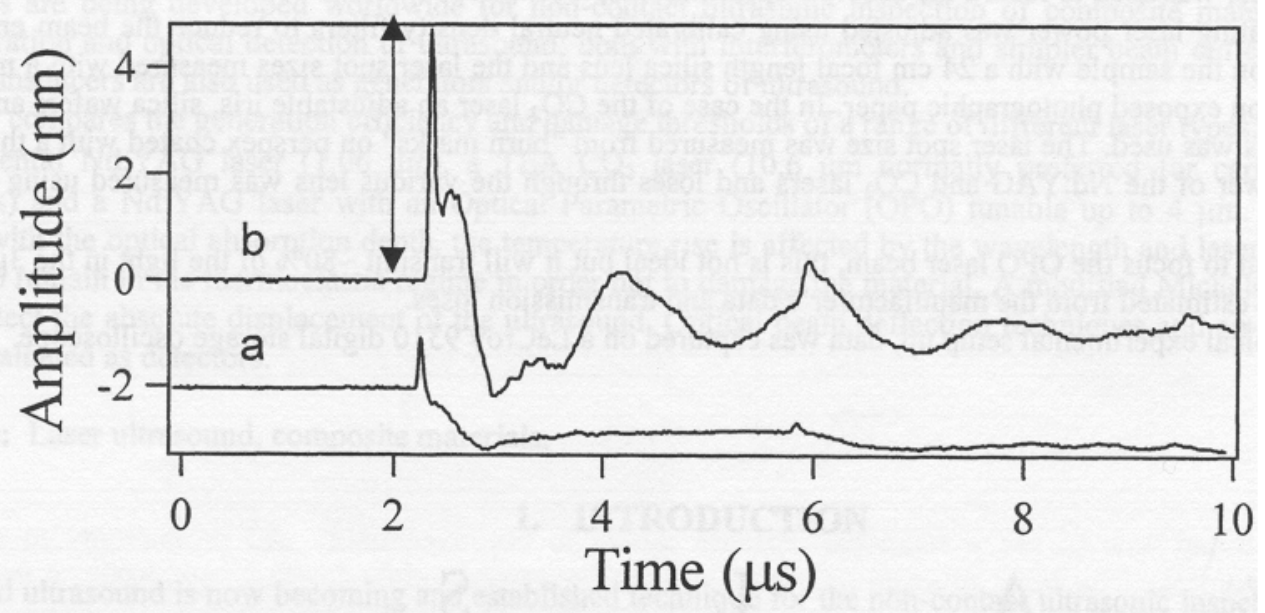

Figure 2.2.1.2 Nd:YAG epicentral waveforms, (a) buried thermoelastic source and (b) above damage threshold (Edwards et al. 2000)

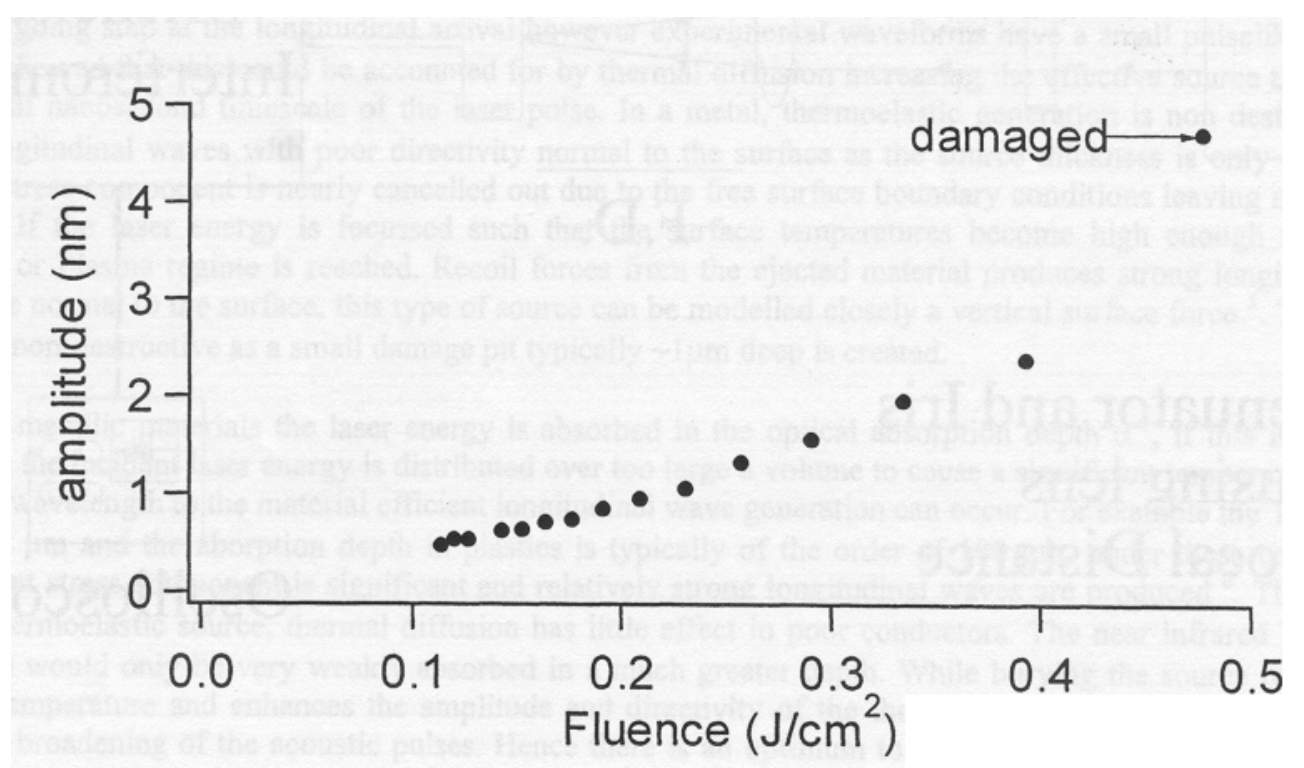

Figure 2.2.1.3 Longitudinal displacement versus laser fluence (energy of 6.2 mJ) (Edwards et al. 2000)

\subsection{TEA $\mathrm{CO}_{2}$}

An adjustable iris and two silicon wafers were used to reduce the power of the TEA $\mathrm{CO}_{2}$ laser to make sure the energy would remain below the damage threshold. Several measurements were taken with different aperture sizes and focal distances. 
Finally, one of the silicon wafers was removed and some measurements were recorded above the damage threshold. Figure 2.2.1.4 shows epicentral waveforms above and below the damage threshold using a $11 \mathrm{~mm}$ aperture. Figure 2.2.1.5 shows the longitudinal displacement using 9,10 , and $11 \mathrm{~mm}$ iris aperture sizes. The risetimes of the longitudinal spikes was typically $40-60 \mathrm{~ns}$. When damage occurred, the risetimes increased to over $70 \mathrm{~ns}$.

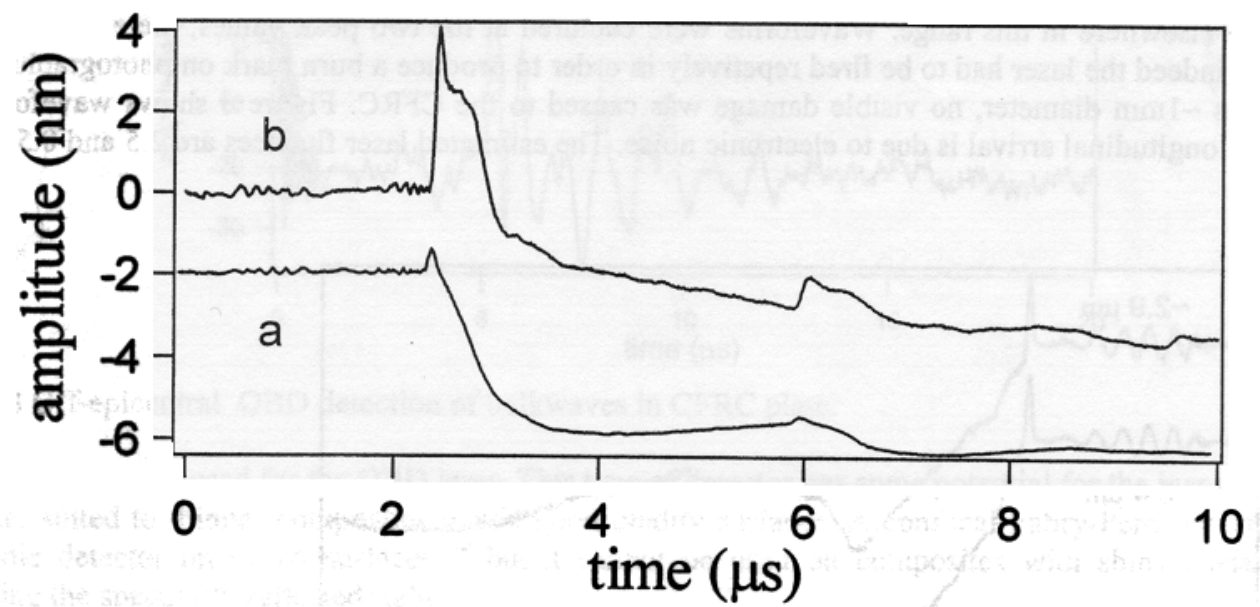

Figure 2.2.1.4 TEA $\mathrm{CO}_{2}$ epicentral waveforms, (a) buried thermoelastic source and (b) above damage threshold (Edwards et al. 2000)

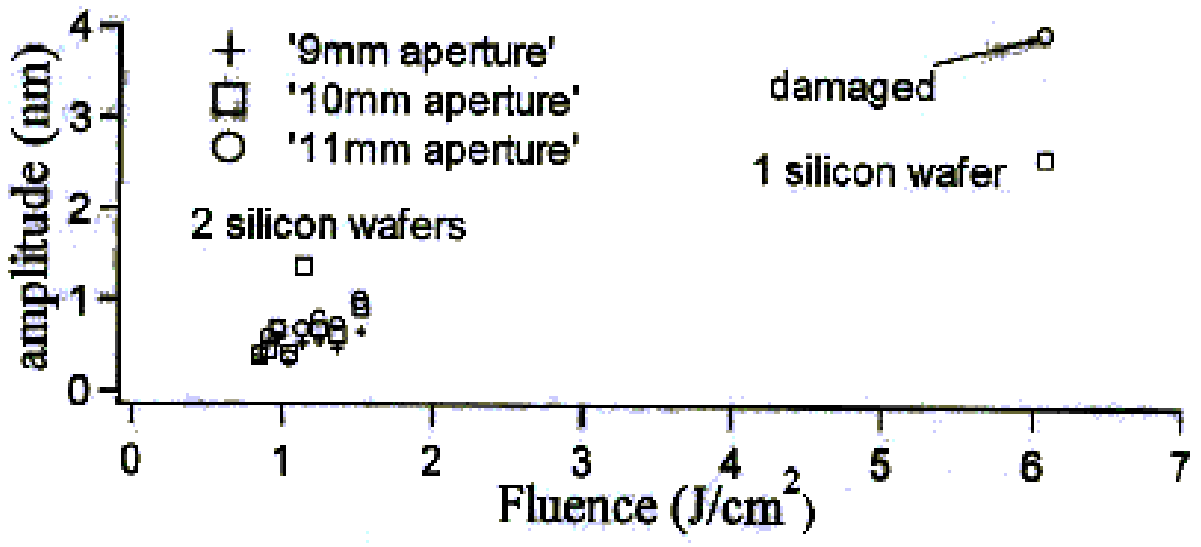

Figure 2.2.1.5 Longitudinal displacement versus laser fluence for 9, 10, and $11 \mathrm{~mm}$ iris aperture sizes (Edwards et al. 2000) 


\subsection{OPO}

Optic Parametric Oscillators are passive non-linear optical devices, which are used with pump lasers to convert the output beam into two discrete beams, a signal beam and idler beam (Edwards et al. 2000). The energy of the combined beams equals that of the initial pump beam. Several measurements were taken with different laser wavelength, which was altered by micrometer adjustments of the OPO. The output power was reduced as the wavelength was increased. The power associated with the different wavelengths was estimated from the manufacturer's data. Two peaks in the generation were found upon tuning the OPO. These peaks occurred at a wavelength just below $3 \mu \mathrm{m}$ and at a wavelength between 3.3 and $3.5 \mu \mathrm{m}$; however, the exact wavelengths could not be measured. Waveforms were captured at the two peak values and there was no visible damage to the composite. Figure 2.2.1.6 shows the waveforms. The periodic signal before the longitudinal arrival is due to electronic noise (Edwards et al. 2000).

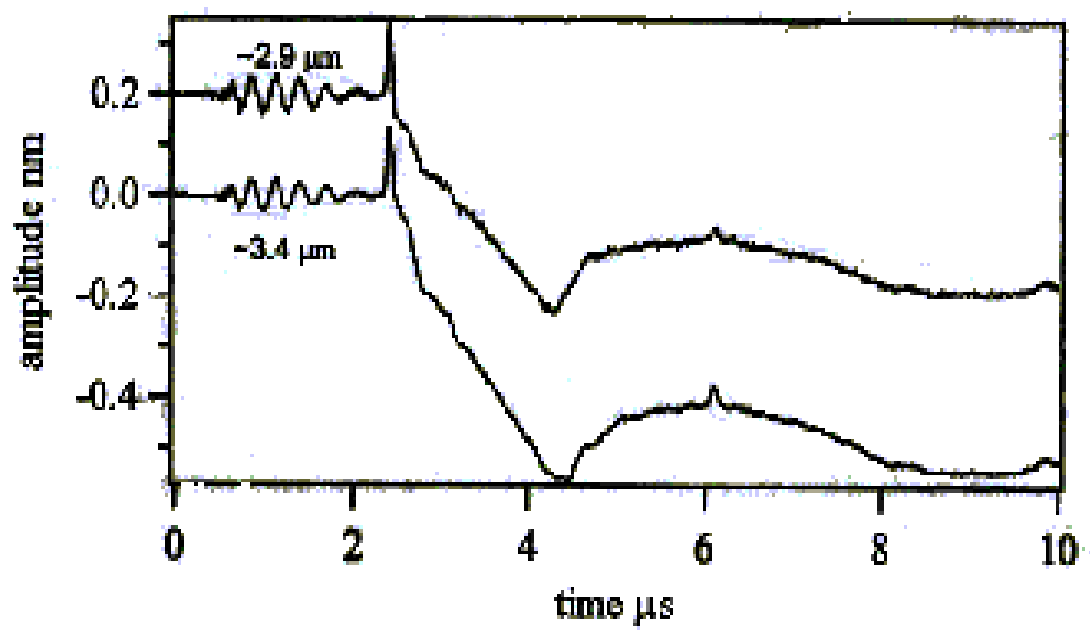

Figure 2.2.1.6 Epicentral waveforms generated at the absorption peaks for the CFRC (Edwards et al. 2000) 


\subsection{OBD}

Ultrasonic waves can cause deflections in a laser beam to reflect off the sample surface, the reflected the laser beam can be captured by a photodiode, which enables the surface tilt to be monitored as shown in Figure 2.2.1.7. The light can then be monitored using a split photodiode fed into a differential pre-amplifier usually used for detecting Rayleigh or Lamb waves. This gives zero output when the reflected beam is centered on the detector, which makes it easy to find the large amplitude, low frequency tilts due to background vibrations.

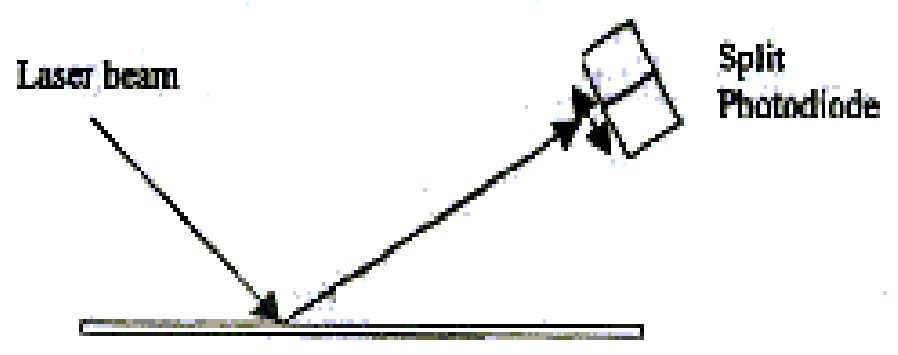

Figure 2.2.1.7 Principle of an Optical Beam Deflection detector (Edwards et al. 2000)

The $200 \mathrm{~mJ} \mathrm{Nd}$ :YAG beam was focussed into a $20 \times 0.5 \mathrm{~mm}$ thick plate line source on a $7 \mathrm{~mm}$ thick CFRC plate. Figure 2.2.1.8 shows the detected waveform. The complexity is due to the highly anisotropic CFRC. The large peak at $5 \mu \mathrm{s}$ is the shear wave. 


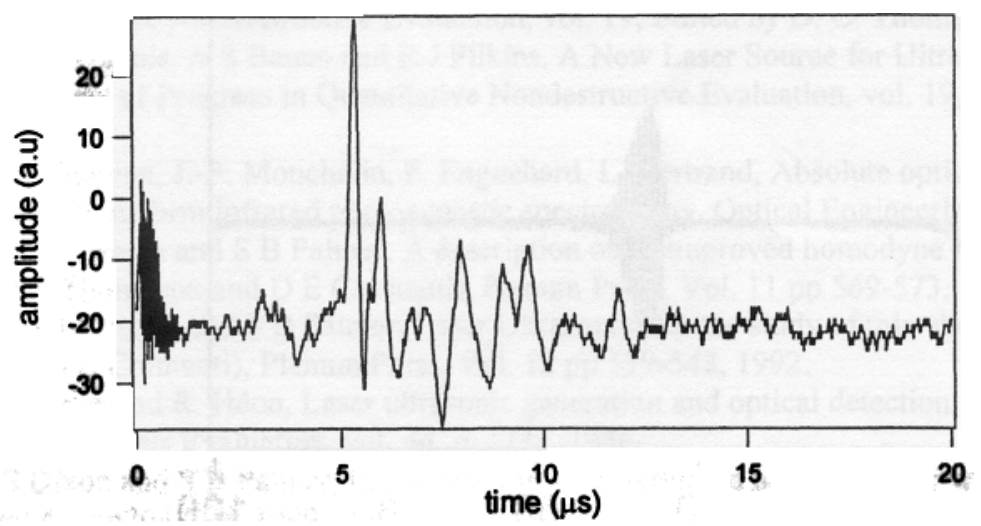

Figure 2.2.1.8 Off-epicentral OBD detection of bulk waves in CFRC plate (Edwards et al. 2000)

A $10 \mathrm{~mW}$ HeNe was used as the OBD laser. This type of detector was determined to be better for the inspection of thinner composites with good quality surfaces.

\subsection{Air Coupled Detection}

Air-coupled ultrasonic detectors also proved to be good detectors of laser generated ultrasound. Standard piezoelectric transducers were used in these experiments performed by Edwards et al. A thin layer of rubber was placed on the transducers to reduce them detection of large acoustic waveforms in the air. When coupled with low noise amplification, ultrasound at a frequency of $\sim 1 \mathrm{MHz}$ could be detected after propagating an appropriate distances through the air. Figure 2.2.1.9 illustrates several echoes that were detected from a CFRC plate by a transducer placed $35 \mathrm{~mm}$ from the plate.

The same transducer was then used as a detector for $\mathrm{CO}_{2}$ generated ultrasound. This experimental setup used a silicon wafer to reduce the beam energy so that the sample was not damaged. The beam was focused to a $10 \times 7 \mathrm{~mm}$ source and the detector was placed $20 \mathrm{~mm}$ away from the sample. Time averaging was used to remove electronic 
noise radiated by the generation laser. Figure 2.2.1.10 shows the detected waveform in a through transmission arrangement.

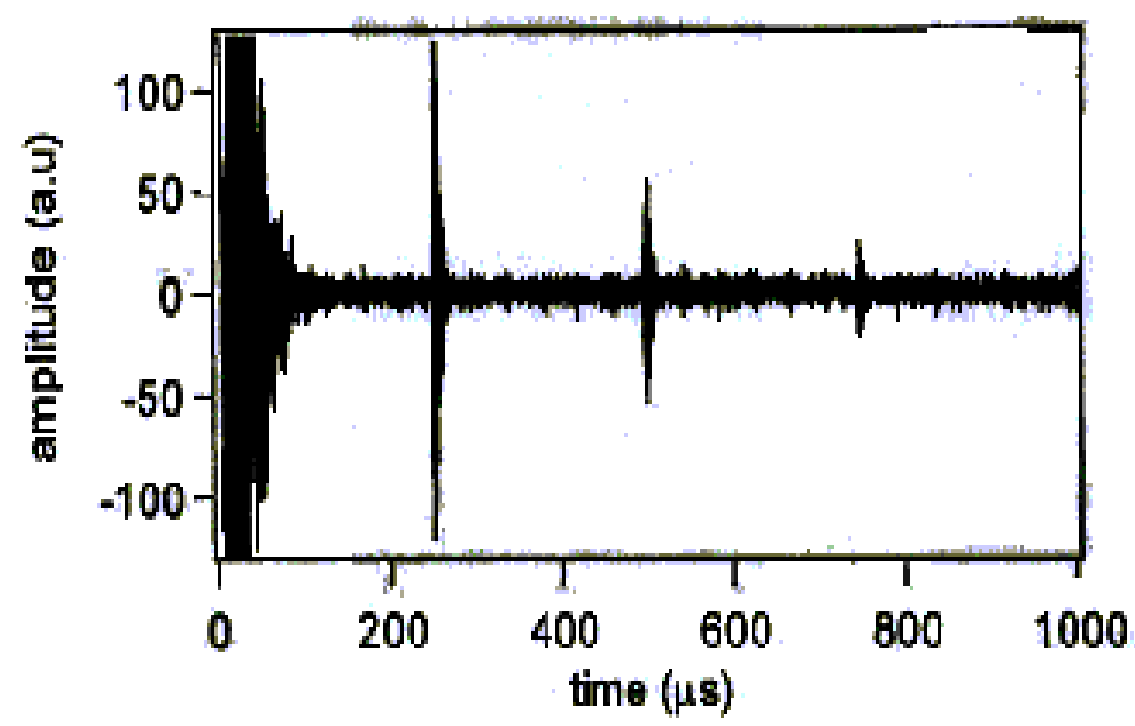

Figure 2.2.1.9 $1 \mathrm{MHz}$ send receive air coupled ultrasound echoes from a CFRC plate (Edwards et al. 2000)

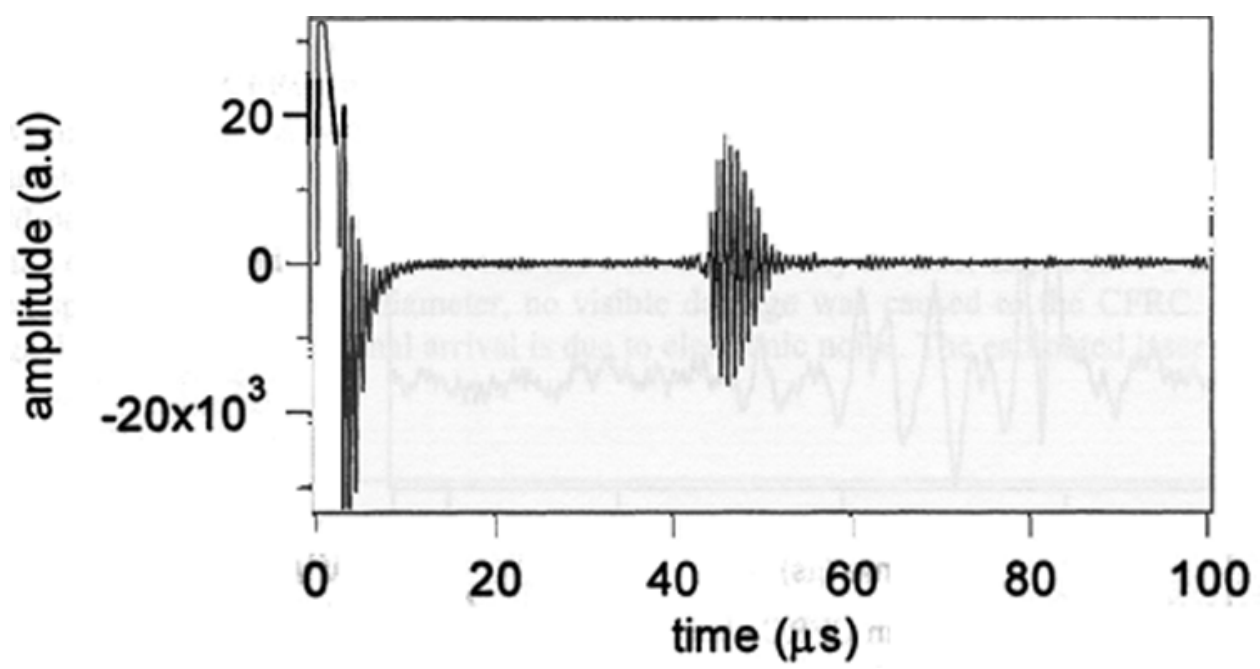

Figure 2.2.1.10 TEA $\mathrm{CO}_{2}$ generated-air coupled detected wave propagated through a $4 \mathrm{~mm}$ thick CFRC Plate (Edwards et al. 2000) 


\subsubsection{Discussion and Conclusion}

The Nd:YAG laser and the TEA $\mathrm{CO}_{2}$ laser were both used to generate nondestructive longitudinal waves of large amplitude in CFRCs. However, it was concluded that the Nd:YAG laser was more efficient for generating non-contact ultrasound in CFRCs (Edwards et al. 2000). It was also confirmed that a tunable OPO laser could be used to generate ultrasound in CFRCs and the absorption peaks observed in the epoxy matrix may be used to evaluate material properties in the future.

Detecting the ultrasound in CFRCs is the next step in developing OBD's and air coupled ultrasound transducers to be used in place of interferometers.

\subsubsection{Ultrasonic Evaluation of Co-Cured Composite Structures (Georgeson, G. E. 2000)}

The aerospace industry has placed significant emphasis upon the development of new composite manufacturing methods. The "Co-curing" of composites has become one of the processes in which non-destructive evaluation is of great interest. The aerospace industry has started using a multi-scan ultrasonic testing (UT) approach for the evaluation of these co-cured composite structures, which could evolve into an approach for evaluating other composite structures such as bridge decks and beams. This section will describe the general approach, as well as the specific techniques of this multi-scan ultrasonic NDT. 


\subsubsection{Introduction}

In co-curing, two or more composite elements are bond or cured together to make a single structure. This technique eliminates fasteners in elements to produce integral joints. This technique is popular in the aerospace industry now but could be an alternative method in the civil engineering industry because there are few limits to the kinds of composite structures that can be co-cured (Georgeson, G. E. 2000).

While there are many advantages to co-curing, there is the disadvantage of inspecting these sections. This is because individual parts can no longer be inspected separately; the entire structure must be handled as a unit, which can be quite difficult to inspect if the structure is large. The areas that are relatively complex and require inspection become much more difficult to reach as well. In a structural design, the stress is often designed to transfer through the joints; therefore, it is critical to identify all joint flaws in the co-cured structure.

\subsubsection{Co-Cured Non-Destructive Inspection}

The difficult inspection of these co-cured composite structures can be simplified through the modified application of UT technology. The UT evaluation for co-cured composite structures, developed by Boeing, combines through-transmission ultrasonics (TTU) and pulse-echo (P/E) ultrasonics. Figure 2.2.2.1 is a schematic of how the two techniques can be used together for evaluating various regions of a structure. 


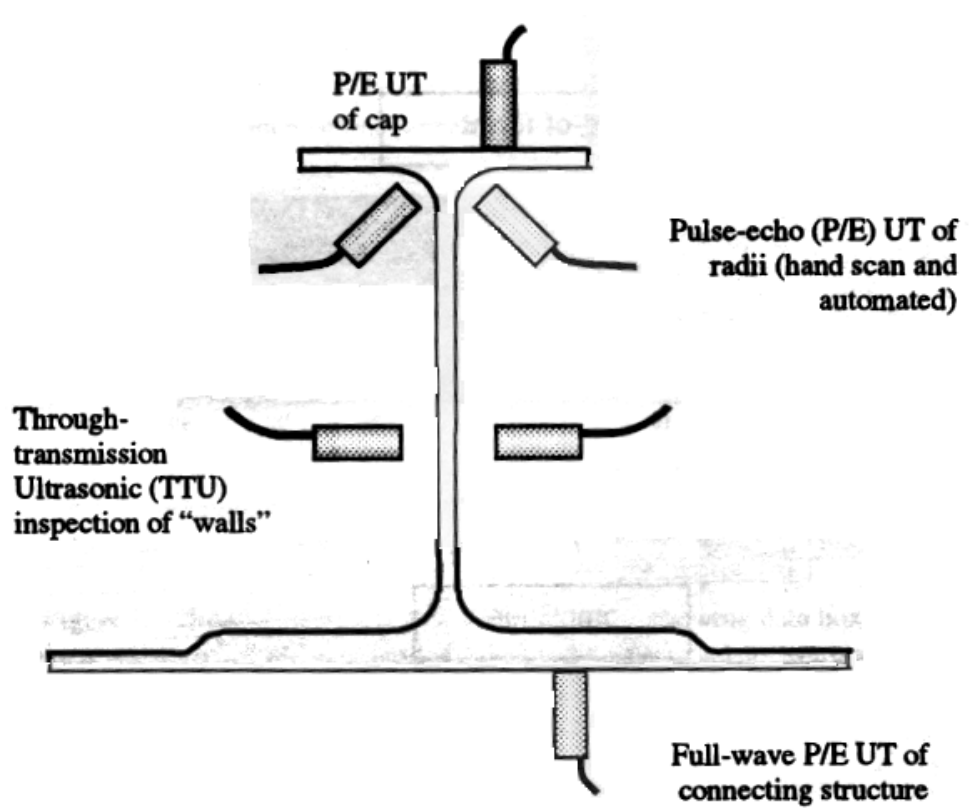

Figure 2.2.2.1 UT methods for inspecting co-cured structure (Georgeson, G. E. 2000)

\subsection{Two-Sided Inspection}

Where transducers can be placed on either side of a structure, TTU is used for inspection. With TTU, the transducer transmitting the ultrasonic signal (transmitter) and the receiving transducer (receiver) are on opposite sides of the section to be inspected. The signal, emitted by the transmitter, goes through the part being inspected to the receiver and measured using a data-collection system.

A flaw in the material between the two transducers will cause a reduction in the signal (attenuation) when it reaches the receiver. If multiple signals are combined incrementally, an image corresponding to the defect can be produced.

\subsection{One-Sided Inspection}

While TTU works well for sections with access to both sides, it is impossible to use for co-cured structures with access to only one side. The pulse-echo (P/E) ultrasonic 
inspection is often done to regions with limited access, allowing for a more complete inspection.

Most connecting structures can only be accessed from one side. The pulse-echo technique can measure the "amplitude" of the signal, which will decrease if the signal is scattered by low levels of porosity, or increase if it is reflected back to the transducer from a delamination or very high levels of porosity. The "time of flight" is also measured using the pulse-echo technique, which is the time it takes for ultrasound to exit the transducer, enter the part, reflect off the back surface or a flaw, and return to the transducer. The time of flight is often used to find depths of flaws in materials.

Some inspections of co-cured joints may require different orientations of the transducer receive a clear image of the flaw. The angles are generally found through trial and error. Figure 2.2.2.2 shows how orienting the transducer at an angle can image cracks perpendicular to the inspection surface. 


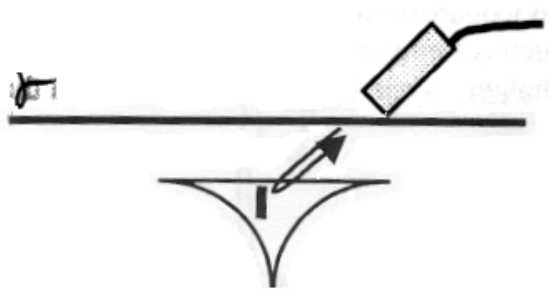

Crack perpendicular to scan surface

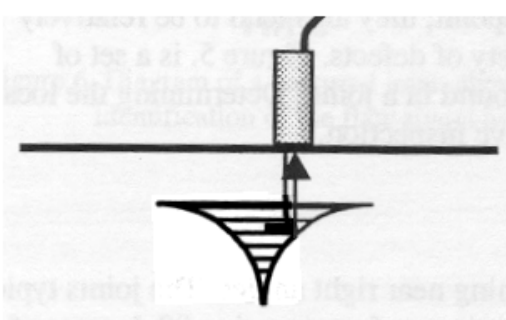

Crack paraillel to scan surface

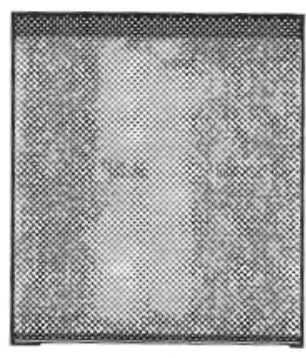

UT amplitude

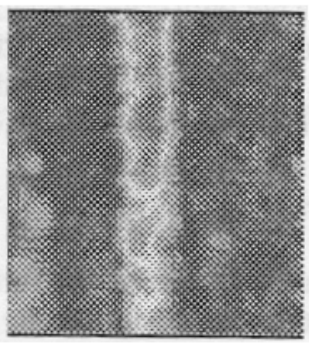

UT amplitude

Figure 2.2.2.2 Examples of the different inspection orientations that can be required to find flaws of different orientations. The images on the right are the corresponding P/E UT amplitude scans of joints containing cracks (Georgeson, G. E. 2000)

\subsection{Joint Inspection}

The co-cured joints are usually the most important regions of inspection because the stress is often transferred there. Flaws or damage in the joints can reduce the strength of the entire structure. Therefore, identify all flaws in the joints a very critical part of the inspection.

\subsubsection{3(a) Potential Joint Defects}

The types of defects found in co-cured structures include disbonds, delaminations, cracks, porosity, voids, and inclusions (similar to those found in the more traditional composite structures). Co-cured joints can be relatively complex. This complexity can cause a variety of defects, which is the reason for such thorough inspection. Figure 2.2.2.3 is a set of photomicrographs that illustrates some of defects 
that might be found in a joint. The more precise the location, size, and orientation of the flaws detected through inspection, the easier it is to correct.

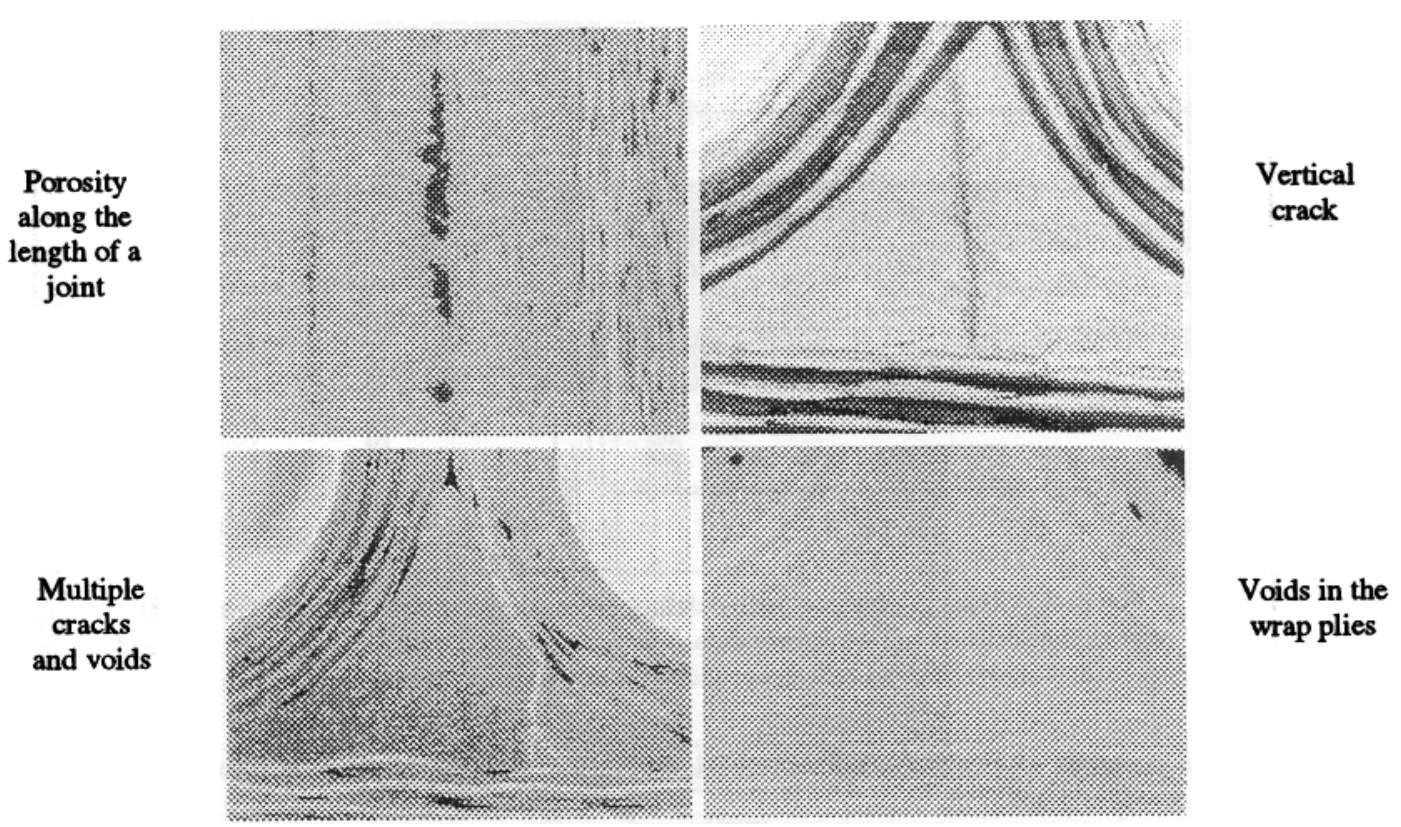

Figure 2.2.2.3 Examples of joint flaws found in co-cured structures (Georgeson, G. E. 2000)

\subsubsection{3(b) Hand-Held Radius Inspection}

A typical joint is a fillet with a radius of curvature ranging from $1 / 8$ to $1 / 2$ inch between two surfaces meeting and joining near right angles. Some of the co-cured joints cannot be thoroughly inspected using P/E UT; therefore, the inspection is done by positioning a transducer to send and receive a signal from the radius corner with the signal reflecting off flaws in the interior. Figure 2.2.2.4 illustrates the typical cross section of a co-cured joint. The ultrasonic beam must inspect the entire $90^{\circ}$ of the arc so the entire interior of the joint is covered and echoes from all flaws are generated. 

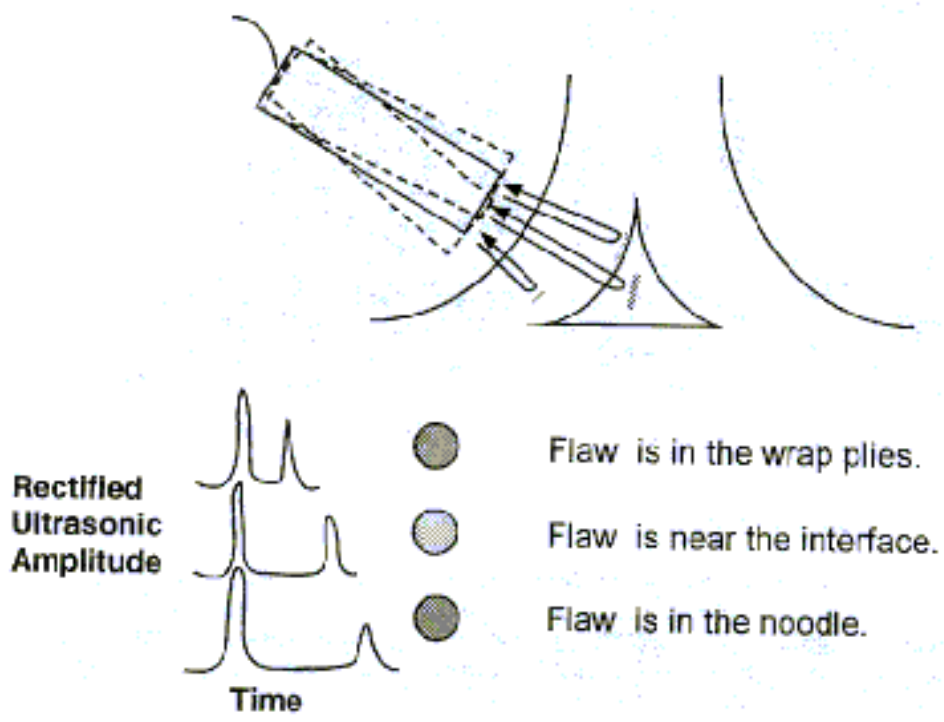

Figure 2.2.2.4 Diagram of a co-cured joint, showing potential locations of flaws. Rotation of the transducer and identification of the flaw signal in time are necessary for locating and sizing of the flaws (Georgeson, G. E. 2000)

An effective non-automated method for inspecting the smallest co-cured joints has been found. This method involves inspection at various angles of rotation. A radius shoe with a hole at the end is attached to the transducer, which uses standing water as the couplant into the joint. This allows for a smooth rotation in the radius up to almost $90^{\circ}$. The transducer is moved along the length of the joint at various angles to ensure thorough inspection of the joints.

\subsubsection{3(c) Automated Radius Scanning}

Hand-held inspection can be time-consuming and expensive. Automated data has proven to be more complete because it produces images and can be stored. The major benefit of automated scanning is that the joints of some structures are not assessable by hand especially those structures that are too large, but can be inspected with the automated scanning technique. 


\section{Straight Joint Inspection Fixture}

For straight joints, a simple ultrasonic test with the help of robotic scanners that would address an immediate need for automated inspection of the radii was developed. The device was attached to the end of a robotic arm, which only requires two axes of motion to function, but allows almost $90^{\circ}$ rotation within a radius. Figure 2.2.2.5 is an UT amplitude image of a joint produced from this fixture for straight joints in co-cured parts.

\section{Curved Joint Inspection Device}

For large co-cured curved joint inspections, a probe that uses mechanical means to generate a rotating ultrasonic beam was used. The ultrasonic beam from a fixed transducer was caused to reflect off a mechanically rotating ultrasonic mirror, formed by cutting a $1 / 4$ inch diameter steel rod at $45^{\circ}$. The rod rotating about its long axis, geared by a DC motor through a timing belt, provided data acquisition pulses keyed to the mirror rotation by connecting a second timing belt to an optical encoder to the motor.

Again, water was used as a couplant being supplied to the transducer and mirror by internal water passages within the probe. A large-scale gantry robot rotated the probe down the length of the curved joint. The robot produced different data acquisition positions as it moved the probe down the length of the joint. A robot is typically used to move the probe down the length of the curved joint so the probe and rotating mirror can be easily positioned for complete inspection of the structure. 
A PC running under Windows 98 acquires the data. A $100 \mathrm{MHz}$ analog to digital conversion board is used to enter ultrasonic data into the PC.

Advantages to this approach include the ready access to all areas needing inspection. In addition, some co-cured structures need to be inspected with a probe mounted on a large-scale robot in order to reach curved joints in the interior because the size of the structure is so large.

\subsubsection{Conclusion and Summary}

This section discusses a multi-scan ultrasonic technique approach for inspecting co-cured composite structures by combining through-transmission ultrasonic and pulseecho ultrasonic techniques. Also, this section proves that large co-cured structures can be inspected with the use of large-scale robots to mount the probes.

\subsubsection{Nondestructive Assessment of the Post-Impact Properties of FRP Composite}

\section{Laminates (Park, S.W. and Zhou, M. 2000)}

Section 2.2.3 discusses the use of nondestructive ultrasonic techniques to assess the mechanical properties of fiber-reinforced polymer (FRP) composites subjected to low-velocity impact. FRP samples impacted at different energy levels are used with an ultrasonic technique to determine the through-thickness wave speeds and the meansquare amplitudes of induced transverse vibration. The post-impact residual strengths of FRP samples subjected to quasi-static extension where also determined. 


\subsubsection{Introduction}

Fiber-reinforced polymer composites are being used in a wide range of engineering applications despite their susceptibility to impact damage. More and more structures are involving FRP composite laminates, especially bridge structures. Since these structures are likely to encounter impacts by foreign objects in their service life, nondestructive evaluation is becoming essential to identify the amount of impact-induced damage and its effect on the residual strength and stiffness of the structure (Park, S.W. and Zhou, M. 2000).

Two types of composites, carbon and glass, were used for the experiments. The dimensions of the tested samples were $152.4 \mathrm{~mm} \times 19.05 \mathrm{~mm} \times 4.47 \mathrm{~mm}$ for the carbon composite and $152.4 \mathrm{~mm} \times 19.05 \mathrm{~mm} \times 3.78 \mathrm{~mm}$ for the glass composite. The samples were impacted using a split Hopkinson pressure bar coupled with a three-point bend loading fixture. The impacted samples were tested ultrasonically. Once ultrasonic testing was performed, quasi-static extension was applied to determine the residual tensile strengths of the samples.

\subsubsection{Nondestructive Ultrasonic Experiments}

Two ultrasonic experiments were conducted on the samples. First, to experiment with the through-thickness wave propagation speed of the damaged samples and second, to experiment with the transverse vibratory response of the impacted specimens. 


\subsection{Through-Thickness Wave Speed Measurement}

A change in the mechanical property of a material (e.g., stiffness, density) caused by a defect or damage in the material will change the propagation speed of mechanical waves through the material. This measurement of wave propagation velocity allows the effects of damage in the material to be conveniently assessed using an ultrasonic measurement. Figure 2.2.3.1 is a schematic of an ultrasonic through-transmission test conducted to measure the longitudinal wave propagation speed of an impact-damaged specimen in the thickness direction. Silicon gel is used to enhance coupling between the PZT transducers and the test samples. The transducers used had a peak frequency of 2.5 $\mathrm{MHz}$ and a bandwidth of $1 \mathrm{KHz}-35 \mathrm{MHz}$ with a 1-MHz single pulse. Figure 2.2.3.2 shows a sample excitation and response signal measured from an impacted carbon FRP sample. The average wave speed of a damaged section was determined by dividing the sample thickness by the measured time it took for a wave to propagate through the thickness.

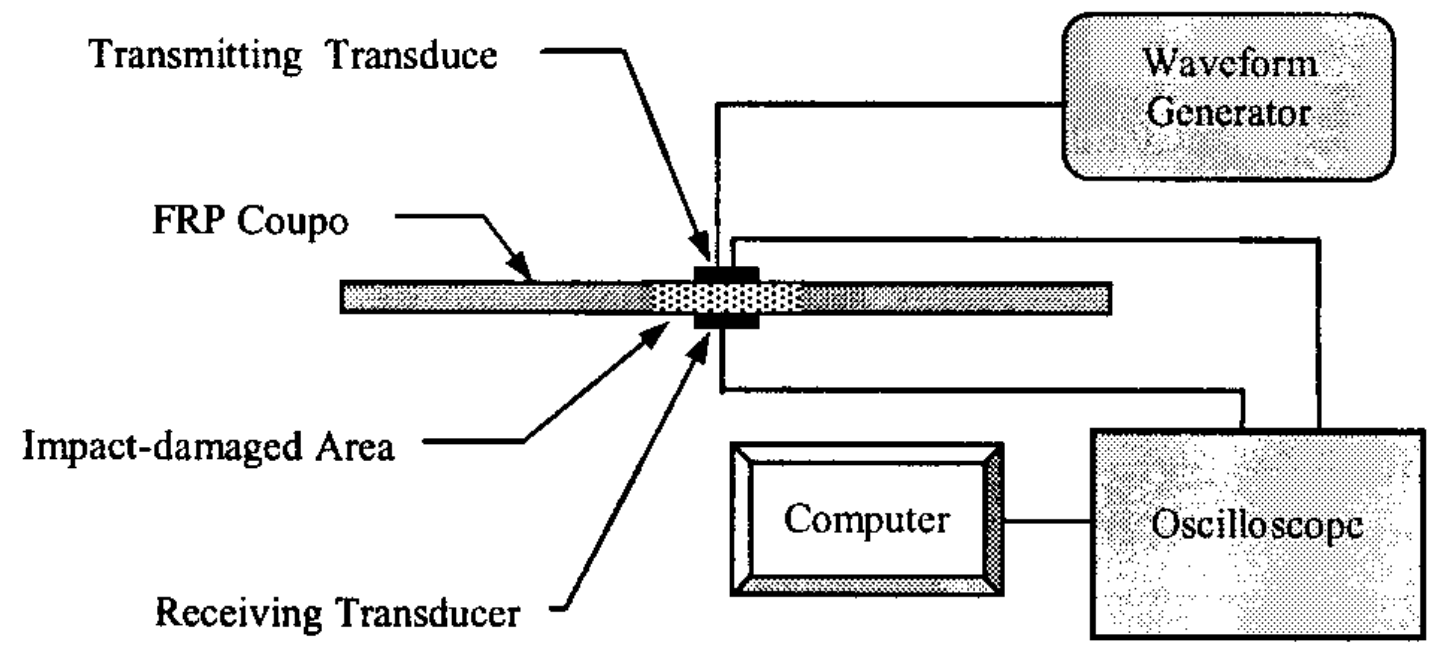

Figure 2.2.3.1 Configuration for through-thickness wave speed measurement test (Park, S.W. and Zhou, M. 2000) 


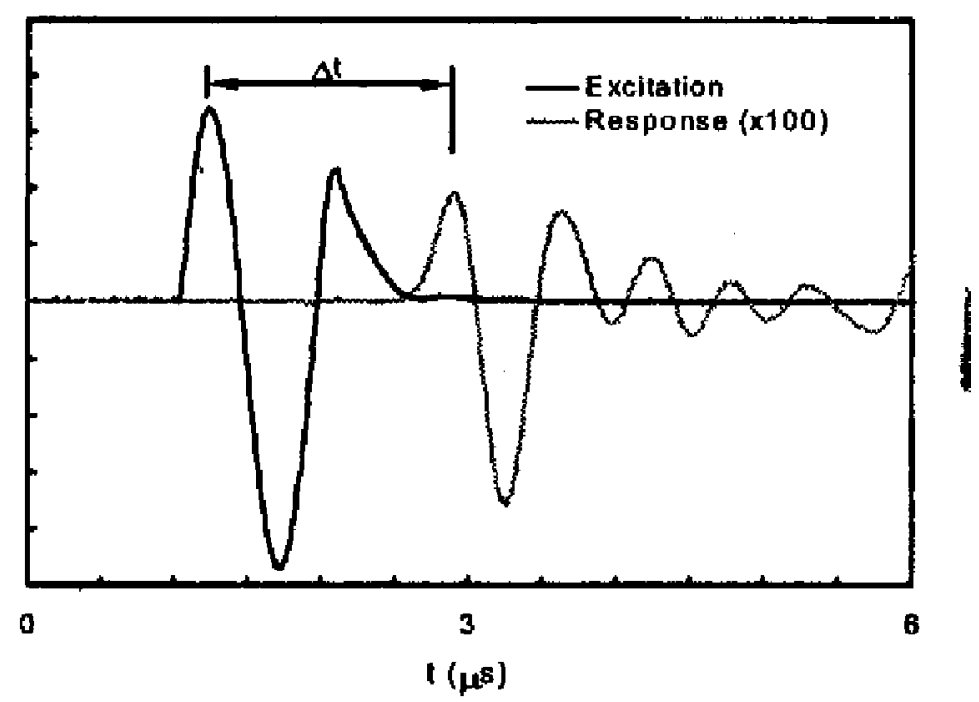

Figure 2.2.3.2 Sample through-thickness excitation and response (Park, S.W. and Zhou, M. 2000)

\subsection{Transverse Vibratory Response Measurement}

Another way to determine a change in structural integrity of a member is to observe changes in the transverse vibratory response of a structural member. The change in the amplitude of induced transverse vibration of impacted specimens was used as an effective parameter to find correlations between acousto-ultrasonic measurements and damage development in graphite-epoxy laminates. Figure 2.2.3.3 illustrates the configuration for transverse vibration measurement tests conducted in a previous experiment. Transmitting and receiving transducers are placed across the damaged section of a simply-supported specimen as illustrated in Figure 2.2.3.3. An excitation frequency of $0.3 \mathrm{MHz}$ with a peak frequency of $2.5 \mathrm{MHz}$ was selected to induce maximum vibration amplitude. Figures 2.2.3.4(a) and (b) show the transient response and the corresponding amplitude spectrum from the carbon FRP sample. The transient waveform for each specimen is obtained through Fast Fourier Transform (FFT). 


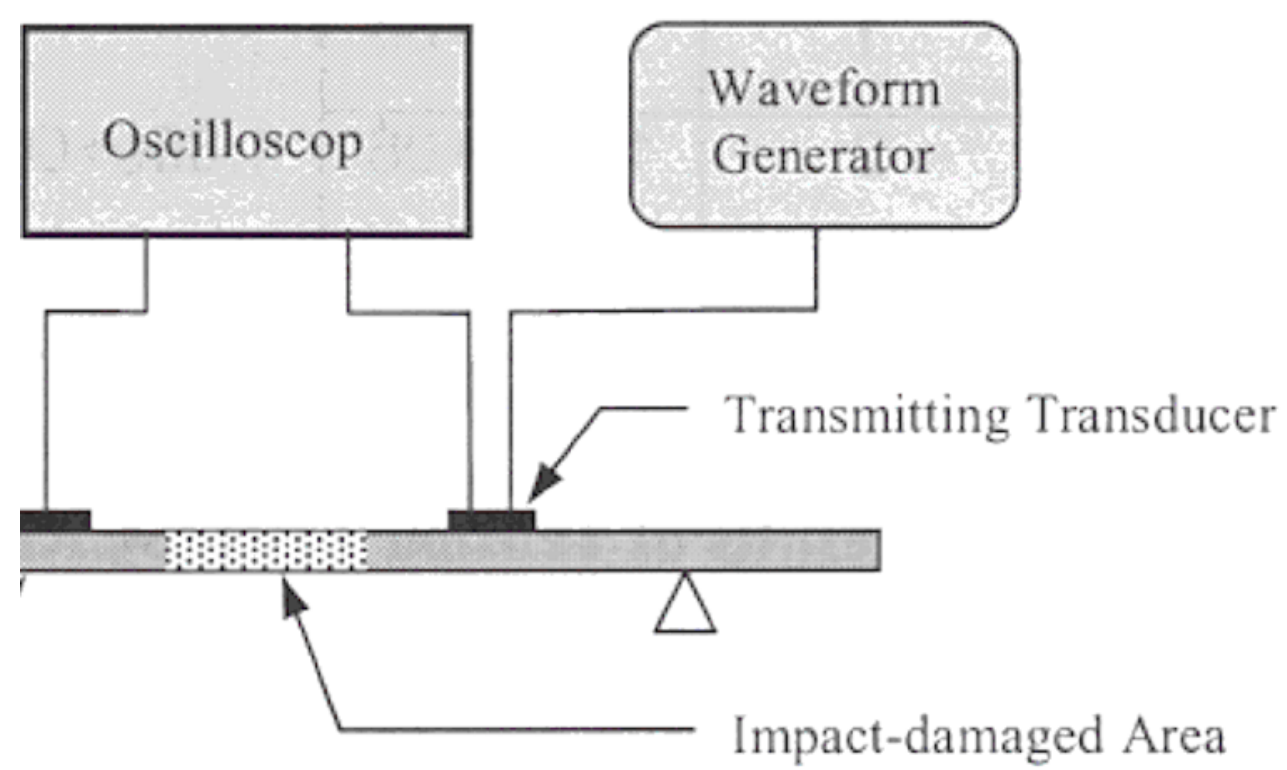

Figure 2.2.3.3 Configuration for transverse vibration measurement test (Park, S.W. and Zhou, M. 2000)

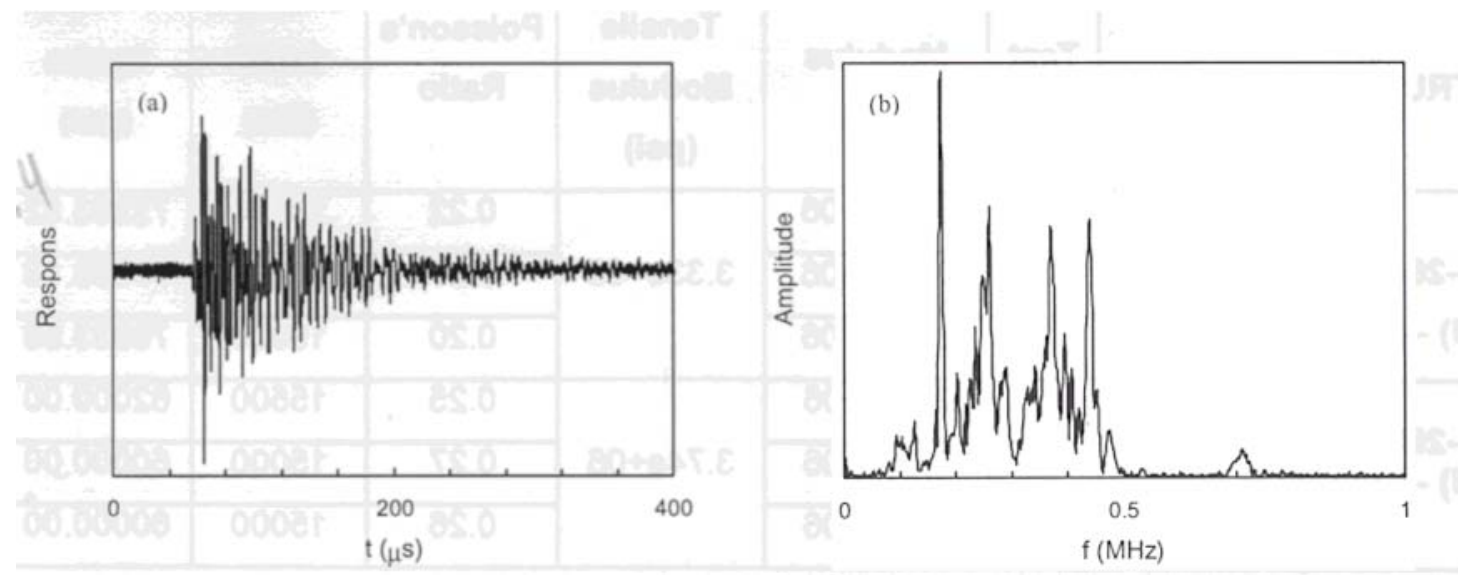

Figure 2.2.3.4 (a) A sample transverse vibration response; (b) the corresponding frequency-amplitude spectrum (Park, S.W. and Zhou, M. 2000)

\subsubsection{Experimental Results and Discussion}

Figure 2.2.3.5(a) plots the measured through-thickness wave propagation speed as a function of impact energy. From Figure 2.2.3.5(a) we observe that wave speed decreases with increasing impact energy, and with decreasing wave speed the normalized residual strength of the material decreases as illustrated in Figure 2.2.3.5(b) (post-impact residual strength plotted as a function of through-thickness wave speed). A definite correlation between the residual strength and wave speed can be observed in the plots. 
This indicates that the through-thickness wave speed could be used to estimate the effects of impact-induced damage on the residual strength of FRP laminates.
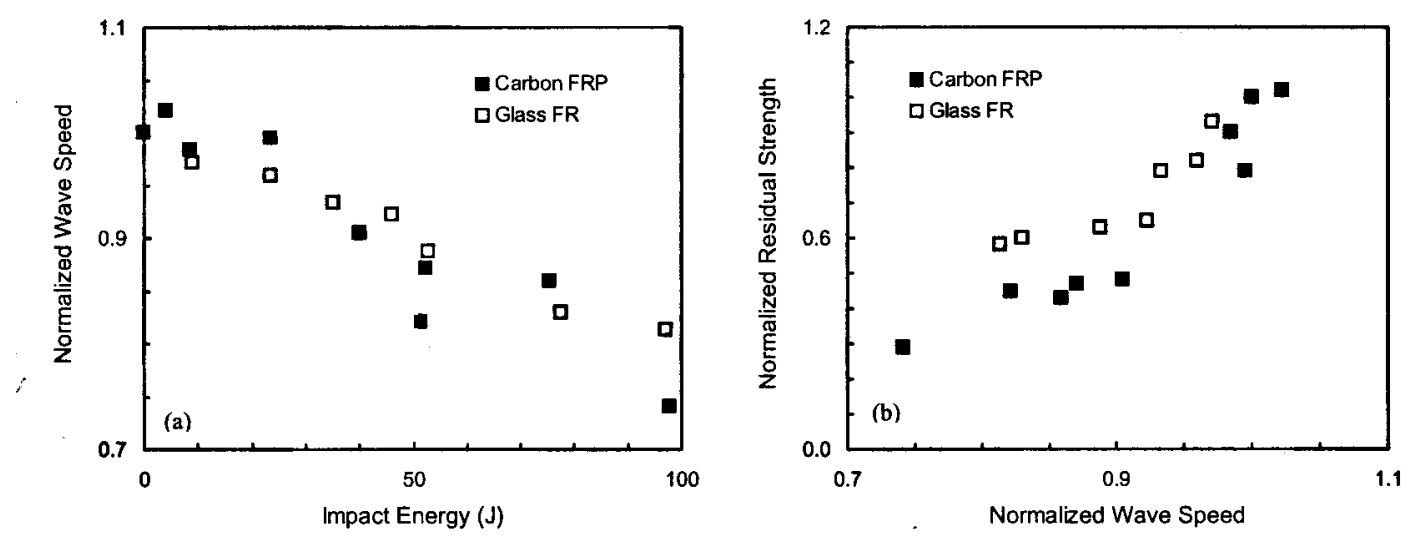

Figure 2.2.3.5 (a) Relationship between normalized through-thickness wave speed and impact energy; (b) relationship between residual strength and through-thickness wave speed (Park, S.W. and Zhou, M. 2000)

The mean-square amplitude was used to quantify the overall intensity of the induced vibration. Figure 2.2.3.6(a) shows normalized mean-square amplitude as a function of impact energy for the two materials. A definite correlation between the mean-square amplitude and the impact energy can also be observed in these plots. Figure 2.2.3.6(b) is a plot of the normalized residual strength as a function of normalized meansquare amplitude. It was concluded that the mean-square amplitude could be used as a measure to estimate the changes in structural integrity of composite laminates subjected to impact. The mean-square amplitude was considered a more reliable means to measure the effects of damage on the residual properties of fiber-reinforced polymer composites because the through-thickness wave speed was not sensitive to fiber damage. 

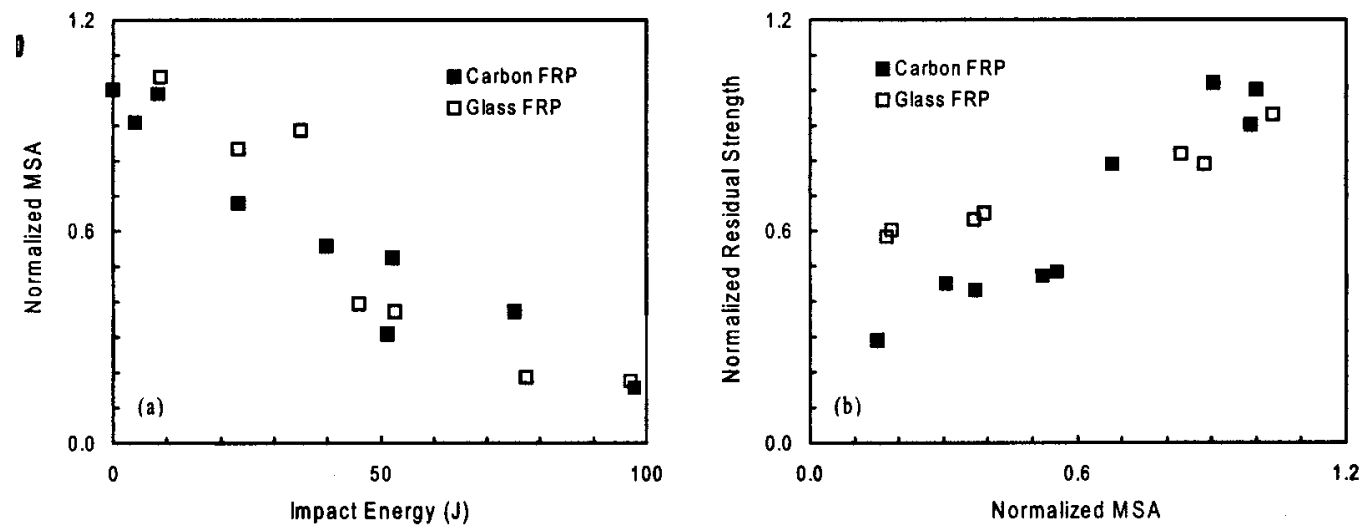

Figure 2.2.3.6 (a) Relationship between normalized mean-square amplitude and impact energy; (b) relationship between residual strength and mean-square amplitude (Park, S.W. and Zhou, M. 2000)

\subsubsection{Conclusions}

Nondestructive ultrasonic testing was previously experimented with to determine the effects of impact-induced damage on the residual strength of FRP (carbon and glass) composite laminates. The results are discussed and illustrated in section 2.2.3. Definite correlations between the applied impact energy and the residual strength of the laminates were found using both through-thickness wave speed and the mean-square amplitude of induced transverse vibration. It was concluded that these correlations could be used to identify damage in laminates subjected to impacts by foreign objects. Although, both the through-thickness wave speed and the mean-square amplitude could be used to identify damages in laminates, the mean-square amplitude method proved a more reliable means of estimating the residual properties of the laminates subjected to impact because of its sensitivity to fiber damage. 


\subsubsection{Nondestructive Evaluation of Critical Pultruded Composite Material Bridge}

\section{Beams (Duke, J.C. et al. 2000)}

Section 2.2.4 discusses the results and details of a comprehensive NDE study of pultruded glass and carbon fiber reinforced vinyl ester bridge beams. The study took place near Blacksburg, Virginia where the composite beams will replace steel I-beams in a short span low traffic road bridge.

\subsubsection{Introduction}

The bridge had a twenty-foot span with a wooden deck overlaid with asphalt. Virginia Transportation Research Council, Virginia Department of Transportation, Morrison Molded Fiberglass Corporation, and Virginia Tech all had a part in the project. The project consisted of mechanical proof testing of the beams, as well as monitoring the degradation of the beams during service using NDE.

Acoustic emission (AE) monitoring and ultrasonic scanning were used during preliminary testing, while continuous AE monitoring, and periodic examination using acousto-ultrasound, infrared thermography, and ultrasonic scanning were experimented with once the beams were in service.

The preliminary tests consisted of a multi-channel Physical Acoustics Corporation Spartan AT system to monitor the beams during proof testing. These preliminary tests consisted of three-point bend tests followed by four point bend tests illustrated in Figure 2.2.4.1. The WDI sensors were attached at locations close to the strain gages bonded to 
the beams. The sensors are placed in regions subjected to the highest induced stresses because of the high attenuation of the composite materials, which makes the AE hard to detect at large distances from the sensors. With the configuration of the sensors illustrated in Figure 2.2.4.1, AE was detected during the initial loading of both beams from all attached sensors. However, upon reloading the beams, the AE was undetectable in all areas until the previous load level was exceeded.

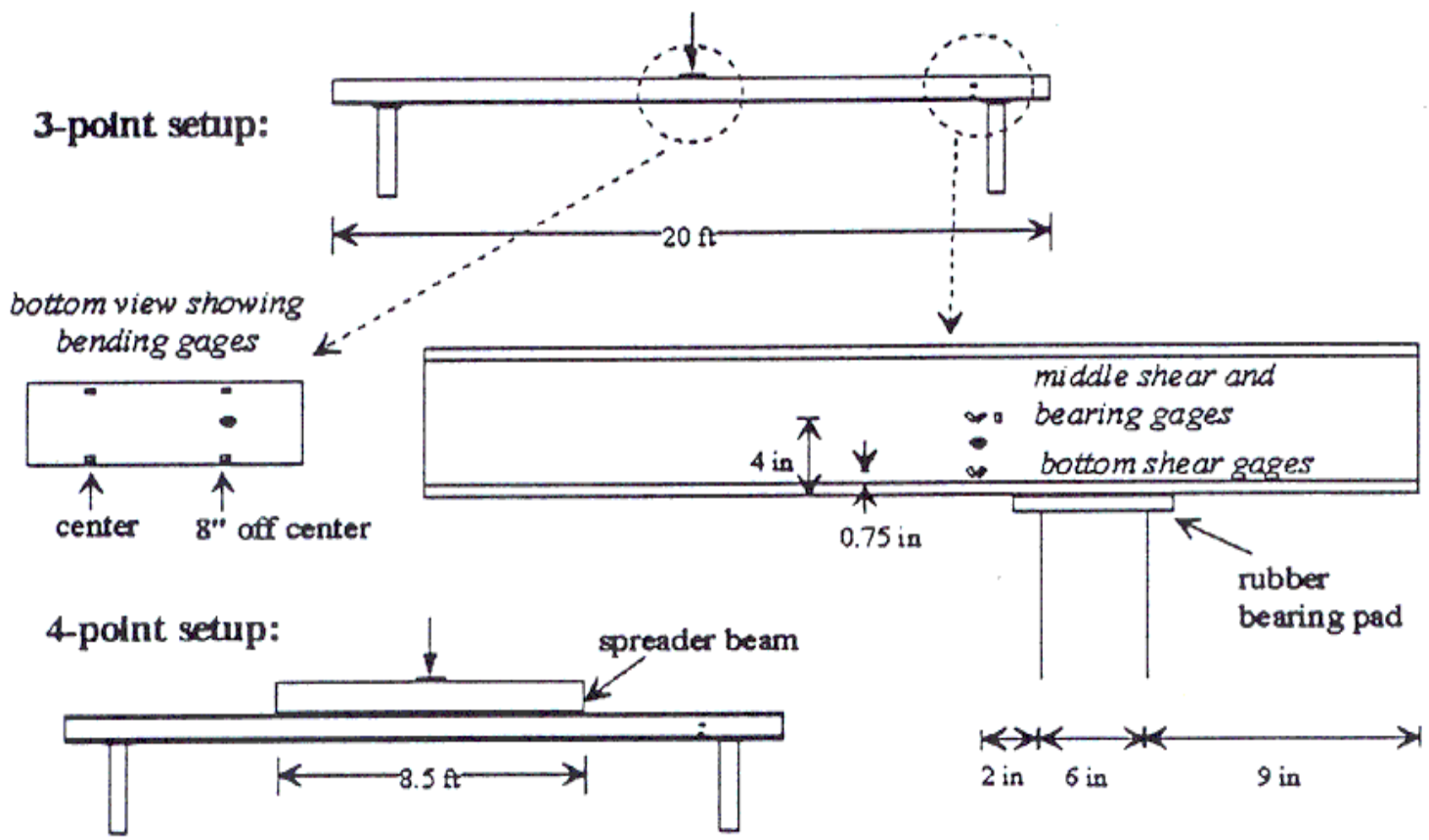

- $A B$ sensors

Figure 2.2.4.1 Schematic of 3 and 4-point setup (Duke, J.C. et al. 2000)

A representative critical area was selected, once in the field, where AE was monitored continuously. The results were remotely reported to the laboratory via a modem. These results are shown in the two plots of Figure 2.2.4.2. 


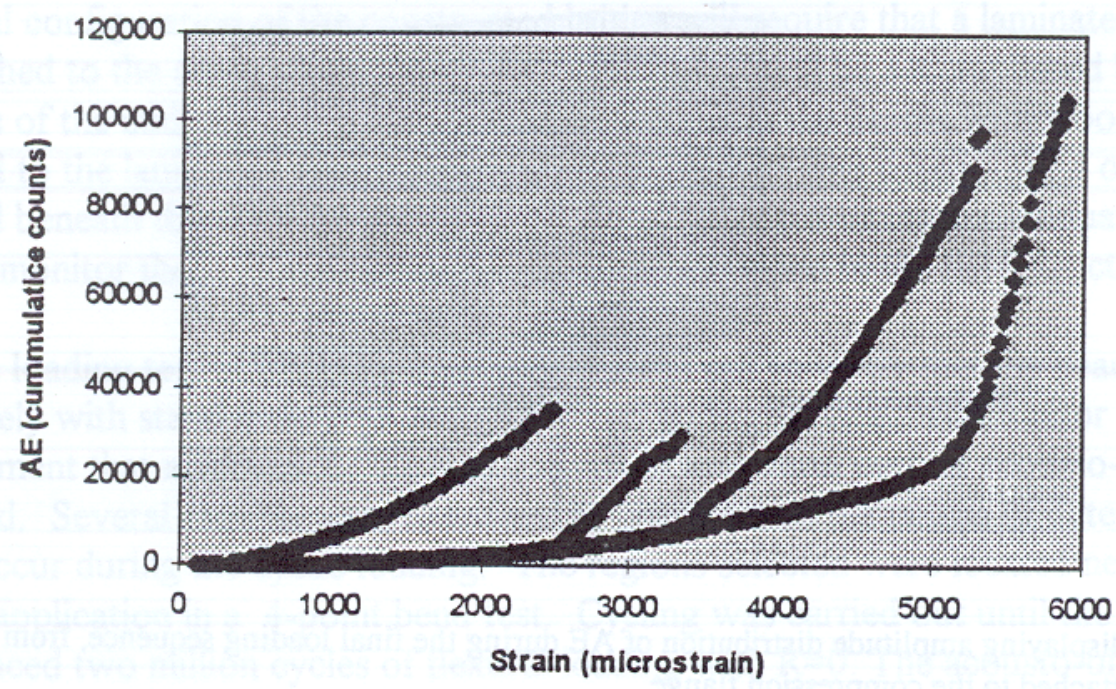

(a)

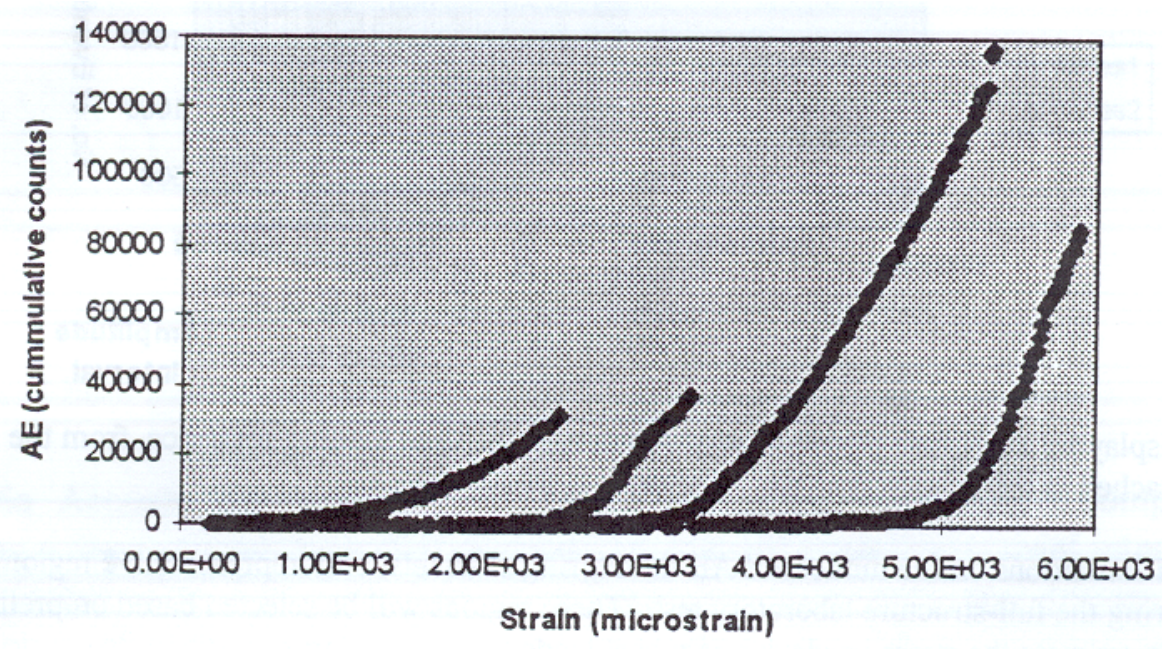

(b)

Figure 2.2.4.2 AE versus Strain (a) compression, and (b) tension (Duke, J.C. et al. 2000)

Other methods were also used periodically to monitor the long-term condition of the bridge since AE coverage is limited and only sensitive to forms of deterioration associated with sudden releases of strain energy (Duke, J.C. et al. 2000). 


\subsubsection{Acoustic-Ultrasonic Monitoring}

Acoustic-ultrasonic evaluation was used to monitor the deterioration of the laminated wood deck clamped to the composite beams. A cyclic loading test was performed on one beam before field installation to verify that the beams could with stand fatigue at the assumed service loads. Acousto-ultrasound was also used to monitor subtle damage development that might occur during this type of long term cycling. For this monitoring, regions located near the points of load application for the 4-point bend test were examined to characterize the nature of deterioration that might occur during the cyclic loading. The acousto-ultrasound data was measured parallel and transverse to the direction of reinforcement, as well as through the thickness of the compression flange. The results obtained during one of the cyclic loading tests are shown in Figure 2.2.4.3.

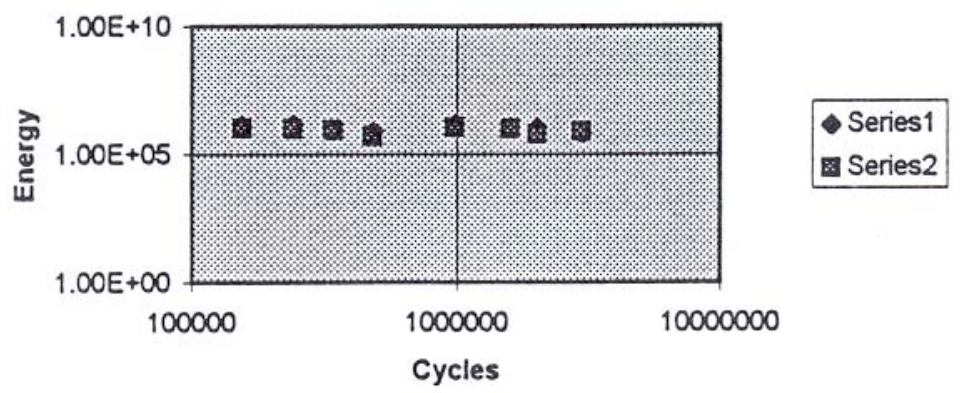

(a)

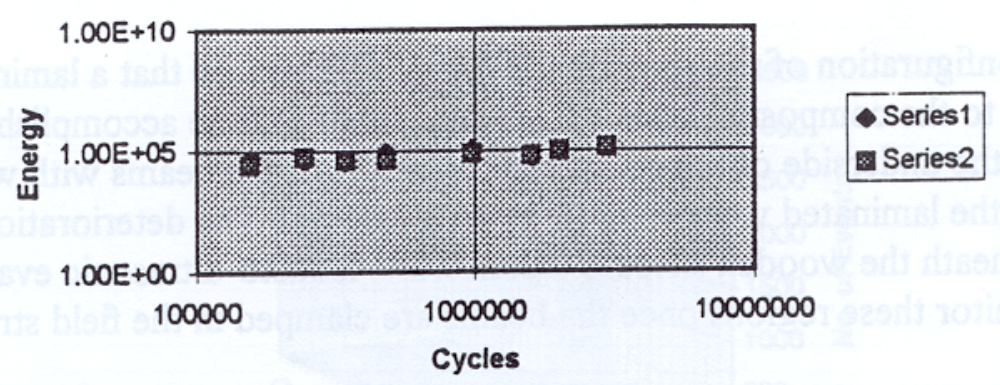

(b)

Figure 2.2.4.3 Acoustic-ultrasonic measurement on the compression flange during a four-point bend flexural fatigue test (a) parallel to the fiber direction, and (b) transverse to the fiber direction (Duke, J.C. et al. 2000) 


\subsubsection{Other NDE}

Infrared thermography was also experimented with to examine the beams. The beams were exposed to a localized elevated source of thermal radiation so variations in heat flux could be monitored.

Other work is being done to develop the capability of ultrasonically scanning portions of the beams. This NDE technique will be used primarily to assess in detail damage that occurs during accelerated hydro-thermal-mechanical laboratory tests.

\subsubsection{Conclusion}

The increasing demand for reinforced composite materials for civil infrastructure applications demands that nondestructive evaluations for assessing the condition of composite materials in the field be developed. Section 2.2.4 describes acoustic-ultrasonic monitoring as a field inspection methodology, which will help in the development of models for predicting long-term durability of composite beams. This section also talks about other NDE methods (e.g., IR thermography and ultrasonic scanning) being experimented with to evaluate composite materials.

\subsubsection{Microwave and Ultrasonic NDE of Thick Glass-Fiber-Reinforced Composites}

\section{(Liu 1998)}

There are many methods used for the NDE of composite materials. The two main techniques that use wave propagation to evaluate material properties are microwave and 
ultrasonic (Liu 1998). The waves propagate through the material and scatter from discrete defects in the material. Ultrasound uses short-pulse excitation, which provides a good separation of reflections from material boundaries and defects. Microwave propagation uses a similar technique as ultrasound except the intrinsic difference in wave impedance between material and small voids are less than that in ultrasound. For composite materials, which are highly inhomogeneous and porous materials, microwave NDE may be a good alternative to ultrasound, which has high signal attenuation from the voids over a long path length. Section 2.2.5 discusses the microwave and ultrasound pulse-echo techniques used to determine defect location, size, and shape in thick composites.

\subsubsection{Introduction}

Composite materials are of great interest to the civil engineering industry because of their ability to reduce the weight and increase the strength of structures. Recently, glass fiber composites (GRPs) are being used instead of graphite fiber composites to build much larger structures due to the decreasing cost of production (Liu 1998). In addition to weight reduction, these coarse microstructures do not corrode like metals, have reduced electrical and magnetic reflections, and can be designed for multifunctional applications.

Many of the NDE techniques for composites have been developed for the inspection of aerospace components made of relatively thin, graphite composites. Ultrasonic techniques have been used for primarily the detection of porosity, 
delaminating, and disbonds (Liu 1998). This technique, however, has high signal attenuation due to the coherent scattering from the coarse microstructure and existence of voids in thick sections. Therefore, a new technique (microwave NDE) is being experimented with thick composites having coarse microstructures. Due to the low microwave attenuation, the signals can penetrate much deeper with a better signal quality.

The pulse-echo technique was used for evaluating thick GRP composites. Data was collected using both the microwave and ultrasound technique. However, the results from the microwave technique had a much better signal-to-noise ratio making the detection of defects much more reliable.

\subsubsection{Experimental}

GRP composites of different thickness where experimented with using ultrasound and microwave pulse echo technique. Some of the samples had defects while others where without defects. The two signals produced from each technique were then compared to determine the advantages and disadvantages of microwave NDE.

\subsection{Ultrasound}

Figure 2.2.5.1 shows the ultrasonic pulse-echo signal obtained from a 0.5 inch thick GRP (second signal in figure) and a 0.25 inch thick GPR (third signal in figure). The first signal (top signal) in Figure 2.2.5.1 is the ultrasonic pulse-echo signal obtained from a 0.5 inch thick homogeneous material (lucite slab). The noticeable noise associated with the second and third signals in Figure 2.2.5.1 (GRP composite) indicated 
the magnitude of scattering noise from the composite microstructure. The transducer used for this experiment had a $1 \mathrm{MHz}$ center frequency and was highly damped to reduce ringing.

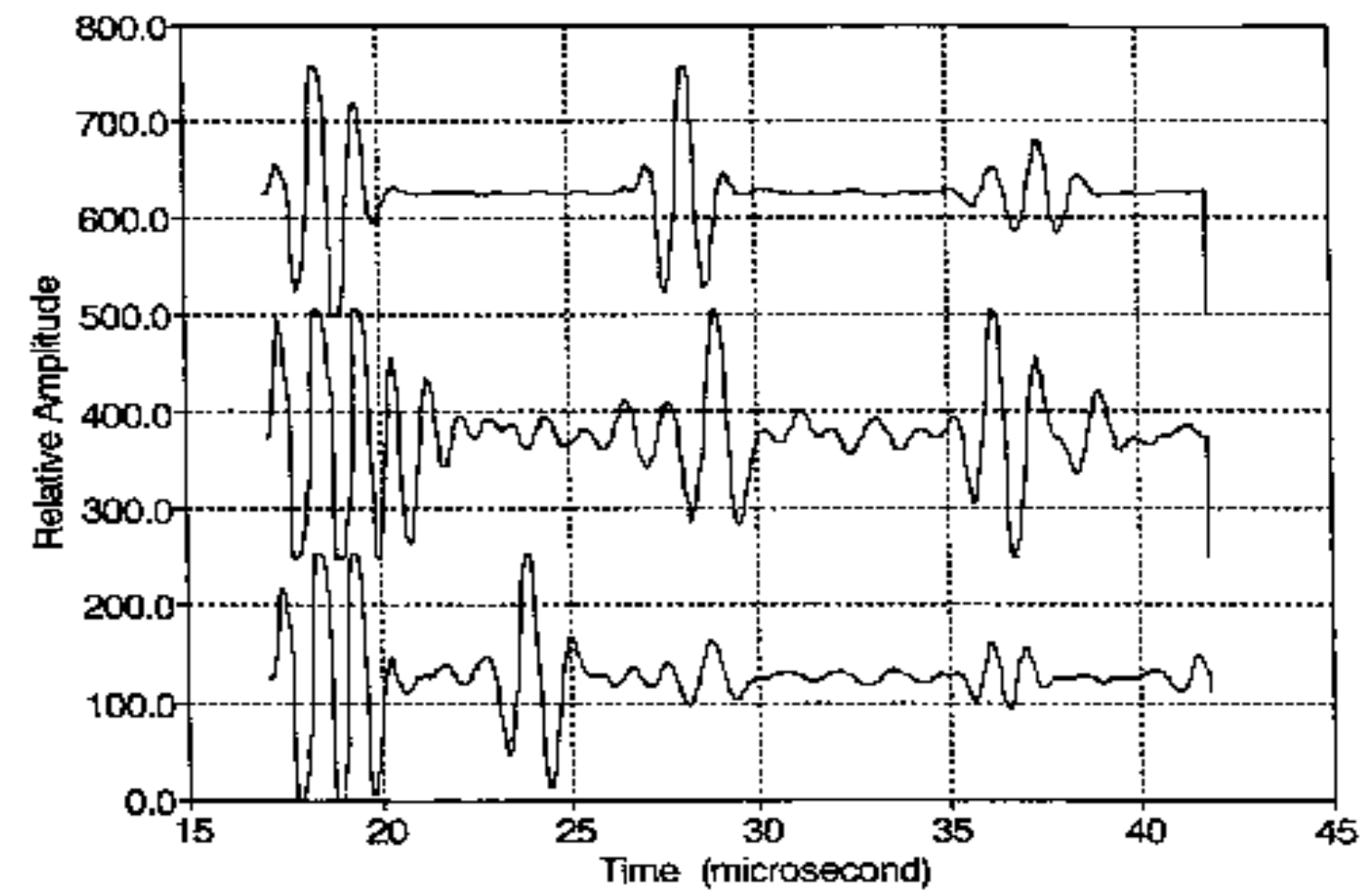

Figure 2.2.5.1 Ultrasonic pulse echo display for a 0.5 inch thick Lucite slab (top signal), a 0.5 inch thick GRP sample (middle signal), and a 0.25 inch thick GRP sample (bottom signal) (Liu 1998)

Figure 2.2.5.2 shows the ultrasonic pulse-echo signal obtained from the 1 inch thick GRP sample. The signal was again compared to the signal of a homogeneous material. The graph indicates that the back wall echo was unclear due to the high scattering noise. It was concluded that any defects in the material would also be unclear due to the large scattering noise in the signal. 


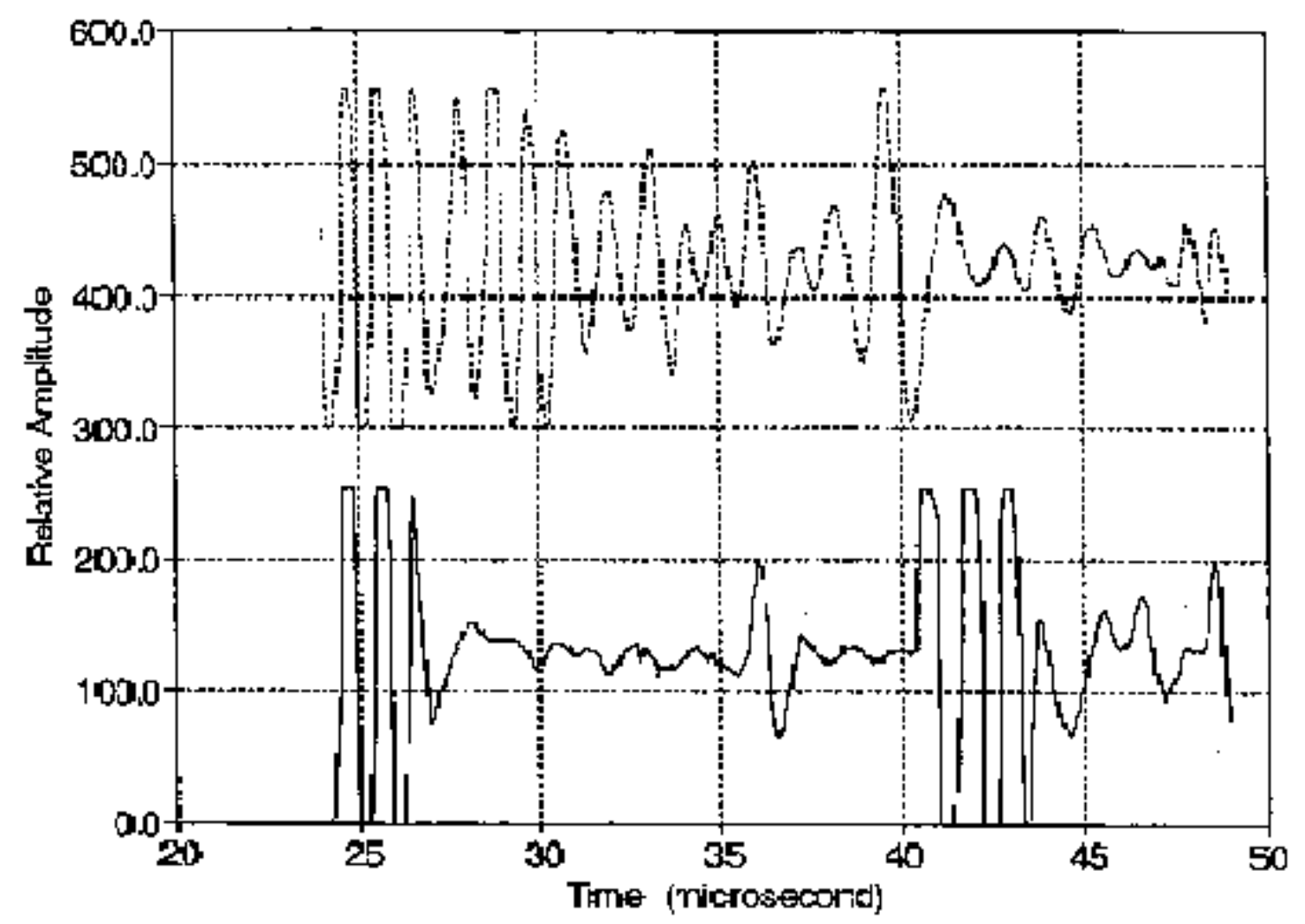

Figure 2.2.5.2 Ultrasonic pulse echo display for Lucite (bottom signal) and GRP sample (top signal). Both specimens were 1.0 inch thick (Liu 1998)

A dual element transducer was used to obtain the signals in the previous two figures. This configuration uses a transducer to inject ultrasonic pulses to the sample and a separate transducer, housed in the same unit, to receive the signal. Figure 2.2.5.3 shows the signals obtain with various sample thickness. The first signal in Figure 2.2.5.3 was received from the homogeneous sample of thickness 0.25 inches. No scattering noise was present preceding the arrival of the back wall echo. However, the scattering noise preceding the back wall echo of the composite samples increases as the thickness increases (illustrated by the second, third, and fourth signals in Figure 2.2.5.3). It was concluded that the dual element transducer would produce unreliable data for detecting defects in thick composites due to the large amount of noise present. 


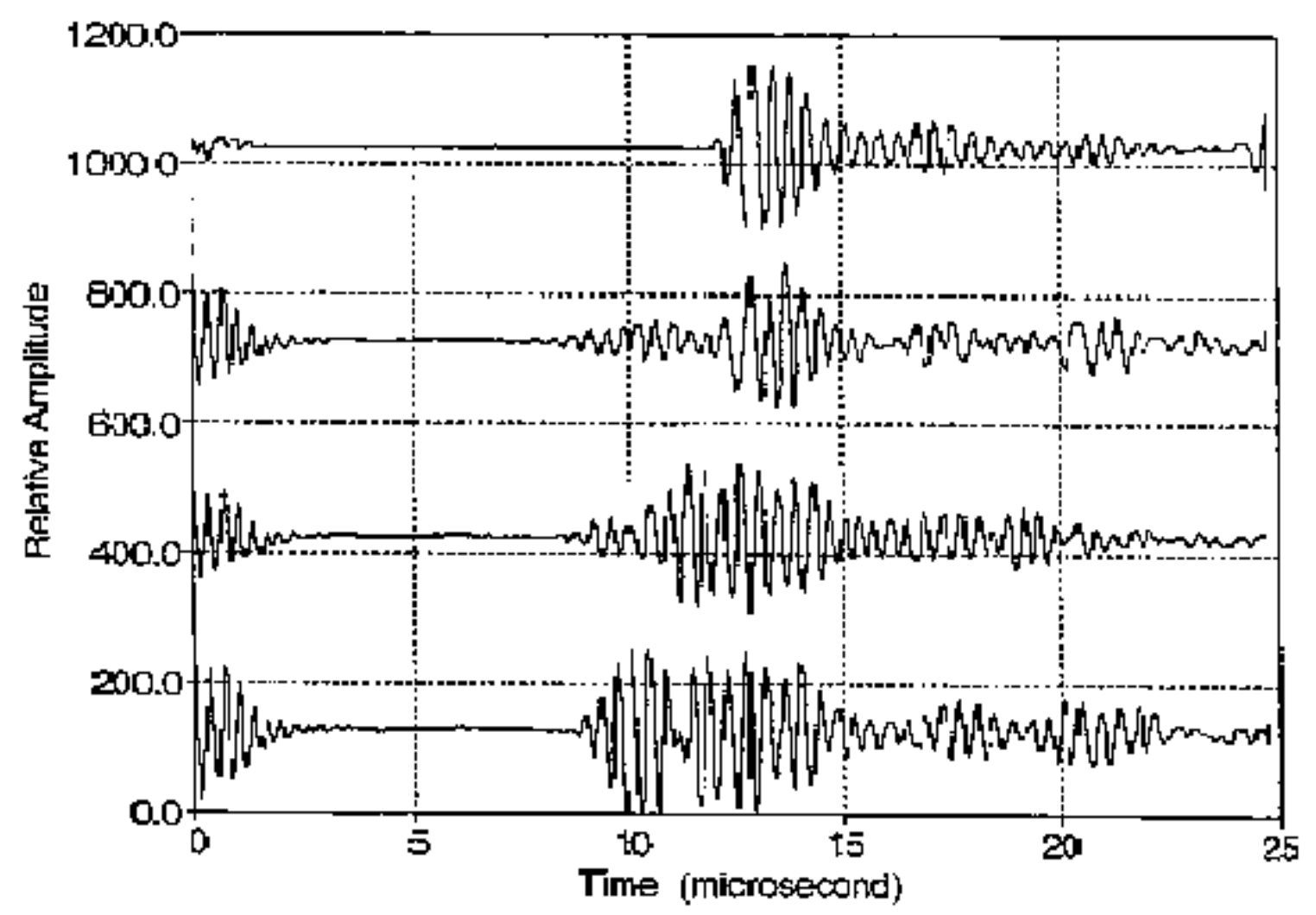

Figure 2.2.5.3 Ultrasonic pulse echoes generated by a dual element transducer at 2.25 MHz. Uppermost trace was in Lucite, 0.25 inch thick; other traces, successively, were GRP, 0.25, 0.5 and 1.0 inch thick (Liu 1998)

A transducer having a center frequency of $500 \mathrm{kHz}$ was used for detecting a 0.375 inch diameter hole representing a defect in a 2 inch thick GRP. The results are illustrated in Figure 2.2.5.4. The hole was detected using ultrasound but the amplitude of the noise was over $30 \%$ that of the defect. 


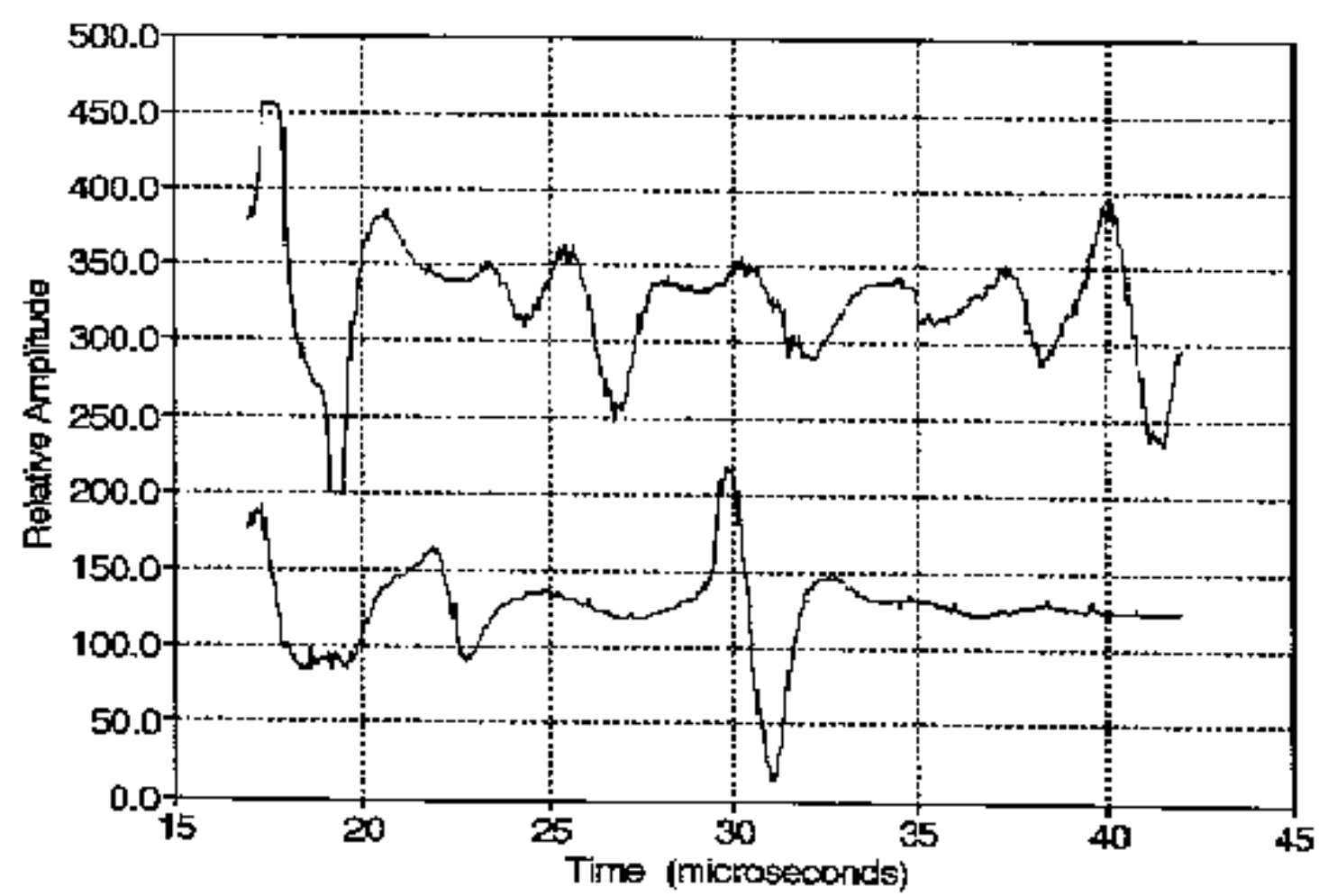

Figure 2.2.5.4 Ultrasonic pulse echo displays $(500 \mathrm{kHz})$ for a 0.375 inch diameter hole (Liu 1998)

\subsection{Microwave Technique}

When using microwaves to evaluate composite materials either a step frequency or a swept frequency technique is necessary because of the high speed of propagation of electromagnetic waves in non-conductive composites. These techniques convert the frequency domain to the time domain. Figure 2.2.5.5 shows the microwave pulse-echo signals observed for the same $0.5,1$, and 2 inch thick composite slabs shown previously. It was observed that the signal-to-noise ratio of the back wall echo was good in all cases. 


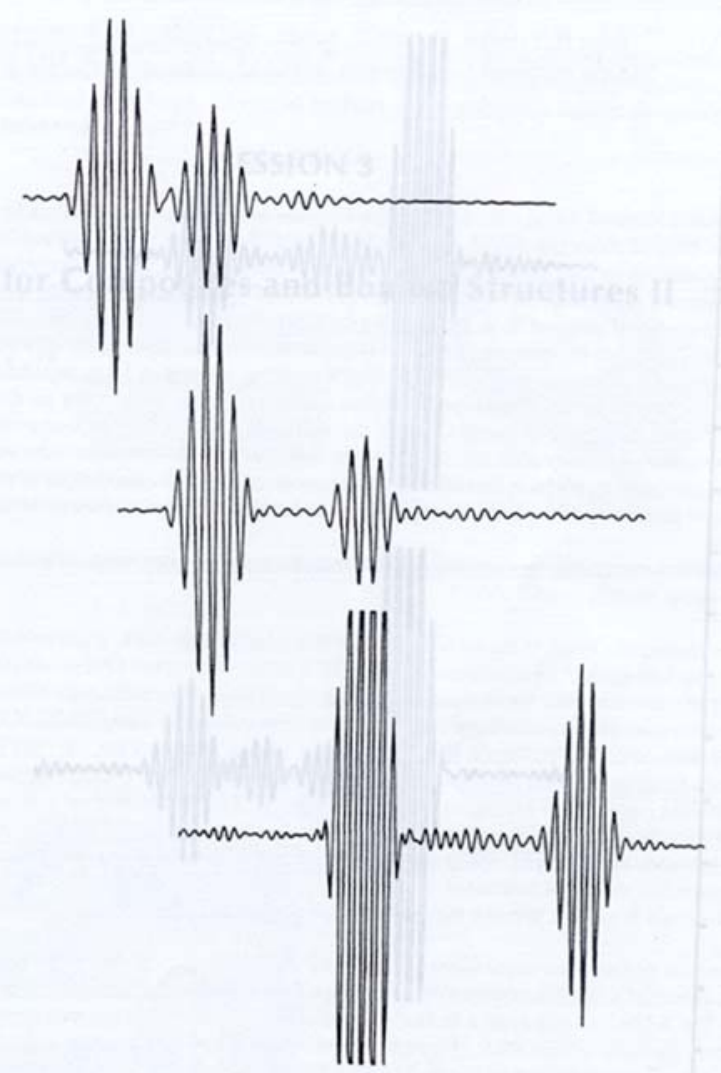

Figure 2.2.5.5 Microwave pulse echo patterns (mean frequency of $30 \mathrm{GHz}$ ) in GRP. Material thickness was 0.5 inch (top signal), 1 inch (middle signal), and 2 inches (bottom signal) (Liu 1998)

Figure 2.2.5.6 shows the results for the drilled hole (same sample used in Figure 2.2.5.4). Because of the reduced scattering noise in the signal, the defect can be detected with a much greater reliability. The microwave NDE was also able to detect flat voids and delaminations, located up to 2 inches from the front surface of GRP samples. 


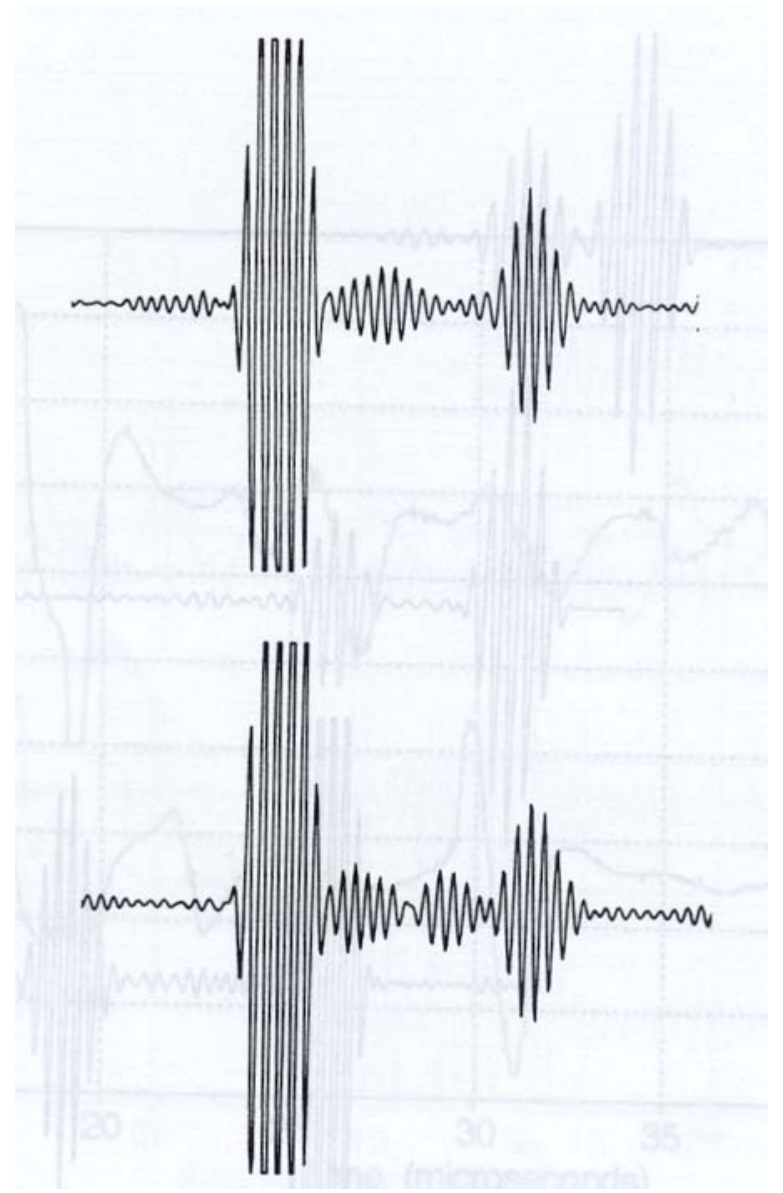

Figure 2.2.5.6 Microwave pulse echo patterns (mean frequency of $30 \mathrm{GHz}$ ) for the 0.375 inch diameter hole. Top signal, drilled hole 0.75 inch from surface; bottom signal, drilled hole 1.25 inch from surface (Liu 1998)

\subsubsection{Conclusion}

Wave propagation techniques have been used for the NDE of composite materials. For thick GRP's of coarse microstructure, noise associated with scattering by the composite microstructure makes using the ultrasonic technique relative unreliable for detecting and locating defects. However, the microwave NDE has great potential of overcoming the scattering noise problems encountered with thick GRP composites. Research needs to be continued to simplify the equipment to facilitate applications in the field. 


\subsubsection{Composite Material Defects Characterization Using Leaky Lamb Wave Dispersion Data (Bar-Cohen et al. 1998)}

Composite materials have been evaluated using Leaky Lamb wave (LLW) propagation. The wave was induced into the material using a pitch-catch technique and the wave spectrum was then inspected to obtain defect and material characteristics. The reflection spectra was acquired in real time while filtering the high frequency noise providing reliable data at amplitude levels that were significantly low. The experimental results was compared to data obtained from a C-scan mounted LLW scanner.

\subsubsection{Introduction}

Finding a nondestructive evaluation technique to determine the integrity, stiffness, and durability of structures is a main priority right now to assure the performance of these structures in service using smaller safety factors. NDE methods have been developed to detect and characterize flaws and to determine the material properties of test specimens. However, composite materials have a multi-layered anisotropic medium, which has posed problems for NDE techniques. This section discusses the LLW technique that has been experimented with for determining elastic properties and defect characteristics.

\subsubsection{Leaky Lamb Wave Phenomenon}

A pitch-catch ultrasonic setup was used to induce leaky Lamb waves into composite materials that were immersed in fluid (water used as a coupling medium). This technique would excite the surface of the material and leak waves into the water that would interfere with the spectral reflection. This phenomenon allows modes to be 
observed and shows the effects that defects have on the modes. The elastic properties could then be determined from the measured dispersion data. Later studies showed how these ultrasonic pulses in the pitch-catch setup where used to determine all five elastic constants fairly accurate (Bar-Cohen et al. 1998).

The experiments performed by the authors of this section used the pitch-catch setup with the leaky Lamb phenomenon. This procedure produces ultrasonic waves that interact with the material and the reflection represents the dispersed spectral characteristics of the layered material. Evaluation of the minima in the reflection spectra at different angles of incidence provide information about the various wave modes. These minima form dispersion curves that can be analyzed.

To obtain quality dispersion curves for composite materials, rapid and accurate control of the angle of incidence/reception and the polar angle with the fibers is required. The recent use of personal computers has increased the quality of these dispersion curves. A computer code was used with the personal computer to control the incidence and polar angles, the height of the transducers from the sample surface, and the transmitted frequency of the pitch-catch setup. The signals were then acquired as a function of the polar and incidence angles and saved in a file for analysis and comparison with the theoretical predictions. The minima from the reflection spectra represented the LLW modes and were used to create the dispersion curves. The incident angle was changed incrementally within the range of $12^{\circ}$ to $50^{\circ}$. The minima of each incident angle was added to the accumulating dispersion curves and plotted simultaneously on the computer 
screen as illustrated in Figure 2.2.6.1 (results for a unidirectional graphite/ epoxy plate).

The inverted elastic and stiffness constants are given on the left.

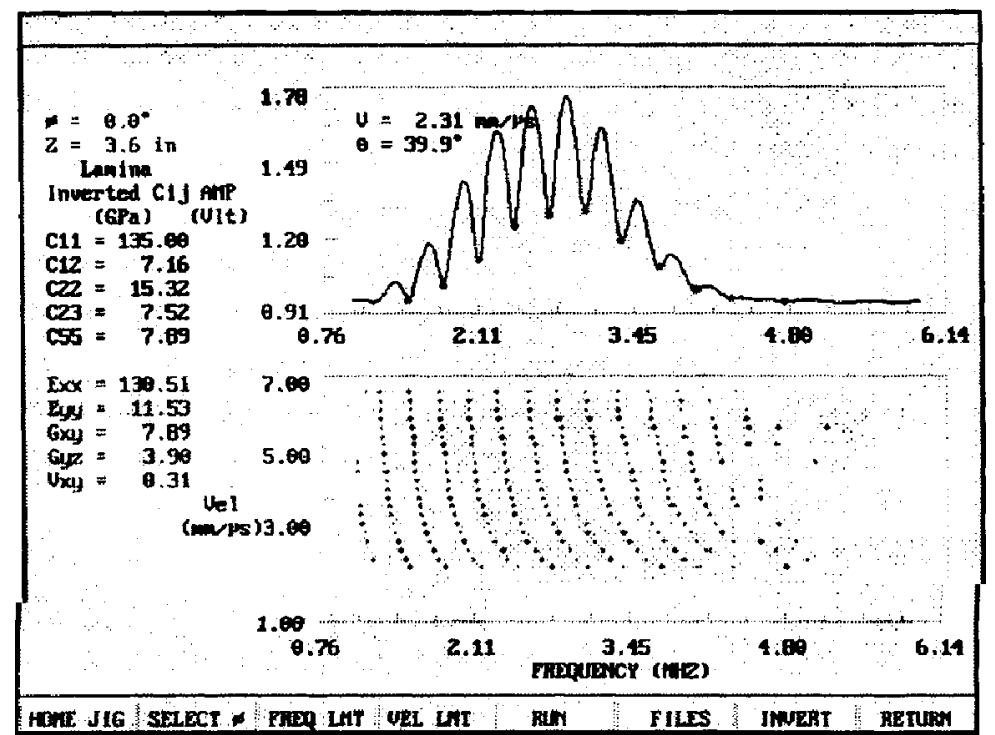

Figure 2.2.6.1 A view of the computer screen with the reflection spectra on the top and the accumulating dispersion curve on the bottom. The inverted elastic stiffness constants are shown on the left

(Bar-Cohen et al. 1998)

\subsubsection{Theory and Data Inversion}

The minima of the each incidence angle are identified because they are highly sensitive to the thickness and the stiffness constants of the composite layers. Therefore, the dispersion curves (made up of these minimas) were used to accurately determine the properties of the material and any changes in their values during service, which were used to characterize any defects.

To demonstrate how these minimas and their dispersion curves can be used to detect changes in material properties, a sample made of a unidirectional 24-ply laminate was heated from room temperature to $480^{\circ} \mathrm{F}$ for 15 minutes. The sample was tested at the same location before and after heat treatment. The measured dispersion curves are illustrated in Figure 2.2.6.2. Distinct differences in the data were observed as seen in the 
figure. These changes in the dispersion data caused significant changes in the matrix dominated stiffness constants.

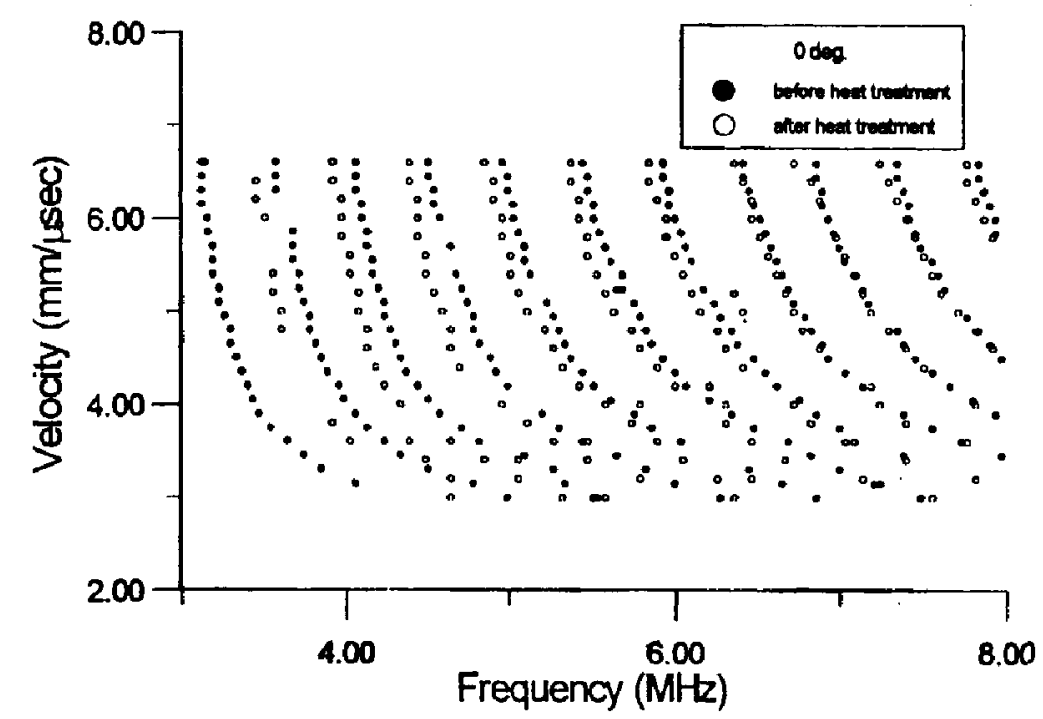

Figure 2.2.6.2 The measured dispersion curves of a unidirectional graphite-epoxy panel before and after heat treatment (Bar-Cohen et al. 1998)

It was concluded that extreme care must be taken in interpreting the numerical results obtained from the inversion of the dispersion data and that only the thickness and matrix dominated constants could be determined accurately from this method.

\subsubsection{LLW Experimental Capability Enhancement}

The LLW technique has had considerable disadvantages in the past that needed to be considered. The complexity of the data acquisition system was a main disadvantage for using the LLW technique in practical situations. Recently, a user friendly control setup that operates on Windows was written that significantly improved the data acquisition process through integration of software and hardware. One disadvantage that needs further investigation is that the inverted material constants assume that the material density was known. However, the inversion algorithm developed for the determination 
of the elastic properties was very successful for unidirectional laminates with known density. The LLW system used for these experiments allows the measurement of dispersion curves at a significantly higher speed than before. A schematic view of the experimental setup is depicted in Figure 2.2.6.3. This setup created a dispersion curve that was based on a set of 20 angles of incidence along a single polar angle. The entire process took about 45 seconds.

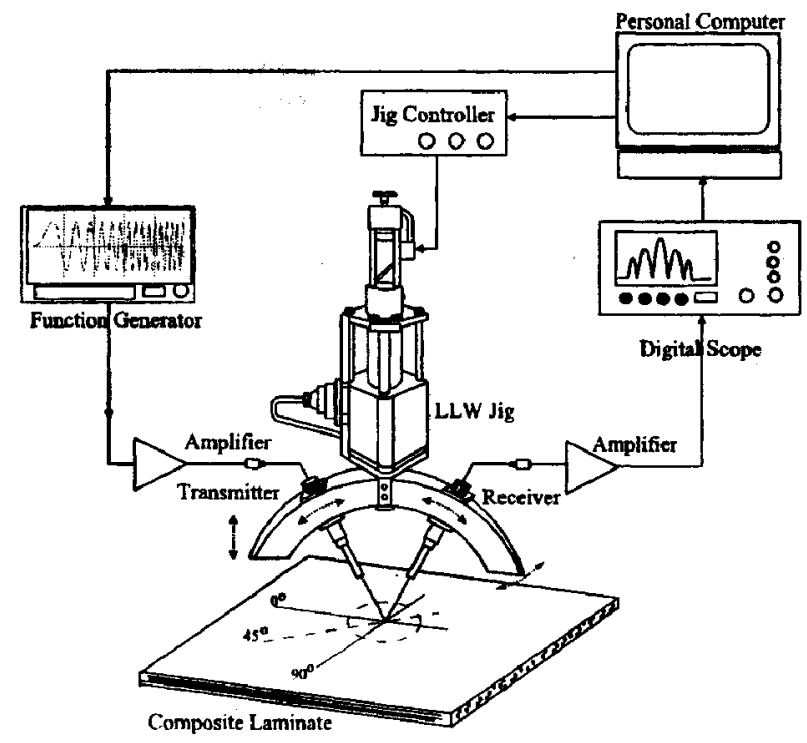

Figure 2.2.6.3 A schematic view of the rapid LLW test system (Bar-Cohen et al. 1998)

This new setup was also used to identify key composite material defects. The reflection spectra was observed to identify known flaws in order to obtain typical representation of their response. A defect free sample was tested to investigate the agreement between experimental results and theoretical dispersion data (Figure 2.2.6.4). Figure 2.2.6.5 shows the results of a porous sample and a defect free sample. The effect of porosity was more noticeable at higher frequencies. Figure 2.2.6.6 shows the dispersion curve of a delaminated sample and a defect free sample. The delamination can be detected at all frequencies. 


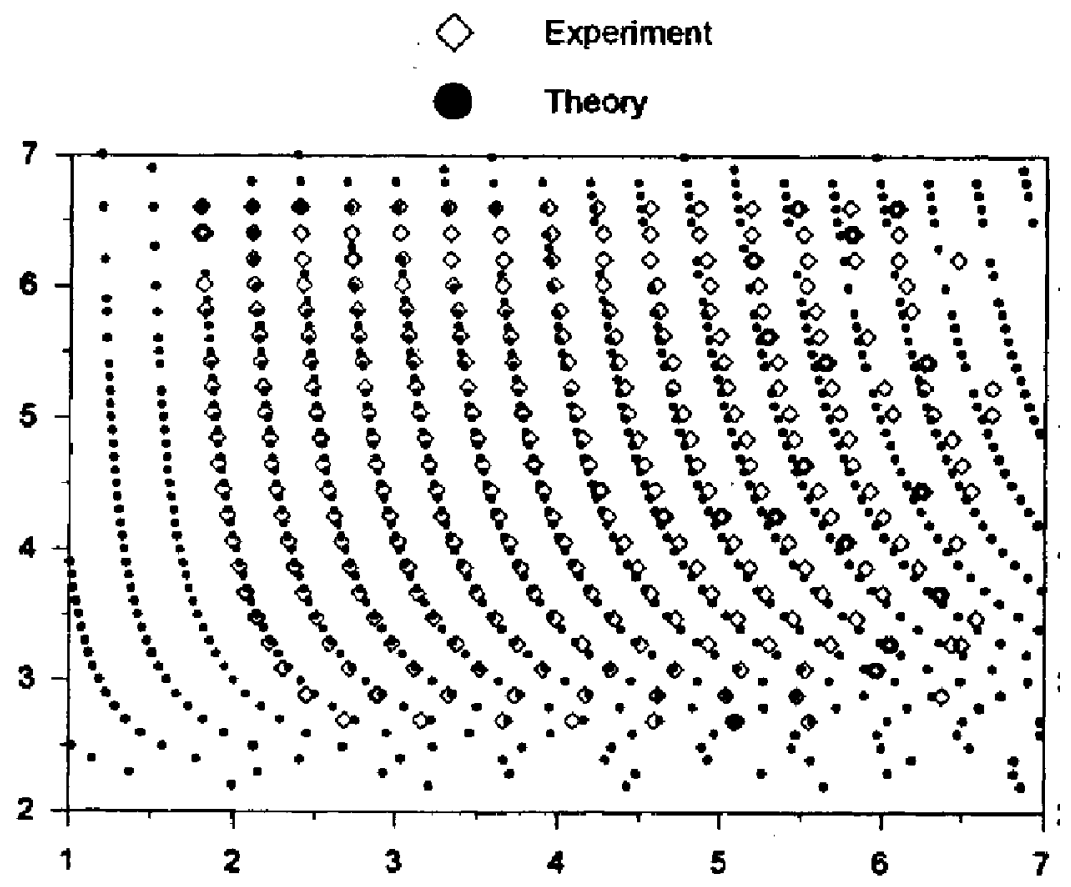

Figure 2.2.6.4 Dispersion data for a defect free 16 layer unidirectional laminate (Bar-Cohen et al. 1998)

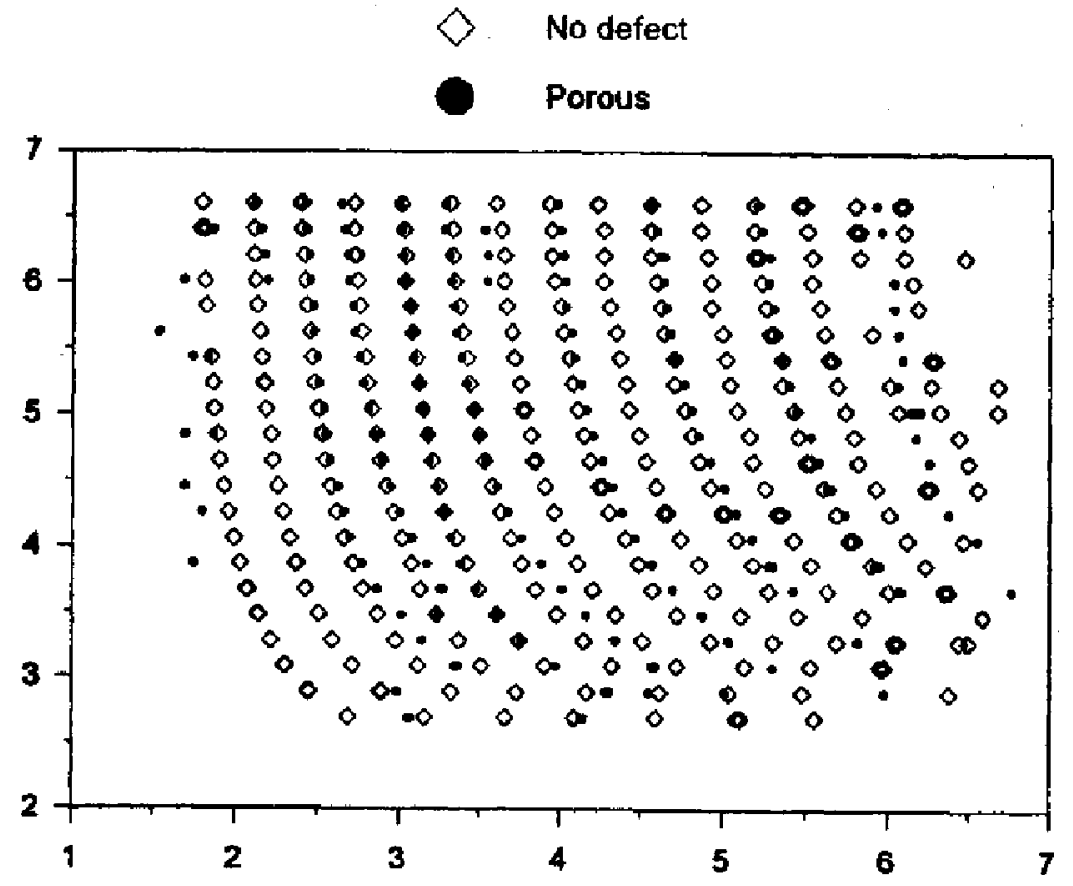

Figure 2.2.6.5 The effect of porosity layer between the $8^{\text {th }}$ and $9^{\text {th }}$ layers (Bar-Cohen et al. 1998) 


\section{$\bigcirc \quad$ No defect}

Delamination

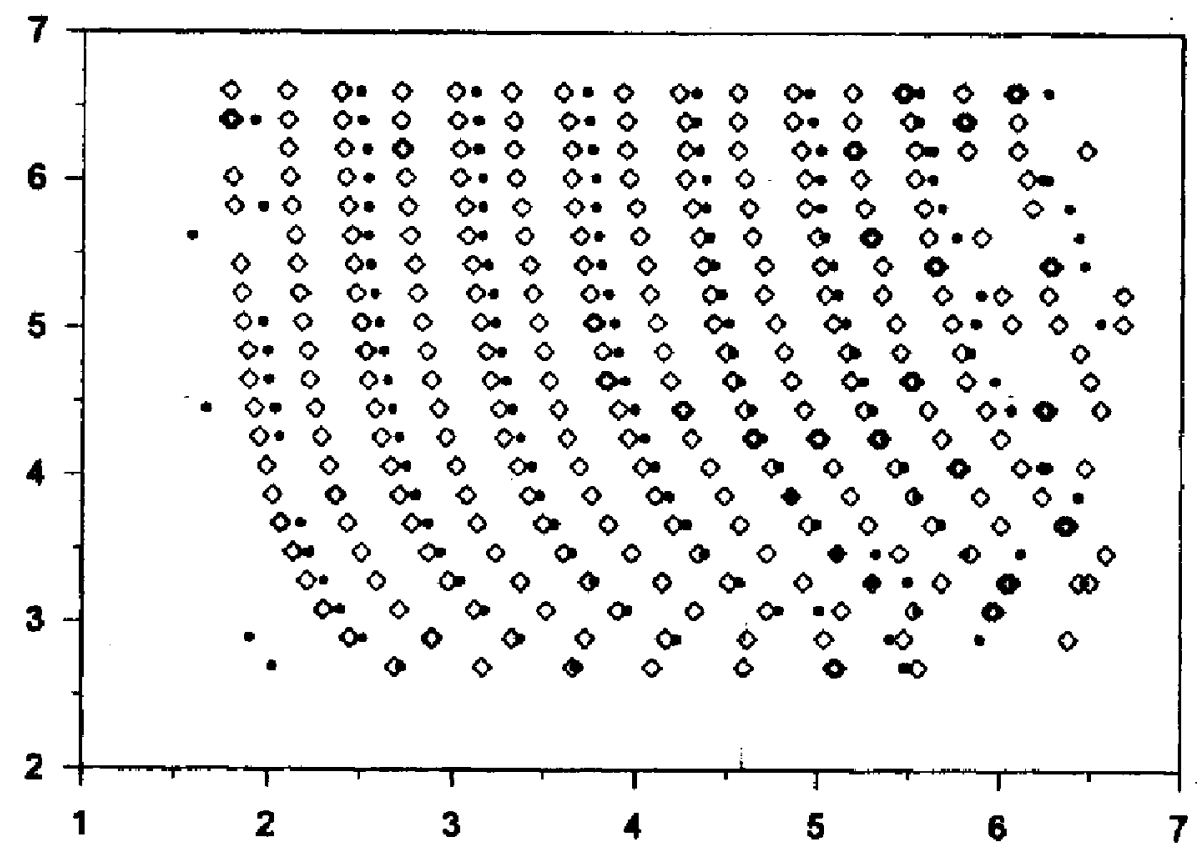

Figure 2.2.6.6 Dispersion curves obtained at a delaminated area between the $8^{\text {th }}$ and $9^{\text {th }}$ layers (Bar-Cohen et al. 1998)

\subsubsection{Conclusions}

Bar-Cohen et al. (1998) has performed significant theoretical and experimental studies of wave behavior in composites. This research has developed a new LLW technique that produces dispersion curves that can be used for both inversion of elastic properties and characterization of flaws in composite materials. Further, this new technique produces rapid and accurate data with a user-friendly data-acquisition system. This development simplifies the process of characterizing flaws in composites and determining the degradation of the material properties. Further investigation of this technique needs to be performed in order for the LLW phenomenon to be employed as a standard quantitative NDE method in the civil engineering industry. 


\subsubsection{Conclusion on Microwave and Ultrasound Wave Propagation}

Based on the literature review of microwave and ultrasonic techniques it was concluded that defects of critical size could be detected. Wave propagating techniques such as microwave and ultrasonic are well developed for the NDE of composite materials. However, a noticeable amount of noise associated with scattering due to the coarse microstructure of the composite material affects the clarity of the signal. This adversely affects the ease of locating and detecting defects. The microwave technique seems to be the best wave propagating method that can potentially overcome some of the noise problems encountered during NDE of composite materials. More research needs to be performed on the microwave technique to develop new wave propagating methods for the NDE of composite materials.

Some of the other disadvantages associated with the ultrasonic inspection technique include:

- Difficulty inspecting composite sections that are irregular in shape and have a rough surface.

- The microstructure of the composite material causes high attenuation of waves.

The main advantages of the ultrasonic inspection technique include:

- $\quad$ The detection of flaws at a great depth due to the superior penetrating power of ultrasound.

- $\quad$ High sensitivity, permitting the detection of small defects. 
- $\quad$ More accurate in determining size, shape, and location of internal defects when compared to other NDE methods.

\subsection{Acoustic Wave Propagation Technique}

This section reviews the NDE technique that uses acoustic waves to penetrate through the material. The acoustic waves use produced using hammers (tap testing) or an acoustic emission (AE) sensor. The signal is then detected using suitable sensors attached to the surface of the structure. The parameters of interest, disadvantages, and advantages of the acoustic emission techniques will be discussed in the section and compared to other NDE methods for evaluating composite materials.

\subsubsection{Blind Deconvolution of Acoustic Emission Signals for Damage Identification in} Composites (Zheng et al. 2000)

Using acoustic emission signals with composites is one of the techniques used for detecting damage and defects in composites. Acoustic emission signals identify the type of damage by using features such as amplitude, frequency, and counts (Zheng et al. 2000). Time-frequency distribution techniques have also been applied to characterize damage where the analysis is on the actual acoustic emission signal itself. The problem is that real information on damage is actually hidden behind the actual signal. This section describes a blind deconvolution method that has been developed to demodulate the signal and identify the damage. Several experiments and their results are discussed in 
this section to determine the validity of the blind deconvolution method as technique for identifying damage in fiber reinforced composites.

\subsubsection{Introduction}

Acoustic emission techniques have been used for condition monitoring and damage detection in many engineering materials. Lately, acoustic emission techniques have been evaluated for the use of damage detection in fiber reinforced composite materials. A significant amount of research has been performed aiming at the technique's ability to identify the damage type; its ability to correlate damages with loading conditions and/or strain conditions; its ability to recognize dominant damage types in specific materials; and its ability to evaluate mechanical properties of specific materials.

Acoustic emission signals are typically described by parameters such as amplitude, duration, counts, events, rise time and energy (Zheng et al. 2000). Past research has tried to correlate these parameters with the technique's ability to identify damage in engineering materials. The relationship between these parameters and damage type is of interest because different damage emits different levels of energy. However, discrepancies in these relationships may occur among different composite specimens and structures due to the attenuation of the stress waves as they propagate through different composite materials. Boundary conditions, size, and many other factors may also result in the inconsistent AE amplitude measurements. With such discrepancies, amplitude analysis based methods for the damage evaluation may not be reliable. 
Other approaches have also been experimented with such as waveform characteristic identification, frequency analyses, and source rise-time measurements but with these methods, detailed experiments must be performed before field analysis can be obtained. This is because for different materials the signal transfer path between the source and the sensor is different and detailed experiments should be performed to ensure satisfactory performance of the AE signal analysis-based condition monitoring system.

The source inversion processing method is another approach that has been used to identify damage in materials. This method extracts information on the wave source by signal demodulation. Because different types of damaged areas generate different energy sources to the AE signal, this approach determines the damage type with more consistency. With the source inversion process, the materials need to be isotropic and elastic. The acoustic and mechanical properties of the material also need to be known for the source inversion process to perform properly. However, composite materials are generally anisotropic making the source inversion processing impractical for analyzing composite materials.

Section 2.3.1 identifies an alternative approach toward wave source analysis of composites. The main difference in this approach from the source inversion processing is that it does not rely on the medium of the material being isotropic and elastic. Using a signal processing approach directly demodulates the AE signal and characterizes the signal by its duration and profile in the time domain. 


\subsubsection{Signal Processing}

The propagation of an acoustic emission, in general, is very complicated because it consists of longitudinal, transverse and Rayleigh wave components together. When acoustic emission is introduced to fiber reinforced composites it becomes even more complicated because of the components generated by reflections and scattering at the boundaries.

The wave component is a function of time and frequency and has parameters related to the material properties. When a time-frequency distribution is introduced, information on the amplitude, frequency and attenuation factor can be obtained. Because different wave types have different traveling speeds and different carrier frequencies, the time-frequency distribution can identify the location of a defect as well as some mechanical properties of the damaged area, such as the damping factor and elastic constant.

The force and the transfer function can be separated in the time domain or in the frequency domain by Fourier transform so the identification of the force can be obtained. The method of separating the wave source information from the transfer function is known as cepstrum (Zheng et al. 2000).

The cepstrum has been used to process AE signals in past experiments but failed to obtain information on the wave source when direct application was used. The direct application of this method failed because the positions of the force and the system 
function were superimposed along the axis defined by the cepstrum. It was also concluded that some characteristics of the AE wave caused problems. Because of these problems, it was determined that a direct application of the cepstrum would be impractical and would not obtain accurate result.

The conventional cepstrum was modified to achieve the separation of the wave source information from that of the system. The first step in modifying this method was to shift the position of the wave source in the cepstrum away from the system's contribution by applying a time-domain shift transformation (Zheng et al. 2000). This step had to be performed before the inverse operation and was eventually called the "lifter" operation. Next, the transient component had to be determined in a step called the deconvolution procedure. This step takes the first arrival wave (longitudinal, transverse, or Rayleigh) as the object for deconvolution. The corresponding transient component would then be constructed from this first arrival wave.

\subsubsection{Experimental}

Three sets of experiments were performed to determine the reliability and quality of the modified processing method to characterize specific damage modes in fiber reinforced composites. The first set of experiments involved the use of 16-layered crossply E-glass/920 epoxy composites which were $200 \mathrm{~mm}$ long, $20 \mathrm{~mm}$ wide, and $2 \mathrm{~mm}$ high. These test experiments presented all possible damage modes. The next group of tests was set up to isolate matrix-cracking damage. These tests were conducted using a cured epoxy tensile test specimen with a $6 \mathrm{~mm}$ diameter hole, located at the center of the gauge 
length. The last set of experiments involved the use of single E-glass fiber tows to isolated fiber/fiber bundle breakage and fiber pullout. These tests were conducted using both single fiber strands and epoxy resin impregnated strands.

The AE sensors with a frequency range of $70-200 \mathrm{kHz}$ were attached to the Eglass/920 test specimens as illustrated in Figure 2.3.1.1. A 20-1200 kHz band-pass filter was applied to all $\mathrm{AE}$ signals to remove low frequency and high frequency noise. The test specimens were loaded to failure while AE signals were recorded on hard disk.

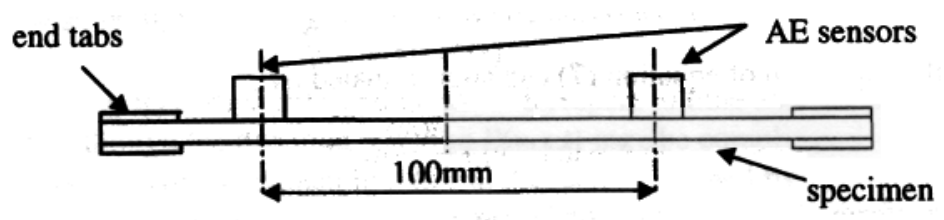

Figure 2.3.1.1 Sensor arrangement (Zheng et al. 2000)

\subsubsection{Results and Discussions}

For the first set of experiments the signal processing results of five signals, whose maximum amplitudes are $47.1 \mathrm{~dB}, 65.34 \mathrm{~dB}, 72.8 \mathrm{~dB}, 90.8 \mathrm{~dB}$, and $99.2 \mathrm{~dB}$, respectively, are shown in Figure 2.3.1.2. Illustrated in the figures, the shorter the wave source duration, the higher the signal amplitude.
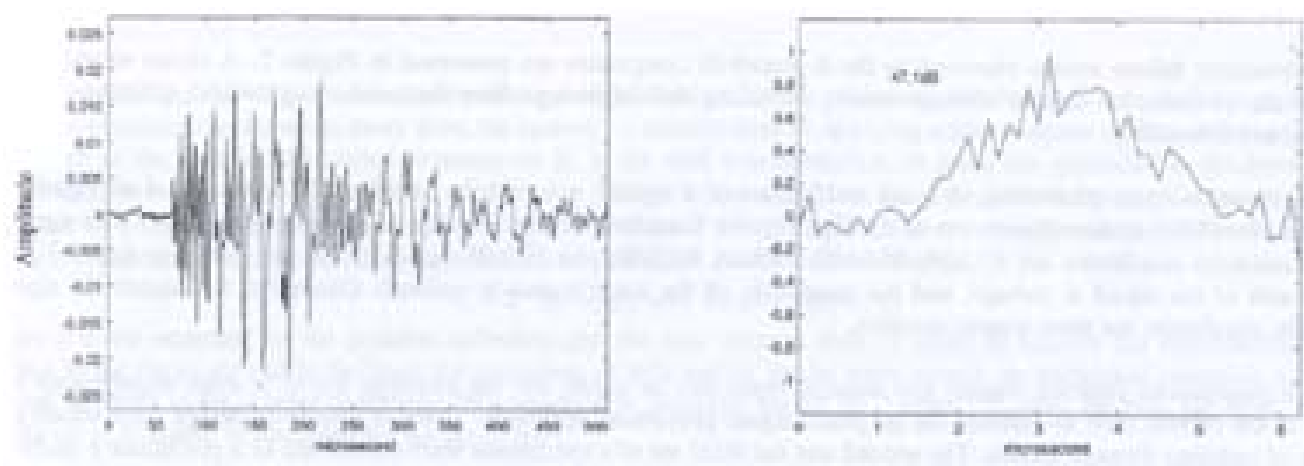

47.1dB signal and its recovered wave source (Zheng et al. 2000) 

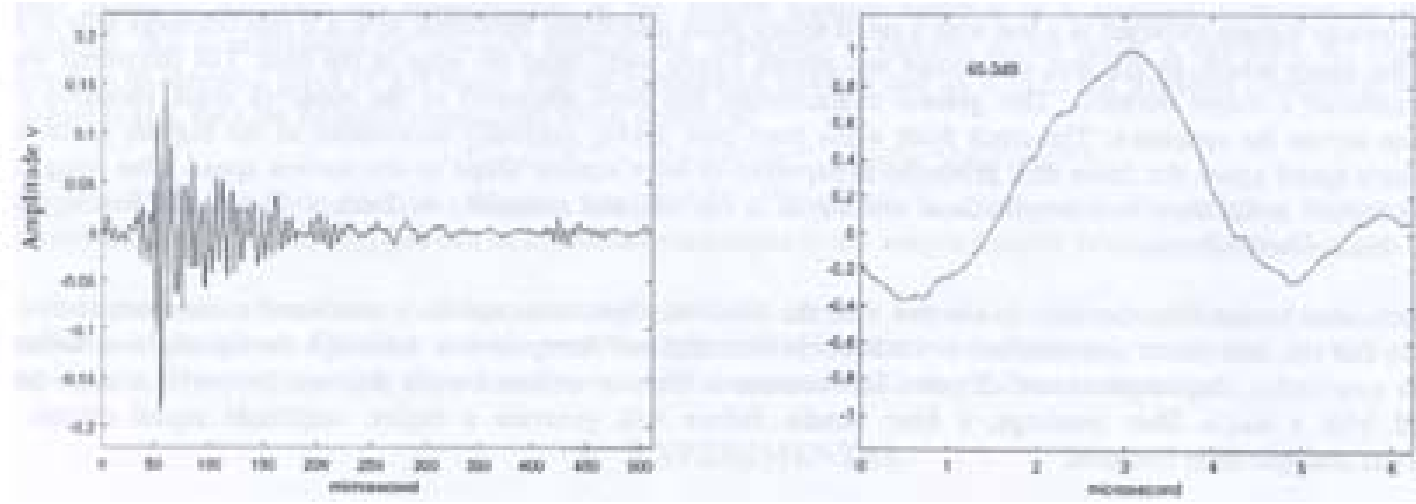

65.3 dB signal and its recovered wave source (Zheng et al. 2000)
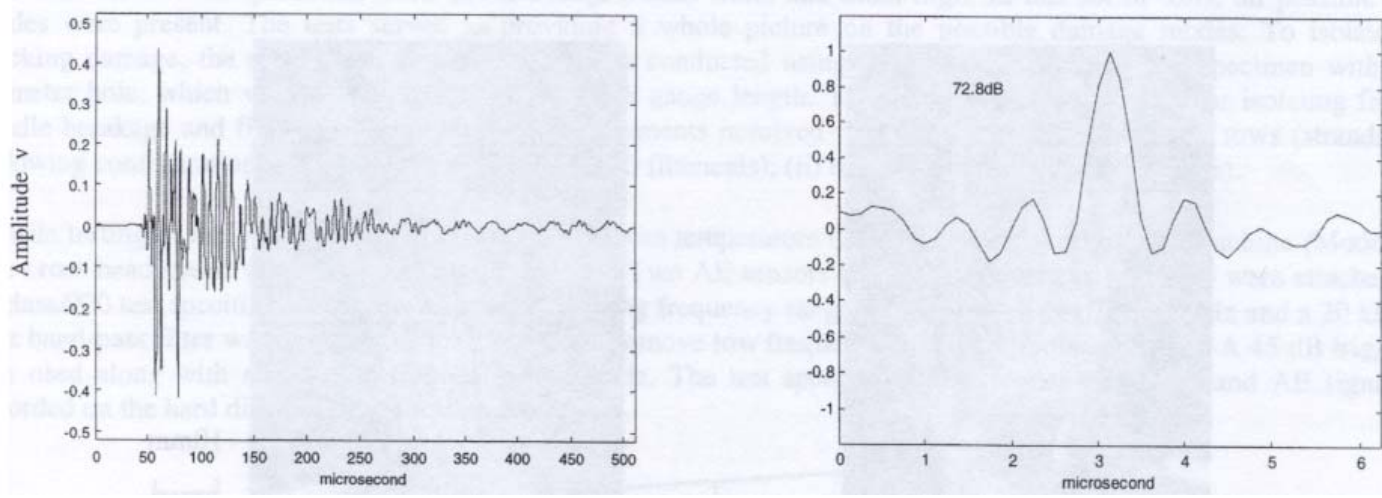

$72.8 \mathrm{~dB}$ signal and its recovered wave source (Zheng et al. 2000)
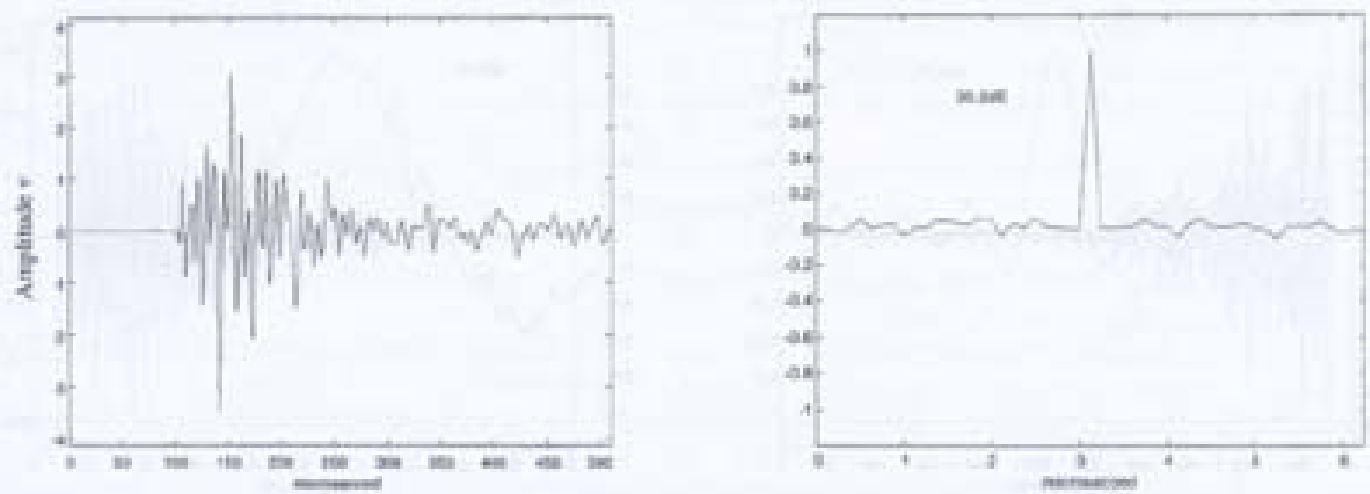

$90.8 \mathrm{~dB}$ signal and its recovered wave source (Zheng et al. 2000) 

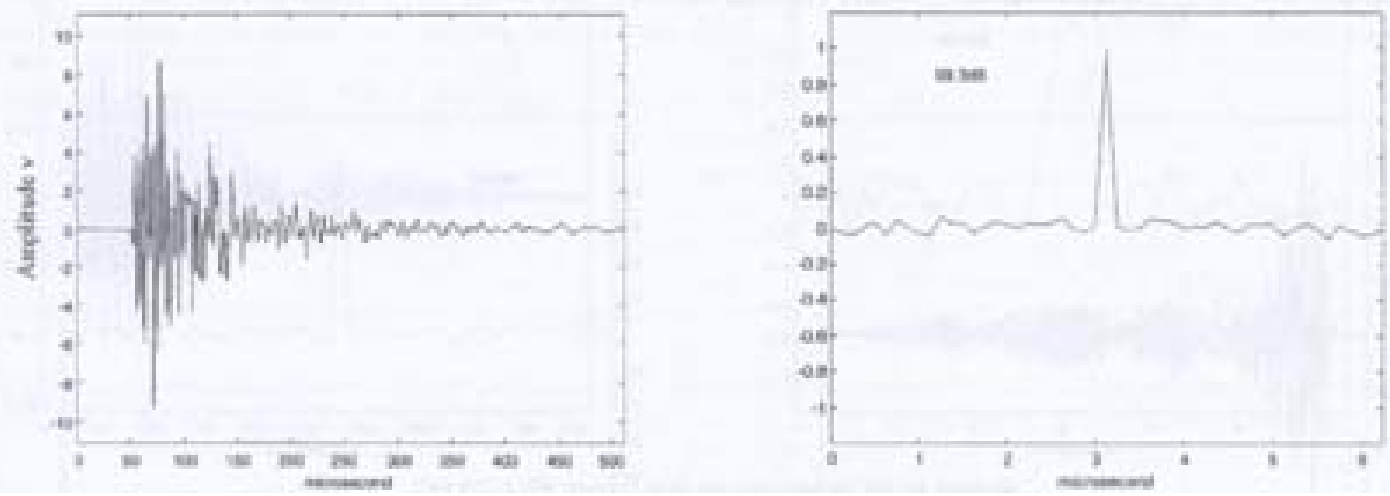

99.3 dB signal and its recovered wave source (Zheng et al. 2000)

Figure 2.3.1.2 AE signals and their recovered wave sources (Zheng et al. 2000)

Figure 2.3.1.3 contains signals recorded in the tests with the cured epoxy resin tensile test specimens. These results were obtained from a sensor placed $15 \mathrm{~mm}$ away from the edge of the hole. The recovered wave sources exhibited a longer duration due to the mode of crack initiation and propagation across the specimen (Zheng et al. 2000).
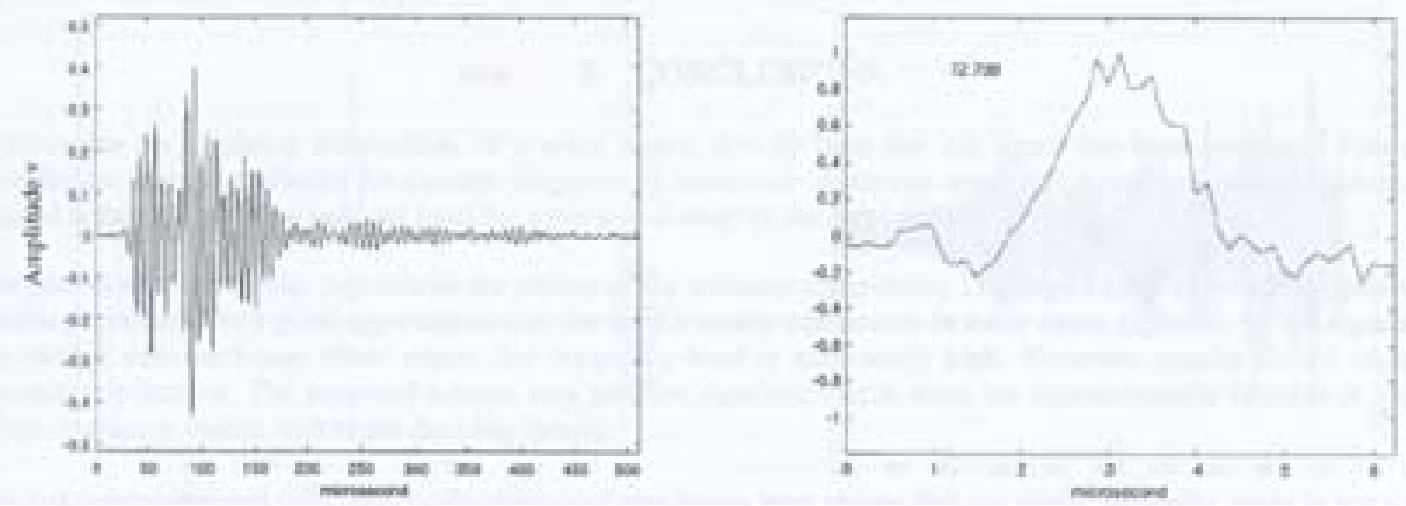

$72.7 \mathrm{~dB}$ signal and its recovered wave source (Zheng et al. 2000) 

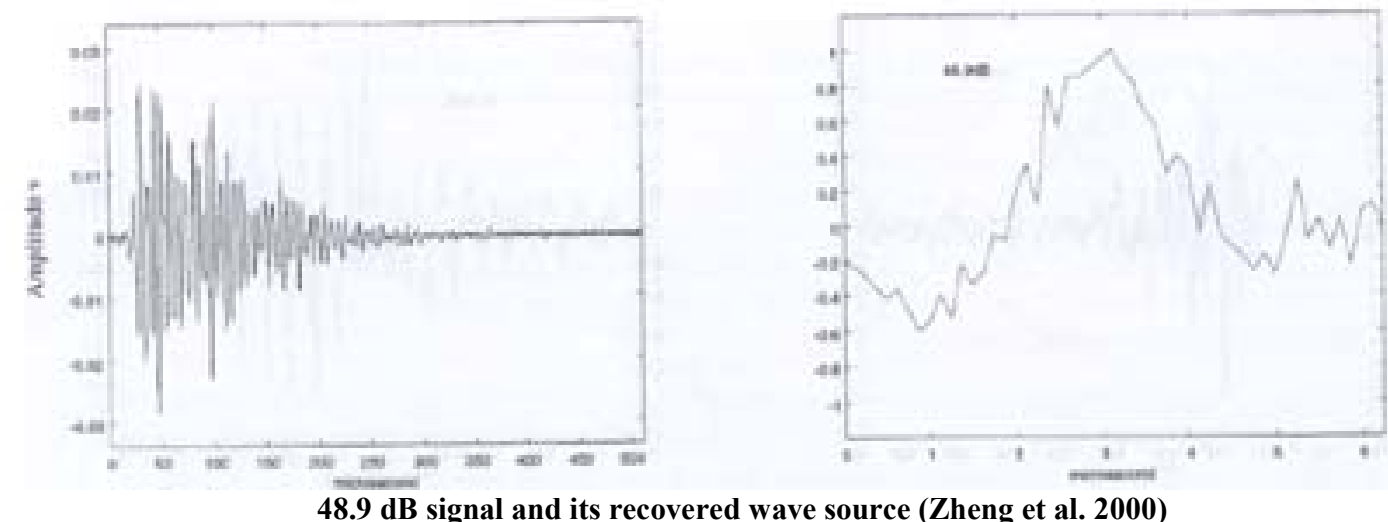

Figure 2.3.1.3 Signals of matrix cracking and their recovered wave sources (Zheng et al. 2000)

Figure 2.3.1.4 illustrates signals generated from the third set of tests (fiber/fiber bundle breakage and fiber pull-out). In this test, one sensor was attached to the fibers in the middle of the specimen. The higher amplitude signal in the fiber bundle failure was caused by the simultaneous multiple fiber fractures.
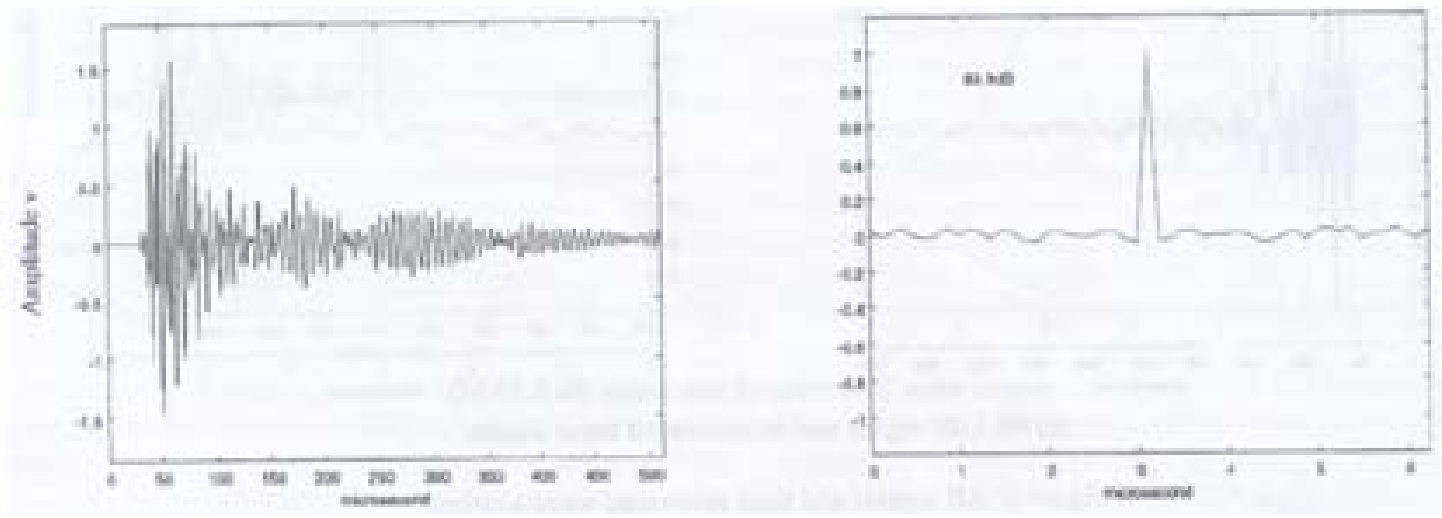

83.9 dB signal and its recovered wave source (Zheng et al. 2000) 

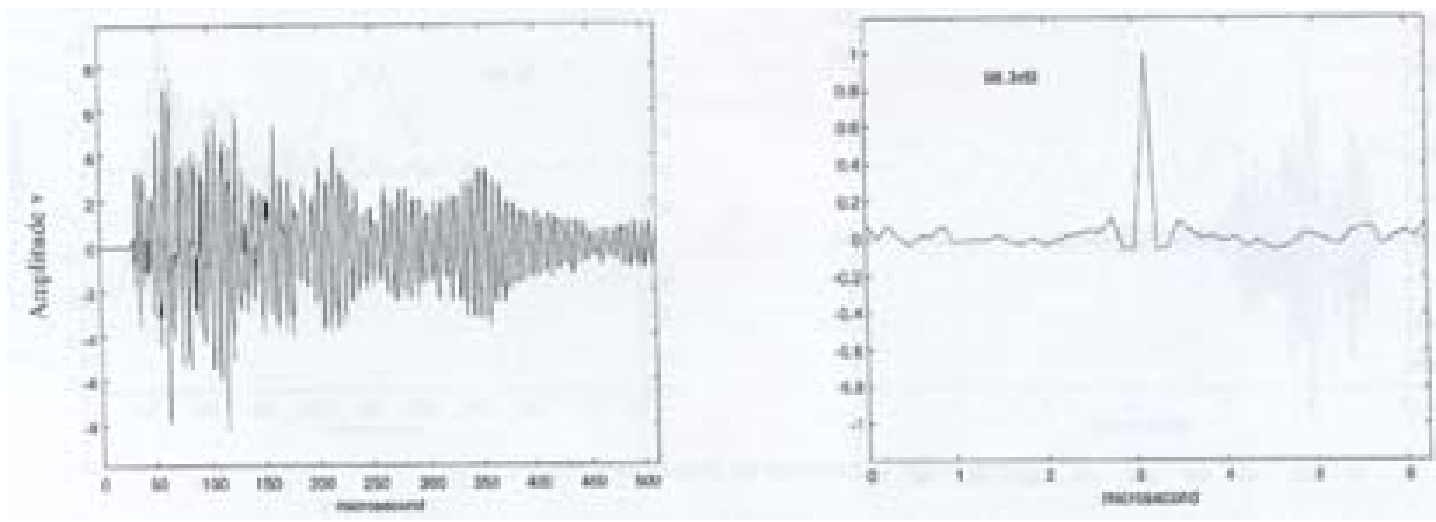

98.3 dB signal and its recovered wave source (Zheng et al. 2000)

Figure 2.3.1.4 Signals of fiber/fiber bundle breakage and recovered wave sources (Zheng et al. 2000)

The signals obtained from fiber pullout are shown in Figure 2.3.1.5. From these figures, it can be seen that the wave sources from the fiber/fiber bundle breakage and fiber pullout have a shorter duration than that of the matrix cracking.
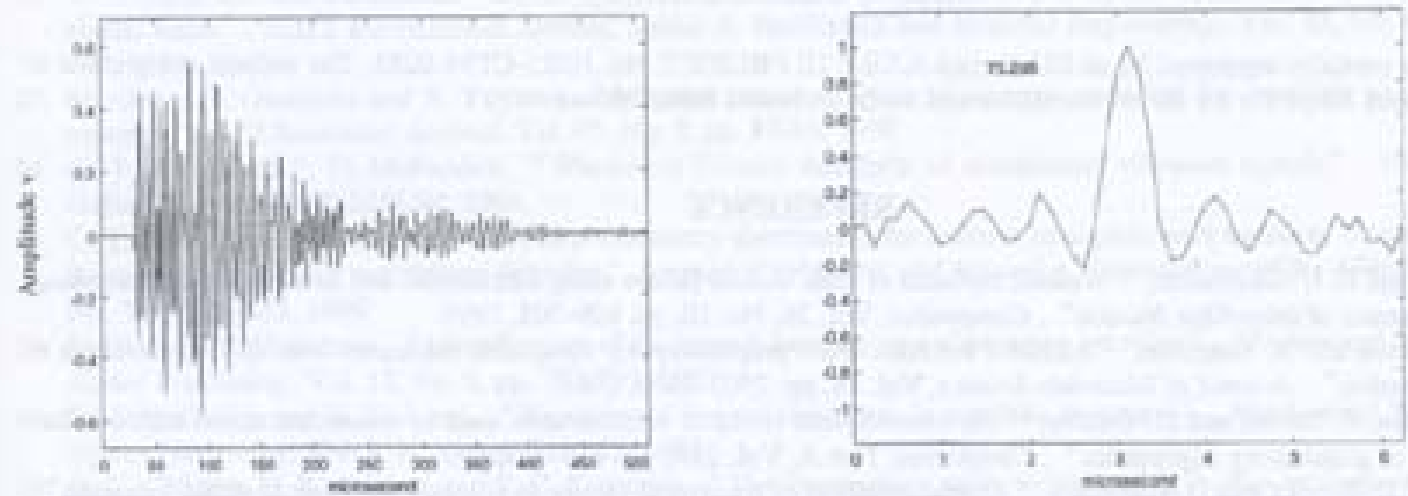

$75.2 \mathrm{~dB}$ signal and its recovered wave source (Zheng et al. 2000) 

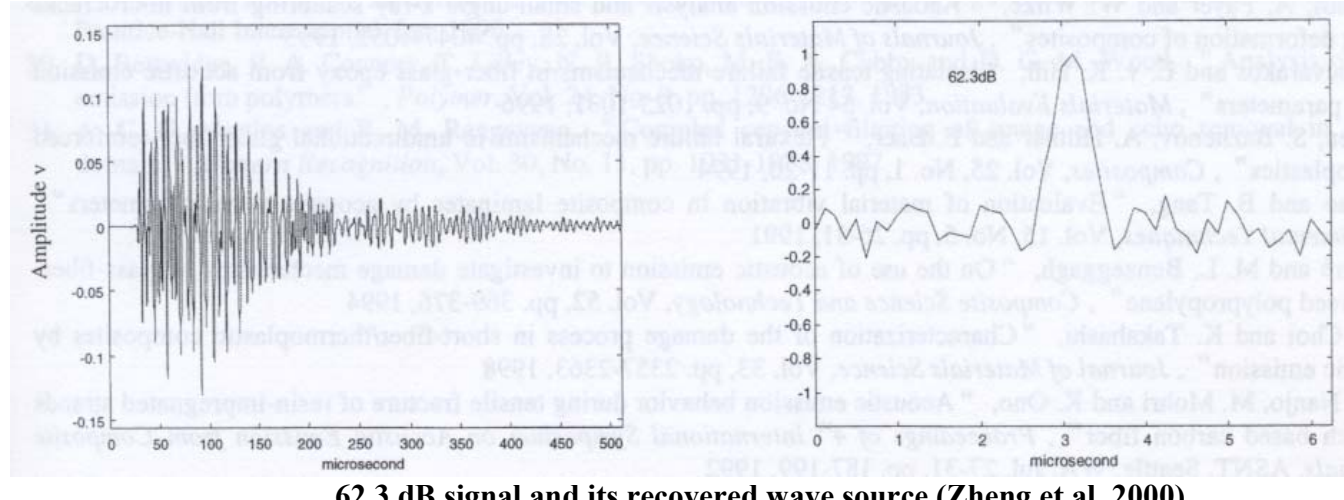

62.3 dB signal and its recovered wave source (Zheng et al. 2000)

Figure 2.3.1.5 Signals of fiber pull-out and recovered wave sources (Zheng et al. 2000)

\subsubsection{Conclusions}

Section 2.3.1 describes a method for damage diagnosis of composite structures using a modified AE signal analysis to obtain information on a wave source directly from the AE signal.

It was concluded that the precision of the results depended on the choice of the transient component; therefore, this approach suggested a good approximation to the ideal transient component with the deconvolution procedure.

Previous experiments have shown that the signal amplitude range was not a reliable indication to the type of damage for specifically fabricated specimens. It is unreliable because similar damage types may generate signals of different amplitude due to the distance between the damage and the sensor, the properties of the material, and the speed of the loading. 


\subsubsection{Experimental Investigation into the Use of Vibration Data for Long Term Monitoring of an All Composite Bridge (Ratcliffe et al. 2000)}

Section 2.3.2 discusses the results of a non-destructive test using broadband vibration data to monitor the structural integrity and health of an all-composite bridge. The 1-351 bridge located in Glasgow, Delaware was replaced with an all-composite bridge in the fall of 1998. The bridge now consists of two E-Glass/vinyl ester sandwich core sections (13ft x $32 \mathrm{ft}$ ) joined by a longitudinal joint in the traffic direction.

The bridge was divided into a mesh of 1050 test points covering the upper and lower surfaces of the composite deck. Using the vibration data, several structural behaviors of the bridge could be observed such as the interactions between the bridge and abutments; the effectiveness of the longitudinal joint to couple the deck sections; the effectiveness of the core to couple the face sheets; and the structural integrity and dynamic consistency of the entire structure. In addition, mode shapes and natural frequencies were determined and correlated with theoretical calculations and vibration analyses conducted for this bridge.

A novel algorithm using the vibration data was being experimented with to detect and locate manufacturing defects, material degradation or service damage in the bridge. This technique has been successful in locating damage in 1-D beam structures, but needed to be experimented with 3-D sandwich structures. 


\subsubsection{Introduction}

The inspection of bridge structures is a necessity in today's engineering process to rehabilitate damaged structures and help in the design of future projects. However, most of these techniques require that the location of the damage be known and typically can only assess damage near the surface of the structures since inspection of the entire structure would require significant time and money.

Composite materials have additional anomalies that affect structural performance, and since this is a relatively new material the types of damage and/or locations of material anomalies affecting performance are not well documented. Section 2.3.2 presents the results of an experimental effort performed by Colin P. Ratcliffe, John W.

Gillespie Jr., Dirk Heider, Douglas A. Eckel II, and Roger M. Crane using modal testing to assess the structural integrity of an all composite bridge.

\subsubsection{Conceptual Design}

The all-FRP bridge is a simply supported $32 \mathrm{ft}$. long bridge. The sectional geometry consists of a web-core sandwich (31 in. high by $26 \mathrm{ft}$. wide) constructed with two E-glass facesheets illustrated in Figure 2.3.2.1. The bridge was manufactured in two $13 \mathrm{ft}$. wide by $32 \mathrm{ft}$. long sections to simplify transportation and field installation. The two sections were then joined using an adhesively bonded vertical butt joint with top and bottom splice plates. Once the sections were installed, a 1.75 in. thick latex modified concrete wear surface was applied to the deck surface. 


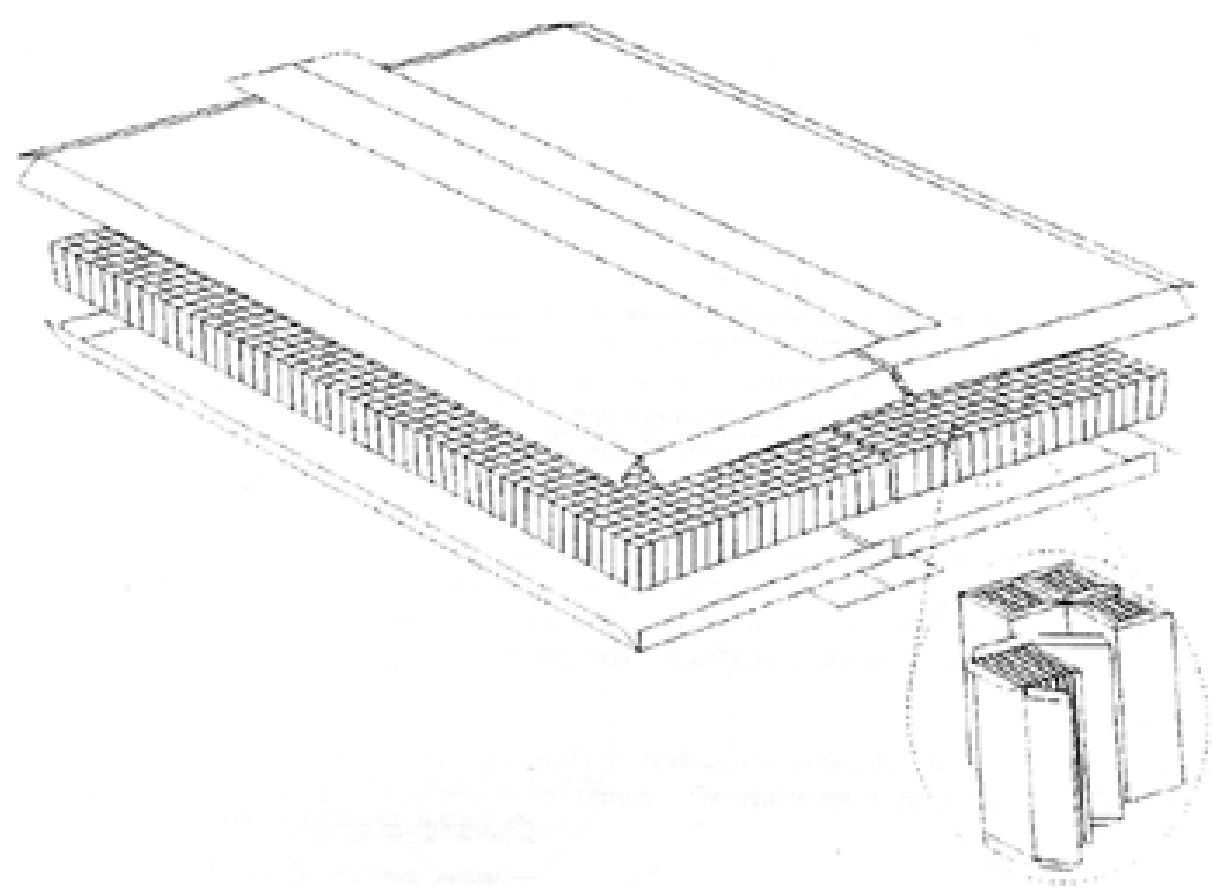

Figure 2.3.2.1 Conceptual design: placement of bottom facesheet, wrapped cores, and top facesheet. The splice plates are adhesively attached on-site (Ratcliffe et al. 2000)

\subsubsection{Fabrication}

The bridge was fabricated using E-glass fabric and vinyl-ester resin, and infused using the patented process known as SCRIMP (Seemann Composite Resin Infusion Molding Process). The fiber preforms having a total thickness of 0.088 inches consisted of five individual layers $(0,+-45$, and 90 orientations $)$ to achieve the design thickness that satisfied the stiffness and strength requirements. The sandwich structure was enclosed with a bottom facesheet $0.7 \mathrm{in.}$ thick and a top facesheet $0.5 \mathrm{in}$. thick. A core consisting of 893 foam rectangular prisms wrapped with one 0.088 in. thick layer of the fiber fabric separated the facesheets of each bridge section (Figure 2.3.2.1). The 8-fiber layers (40 plies) of the bottom tension facesheet were placed against a tooled surface, the prisms comprising the core were placed next, and the 6-fiber layers (30 plies) comprising the top 
compression facesheet were placed last. Once the entire section was sealed with a vacuum bag, the liquid resin was allowed to flow or infuse into the dry fiber preform.

This project focused on collecting a large quantity of frequency-based vibration data from the bridge deck to develop a damage detection procedure based on broadband vibration data. The data will also be used for comparison with future projects.

\subsubsection{Modal Testing}

Test grid points were arranged at both the top surface and bottom surface of the deck. Both top and bottom alignments were uniform, with an 18 in. spacing in the $\mathrm{X}$ -

direction (north-south), and a twelve in. spacing in the Y-direction (east-west). The grid was numbered basically south to north starting with number 5 on the top surface because numbers 1 through 4 were reserved for accelerometer positions. The highest numbered grid point on the top surface was number 550 .

The grid points for the lower surface were directly under the points on the top surface, however the lower surface has one less line of grid points at both the north and south ends because the deck extends over the supporting abutments were it was not possible to test. There is a plan view of the grid points for the southeast corner of the upper surface in Figure 2.3.2.2. 


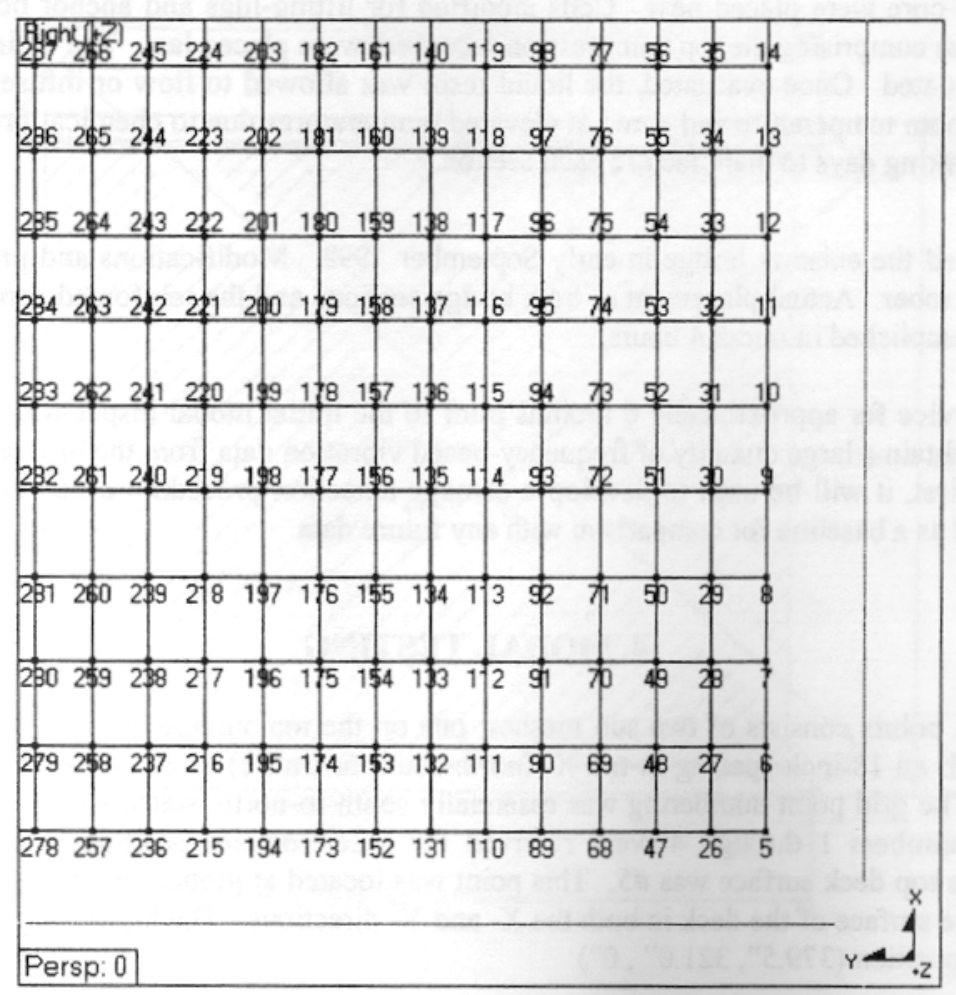

Figure 2.3.2.2 Southeast portion of grid pattern for upper deck surface (Ratcliffe et al. 2000)

\subsubsection{Equipment}

\subsection{Accelerometers}

There are two accelerometers located on the top surface, and two on the bottom surface of the bridge deck. They were placed approximately six feet from each corner. All the accelerometers used were PCB ICB Type 353B33 with a nominal sensitivity of $100 \mathrm{mV} / \mathrm{g}$. The upper surface accelerometers were secured to the deck through a two stage fastener while the accelerometers on the lower surface were super-glued directly to the surface. The accelerometers were able to be super-glued to the bottom surface of the composite face sheet because it was relatively smooth with no concrete wear surface on it. 


\subsection{Excitation and Force Gage}

A ball peen sized hammer was used to provide an adequate level of excitation so the general resonant behavior of the bridge could be assessed. The hammer was used with the third softest of the four available tips. This tip was capable of producing energy in the frequency range of interest.

\subsection{Analyzer}

The analyzer was an Oros, Inc., 16-channel PC-PACK analyzer. This analyzer incorporates ICP signal conditioning and was used because of its high dynamic range (90 $\mathrm{dB}$ per channel). Channel one was used for the hammer and channels two through five were used to capture data from the accelerometers.

\subsubsection{Data Capture}

The impact excitation test was determined to be the only test method that could capture the data needed in a cost and time effective manner. To eliminate the amount of error, each coordinate was impacted twice with the hammer and the frequency responses were averaged. More impacts would have been preferred but two impacts allowed the entire structure to be tested in the time allowed. The final results indicated that two impacts were sufficient in identifying the modal behavior of the structure (Ratcliffe et al. 2000). The data captured was in the range of $0-1 \mathrm{kHz}$, with a frequency resolution of $0.625 \mathrm{~Hz}$. The real-time measurement was determined to be 1.6 seconds per impact. Since the structure's ring down was nearly complete at the end of the 1.6 second time measurement, the settling time was negligible. 


\subsubsection{Modal Analysis}

The modal analysis was performed using a commercial program known as VES. The program used the data captured by the four reference accelerometers to perform modal analysis. It was concluded at the end of the modal analysis that one reference accelerometer would have produced enough data to analyze the modal. Due to the large number of test points and quantity of data, it took the program more than 30 hours to complete each phase of the analysis.

\subsubsection{Final Modal Results}

Once the final modal analysis was complete a total of 20 modes in the frequency range $18-216 \mathrm{~Hz}$ were determined. The natural frequencies and modal viscous damping ratios determined from the analysis are presented in Table 2.3.2.1, and eight of the mode shapes are illustrated in Figure 2.3.2.3. 
Table 2.3.2.1 Natural Frequencies and Corrected Viscous Damping Ratios for Full Composite Bridge Deck (Ratcliffe et al. 2000)

\begin{tabular}{|c|c|c|}
\hline Mode & $\begin{array}{c}\text { Natural Frequency } \\
(\mathrm{Hz})\end{array}$ & $\begin{array}{c}\text { Corrected Viscous } \\
\text { Damping Ratio } \\
(\%)\end{array}$ \\
\hline 1 & 18.74 & 4.6 \\
\hline 2 & 24.10 & 2.6 \\
\hline 3 & 44.67 & 3.7 \\
\hline 4 & 51.82 & 1.6 \\
\hline 5 & 76.61 & 1.3 \\
\hline 6 & 87.17 & 1.5 \\
\hline 7 & 90.57 & 0.8 \\
\hline 8 & 107.51 & 0.9 \\
\hline 9 & 117.18 & 1.5 \\
\hline 10 & 132.63 & 0.7 \\
\hline 11 & 136.43 & 1.3 \\
\hline 12 & 146.83 & 1.2 \\
\hline 13 & 149.42 & 1.3 \\
\hline 14 & 155.50 & 1.2 \\
\hline 15 & 163.34 & 1.1 \\
\hline 16 & 165.87 & 0.7 \\
\hline 17 & 170.54 & 0.8 \\
\hline 18 & 178.36 & 0.9 \\
\hline 19 & 207.90 & 1.1 \\
\hline 20 & 215.97 & 1.5 \\
\hline
\end{tabular}

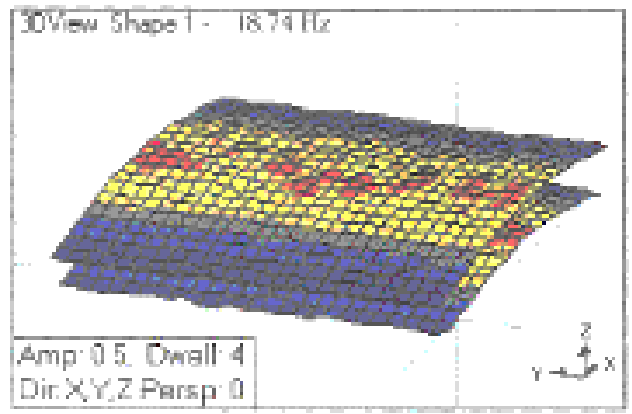

(a)

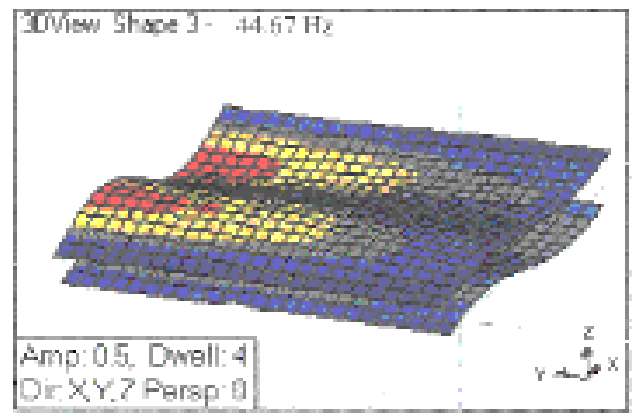

(b) 


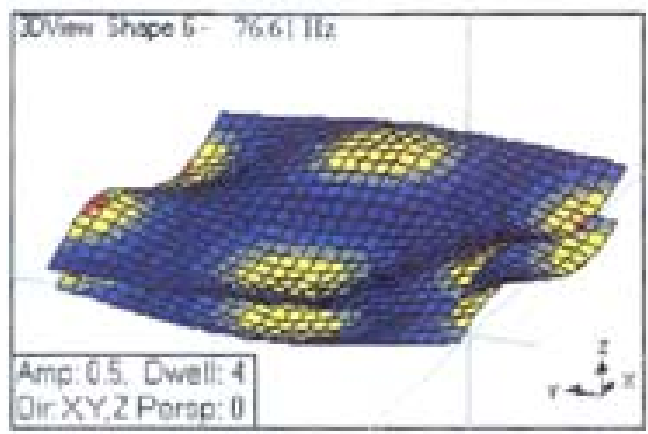

(c)

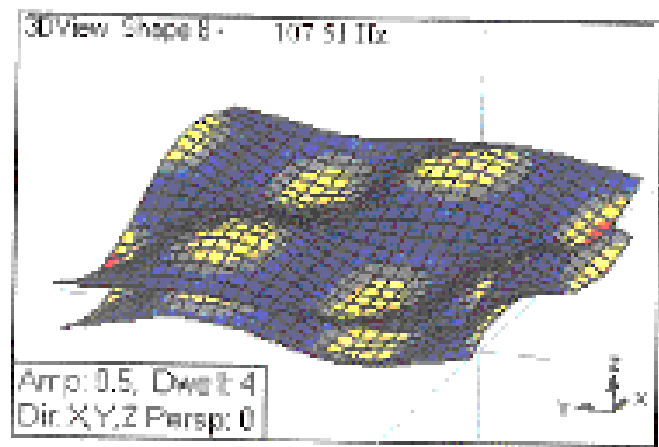

(e)

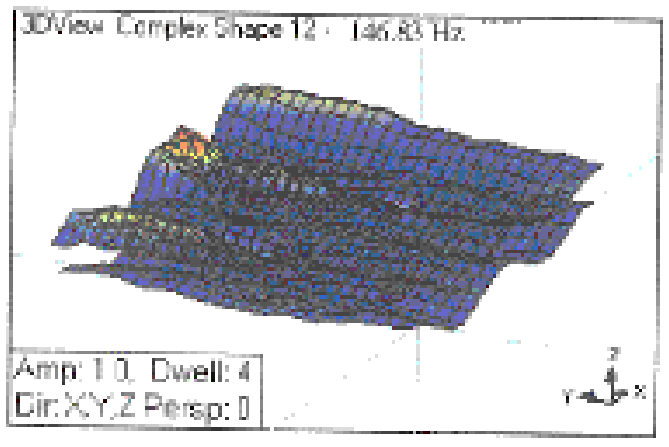

(g)

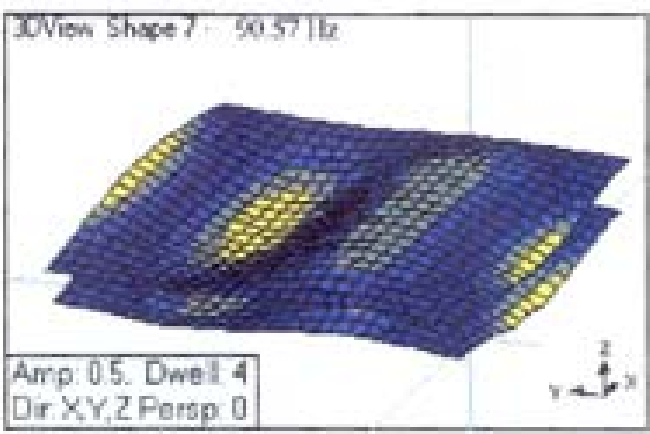

(d)

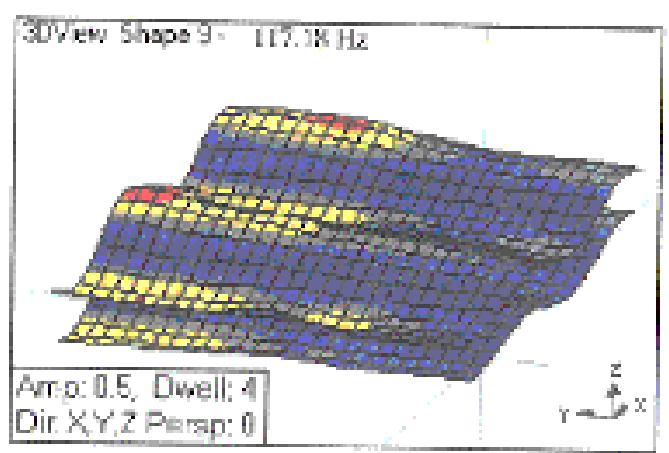

(f)

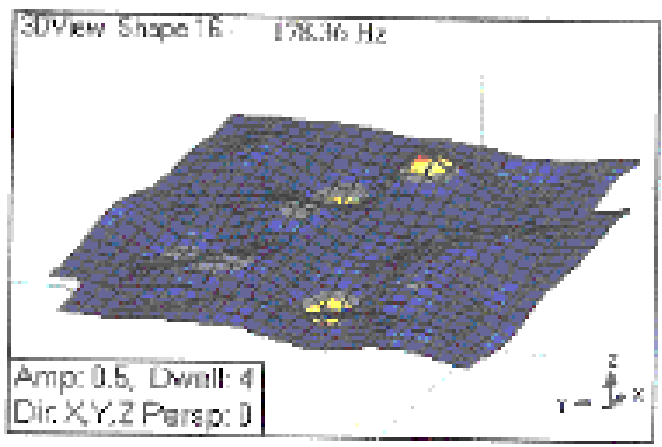

(h)

Figure 2.3.2.3 Mode shape for full scale bridge section with (a) 18.6 Hz resonant frequency, (b) $45.0 \mathrm{~Hz}$ resonant frequency, (c) $76.6 \mathrm{~Hz}$ resonant frequency, (d) $90.5 \mathrm{~Hz}$ resonant frequency, (e) $107.51 \mathrm{~Hz}$ resonant frequency, (f) $117.3 \mathrm{~Hz}$ resonant frequency, (g) $146.83 \mathrm{~Hz}$ resonant frequency, and (h) $178.36 \mathrm{~Hz}$ resonant frequency (Ratcliffe et al. 2000) 


\subsubsection{Analytical Determination of Fundamental Resonant Frequency}

The natural frequency of the bridge was determined using anisotropic beam theory and the governing fourth order differential equation. Simply supported boundary conditions are used to find the natural frequencies for free vibration. Once the theoretical fundamental frequency is calculated, it was compared to the natural frequency found from modal testing to determine the structural stiffness of the bridge. The natural frequencies will be useful in determining structural properties because it is a function of the flexural rigidity, cross-sectional area, span and the mass densities of the materials from the bridge (Ratcliffe et al. 2000). The theoretical value for the flexural rigidity of the bridge was determined to be $5.7 \times 10^{9} \mathrm{lb}$.-in. ${ }^{2}$, and the fundamental natural frequency for the bridge was analytically calculated to be $18.6 \mathrm{~Hz}$.

\subsubsection{Discussion of Modal Results}

Illustrated in Figure 2.3.2.3, all the mode shapes were smooth and continuous, indicating quality data and analysis. For low frequencies (e.g., below $75 \mathrm{~Hz}$ ), simply supported boundary conditions were observed, but for frequencies above $75 \mathrm{~Hz}$ some relaxation of the boundaries occurred, indicating some motion along the y-direction of the abutments. The first natural frequency analytically calculated $(18.6 \mathrm{~Hz})$ was in excellent agreement with the first natural frequency experimentally determined (18.74 $\mathrm{Hz})$.

The results, however, indicated some variation in motion between the top and bottom surfaces. This was of some interest because it could give some clues about the 
effectiveness of the core to couple the face sheets. The two conclusions of why there were some variations in the top and bottom surfaces were that the bridge panels may have had some relative motion between the top and bottom surfaces, or there was some difference in ambient conditions (e.g., the top surface was measured on a hot day and the bottom surface measured on a cool day). It was considered more likely that first of the two conclusions caused the variation indicating manufacturing imperfections.

\subsubsection{Data Quality}

The data quality of the overall project proved to be extremely good. The quality of the in-service monitoring was typically at $98 \%$ or better, with virtually no drop-outs. However, the impact-generated data proved to have a higher signal noise above about $500 \mathrm{~Hz}$, but was still acceptable for many applications.

\subsubsection{Conclusion}

The experimental observed fundamental resonance $(18.74 \mathrm{~Hz})$ correlates well with the theoretical fundamental natural frequency $(18.6 \mathrm{~Hz})$.

The abutments on the bridge provided good simply supported boundary conditions up to about $75 \mathrm{~Hz}$. The motion along the y-direction of the abutments was probably due to the rubber pad placed between the abutments and lower surface of the deck where a gap was visually observed. This was actually captured by the modal analysis at frequencies above $75 \mathrm{~Hz}$. The final conclusion was that the inspection of large structures may be accomplished with the modal technique discussed in this section. 


\subsubsection{Conclusion on Acoustic Wave Propagation}

Based on the literature review of acoustic emission it was concluded that defects could be detected using this method. This method has shown considerable promise for evaluating cracks and other defects in composite members.

The advantages of acoustic techniques include:

- $\quad$ Acoustic pulses have lower frequencies than pulses generated by ultrasonics and can penetrate deeper into the composite material.

- $\quad$ AE sensors can be embedded into the structure to provide continuous (real time) sensing of emitted signals.

The disadvantages of acoustic techniques include:

- $\quad$ The AE signal is affected by many external noises such as mechanical noise, electrical background noise and environmental noise.

- $\quad$ Acoustic pulses generated by impact (hammers, etc.) propagate in all directions rather than as a focussed beam such as in ultrasonics.

- $\quad$ Control of impact pulse parameters such as frequency and frequency bandwidth is difficult and limited to impact duration and intensity. 


\subsection{Load Testing with Strain Gages}

This section describes the evaluation of civil structures (bridges) through load testing. The load testing was performed on all-composite bridges and also on bridges rehabilitated with composite wraps. Strain gages were used to collect experimental data, which were compared to calculated theoretical stresses.

\subsubsection{Evaluating Effectiveness of FRP Composites for Bridge Rehabilitation through Load Testing (Hag-Elsafi et al. 2000)}

FRP composite laminates are now being used to rehabilitate deteriorating bridges such as the seventy-year old reinforced concrete bridge in Rensselaer County, New York. This section discusses the load tests conducted before and after installation of the FRP laminates, which evaluated the effectiveness of the rehabilitation system in strengthening the bridge structure.

\subsubsection{Introduction}

The bridge used to conduct this research was a simple span, reinforced concrete T-beam structure. The bridge has 26 beams spaced at $4.5 \mathrm{ft}$ center to center, and has five traffic lanes. Visual inspections indicated that many of the beams had large defected areas consisting of freeze-thaw cracking, and delaminations. FRP laminates was used to rehabilitate the bridge to avoid temporary closure of the bridge and disruption to traffic. FRP laminates could also improve shear and axial capacities as well as improving flexural strength to the reinforced concrete beams. The bridge was tested before 
installation of the laminates and again after installation to evaluate the effectiveness of FRP composites.

\subsubsection{Service Load Stresses}

The service load stresses for the main steel rebars and concrete were estimated by conservatively assuming the usual steel strength, $F_{y}=30 \mathrm{ksi}$, and concrete strength, $\mathrm{f}_{\mathrm{c}}=$ $3 \mathrm{ksi}$. Once the stresses were calculated using these values, they were considerably lower than the corresponding allowable stresses for steel and concrete according to Guide Specifications for Highway Bridges (Hag-Elsafi et al. 2000). Although the estimated service load stresses and load effects were relatively low based on the assumptions, the bridge still needed rehabilitation because of an average $15 \%$ loss due to corrosion in the steel rebar area (Hag-Elsafi et al. 2000). The webs were strengthened using FRP Ujackets to eliminate further delamination and freeze-thaw cracking. The FRP laminates used were unidirectional carbon fibers saturated with epoxy resin that was applied in the field by a method known as Awet lay-up ${ }^{\circledR}$ process. The strengthening scheme, based on a 15\% loss criterion, is illustrated in Figure 2.4.1.1. 


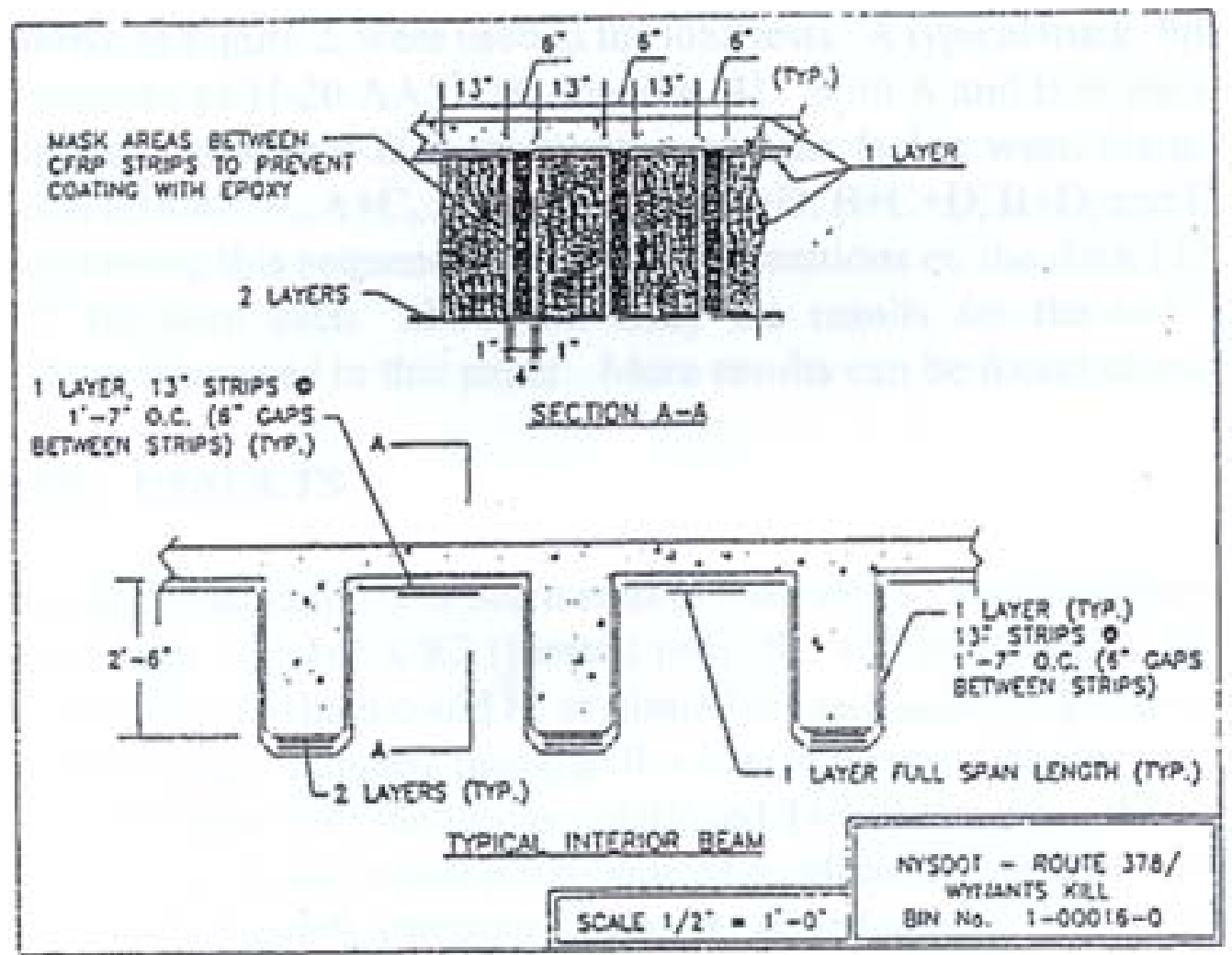

Figure 2.4.1.1 Details of the FRP laminate strengthening system (Hag-Elsafi et al. 2000)

\subsubsection{Load Testing}

Nine beams with strain gages were used to obtain live load distribution. Figure 2.4.1.2 shows the beams with the location of strain gages to measure flexural strains in main reinforcing steel and the FRP laminates. 

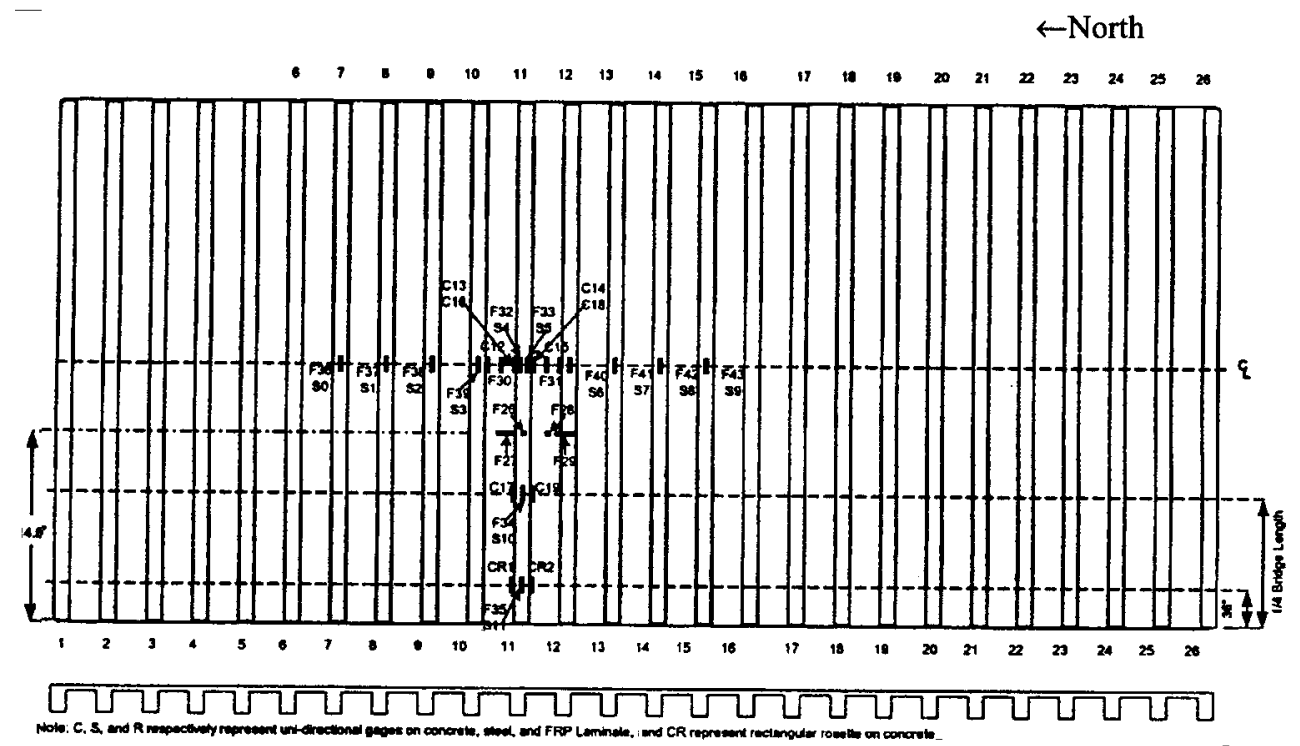

Figure. 3a

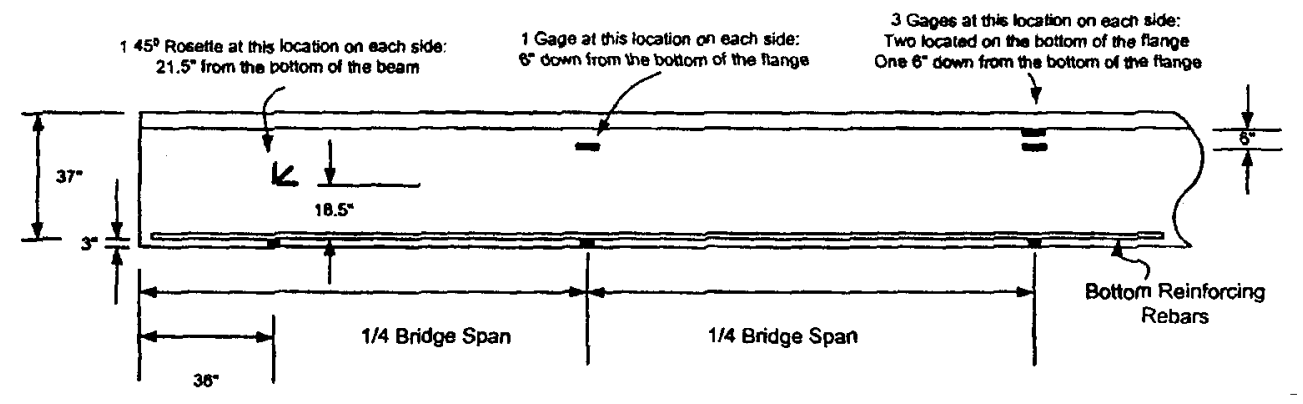

Figure. $3 b$

Figure 2.4.1.2 Instrumentation Plan. (3a) Gage numbers and location, (3b) Gage locations on center beam (Hag-Elsafi et al. 2000)

Conventional foil strain gages were used to measure the strains. For the "Before" installation test, 10 gages on the steel and 13 gages on the concrete were used, and an additional 18 gages on FRP laminates were used for the "After" installation test. Four conventional trucks, shown in Figure 2.4.1.3, were used in the load tests. Trucks A and B were used for the tests in the eastbound lane facing east, and C and D were used for tests in the westbound lane facing west. 


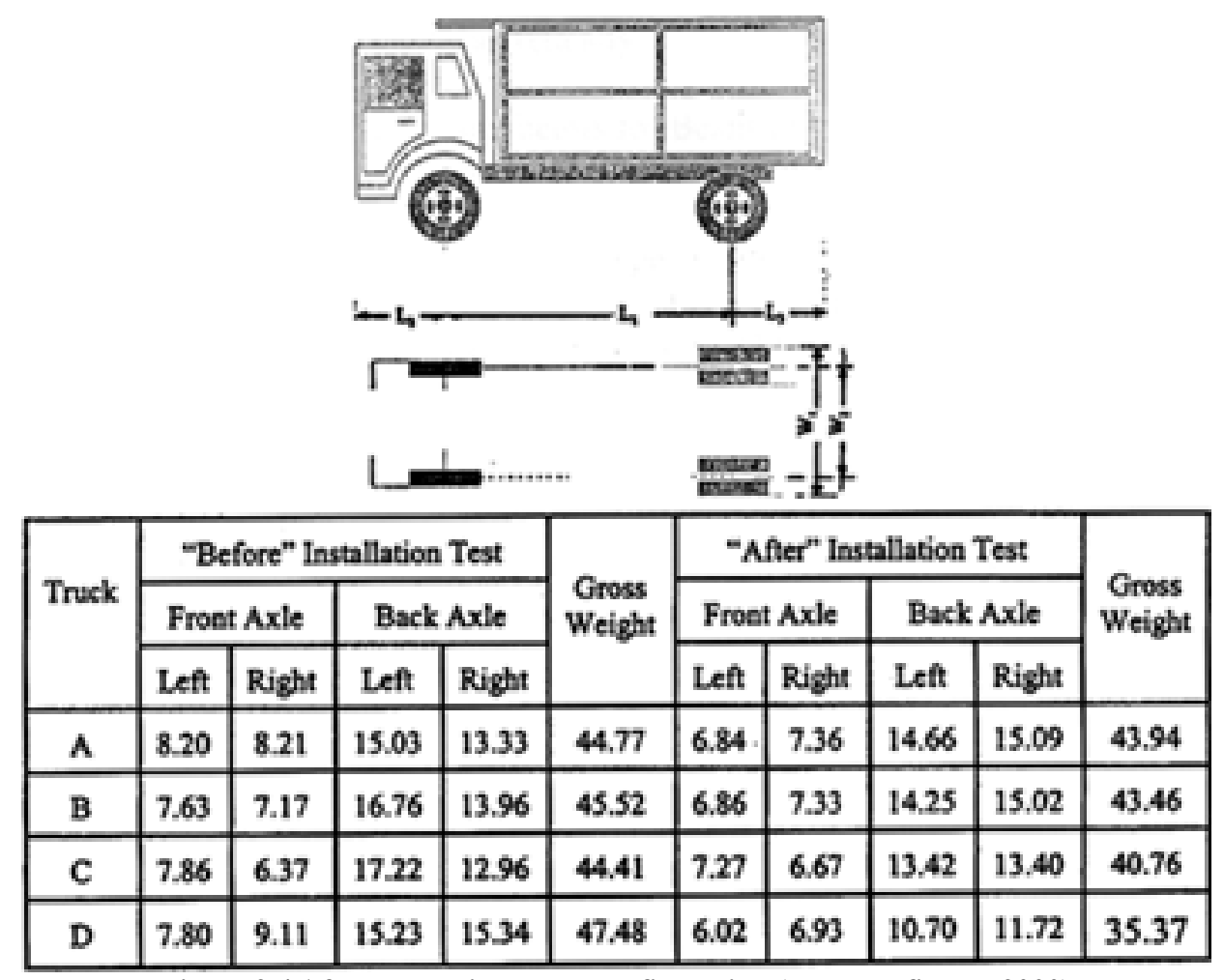

Figure 2.4.1.3 Truck weights and configuration (Hag-Elsafi et al. 2000)

\subsubsection{Load Test Results}

The results discussed in section 2.8 are from the gages located on Beam 11. The total moments at midspan before and after rehabilitation are given in Table 2.4.1.1. The calculated live load distribution factors for Beam 11 are also given in Table 2.4.1.1. The test results revealed fixity of beam ends indicated by the compressive strains measured by the gages located near the end of Beam 11 (S11 and CR2), illustrated in Figure 2.4.1.2. The test results indicated an average decrease of $11.4 \%$ in the "Before" rehabilitation calculated live load distribution factors for Beam 11; therefore, the installation of the transverse FRP laminates between the beams beneath the deck improved transverse load distribution. Figure 2.4.1.4 shows the calculated theoretical stresses and the measured stresses in the main steel rebar before and after rehabilitation. The graphs show good agreement between the theoretical values and the measured values. Illustrated in Figure 
2.4.1.4(c), a $13 \%$ decrease in the main steel live load stresses after rehabilitation with the FRP laminates is determined.

Table 2.4.1.1 Before and After live load distribution factors for Beam 11 (Hag-Elsafi et al. 2000)

\begin{tabular}{|c|c|c|c|c|}
\hline \multirow{2}{*}{$\begin{array}{c}\text { Iluxks } \\
\text { Posilion } \\
\text { ftom } \\
\text { Abmitmentx } \\
\text { fit }\end{array}$} & \multicolumn{2}{|c|}{ AEcforse Rchabilitation } & \multicolumn{2}{|c|}{ Asfter Retubilitation } \\
\hline & 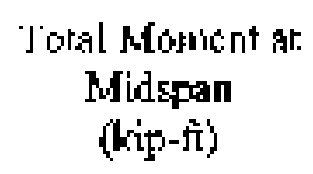 & $\begin{array}{l}\text { Liver I.gad } \\
\text { [jistribution } \\
\text { Fiastor }\end{array}$ & 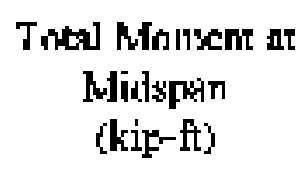 & $\begin{array}{l}\text { T.jue Load } \\
\text { Diulribution } \\
\text { Fastar }\end{array}$ \\
\hline $12.0 \mathrm{ft}$ & 162.6 & 0.230 & 151,0 & [D.L 10 \\
\hline 13.5 & 205.3 & 0.214 & 191.1 & 0.190 \\
\hline 74.5 & 236.8 & 0.236 & 220.5 & 0.204 \\
\hline
\end{tabular}
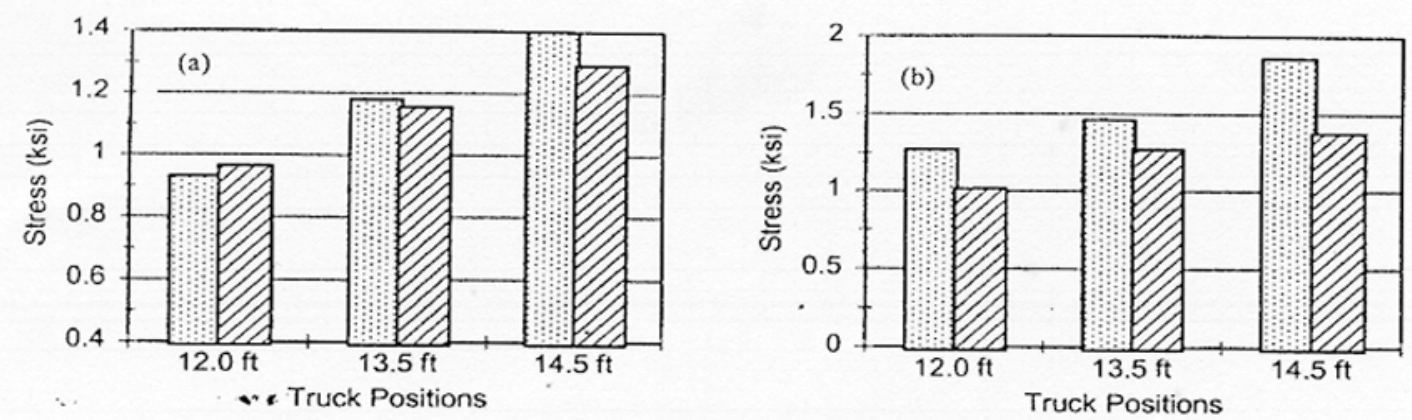

Predicted "After" $\square$ Measured "After"

Dredicted "Before" $\square$ Measured "Before"

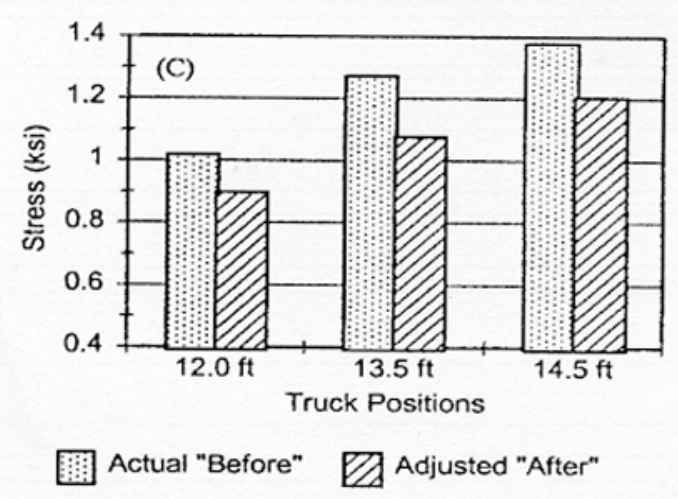

Figure 2.4.1.4 Comparison of main rebar stresses: (a) and (b) Predicted Vs measured for a "Before" and "After" installation, (c) "Before" Vs "After" (Hag-Elsafi et al. 2000) 


\subsubsection{Conclusion}

Before and After FRP installation test were performed on a seventy-year old reinforced concrete T-beam bridge. The results revealed an improvement in live-load distribution, an increased effective width of the beams flanges, and fixity of the beam ends, which was unintended. The FRP laminates demonstrated a feasible, cost-effective bridge rehabilitation technique. As illustrated by the values in the graphs and tables, live load testing is a good NDE technique to evaluate the effectiveness of the rehabilitation with FRP laminates.

\subsubsection{Proof Load Testing and Monitoring of an FRP Composite Bridge (Yannotti,}

\section{A.P. et al. 2000)}

A prefabricated E-glass FRP composite bridge replaced a badly deteriorated concrete slab superstructure in New York State, which opened in October 1998. Once the composite bridge was constructed, proof load testing was performed where strains and deflections were monitored and compared to theoretical values obtained from a design model. Section 2.4.2 describes the bridge design and field test results.

\subsubsection{Introduction}

Deterioration of steel and concrete bridges due to freeze-thaw conditions and the application of road salts during the winter seasons have presented a significant problem in the management and maintenance of the American highway infrastructure. As a result, 
fiber-reinforced polymer composites that appear to be less vulnerable to environmental damages have been considered as an alternative material (Yannotti, A.P. et al. 2000).

Since FRP composites are new materials, and their behavior and life service is not yet completely understood; it is necessary to perform NDE on different composite structures in service to achieve a better understanding of FRP composite materials. Such evaluations would produce better and more cost-effective designs in the future. Therefore, Yannotti, A.P. et al. performed different load tests on the composite bridge shown in Figure 2.4.2.1 to collect data that could refine the design and construction process of future composite bridges. The data collected consisted of strains and deflections measured under truckloads of known weight

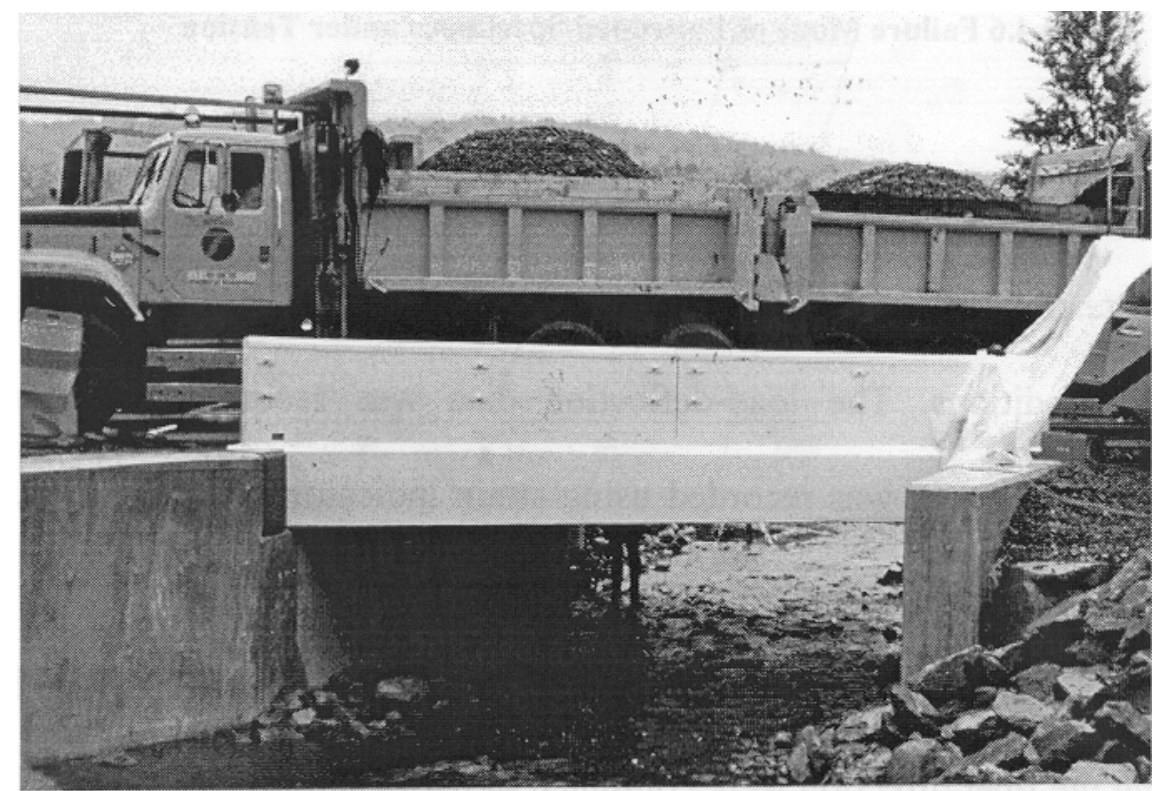

Figure 2.4.2.1 Proof load testing of the FRP superstructure (Yannotti, A.P. et al. 2000)

\subsubsection{Bridge Structure}

A reinforced concrete slab bridge over Bennett's Creek in Steuben County was replaced with New York's first FRP bridge. The bridge was load-posted for ten tons due 
to very poor conditions. The minimum traffic on the bridge made it an ideal experimental site for an FRP bridge.

\subsubsection{Design and Fabrication}

The $7 \mathrm{~m}$ long and $10 \mathrm{~m}$ wide bridge was fabricated using E-glass/vinyl ester material. The bridge was fabricated in two pieces with a longitudinal centerline joint and connected with a mechanical shear key filled with an epoxy resin. The design criteria limited the service load stresses to $20 \%$ of ultimate strength and live load deflections were limited to $\mathrm{L} / 800$.

\subsubsection{Sensors and Instrumentation}

The strain gages used for measuring the strains were general-purpose $120 \Omega$, selftemperature compensating, constantan foil-strain gages. These gages were mounted directly on the bottom laminate. Twenty-four gages were used in all, 18 placed in the longitudinal direction and 6 in the transverse direction illustrated in Figure 2.4.2.2. The gages were placed at the span midpoints and quarter points so they could be easily compared to the theoretical values. Also, the strain gages at the span midpoints could verify strain compatibility across the center longitudinal joint. All the gages were protected from the environment so long-term monitoring could be achieved. Midspan deflections were also measured using LVDTs, and compared to conventional field survey measurements. 


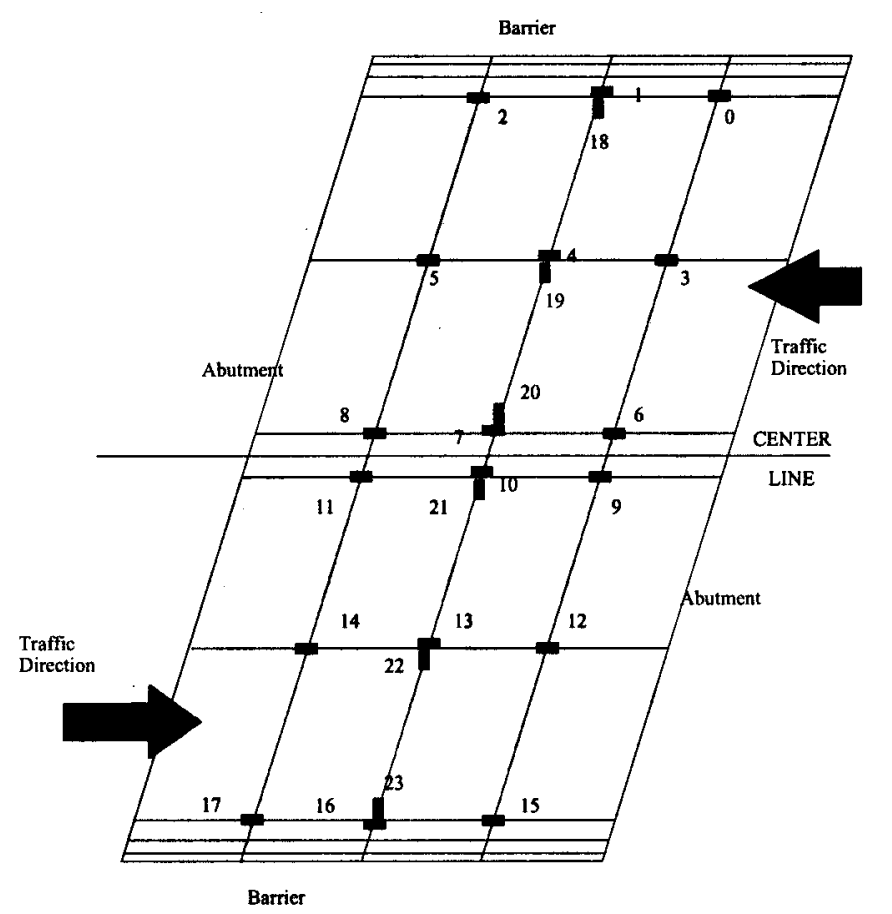

Figure 2.4.2.2 Locations of strain gages (Yannotti, A.P. et al. 2000)

\subsubsection{Proof Load Test}

Four different load cases were performed during the load testing conducted in October 1998. Case 1 consisted of two empty trucks, case 2 had two trucks carrying service loads, case 3 had four trucks carrying service loads, and case 4 had four overloaded trucks. During testing (case 4), the bridge was loaded 1.4 times heavier than the design load. The trucks used were ten-wheel dump trucks of known weight. To eliminate random errors, each case was repeated 3 times and an average of three values was taken. The trucks were placed at pre-marked positions on the bridge to maximize bending moment. 


\subsubsection{Test Results}

The maximum strain recorded on Gage 1 during Load Case 4 was $204 \mu \varepsilon$, which was considerably less than the expected $600 \mu \varepsilon$. This suggests a greater load capacity than determined through analysis. The design deflection limitation $(\mathrm{L} / 800)$ was calculated to be $8.8 \mathrm{~mm}$ and the maximum deflection measured at midspan for Load Case 4 was $3.5 \mathrm{~mm}$, which was considerable less than the limitation value. Table 2.4.2.1 shows all the gages and the strains associated with Load Case 4. Figure 2.4.2.3 shows the consistency of the results between adjacent pairs of gages located across the centerline longitudinal joint. This suggests an effective transfer of the loads across the centerline longitudinal shear key.

Table 2.4.2.1 Strain data from Load Case 4 (Yannotti, A.P. et al. 2000)

\begin{tabular}{|l|l||l|l||l|l||l|l|}
\hline Gage & $\begin{array}{l}\text { Strain } \\
(\mu \varepsilon)\end{array}$ & Gage & $\begin{array}{l}\text { Strain } \\
(\mu \varepsilon)\end{array}$ & Gage & $\begin{array}{l}\text { Strain } \\
(\mu \varepsilon)\end{array}$ & Gage & $\begin{array}{l}\text { Strain } \\
(\mu \varepsilon)\end{array}$ \\
\hline 0 & 33 & 6 & 127 & 12 & 62 & 18 & -30 \\
\hline 1 & 204 & 7 & 147 & 13 & 134 & 19 & 40 \\
\hline 2 & 67 & 8 & 139 & 14 & 64 & 20 & 17 \\
\hline 3 & 81 & 9 & 132 & 15 & 67 & 21 & -14 \\
\hline 4 & 102 & 10 & 156 & 16 & 156 & 22 & 44 \\
\hline 5 & 157 & 11 & 113 & 17 & 14 & 23 & -22 \\
\hline
\end{tabular}




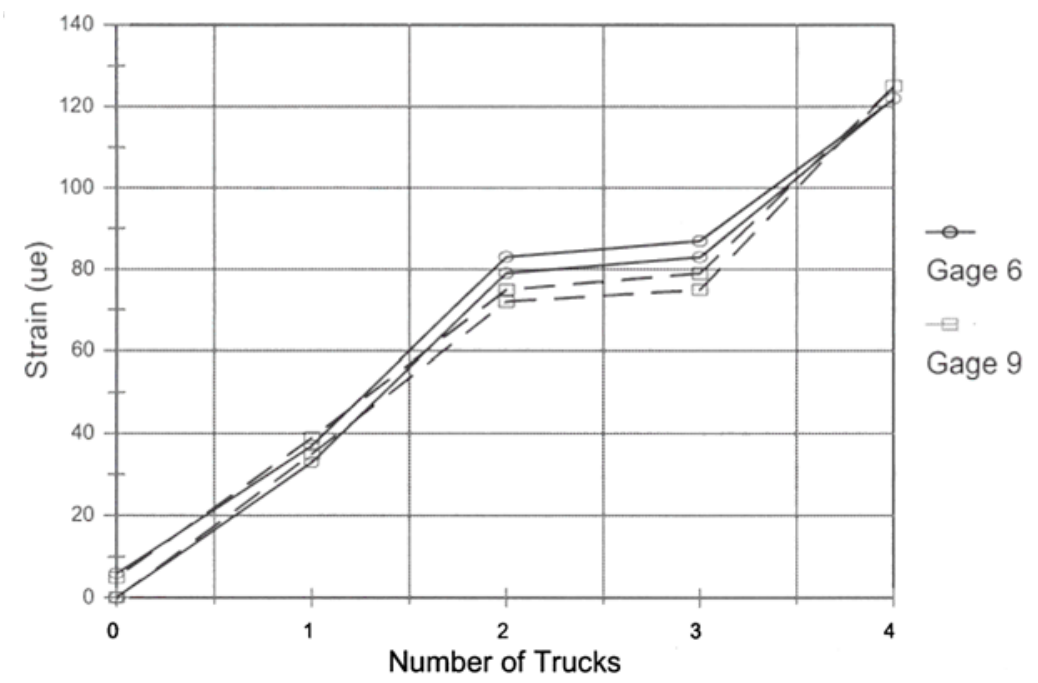

Figure 2.4.2.3 Test results showing the effectiveness of the shear-key (Yannotti, A.P. et al. 2000)

Load Case 4 produced significantly higher strains at gage 1 than at the midspan gages 4 and 7. It was concluded that gage 1 had some problems. Once gage 1 was corrected the second load test had more accurate results.

\subsubsection{Visual Inspection}

Frequent visual inspections were also performed to check for delaminations in the FRP materials. These inspections consisted of tapping the underside of the bridge surface with a rubber mallet or a small rounded stone. Monitoring also included checking for cracks in the FRP materials and any signs of ultraviolet or moisture deterioration. Chain dragging was also performed to identify any delaminations between polymer concrete wearing surface and the FRP structure. Some damage to the polymer concrete wearing surface from the snowplows used during the winter season has been identified already. In addition to the continuos visual inspections, load test were repeated every six-months to obtain sufficient data. 


\subsubsection{Conclusions}

Load testing of the fiber-reinforced-polymer composite bridge, in New York, has evaluated its performance and the results were compared with design values from an analytical model. The results indicated that the FRP superstructure is performing much better than the previous concrete bridge and the stresses and deflections measured are well below the calculated values obtained by the preliminary finite element analysis model (Yannotti, A.P. et al. 2000). This data can be used to calibrate and refine analytical models of FRP superstructures in the future.

\subsubsection{Conclusion on Load Testing with Strain Gages}

Based on the literature review, it was concluded that load testing with strain gages was able to effectively evaluated the stresses and deflections of bridges made of composite materials or rehabilitated with composite wraps. Live load testing was also successful in collecting data before and after rehabilitation for comparison. However, using strain gages was unable to detect internal defects, which could cause problems. Using strain gages to measure stresses under different load conditions will help calibrate and refine analytical models for the design of composite bridges in the future. However, structures may need to be tested to failure to obtain significant information. In which case it could not be considered a nondestructive evaluation. 


\subsection{Fiber Optic Health Monitoring Systems}

This section talks about a new NDE technique that uses fiber optics to evaluate the performance of composite structures and materials. Although this technique was developed for the evaluation of composite components used in the aerospace industry, it is now being used to evaluate the performance of civil infrastructures such as composite bridges. This section talks about the parameters of interest, advantages, and disadvantages of using fiber optics for evaluating composite structures and materials.

\subsubsection{A Composite Bridge Health Monitoring System (Thomas 1999)}

\subsubsection{Introduction}

Fiber composites have become one of the most promising technologies of today's infrastructures. Since the aircraft and boating industries have been successful with these materials, they are now being considered for the use in bridge design and other civil infrastructures (Thomas 1999). Since this is a new material and technology in the civil

engineering field, a significant number of projects must be tested and monitored to generate the experience database that will be used in any new codes. The "Tech 21" bridge is of the more recent projects, which uses fiber optic sensors for tracking the condition of the field bonded joints and measuring the strain in the bridge structure.

\subsubsection{System Description}

\subsection{Bridge}

This all-composite bridge, which is referred to as the "Tech 21 Bridge", is a twolane vehicle bridge that spans $30 \mathrm{ft}$ and has overall dimensions of $33 \mathrm{ft}$ in length and $24 \mathrm{ft}$ 
in width. The finished bridge is shown in Figure 2.5.1.1. It was fabricated using E-glass fibers and polyester resin through a combination of pultrusion and wet lay-up processes. The basic cross section of the bridge is shown in Figure 2.5.1.2.

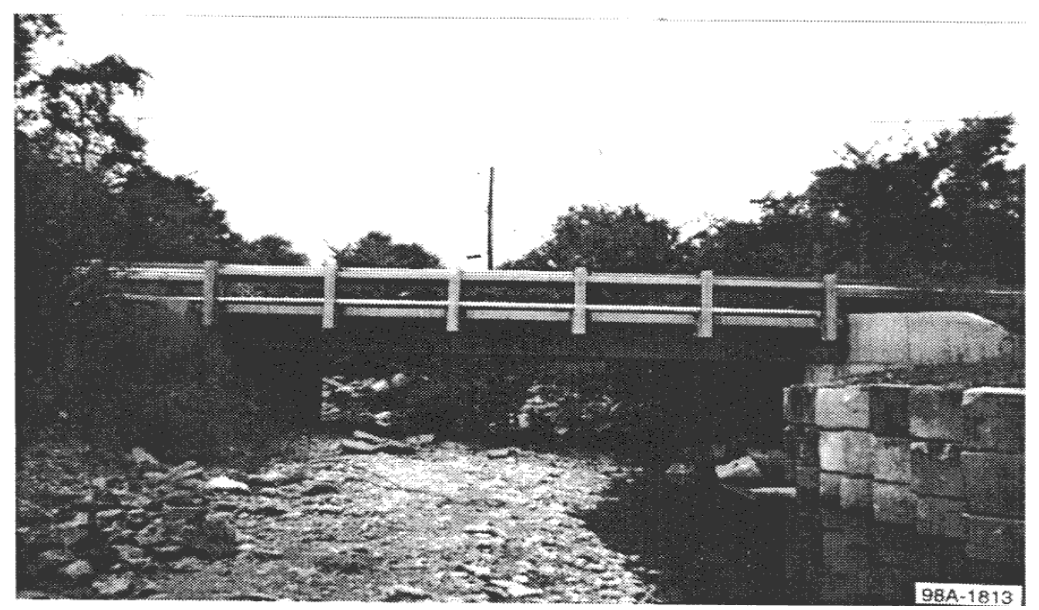

Figure 2.5.1.1 "Tech-21" all composite Bridge (Thomas 1999)
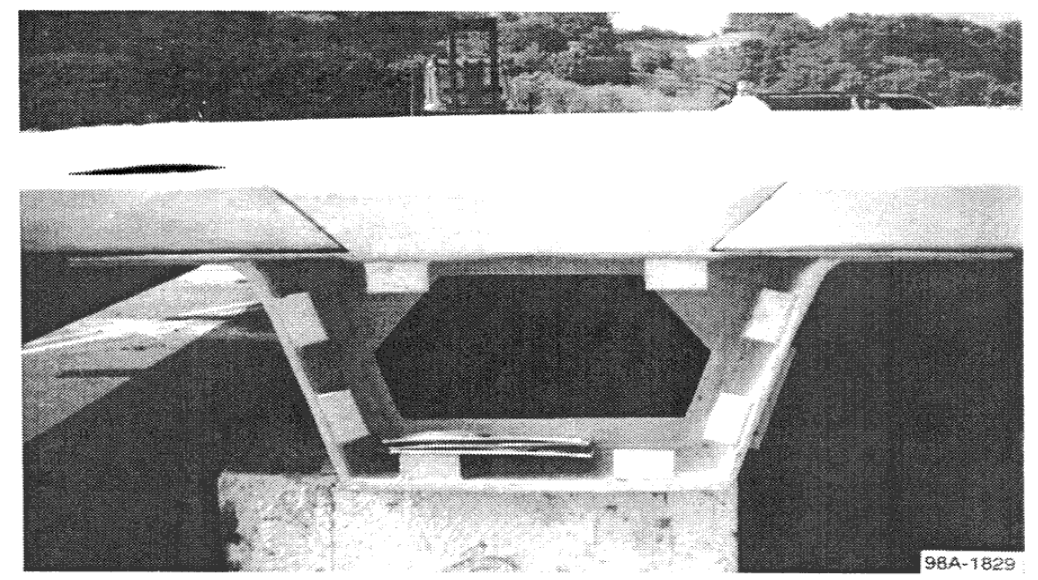

Figure 2.5.1.2 Cross-Section of the Bridge (Thomas 1999)

\subsection{Fiber Optic Instrumentation}

A complete, embedded fiber optic sensor system for monitoring strain and the condition of the field bond lines was designed and installed in the bridge system. The system included Bragg grating strain sensors, Sapphire fiber chemical sensors, and 
portable on-site data acquisition equipment. Also, a new chemical sensor system for monitoring the bondlines was installed.

\subsubsection{2(a) Bragg Grating Strain Sensors}

\section{Sensors}

The Bragg grating fiber optic sensors detects strain by directly measuring the shift of a specific wavelength of light that is filtered by the sensor. The gratings are regions of varying index of refraction, which are placed in a germanium doped glass fiber. A broadband IR signal is introduced to the fiber and the sensor reflects a narrow band signal about a center wavelength, as shown schematically in Figure 2.5.1.3. The sensors are single glass fibers having a center wavelength of approximately $1330 \mathrm{~nm}$, which are essentially the same material as the bridge structural fibers, making it easy to incorporate within the laminate without effecting the structure's properties.

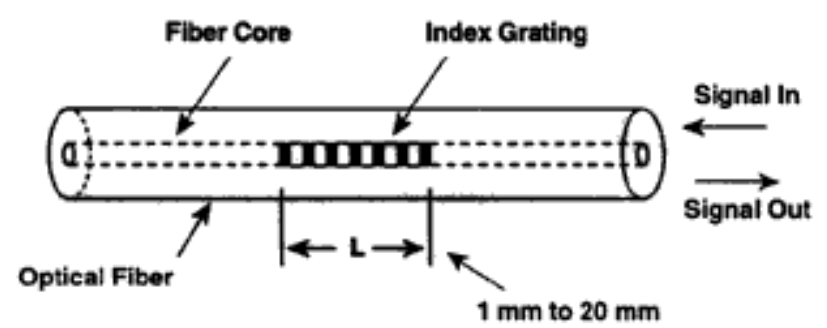

- Physical strain on the fiber causes shift in wavelength of the filter function, which can then be measured to provide a direct strain measurement
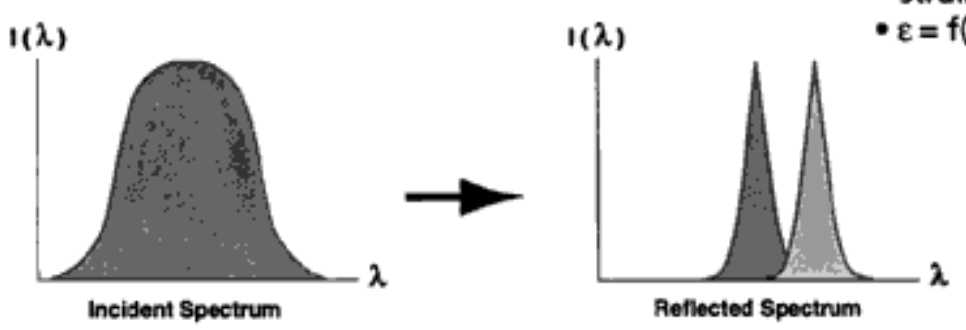

Figure 2.5.1.3 Bragg grating strain sensor operation (Thomas 1999) 
Eighteen Bragg grating sensors were installed on the bridge at nine locations illustrated in Figure 2.5.1.4. At each of the nine locations, a sensor was installed in the beams during fabrication, and a sensor was placed on the upper (inside) surface of the lower flange of the beams once the bridge was constructed as illustrated in Figure 2.5.1.5. Both sensors were painted with a urethane primer to improve adhesion and secured using a wet lay-up of random mat fiberglass and polyester resin.

\section{Instrumentation}

The strain sensor cables were routed through the beam sidewalls to the interior of the beams on the west end of the bridge where they were terminated in junction boxes. Fiber optic patch cables were used for connecting the sensors to the data acquisition cabinet. An EPC model FLS3100 signal processor system was used to process the fiber optic strain sensor data. A Campbell Scientific data acquisition system was used for the long-term monitoring of the bridge. Data was collected at a rate of one sample per minute and averaged over $1 \mathrm{hr}$ periods. The engineers in charge received these $1 \mathrm{hr}$ averages for each channel once a day via modem.

To reduce cost and on-site hardware, optical multiplexing was used. This method records multiple sensors through a single processor by optically switching between multiple single sensor fibers. To provide long-term monitoring of 13 Bragg grating strain sensors three, one by four optical switches were used. The switches were remotely controlled by the acquisition system to cycle through all the channels automatically. The complete system is shown in Figure 2.5.1.6. 


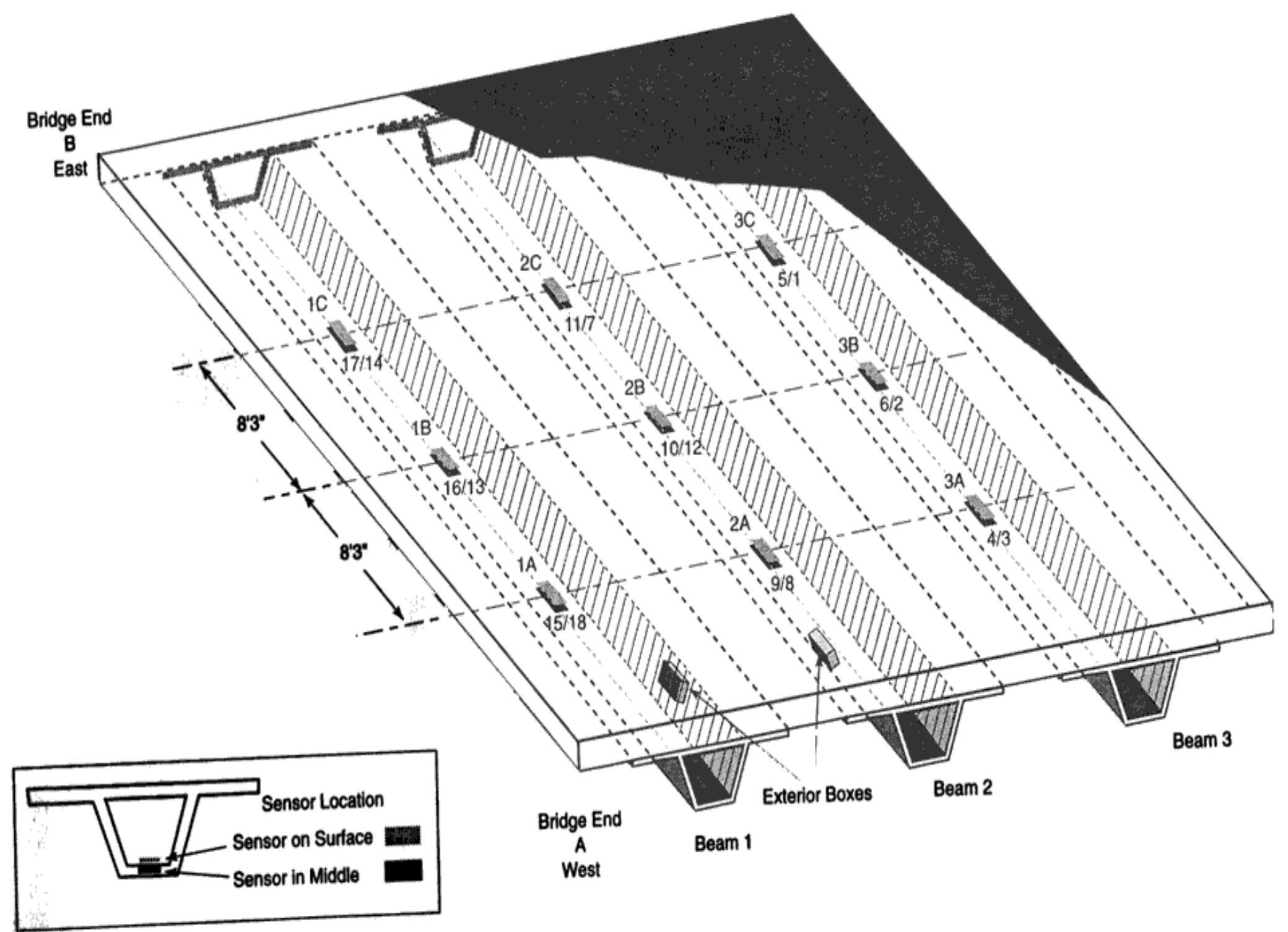

Figure 2.5.1.4 Locations of the Bragg grating strain sensors (Thomas 1999) 


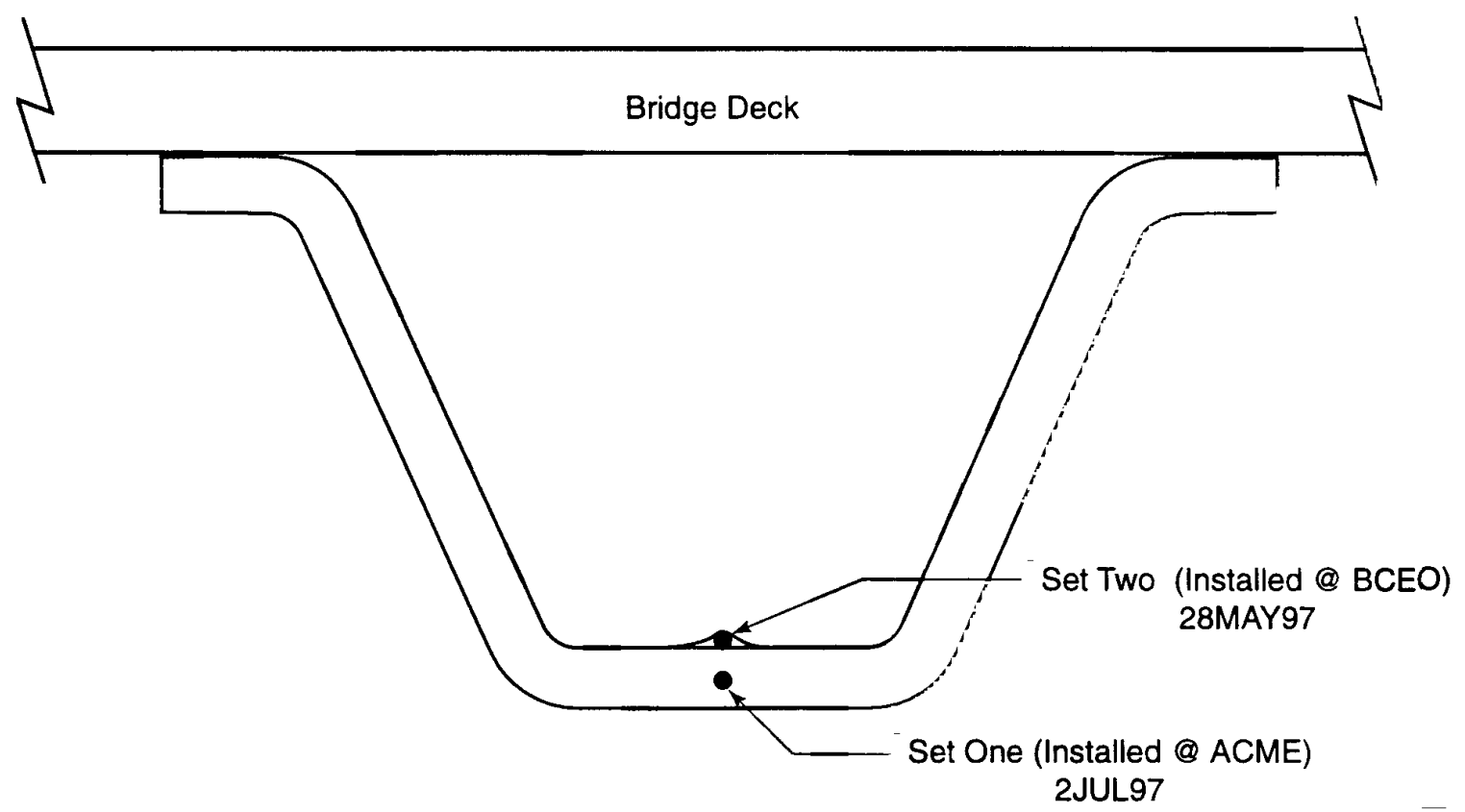

Figure 2.5.1.5 Bragg grating sensor locations (Thomas 1999)

\subsubsection{2(b) Sapphire Fiber Chemical Sensors}

\section{Infrared (IR) Absorption Spectroscopy}

Infrared absorption spectroscopy is one of the analytical methods used for measuring compositional changes in hydrocarbons that has been favored in NDT (Thomas 1999). Recent improvements in IR fiber technology have pioneered the coupling of optical fibers to Fourier Transform Infrared Spectrometers (FTIR) in the midIR with improved sapphire and chalcogenide fibers.

Infrared transmitting optical fibers are being used to obtain measurements in difficult environments since remote IR spectroscopic measurements can be taken at distances up to $60 \mathrm{ft}$ from the spectrometer. However, it is necessary to perform reflectance measurements on the epoxy adhesives where the IR beam only penetrates a few wavelengths of light into the adhesive. This is necessary since epoxy adhesives 
(opaque materials) are not amenable to transmission measurements. One technique of reflectance measurement used with opaque materials is attenuated total reflectance (ATR). This is known as evanescent wave spectroscopy when applied to optical fibers because at each point of internal reflection, an evanescent wave penetrates a fraction of the wavelength outside the core of the fiber into the surrounding material.

\section{Bridge Sensors}

To monitor the cure and long-term performance of the bridge bondlines, an ATR system consisting of Sapphire fibers was constructed. As broadband IR light passes

along the sensor, different wavelengths are absorbed due to the changing chemistry of the surrounding material. A spectrometer then analyzes the output from the remaining IR light that has passed through the fiber. This signal can be analyzed by subtracting the output signal from the background signal to show peaks that indicate regions of high absorbency, as shown in Figure 2.5.1.7. The main purpose of locating these regions of high absorbency is to provide an indication of any moisture that penetrates the bondline and could result in freeze thaw cycle problems. These chemical sensors also monitor the condition of the two field bonds including cure monitoring of the epoxy to detect any epoxy degradation after installation. 

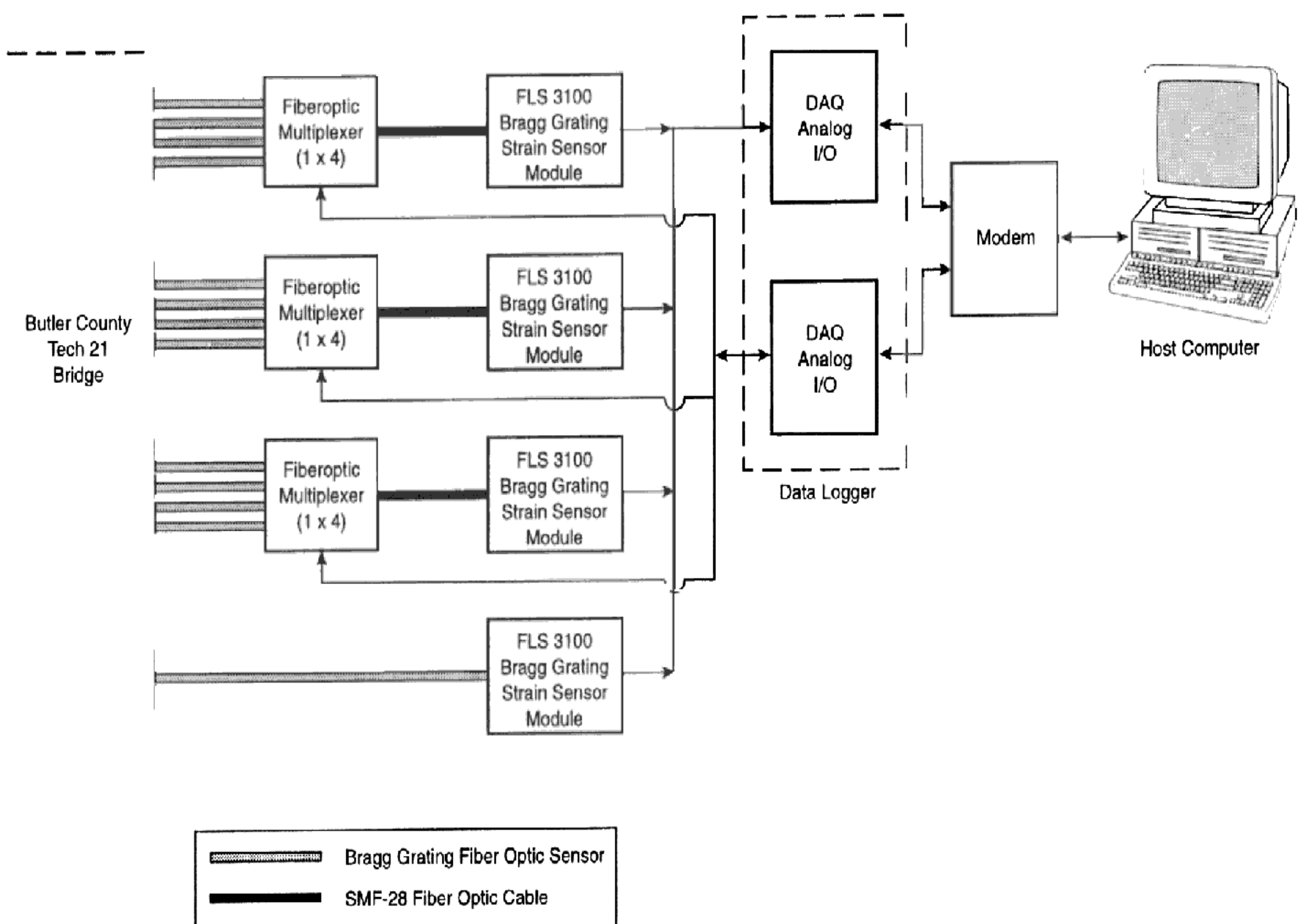

Figure 2.5.1.6 Schematic of Bragg grating sensor system (Thomas 1999) 


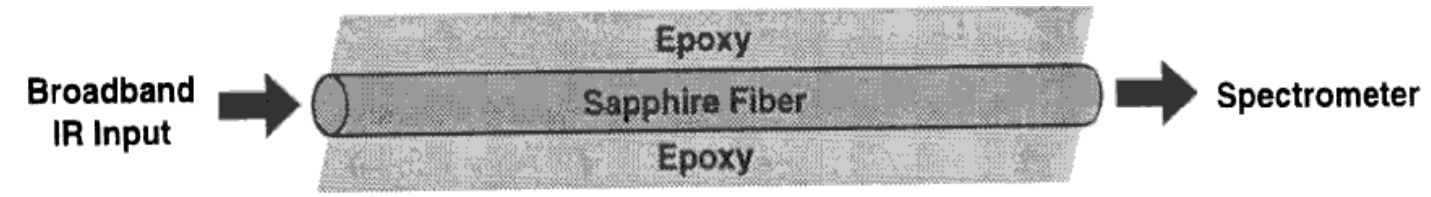

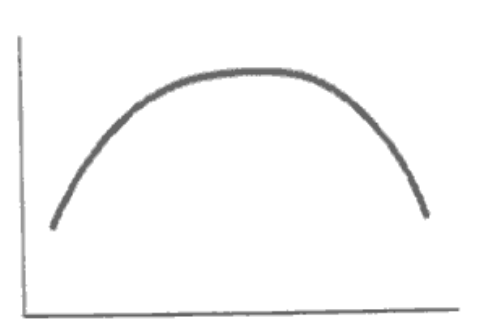

$\lambda$

Background Signal

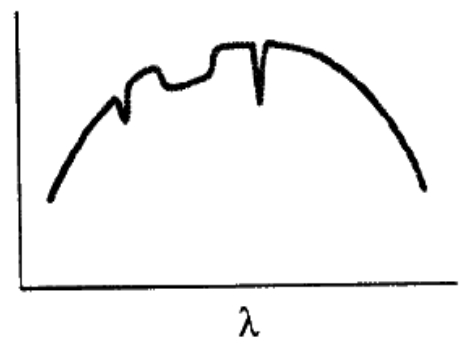

In Bondline/Composite

Figure 2.5.1.7 Schematic of chemical sensor operation (Thomas 1999)

The sensors installed in the bridge consisted of a 2-meter section of $150 \mu$ Sapphire fiber packaged inside a 0.08 -in. diameter stainless steel tube. The stainless steel tube consisted of multiple machined "windows" to provide contact between the bare sensor fiber and the epoxy, illustrated in Figure 2.5.1.8. A second fiber of $150 \mu$ Zirconium Fluoride $(\mathrm{ZrF})$ was packaged in stainless steel tubing in lengths of $8 \mathrm{ft}$ and 16 $\mathrm{ft}$ without windows for assembly of a $30 \mathrm{ft}$ long sensor. The fabricated subcomponents were assembled into four, 30 -ft long sensors installed in pairs in the two bridge bondlines as shown in Figure 2.5.1.9. The sensor pairs were placed in the grooves as shown in Figure 2.5.1.9 and secured with small amounts of LPL epoxy. However, two out of the four sensors where damaged in shipment to the site. In future projects, this can be avoided by installing the sensors during bridge manufacturing at the site. 


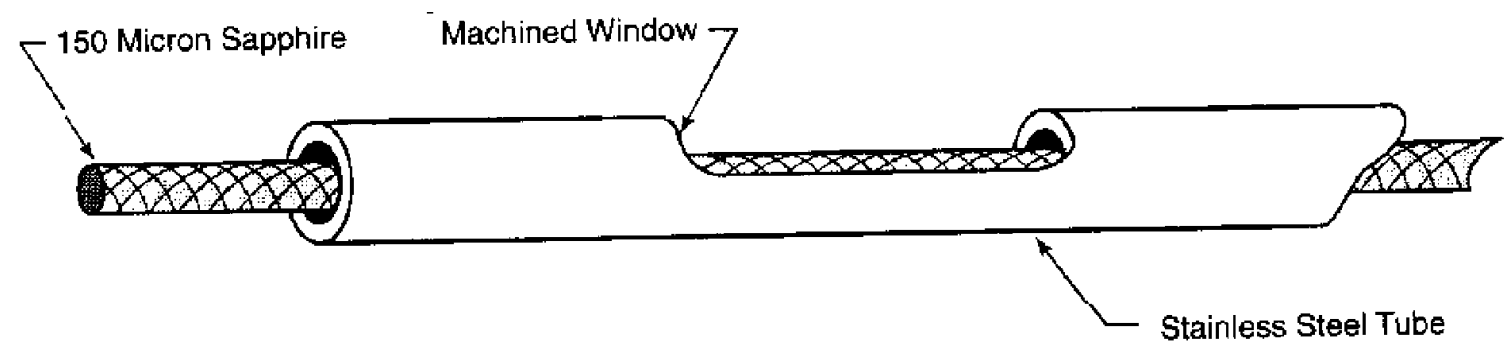

Figure 2.5.1.8 Sapphire fiber sensor packaging (Thomas 1999)

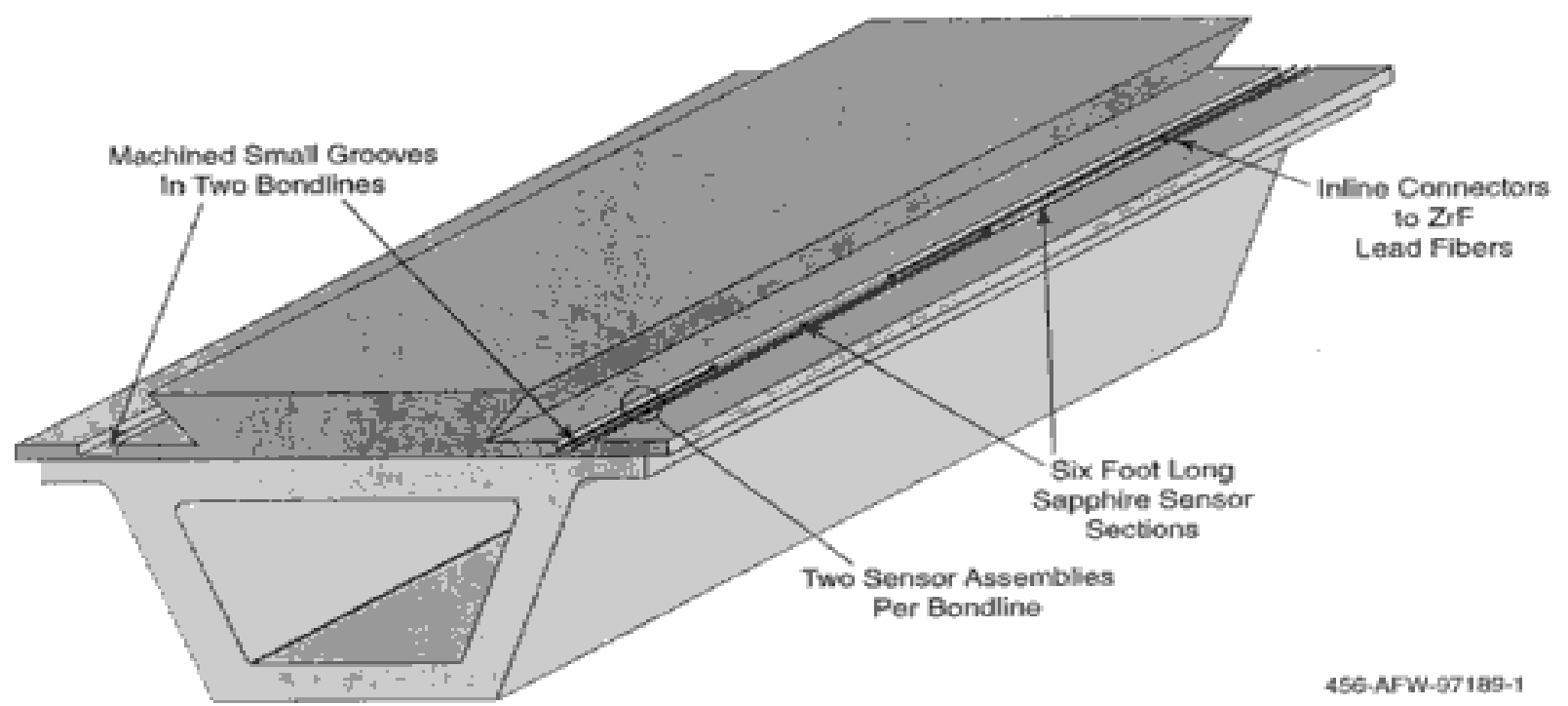

Figure 2.5.1.9 Layout of bondline sensor assemblies (Thomas 19999)

\section{Instrumentation}

The data from the bondline chemical sensors was obtained and analyzed using a laboratory spectrometer along with software previously developed for such data analysis. In addition, a small, low cost, portable spectrometer was developed as the basis for future acquisition of such data.

\subsubsection{Field Tests}

The field tests associated with the "Tech-21" bridge consisted of live load tests as well as long-term monitoring of the bridge. The live load tests were performed using individual vehicle crossings to record data from the sensors at high rates. Strain sensors 
continuously recorded the strains due to diurnal and seasonal cycling for the long term monitoring.

\subsection{Live Load Tests}

\subsubsection{1(a) Winter Tests - January 1998}

For maximum seasonal variation, two live load tests were performed six months apart using the fiber optics system previously discussed. Both tests were compared to a conventional strain measurement system that was temporarily installed for the live load tests and showed good agreement.

The first test consisted of a 24 ton, three-axle dump truck driven over the bridge as readings from the sensors were collected with the data acquisition system. Multiple truck passes had to be made in order to record data from all 18-strain sensors. Each pair of sensors at the nine locations had slightly different stain readings due to their location in the laminate illustrated previously in Figure 2.5.1.4 and Figure 2.5.1.5. The average value of the two sensors at each location is used to plot the data.

As a check, the fiber optic strain readings from 6/2,10/12, and 16/13 (shown in Figure 2.5.1.4) were compared to the readings from the three BDI strain transducers located at the center span line (16 ft 6 in.). These comparisons showed that the fiber

optic sensors provided reasonable strain readings for all of the tests. Figure 2.5.1.10 and Figure 2.5.1.11 display the strain readings from the fiber optic sensors along with the 
average reading of the BDI strain transducers. The comparison between the center fiber optic strain sensors and the center BDI sensors can be observed from these two figures.

\section{Test Consistency}

Consistency between loading conditions was important since four fiber optic strain gages were recorded in a test run. Other than slight differences on the graphs due to different rates of speed traveled by the trucks, each of the multiple tests for a given lane produced the same peak strains as verified by the BDI strain transducers. The different speeds of the trucks caused the peak strains to occur at different times but at the same physical location. These slight differences were minor and any changes in the system deemed excessive. The good consistency between the multiple passes can be observed in Figure 2.5.1.12.

\section{Redundant Gage Comparison}

Some slight differences in the strains recorded from fiber optic sensors in the same locations where observed. This could be a factor of localized bending or the fiber optic sensors may be slightly out of alignment with one another. The theory for a beam in bending suggests that the strains on the innermost surface of the beams should be lower than the strains in the middle of the beams (Thomas 1999). Many of the strain readings agreed with this theory, but others showed that the strain was lower in the middle. However, the differences were typically less than 10 percent. 


\section{Beam 1}

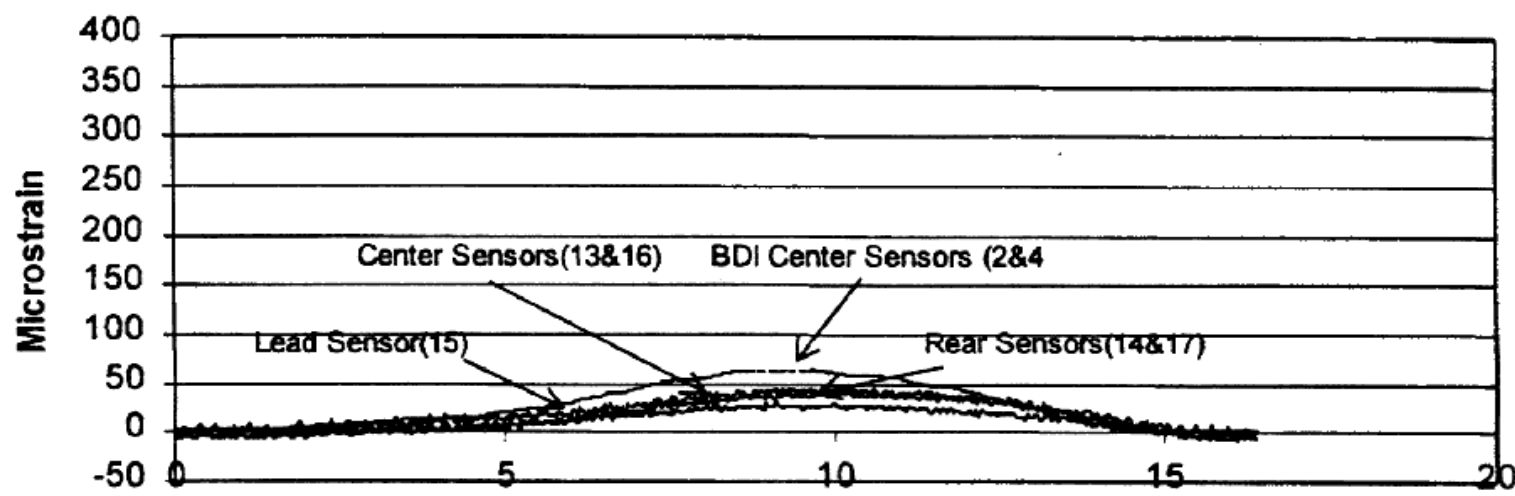

Time (s)

\section{Beam 2}

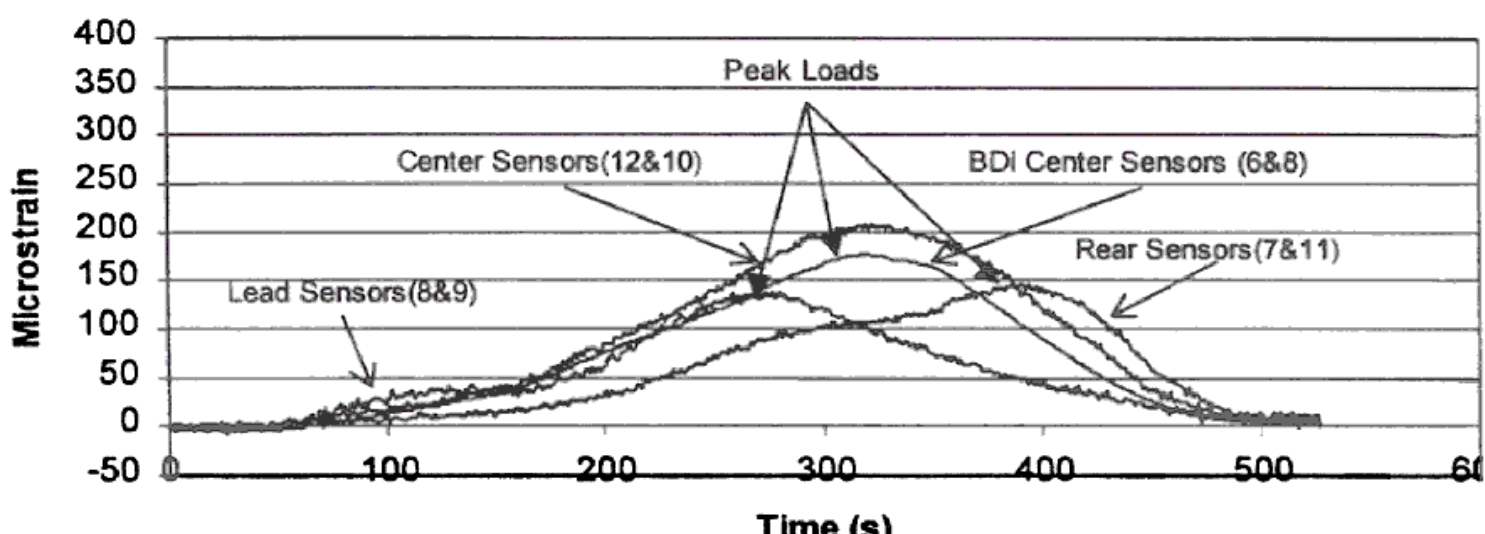

Beam 3

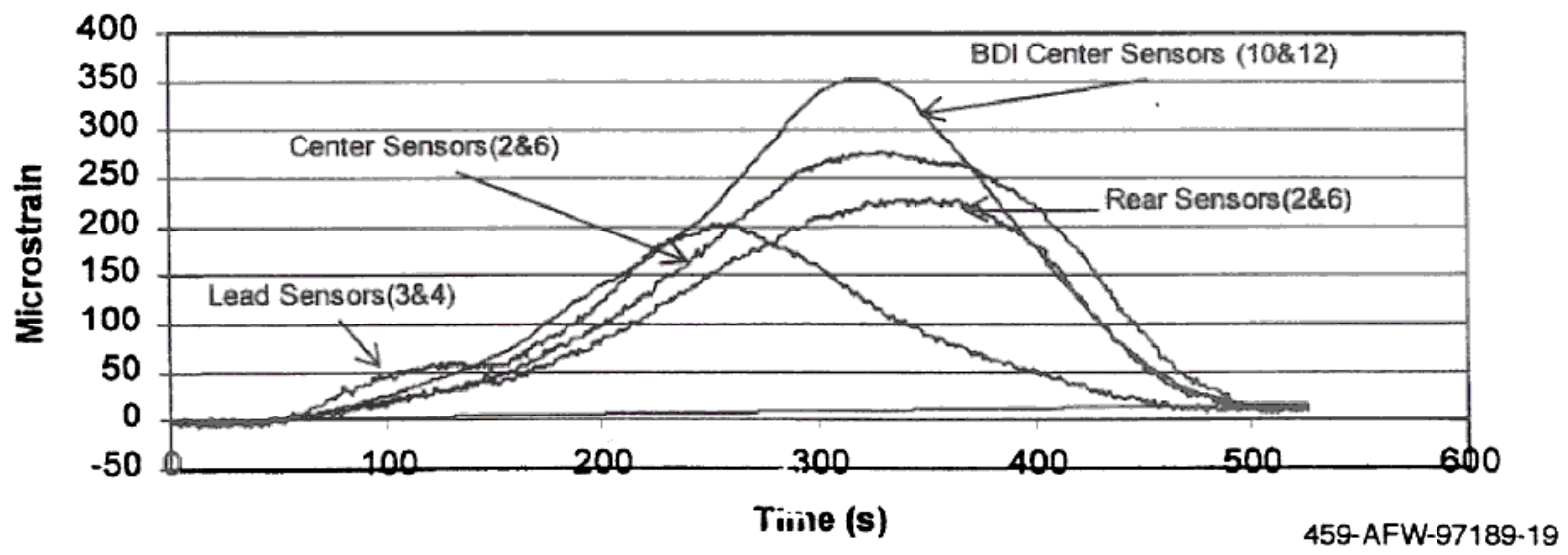

Figure 2.5.1.10 Bridge response - truck traveling east, right lane - January 1998 (Thomas 1999) 


\section{Beam 1}

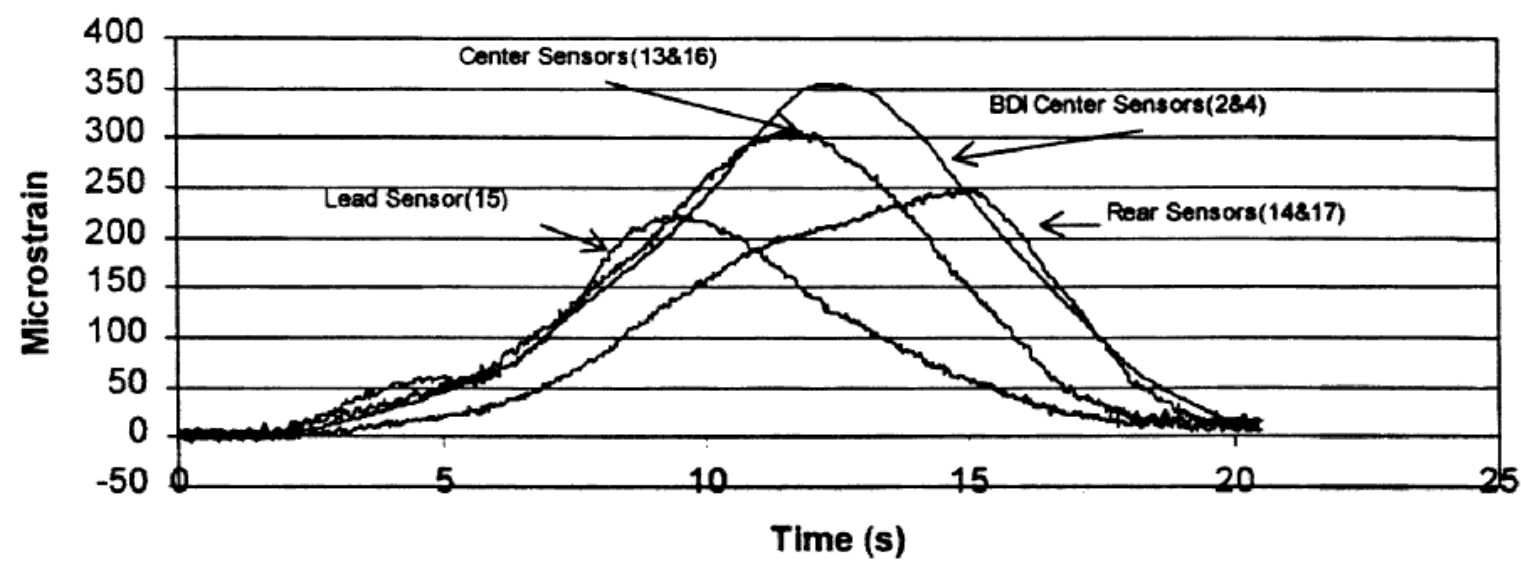

Beam 2

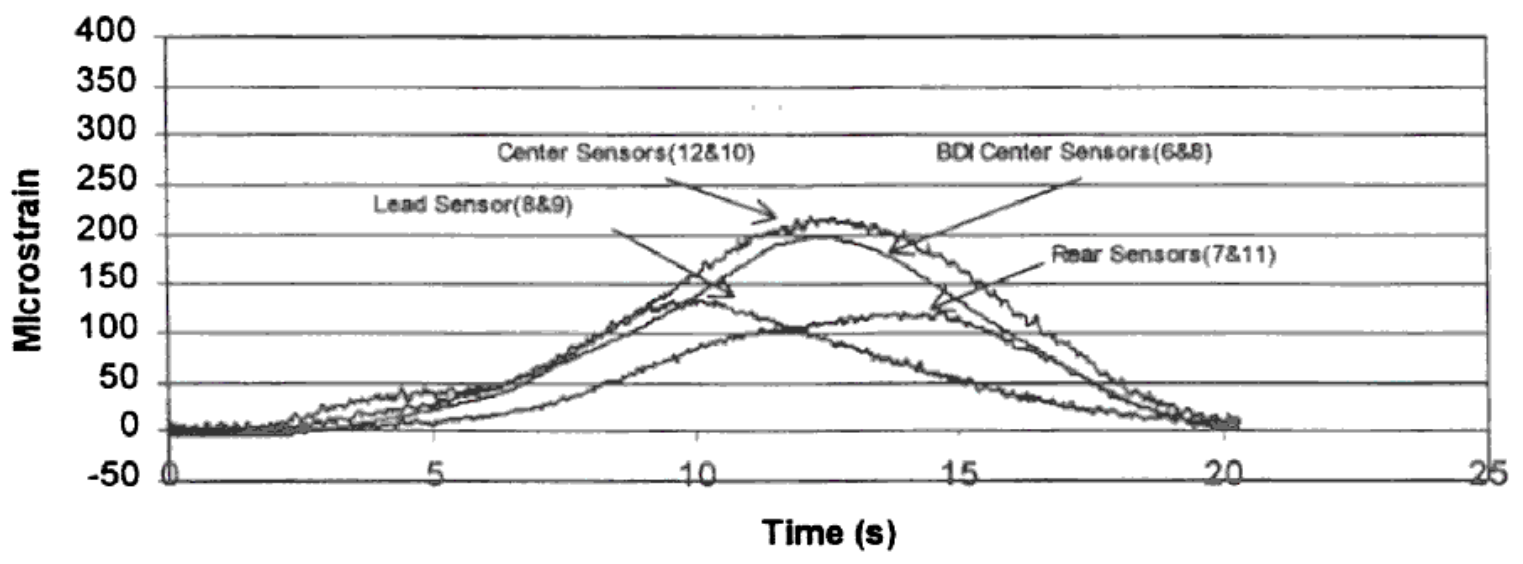

Beam 3

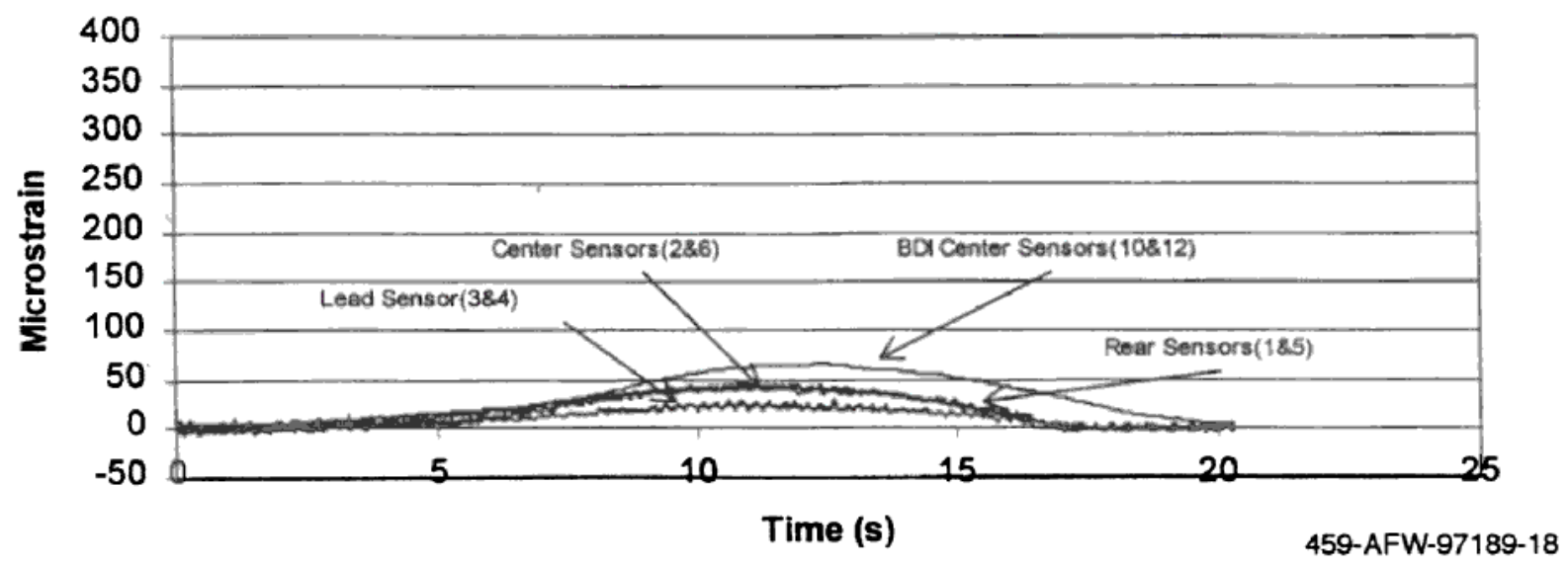

Figure 2.5.1.11 Bridge response - truck traveling east, left lane, January 1998 (Thomas 1999) 


\section{Channel 12 Test $1,3,5,7 \& 9$}

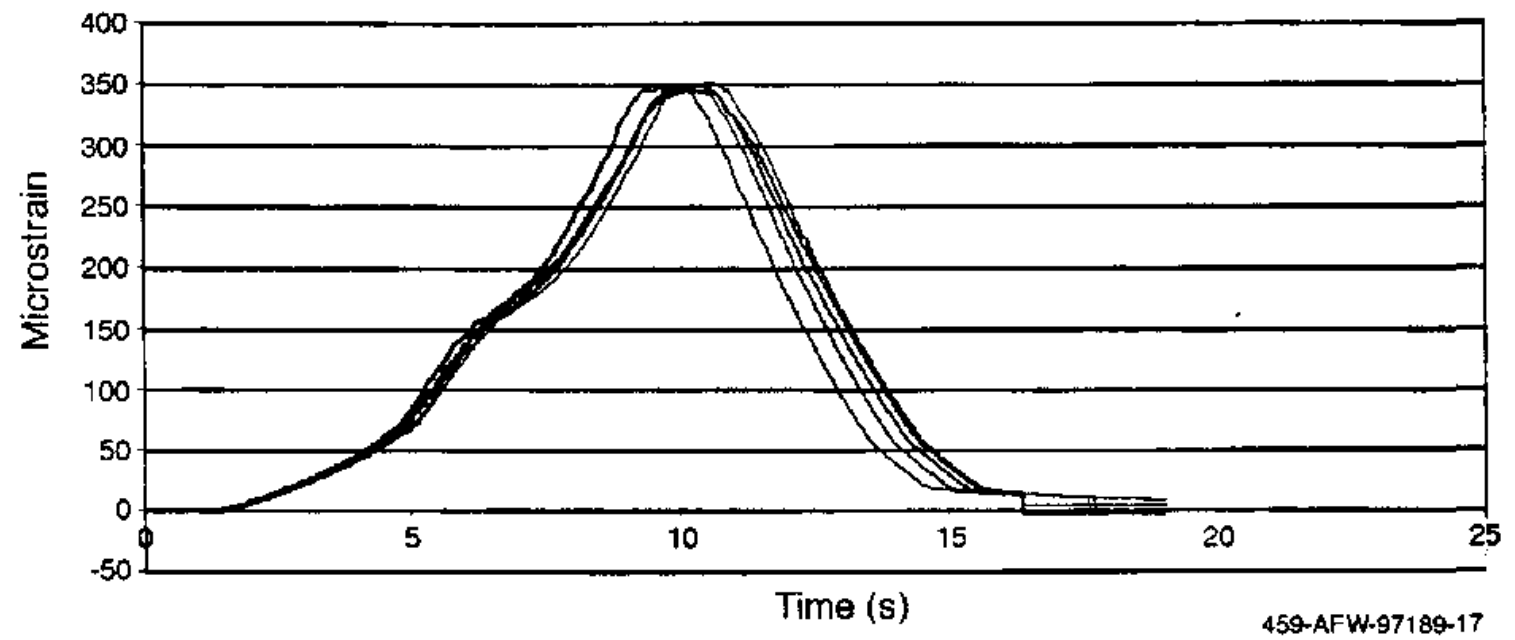

Figure 2.5.1.12 Test repeatability (Thomas 1999)

\section{Apparent Noise}

The "noise" in the fiber optic data was a result of limited resolution. Using Bragg grating fiber optic sensors that were configured to measure strains up to approximately $5000 \mu \mathrm{e}$ resulted in the limited bit resolution of the acquired signal in the low range of strain seen in the bridge. This is shown in Figure 2.5.1.13. Reducing the input range of the fiber optic system could help reduce this "noise". However, such a hardware change is not necessary since it has minor effect on the data collected.

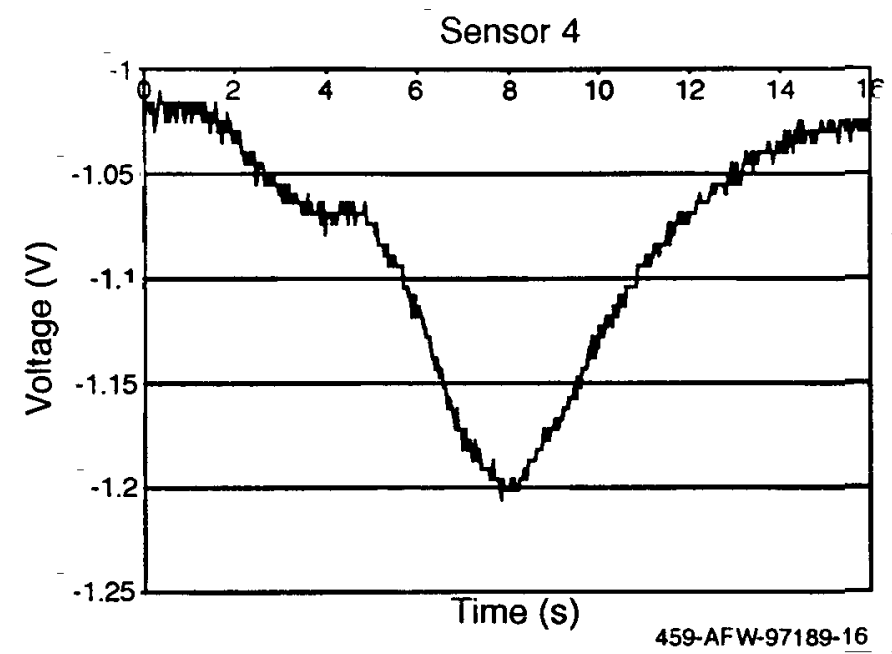

Figure 2.5.1.13 Voltage plot showing limited resolution (Thomas 1999) 


\subsubsection{1(b) Summer Tests - August 1998}

The Bragg grating strain sensor system was connected directly to a portable data acquisition system to collect the data for the summer tests. These test series consisted of loading conditions well beyond what could be expected in service. Along with the single truck tests perform in the same manner as the winter tests, two, three-axle trucks were driven side by side over the bridge. Truck No. 1 weighed 71,920 lb. and truck No. 2 weighed 70,600 lb. The heavier truck was used for the single truck tests. The single truck tests were performed at $5 \mathrm{mph}$ over the eastbound (right) lane and then at $5 \mathrm{mph}$ over the westbound (left) lane. Again, the operating speed varied from the target $5 \mathrm{mph}$ causing the timing of data peaks between runs to be slightly different. However, the repeated tests provided good data for evaluating the bridge conditions.

The bridge response to the truck travelling in the right lane is shown in the three plots of Figure 2.5.1.14. The highest strains are recorded in beam 3 as could be expected.

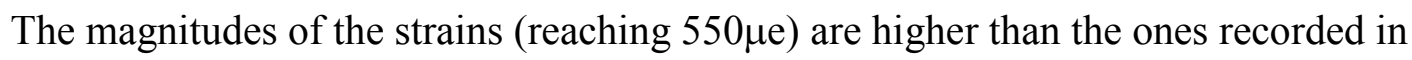
January, due to the 36-ton truck used instead of the 24-ton truck. The bridge response to the truck travelling in the left lane is shown in the three plots of Figure 2.5.1.15. Similar results occurred in the beams as expected. Figure 2.5.1.16 shows the data from the two crossing tests with both trucks traveling side by side. The peak strains are significantly higher than those recorded in the single truck pass.

For these tests, a secondary source of "noise" is apparent in the signals. This noise comes from performing the tests on a hot and humid day. This could have 
degraded the performance of some channels since there are a number of physical connections associated with the fiber optic cables required to cycle through all of the strain sensors. The signal quality on channels 3,15 , and 18 was significantly degraded by poor optical cleanliness. However, the rest of the data from the channels was generally clear and relevant to the evaluation of the bridge. Minimizing the number of connections in the system and cleaning the contact face of the fiber optics will significantly improve the quality of the signal (Thomson 1999).

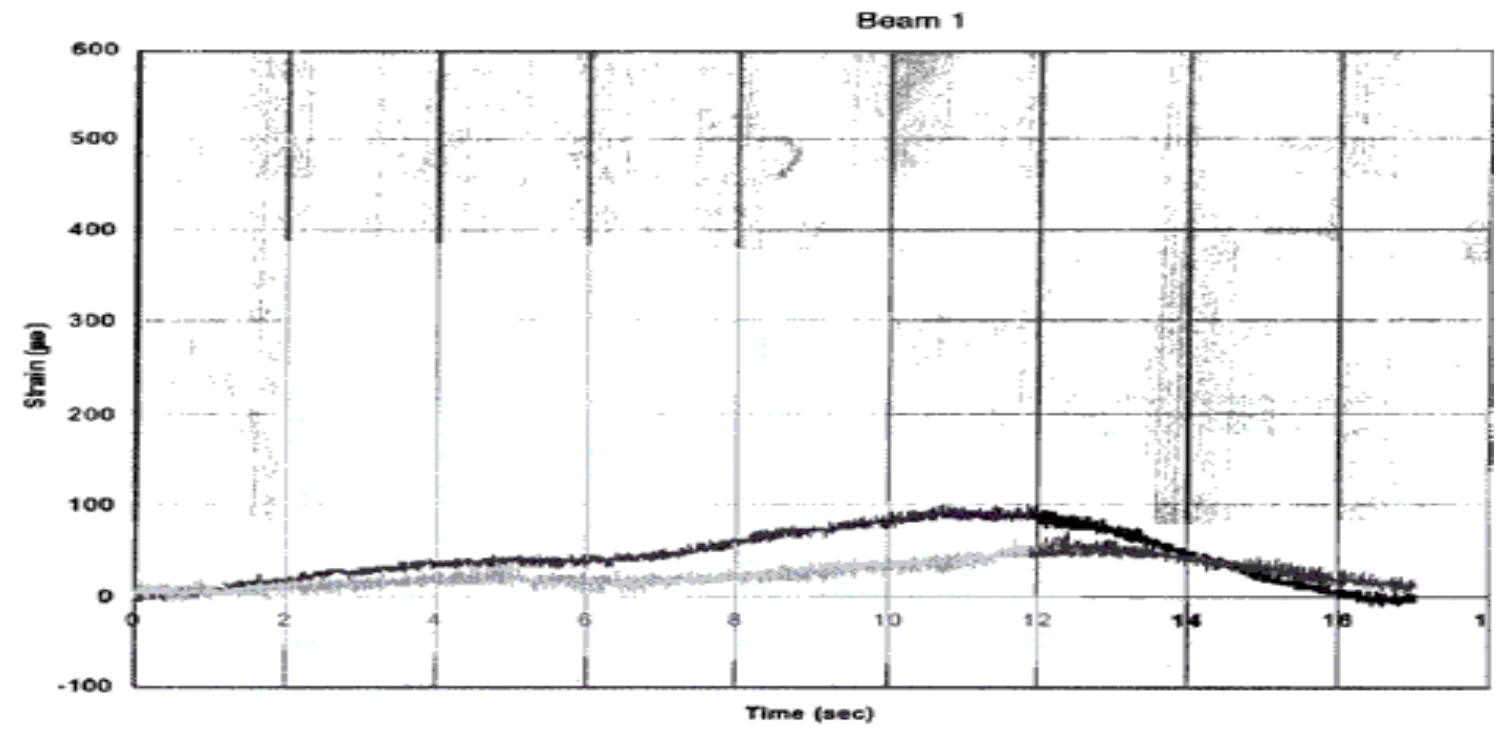

Figure 2.5.1.14(a) Bridge response - truck traveling east, right lane - August 1998 (Thomas 1999) 


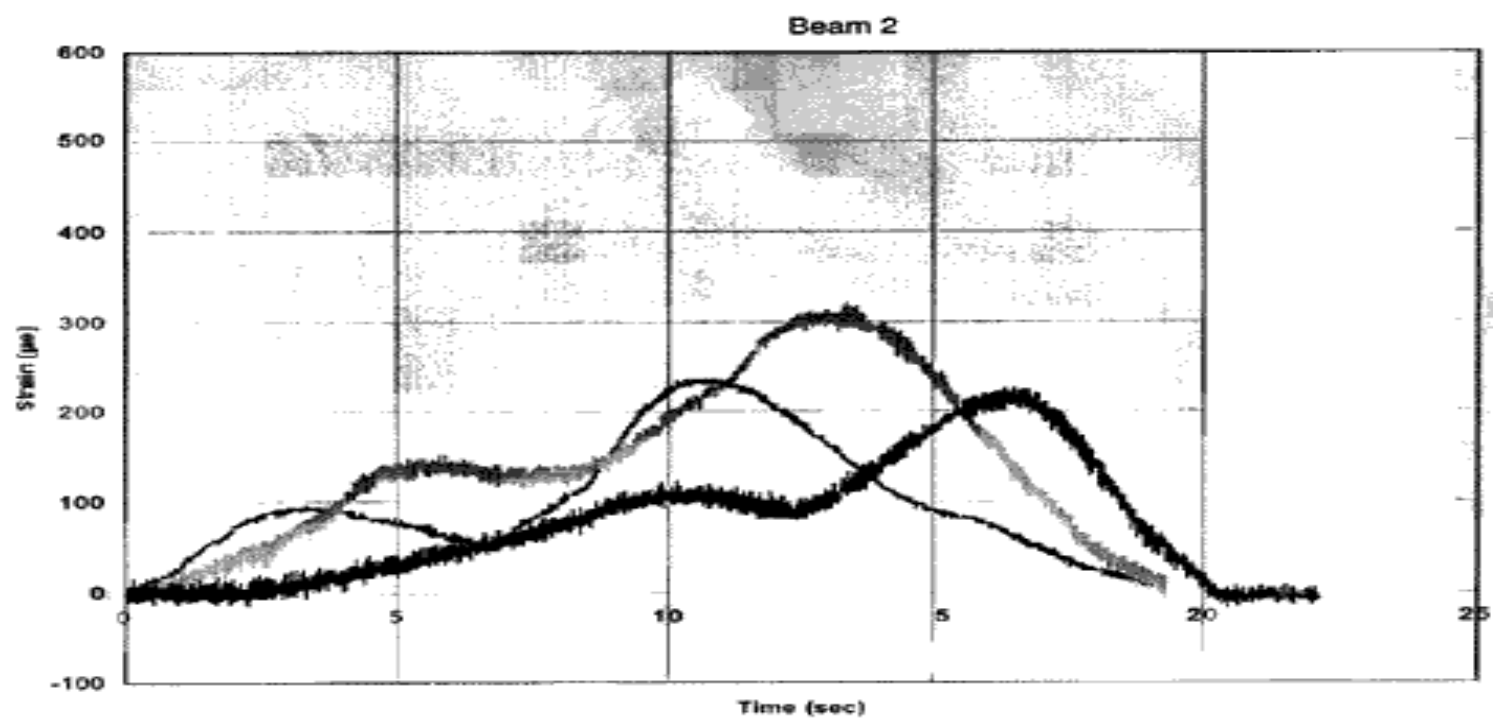

Figure 2.5.1.14(b) Bridge response - truck traveling east, right lane - August 1998 continued (Thomas 1999)

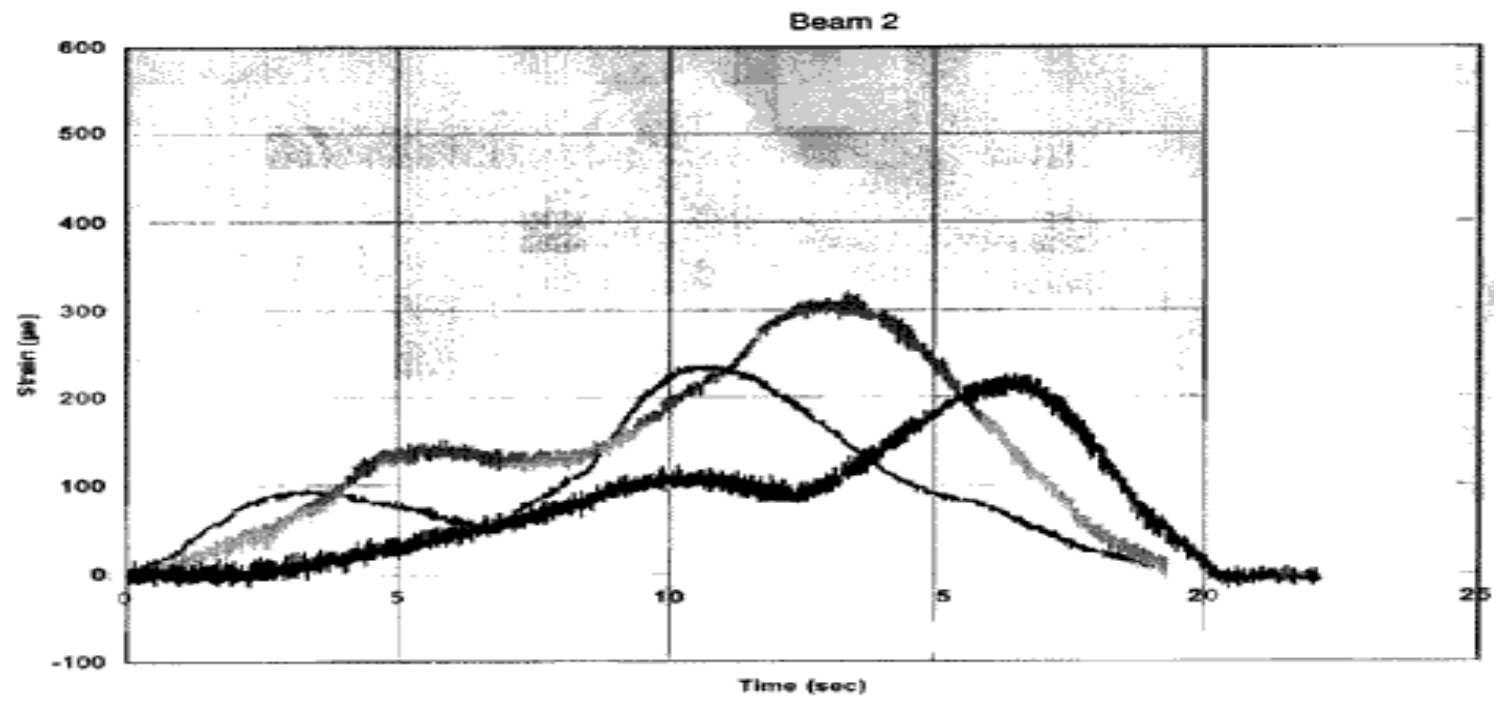

Figure 2.5.1.14(c) Bridge response - truck traveling east, right lane - August 1998 continued (Thomas 1999) 


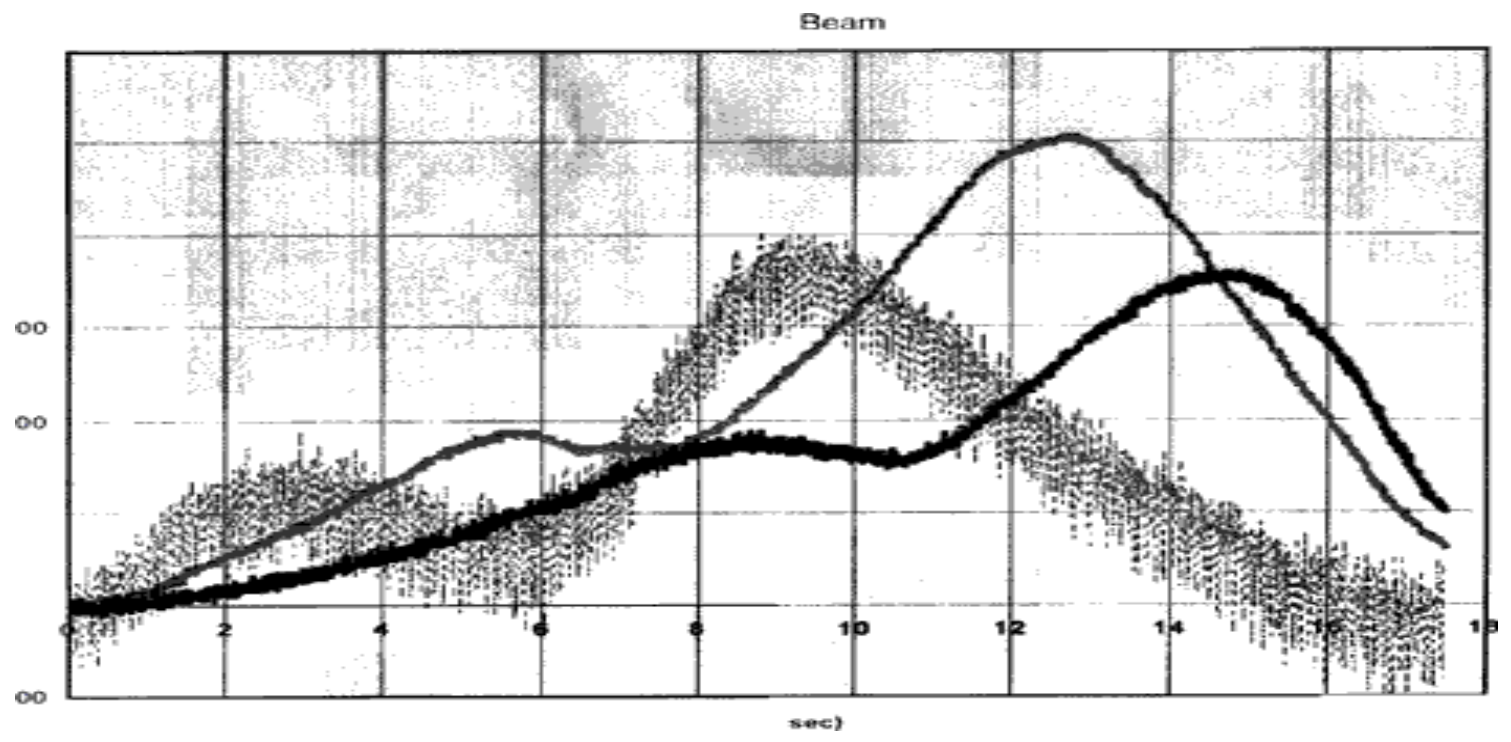

Figure 2.5.1.15(a) Bridge response - truck traveling east, left lane - August 1998 (Thomas 1999)

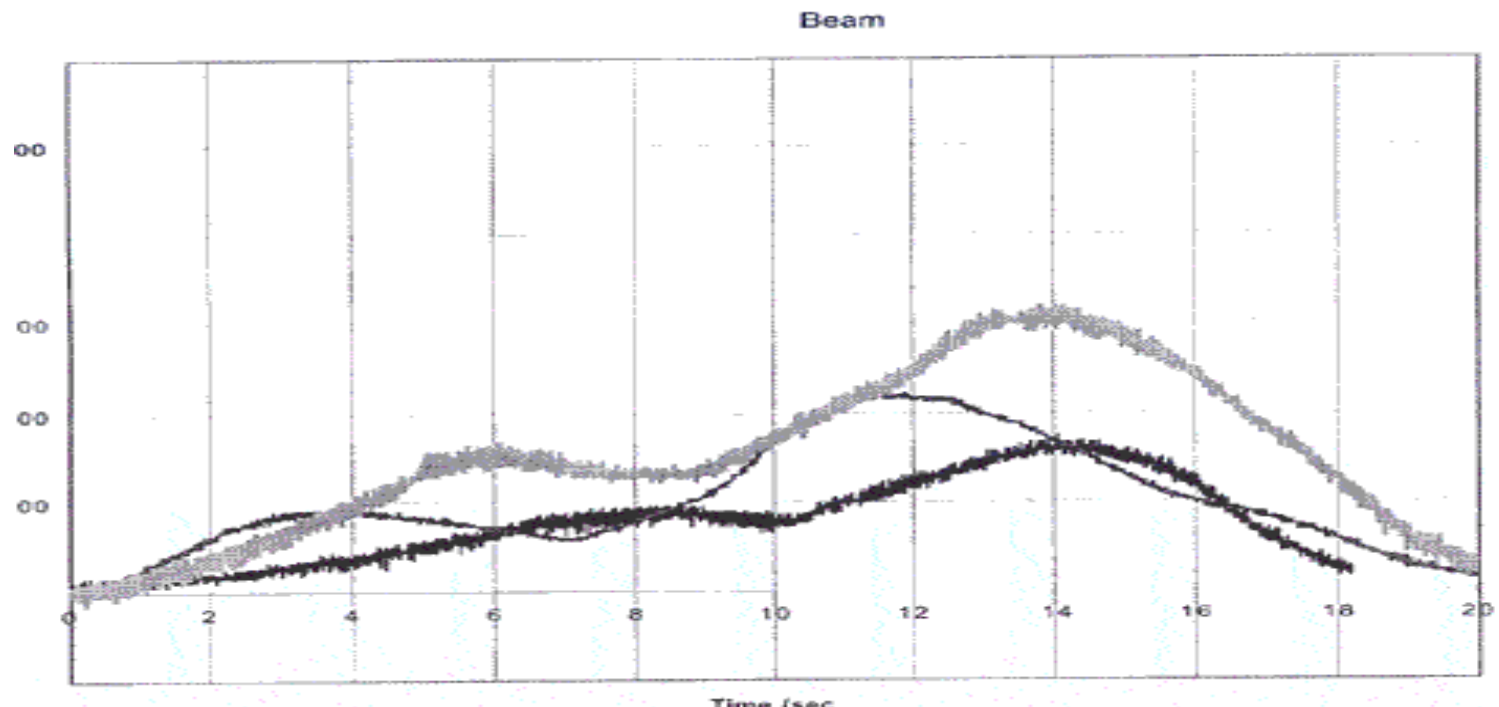

Figure 2.5.1.15(b) Bridge response - truck traveling east, left lane - August 1998 continued (Thomas 1999) 


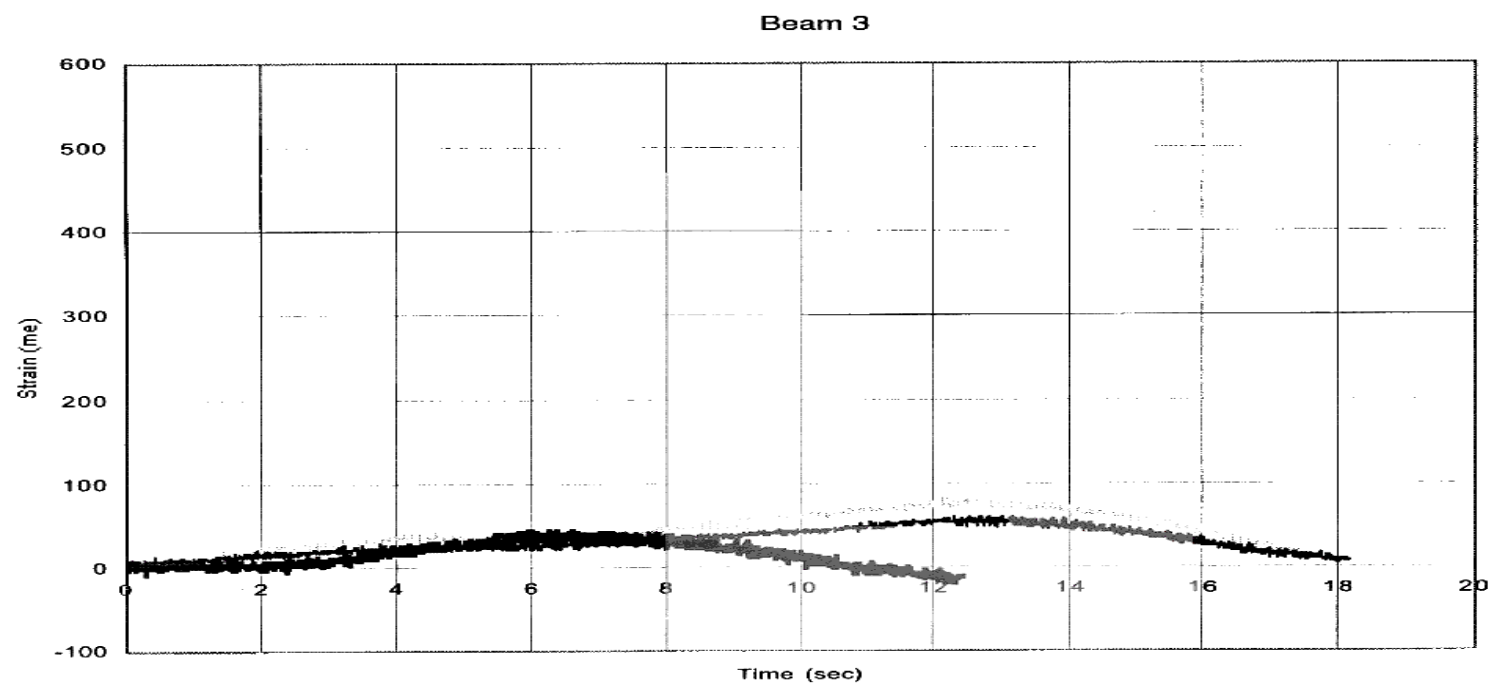

Figure 2.5.1.15(c) Bridge response - truck traveling east, left lane - August 1998 continued (Thomas 1999)
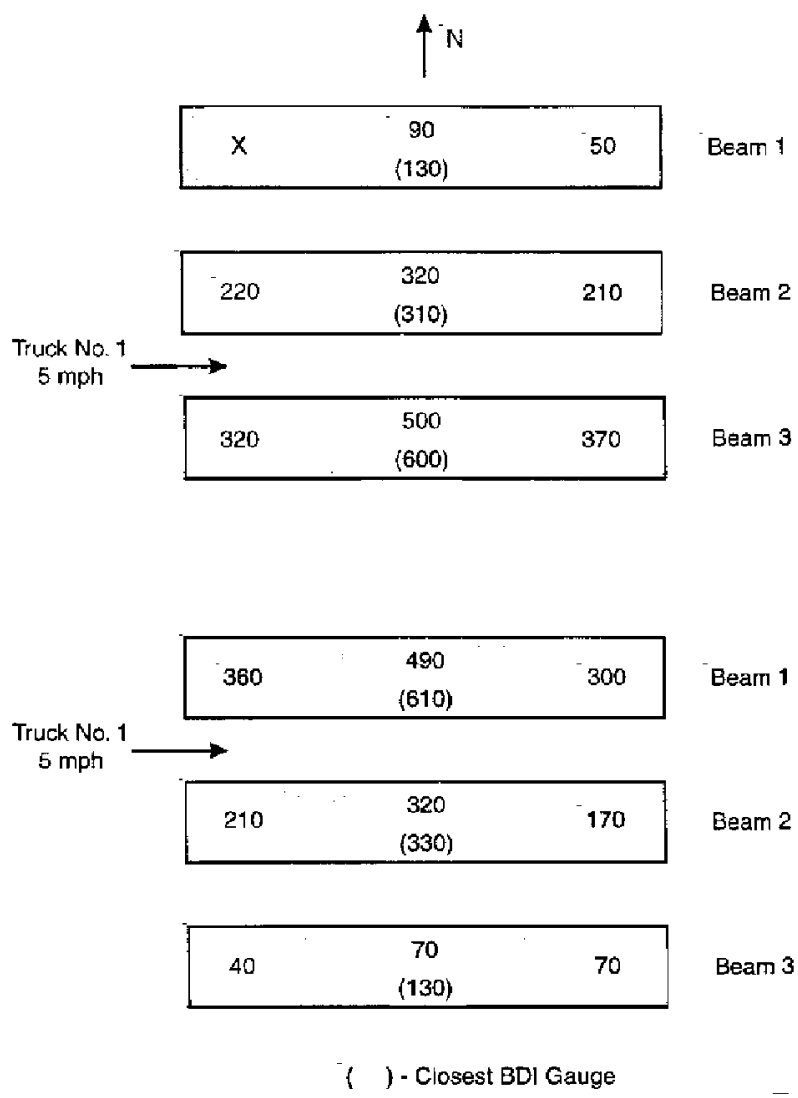

Figure 2.5.1.16 Live load test peak strains - August 1998 (Thomas 1999) 
A good illustration of the response of the bridge for the single truck tests is shown in Figure 2.5.1.16. At the locations where two strain sensors were operating, an average of the strain magnitude is shown. The peaks recorded by the BDI transducers are shown in parenthesis. The BDI transducers were close enough to the fiber optic sensors to provide a good comparison between the two sensor systems. Figure 2.5.1.17(a) and (b) is a further comparison of the fiber optic sensor system to the conventional BDI system.

The bridge response from the tests with two trucks side by side is illustrated in Figure 2.5.1.18. The peak strain levels recorded in Figure 2.5.1.19 are equal to the summation of the peak strain values in Figure 2.5.1.16, as one would expect.

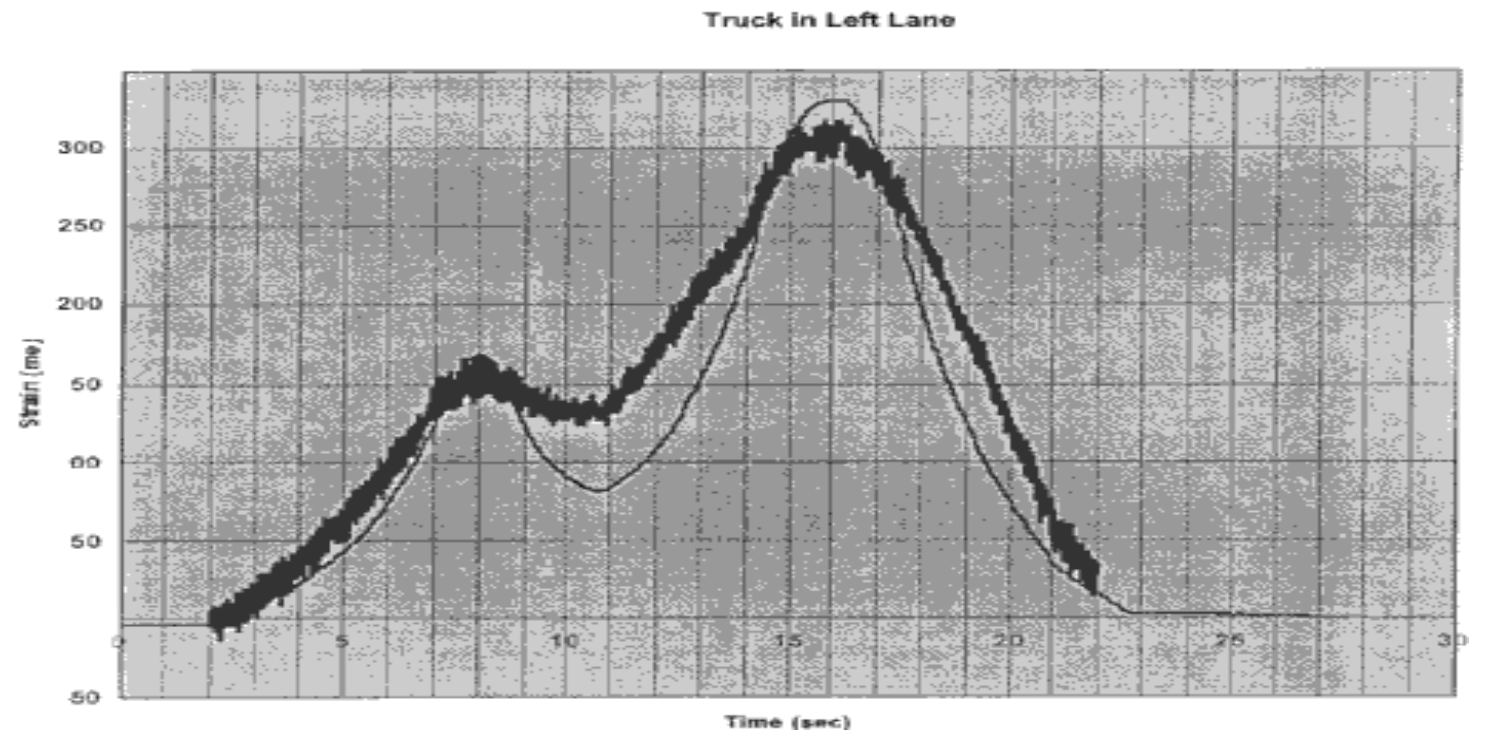

Figure 2.5.1.17(a) Comparison of conventional and fiber optic sensor results (Thomas 1999) 


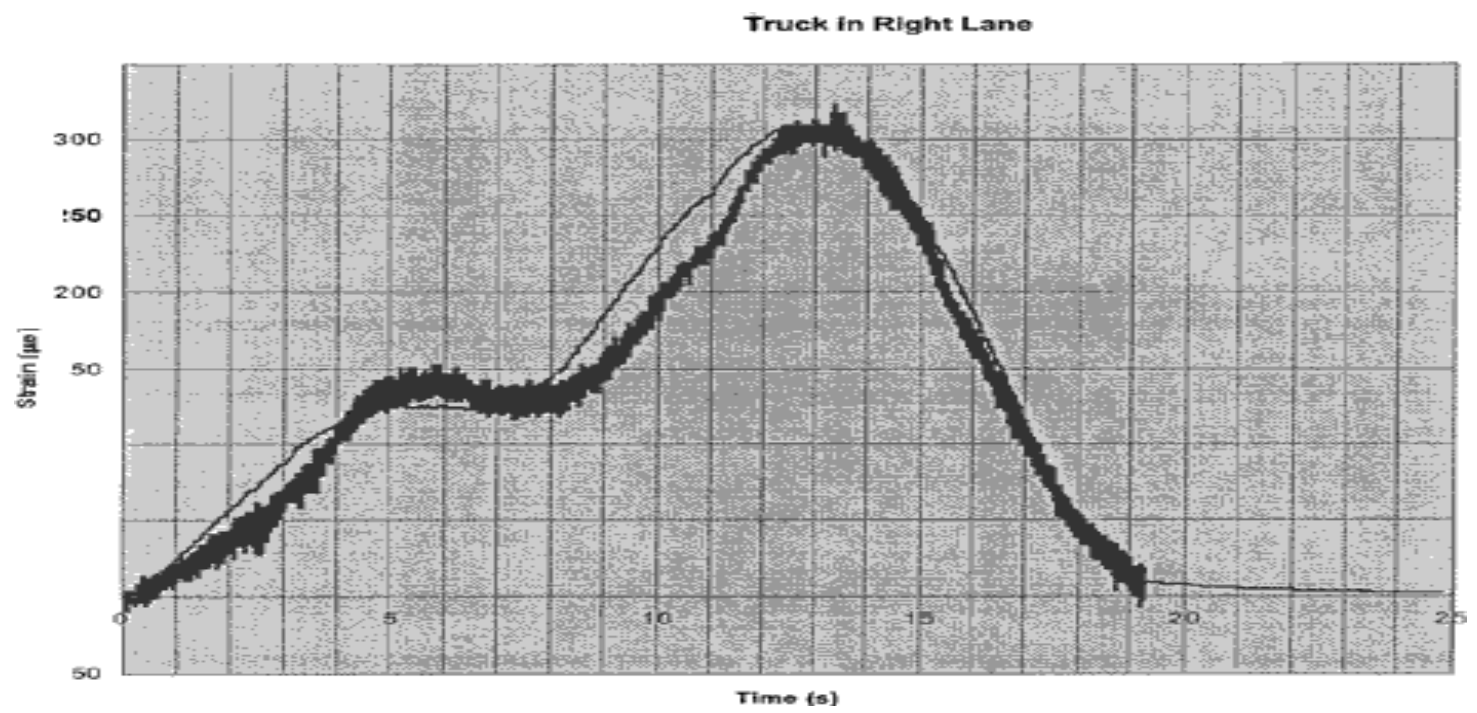

Figure 2.5.1.17(b) Comparison of conventional and fiber optic sensor results continued (Thomas 1999)

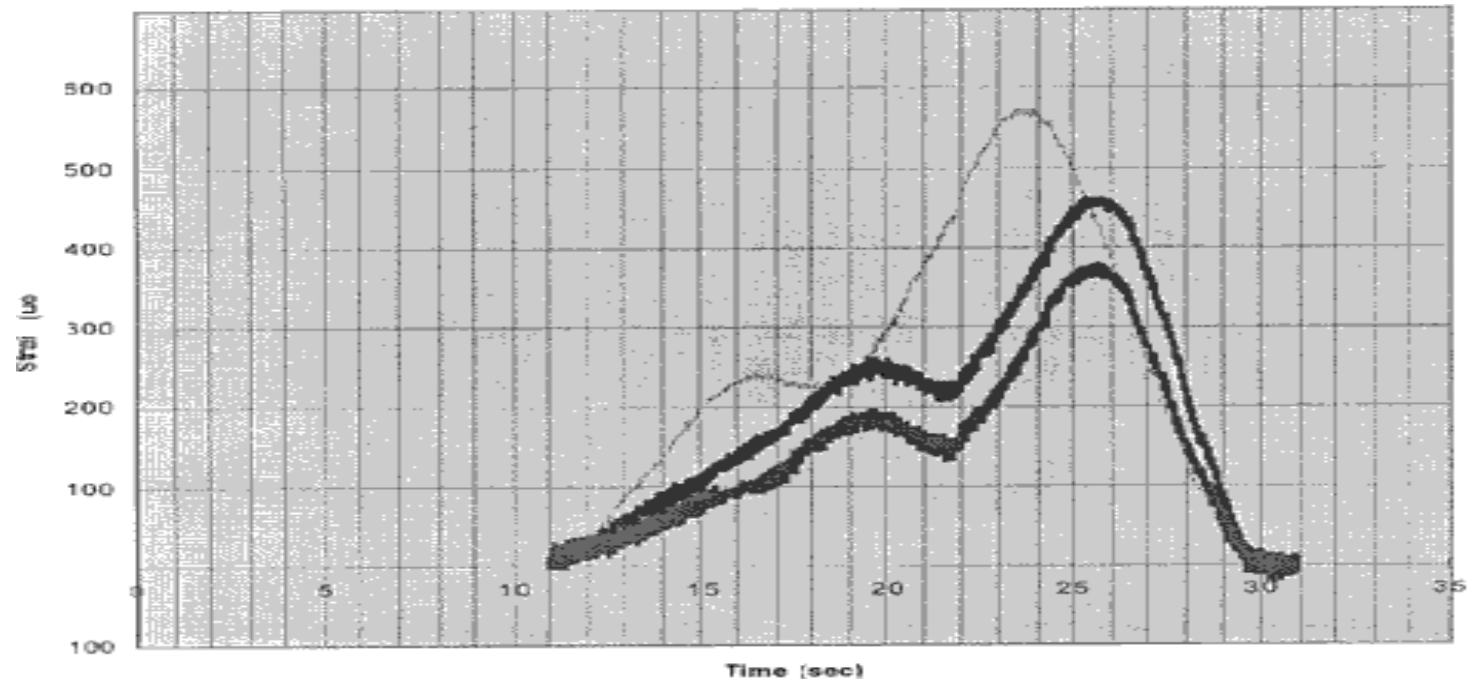

Figure 2.5.1.18 Data from two truck tests - August 1998 (Thomas 1999) 


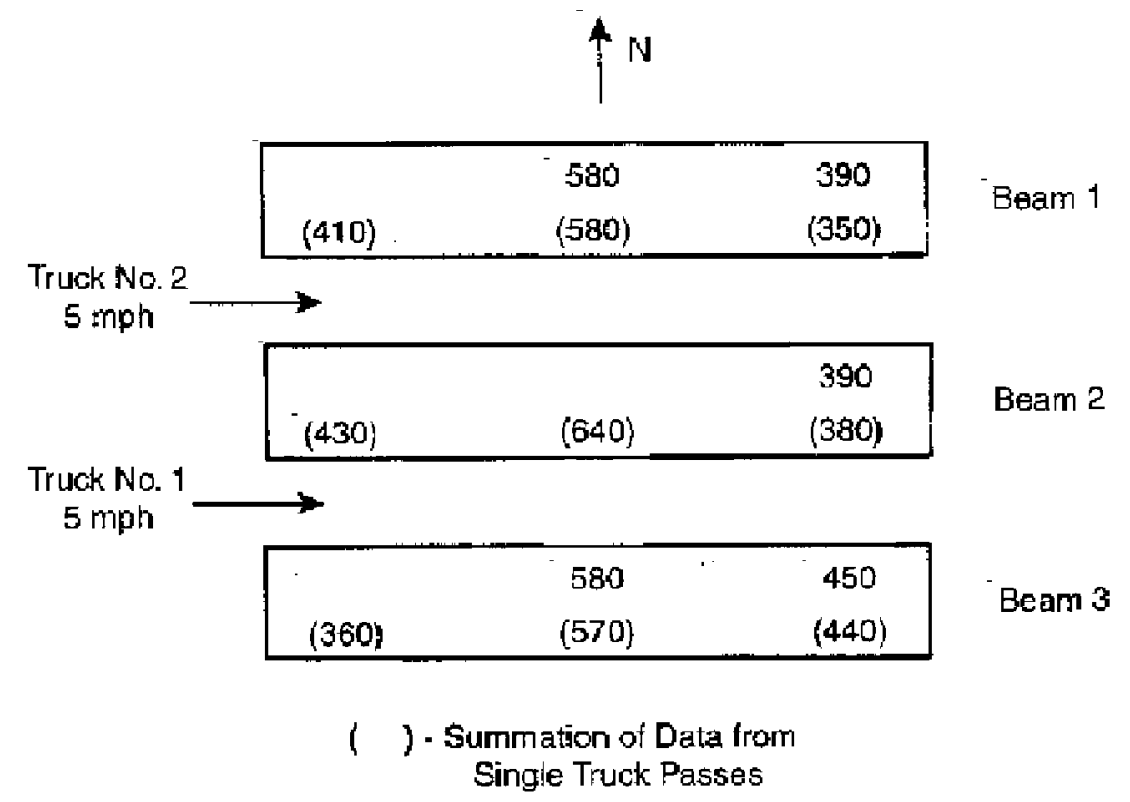

Figure 2.5.1.19 Comparison of single and two truck passes (Thomas 1999)

\subsection{Long - Term Monitoring}

The long-term strain readings have been taken since January 1998 for an eightmonth recording period. Data has been recorded periodically since then and indicate that the bridge is performing consistently. With the optical multiplexing discussed previously, the data includes 13 channels. A typical data plot for a 30 -day period is illustrated in Figure 2.5.1.20. This plot shows how the strain readings are directly related to the diurnal temperature cycling. This does not indicate live loading of the bridge because of the slow data sampling and one-hour averaging. The entire strain of the bridge over the eight-month recording period is shown in Figure 2.5.1.21. Along with the consistency of the diurnal temperature cycling, a long-term increase in compression strain is also clear. Since any long-term creep of the bridge would produce tensile strain, this data was questionable. 
The compression strain could have been caused by only two known sources. If the bridge were thermally expanding in excess of the gaps provided at the abutment, the bridge would be constrained and further thermal expansion would result in the compression strain. The second source would be if the deck structure were experiencing thermal expansion significantly greater than the beams, which would yield upward bending causing compressive, strain in the lower flanges. However, the temperature trend over the measured period is noticeably flat and would not cause continued bridge expansion. The second source mentioned is not likely to occur over a long-term period but would certainly occur in daily cycling where the deck was warmer than the beams. This leaves the concern of drift in the data acquisition equipment.

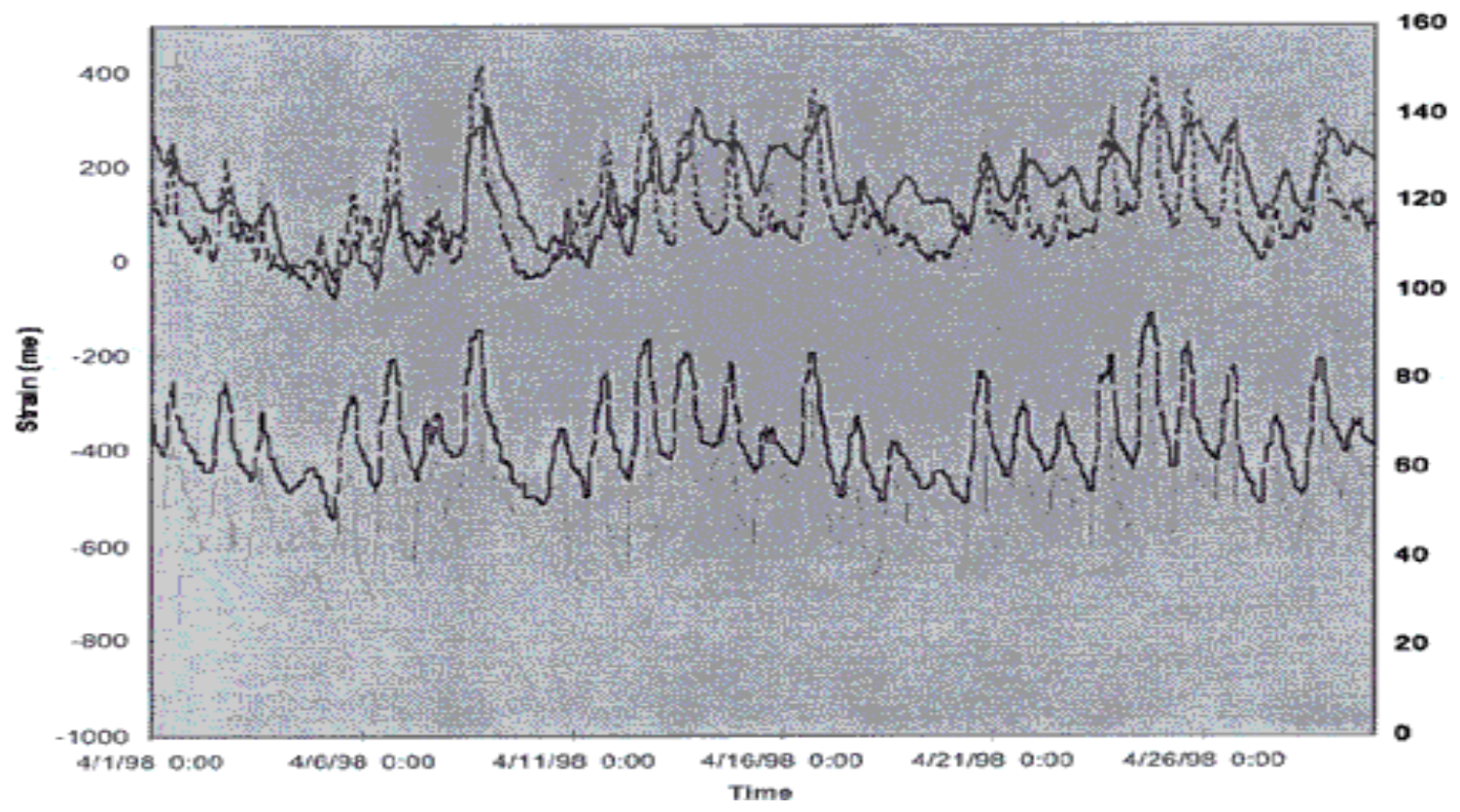

Figure 2.5.1.20 Long - term strain data for April 1998 (Thomas 1999) 


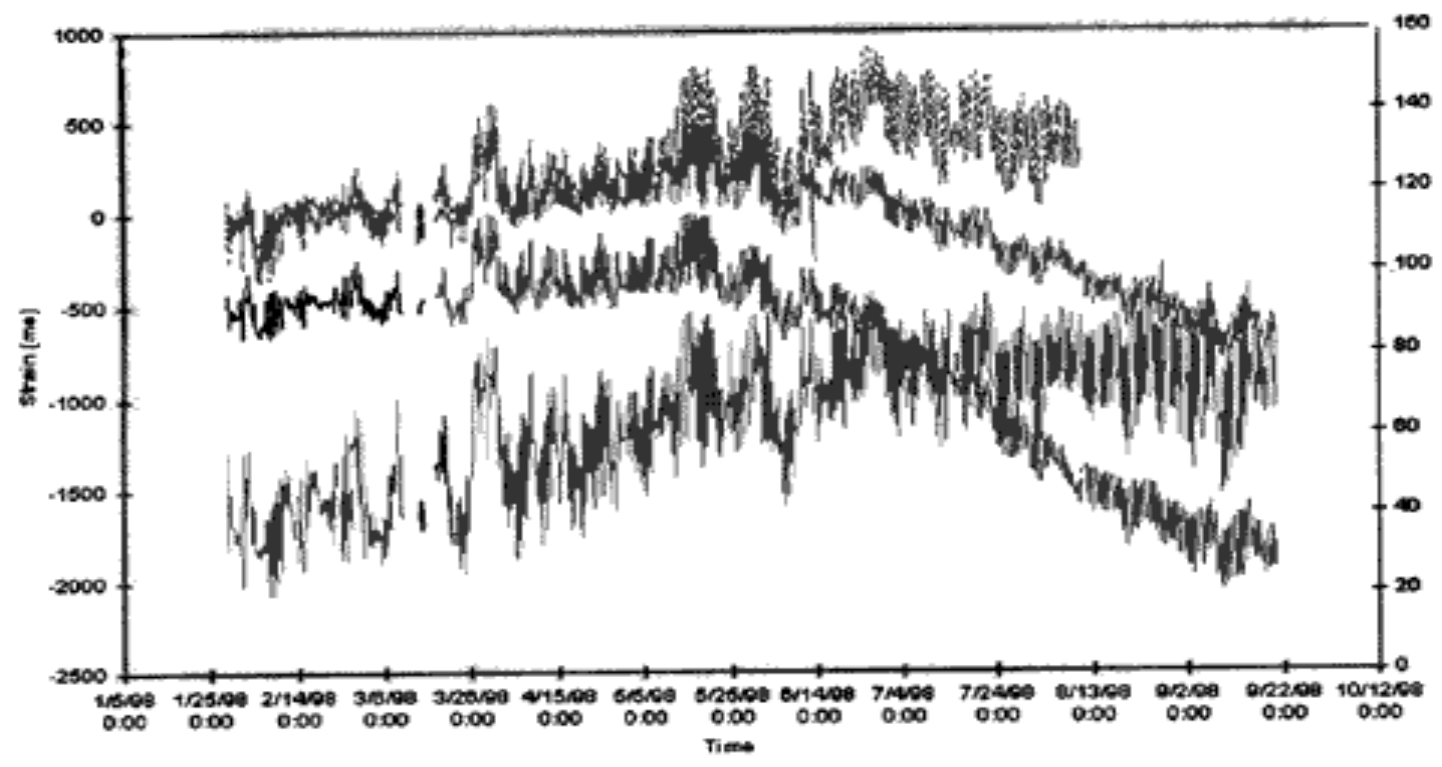

Figure 2.5.1.21 Long - term monitoring data - January to September 1998 (Thomas 1999)

Some other difficulties with the data acquisition system existed because the range of the system was between zero to $4 \mathrm{~V}$, and the EPC Bragg grating system (BDI) provides an output of zero to $10 \mathrm{~V}$. Therefore, a modification had to be made to the acquisition system to allow the addition of an offset voltage so it was within the range of the BDI system. It is recommended that these voltage ranges be equal to eliminate this issue (Thomson 1999).

\subsection{Bondline Condition Monitoring}

The chemical sensors in the south bondline where damaged during installation but the sensors in the north bondline indicated that the bondline was in stable condition. Data was taken at the site on four occasions (July $8^{\text {th }} 1997$, Nov $17^{\text {th }} 1997$, Jan $28^{\text {th }} 1998$, and Aug $27^{\text {th }} 1998$ ) illustrated in Figure 2.5.1.22 and Figure 2.5.1.23. There was significant curing of the bondline epoxy following initial installation but minor, insignificant changes from November 1997 to August 1998. 


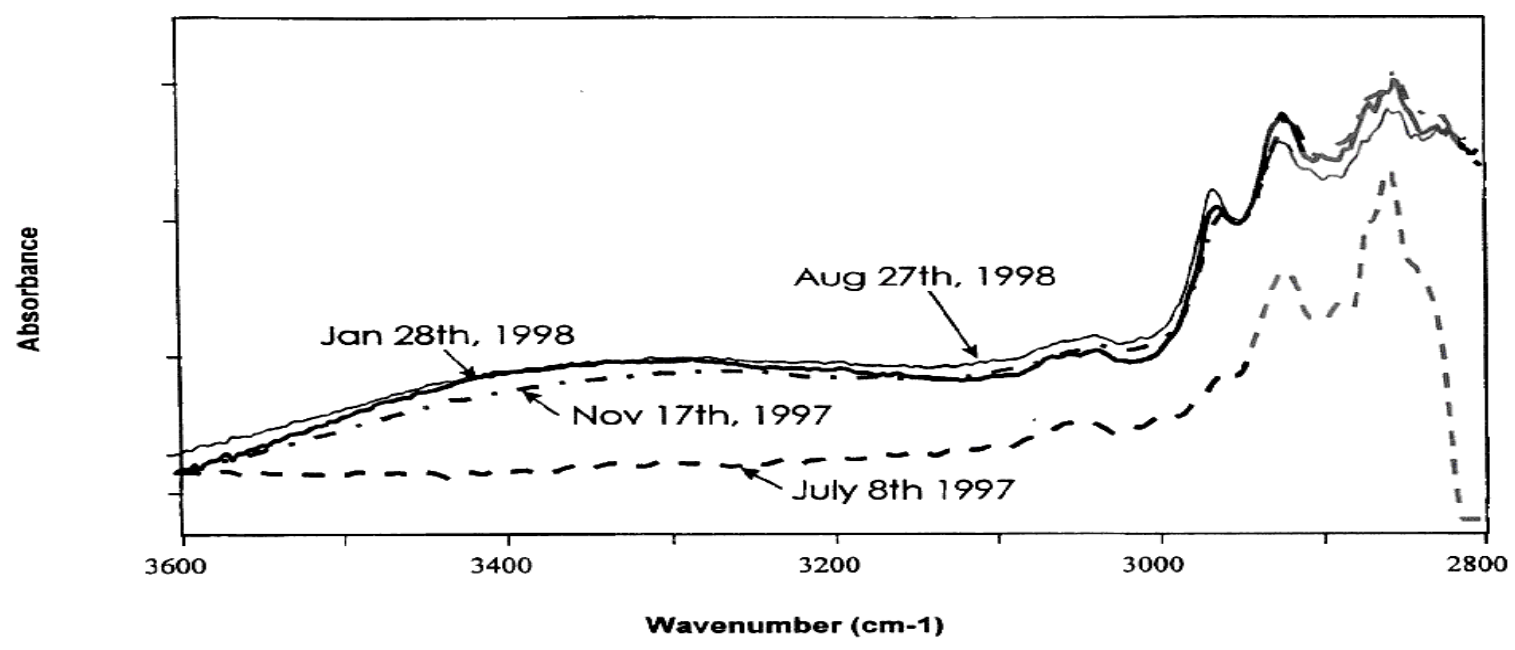

Figure 2.5.1.22 Bondline chemical sensor data - east half (Thomas 1999)

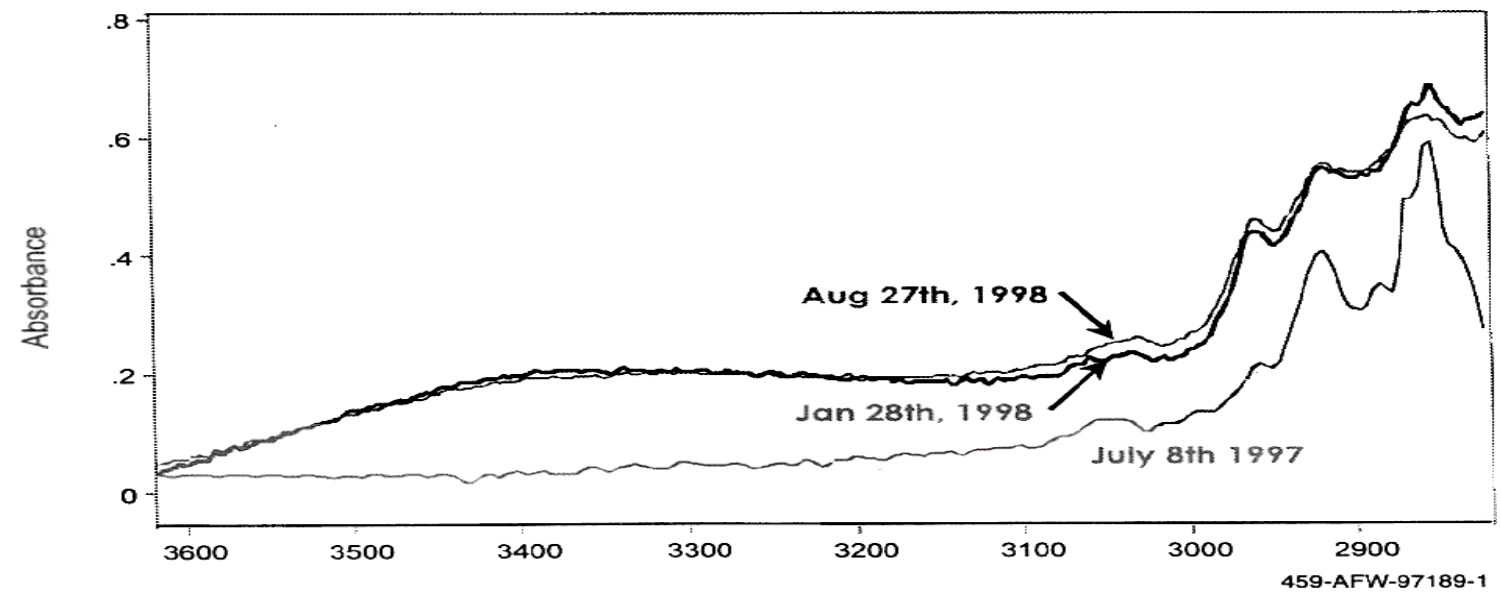

Figure 2.5.1.23 Bondline chemical sensor data - west half (Thomas 1999)

The lower wavenumber peaks in the graphs indicate little or no change in the various carbon bonds. As illustrated in the graphs there was no significant change in the curing for the July $8^{\text {th }}$ results. Some slight changes were seen in the broad oxygenhydrogen bands between 3200 and 3600 wavenumbers. This was considered the result of minor changes in the background-input signal. 
One point that should be noted regarding the bondline data analysis is that the operator should have some knowledge of spectral analysis (Thomson 1999). This would help in identifying changes in the various carbon bonds.

\subsubsection{Commercial System Design}

The main goal of monitoring the "Tech-21" bridge was to provide a complete, commercially viable fiber optic sensor system for use on future composite bridge installations and long-term monitoring. This resulted in the recommended design of two distinct systems, specified below.

These two systems, the bondline chemical sensors and the Bragg grating strain sensors, provide significantly different information and can be used together or separately on future installations. In the future, it would be desirable to have continuous tracking of strain data using the bondline chemical sensors over longer and more regular intervals. This would allow the use of one portable spectrometer system for measurements at several installations going on at the same time.

\subsection{Bondline Chemical Sensor System}

The key enabling technology for this new commercial system is the compact, high resolution IR spectrometer that can be easily transported to field test sites. The expensive sapphire fiber sensor can be replaced with a more inexpensive calgogenite fiber sensor and is recommended for future tests (Thomson 1999). The output of these fibers is connected directly to a laptop computer through a PCMCIA card data acquisition unit. 
A mid-infrared $8 \mathrm{~cm}^{-1}$ resolution discrete peak spectrometer was developed to monitor a wide range of materials and measure up to 12 different wavelengths. Other commercial opportunities for this technology can be used for dedicated applications and are being explored for future use.

\subsection{Bragg Grating Strain Sensor System}

The Bragg Grating Strain Sensor System consists of sensors and hardware that can be purchased as commercial off-the-shelf products. While the recommended system is commercially available now and represents the most cost-effective option at this time, substantial advancements are being made to reduced cost hardware and expanded capabilities (such as fiber multiplexing) in the near future (Thomson 1999). The components used for this system are listed in Table 2.5.1.1. A 12-channel system consisting of these components would cost approximately $\$ 25,000$.

Table 2.5.11 Bragg grating strain sensor system (Thomas 1999)

\begin{tabular}{lll}
\hline \multicolumn{1}{c}{ Component } & \multicolumn{1}{c}{ Supplier } & \multicolumn{1}{c}{ Model } \\
\hline Strain Sensor & EPC & FGS-10-003-FC-A \\
Signal Processor & EPC & FLS3100 \\
$1 \times 4$ Switch & E-Tek & MFSW5014043 \\
On-Site DAQ & Campbell Scientific & CR10-X \\
Multiplexer & Campbell Scientific & AM-416 \\
Modem & Numerous & \\
Portable DAQ & National Instruments & 8 channelPCMCIA \\
\hline
\end{tabular}

\subsubsection{Conclusion and Recommendations}

This fiber optic health monitoring system, along with the bridge itself, is an example of how technology initial used in the aerospace industry is now being transitioned to civil infrastructure applications for widespread, commercial benefits. The 
new, low cost commercial chemical sensing system for bondline monitoring is a significant development, since it now permits advanced infrared spectral analysis of the specific chemistry of a material through direct field measurements.

The program also completed a commercial system for the Bragg grating strain sensors. This complete system includes the sensors, multiplexing switches and all data acquisition and transmission hardware and software mentioned in Table 2.5.1.1.

The main conclusions of the fiber optic sensors and the bridge performance itself were as follows:

- $\quad$ The all-composite bridge performed well after a year of monitored service and proved to be able to withstand live loads well in excess of the legal truck limit for its location.

- $\quad$ The Bragg grating sensors produced data that agreed well with standard strain gauges and predicted values.

- The diurnal cycling indicated by the temperature data coincided with the long-term data from the Bragg grating sensors.

- The bondline chemical sensors that functioned showed the adhesive had fully cured and that water and other chemicals did not penetrate the bondline.

- $\quad$ The Mini-Spectrometer system that was developed will provide major cost and convenience advantages; therefore, making field analysis more practical in the future. 
Two operational procedures were recommended for future installations (Thomson 1999):

- $\quad$ All sensors should be installed and checked at the factory during bridge fabrication.

- $\quad$ The disconnection and reconnection of fiber optic leads should be avoided as much as possible in the field and all leads should be cleaned before reattachment to avoid contamination and "noise" interference.

The "Tech-21" program has demonstrated the potential advantages of a fiber optic instrumentation in civil infrastructure applications of composite materials. The commercially available fiber optic strain measurement system and chemical analysis system can provide an effective means of monitoring the response of future bridges and a wide range of other applications.

\subsubsection{Application of Optical Fiber Sensors to the Intelligent Processing of Materials (Claus and Arya 1998)}

Using sensors for the sensing of critical components and process parameters in analytical or empirical models has become common for the control of a material's production process. This adaptive control of a material production process is referred to as Intelligent Processing of Materials (IPM). This process, however, has been hindered by the lack of suitable feedback sensors and instrumentation that can survive the harsh processing environment (Claus and Arya 1998). Fiber optic sensors are much more 
durable, which present an attractive option for application in these harsh processing environments. Three specific applications are discussed in section 2.2 where optical fiber sensor-based instrumentation are proposed and implemented to (Claus and Arya 1998):

(1) monitor epoxy processing in advanced polymer-matrix composites,

(2) monitor internal strain during fabrication of multilayered ceramic actuator (MCA) elements, and

(3) record in situ temperature and strain during the fabrication of titaniummatrix composites.

\subsubsection{Introduction}

The current demand for high strength composite materials, which began in the aerospace application and are now emerging into civil structure applications has increased the demand for novel material fabrication and development techniques. To successfully develop these high strength composite materials, micro- and macro-level monitoring of the manufacturing process needs to be developed. This process has started using fiber optics combined with other technologies to act as the nerves and the muscles of novel smart materials.

Smart materials are composites that incorporate sensors, actuators, and adaptive signal processing functions within the structural material components. By incorporating these technologies within the material's components, the material has the ability to detect changes in its environment and/or chemical and physical characteristics. The basic limitation to the advancement of smart material technologies is that the complex 
processing requires high cost components. Decreasing costs will increase the use of smart materials in applications such as automotive engines, electric power plant structures, high temperature furnaces, and lightweight bridges (Claus and Arya 1998).

Optical fiber-based sensors have been used, in the past, primarily for applications in the instrumentation of aerospace and hydrospace vehicles, materials and systems. Such fiber-based sensors and optical methods are being considered for the use of monitoring the fabrication of high strength composite materials due to advantages such as (Claus and Arya 1998),

- their inherent immunity to high levels of electromagnetic interference, which are present in and/or near the processing system,

- their small size and weight,

- their capability of using metal-coated silica fibers and sapphire fibers for high temperature process control between $800-2000{ }^{\circ} \mathrm{C}$,

- their ability to incorporate in-line optical signal processing functions,

- their multiplexing system arrangements,

- their high sensitivity, resolution, and dynamic range, and,

- their ability to be structurally integrated within advance composite materials

Figure 2.5.2.1 illustrates the different monitoring functions that the fiber optic sensors could be used for during its fabrication, and in-service periods. 


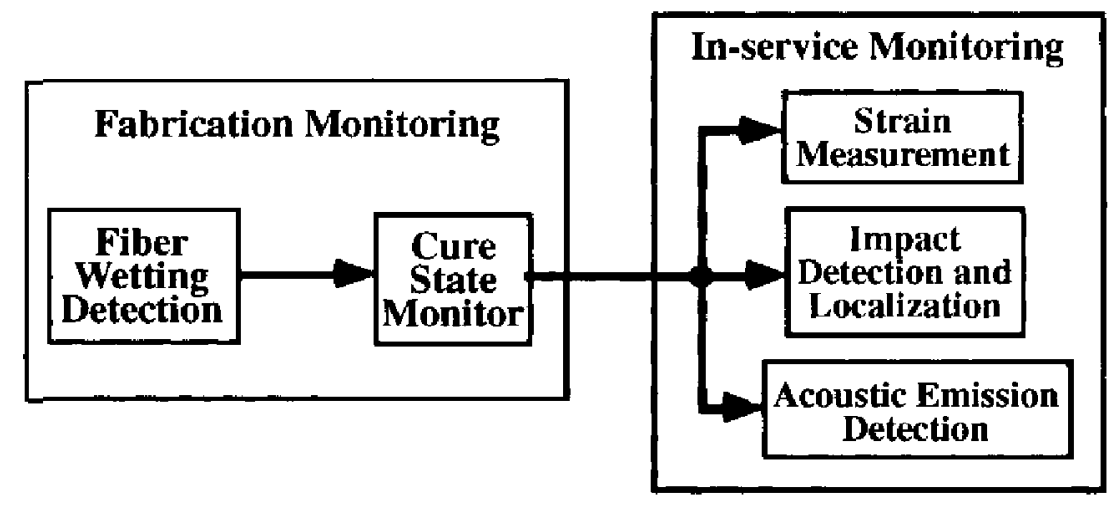

Figure 2.5.2.1 IPM application for optical fiber sensors (Claus and Arya 1998)

\subsubsection{Intelligent Processing of Materials - Polymer-Matrix Composites}

Composite materials are macroscopic combinations of two or more distinct materials that have a recognizable interface between them (Claus and Arya 1998). These materials typically comprise reinforcement such as fibers, supported by a binder or matrix material. Usually the matrix used in composite fabrication includes polymeric compounds such as polyester and vinyl ester resins, epoxy resins, or thermoplastic resins. These resins help transfer the load to and between the fibers.

The use of composites as engineering materials for large structures as well as other applications has been increasing due to the advantages that composites offer over conventional materials such as metals and alloys. Some of these advantages include high strength and stiffness-to-weight ratios, fatigue resistance, small cross-section, excellent corrosion resistance, and the ability to adjust mechanical properties geometrically (Claus and Arya 1998). The disadvantage of these composite materials is the high manufacturing costs as well as the uncertainty associated with the reliability of such members. The need for low-cost manufacturing is of major importance, which requires 
the use of effective process analysis and feedback control sensors instrumentation to minimize both processing times and the percentage of wasted material scrap.

\subsection{Epoxy - Matrix Composites}

The fabrication of epoxy matrix composites consists of a matrix that penetrates, surrounds and wets reinforcing fibers, and then cures. This curing process is a critical step in the fabrication of composite materials, since the mechanical properties are a direct result from the degree of crosslinking achieved in the process. This curing process usually uses heat and/or pressure to speed up the chemical reaction, which is usually energy intensive and expensive. Despite the expense, composites are often over-cured, in order to insure complete crosslinking of the thermoset resin. Over-curing (heating too fast and with too high of a temperature) causes the matrix to become brittle and weak. To optimize cure time without over-curing, sensors are being developed that sense when the cure is complete (Claus and Arya 1998). These cure-monitoring sensors are being used with conventional controllers to form intelligent control systems (Claus and Arya 1998).

\subsection{EFPI-based Optical Fiber Sensors}

The multifunctional sensor discussed in this section is an advanced form of the extrinsic Fabry-Perot interferometric (EFPI) fiber optic sensor. The EFPI sensor measures surface strains and internal strains in composite materials, which have been validated in several tests. The EFPI sensor was redesigned to make it capable of measuring the cure state during fabrication, while still permitting the sensor to function as a strain sensor after fabrication. The redesign included changing the type of glass fiber 
used for certain parts of the sensor, and employing a low-coherence light source, which measured the refractive index of the epoxy matrix during cure. It is possible to monitor the curing of a composite structure with these measurements because the refractive index of an epoxy directly depends on the cure state.

Figure 2.5.2.2 shows the basic EFPI sensor geometry, which implement short gage length strain and ultrasonic stress wave sensors. The figure shows a single mode fiber, used as the input/output fiber, and a multimode fiber, used as a reflector, forming an air gap that acts as a low-finesse Fabry-Perot cavity. A glass capillary tube (hollowcore fiber) serves to align the two fibers colinearly. A Fresnel reflection is caused from the air/glass interface (reference reflection) at the front of the air gap and the air/glass interface (sensing reflection) at the far end of the air gap, which interfere with one another in the input/output fiber. The end of the multimode fiber is typically shattered to eliminate any unwanted reflections from light that passes through the air gap by scattering the light from the fiber. As the sensor is strained, the air gap changes in length causing a change in the phase difference between the reference reflection and the sensing reflection. This changes the intensity of the light measured at the output arm of a coupler. 


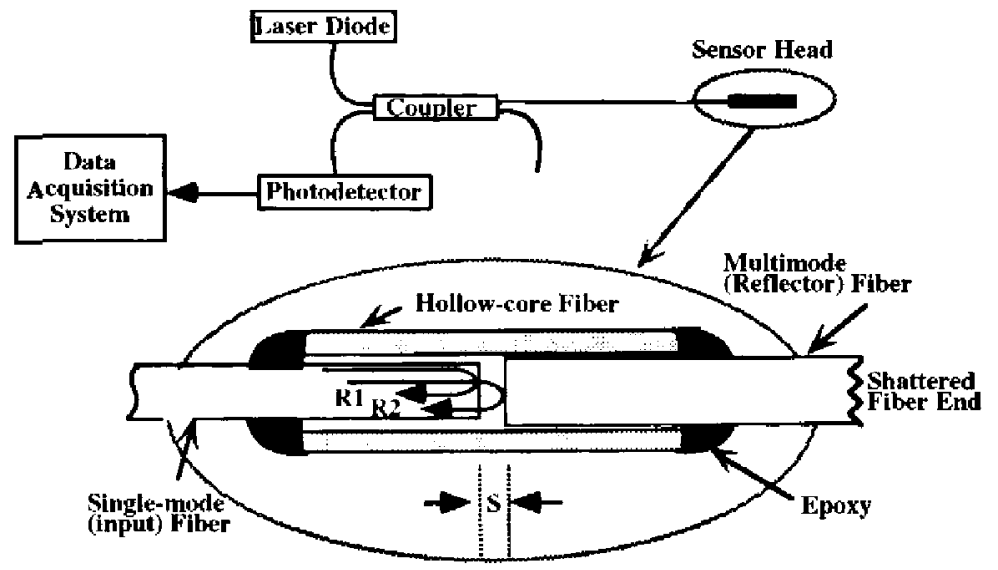

Figure 2.5.2.2 Geometry of conventional EFPI strain sensor (Claus and Arya 1998)

The output wave of the EFPI sensor was evaluated in terms of a plane-wave approximation. The observed intensity at the detector is a superposition of the reflections (Claus and Arya 1998). The different intensities due to gap-separation $s$ are illustrated in Figure 2.5.2.3. The decaying amplitude is due to losses or attenuation from the separation of the single mode fiber and the multimode fiber.

The gage length may be set during fabrication to be several millimeters or less. The strain was determined by a ratio between the endface displacement measured and the gage length. The sensors typically exhibit a $30 \mathrm{~dB}$ signal-to-noise ratio, which means an air gap elongation of roughly $4.6 \mathrm{~nm}$ is resolvable for $1,300 \mathrm{~nm}$ operations. For longer gage lengths, the resolution improves. 


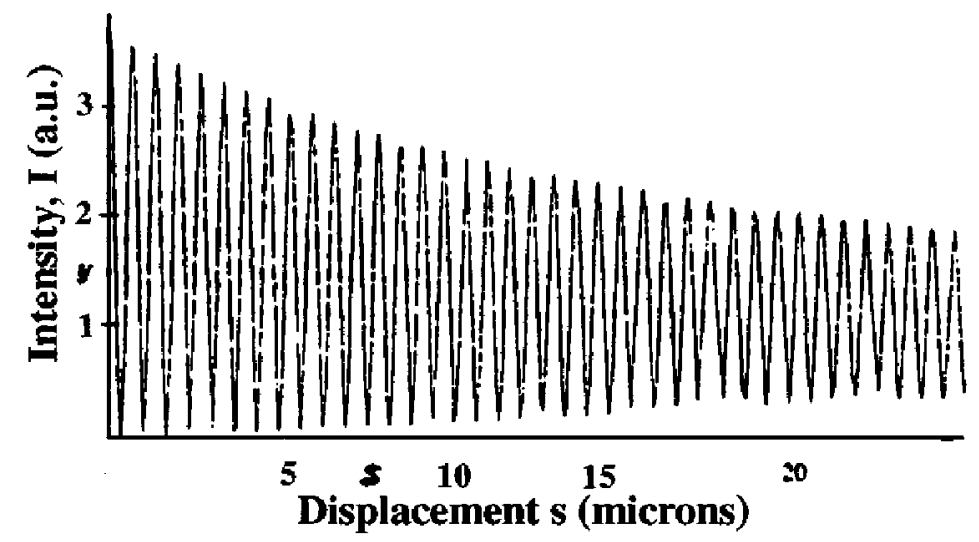

Figure 2.5.2.3 Variation of output intensity y (in arbitrary units) with changes in gap separations (in $\mathrm{mm}$ ) (Claus and Arya 1998)

\subsection{Epoxy Matrix Processing using EFPI Sensors}

The manufacturing of epoxy matrix composites is quit complex involving many steps, of which the curing process is the most important because improperly cured composites are likely to fail much more easily. This curing process transforms layers of reinforcing fibers emerged in soft tacky resin into hard structural members. When the epoxy is ready to be hardened, a curing agent is mixed into the epoxy resin, which polymerizes the resin and forms a solid crosslinked network between the fibers and epoxy.

A major objective in manufacturing composite parts is to reduce the cost, which can be achieved by minimizing cure time. One way to reduce curing time is to heat the composite more quickly and at higher temperatures, but hazards such as thermal runaway and thermal degradation can occur. Conventional cure cycles that have evolved from trial and error are inflexible, unadaptable and do not necessarily minimize cure time. Thus, a sensor that can be directly integrated in the composite to monitor composite 
curing would help develop flexible and intelligent controllers for minimizing time (Claus and Arya 1998).

Using modified reflector fibers in the EFPI sensor makes it is possible to monitor the cure state by reflectometry. This setup replaces the multimode reflector fiber used in the conventional EFPI sensor with an unclad homogeneous glass fiber, as shown in Figure 2.5.2.4. The glass type varied with the particular type of epoxy used to assure the glass refractive index was higher than that of the uncured epoxy, but less than or equal to that of the fully cured epoxy. This EFPI sensor was then made the same way as the conventional sensor except the free end of the reflector fiber was not shattered, but instead cleaved in order to produce a smooth reflective surface perpendicular to the fiber axis.

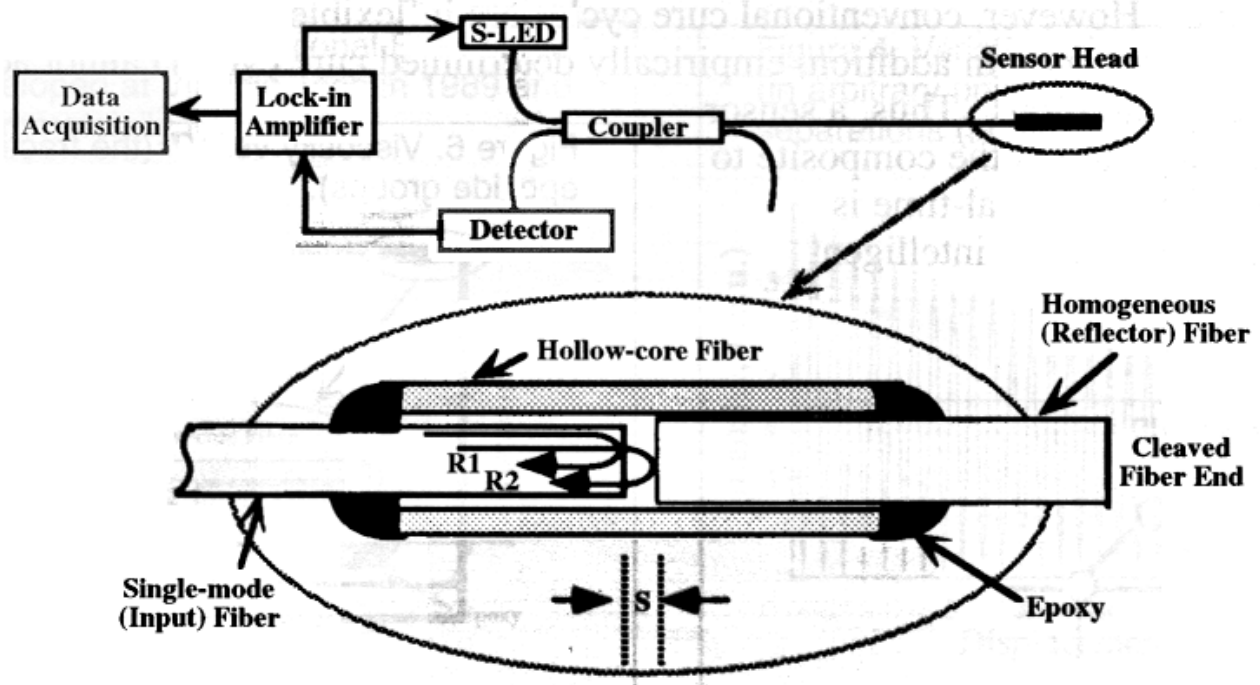

Figure 2.5.2.4 Modifications to EFPI sensor for cure state monitoring (Claus and Arya 1998)

When the sensor operated as a cure monitor, the input end of the sensor was connected to a superluminescent diode (S-LED) shown in Figure 2.5.2.4. A 2 x 2 fiber optic coupler was used for connecting the input fiber to both the source and a 
photodetector, which will detect the light reflections from the different interfaces. When the epoxy is cured, the refractive index of the epoxy resin will rise to a value equal to that of the reflector fiber.

The reflections from the reflector fiber are guided to the photodetector by the input fiber and the $2 \times 2$ coupler where they are converted to an electrical signal. By monitoring these reflections from the reflector fiber, the state of cure was determined by the measured refractive index of the curing epoxy.

Following the cure and fabrication of the composite material, the electronics and data acquisition for the cure monitor where disconnected so the sensors could be connected to a laser diode, avalanche photodiode, and data acquisition system to function as a conventional EFPI sensor. This system was able to measure static or dynamic strains, and stress waves originating from acoustic sources, such as ultrasonic NDE sources.

\subsection{Experiments}

To validate the use of a modified EFPI sensor for monitoring the cure state, Devcon 5-Minute Epoxy ${ }^{\circledR}$ was used because of its fast cure time. First, a fiber optic reflectometer, which was calibrated using Cargille certified refractive index liquids, was made and used to determine the refractive index of the 5-minute epoxy as a function of the cure state (Figure 2.5.2.5). Next, the reflectometer fiber was immersed in a batch of freshly mixed 5-minute epoxy to determine the refractive index as a function of time, 
which is graphed in Figure 2.5.2.6. Using the data from Figure 2.5.2.5 and Figure 2.5.2.6, the refractive index of uncured 5-minute epoxy was determined to be approximately 1.54 and that of fully cured 5-minute epoxy was approximately 1.58.

A glass fiber having a refractive index greater than 1.54 and less than or equal to 1.58 was required to fabricate a prototype multifunctional sensor. Corning 0120 glass with a reflective index of 1.56 was used for the initial experiments. Cutting a short length of the fiber and mechanically splicing it to a multimode optical fiber tested the glass. The assembly worked effectively as a cure monitor, but not as an interferometric strain sensor, since it lacked a Fabry-Perot cavity.

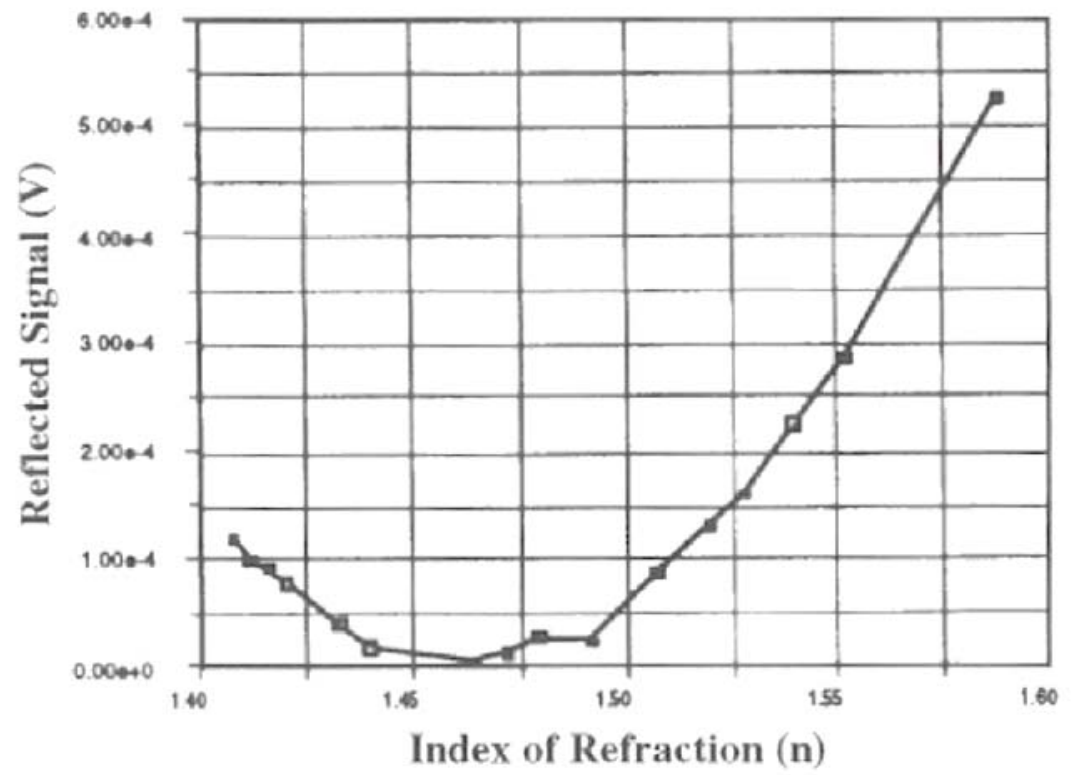

Figure 2.5.2.5 Reflectometer output as a function of refractive index (Claus and Arya 1998) 


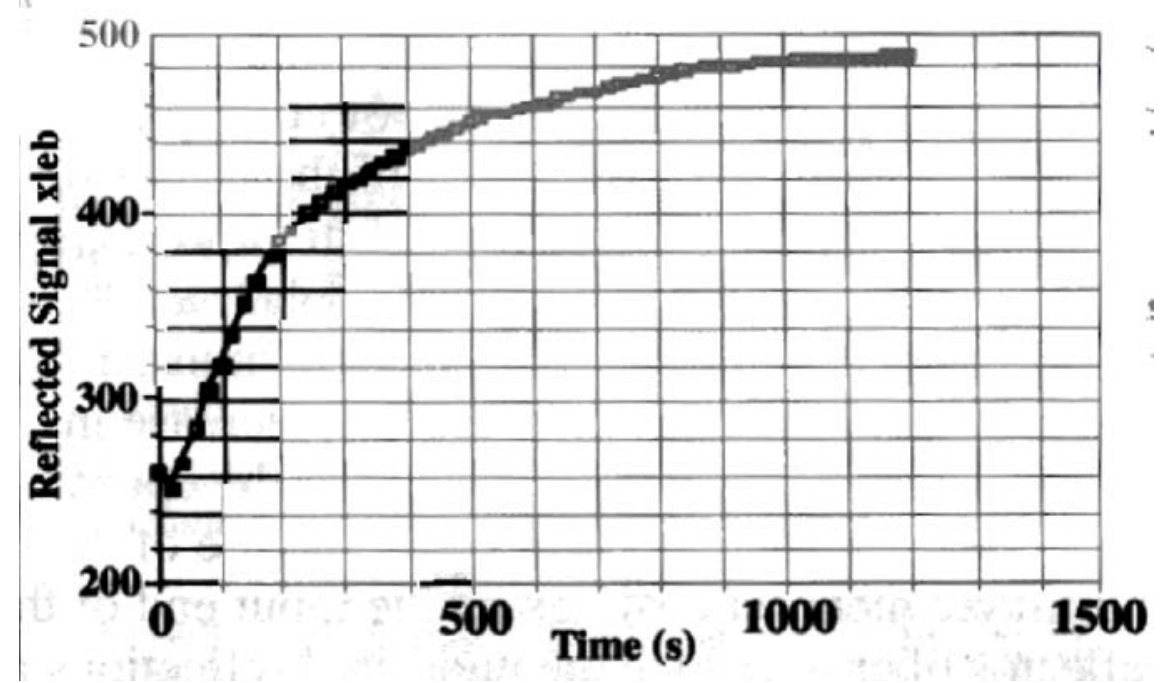

Figure 2.5.2.6 Output of reflectometer for curing 5-minute epoxy (Claus and Arya 1998)

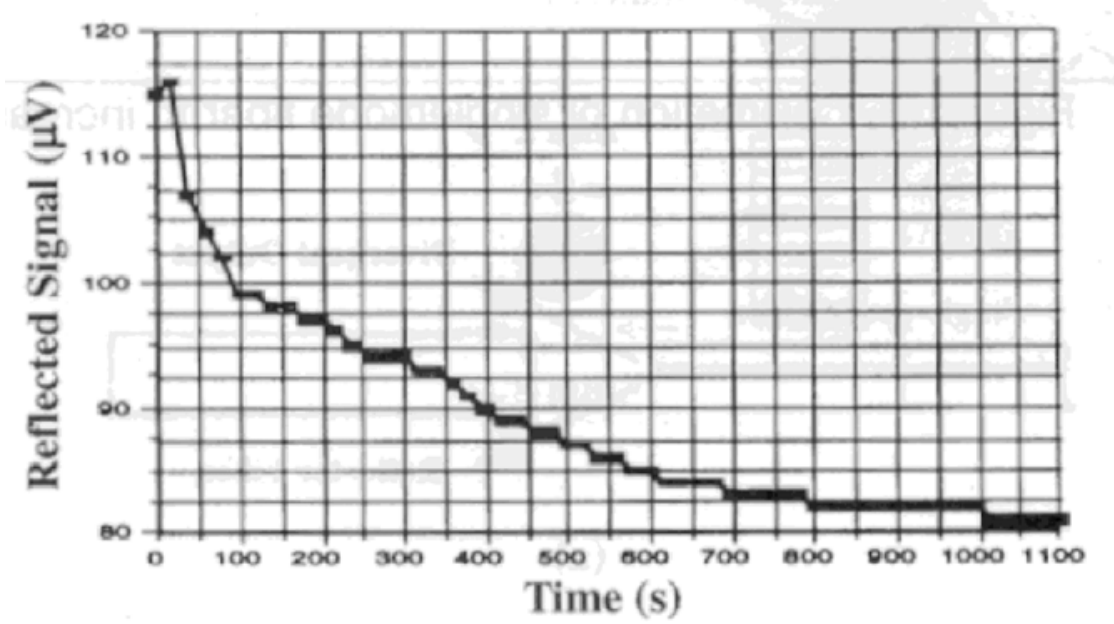

Figure 2.5.2.7 Cure monitor sensor output (without air gap) for curing 5-minute epoxy (Claus and Arya 1998)

A test was performed by placing the distal end of this cure monitor into freshly mixed 5-minute epoxy and connecting the input fiber to a coupler leading to a LED, and a photodetector connected to a lock-in amplifier. The collected data is given in Figure 2.5.2.7 and shows that the sensor output declined with progressing cure state. This confirmed that the Corning 0120 glass with a refractive index of 1.56 was a good choice for the reflector fiber. The EFPI sensor was then assembled using a single mode fiber as the input fiber, and a 1-cm long piece of Corning 0120 was used for the reflector fiber. In 
order to align the fibers, a glass capillary tube was bonded to the fibers using epoxy leaving a $50 \mu \mathrm{m}$ gap separating the fiber ends

When the sensor was used as a strain sensor, interferometric fringes were detected at the output of the sensor, but functioned normal when used to monitor the cure state. Due to the divergence of the light emerging from the end of the single mode fiber, illustrated in Figure 2.5.2.8(a), the desired signal that was reflected from the far end of the reflector fiber was swamped by the reflection from the single mode fiber. A miniature gradient index lens was constructed and spliced to the end of the single mode fiber to reduce the divergence of the fiber output illustrated in Figure 2.5.2.8(b). A reflective silver coating was then applied to the far end of the 0120 glass reflector fiber by vacuum evaporation to increase the reflection of light transmitted by the fiber.

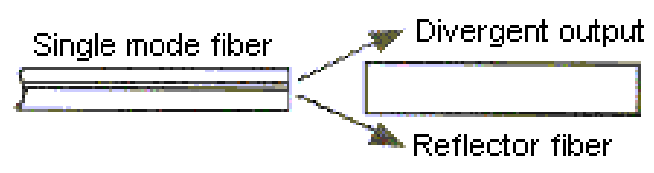

(a)

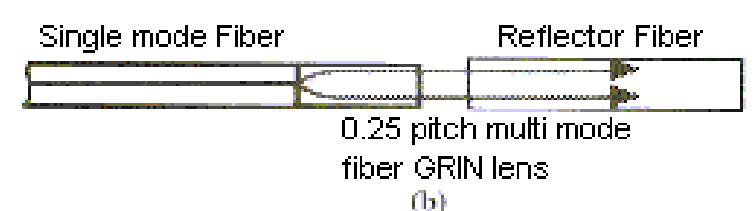

(b)

Figure 2.5.2.8 Collimation of single mode fiber to increase sensor signal (Claus and Arya 1998)

The output signal obtained from this sensor when placed in a freshly mixed 5minute epoxy is shown in Figure 2.5.2.9. There was an $8 \%$ increase in the signal in the first five minutes, which could not be explained. The signal then decreased to a value $5 \%$ lower than the initial output after 60 minutes due to the increased attenuation of the reflector fiber caused by the increased surrounding epoxy. The sinusoidal fluctuations in the sensor output resulted from instabilities in the LED. Once the epoxy had fully cured, the LED was disconnected and a coherent source was connected to the input of the single 
mode fiber where stress produced by the hand was measured. This test gave good signalto-noise ratio at the sensor output.

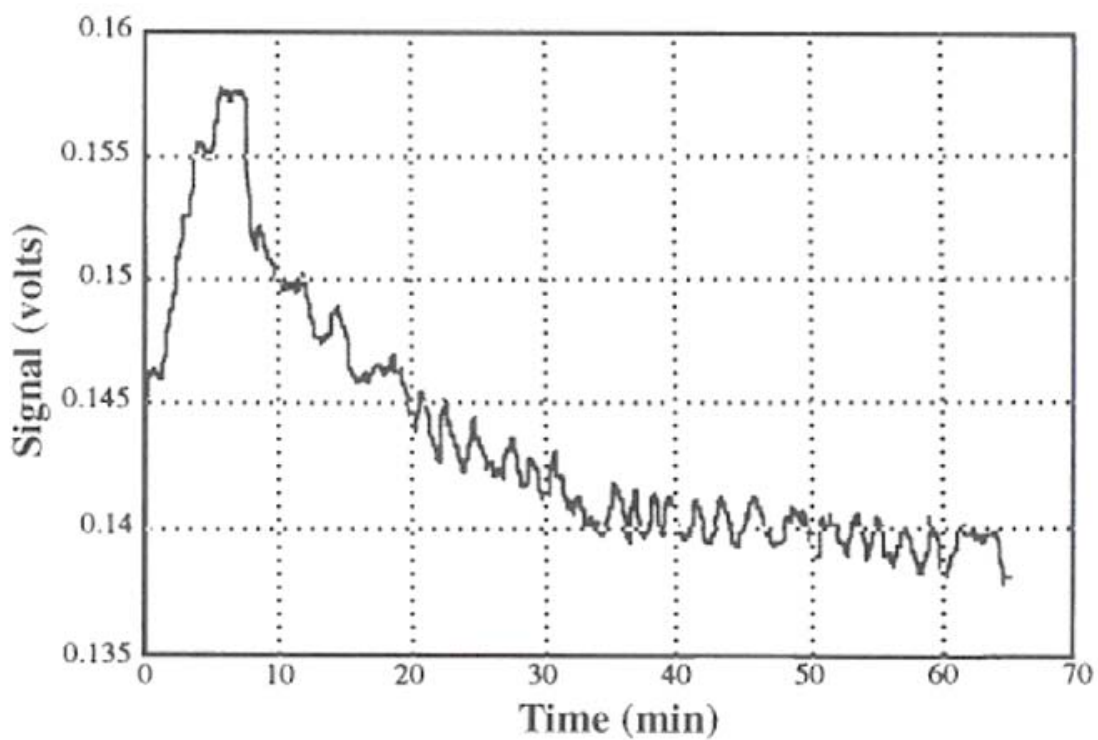

Figure 2.5.2.9 Output of collimated cure monitor for curing Devcon 5-minute ${ }^{\circledR}$ epoxy (Claus and Arya 1998)

These experimental results demonstrated that the modified extrinsic Fabry-Perot interferometer sensor could be embedded in epoxy to measure the state of cure, while retaining its ability to measure strain in the epoxy after cure.

\subsubsection{Intelligent Processing of Materials - Multilayer Ceramic Actuator Elements}

Ceramic actuators are often implemented in the multiple layers of smart systems to complement the other sensor instrumentation. The process of making such multilayer ceramic actuator (MCA) elements involves the mixing of a slurry, tape casting, printing electrodes, laminating the tape, burning out residual binder introduced in the slurry, sintering, terminating, and packaging of the actuator element. The final yield of the material relies heavily on the burnout process. These optimal burnout and sintering schedules have been conventionally obtained through empirical trials. The binder 
burnout process in MCA manufacturing demands sensor instrumentation (Intelligent Processing of Materials), which monitors real-time temperature and strain states. Due to the high temperatures of this process $\left(400^{\circ} \mathrm{C}\right)$, gold-coated single-mode silica fibers that can withstand long-term exposure at temperatures up to approximately $850-900{ }^{\circ} \mathrm{C}$ were used to fabricate the EFPI sensors. The experimental setup for sensor and MCA element placement in the "smart" kiln is illustrated schematically in Figure 2.5.2.10.
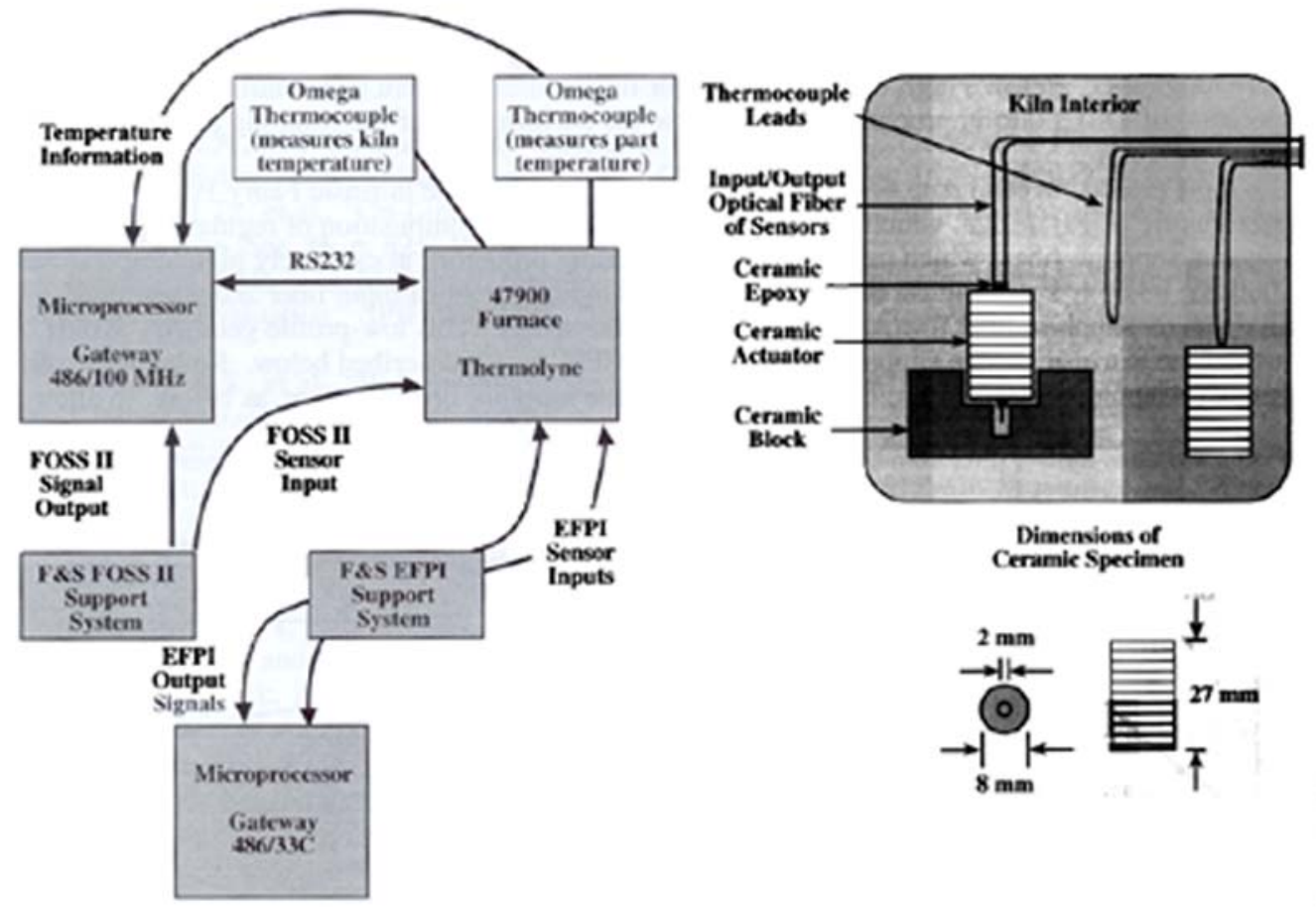

Figure 2.5.2.10 Schematic of the experimental arrangement used in the IPM of MCA elements (Claus and Arya 1998)

Figure 2.5.2.11 illustrates the typical EFPI experimental results of the IPM that were obtained. The anomaly between the displacement-versus-time plot was due to intentional heating the MCA too fast. Heating the element too fast caused a rapid destructive expansion of the matrix caused by outgassing. This will be avoided in the future through feedback control of temperature and pressure. 


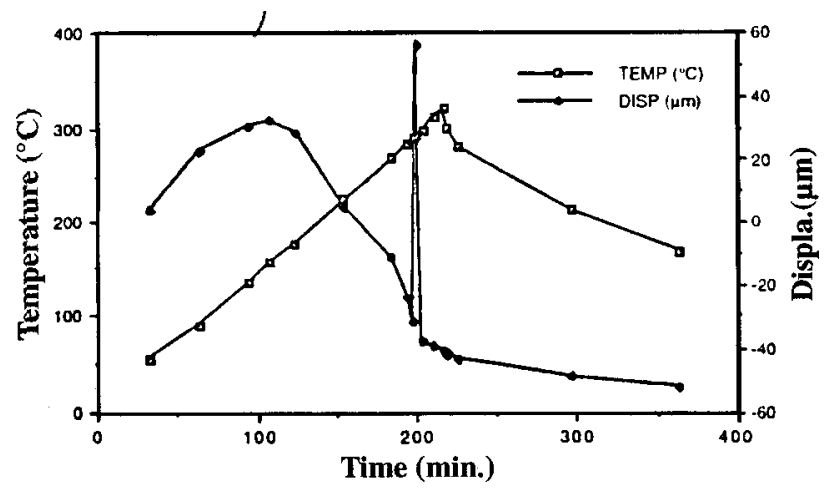

Figure 2.5.2.11 Fiber sensor data from MCA burnout tests (Claus and Arya 1998)

\subsubsection{High Temperature Optical Fiber Sensors for the Intelligent Processing of Materials -Titanium Matrix Composites}

Titanium matrix composites (TMC) reinforced with continuous filaments are well suited for structural elements subjected to fatigue loading in high temperature environments because of their high interlaminar shear strength, high fracture toughness, and high transverse tensile strength (Claus and Arya 1998). However, the processes for these TMCs are expensive. This process was improved by adding high temperature sapphire-based optical fiber sensors for process monitoring inside the metal matrix to reduce scrap, energy costs, and improve uniformity.

The high physical strengths of these TMCs result from the adhesion of the reinforcing fibers to the matrix. Many of the current procedures use high temperatures to insure uniform consolidation in the matrix, which can change the mechanical properties of the composite. Distributing temperature sensors throughout the specimen can guarantee the uniformity of the consolidation at lower temperatures; thus reducing the processing cost due to lower energy consumption. 
A good candidate for monitoring this process is the intrinsic Fabry-Perot interferometric design (illustrated in Figure 2.5.2.12), which combines regular silica fibers, and special sapphire fibers that can maintain light guiding properties at extremely high temperatures $\left(\sim 2,000^{\circ} \mathrm{C}\right)$. Reflections from the silica-to-sapphire interface and the opposite end of the sapphire fiber interface allow relative phase measurement. This intrinsic device differs from the EFPI sensor described before in that the change in phase in the output signal is due to both strain-induced relative displacements between the opposite ends of the sapphire fiber, and changes in the index of refraction of the sapphire fiber material. Due to index changes caused by both photoelastic effects and temperature changes, the analysis of the signal for strain measurements is complicated.

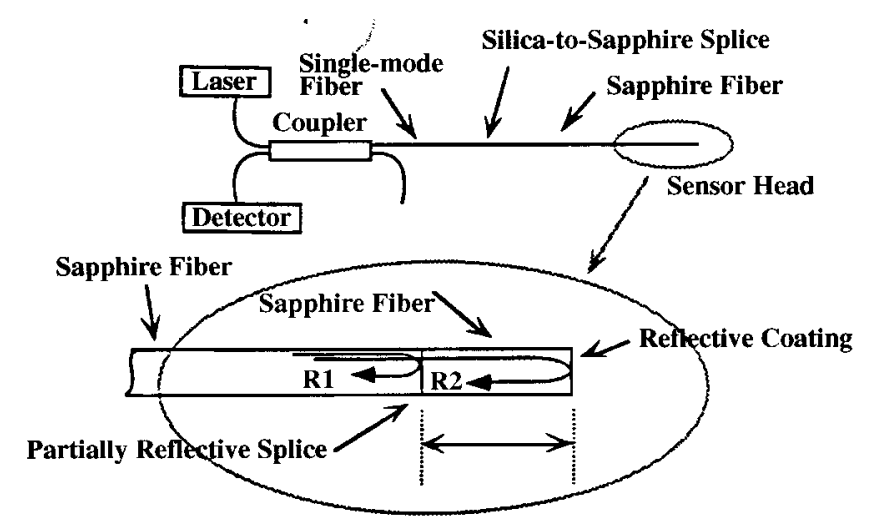

Figure 2.5.2.12 Intrinsic Fabry - Perot interferometer design (Claus and Arya 1998)

\subsubsection{Conclusions}

Novel optical fiber configurations were demonstrated to be extremely effective for instrumentation purposes in specific IPM applications. The extrinsic and intrinsic Fabry-Perot interferometers were both utilized for the IPM of polymer-matrix composites, titanium-matrix composites, and for monitoring the binder burnout process in MCA elements. These fiber optic sensors proved to increase performance of the resulting 
parts while lowering costs, making the advancement of smart material technologies applicable where cost is a major consideration.

\subsubsection{Fiber Optic Health Monitoring System for Composite Bridge}

\section{Decks (Furrow et al. 2000)}

About 112,000 bridges in the United States are rated as substandard through either deterioration or obsolescence, and there are over 578,000 bridges on the public roads, which means that the number of substandard bridges will probably keep increasing (Furrow et al. 2000). Fiber reinforced polymer composites have been an increasing material used for building bridges due to there advantages such as reduced weight, decreased effects from the environment, and speed of installation. With the increasing use of this material, the development and implementation of an embeddable fiber optic health monitoring system to monitor strain in composite bridge decks over time has become necessary. Luna Innovations has successfully embedded fiber optic strain sensors in three composite bridge decks.

\subsubsection{Introduction}

The research performed by Luna Innovations along with researchers at Atlantic Research Corporation developed an optical fiber sensor system capable of measuring deformation of modular composite bridge decks over time. A system to monitor both real time measurement and periodic interrogation over a period of years was designed. The fiber optic sensors were installed in a total of four composite bridge decks in the top 
and bottom face sheets and between the face sheets and the triangular support structure. The sensors were also used in laboratory three-point bending tests to compare to the data obtained from the sensors used in the field tests.

During evaluation of the sensing and measurement instrumentation, several sensor types were considered; however, the extrinsic Fabry-Perot interferometric (EFPI) sensors proved to give the most accurate and reliable readings. These sensors were then tested in the laboratory on a triangular section with three-point-bend testing. The section was tested until fracture and the sensor output data successfully illustrated the load bearing capability of the support structure.

The sensors were then installed in four bridge decks. Two of the bridge decks were manufactured and tested at Georgia Tech and two of the bridge decks were manufactured and placed in the on ramp at the truck weigh station near Roanoke, Virginia. One bridge deck at Georgia Tech had 14 Extrinsic Fabry-Perot Interferometer strain sensors installed in it and the other bridge deck had 11 EFPI sensors installed in the deck at similar locations. Both bridges had the sensors positioned such that 3-pointbending would be monitored. Both the decks at the Virginia weigh station were instrumented with 12 EFPI sensors to monitor the health of the bridge decks over a period of time. 


\subsubsection{Evaluation and Down-Selection of Sensors and Instrumentation}

\subsection{Sensor Classification and Selection}

Intrinsic optical fiber-based sensors such as the sapphire intrinsic Fabry-Perot interferometric sensors (IFPI) are referred to as sensors with the sensing region inside the fiber where the optical energy is affected directly by disturbances being measured. An indication of the magnitude of the disturbance can be determined by the changes in the output intensity. Extrinsic optical fiber-based sensors are sensors with the sensing region outside the fiber. Here, the fibers only serve to carry optical power to, and sensing information from, an external region, usually air. The extrinsic Fabry-Perot interferometric (EFPI) sensors are usually immune to vibration and temperature fluctuations.

The extrinsic-type optical fiber sensors used in these previous applications are suggested to be more reliable for embedded applications than intrinsic-type optical fiber sensors (Furrow et al. 2000). From the results obtained by Sirkis, the EFPI sensor was the only sensor that was sensitive to only the axial strain components; and unlike the intrinsic sensors, the EFPI sensor was relatively insensitive to temperature variations (Furrow et al. 2000).

Fiber sensors are also classified according to the transduction mechanism, such as intensity, phase, polarization, modal content, etc. Intensity based fiber sensors offer the advantages of ease of fabrication, robustness, and simplicity of signal processing, while the interferometric based sensor offers extremely high levels of sensitivity. This sensor 
usually consists of a reference fiber and a sensing fiber. Since the light source and other external fluctuations affect both the sensing and reference fibers equally, the differential interference offers a self-referencing mechanism; therefore, making it a more sensitive and accurate sensor than the intensity based sensors. However, they often involve a more complex output signal-processing scheme. The interferometer scheme used for these applications was the Fabry-Perot.

\section{Extrinsic Fabry-Perot Interferometer (EFPI)}

The developed and patented EFPI by Luna consists of a lead-in, single-mode fiber and reflector fiber held in alignment by a small diameter capillary tube as shown in Figure 2.5.3.1(a). This basic EFPI sensor measures displacement. Light from a laser enters the sensing head through a fused biconical tapered coupler, which cause two Fresnel reflections to originate at each air/glass interface within the Fabry-Perot cavity. The intensity fringes resulting from the interference are then monitored by a photodetector. The sensor output signal, in normal sensor operation, varies sinusoidally when strain, crack size, or temperature in the material changes the air-gap cavity spacing. These changes cause a phase delay between the two backward-propagating reflections. The sinusoidal pattern of this configuration is shown in Figure 2.5.3.1(b). The periodicity of this sinusoidal pattern was dependent upon the gap length. 

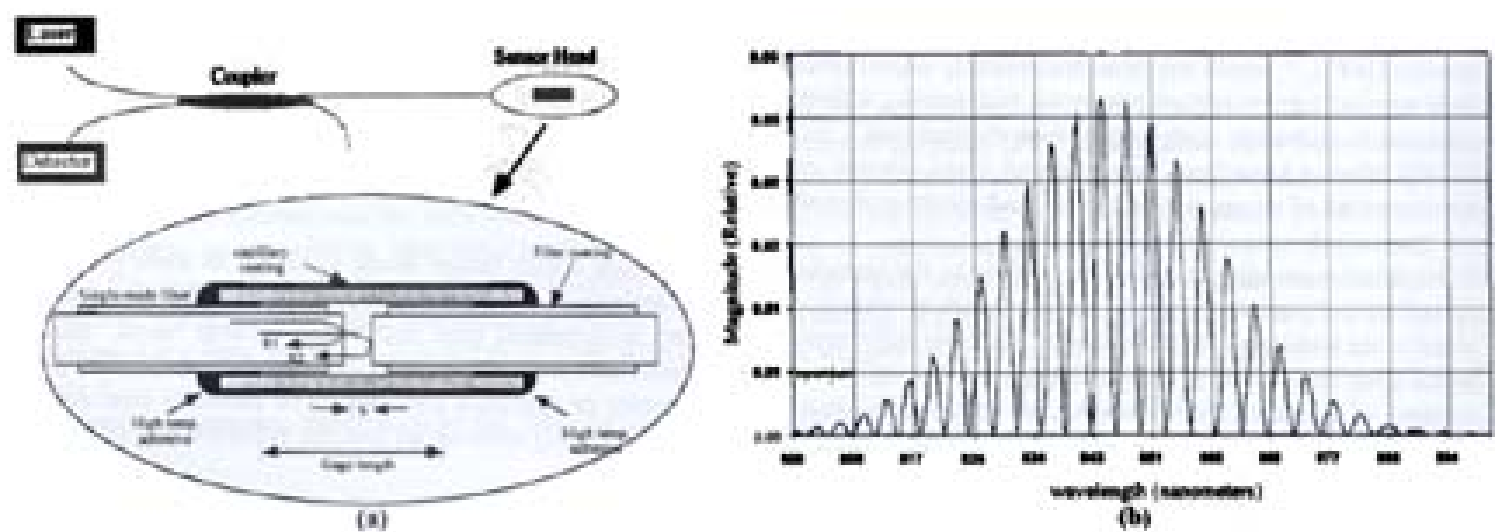

Figure 2.5.3.1 (a) Schematic of the Luna strain, crack opening, and temperature sensor system. (b) Modulated spectral output characteristic of absolute EFPIs (Furrow et al. 2000)

\subsubsection{Laboratory Demonstration of the EFPI Sensor}

Two sensors were attached to the surface of the laboratory bridge decks to demonstrate the capability of the EFPI sensor. One sensor was placed in the axial direction while another was placed in the transverse direction. The test section was then placed in an Instron machine and loaded until failure. The test specimen was loaded to $20,000 \mathrm{~N}$ then to $30,000 \mathrm{~N}$ and finally to $50,000 \mathrm{~N}$ at a rate of $2 \mathrm{~mm} / \mathrm{min}$. The results can be seen in Figure 2.5.3.2(a), 2.5.3.2(b), and 2.5.3.2(c), respectively. As expected, the displacement is greater as the load increases causing more of a fringe during the same time period in the EFPI output. Once fracture occurred, the specimen began to experience much larger displacements as shown by the increase in the number of fringes (Figure 2.5.3.2(c)). 


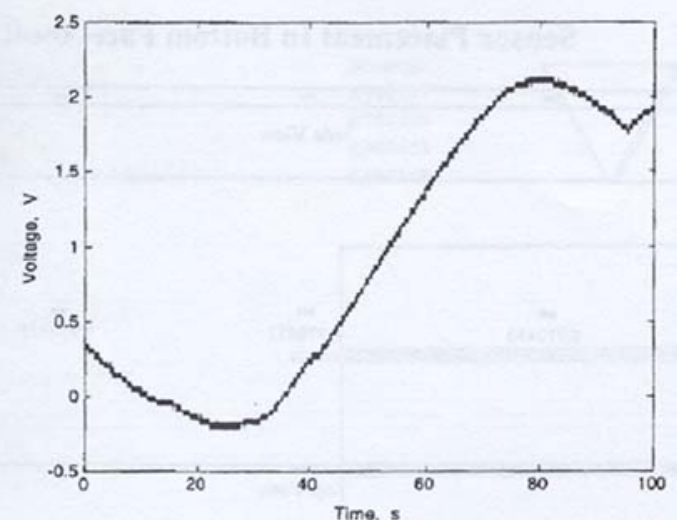

(a)

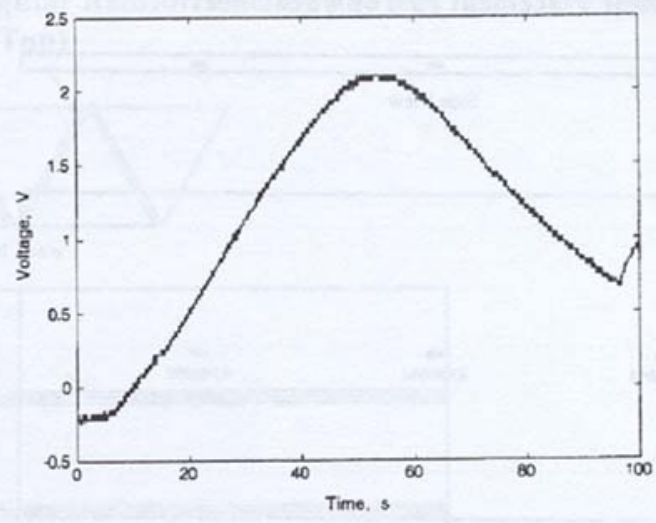

(b)

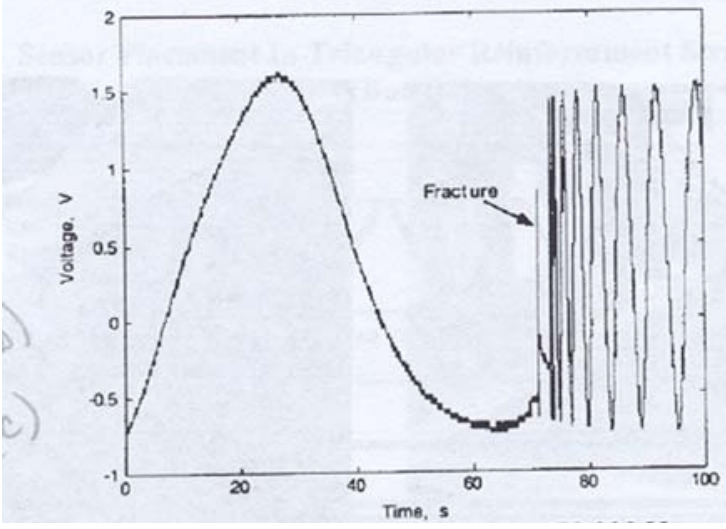

(c)

Figure 2.5.3.2 Sensor response as the specimen was loaded to (a) $20,00 \mathrm{~N}$ at a rate of $2 \mathrm{~mm} / \mathrm{min}$, (b) $30,000 \mathrm{~N}$ at a rate of $2 \mathrm{~mm} / \mathrm{min}$, and (c) $50,000 \mathrm{~N}$ at a rate of $4 \mathrm{~mm} / \mathrm{min}$ (Furrow et al. 2000)

\subsubsection{Sensor Installation and Testing}

\subsection{Sensor Installation and Testing of the First Three-Point-Bend}

\section{Composite Bridge Deck Specimen}

\section{Facesheet Sensor Installation}

Luna installed, in the composite bridge deck 3-point-bend test specimen, three strain sensors in both the top and bottom knitted facesheets and eight strain sensors along the transverse centerline of the support structure consisting of five pultruded triangular sections making a total of 14 sensors. The top facesheet consisting of 13 plies was 
instrumented with three EFPI sensors embedded between the $6^{\text {th }}$ and $7^{\text {th }}$ plies. The bottom facesheet, a thin 4 - ply panel, was also instrumented with three sensors placed between the $2^{\text {nd }}$ and $3^{\text {rd }}$ plies. All sensors were installed along the facesheets longitudinal centerline.

\section{Support Structure Sensor Installation}

Creative Pultrusions in Alum Bank, Pennsylvania placed the eight sensors along the transverse centerline of the structure, four on top of the structure and four on bottom of the structure. The sensors were epoxied on the surface of the support structure with the facesheets being placed over the sensors.

\section{Sensor Checkout}

All of the 14 sensors were connected to a data acquisition system to determine their working condition. Eight of the fourteen sensors produced good signals, five sensors had bad signals, and one sensor was broken. The sensors embedded in the top and bottom facesheets presented the most problems because it was difficult to lay up the facesheets without damaging the sensors.

\section{Structural and Durability Testing}

From the sensors that were not damaged, real-time strain data was taken during structural testing of the composite bridge deck section. The EFPI strain data was then compared to electrical strain gage data, which proved to be consistent with one another. The only difference was that the EFPI data was slightly less than the electrical strain gage 
data because the EFPI's were embedded closer to the neutral axis of the test specimen while the electrical strain gages were attached to the surface.

\subsection{Sensor Installation and Testing of the Second Three-Point-Bend Composite Bridge Deck Test Specimen \\ Facesheet Sensor Installation}

This composite bridge deck had two sensors embedded along the longitudinal centerline in the 13-ply 10 -ft. by 10 -ft. facesheet. USCONEC connectors protected by square brass tubing were used to improve survivability of the facesheet sensors. An identical connector was mated to the connector inside the brass tube and attached to a demodulation system so the EFPI sensors could be monitored.

\section{Support Structure Sensor Installation}

ARC personnel attached eleven EFPI sensors to the triangular support structure.

Once the sensors were epoxied to the surface of the support structure, the facesheets were placed on top of the sensors.

\section{Sensor Checkout}

The eleven sensors on the support structure were then connected to Luna's AFSS data acquisition system to test the sensor's performance where ten of the eleven sensors displayed good signals. The brass tubes of the two-facesheet sensors were crushed during the manufacturing process making it impossible to test the sensors. 


\section{Structural and Durability Testing}

After sensor checkout, eight EFPI sensors were connected to an AFSS

demodulation system, which connected the sensors to the data acquisition system. Only eight of the ten working sensors were used during the test because the AFSS system only has eight inputs. Three-point-bend testing was performed on the bridge deck having a span length of 9'7' and a load applied at the midspan covering an area of 20' x 13 '”. The tests consisted of a load rate of $0.1 \mathrm{~mm} / \mathrm{min}$. The specimen was initially loaded to 80 kips, unloaded, and then loaded to 140 kips and unloaded. The results are illustrated in Figure 2.5.3.3. $800 \mu \varepsilon$ were the maximum tensile strain at the bottom of the deck and $400 \mu \varepsilon$ was the maximum compressive strain at the top of the deck.

\subsubsection{Field Testing}

There were two $10 \mathrm{ft}$. $\mathrm{x} 20 \mathrm{ft}$. composite bridge decks instrumented by Luna for field-testing. Both bridge decks had three EFPI sensors embedded along the longitudinal centerline and nine EFPI sensors attached to the bottom of the triangular support structure for a total of six facesheet sensors and eighteen support structure sensors. Only sixteen out of the eighteen support structure sensors produced signals and all six of the facesheet sensors were unable to produce signals due to the failure of the brass tubes. Once the working sensors were located, the bridge decks were installed into the on ramp of a truck weight station located on Interstate 81 near Roanoke, VA. Strain data was recorded from only eight of the working sensors because of the limited inputs. Data was taken on December 3, 1999 and again on December 9, 1999 for approximately 6 hours each. The data from each channel is shown from Figure 2.5.3.4 to Figure 2.5.3.7 (the maximum 
strain is seen in sensors 2 and 6). The strain spikes were caused from trucks travelling over the bridge. The consistency of the data from week to week indicates that no major structural damage occurred during the tests. The slight drift in the data is suggested to be a result of thermal expansion of the bridge deck due to the increasing temperature throughout the day (Furrow et al. 2000).

\subsubsection{Future Testing}

The field tests were continued by Luna in the following months and the data was reported to ARC in March of 2000. The tests were performed to determine the cause of the sensor drift, whether changing temperatures or actual permanent strain in the bridge deck was the cause.

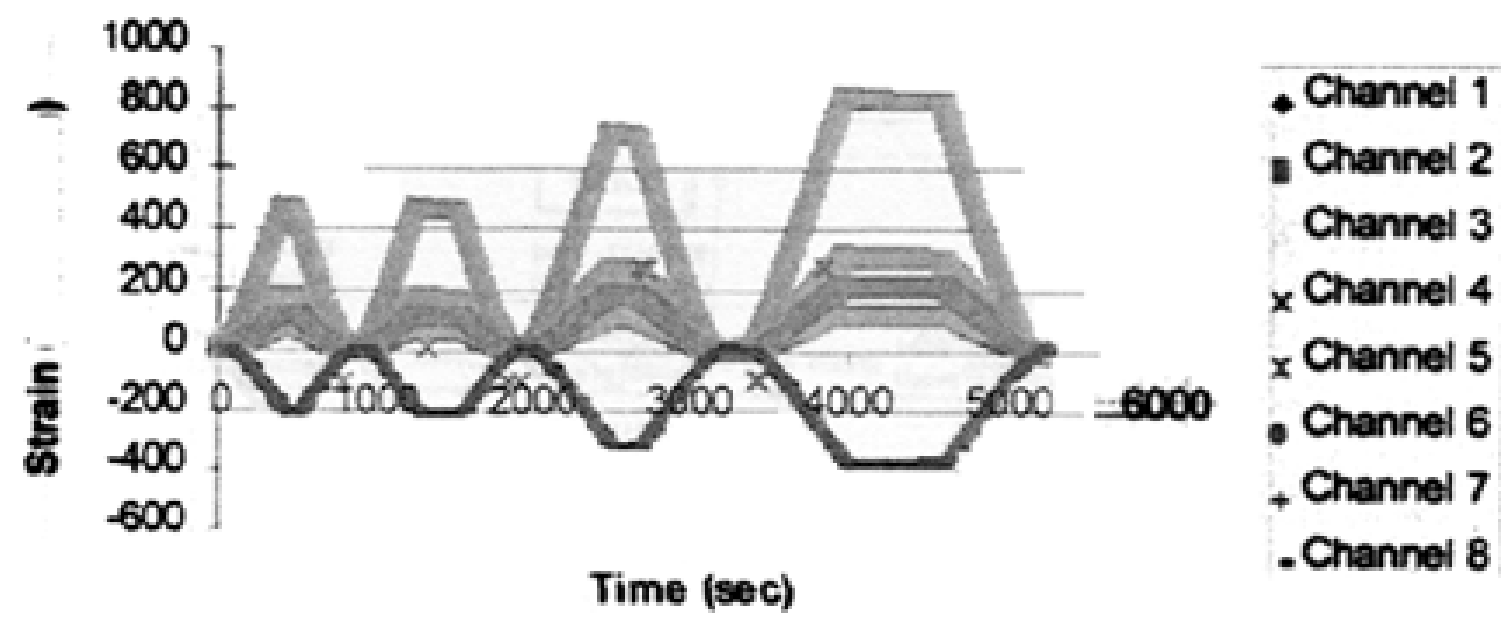

Figure 2.5.3.3 Plot of strain versus time during 3-point -bending of composite bridge deck (Furrow et al. 2000) 


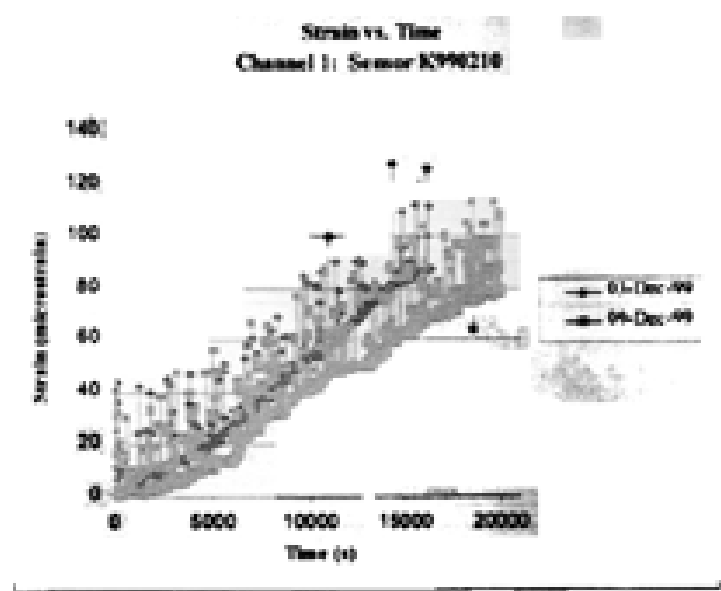

(a)

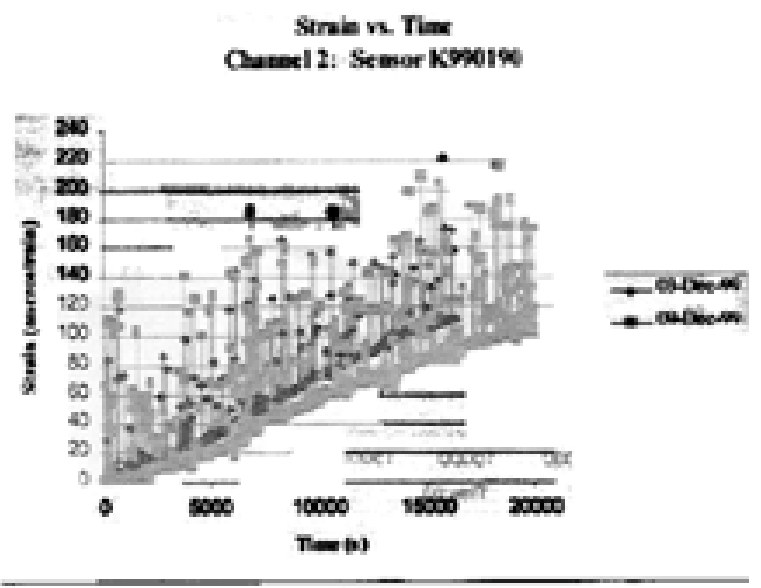

(b)

Figure 2.5.3.4 Stain vs. time data from (a) sensor 1 and (b) sensor 2 (Furrow et al. 2000)

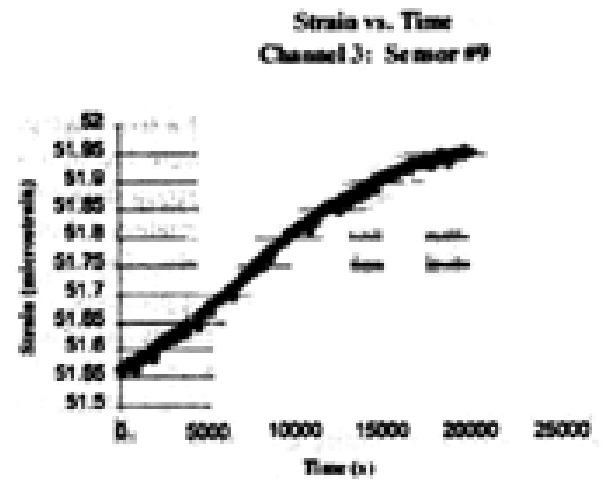

(a)

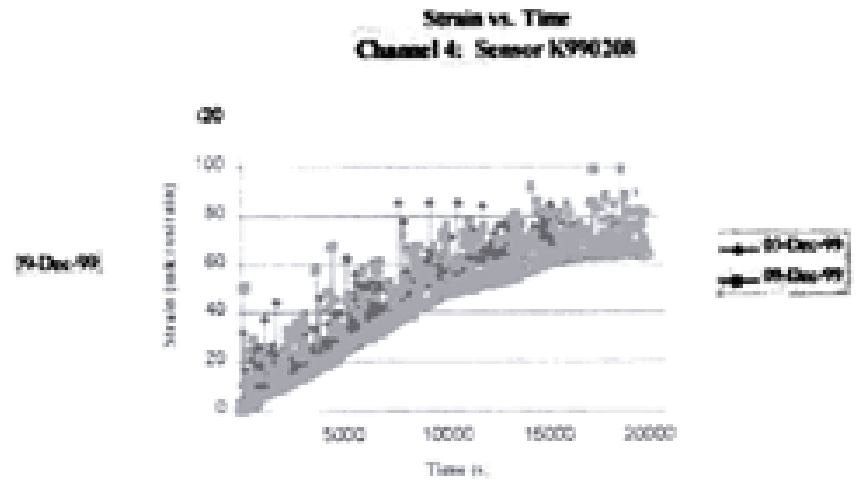

(b)

Figure 2.5.3.5 Stain vs. time data from (a) sensor 9 and (b) sensor 4 (Furrow et al. 2000) 


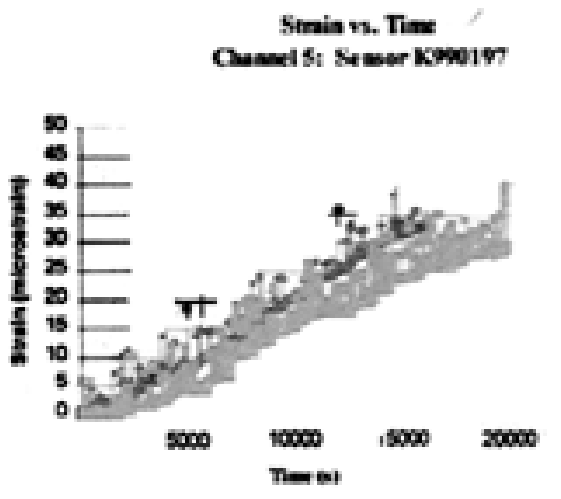

(6)

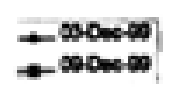

Figure 2.5.3.6 Strain vs. time data from (a) sensor 5 and (b) sensor 6 (Furrow et al. 2000)

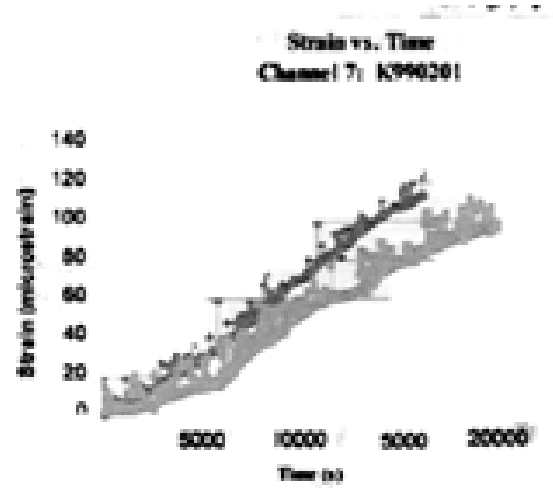

(a)

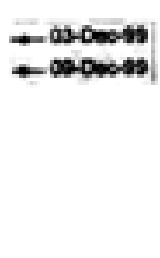

Figure 2.5.3.7 Strain vs. time data from (a) sensor 7 and (b) sensor 8 (Furrow et al. 2000)

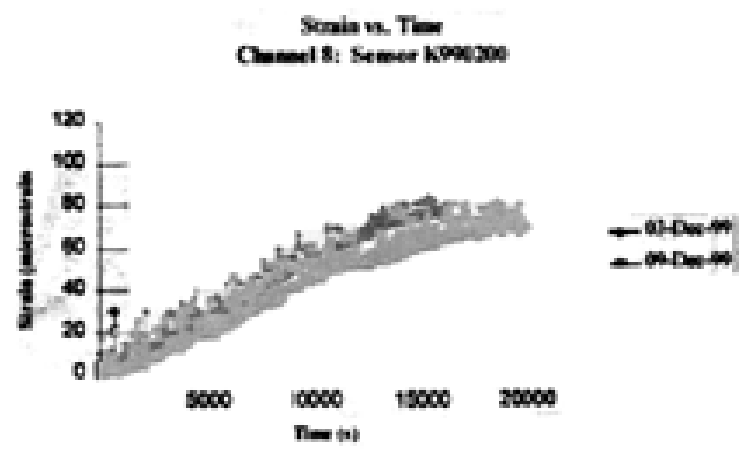

(b)

\subsubsection{Conclusions}

Luna Innovations successfully evaluated and down-selected a sensing and measurement instrumentation system. Luna was able to install fiber optic sensors in four composite bridge decks and demonstrated the performance of the selected sensing system. Finally, data was captured and analyzed during two laboratory three-point bend tests, and a field test performed on a composite bridge deck installed into the on ramp of a truck weight station. 


\subsubsection{Conclusion on Fiber Optics Health Monitoring Systems}

Based on this literature review, it was concluded that the use of fiber optics is a promising NDE technique to evaluate composite structures and materials. The main disadvantage with fiber optic health monitoring systems is that if damaged they are hard to repair and the necessary data is unable to be collected. The fiber optics can be damaged during fabrication and/or transportation to the field site. It was concluded that more research needs to be done with fiber optics in order to develop a more durable system that can withstand fabrication and transportation to the field site. The fiber optic health monitoring system is ideal for evaluating a structure's performance over a period of time but is not well suited for locating and detecting internal defects that might develop.

Other disadvantages include:

- $\quad$ There are a number of cables with connectors, which can cause problems if they are not kept clean (a major disadvantage for field applications).

- $\quad$ The system is almost impossible to repair if it is damaged.

- It can become expensive to install a fiber optic health monitoring system.

Some advantages of installing a fiber optic health monitoring system includes:

- $\quad$ It is ideal for long term (real-time) monitoring of a structure.

- $\quad$ The data is relatively easy to analyze.

- $\quad$ The system can be installed during fabrication of the bridge. 


\subsection{New NDE Techniques}

With the increasing demand for fiber reinforced plastic (FRP composites), newer and faster methods for evaluating composite materials are being developed. This section talks about some of the new NDE methods being experimented with. Although most of these new techniques are being used in the aerospace industry, we may be able to incorporate their ideas with our technology to evaluate civil infrastructures made of composite materials. Most of the new techniques talked about in this section use lasers.

\subsubsection{Characterization of Composite Microstructure and Damage Using Optical Coherence Tomography (Dunkers et al. 1999)}

A new non-destructive and non-contact technique that images microstructures within scattering media is being experimented with. Optical coherence tomography (OCT) was used to evaluate an epoxy/unidirectional E-glass composite and an epoxy/0$90^{\circ}$ woven E-glass composite through tomographic reconstruction. The advantages and limitations of optical coherence tomography (OCT) will be discussed in this section.

\subsubsection{Introduction}

The capability to measure damage non-destructively in structures is of great interest since it can monitor and even control the damage. The ability to perform these evaluations with a single technique is even more advantageous because it would eliminate the complications involved with combining data from different sources. This section talks about a new measurement method (optical coherence tomography) that can 
characterize both microstructure and damage with high resolution and good penetration depth.

This new method (OCT) uses light in a similar way that ultrasound uses sound to image a defect or damage in a material. The same way ultrasound uses sound to penetrate a material and back-scatter, OCT uses light to penetrate a material and backscatter. This method uses a fiber optic interferometer and a broadband laser to produce a light source. The fiber optic interferometer consists of a single-mode optical fiber coupled with a 50/50 fiber optic splitter that illuminates both the sample and a linearly translating reference mirror. The reference mirror reflects light into the 50/50 splitter with light reflected back from the sample to create a temporal interference pattern, which is measured with a photodiode detector. This method is repeated at different transverse positions until a two dimensional array which represents the backscattering of a cross sectional plane of the material is created.

This technique compares very favorable to other NDE techniques used to evaluate composite materials. The main advantage is the greater resolution and penetration depth that can be achieved with OCT. The one draw back that this technique has is that it can not evaluate carbon fiber composites since it is an optical technique. OCT offers an excellent combination of spatial resolution, depth of field, and imaging accuracy (Dunkers et al. 1999). 
This section discusses the potential of OCT for imaging both microstructure and damage of composite materials. The OCT imaging of composite damage was performed using a tomographic reconstruction of impact damage along the thickness of the composite.

\subsubsection{Experimental}

\subsection{Materials}

The epoxy resin system used in the composite consisted of a diglycidyl ether and two amines. The fiber volume fraction was $44 \%$ for the epoxy/unidirectional E-glass composite and $46 \%$ for the epoxy $/ 0-90^{\circ}$ woven E-glass composite. The refractive index of the resin and fibers was 1.552 and 1.554 , respectively.

\subsection{Instrumentation}

Figure 2.6.1.1 is a schematic of the imaging system used to test the two composite materials. A commercial superluminescent light operating at $1.3 \mu \mathrm{m}$ with an output power of up to $15 \mathrm{~mW}$ and a spectral bandwidth of $40 \mathrm{~nm}$ was used to produce the laser light. A single-mode fiber-optic Michelson interferometer was used to diverge the light to the reference mirror and the sample. Once the signal was filtered, it was digitized and stored on a computer. Mapping the logarithm of the signal strength to a gray scale lookup table created the image. The whole imaging process took approximately $1 \mathrm{~min}$. The laser beam was focused to about a $30 \mu \mathrm{m}$ diameter spot to a depth of about $1000 \mu \mathrm{m}$. 


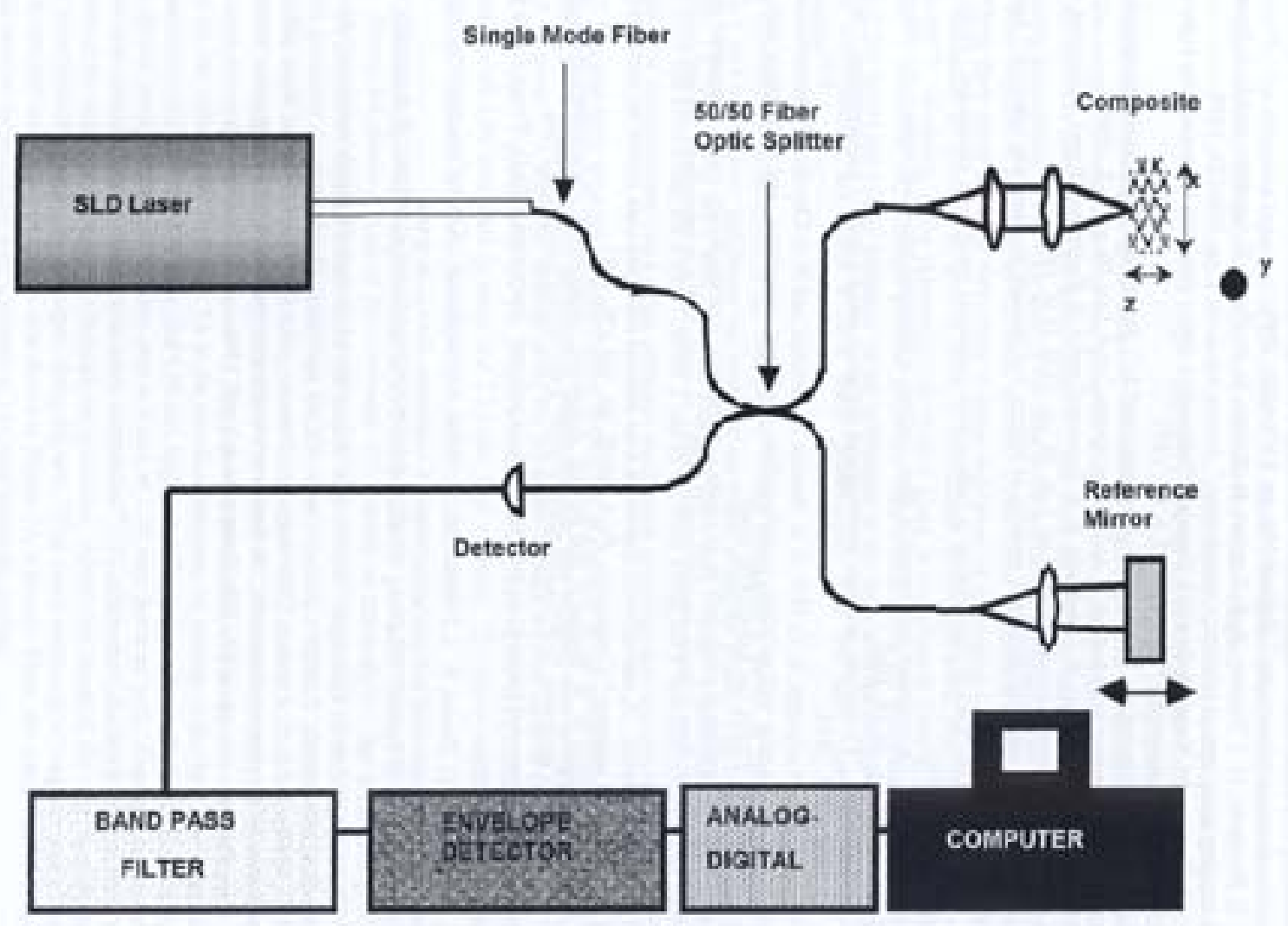

Figure 2.6.1.1 Schematic representation of the solid state laser and OCT system layout (Dunkers et al. 1999)

\subsubsection{Results and Discussion}

Figure 2.6.1.2 shows the OCT image of the epoxy/unidirectional E-glass composite. The two black arrows are pointing out the polyester stitching that holds a single layer of tows together. The small dark areas are the voids that were detected. These voids could have been caused by air pockets entrapped during the molding process because of insufficient driving pressure, high resin viscosity or low reinforcement permeability (Dunkers et al. 1999). The dark areas were verified to be voids in the fiber tows by slicing the sample along the $\mathrm{x}-\mathrm{y}$ plane. The OCT image was also able to provide information about the permeability of the reinforcement by indicating if the fibers had a nested configuration or a stacked configuration. The permeability of the reinforcement would be about $50 \%$ lower if the configuration was nested. 


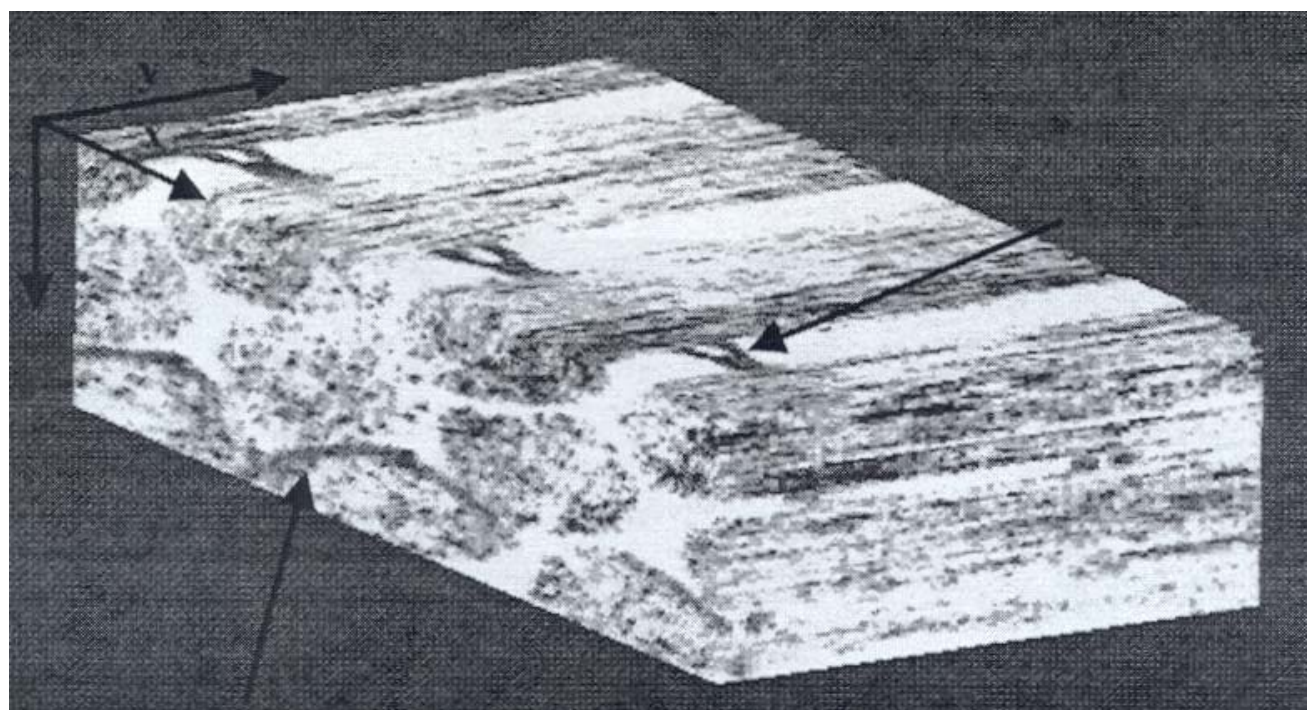

Figure 2.6.1.2 OCT volumetric reconstruction of an epoxy/unidirectional E-glass composite (Dunkers et al. 1999)

The OCT image of the epoxy $/ 0-90^{\circ}$ woven composite is illustrated in Figure

2.6.1.3. The arrow towards the top of the figure indicates the tows along the y-axis and the arrow in the middle of the figure indicates the tows along the $\mathrm{x}$-axis that are crossing over the tows along the y-axis.

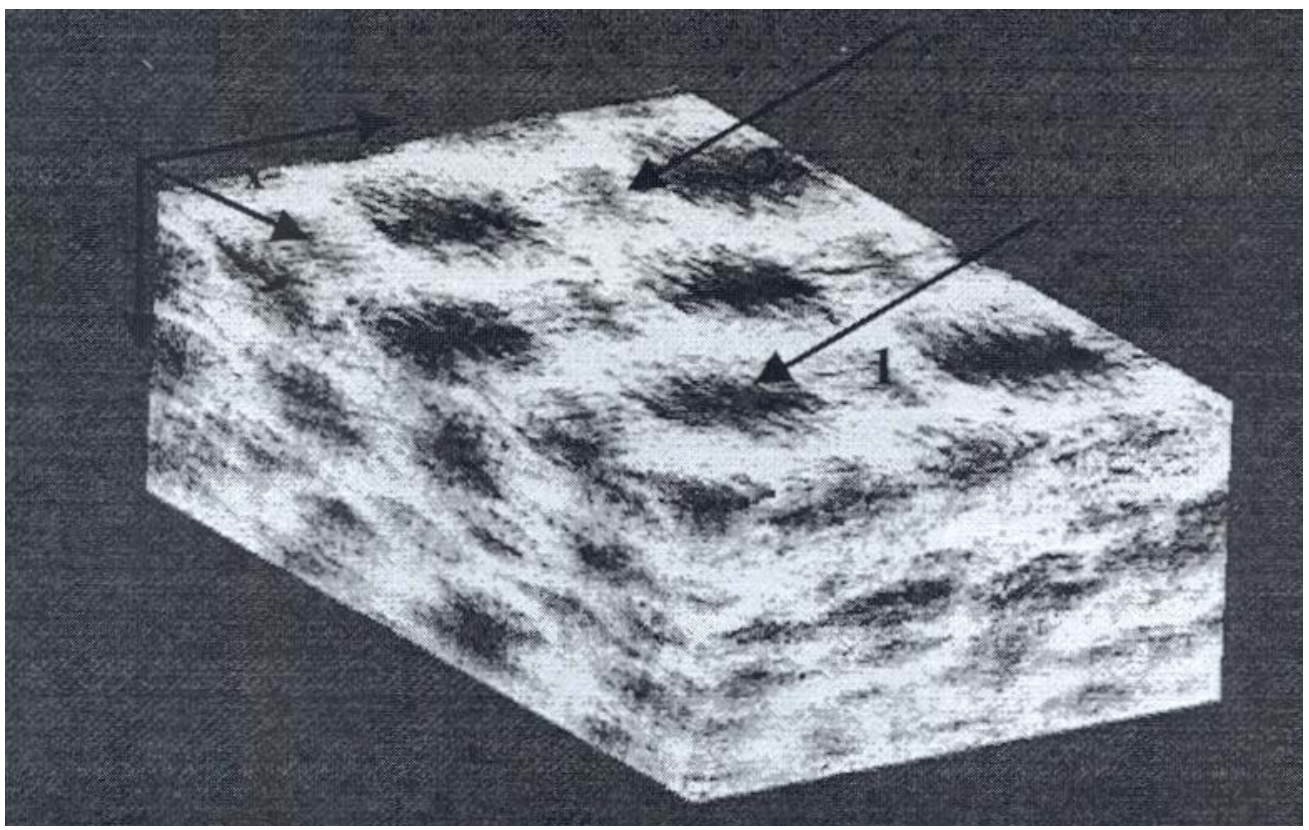

Figure 2.6.1.3 OCT volumetric reconstruction of an epoxy $/ 0-90^{\circ}$ woven composite (Dunkers et al. 1999) 
The OCT technique was also used to create images of composite samples

subjected to impact damage. Figure 2.6.1.4 shows the damage along the $x-y$ plane at the surface of the composite. Figure 2.6.1.5 shows the damage along the x-y plane $337 \mu \mathrm{m}$ below the surface. Arrows one and two in Figure 2.6.1.5 point to cracks propagating through the fiber tow and arrow three indicates the polyester crossing threads that hold the top layer together. Figure 2.6.1.6 shows a delamination in the $x-y$ plane at a depth of $652 \mu \mathrm{m}$ from the surface indicated by the arrow.

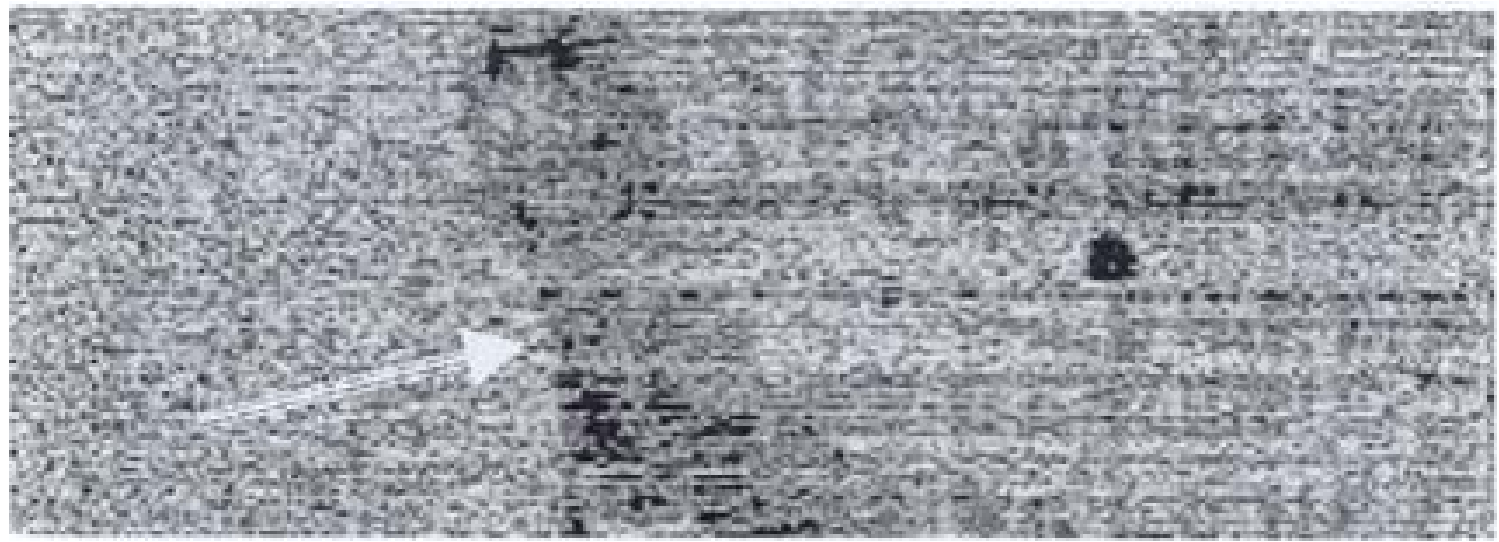

Figure 2.6.1.4 OCT image of impact damaged epoxy/unidirectional E-glass composite at the surface (Dunkers et al. 1999)

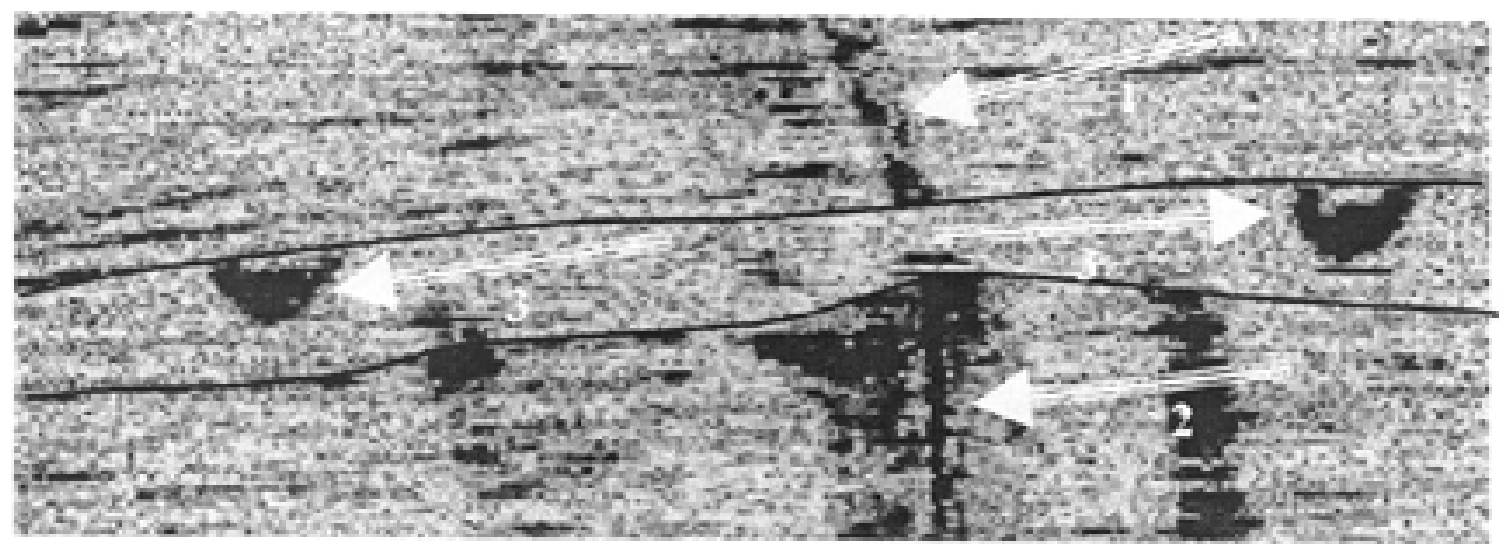

Figure 2.6.1.5 OCT image of impact damaged epoxy/unidirectional E-glass composite $337 \mu \mathrm{m}$ from surface (Dunkers et al. 1999) 


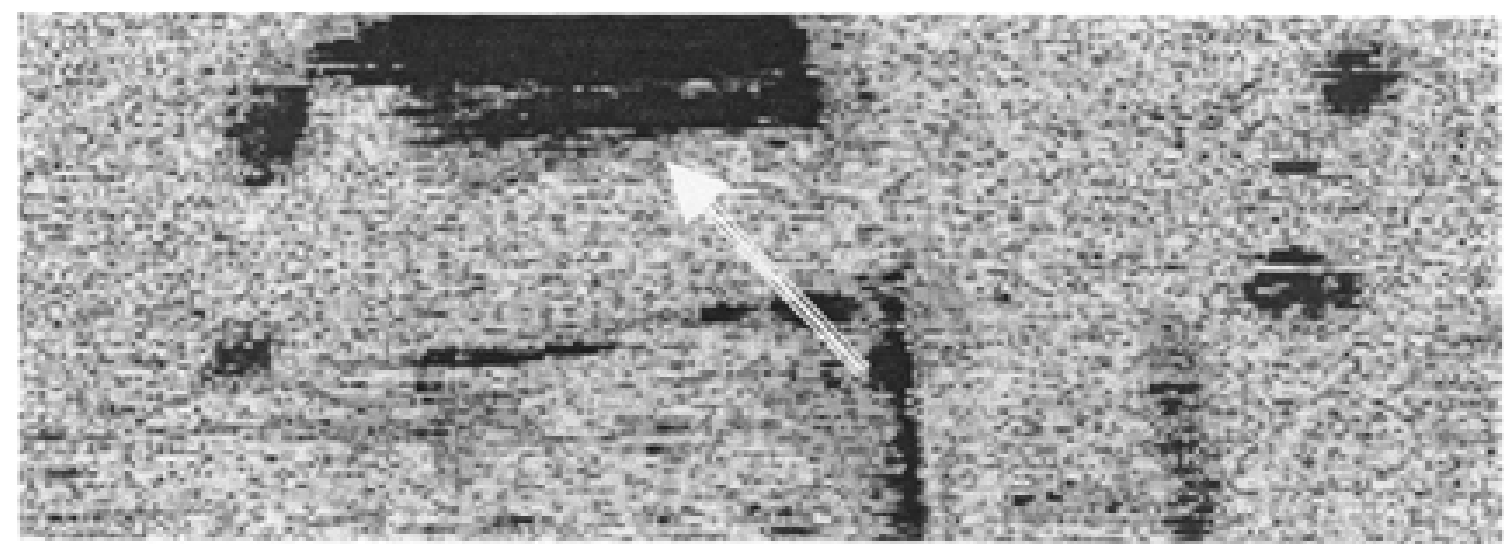

Figure 2.6.1.6 OCT image of impact damaged epoxy/unidirectional E-glass composite $652 \mu \mathrm{m}$ from surface (Dunkers et al. 1999)

\subsubsection{Non-contact Laser Based Computation Method of NDE of Composites and other Structures (Thevar et al. 1999)}

Due to the recent demand for composite materials in the civil engineering field, more and more NDE techniques are being experimented with. This section talks about a new laser based remote NDE method named RAID. This section discusses its ability to detect defects in solid laminates and honeycomb/carbon composites, and also its ability to detect bonding defects and cracking in ceramic and carbon foam materials.

\subsubsection{Introduction}

A technique using a Remote Acoustic Impact Doppler (RAID) for NDE has been experimented with. The results of using this technique to evaluate composite materials is discussed in section 2.6.2. This technique analyses the surface relaxation frequencies generated by acoustic impact excitation with a customized laser Doppler scanning system. The main advantage of this system is that it can be remotely activated making it a non-contact NDE method. 
It has proven to be a very effective method in evaluating different types of composite structures.

\subsubsection{Description of the RAID NDE Technique}

\subsection{System Description}

The RAID technique uses an acoustic transducer to produce an air coupled shock or pressure wave that could be remotely activated from a distance up to four meters away making it totally non-contacting. A schematic of the transducer is illustrated in Figure 2.6.2.1. The shock wave excites the natural relaxation frequencies of the object being tested. This natural relaxation frequency was of interest because it is dependent upon the underlying substructure for any given material (Thevar et al. 1999). One advantage of the RAID technique was that it could inspect a large area of the structure.

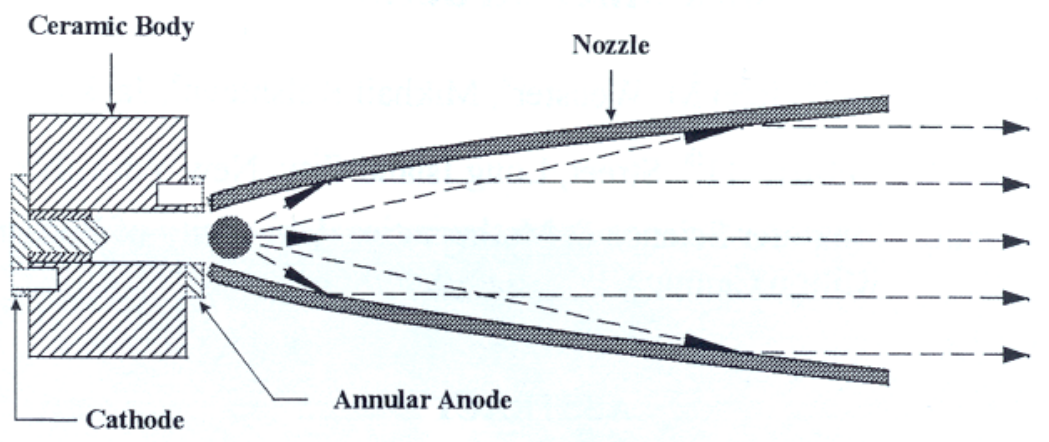

Figure 2.6.2.1 Schematic diagram of the acoustic transducer without shielding (Thevar et al. 1999)

Once the transducer was remotely activated the area was scanned using a customized scanning laser Doppler velocimeter. A He-Ne laser was used to scan the surface, which could collect up to $512 \times 512$ data acquisition points. The analog time 
domain velocity signal received from each data point was then converted into frequency domain by Fast Fourier Transform (FFT) and then stored into a computer as individual frequency bands. An image was then computed and presented on a monitor. Figure

\subsubsection{2 shows a schematic of this system.}

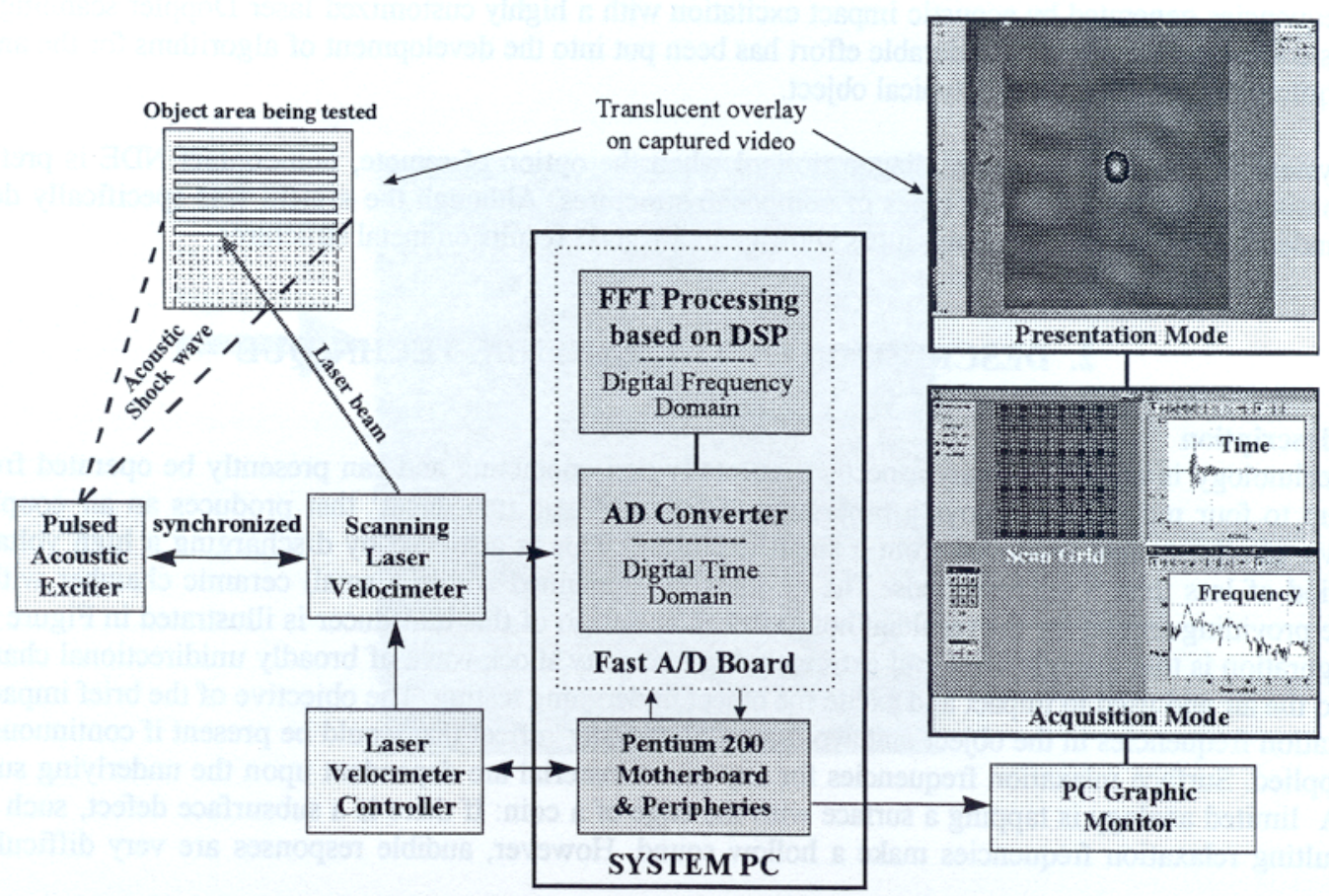

Figure 2.6.2.2 Schematic diagram of the remote acoustic Doppler NDT system (Thevar et al. 1999)

\subsection{Methodology of Defect Detection}

Once the number of scan points was set (up to the maximum 512x512) a vibrometer measured each data points time domain velocity signal. Each signal was then converted to frequency domain and stored in the computer as individual frequency bands over the pre-selected frequency range (up to $200 \mathrm{kHz}$ ). The number of frequency bands or FFT lines to be displayed was then programmed into the system ( 800 lines proved to be sufficient for most cases). An object scanned with 800 lines over a frequency range of 0 to $50 \mathrm{kHz}$ is represented in Figure 2.6.2.3. The different magnitudes show information 
generated by background noise, bending modes, resonant oscillations, and defects (Thevar et al. 1999).

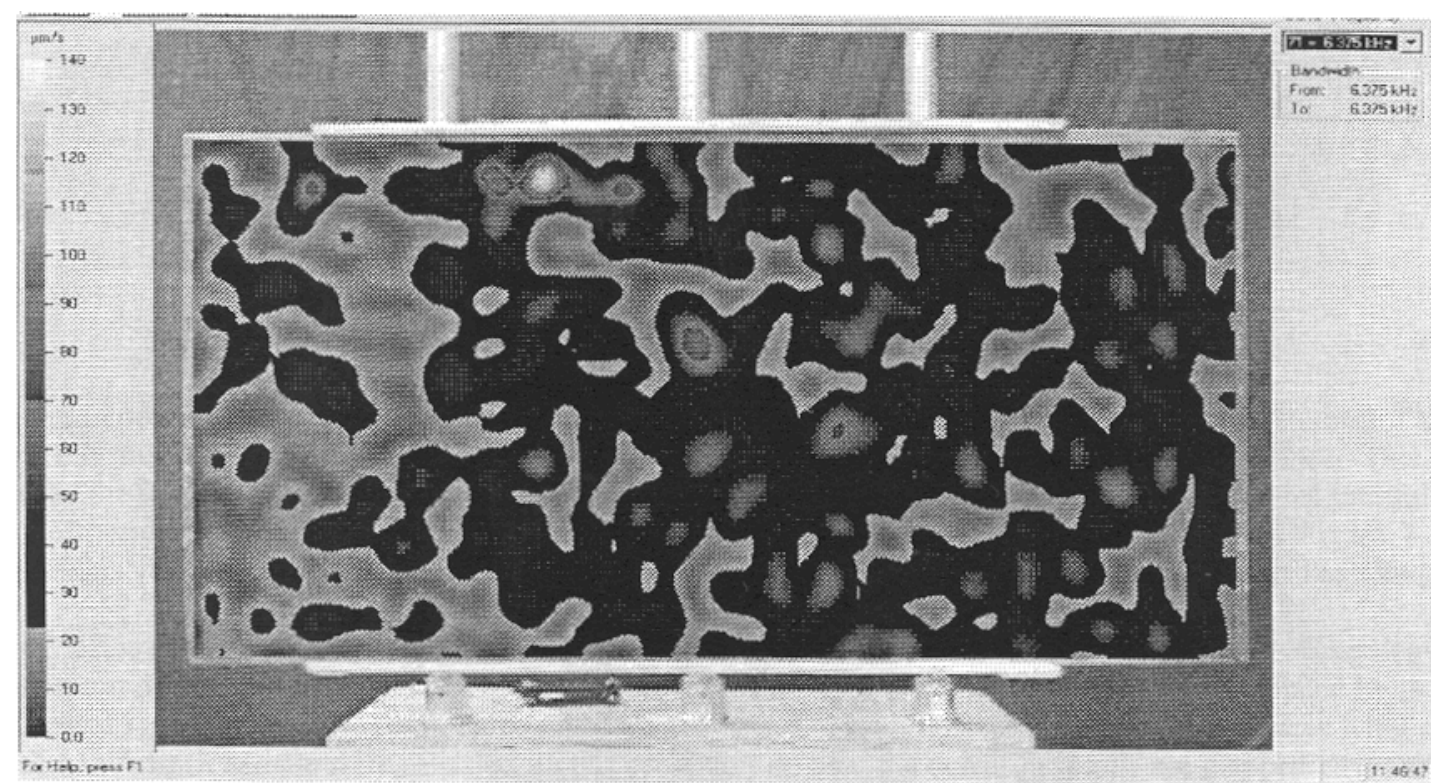

Figure 2.6.2.3 Velocity color map at a single frequency line (Thevar et al. 1999)

Defects generally have signals with a magnitude higher than the surrounding and are found to exist in a number of consecutive frequency bands; noise signals generally have random magnitudes and do not reproduce themselves in as many adjacent frequency bands; bending mode signals generally occur at low frequencies (Thevar et al. 1999). The bending mode signals are avoided by ignoring data below certain frequencies (typically $2 \mathrm{kHz}$ ).

Defects are determined using a software program to identify a cluster of data points with very close magnitudes at a particular frequency. Once a cluster of data points are identified in an individual FFT line, the program will combine FFT lines vertically linking clusters to determine the size and depth of the defect (Figure 2.6.2.4). 


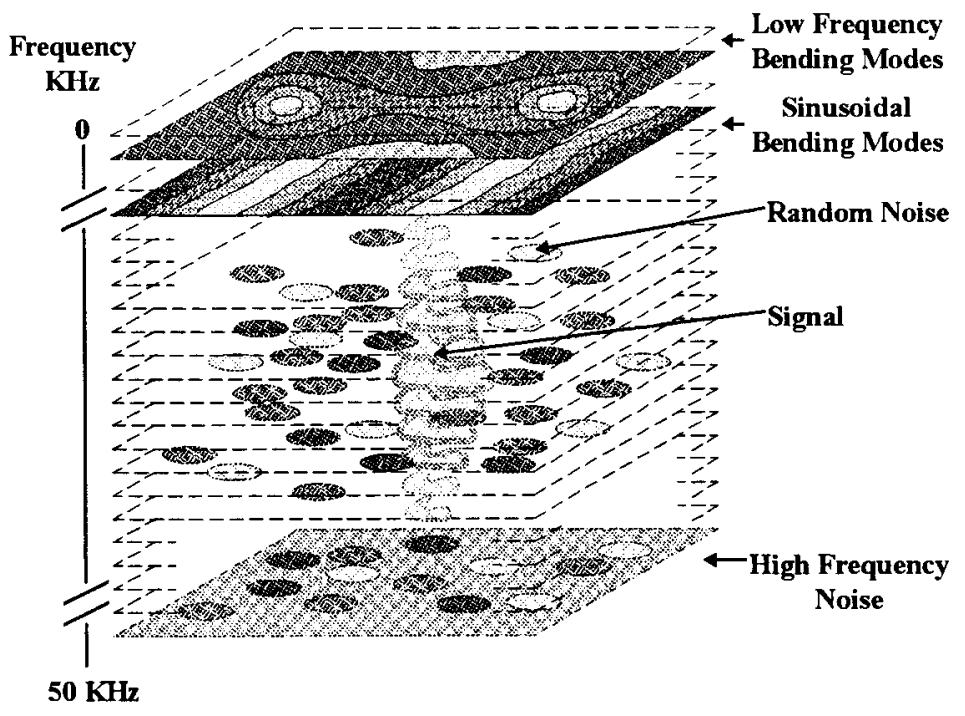

Figure 2.6.2.4 Three-dimensional representation of the analysis concept (Thevar et al. 1999)

\subsubsection{Application of RAID Technology to Composites}

The composite materials used for this project were mainly composites from aircraft panels. However, defects were successfully detected at a depth up to fourteen layers below the surface.

The results from two different test samples are presented in section 2.6.2. The test samples consisted of a carbon composite honeycomb scarf repair panel with defects imitated by the inclusion of shims, and a carbon foam composite with fractures beneath the surface. Figure 2.6.2.5 shows one of the images obtained t the fault regions are shown as isolines overlaid on the image of the object. Figure 2.6.2.6 illustrates a velocity color map of the carbon foam specimen. The bright yellow region indicates the defect. 


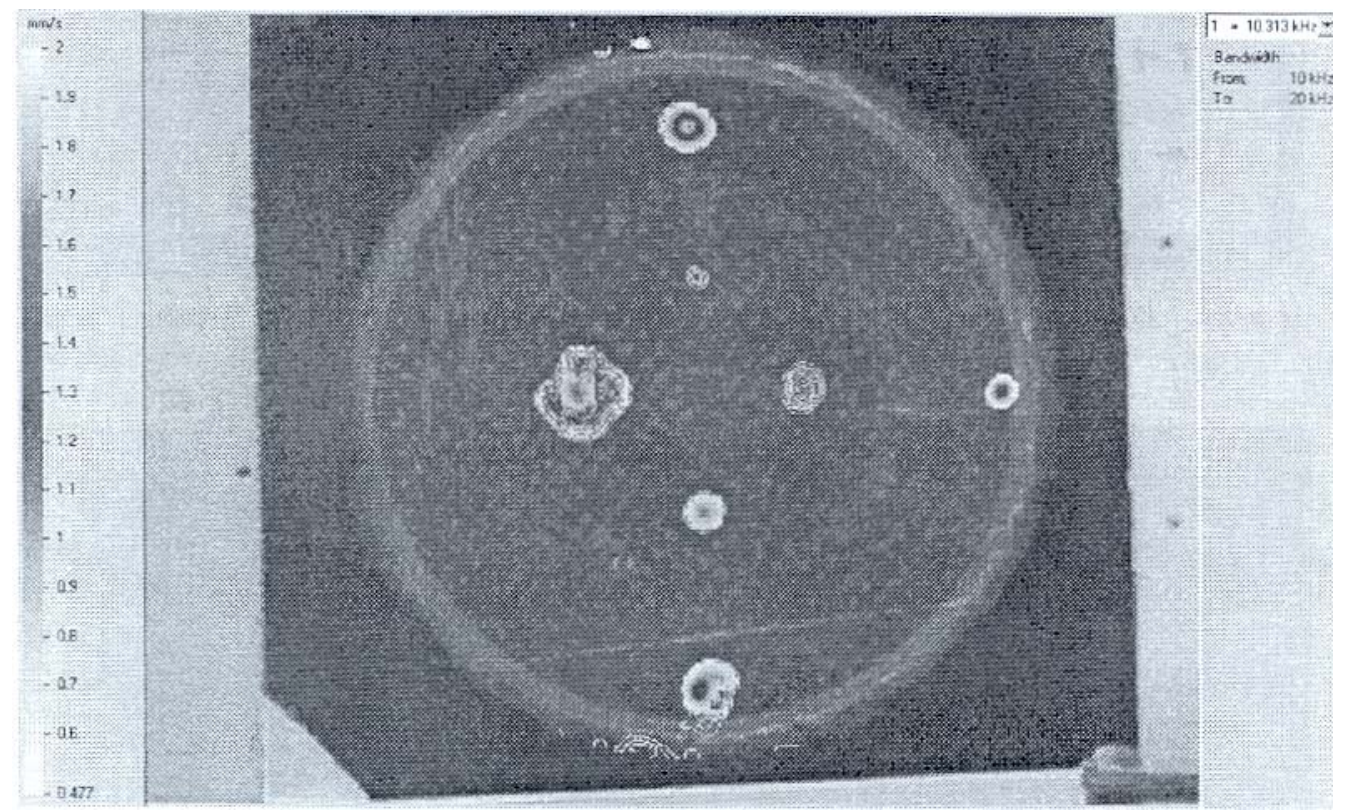

Figure 2.6.2.5 Seven defects in the parametric repair panel revealed (Thevar et al. 1999)

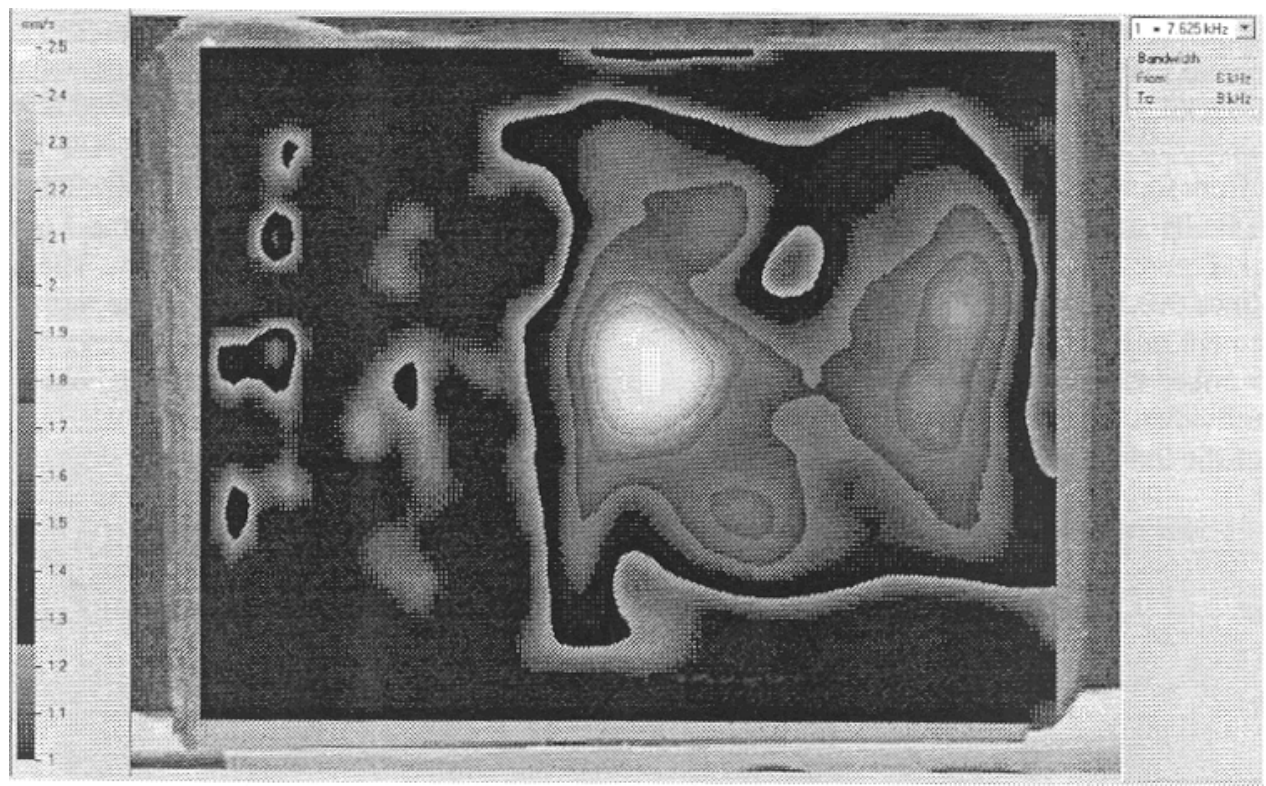

Figure 2.6.2.6 Detection of defect in carbon foam composite (Thevar et al. 1999)

\subsubsection{Conclusions}

It was successfully concluded that the RAID system is a non-destructive, noncontact method that can detect defects in various composite materials. Future investigations into developing prototype software programs could help improve and speed up the RAID technique. 


\subsubsection{FOPSESPI for Non-Destructive Evaluation (NDE) of Composites}

\section{(Murukeshan et al. 1999)}

Fiber optics is now being used for the non-destructive testing of composite laminates. A new system know as fiber optic phase shifting Electronic Speckle Pattern Interferometer (FOPSESPI) has been experimented with for the NDE of composites. The technique of this new system and the results of the tests are discussed in section 2.6.3. The FOPSESPI technique was used with GFRP (glass fiber reinforced plastic) to determine delaminations and fiber breakage by analyzing the fringe patterns created by phase shifting.

\subsubsection{Introduction}

Speckle interferometry allows electronic detection with phase shifting for obtaining measurements of surface deformation, displacements, slope etc. (Murukeshan et al. 1999). These defects are detected in the fringe pattern. Recently, fiber optics has made this technique more versatile.

There are basically two types of defects in composite materials that decrease the strength and stiffness of the structure e.g. delamination and fiber breakage (Murukeshan et al. 1999). These defects can occur during fabrication or during service loading. This section talks about a FOPSESPI system used for the non-destructive evaluation of GFRP laminates. The GFRP laminates tested using this technique are mainly used in the aerospace industry. However, this technique could eventually be tested on composite materials used in the civil engineering industry. 


\subsubsection{Fiber Optic Phase Shifting ESPI System}

Figure 2.6.3.1 shows the schematic of the optical head in the ESPI system developed. A single mode laser diode of $40 \mathrm{~mW}$ output power with an emission wavelength of $832 \mathrm{~nm}$ was used for the coherent source. A standard single mode glass fiber was used to couple the laser beam and a $2 / 2$ fiber coupler was used to split the coupled laser beam into two output fibers, which are connected to the input ends of a two phase modulator. Wrapping a few turns of standard single mode fibers around a PZT cylinder made the phase modulators. This induces a change in the phase of the guided light wave because the fibber length or the refractive index of the guided wave inside the fiber is changed by a voltage to the PZT cylinders. An image was then captured by a CCD camera. A digital image was processed and stored into a personal computer using a processing card.

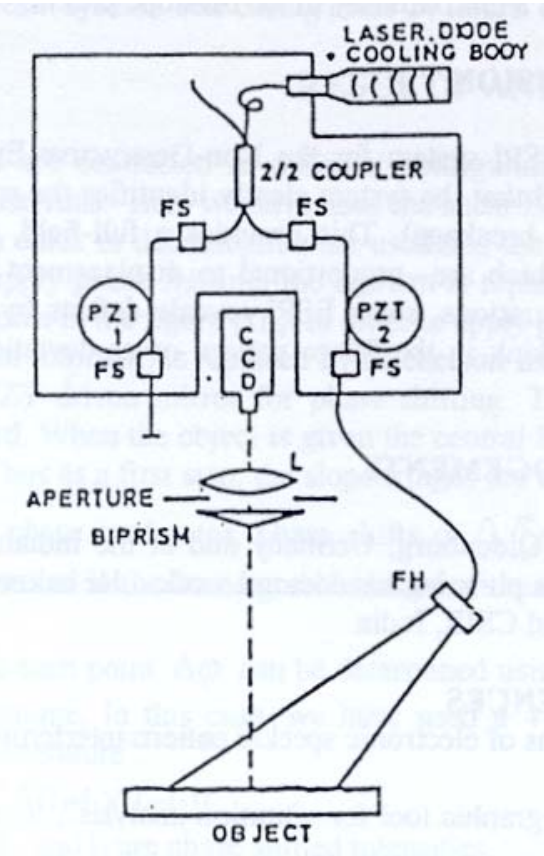

FS $=$ Finger splices/ Fiber connector $\mathrm{CCD}=$ Charge Coupled Device $\mathrm{FH}=$ Fiber holder

PZT 1 \& PZT 2 = Phase modulators

Figure 2.6.3.1 Schematic of the optical head for the developed ESPI system (Murukeshan et al. 1999) 
The complete system allows electronic detection and phase shifting, which is highly sensitive to surface and subsurface defects. The defects are seen as irregularities in the fringe pattern. The use of fiber optics makes this a more versatile technique, which is compact, lightweight and capable of exploring optically in accessible areas (Murukeshan et al. 1999).

\subsubsection{ESPI - Principle}

When an object has a defect or deformation, a phase difference is introduced causing a phase change in the object wave. A CCD camera captures the signal image and displays it on a TV monitor. However, the voltage signals from the CCD camera have to be subtracted from the captured image and the signal is rectified before displayed on the monitor. The low frequency noise was removed from the difference signal using a high pass filter to enhance the fringe contrast. The evaluation of the object deformation can be done quantitatively with the phase measuring approach.

\subsubsection{Specimen Preparation}

The FOPSESPI technique was used on several GFRP specimens prepared with unidirectional glass fiber mat and epoxy resin. Some of the specimens had a thin Teflon film placed between the layers of glass fiber mats during the lamination to represent defects. A total of four glass fiber mat layers were used to make the laminate. 


\subsubsection{Results and Discussions}

Specimens with either a crack or delamination were tested using the FOPSESPI configuration and compared to the results of defect free specimens. Phase shifting was accomplished by replacing the biprism with a Michelson interferometer and a PZT driven mirror illustrated in Figure 2.6.3.2. First the object, before deformation, was stored in the frame grabber board of the associated image processing card. Once the object was loaded, the correlation fringes and their slopes were recorded in real-time on the TV monitor. The PZT driven phase modulator creates four phase shifts $(0, \pi / 2, \pi, 3 \pi / 2)$ and the phase shifted fringe patterns were then stored in the frame grabber board.

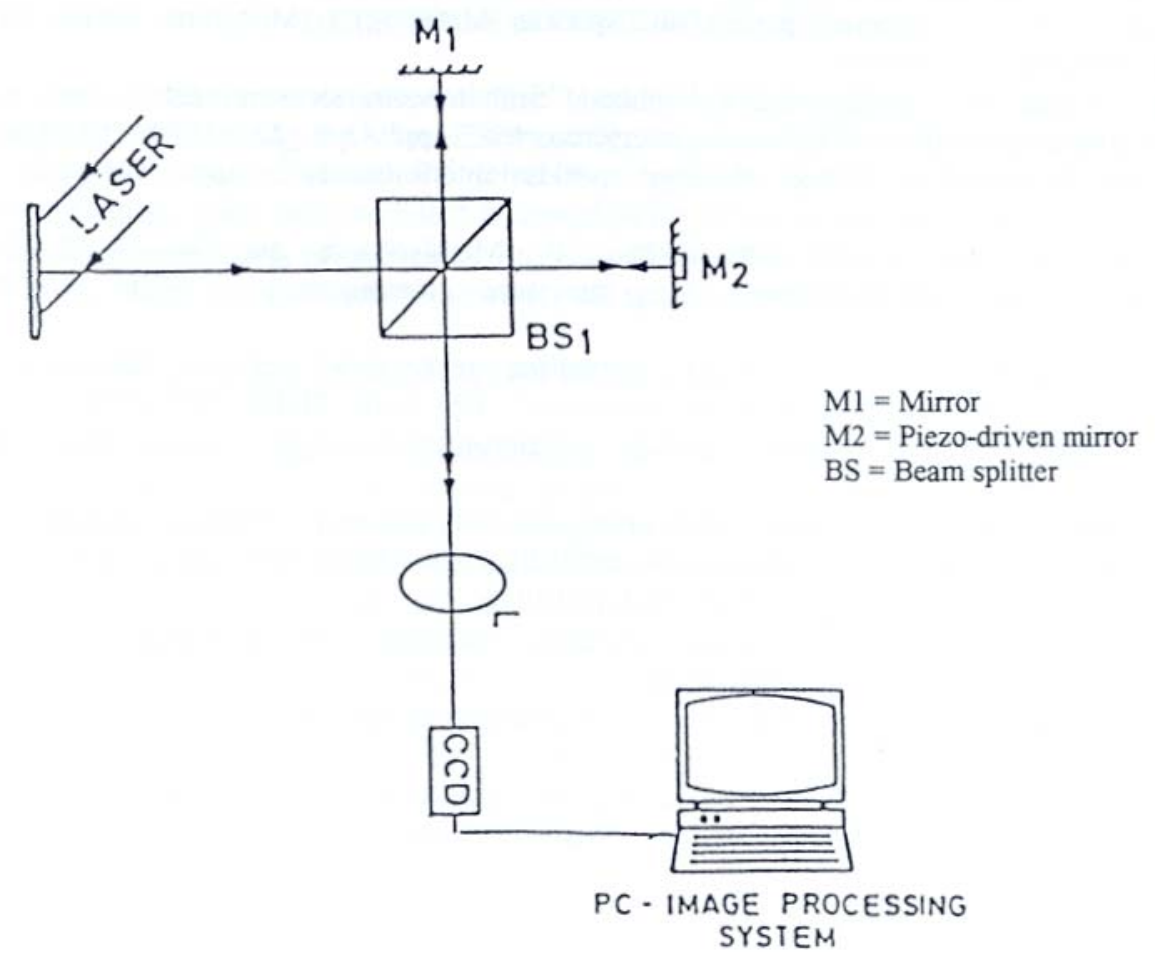

Figure 2.6.3.2 Schematic of the developed ESPI System (Murukeshan et al. 1999)

A 4-step phase shifting algorithm was used with the phase shifting technique to determine the phase at each point. 


\subsubsection{Detection of Fiber Breakage}

The system was used to detect fiber breakage inside the GFRP specimens. The images that were captured are illustrated in Figure 2.6.3.3. The change in fringe shape at the defect site (where fiber breakage was programmed) indicates that the FOPSESPI configuration could detect fiber breakage.

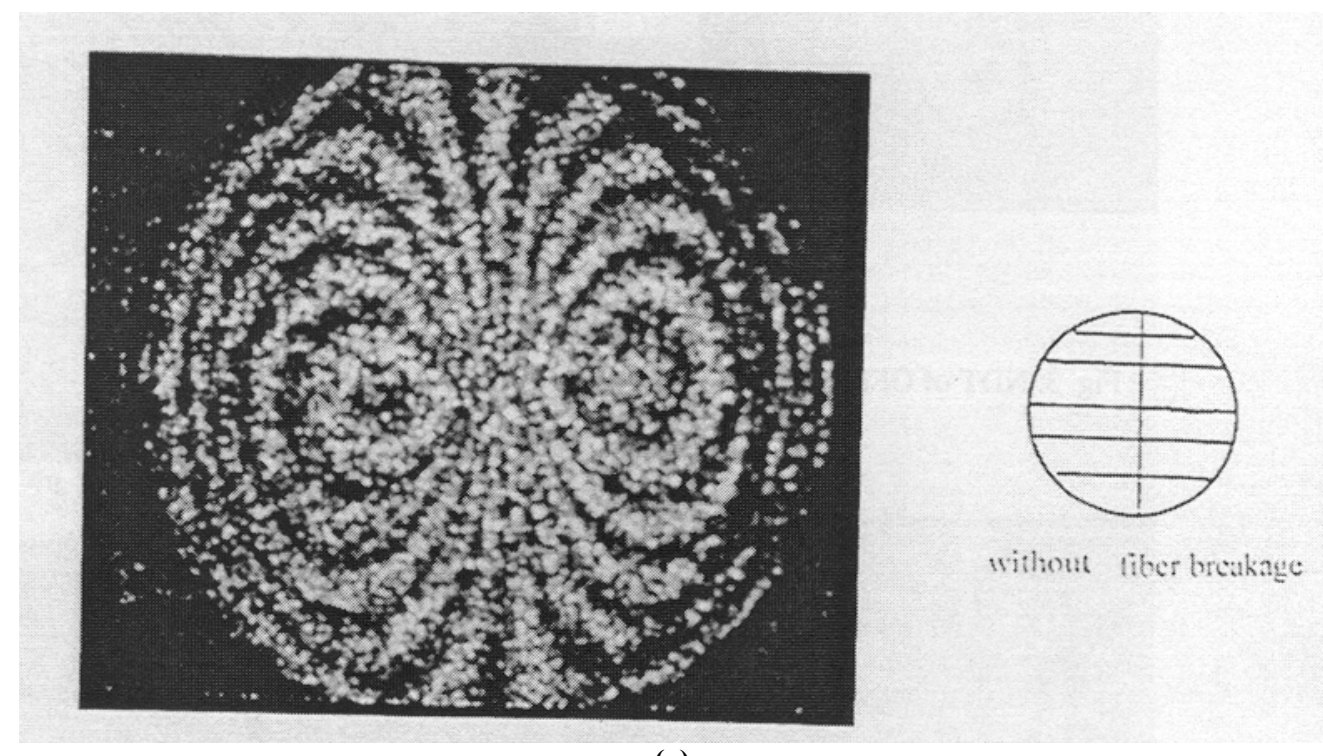

(a)

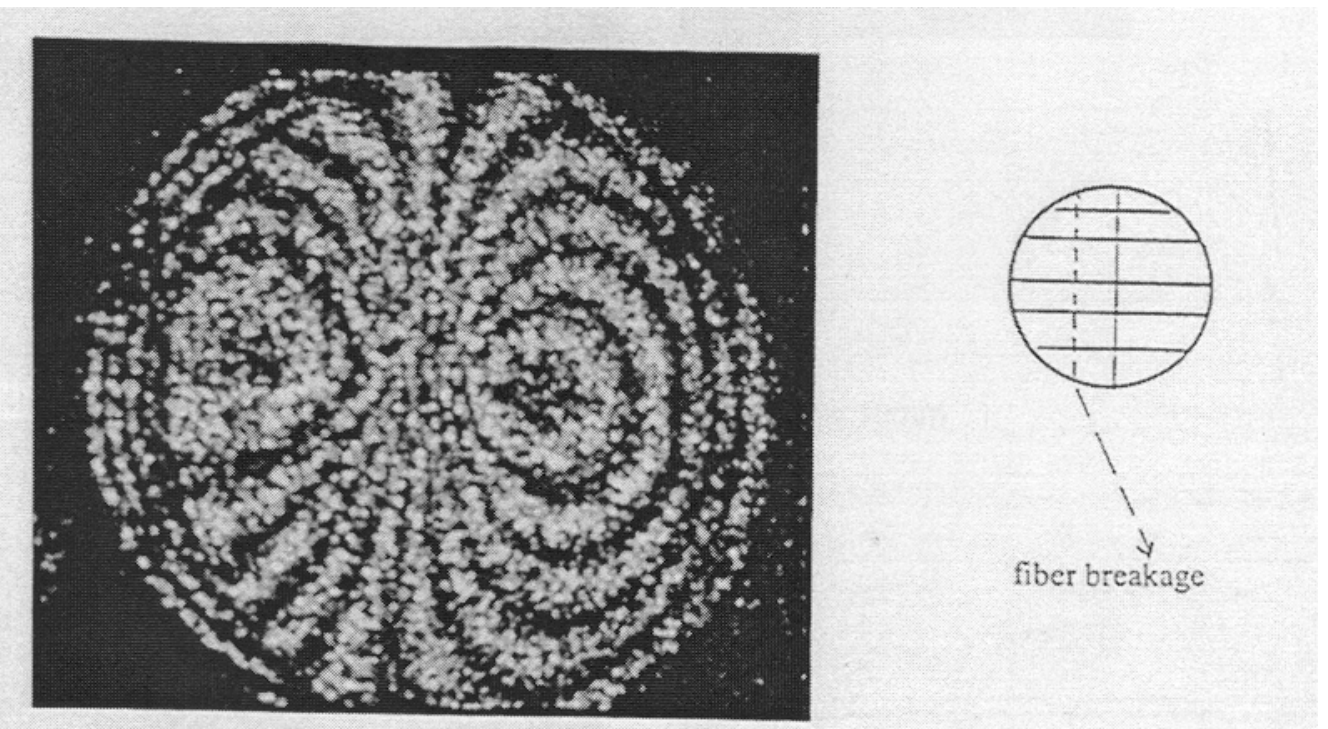

(b)

Figure 2.6.3.3 (a) Shear (slope) fringes for a defect free diaphragm, and (b) shear (slope) fringes obtained for the same specimen with fiber breakage (Murukeshan et al. 1999) 


\subsubsection{NDT of GFRP Specimens with Programmed Delamination}

The FOPSESPI system was also experimented with to locate delaminations in GFRP materials. Placing 5, 10, and $15 \mathrm{~mm}$ diameter Teflon film between the third and fourth layers of the glass fiber mats during lamination represented the delaminations. The specimens were then loaded at the center. Figure 2.6.3.4 shows the fringe patterns obtained from these defects. It was concluded that the size of the defects affects the fringe quality dramatically. Figure 2.6.3.5 shows the different slope distributions obtained when the phase map was scanned through the defected areas. It was clearly visible that the slope distribution was disturbed by the defects. It was also concluded that the FOPSESPI configuration was able to detect delaminations in the laminates.

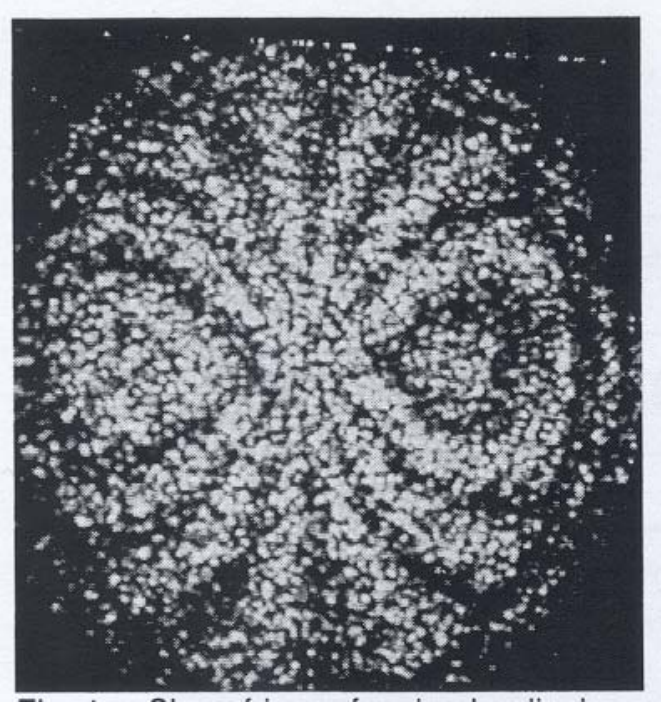

(a) 


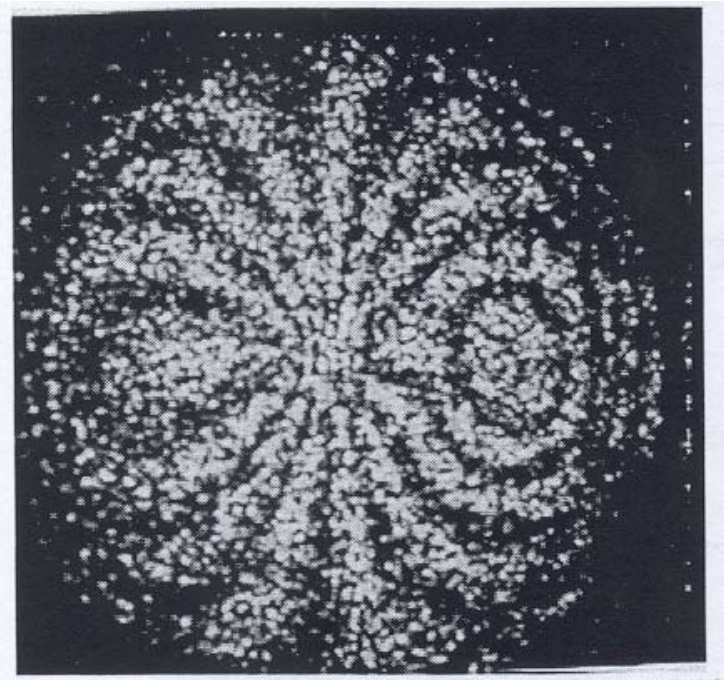

(b)

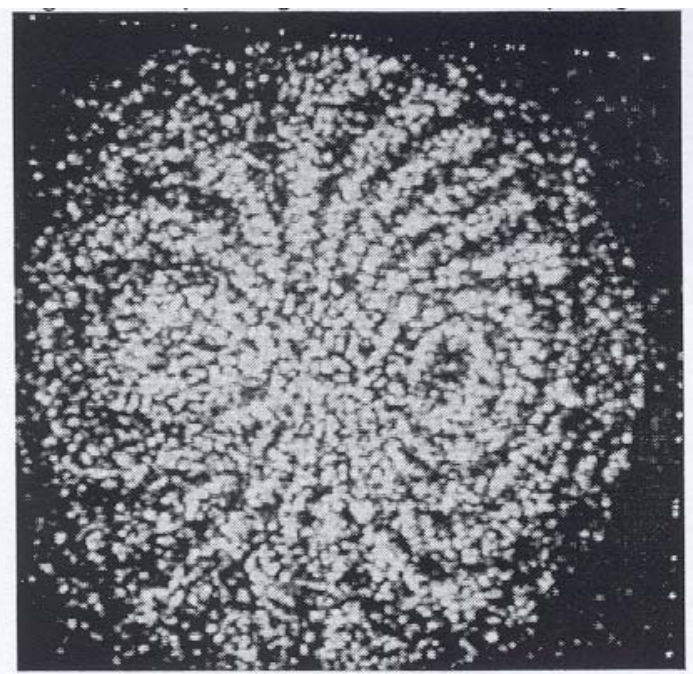

(c)

Figure 2.6.3.4 Slope fringes for circular diaphragm with defect size (a) $5 \mathrm{~mm}$, (b) $10 \mathrm{~mm}$, and (c) $15 \mathrm{~mm}$ (Murukeshan et al. 1999) 


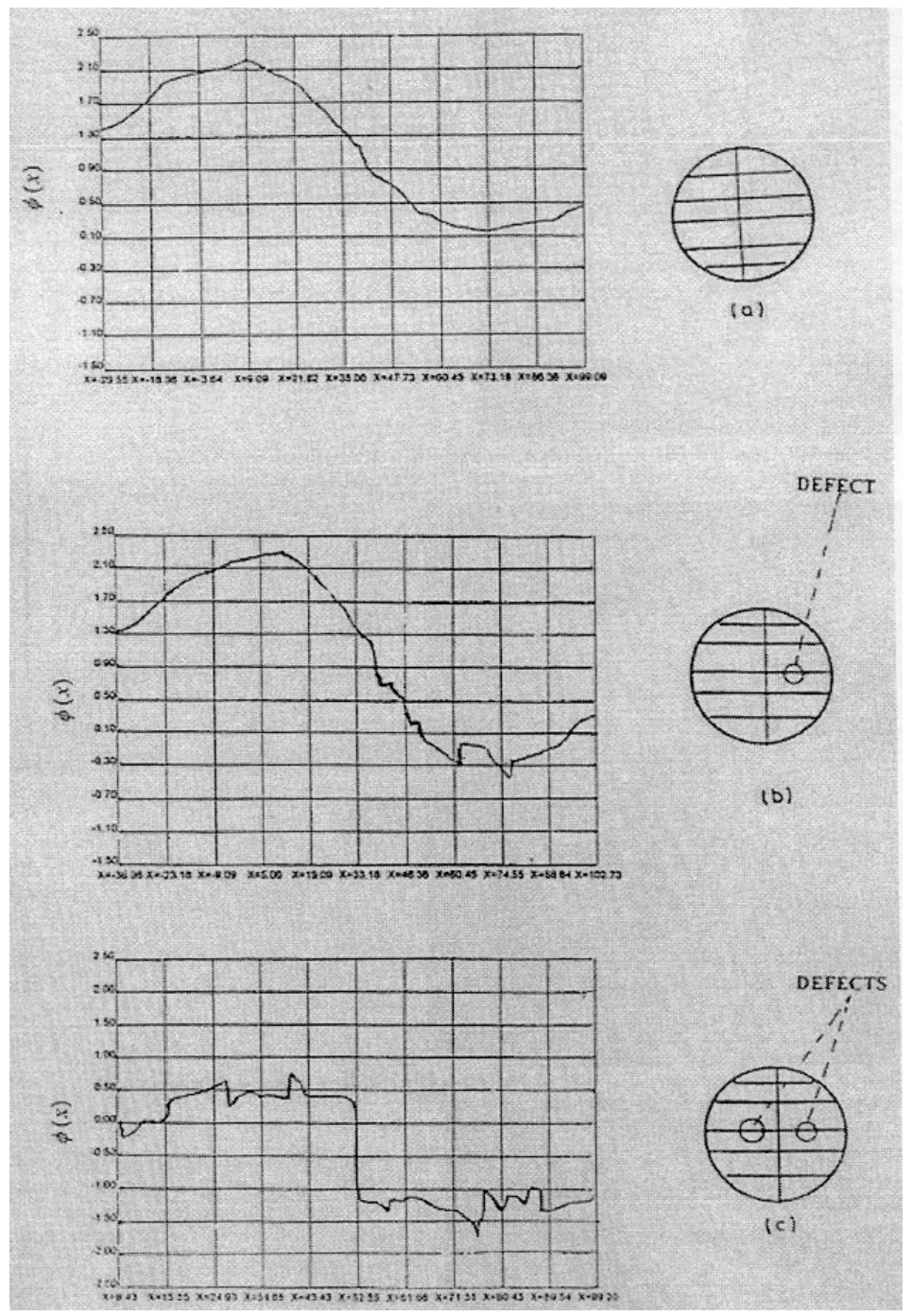

Figure 2.6.3.5 Slope profiles of defective GFRP circular diaphragm (Murukeshan et al. 1999)

\subsubsection{Conclusion}

It was concluded that the FOPSESPI system was a truly non-destructive test that clearly identified subsurface and surface defects in laminate composites. The defects were revealed using the shear ESPI configuration by identifying anomalies in strain concentrations. 


\subsubsection{Cost Effective Advances in Portable Radioscopic NDT of Composite Materials (Hall 1998)}

Recently advances in Radioscopic Testing have occurred due to the availability of the ASTM radioscopic specifications and new equipment designs. Improvements in image quality and cost reductions are a direct result from these advances. These new advances allow for future reference and failure analysis.

\subsubsection{Advances in Radioscopic Imaging}

Radioscopic imaging of composite materials has had several major advances over the last ten years (Hall 1998). These advances have increased the performance and versatility of the systems, and have reduced the cost of radioscopic imaging. X-ray systems are designed to be more efficient with improved beam quality and reduction in generation of signal noise.

A new technology using amorphous silicon flat panel sensors and displays has developed a portable radioscopic system. This system is not only very versatile but offers an improved image contrast and resolution. The technology also has the

advantages of being digital, and opening the door for image transmission via LAN (local area network) for applications requiring remote viewing and analysis.

\subsubsection{NDE / NDT Training}

Training personnel for radioscopic NDT is becoming a requirement due to the complex nature of technique. Radioscopic NDT must be specifically dealt with in the 
Written Practice and tested according to those guidelines in compliance with the specific ASTM specifications.

\subsubsection{Composite Radioscopic Technique}

Radioscopic NDT of composites presents unique considerations due to the materials complexity. Radiation can be very dangerous during radioscopic NDT so the appropriate safety requires should be followed during testing (e.g. lead apron, etc.).

\subsubsection{Radioscopic Applications}

There is a wide range of composite portable radioscopic NDT applications to determine present strength. A main advantage of these portable systems is the instantaneous feedback on the material. A thermal printer, $8 \mathrm{~mm}$ recorder, or VHS can store the images from radioscopic NDT. Digital image storage is more expensive; however, image storage and file management becomes very simple if the entire system is digital computer based. The more features a system has (e.g. electronic magnification, edge enhancement, frame averaging, etc.) the higher the cost.

\subsubsection{Cost Consideration}

The portable radioscopic system increases the application potential without the drawbacks of a permanent radioscopic inspection system. The instant feedback allows for production process correction without production delays. 
Radioscopic testing can detect the exact point where an error occurs in a timely manner, which will confirm the quality of the structure and reduce the cost of the inspection.

\subsubsection{Conclusion}

The cost effectiveness of portable radioscopic NDT is due to the flexibility of instant feedback without the drawbacks of a fixed site location. The ability to retrieve information from the NDT radioscopic images can be very valuable for engineering analysis of field failures. There will be significant improvements in the field of portable radioscopic NDT in the near future that will have great advantages in expanding the applications (Hall 1998).

\subsubsection{Nondestructive Characterization of Layered Composite Materials with a Laser Optoacoustic Sensor (Karabutov et al. 1998)}

A new technique that employs laser pulses for generating wide-band acoustic transients in the ultrasonic frequency range of $100 \mathrm{kHz}$ to $10 \mathrm{MHz}$ is discussed in section 2.6.5. The laser pulses were produced from a laser optoacoustic sensor (LOAS) and are totally nondestructive. This form of ultrasonic testing can be performed in either transmission mode (LOAS-T) or in reflection mode (LOAS-R).

This section discusses the test results of LOAS on layered graphite-epoxy composite materials. The specimens had different volume percentages of micropores 
ranging from 0 to $1.2 \%$ and were tested in the frequency range of 1 to $5 \mathrm{MHz}$. Section 2.6.5 discusses the possibility of using the LOAS technique as a diagnostic system for the quantitative characterization of composite materials.

\subsubsection{Introduction}

Nondestructive evaluation of composite materials has become a major issue since defects may reduce mechanical strengths of composite materials. An ideal NDE method would be capable of determining the quality of the composite structure during manufacturing, but also during service (Karabutov et al. 1998).

The most common defects in composite structures that need to be evaluated are fiber breaks, microcracks, microsplits and pores in the bounding medium, and detachment of fibers from the bounding material. These defects can be detected using ultrasound because changes in the acoustic attenuation and the speed of sound in composite materials are affected by these defects (Karabutov et al. 1998).

Ultrasonic evaluation is very sensitive and may be a difficult NDE method to use with an acoustically heterogeneous material such as composite materials. Therefore, a laser optoacoustic system used to produce powerful wide-band ultrasonic pulses was experimented with to evaluate mechanical properties of graphite-epoxy composites.

The laser optoacoustic system produces a short laser pulse with a given intensity that excites the surface of the material under investigation. The excitation of the surface 
results in the nonstationary heating of subsurface layers causing thermoelastic expansion. This expansion yields acoustic pressure waves propagating into the material as ultrasonic pulses (USP). These ultrasonic pulses are measured to determine the speed of sound and the acoustic attenuation coefficient. Changes in the material mechanical properties will yield transformations in the measured speed of sound and acoustic attenuation coefficient. The detection of acoustic waves is called forward signal propagation mode when the acoustic transducer is located on the opposite side of the surface being excited and backward signal propagation mode when the acoustic transducer is located on the same side as the surface being excited (Figure 2.6.5.1).
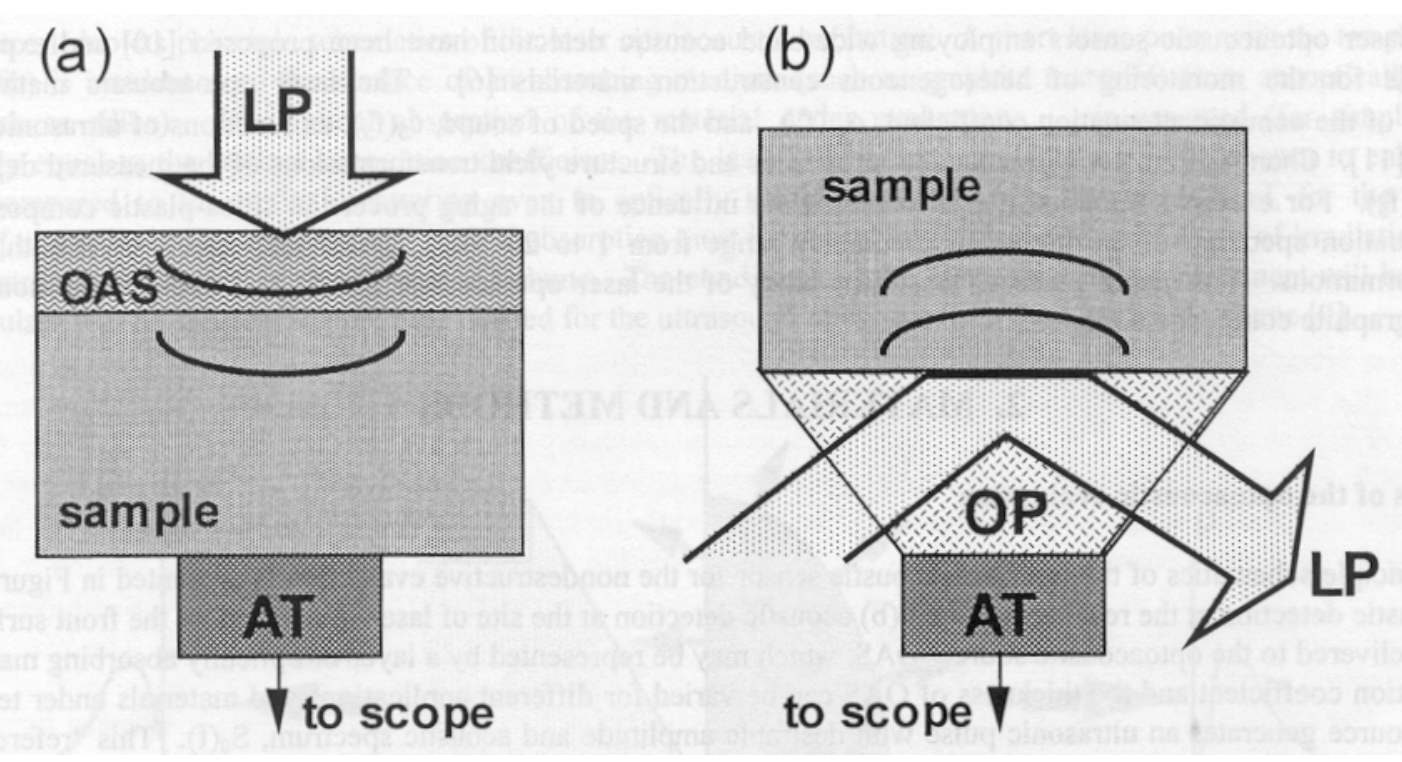

Figure 2.6.5.1 Principle schematics of the laser optoacoustic sensors for detection in (a) "forward" signal propagation mode (LOAS-F), and (b) "backward" signal propagation mode (LOAS-B) (Karabutov et al. 1998) 


\subsubsection{Materials and Methods}

\subsection{Principles of the Optoacoustic Evaluation}

The laser optoacoustic system used a layer of optically absorbing material for coupling represented as OAS in Figure 2.6.5.1. This layer received the laser pulses from the system to excite the surface of the material being tested. The pulse then propagates into the sample and was detected using a wide-band piezotransducer. The acoustic attenuation coefficient was then determined using the acoustic spectrum of the reference pulse (initial pulse before propagating through sample) and the acoustic spectrum of the transmitted pulse (pulse after propagating through sample). Once the acoustic attenuation coefficient was determined the acoustic attenuation was measured as a function of ultrasonic frequency.

When the laser causes thermoelastic excitation, ultrasonic pulses propagate in two directions. The pulses propagate into the sample and back to the transducer. The pulse that propagates towards the acoustic transducer serves as a reference signal. The pulses that propagate into the material are scattered by defects and other material properties (layers, fibers, etc.), that are detected by the acoustic transducer after a certain delay relative to the reference ultrasonic pulse.

The measured arrival time for the back-scattered ultrasonic pulses was used to determine the location of defects in the composite sample. The back-scattered ultrasonic frequency spectrum also gave information about the sample heterogeneity as a whole. 


\subsection{Components of the Optoacoustic Sensor}

Section 2.6.5 describes a laser optoacoustic sensing system operating in both the forward and backward detection modes. The evaluation of epoxy-graphite composite samples was performed with the laser optoacoustic sensor system in the frequency range of $0.1 \mathrm{MHz}$ to $10 \mathrm{MHz}$. A Q-switched ND:YAG laser was used to produce laser pulses with wavelength of $1064 \mathrm{~nm}$ and a pulse duration of $12 \mathrm{~ns}$. The transducers used to detect the ultrasonic pulses were wide-band lithium niobate acoustic transducers. Once the transducer detected the signals, the data was acquired with a 12 bit A/D electronic card and processed with a personal computer.

\subsection{Epoxy-Graphite Samples}

There were three different epoxy-graphite composite samples used with the laser optoacoustic sensing system. The table below (Table 2.6.5.1) gives the three sample's mechanical and acoustic properties.

Table 2.6.5.1 Parameters of the evaluated samples of graphite-epoxy composite material (Karabutov et al. 1998).

\begin{tabular}{|c|c|c|c|c|}
\hline $\begin{array}{c}\text { Sample } \\
\text { Number }\end{array}$ & $\begin{array}{c}\text { Matrix, } \\
\%\end{array}$ & $\begin{array}{c}\text { Porosity, } \\
\%\end{array}$ & $\begin{array}{c}\text { Speed of Sound, } \\
\mathrm{m} / \mathrm{s}\end{array}$ & $\begin{array}{c}\text { Sample Thickness, } \\
\mathrm{mm}\end{array}$ \\
\hline 1 & 36.2 & $<0.1$ & 3041 & 17.5 \\
\hline 2 & 41.4 & 0.4 & 2961 & 18.5 \\
\hline 3 & 31.5 & 1.2 & 2891 & 16.8 \\
\hline
\end{tabular}

\subsubsection{Results}

Figure 2.6.5.2 shows the acoustic attenuation in the three different samples as a function of ultrasonic frequency. Forward detection mode was used to obtain the measurements. Greater acoustic attenuation was observed in the samples with a greater porosity of composite. The acoustic attenuation coefficient was determined by combining the scattering of ultrasound by structural heterogeneities, scattering on pores, 
and acoustic absorption within the sample. Optoacoustic evaluation using forward detection mode required measurements of small variation of high amplitude ultrasonic pulses because the scattered acoustic energy was usually small relative to the total energy of the propagating ultrasonic pulse (low signal-to-noise ratio) (Karabutov et al. 1998). However, the ultrasonic pulse scattered back from defects and measured using the backward detection mode was less affected by acoustic absorption (high signal-to-noise ration) producing a signal of higher quality.

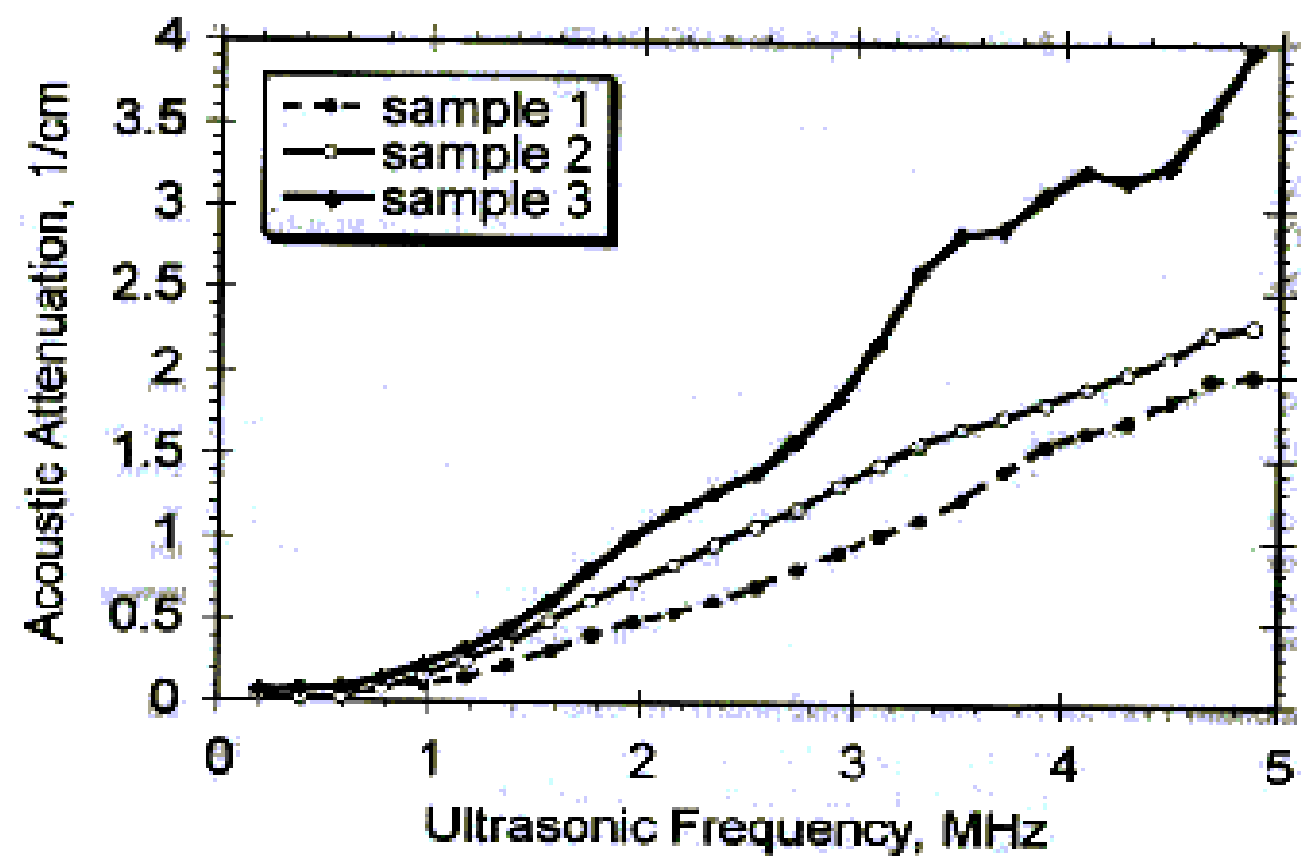

Figure 2.6.5.2 Ultrasonic frequency spectra of the acoustic attenuation coefficient measured in layered graphiteepoxy composite samples with different porosity $(<0.1 \%$ in sample $\# 1,0.4 \%$ in sample $\# 2$, and $1.2 \%$ in sample \#3) (Karabutov et al. 1998)

Figure 2.6.5.3 presents the temporal profiles of ultrasonic pulses detected from samples with a low concentration of micropores (sample 1) and also a sample with a high concentration of micropores (sample 3). Both measurements were taken using the backward detection mode. It was concluded that the two samples had similar acoustic 
profiles indicating that the internal structure and composition of the two samples were similar. However, the profiles detected from the depth of each sample are different.

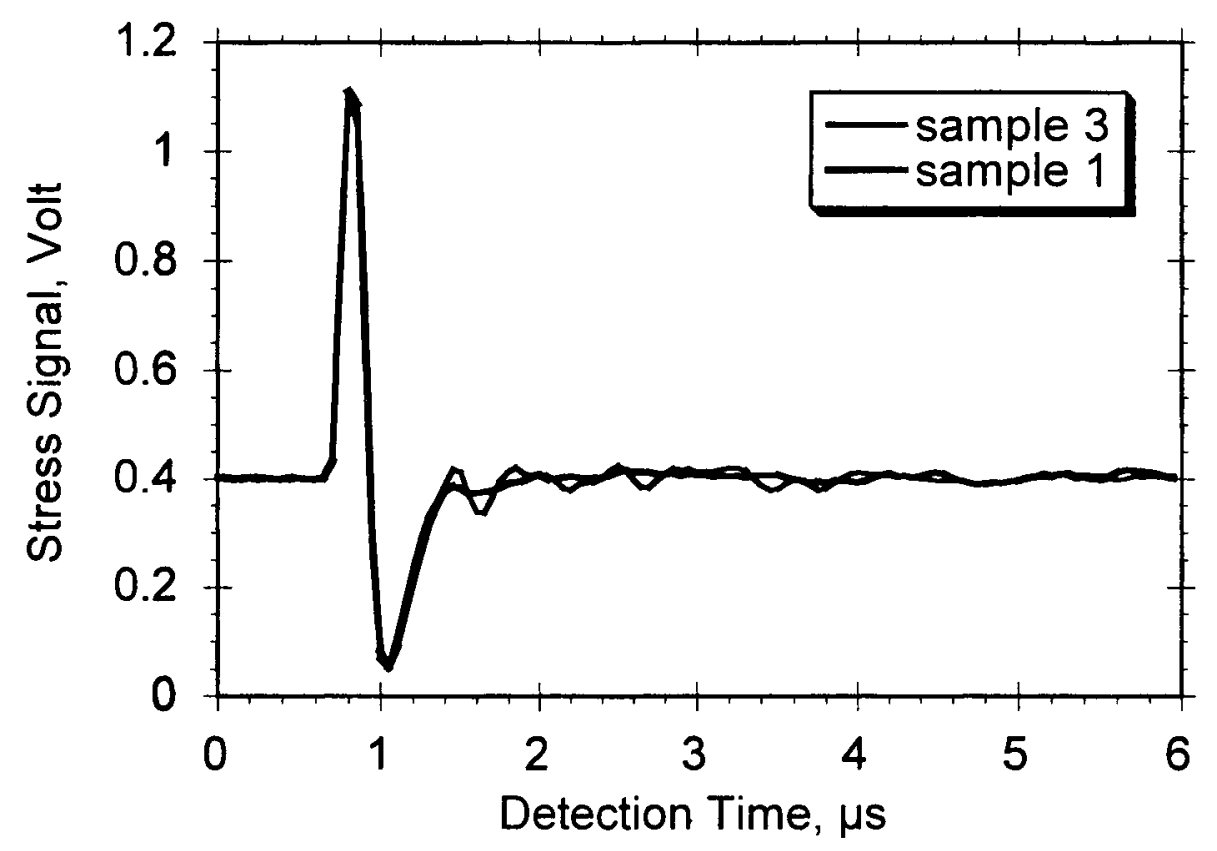

Figure 2.6.5.3 Temporal profiles of ultrasonic pulses measured in backward detection mode for graphite composite samples with two different percentage of porosity $(<0.1 \%$ in sample \#1, and $1.2 \%$ in sample \#2) (Karabutov et al. 1998)

Figure 2.6.5.4 is the acoustic spectra of backscattered ultrasonic pulses. This ultrasonic spectrum of the signals, measured with the laser optoacoustic sensor, gave a better visual representation of the heterogeneous material. The smooth component (major) of the signal was defined by the spectral sensitivity of an acoustic transducer in the laser optoacoustic sensor and the irregular component (noise-type) was defined by efficiency of ultrasonic backscattering by structural heterogeneities. The total power of the noise-type component in the measured ultrasonic spectrum can be used as a parameter of material porosity (Karabutov et al. 1998). This idea was used for further analysis of the laser-induced ultrasonic signal. 


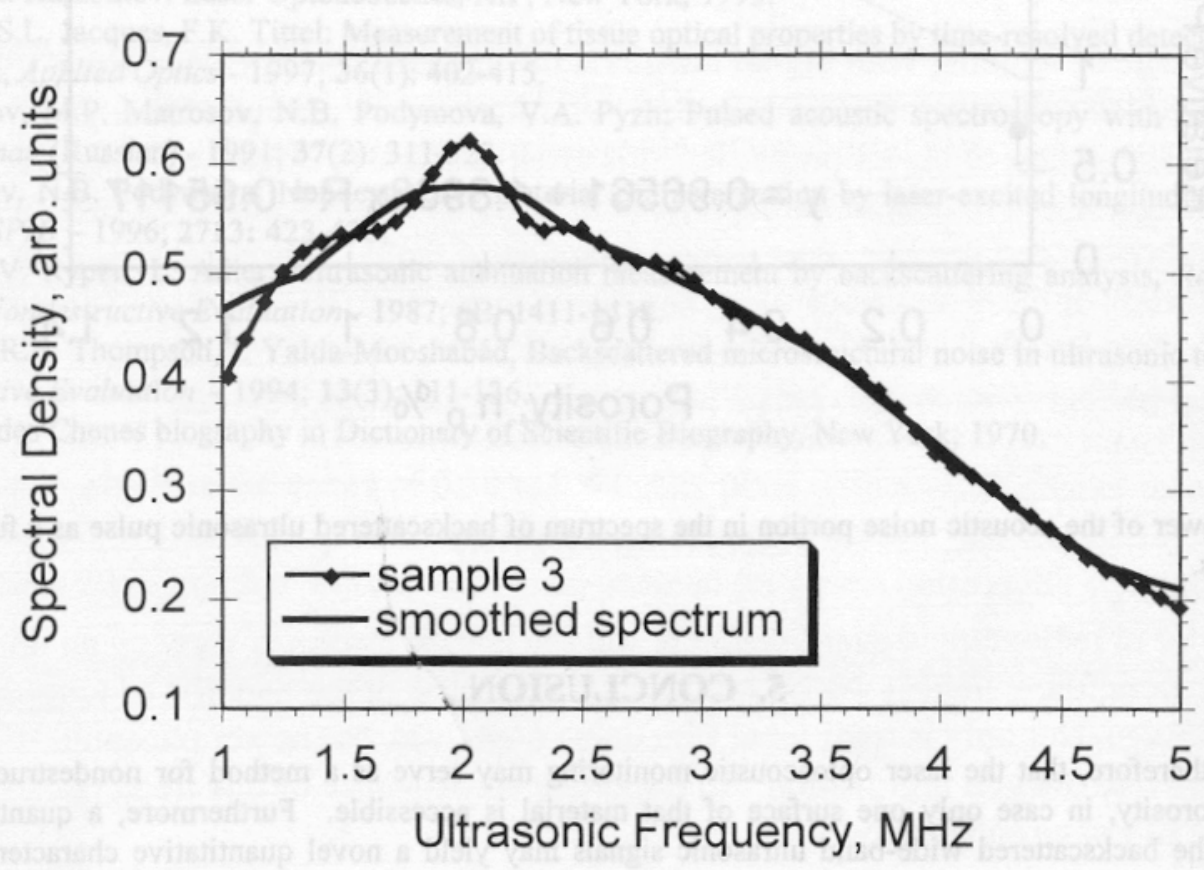

Figure 2.6.5.4 Measured and smoothed acoustic spectra of backscattered ultrasonic pulses, determined for the graphite composite sample with porosity of $1.2 \%$ (Karabutov et al. 1998)

\subsubsection{Discussion}

The total power of the acoustic noise-type component has a sharp increase with an increase of micropore concentration in the samples. From this observation it was concluded that the amount of micropore concentration could be determined from the noise component of the backscattered ultrasonic signal.

\subsubsection{Conclusion}

It was concluded that laser optoacoustic monitoring could be used for a nondestructive evaluation method to determine the amount of porosity in composite materials. A quantitative analysis of temporal profiles of the backscattered ultrasonic signals from the laser optoacoustic method could also give characteristics of composite materials such as concentration and mechanical properties of structural heterogeneities. Therefore, this method proved to be a good nondestructive technique for detecting 
structural heterogeneities. With further experimentation of this method it could potentially reveal the nature of defects, such as porosity or layered separations.

\subsubsection{Nondestructive Evaluation of Fatigue Changes of Composite Structure by Laser-Excited Ultrasonic Waves (Karabutov et al. 1998).}

Nondestructive testing of reinforced composite materials has been experimented with using acoustic spectroscopy with laser excitation of ultrasound. The ultrasound was measured in the frequency range of 1 to $20 \mathrm{MHz}$. The signal was transformed into an ultrasound attenuation spectrum to observe the affects of fatigue on the structure of composites.

\subsubsection{Introduction}

Changes in composite material's structure due to fatigue changes or damages caused by mechanical, heat, electrical etc. loading is of great interest. Typical damages that can occur within fiber reinforced composites are fiber breaks, cracks of matrix, and delaminations of fibers from matrix (Karabutov et al. 1998). These changes in the composite material's structure will cause changes in the attenuation and velocity of ultrasonic waves. Therefore, acoustic spectroscopy methods have great potential for evaluating composite materials. These acoustic spectroscopy techniques measure frequency dependencies of attenuation coefficient and velocity of ultrasonic waves in a sample. These dependencies carry information about the size and location of defects within the samples. 
The traditional acoustic spectroscopy methods have had difficulties measuring ultrasonic waves in composites due to their heterogeneous medium. A wide-band acoustic spectroscopy setup with laser-excited ultrasonic pulses has been developed to overcome the difficulties associated with heterogeneous materials. This method produces nonuniform transient heating of the medium and thermal expansion takes place and a pulse of acoustic pressure is irradiated into the medium.

\subsubsection{Experimental Setup}

Figure 2.6.6.1 shows a schematic of the acoustic spectroscopy setup with laser opto-acoustic (OA) source developed. A pulsed Q-switched Nd: YAG laser was used to excite acoustic pulses. Two types of OA sources were used with the laser: mercury in quartz chamber (rigid boundary of mercury) and an optical filter - blue-green glass with free boundary. The acoustic pulses propagated through composite samples and were received using a wide-band piezoelectric receiving transducer. The signal-to-noise ratio of the received electric pulses was 20-30 dB. Standard FFT was used to calculate the spectra of acoustic pulses. 


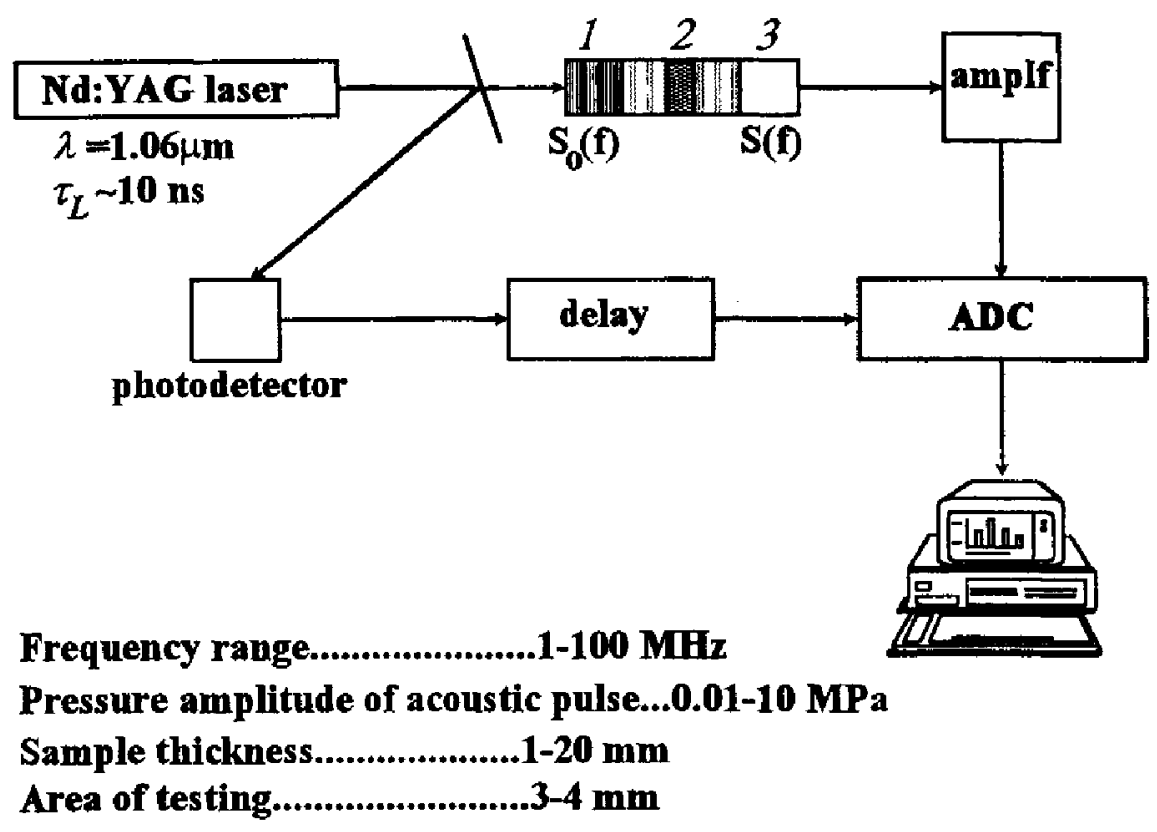

Figure 2.6.6.1 Wide-band acoustic spectrometer with laser excitation of ultrasound (1- OA source, 2investigated sample, 3- piezoelectric receiving transducer) (Karabutov et al. 1998)

\subsubsection{Investigated Samples}

Glass-fiber-reinforced composite samples made up of 24 layers of 300 micron glass fibers in a polymer matrix were tested. Cyclic loading and tensile loading tests were performed to several different composite samples.

The samples were notched at the center to model the microplastic fatigue changes of composite structure (Figure 2.6.6.2). Due to the high stresses at the center of the samples (caused by the notches) the outer sections of the samples (section A) would appear unloaded, which was compared to the loaded section (section B). Acoustic flaw detectors were attached to both section B and section A of each sample to measure the amplitude of the acoustic signal during static loading. The amplitude decreased linearly with increasing tensile strength until drastically decreasing at the critical tensile strength (Figure 2.6.6.3). Once the tensile load was removed, the acoustic wave amplitude 
exhibited a hysterisis-like behavior (Figure 2.6.6.3). The final measured amplitude was $20 \%$ lower that the initial measured amplitude. This reduction was due to microplastic fatigue changes in the composite structure.

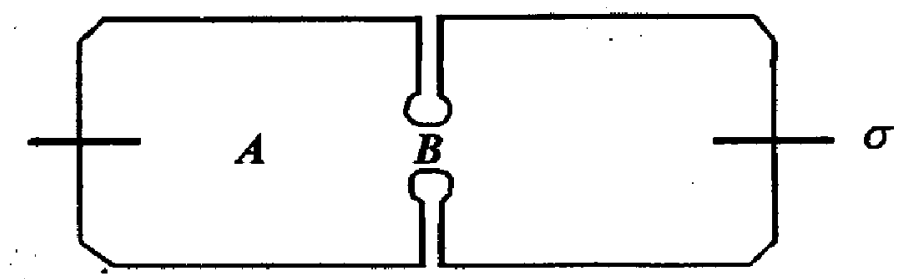

Figure 2.6.6.2 Glass-fiber-reinforced composite sample. A - unloaded section, B - statically loaded section (Karabutov et al. 1998)

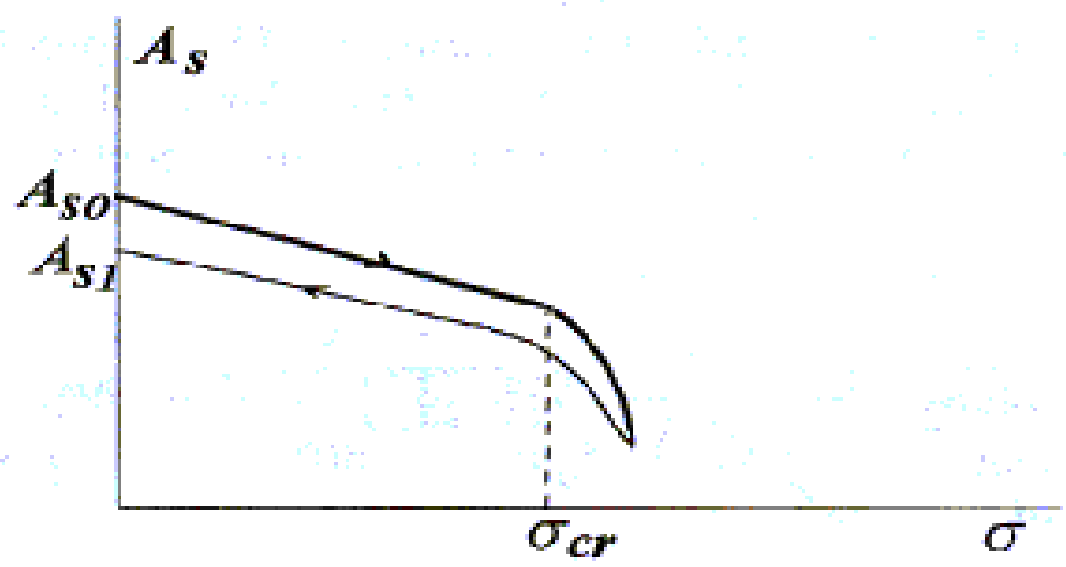

Figure 2.6.6.3 Static loading curve for composite samples (Karabutov et al. 1998)

\subsubsection{Experimental Results and Discussion}

Measurements were taken in both section A and section B of the sample (Figure 2.6.6.3). The ultrasound attenuation coefficient was measured for each sample in both sections and the absolute value was determined in the frequency range 1-20 MHz. This frequency range was used because of the strong scattering of high frequency harmonics of ultrasound pulses in composites. 


\subsection{Attenuation of Ultrasound in Green-State (Before Cyclic Loading) Composite Sample}

Figure 2.6.6.4 shows the frequency dependencies of ultrasound attenuation coefficient of two glass-fiber reinforced composite samples in both the loaded section (section B) and unloaded section (section A). The high and narrow resonance peaks of attenuation for the unloaded sections (section A) occurred at a frequency around $16 \mathrm{MHz}$ for both samples. The difference in the two resonance frequency values could have been due to some imperfections from the composite manufacturing. This was probably the reason for the different values of critical strengths $\left(\sigma_{\mathrm{cr}}\right)$ as well.
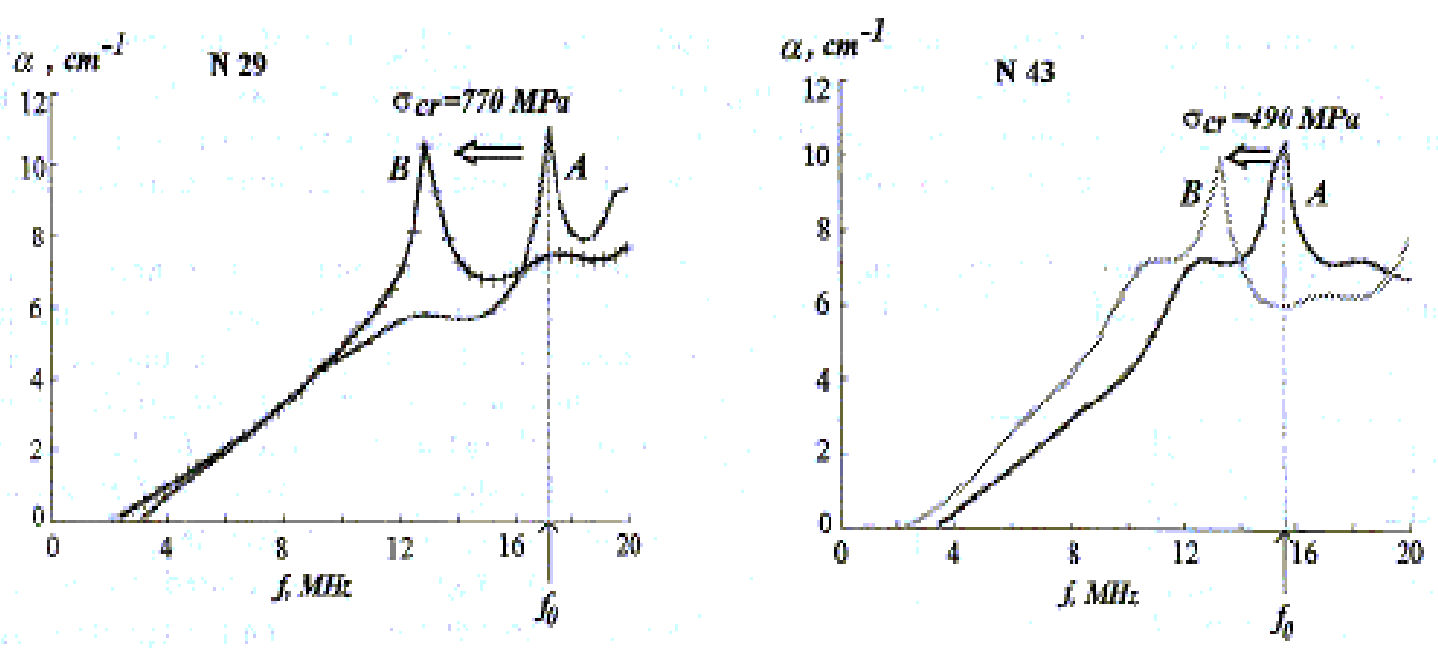

Figure 2.6.6.4 Frequency dependencies of ultrasound attenuation coefficient in green-state composite samples. Signal A - unloaded section, and signal B - statically loaded section (Karabutov et al. 1998)

The resonance peak of attenuation of the loaded sections occurred at the same frequency. However, the central frequency of the loaded sections B have shifted into the low frequency region of the spectra of ultrasound attenuation and decreased approximately $20 \%$. This shift in frequency was caused by a decrease of ultrasonic wave velocity in the statically loaded section of the samples. It was concluded that a decrease in acoustic wave velocity indicated a decrease of elastic moduli. 


\subsection{Attenuation of Ultrasound in Composite Samples After Service Life Testing}

Three different composite samples were subjected to cyclic loading before the static tensile loading (described in section 2.6.6.4.1). The attenuation coefficient was again measured in both section A and section B of Figure 2.6.6.2. Figure 2.6.6.5 shows the frequency dependencies of ultrasound attenuation coefficient in two different samples that experienced cyclic loading before the static loading was applied. When the graphs of Figure 2.6.6.5 were compared to the graphs of Figure 2.6.6.4 obvious differences in the signals were observed. The main difference is that there is no distinct resonance peaks of ultrasound attenuation. Microcracks in the composite structure caused by the cyclic loading could have been the reason for no distinct resonance peaks.
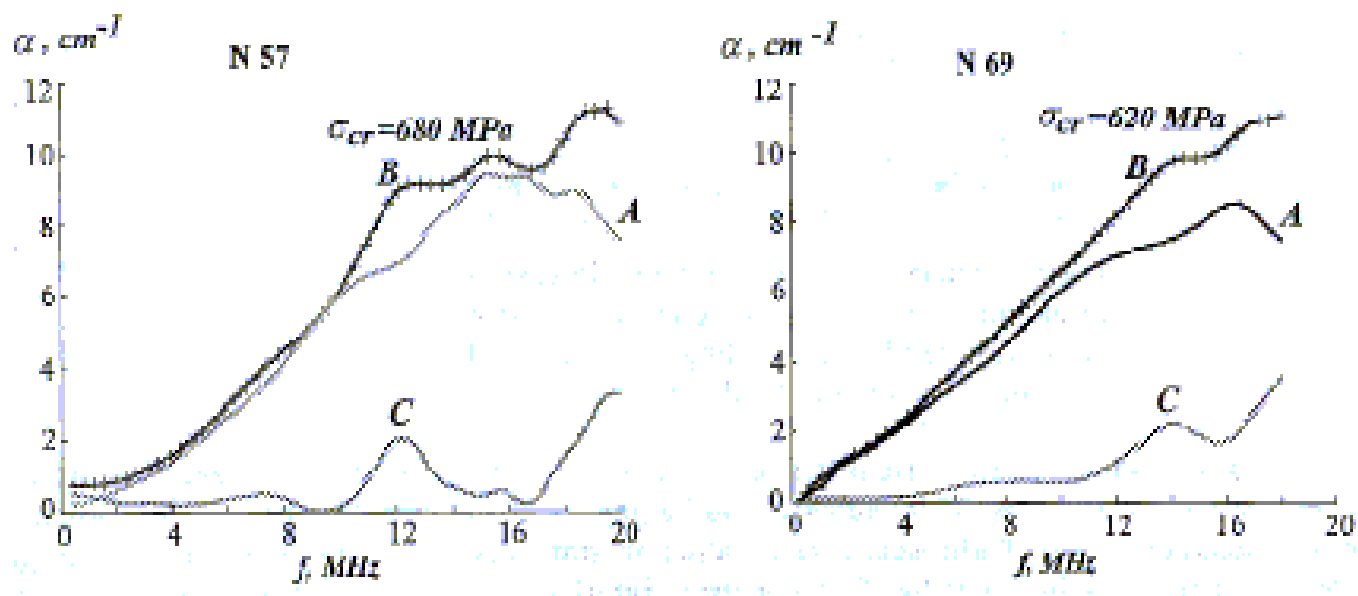

Figure 2.6.6.5 Frequency dependencies of ultrasound attenuation coefficient in composite samples after service life testing. Signal A - unloaded section, signal B - statically loaded section, and signal C - difference of attenuation spectra in sections $B$ and $A$ (Karabutov et al. 1998)

It was suggested that the microcracks formed during cyclic loading migrated during static loading and a concentration of nearby fiber-matrix interfaces occurred (Karabutov et al. 1998). This would have caused a higher degree of periodicity in section B, which was confirmed by the frequency dependence of "differential " attenuation of ultrasound (signal C in Figure 2.6.6.5). From this signal (signal C) the resonance peak 
was clearly seen. This resonance peak occurs at a frequency of about $12 \mathrm{MHz}$ practically corresponding to the resonant frequency for the green-state samples. It was then concluded that the structure of statically loaded sections of composite samples after service life testing (cyclic loading) had a higher degree of periodicity caused by the migration of microcracks during static loading and its concentration of nearby fibermatrix interfaces.

A third composite sample was statically loaded to failure after cyclic loading was performed. The frequency dependencies of ultrasound attenuation coefficient for this sample are shown in Figure 2.6.6.6. Resonant peaks were observed in both signals. The relation between the resonant frequencies of attenuation in sections $\mathrm{A}$ and $\mathrm{B}$ showed a decrease of acoustic wave velocity in section B by about $22 \%$. This indicated the beginning of sample failure. A high degree of defects in the loaded section (section B) was indicated by the series of resonance peaks of ultrasound attenuation in signal B of Figure 2.6.6.6.

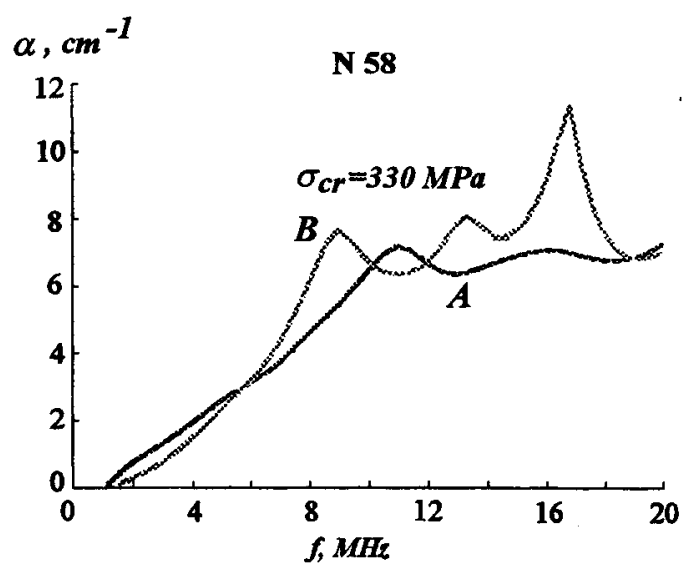

Figure 2.6.6.6 Frequency dependence of ultrasound attenuation coefficient in a composite sample after service life testing (fatigue testing). Signal A - unloaded section, and signal B - statically loaded section (Karabutov et al. 1998) 


\subsection{Classification of Composite Samples According to the Aging}

The obtained ultrasonic data correlated well with the typically behavior of aging composites. It was concluded that the resonance peaks of ultrasonic attenuation gave important information about the composite's structure. The resonance peaks of ultrasound attenuation in green-state samples gave information about the composites initial periodical structure; these peaks were shifted into the low frequency region at the first stage of fatigue changes. The resonance peaks of the samples, which experienced cyclic loading before static loading, also gave information about the composite's structure. Figure 2.6.6.7 classifies the samples according to aging (number of cycles). It was concluded that spectroscopy analysis could be used to quantitatively evaluate composites undergoing cyclic and static loading.

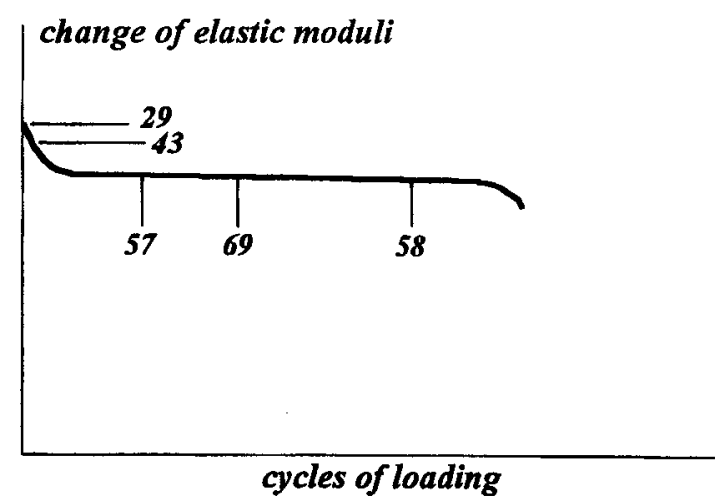

Figure 2.6.6.7 Qualitative classification of composite samples according to the aging (Karabutov et al. 1998)

\subsubsection{Conclusions}

Wide-band acoustic spectroscopy with laser excitation of ultrasound was experimented with. Good correlation between the results obtained from wide-band ultrasonic investigation of composite's structure and typical fatigue changes of elastic properties of composites was established. The wide-band frequency spectra of ultrasound 
attenuation carried useful information about the initial structure of composites and about fatigue changes in the composite's structure during loading.

\subsubsection{Conclusion on New NDE Techniques}

Based on this literature review, it was concluded that there are many new techniques that can evaluate the performance of composite materials and detect/locate defects within composite materials. However, most of these techniques are being used in the aerospace industry and need to be modified so they could be used to evaluate civil infrastructures on site. The main disadvantage that some of these new techniques have is that they are stationary and can only be used in the laboratories. More research needs to be performed on these techniques to make them applicable for field use. The main advantage that all these techniques share is the speed that they can evaluate composite materials.

\subsection{Final Thoughts on All NDT Methods Used for Evaluating Composite Materials and Structures}

Many NDT techniques for evaluating composite materials and structures have been reviewed and discussed in this chapter. Based on the literature review, it was concluded that all NDT techniques discussed in this chapter have many advantages, but also some disadvantages. However, this chapter has indicated that many NDT techniques have the ability to detect and characterize defects in structures made entirely of composite materials and also structures rehabilitated with FRP wraps. It was also 
concluded that future research needs to be conducted on all NDT methods for evaluating composite materials to ensure reliable results.

\subsubsection{Infrared Thermography}

Based on the literature review in this chapter on IR thermography, it can be concluded that this is a promising technique for evaluating composite materials and structures. This technique seems to be more effective for evaluating structures that have been wrapped with FRP composites (columns, beams, etc.). IR thermography has proved to be very successful in detecting defects such as disbonds and delaminations close to the surface. However, this technique could be used with other NDT techniques such as ground penetrating radar (GPR) or wave propagating techniques to detect defects deep within the structure as well as defects close to the surface. The main disadvantages this technique has is the cost of buying an infrared camera and other equipment is quit expenses.

\subsubsection{Wave Propagating NDE Techniques (Acoustic, Ultrasound, Microwave, Etc.)}

NDT methods that use wave propagation to detect defects have been around for many years. This technique uses sound, microwave, or ultrasound waves to propagate through a material, which is caused by the vibration of particles. These NDT methods have mainly been used on homogeneous materials such as steel. When this technique was introduced to heterogeneous or porous materials such as composites, wave attenuation (loss of energy per unit distance) became a factor. Also, the amount of noise associated with the ultrasonic signals have created problems evaluating heterogeneous 
materials. Microwave signals have been more successful in evaluating materials that are highly heterogeneous (composite materials) because the noise-to-signal ratio is much lower.

New techniques that use lasers to generate ultrasound wave propagation have been successful in evaluating composite materials. These techniques use the amount of wave attenuation to determine the quality of the composite material. The higher the attenuation the more defects or pores the material has. This technique seems promising for evaluating composite materials. However, future research needs to be performed to set a standard for determining the quality of the composite material or structure.

\subsubsection{Load Testing with Strain Gages}

Using strain gages for long term monitoring and evaluating a structures performance under different load conditions has its advantages but for detecting and locating defects within a structure it is not very useful. This technique is very useful for developing analytical models for future designs. However, a structure or a member of the structure may need to be tested to failure in order to collect sufficient data.

\subsubsection{Fiber Optic Health Monitoring Systems}

All-composite bridges have the advantage of being manufactured with fiber optic sensors installed into the structure permanently. The main advantage of this NDT technique is it's long term monitoring ability and the results obtained are usually very accurate. This system can monitor strains and deflections in a structure during 
installation and also over a long period of time. However, fiber optic systems are not very useful for detecting and locating defects that might develop within the structure. The fiber optic sensors are very delicate and can be damaged very easily during installation or shipment to the site. Also, there are many connectors and wires involved with a fiber optic sensor system that can cause problems. The connectors and wires need to be kept clean and this is very difficult to do in the field. A fiber optic sensor system can be very effective if the sensors are all working properly. Future research needs to be conducted to create a more durable fiber optic sensor system.

\subsubsection{New NDE Techniques Being Developed}

There are many new NDT techniques that are being developed by the aerospace industry to evaluate composite components for aircrafts. These techniques could someday be used to evaluate composite materials and/or structures used in the civil engineering industry. Many of these techniques use lasers and stationary machinery to thoroughly evaluate composite materials. Other research has developed new ways to use fiber optics to evaluate composite materials. There has been extensive research done by the aerospace industry to develop NDT methods that evaluate the quality of composite components during the manufacturing process. Future research needs to be conducted on these new NDT techniques to develop equipment and methods for evaluating composite materials used in the civil engineering industry. New NDT Techniques need to be developed for evaluating these composite materials during manufacturing and also when in service. 


\section{CHAPTER 3}

\section{BASIC THEORY OF INFRARED THERMOGRAPHY}

\subsection{Introduction}

This chapter is intended to provide a brief introduction to the theory behind infrared thermography for nondestructive evaluation (NDE). Infrared thermography is used to discover subsurface features (such as subsurface thermal properties, presence of subsurface anomalies/defects), due to relevant temperature differences observed on the surface with an infrared (IR) camera. In general, the surface being inspected is heated and the local infrared emission from the surface, which is in accordance with the StefanBoltzmann law, is recorded by an infrared detector to provide information about the internal structure of the specimen (Maldague et al. 1993).

The term "infrared" refers to a broad range of frequencies, beginning at the top end of those frequencies used for communication $\left(.003 \times 10^{14} \mathrm{~Hz}\right)$ and extending up to the low frequency (red) end of the visible spectrum $\left(4 \times 10^{14} \mathrm{~Hz}\right)$. The wavelength of infrared radiation ranges from about 1 millimeter down to $.75 \mu \mathrm{m}$ (Maldague et al. 1993). Figure 3.1.1 shows the electromagnetic spectrum of infrared in relation to other wavelength regions. The electromagnetic band width of infrared is usually associated with energy produced by object temperatures of $-20^{\circ} \mathrm{C}$ and above. 


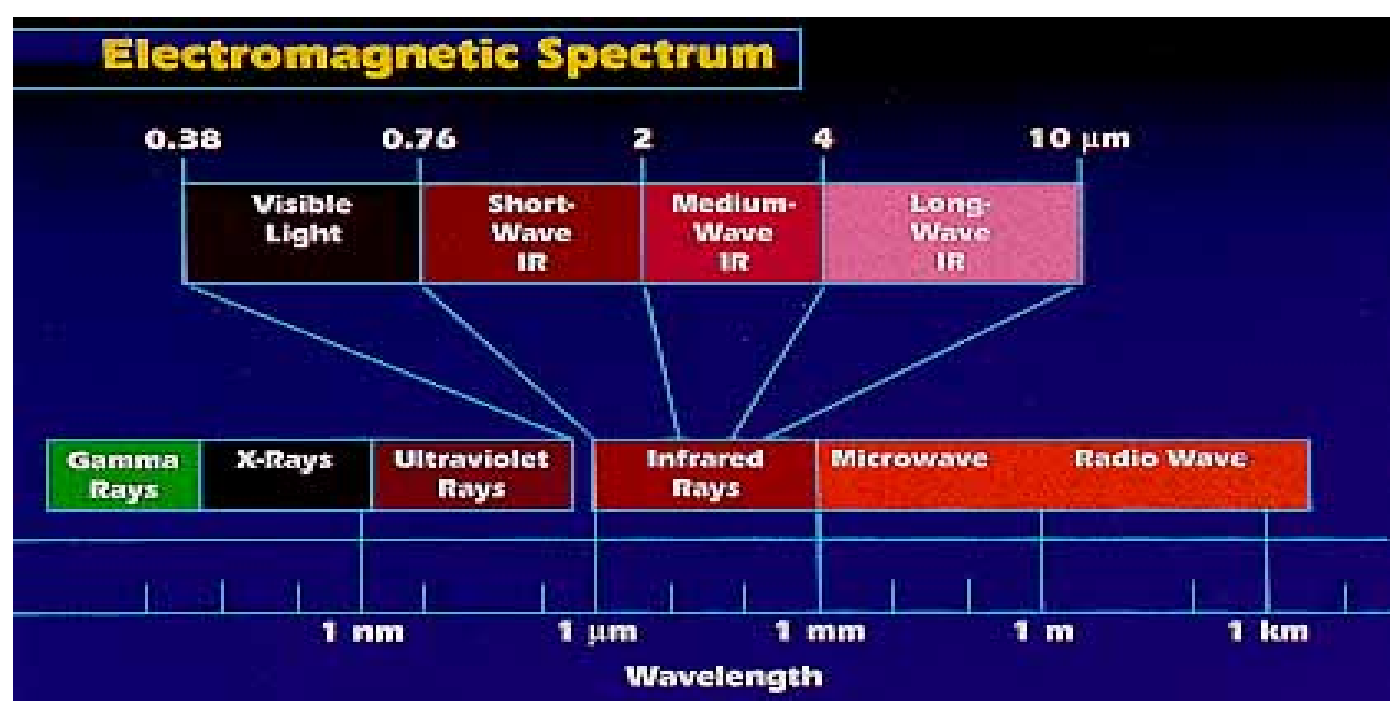

Figure 3.1.1 The Electromagnetic Spectrum

\subsection{Infrared Thermography}

As the name infers, infrared thermography is a technique that produces a visible graph or thermographic image of thermal energy radiated from surfaces. The energy emitted by a surface at a given temperature is called the spectral radiance and is defined by the Planck's law. All infrared systems are sensitive to infrared radiated energy only. They do not actually measure temperature. They are useful in applications where a variation in temperature, reflection, surface condition, or material may cause a difference in the radiated energy level detected by the infrared camera.

\subsubsection{Infrared Cameras}

Prior to the recent advances in microelectronics, the basic infrared camera consisted of a single detector piece with an electromechanical scanning system. These cameras had very high noise and the performance of the cameras was limited. The new generation of infrared cameras are known as Focal Plane Arrays (FPAs). These new instruments have quantum (cooled) detectors with direct digital interface, low noise, and 
high spatial resolution. Different infrared cameras use different methods of cooling such as electronic cooling, fans, or liquid nitrogen. The liquid nitrogen can cool the instrumentation to $-196^{\circ} \mathrm{C}$, allowing the detection of temperature differences as small as $0.01{ }^{\circ} \mathrm{C}$ (Weil 1989). The new infrared cameras use electronic cooling rather than liquid nitrogen and provide a temperature resolution of $0.1^{\circ} \mathrm{C}$, which is adequate for most civil engineering applications. Although these new high performance cameras are quite expensive, they are being used more and more for all types of infrared thermography applications.

\subsection{Basic Theory}

As stated previously, infrared thermography is a non-contact sensing method concerned with the measurement of radiated electromagnetic energy. An infrared camera is just a spectral radiometer that measures this energy. This technique uses an infrared camera to create fully analyzable images from the thermal radiation given off by a surface. Infrared measurement depends strongly on emissivity $(\varepsilon)$ and surface temperature. The theory of measuring infrared radiation is mainly based on the StefanBoltzmann law and Planck's law.

\subsubsection{Emissivity}

Emissivity is a surface property that specifies the ability of a surface to emit energy. The values of emissivity vary between 0 (for a perfect reflector) to 1 (for a perfect emitter, typically called a 'black body'). An emissivity factor of 0.9 absorbs $90 \%$ 
of the received energy and reflects $10 \%$. Emissivity has dependencies with surface orientation, temperature, and wavelength.

One can think of emissivity as a pipe connecting two water tanks. The first tank initially has a higher water level than the second tank so gravity will equalize the levels via the pipe. If the pipe is small, i.e., low emissivity, the time taken to equalize the two tanks will be longer than if the pipe was large, i.e., high emissivity. Water will always be transferred between the two tanks as long as there is a difference in water level and the size of the pipe will control the rate of water transfer. One can think of emissivity the same way. As long as there is a temperature difference, energy will be transferred and emissivity will control the rate of energy transfer.

\subsubsection{The Stefan-Boltzmann Law}

The Stefan-Boltzmann law states that the total energy radiated per unit surface area of a black body in unit time is proportional to the fourth power of its thermodynamic temperature (in Kelvin):

$$
\operatorname{Energy} \operatorname{Flux}(\mathbf{E})=\sigma \mathbf{T}^{4} \mathrm{j} / \mathrm{m}^{2} \mathrm{~s} \quad \ldots \quad \ldots \quad . \quad . \quad .
$$

Where $\sigma$ is Stefan's constant and approximately $5.6703 \times 10^{-8}$ watt $/ \mathrm{m}^{2} \mathrm{~K}^{4}$. Note that the amount of energy emitted depends very strongly on the temperature (e.g. if the temperature doubles the energy emitted by the black body will be 16 times greater). 


\subsubsection{Planck's Law}

This law gives the distribution of spectral-energy radiated by a black body. As stated before, a black body is a hypothetical body that completely absorbs all radiant energy falling upon it, reaches some equilibrium temperature, and then re-emits that energy as quickly as it was absorbed. Planck hypothesized that when an oscillator changes form a state of energy, $E_{1}$, to a lower state of energy, $E_{2}$, the discrete amount of energy $E_{1}-E_{2}$ is emitted as a quantum of radiation. The energy of this quantum is equal to the product of the frequency of the radiation, $v$, and Planck's constant, $h$ :

$$
E_{1}-E_{2}=h v \ldots \ldots \ldots
$$

Planck's law for the energy, $E$, radiated per unit volume by a cavity of a black body in a wavelength interval is as follows:

$$
E_{\lambda}=\frac{8 \pi h c}{\lambda^{5}} \times \frac{1}{\exp (h c / k T \lambda)-1}
$$

where,

$$
\begin{aligned}
& h=\text { Planck's constant }\left(6.626 \times 10^{-34} \mathrm{~J}^{*} \mathrm{~s}\right) \\
& c=\text { the speed of light }\left(2.99 \times 10^{8} \mathrm{~m} / \mathrm{s}\right) \\
& k=\text { the Boltzmann constant }\left(5.6703 \times 10^{-8} \mathrm{watt} / \mathrm{m}^{2} \mathrm{~K}^{4}\right) \\
& T=\text { the absolute temperature (in Kelvin). }
\end{aligned}
$$

The wavelength of the emitted radiation is inversely proportional to its frequency:

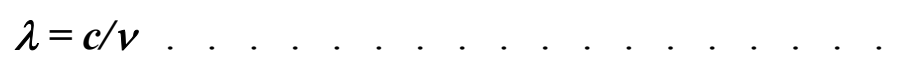

Figure 3.3.1 shows the variation of energy for different wavelengths at different temperatures. 


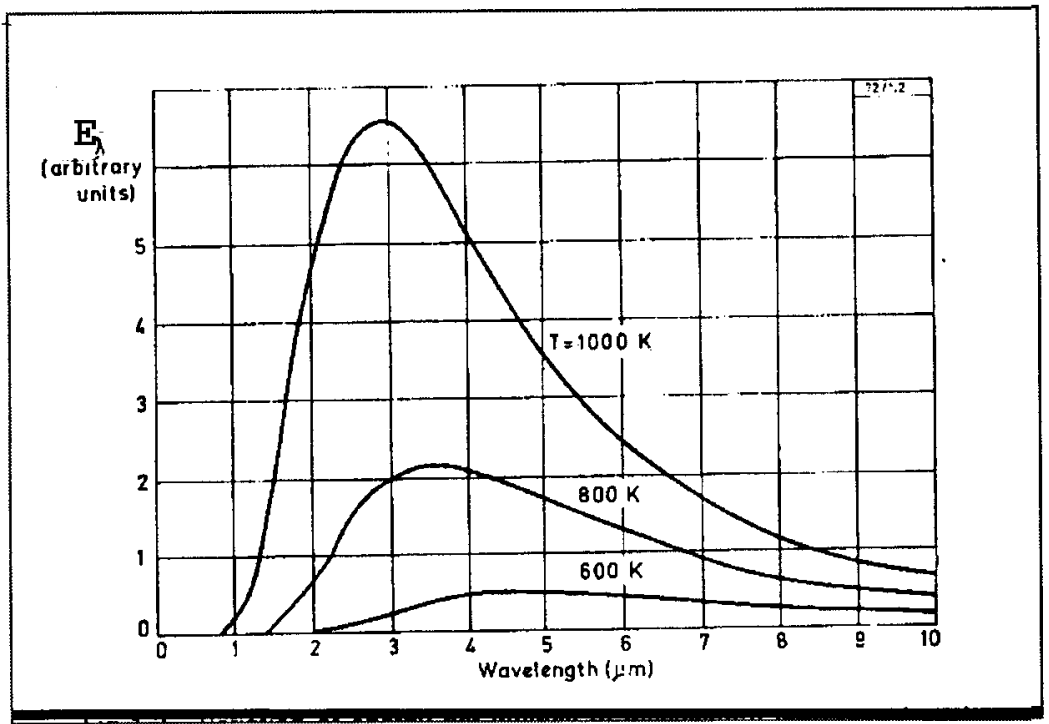

Figure 3.3.1 variation of energy per interval of wavelength at different temperatures (Morten 1971).

\subsection{Heat Transfer}

Heat moves from high temperature objects to lower temperature objects. Heat transfer changes the internal energy of both objects involved according to the First Law of Thermodynamics. There are three mechanisms involved in the transfer of heat, which include radiation, conduction, and convection (Halabe 1999). The main environmental factor that affects these three mechanisms is solar radiation. The amount of solar radiation that an object receives depends on the time of the year and day.

\subsubsection{Radiation}

Radiation is heat transfer by the emission of electromagnetic waves, which carry energy away from the emitting object. All surfaces not only radiate heat to the atmosphere but also simultaneously receive radiation back from the atmosphere. For ordinary temperatures (less than red hot), the radiation falls in the infrared region of the electromagnetic spectrum. The relationship governing radiation from hot objects according to the Stefan-Boltzmann law is: 


$$
\mathbf{P}=\varepsilon \sigma A\left(\mathbf{T}^{4}-\mathbf{T}_{\mathrm{c}}{ }^{4}\right) \ldots \ldots \ldots
$$

where,

$$
\begin{aligned}
& \mathrm{P}=\text { radiated power }(\text { watts }) \\
& A=\text { radiating area }\left(\mathrm{m}^{2}\right) \\
& \sigma=\text { Stefan's constant }\left(5.6703 \times 10^{-8} \mathrm{watt} / \mathrm{m}^{2} / \mathrm{K} 4\right) \\
& \varepsilon=\text { emissivity }(=1 \text { for ideal black body) } \\
& T=\text { temperature of radiator (in Kelvin) } \\
& T_{\mathrm{c}}=\text { temperature of surroundings (in Kelvin) }
\end{aligned}
$$

\subsubsection{Conduction}

Conduction is heat transfer by means of molecular agitation within a material without any motion of the material as a whole. When a material is heated, the energy will be transferred to colder regions because the higher speed particles will collide with the slower ones with a net transfer of energy to the slower particles. For heat transfer between two plane surfaces the rate of conduction heat transfer is:

$$
\frac{Q}{t}=\frac{\kappa A\left(T_{\text {hot }}-T_{\text {cold }}\right)}{d}
$$

where,

$$
\begin{aligned}
& \mathrm{Q}=\text { heat transferred (joules) } \\
& \mathrm{t}=\text { unit of time (seconds) } \\
& \mathrm{K}=\text { thermal conductivity of the barrier }\left(\mathrm{J} / \mathrm{m} / \mathrm{sec} / \mathrm{K} \text { or watt } / \mathrm{m} / \mathrm{K} \text { or } \mathrm{watt} / \mathrm{m} /{ }^{\circ} \mathrm{C}\right) \\
& \mathrm{A}=\operatorname{area}\left(\mathrm{m}^{2}\right) \\
& \mathrm{T}=\text { temperature (in Kelvin) }
\end{aligned}
$$




$$
\mathrm{d}=\text { thickness of barrier }(\mathrm{m})
$$

\subsubsection{Convection}

Convection is heat transfer by motion of a fluid such as air or water. Once the fluid is heated it is forced to move away from the source of heat, carrying energy with it. The most common example of convection takes place between a hot surface and the surrounding air. Convection above a hot surface occurs because hot air expands, becomes less dense, and rises. This causes convection currents, which transports energy. The main parameters that determine the amount of heat flow by convection are the temperature of the surface, the temperature of the surrounding air (ambient temperature), and the wind speed.

\subsection{Application of Infrared (IR) Thermography to Rehabilitated Structures with Composite Wraps}

Infrared thermography can be used to detect defects present in composite retrofits such as FRP wraps. The main parameters that affect the detection of these defects with IR thermography include ambient air conditions, wind velocity, composite thickness, depth of debond, and the thermal properties of the different layers. Underlying composite defects (such as debonds, porosity, etc.) result in localized thermal conductivity deviations which give rise to localized hot or cold spots on the test surface. These hot or cold spots can be detected using the infrared camera and, hence, pinpoint the location, shape, and approximate size of a flaw. The best results occurred when the test surface has been thermally stimulated using either a hot or cold heating method. 
A delamination in a composite retrofit wrap may be filled with air, which acts as a thermal insulator. An insulator stops heat flow and returns the heat to the surface causing the surface above the insulator to be hotter than the surrounding areas (Figure 3.5.1).

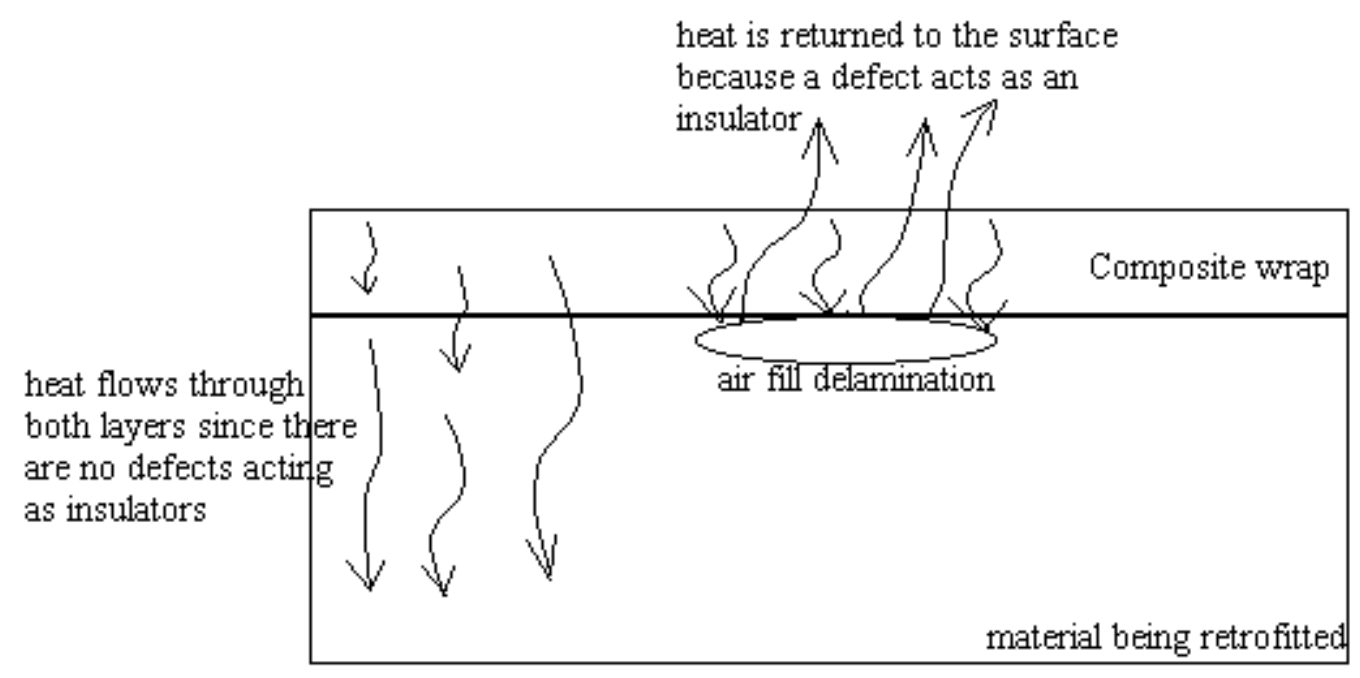

Figure 3.5.1 Illustration of how a defect affects heat flow

The surface temperature differences between the sound and delaminated areas are caused by the differences in thermal behavior due to different properties of heat conduction and/or heat capacity. This concept can be seen from the data collected by Manning and Holt (1980) for concrete deck surfaces (Figure 3.5.2). 


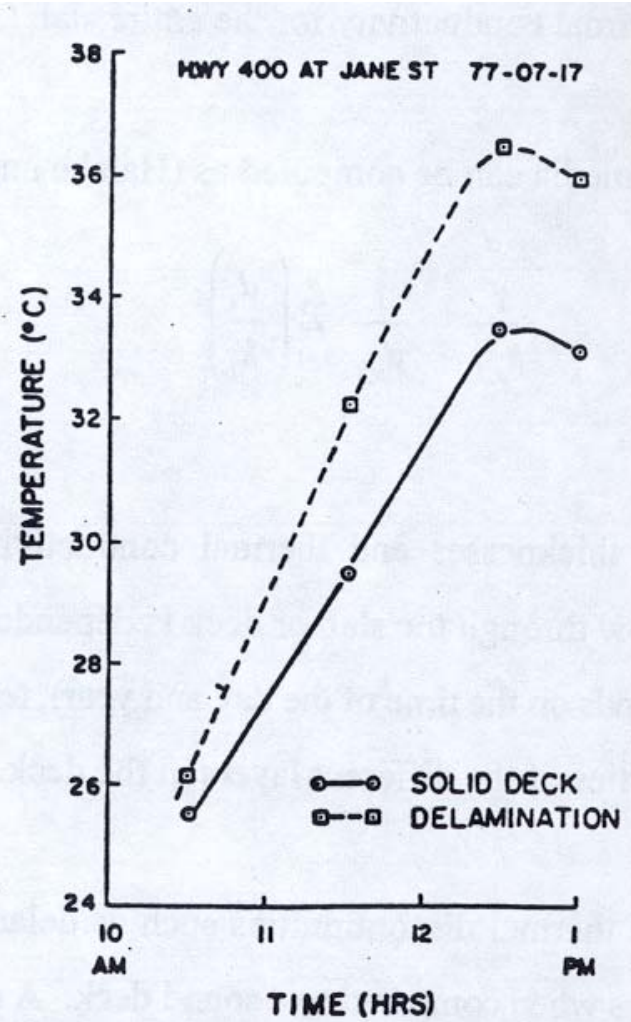

Figure 3.5.2 Field data for temperature differences between solid and delaminated area in bridge deck (Manning and Holt 1980)

There are many variables that need to be considered when performing infrared thermography in an uncontrolled environment. Any external variable that changes the image of a defect, with respect to an undamaged area, degrades the sensitivity and reliability of the technique. Infrared measurements are severely affected by the unpredictable changes in weather conditions, and also uneven heating of the surface. Although the unpredictable changes in weather conditions cannot be controlled, using uniform heating methods to heat the surface can minimize heating variations. When structures are wrapped with composite material there are sometimes variations in the thickness of the wrap. The degree of thickness variation in the composite material or adhesive affects the quality of the thermographic image. The surface being inspected should always be cleaned before infrared thermography is used. The emissivity of the 
surface under inspection can change due to the dirt particles clinging to the surface (Hawkins et al. 1999). One way to produce more reliable data is to take thermographic images at different times and compare the defects observed. This will reduce the emissivity variations greatly, but it comes at a price. Making several trips to the site with all the equipment will add to the labor time, which will increase the cost of the inspection. 


\section{CHAPTER 4}

\section{LABORATORY EXPERIMENTS}

Infrared thermography was experimented with and the results are discussed in this chapter. The experimental set-ups, specimen configurations, and the results of the infrared testing will all be presented in this chapter.

\subsection{Experimental Set-up}

Infrared thermography basically consists of two phases. First a thermal gradient needs to be established using a heat gun, a warm water soak, a heat lamp, solar heat, etc. Then the surface temperature of the structure needs to be monitored as it returns to thermal equilibrium. This technique is illustrated in Figure 4.1.1.

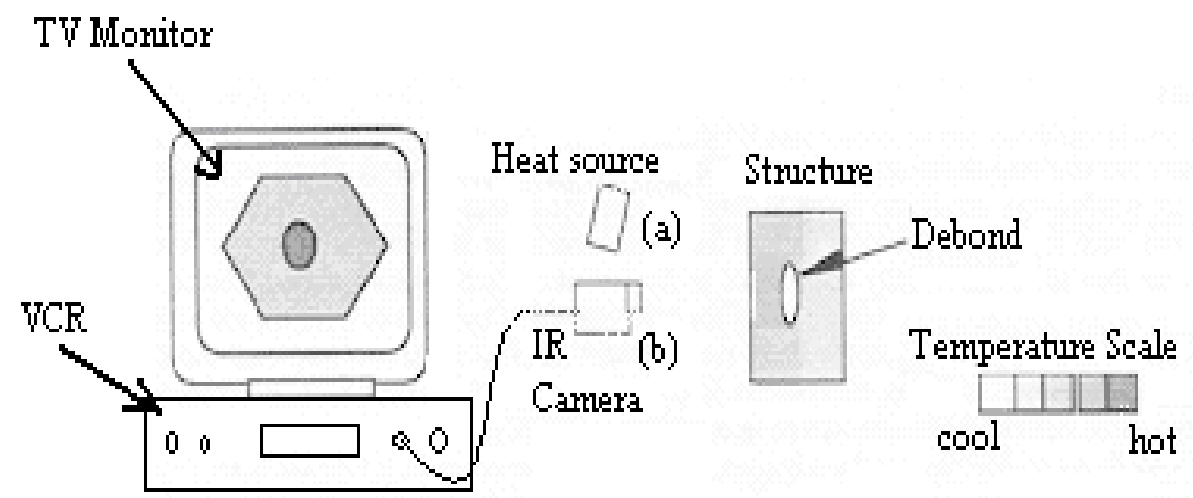

Figure 4.1.1 The two phases of infrared thermography: (a) creating thermal gradients with a heat source, and (b) capturing the thermal gradients with an infrared imaging device 


\subsubsection{Heating Sources}

There were several heating methods used to create thermal gradients. A quartz heater, solar radiation, and solar radiation combined with plastic wrap to create a greenhouse effect were all experimented with.

\subsubsection{Quartz Tower Heater}

A picture of the quartz tower heater is illustrated in Figure 4.1.2. The quartz heater had two temperature settings, 750 and 1500 watts. The 1500-watt setting was used to heat the specimens. The heater was placed approximately one foot from the surface of the specimen being heated. This allowed the specimens to be heated uniformly. The specimens were heated to approximately $65^{\circ} \mathrm{C}$. Thermal images were then taken as the surface temperature returned to thermal equilibrium.

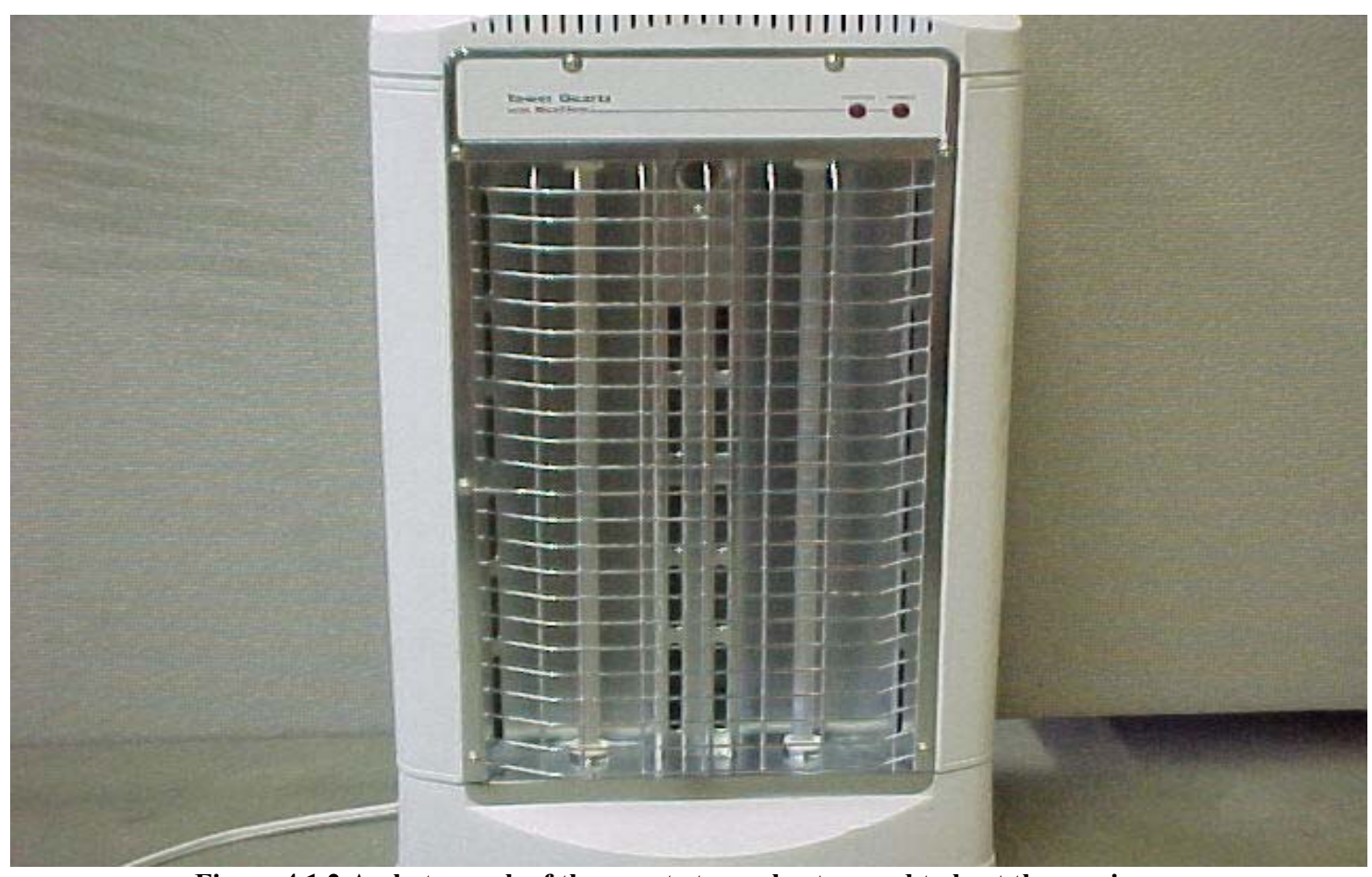

Figure 4.1.2 A photograph of the quartz tower heater used to heat the specimens 


\subsubsection{Solar Radiation}

The specimens were also heated using solar radiation. The specimens were placed in the sun between 11:00 AM and 3:00 PM when solar radiation is the greatest. This type of heat source heated the specimen's surface temperature to about 30 or $35^{\circ} \mathrm{C}$. The specimens were heated in the sun for one or two hours before thermal imaging was performed (Figure 4.1.3).

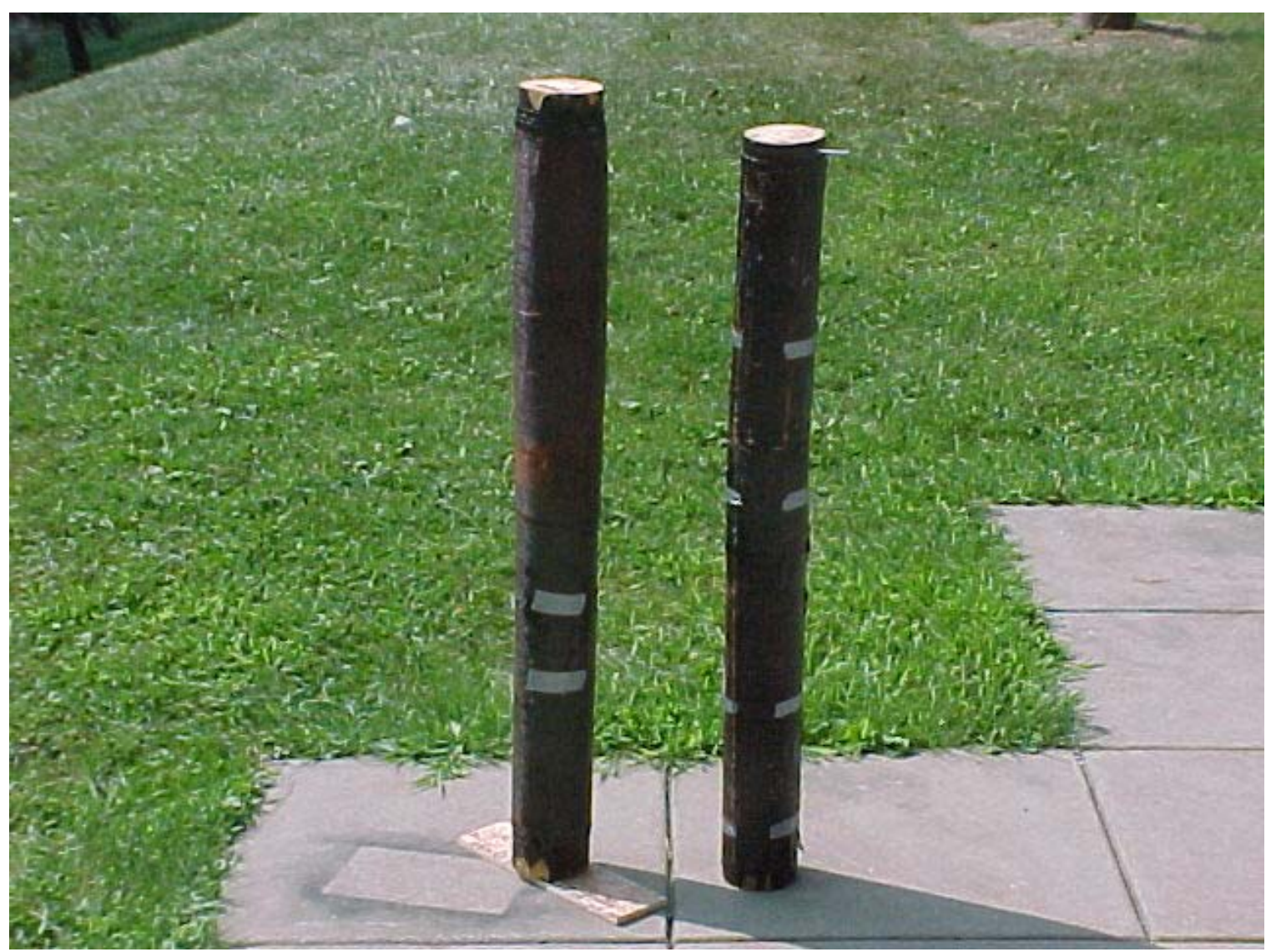

Figure 4.1.3 Specimens heated by solar radiation

\subsubsection{Greenhouse Effect}

A box made of clear plastic was created to produce a greenhouse effect (Figure 4.1.4). Specimens were placed in this simulated greenhouse during hot, sunny days between 11:00 AM and 3:00 PM. This method of heating produced surface temperatures 
around 40 or $45^{\circ} \mathrm{C}$. The temperature inside the simulated greenhouse was approximately 10 to 15 degrees warmer than the outside temperature. The specimens remained in the plastic greenhouse for approximately one hour. The simulated greenhouse was then removed and thermal imaging was performed.

The greenhouse effect refers to circumstances where the short wavelengths of visible light from the sun pass through a transparent medium (such as clear plastic) and are absorbed, but the longer wavelengths of infrared radiating from the heated objects are unable to pass through that medium. The trapping of this radiation leads to more heating and a higher resultant temperature.

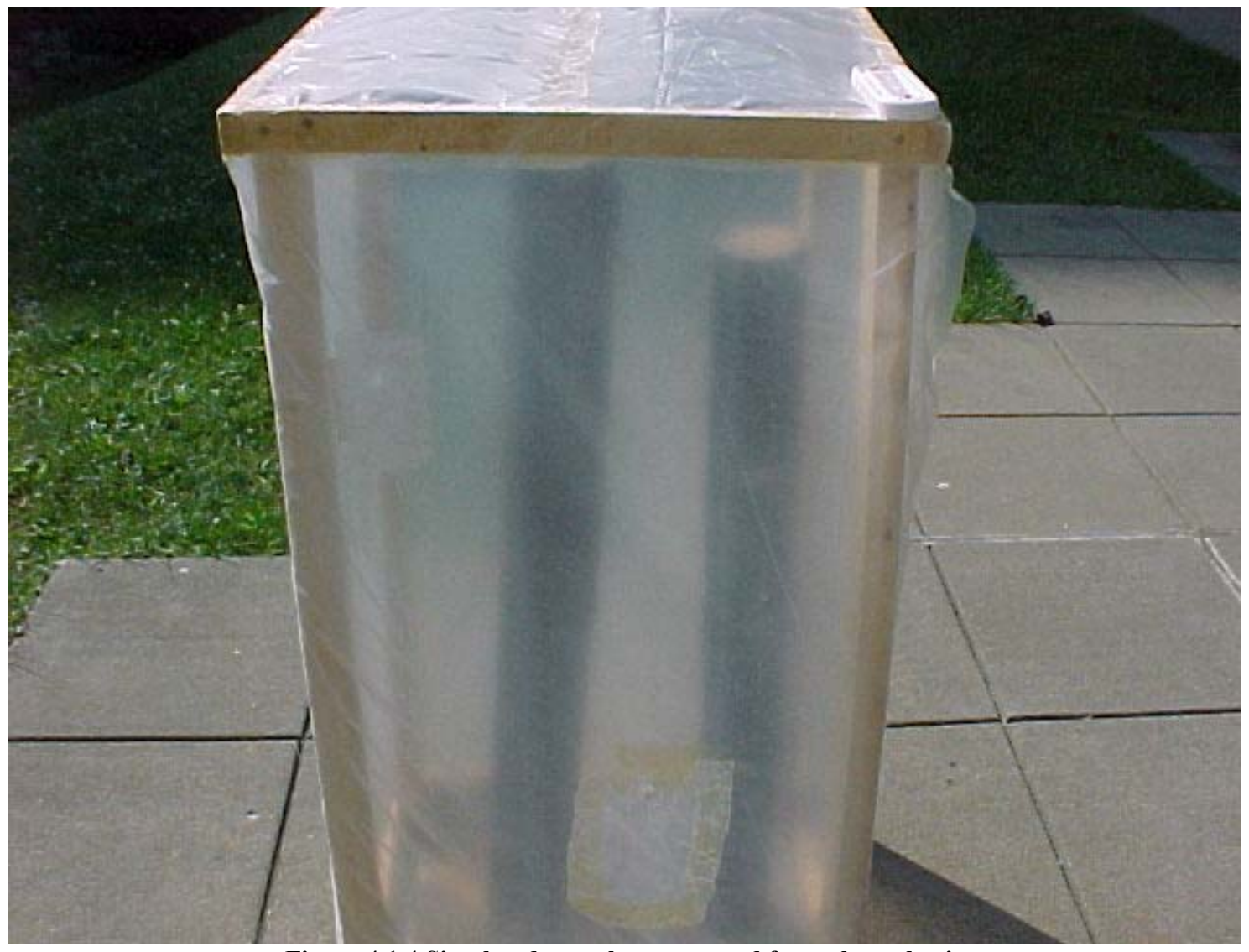

Figure 4.1.4 Simulated greenhouse created from clear plastic 


\subsubsection{Infrared Imaging System}

The thermal imager used for all experiments was the FLIR Prism Single Point Infrared Camera. This infrared camera is a Focal Plane Array (FPA) camera, which has a resolution of $0.2^{\circ} \mathrm{C}$ and can detect radiation in the medium wavelength ( 3 to 5 microns) spectral region. In addition, the camera provides the ability to remotely measure the absolute temperature of a specific point on the specimen in one of three different operating configurations (Live Crosspoint Temperature Display, Temperature Hold, or Peak Temperature Display). For these experiments the Live Crosspoint Temperature Display mode was used to measure the specimens surface temperature in Celsius.

This infrared camera provides two control buttons, black level and gain control buttons. The black level control allows the operator to select the particular temperature at which the viewed specimen becomes visible on the display screen, while the gain control allows the operator to select the width of the temperature spread to be viewed. The thermal images can then be viewed in a black and white mode or a color mode. The black and white mode is a gray scale, ranging from white (which is hot) to black (which is cold). The color mode gives different colors (white, pink, green, blue, black, etc.) with different intensities. As the surface temperature of the specimen increases, the color intensity increases. The color mode was mainly used for all thermal imaging.

A real-time image showing the relative intensity of the radiation was displayed on an 8-inch TV monitor. The TV monitor had a built-in VCR, which was connected to the infrared camera (Figure 4.1.1). The monitor was used to view the thermal images 
produced from the specimens during the heating cycles, while the VCR recorded the images. The infrared camera was mounted on a tripod to control the distance between the thermal imager and the heated samples. Figure 4.1.5 and Figure 4.1.6 shows photographs of the experimental set-ups.

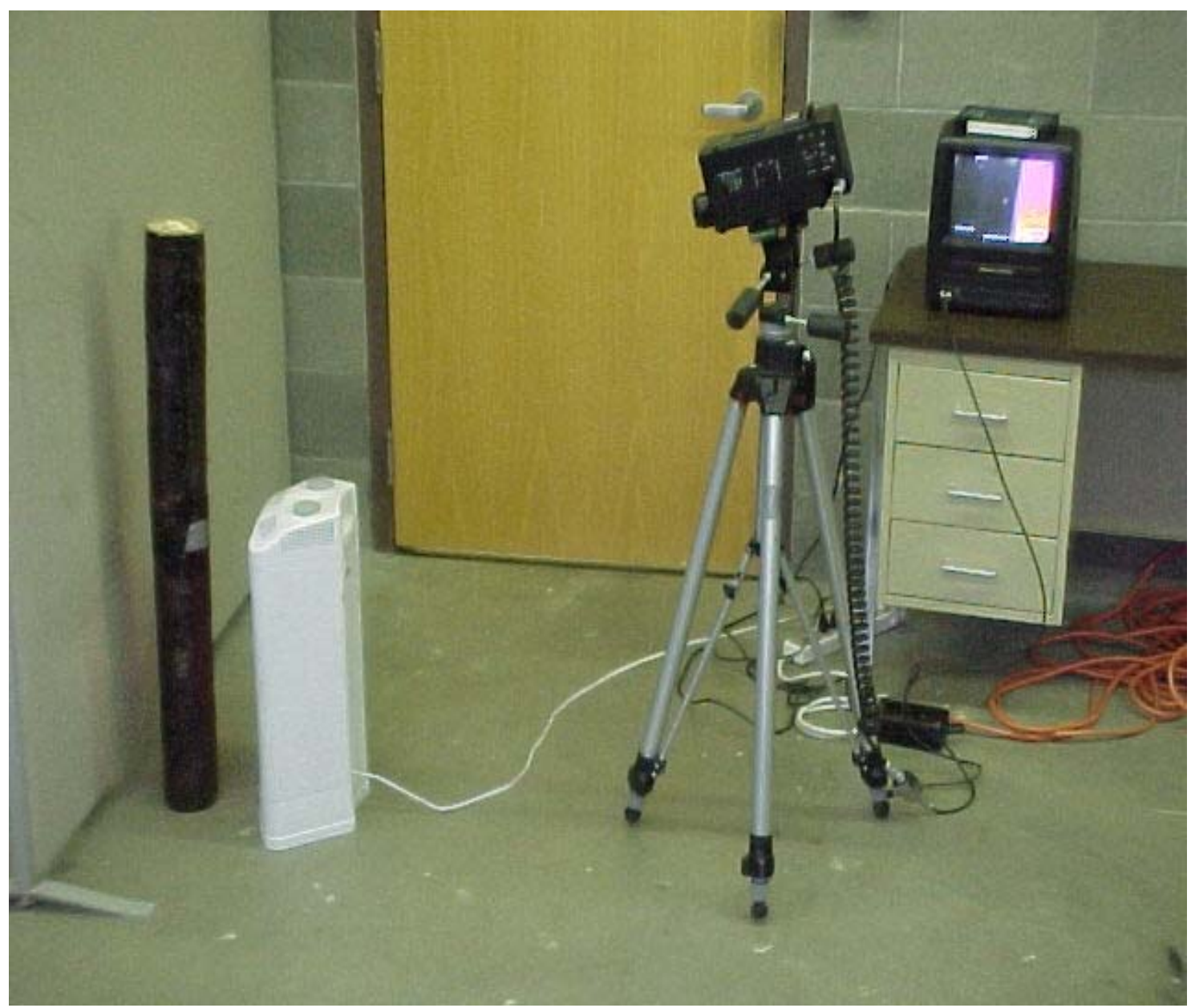

Figure 4.1.5 Experimental set-up using the quartz heater to heat specimens 


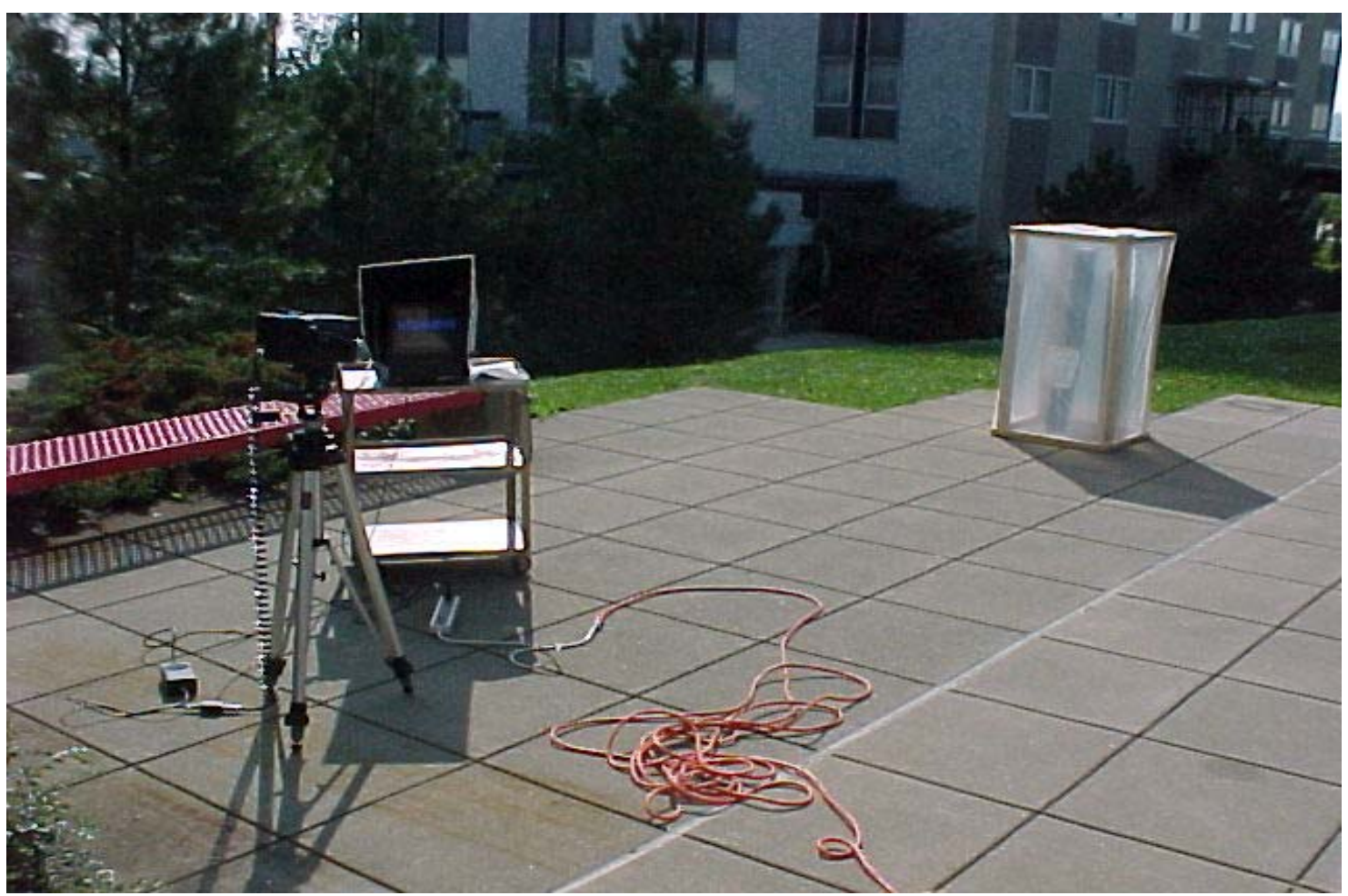

Figure 4.1.6 Experimental set-up using the sunlight and a simulated greenhouse to heat the specimens

\subsection{Specimen Set-up}

Three timber piles wrapped with GFRP composite fiber were tested in the laboratory. The timber piles were approximately 4 inches in diameter. The composite wrap was approximately $1 / 8$ inch thick and made of glass reinforced fabrics and phenolic resin composites. Two timber piles were created with simulated delaminations, while the third specimen had no known defects. The delaminations were created with different sizes and severity to study the effects of these variables and others on the infrared nondestructive testing technique.

\subsubsection{Specimen 1}

This timber pile had four simulated delaminations created under the composite wrap. The delaminations were of different size and thickness. Figure 4.2.1 shows the 
specimen with the composite wrap removed. A cordless saw and a screwdriver were used to remove the wrap from the timber pile so the delaminations could be created. A cordless screwdriver with a $1-1 / 2$ inch drill bit was used to create delaminations beneath the composite wrap (illustrated in Figure 4.2.2). All the delaminations were 0.125" below the surface; however, the delaminations ranged in size from 1.5" in diameter to 3 " $\mathrm{x} 1.5$ " in size, and from 0.125 " thick delaminations to 0.375 " thick delaminations. To prevent the simulated delaminations from filling with resin, a thin plastic film was used to cover the pockets (illustrated in Figure 4.2.3). Figure 4.2.4 shows the specimen once the composite wrap was reapplied.

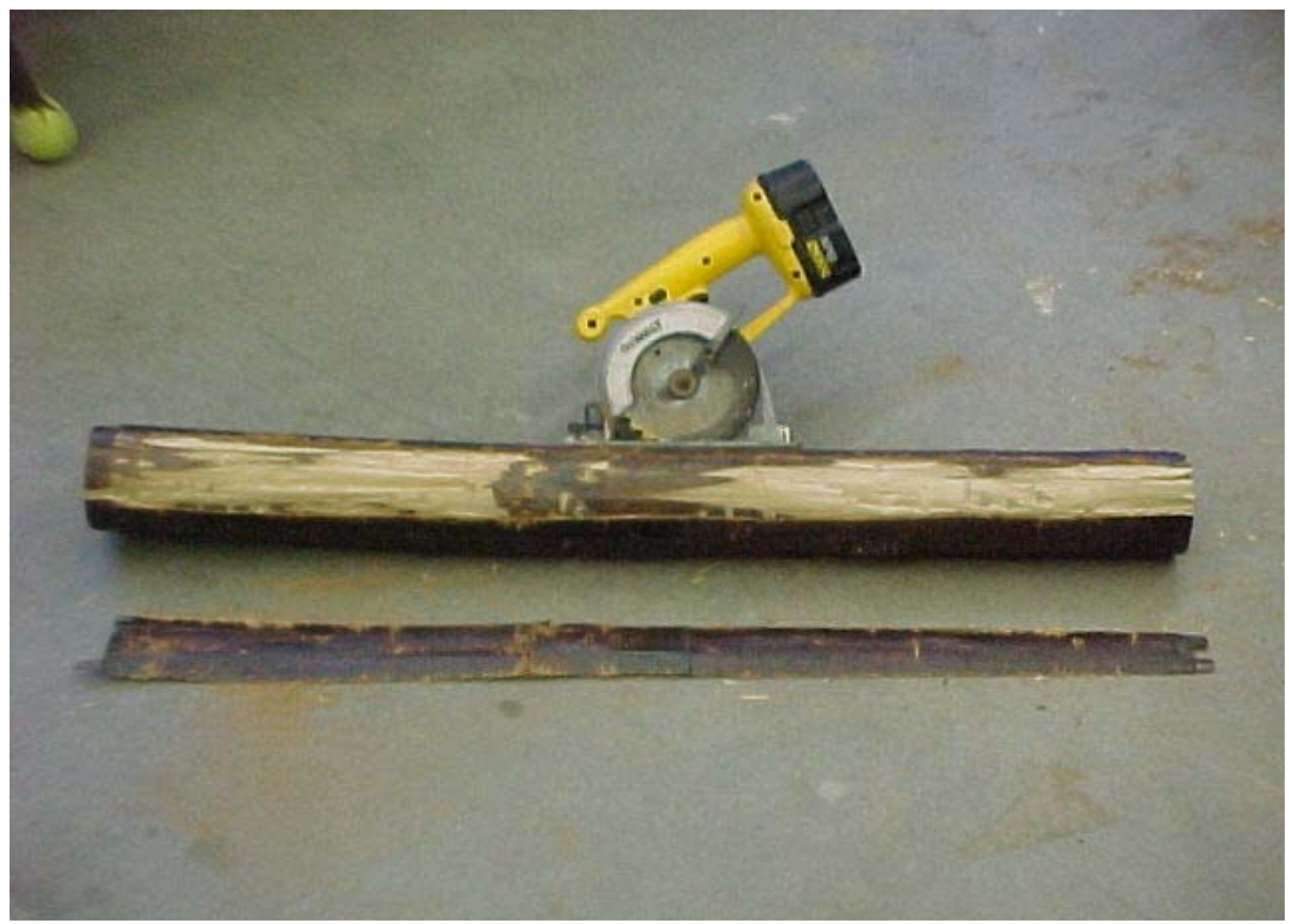

Figure 4.2.1 Cordless saw used to remove composite wrap 


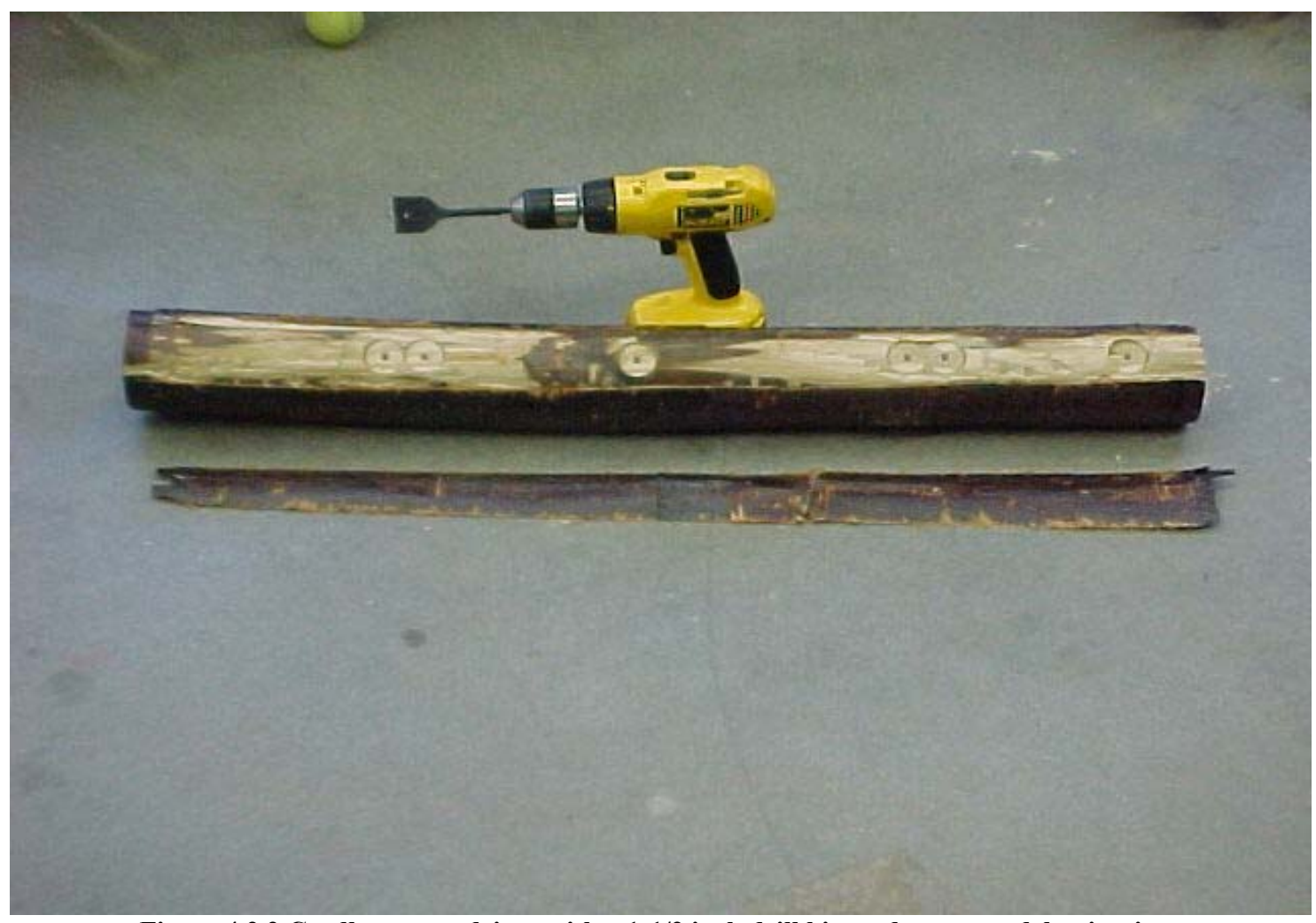

Figure 4.2.2 Cordless screwdriver with a 1-1/2 inch drill bit used to create delaminations

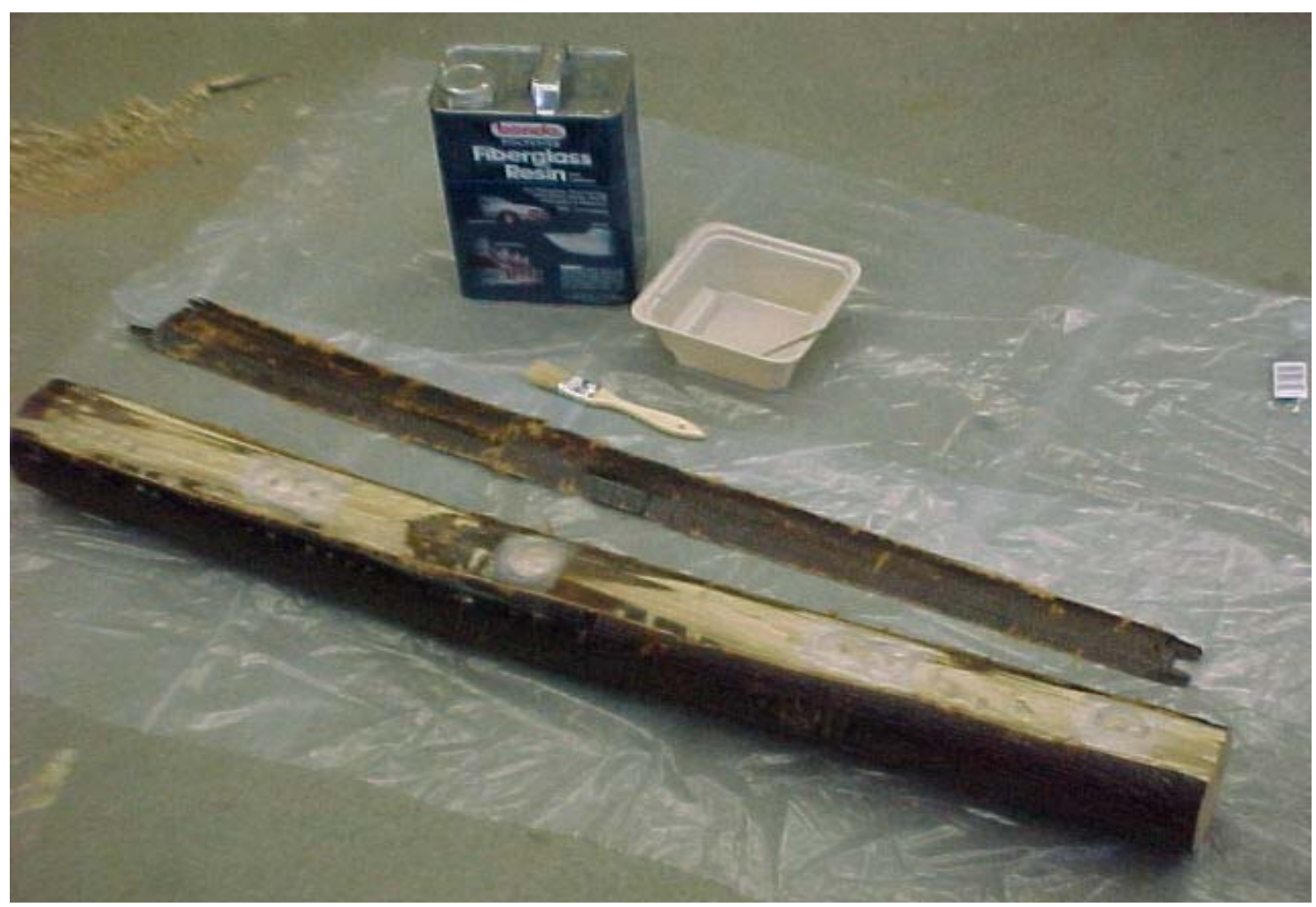

Figure 4.2.3 A thin plastic film was used to cover the pockets to prevent them from filling with resin 


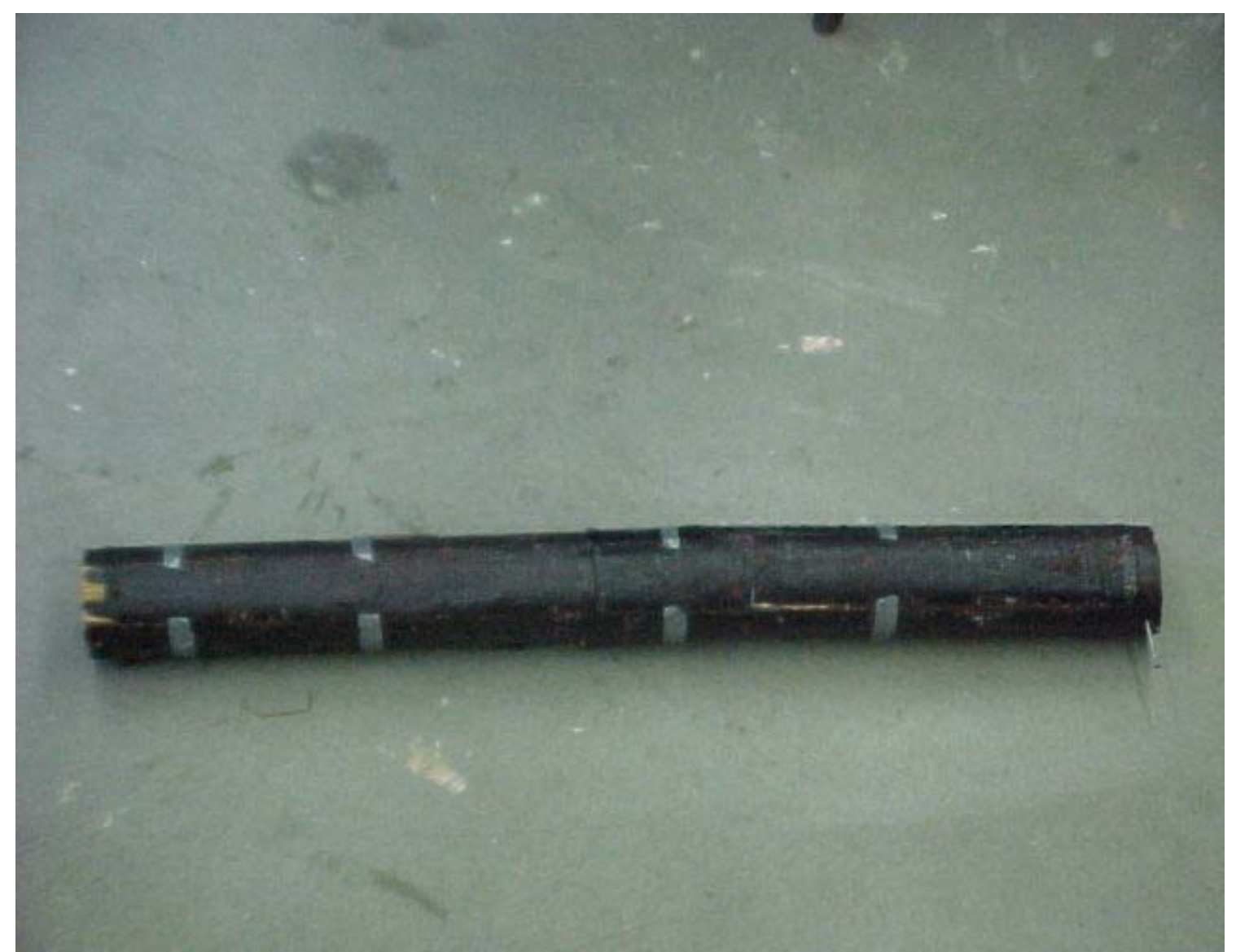

Figure 4.2.4 Photograph of the specimen with the composite wrap reapplied and duct tape marking the delaminations

\subsubsection{Specimen 2}

This specimen also had a simulated delamination. The delamination was approximately 3 " x 3", which was created using a screwdriver to pry apart the composite wrap from the timber surface (Figure 4.2.5). The composite wrap was an 1/8 inch thick; therefore, the delamination was approximately $1 / 8$ inch deep. Once the delamination was created the cracked edges were filled in with resin to prevent air from entering or escaping the delamination. 


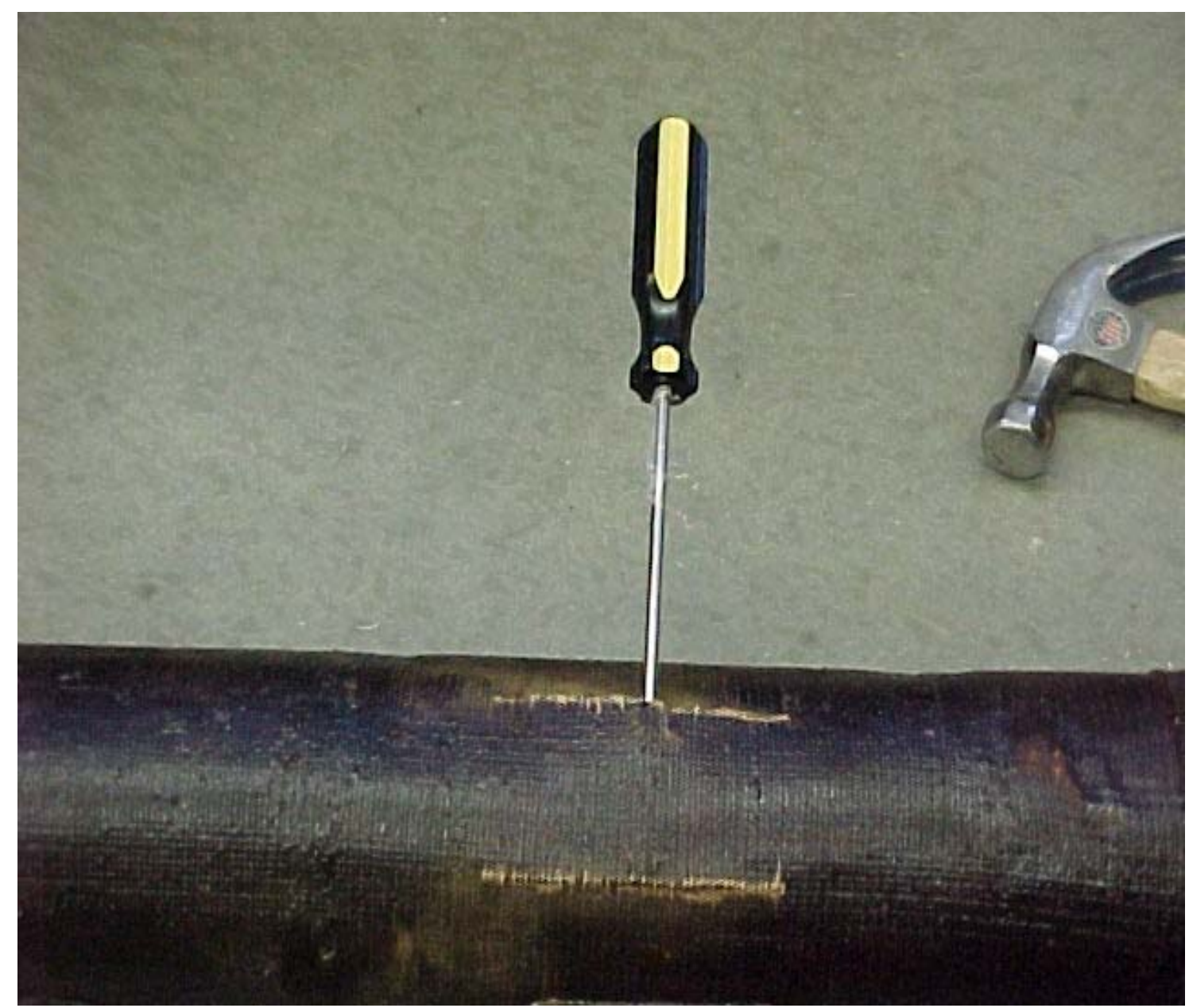

Figure 4.2.5 Creating a delamination by prying composite wrap away from timber surface

\subsubsection{Specimen 3}

The third specimen had no known defects. It had the same composite wrap as the other two specimens ( $1 / 8$ inch thick). This specimen was used to compare the thermal images of damaged timber piles wrapped with GFRP fabric with the thermal images of an undamaged timber pile wrapped with GFRP fabric. 


\subsection{Experimental Results}

All three specimens were subjected to three different heating methods. The following three sections discuss experimental results obtained using the different heating methods.

\subsubsection{Quartz Tower Heater}

The three specimens were tested using a quartz tower heater to create thermal gradients and a FLIR Prism Single Point Infrared Camera to capture the thermal images. The three specimens were all heated to approximately $65^{\circ} \mathrm{C}$ before thermal imaging was performed. All the simulated delaminations were detected using this type of heating method.

The first specimen (specimen 1) had four simulated delaminations and Figure 4.3.1 is the thermal image of the first simulated delamination. The surface temperature of the first defect, recorded by the infrared camera, was $66.1{ }^{\circ} \mathrm{C}$. Figure 4.3 .2 shows the thermal image of the second simulated delamination. The surface temperature of this defect was $57.9{ }^{\circ} \mathrm{C}$. The first delamination was $3 " \mathrm{x} 1.5$ " and 0.375 " thick causing it to have a higher surface temperature than the second delamination, which was 1.5 " in diameter and 0.125 " thick. The surface temperature of the bonded area, between the first and second delamination was $49.0^{\circ} \mathrm{C}$ (illustrated in Figure 4.3.3). Figure 4.3.4 shows the section of the specimen that was tested first. The piece of duct tape closest to the floor in Figure 4.3.4 represents the first delaminated area and the piece of duct tape near the center of the specimen represents the second delaminated area. 


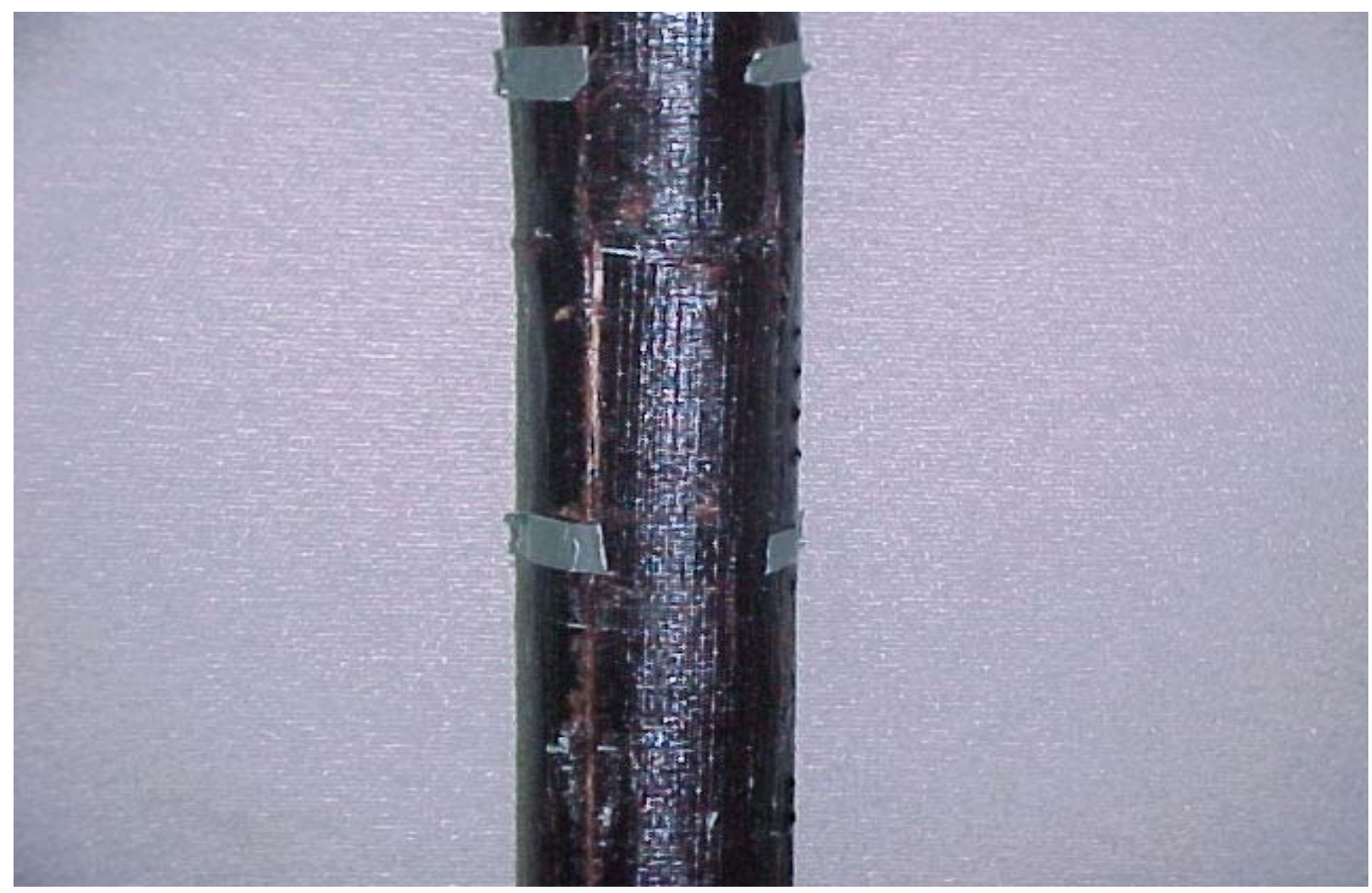

(a)

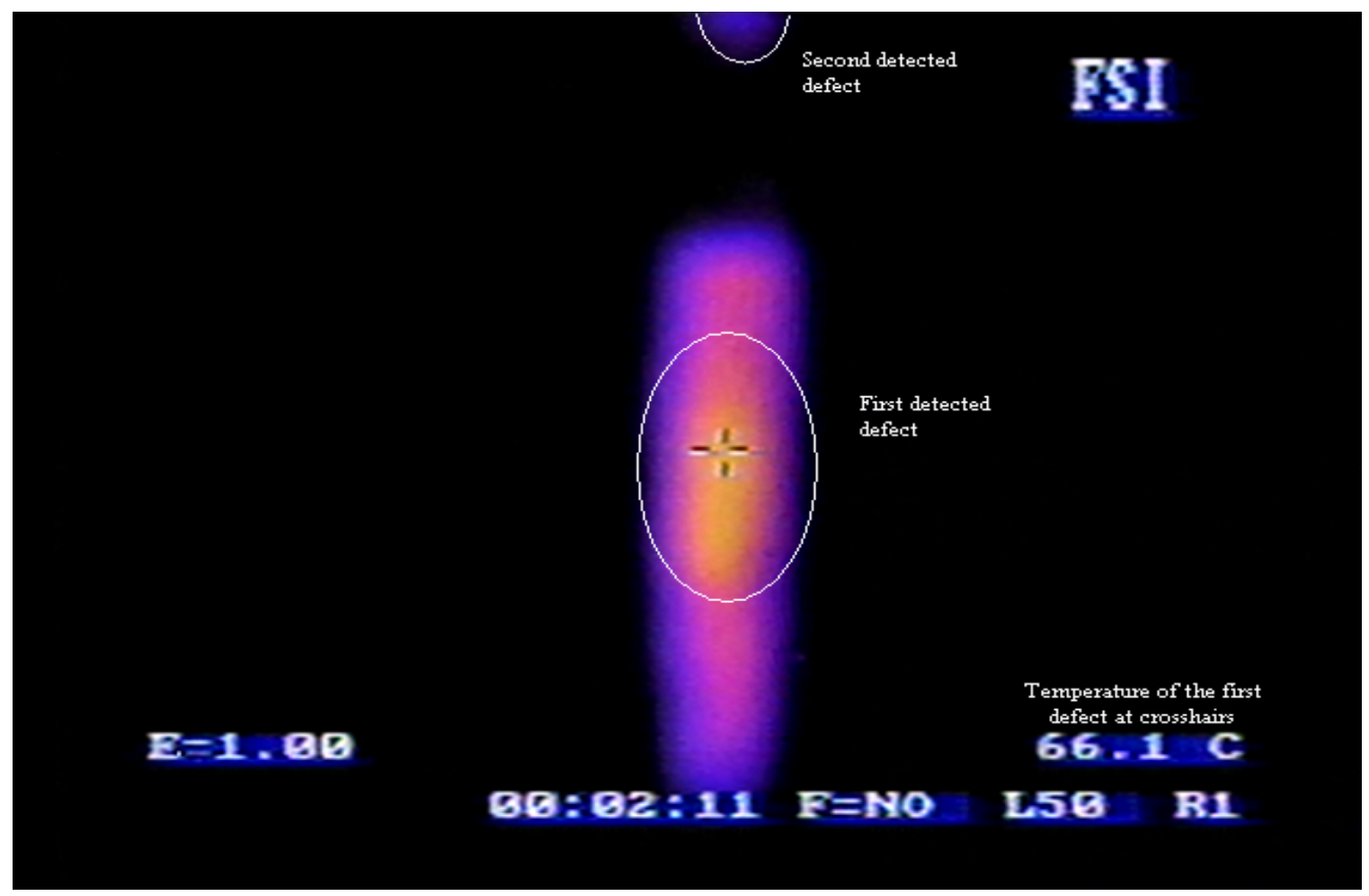

(b)

Figure 4.3.1 (a) Photograph of the surface where the first simulated delamination was placed on specimen 1, and (b) thermal image of the first simulated delamination detected by the infrared camera 


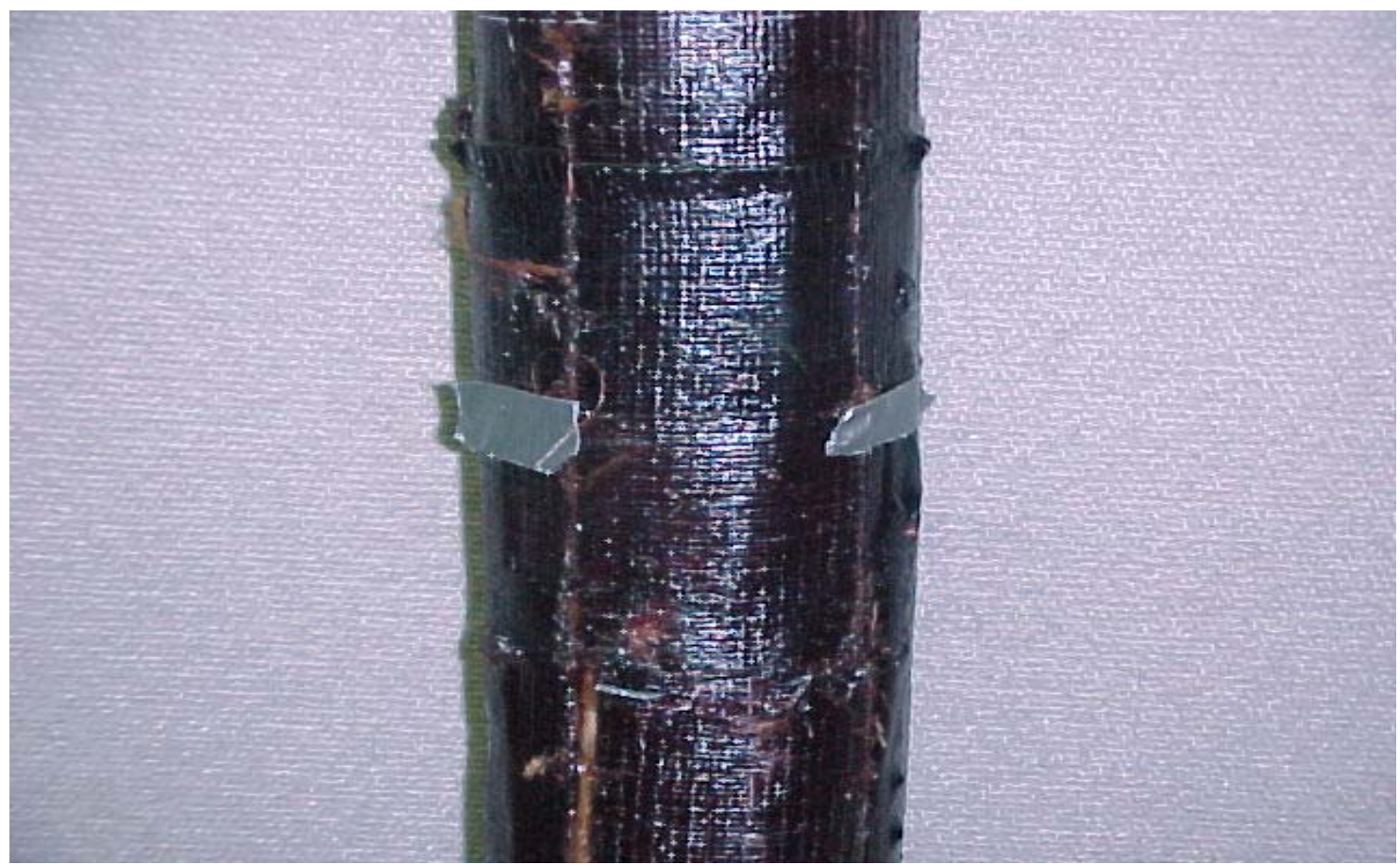

(a)

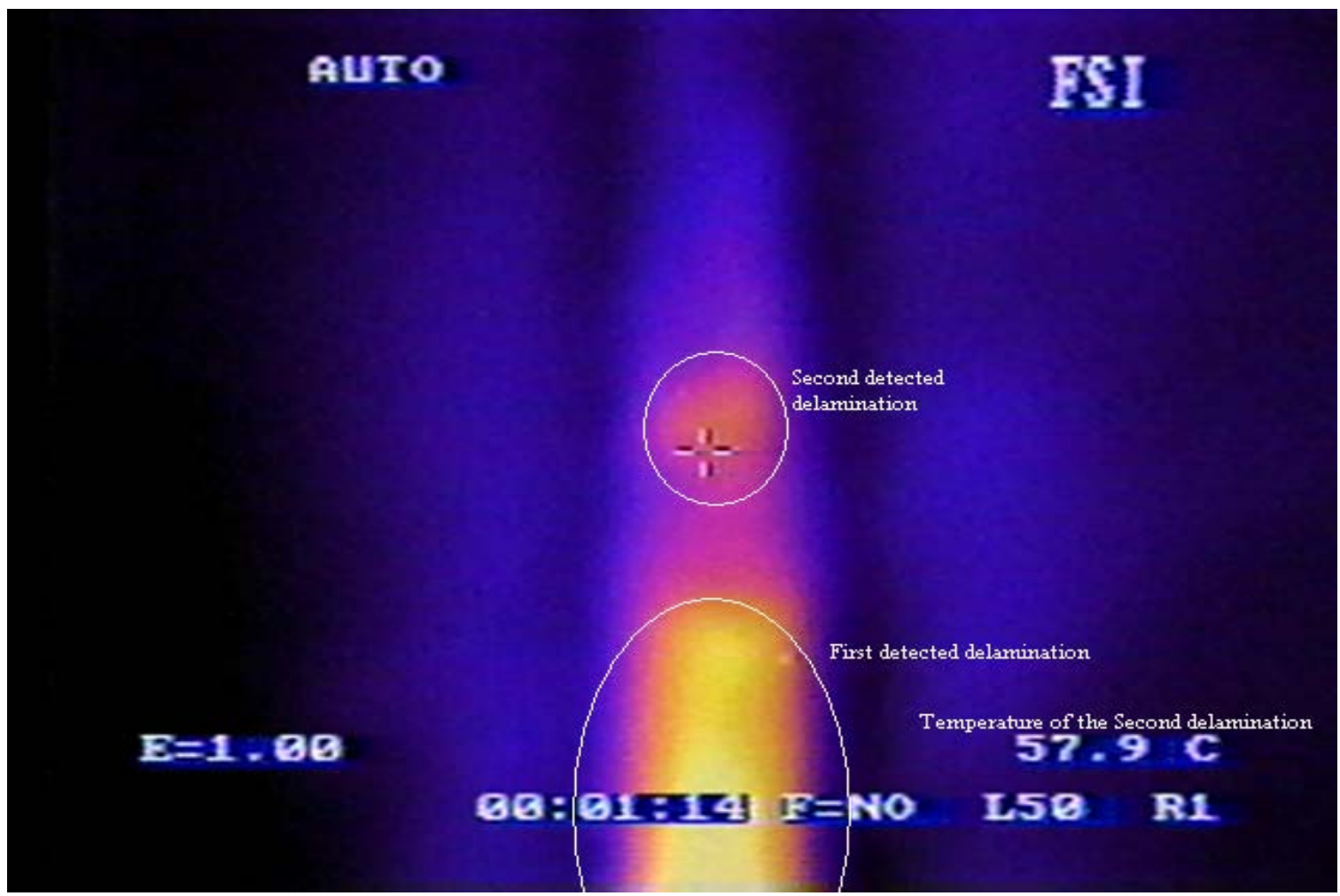

(b)

Figure 4.3.2 (a) Photograph of the surface where the second simulated delamination was placed on specimen 1, and (b) thermal image of the second simulated delamination detected by the infrared camera 


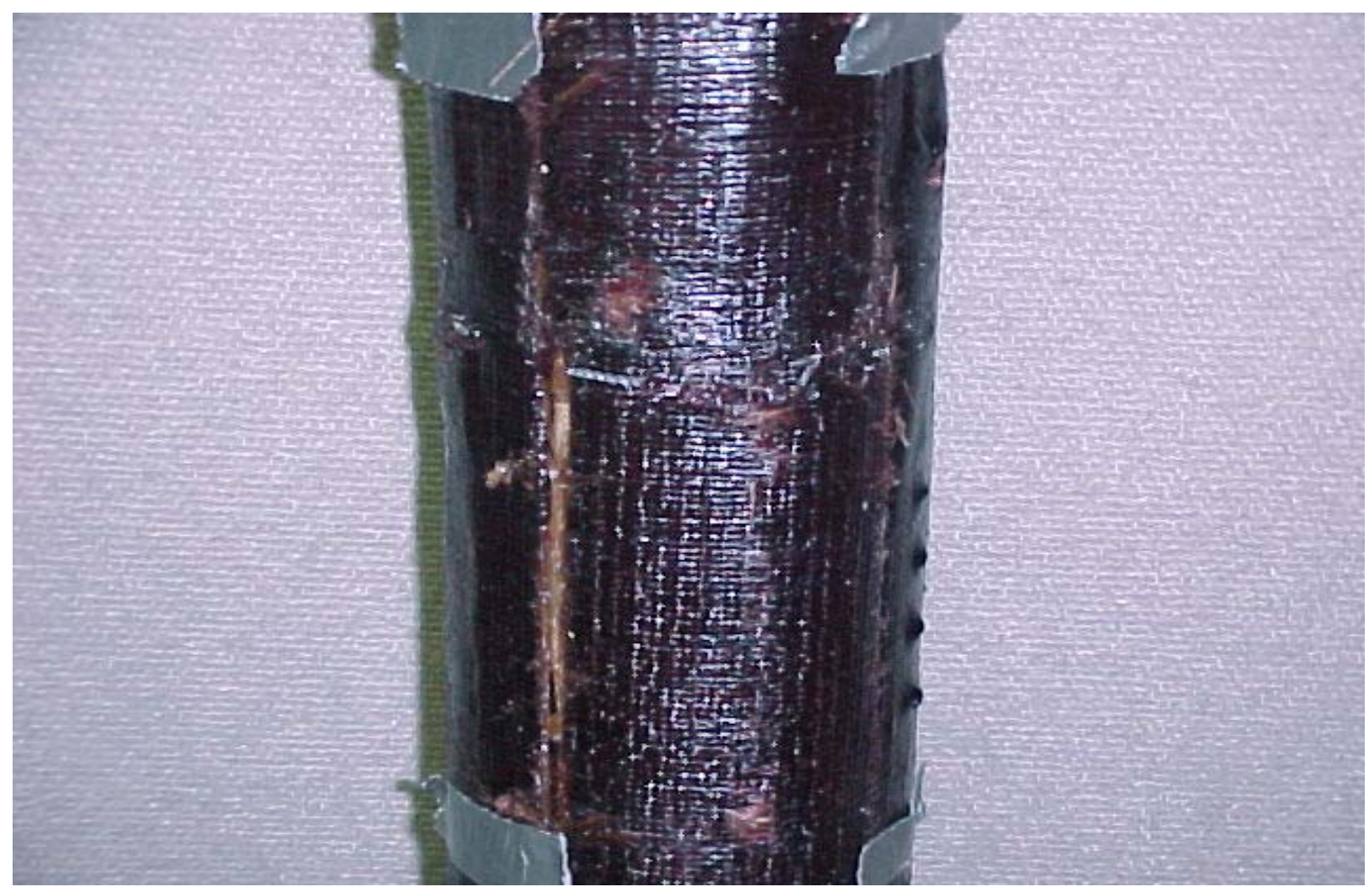

(a)

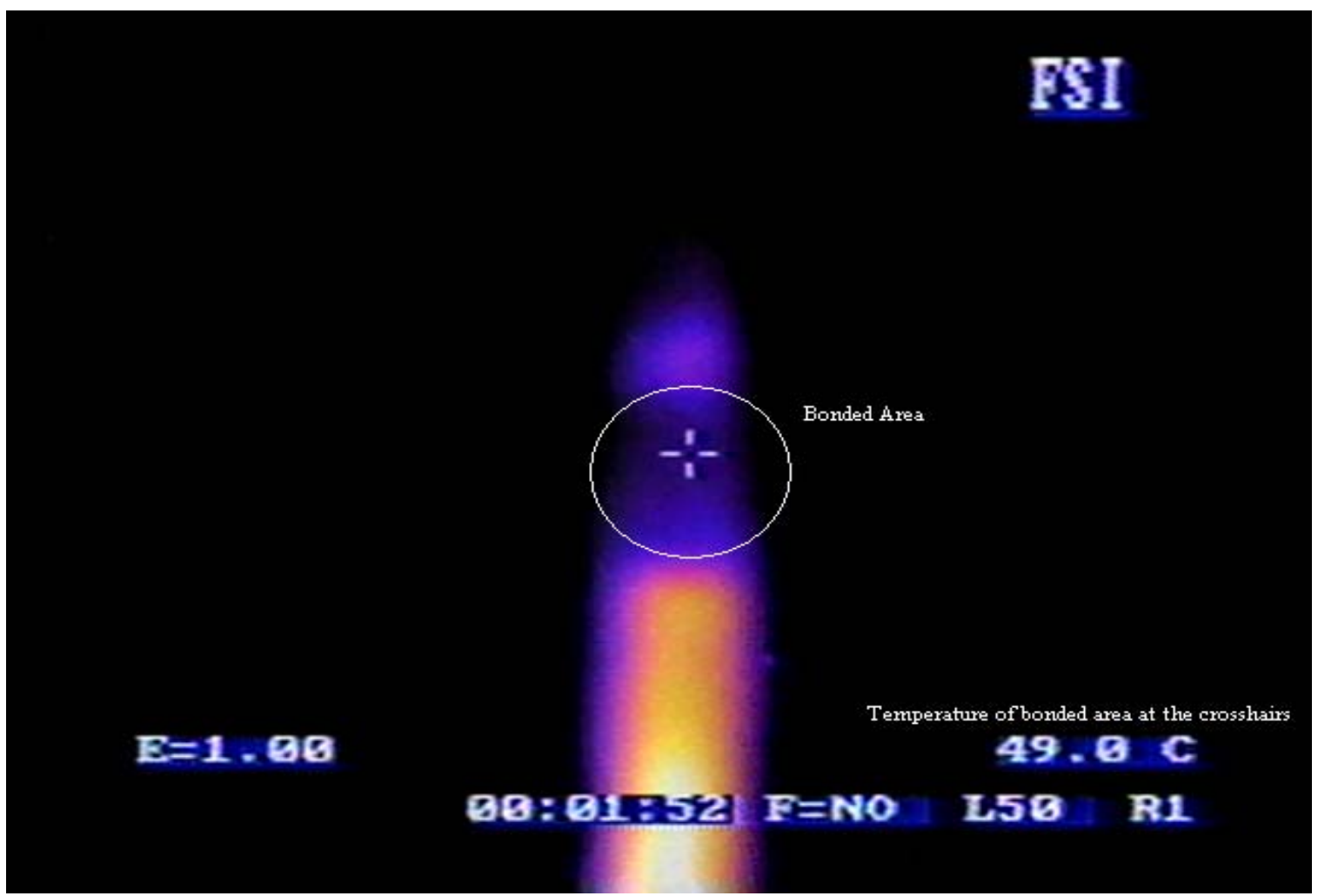

(b)

Figure 4.3.3 (a) Photograph of the surface between the first and second delamination on specimen 1, and (b) thermal image of the bonded area between the first and second delamination 


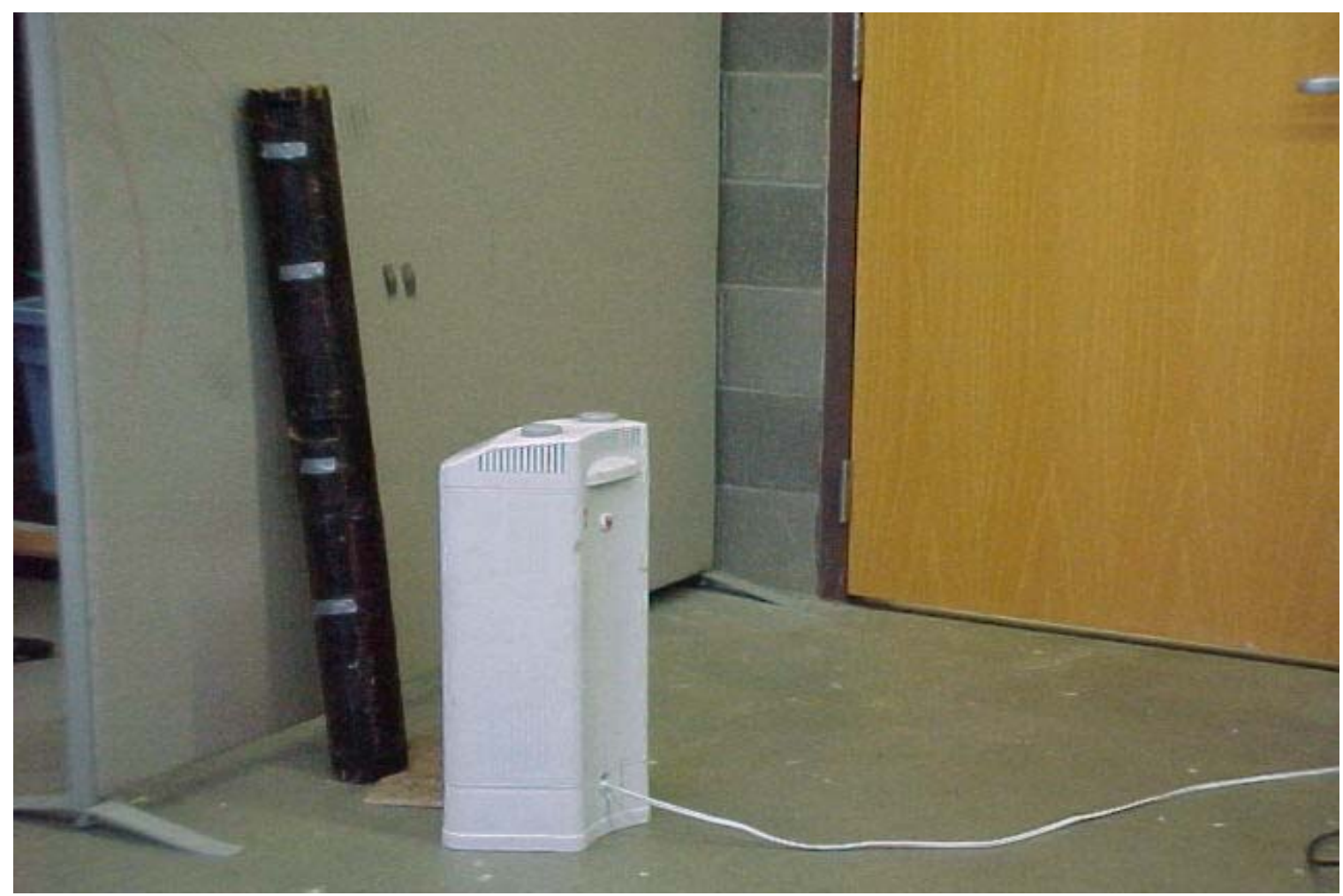

Figure 4.3.4 First and second delamination on specimen 1 (marked with duct tape) being heated by the quartz tower heater

The first specimen was then rotated 180 degrees so that the third and fourth delaminations were next to the floor (illustrated in Figure 4.3.8). In this case, the fourth delamination was marked with the piece of duct tape closest to the floor and the third delamination was marked with the piece of duct tape second from the floor (illustrated in Figure 4.3.8). Figure 4.3.5 shows the thermal image of the third simulated delamination. This delamination had a surface temperature of $62.0{ }^{\circ} \mathrm{C}$. The thermal image of the fourth delamination is shown in Figure 4.3.6. The surface temperature for the fourth delamination was $52.2^{\circ} \mathrm{C}$. The size of the third delamination was 3 " $\mathrm{x} 1.5$ " and 0.125 " thick, and the size of the fourth delamination was 1.5 " in diameter and 0.375 " thick. The thermal image of the bonded area between the third and fourth delamination is shown in Figure 4.3.7. This area had a surface temperature of $46.7^{\circ} \mathrm{C}$. 


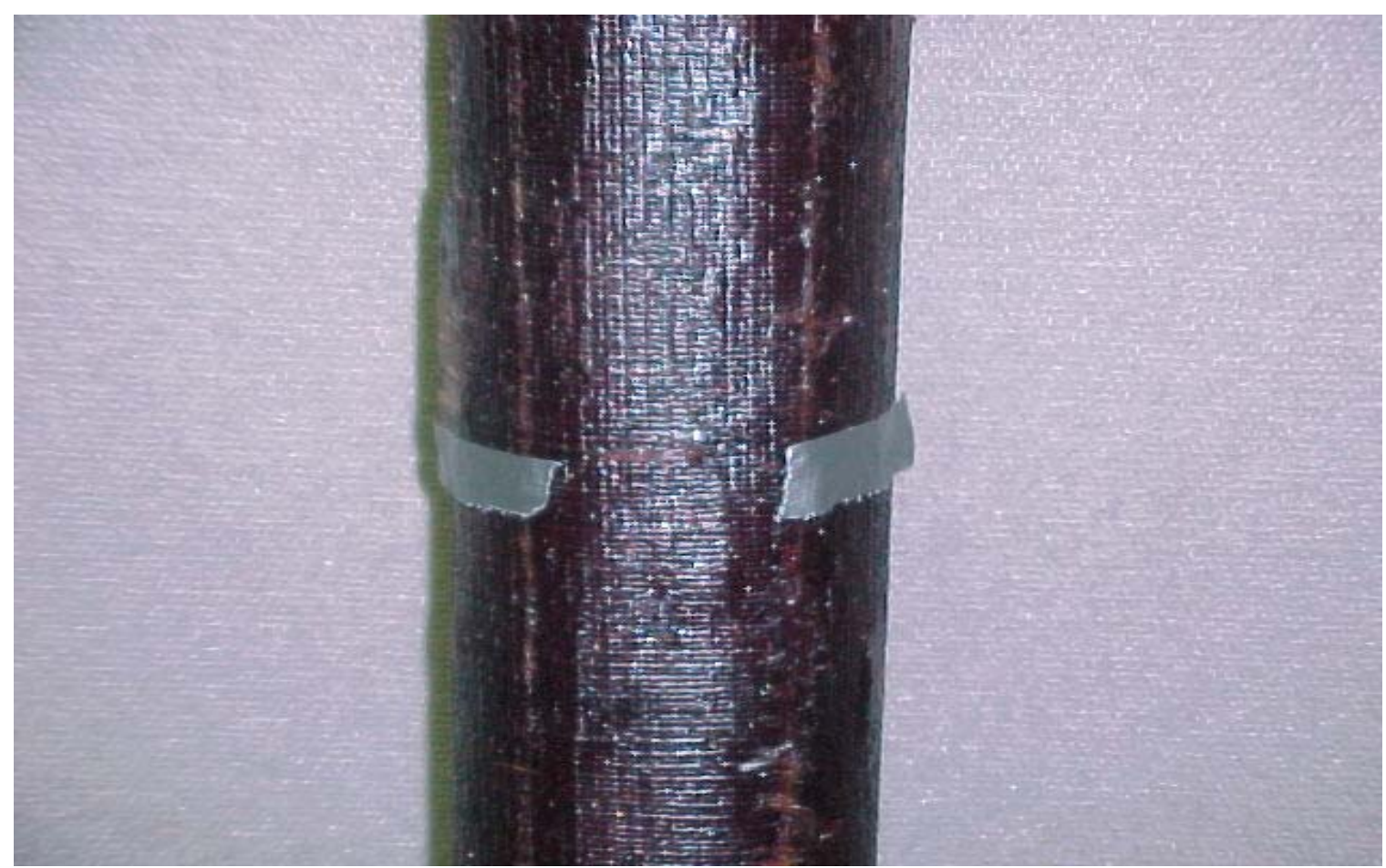

(a)

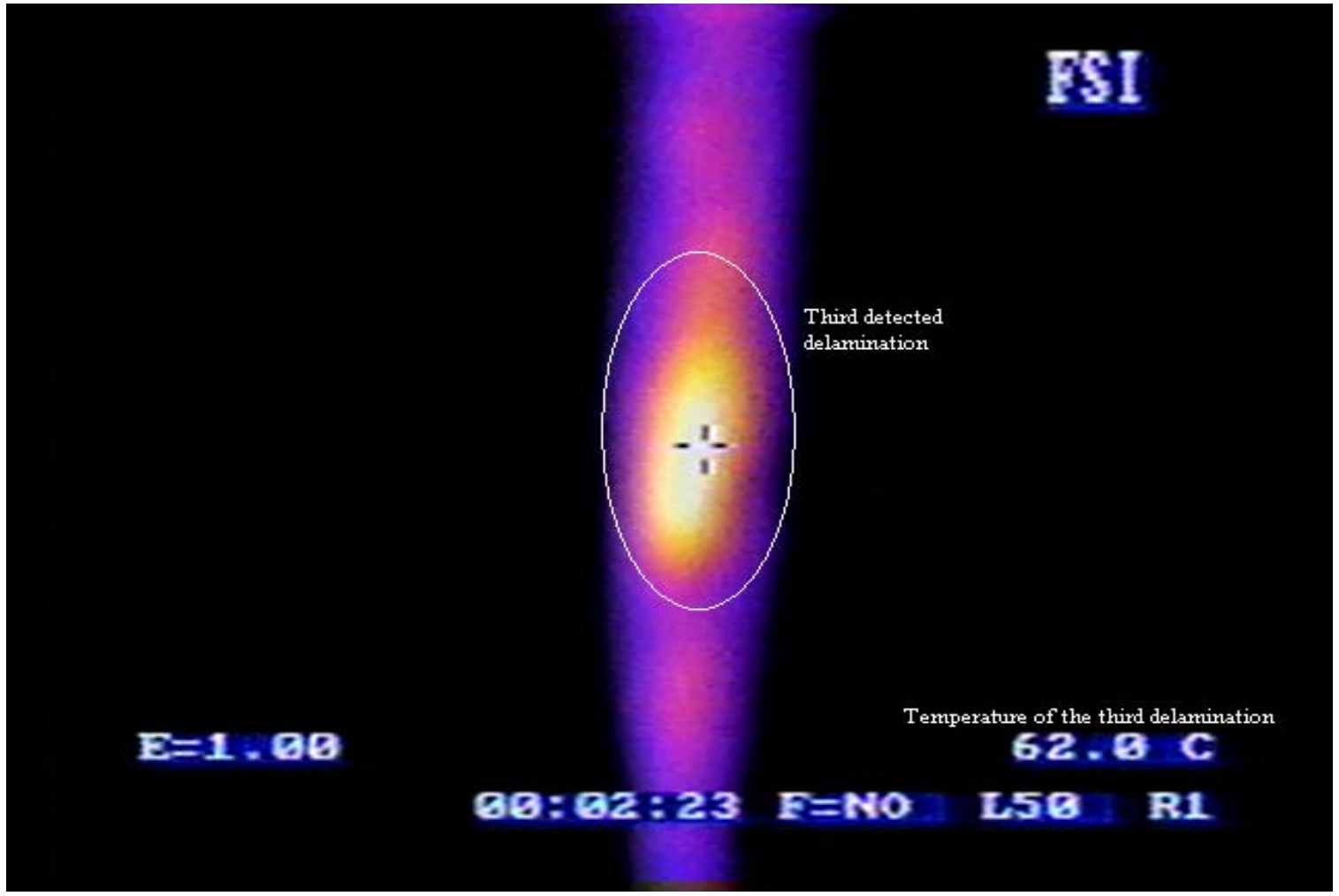

(b)

Figure 4.3.5 (a) Photograph of the surface where the third simulated delamination was placed on specimen 1, and (b) thermal image of the third simulated delamination detected by the infrared camera 


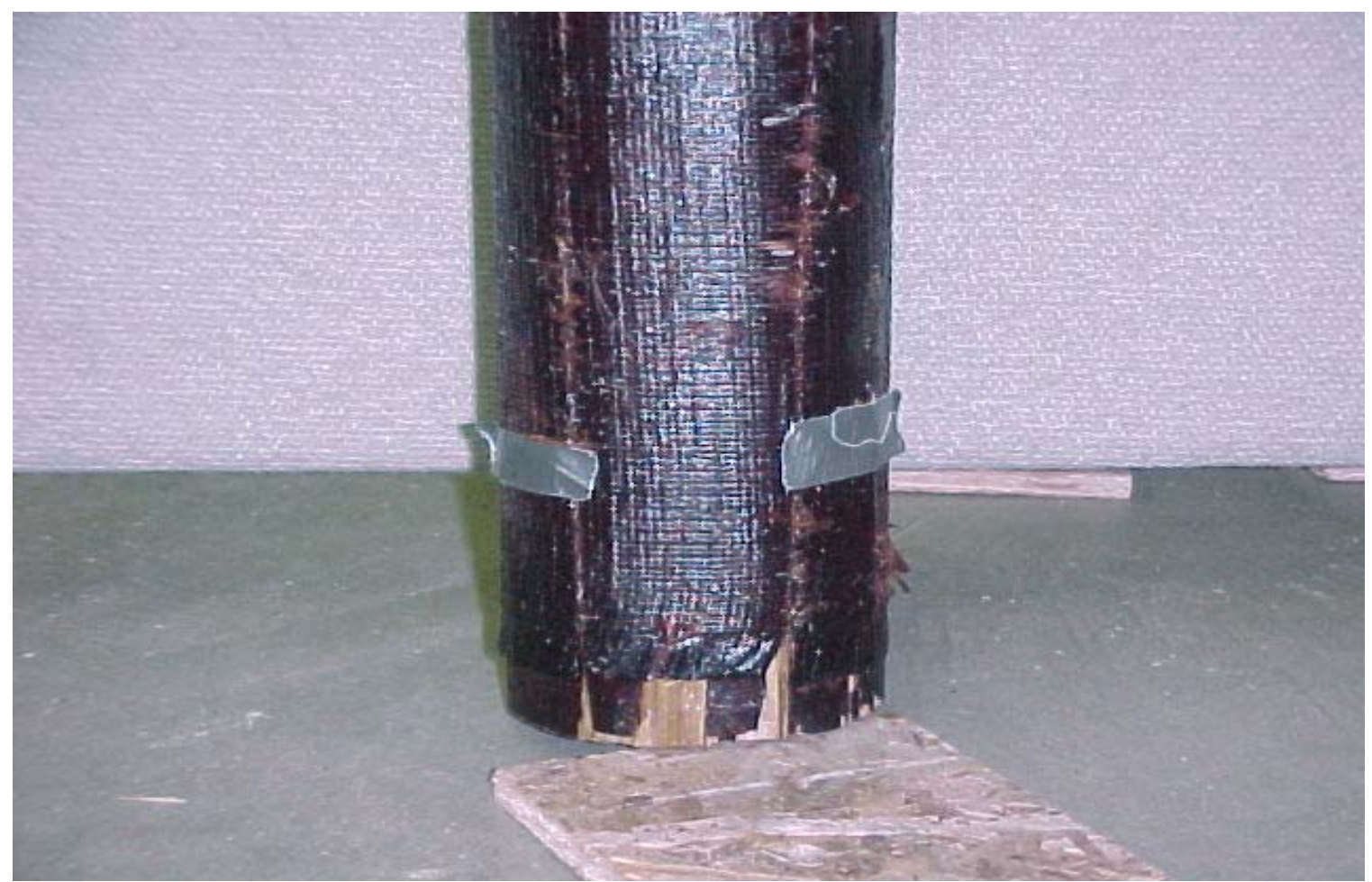

(a)

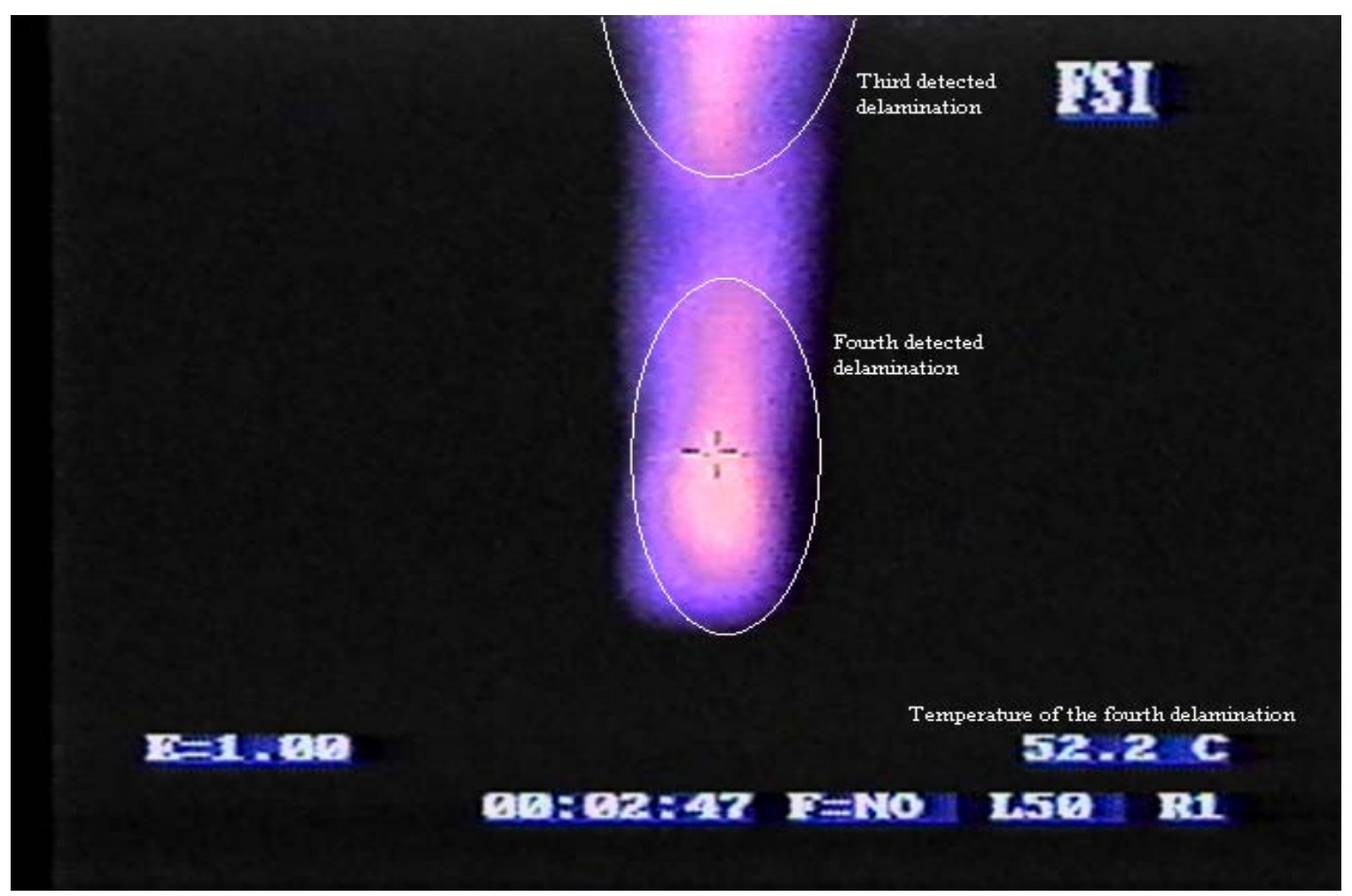

(b)

Figure 4.3.6 (a) Photograph of the surface where the fourth simulated delamination was placed on specimen 1, and (b) thermal image of the fourth simulated delamination detected by the infrared camera 


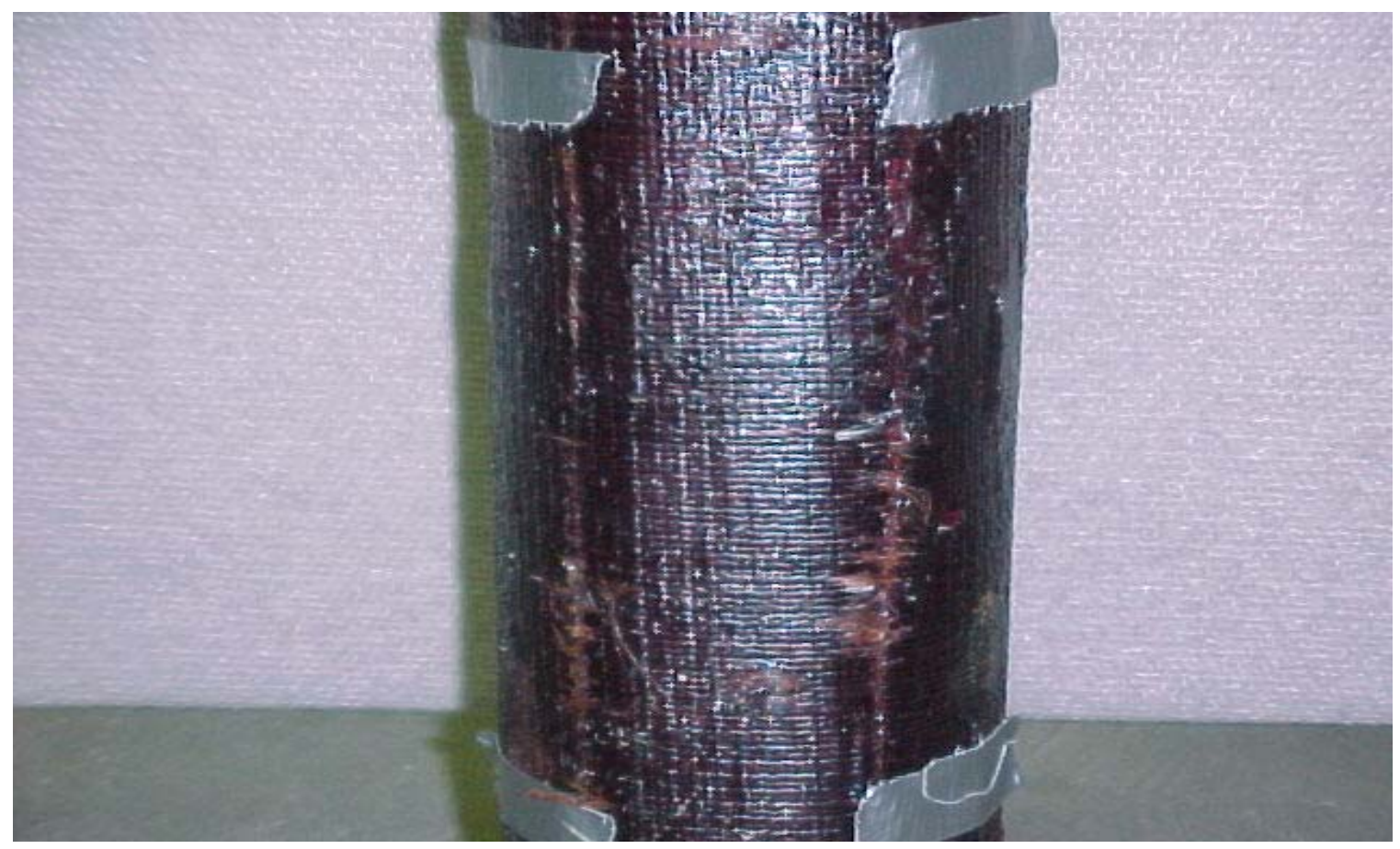

(a)

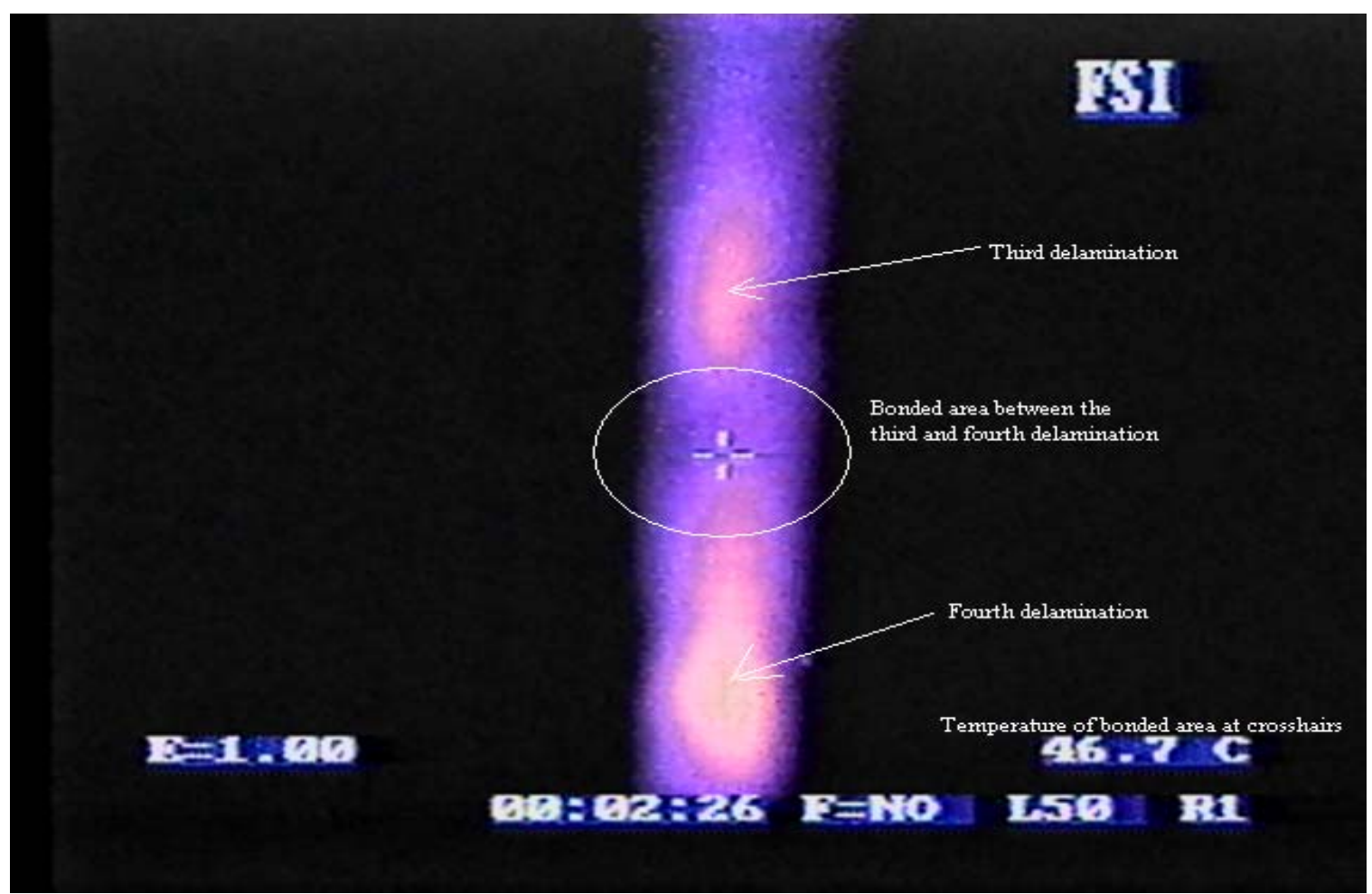

(b)

Figure 4.3.7 (a) Photograph of the surface between the third and fourth delamination on specimen 1, and (b) thermal image of the bonded area between the third and fourth delamination 


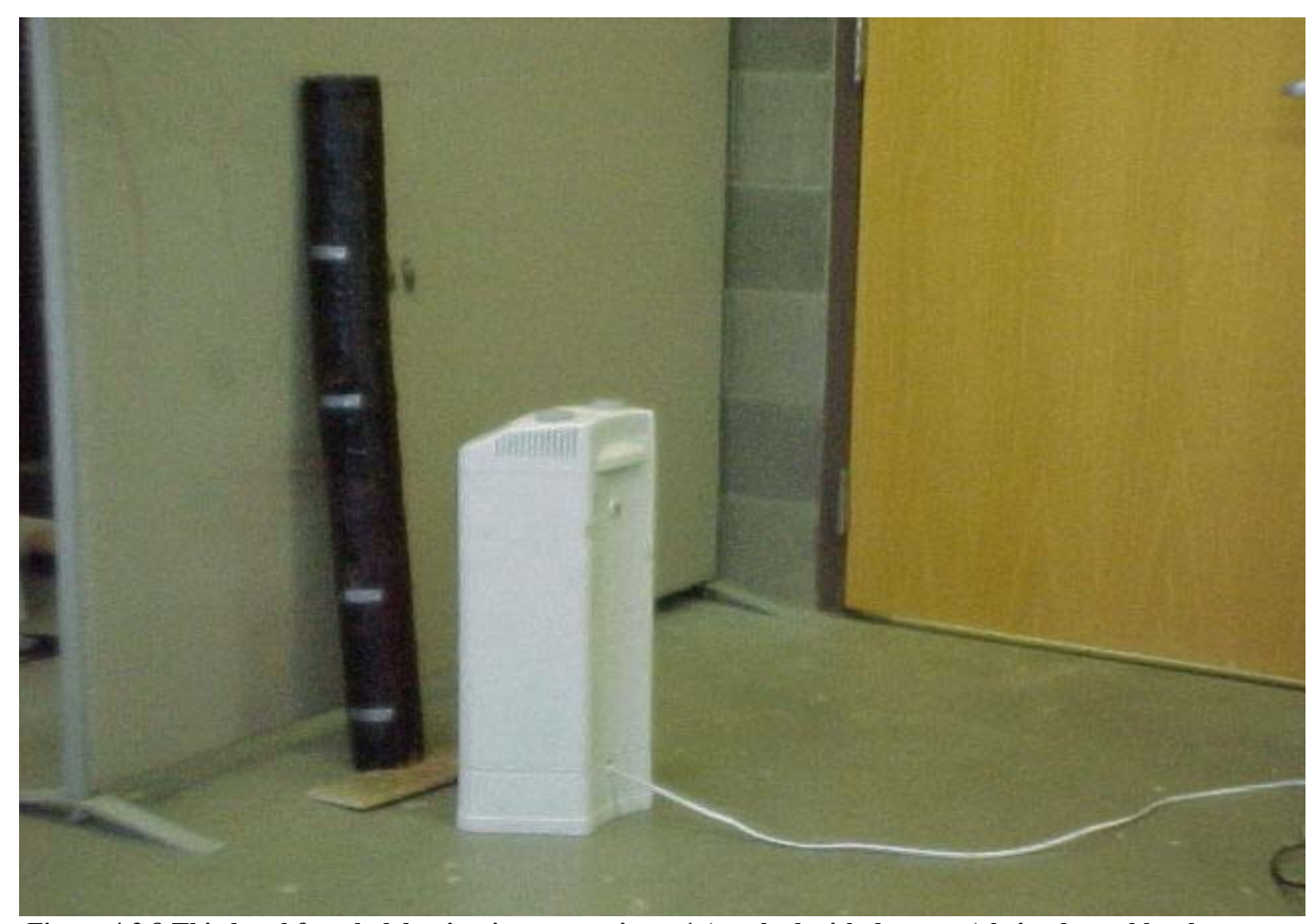

Figure 4.3.8 Third and fourth delamination on specimen 1 (marked with duct tape) being heated by the quartz tower heater

The next specimen that was tested (specimen 2) had one simulated defect (see

Figure 4.2.5). Again, this defect was easily detected using the quartz tower heater and the FLIR Prism Single Point Infrared Camera. Figure 4.3.9 shows the thermal image recorded by the infrared camera. This delamination had a surface temperature of $53.3{ }^{\circ} \mathrm{C}$. The size of this defect was approximately 3" x 3" with a relatively small thickness (approximately $0.125 "$ thick). 


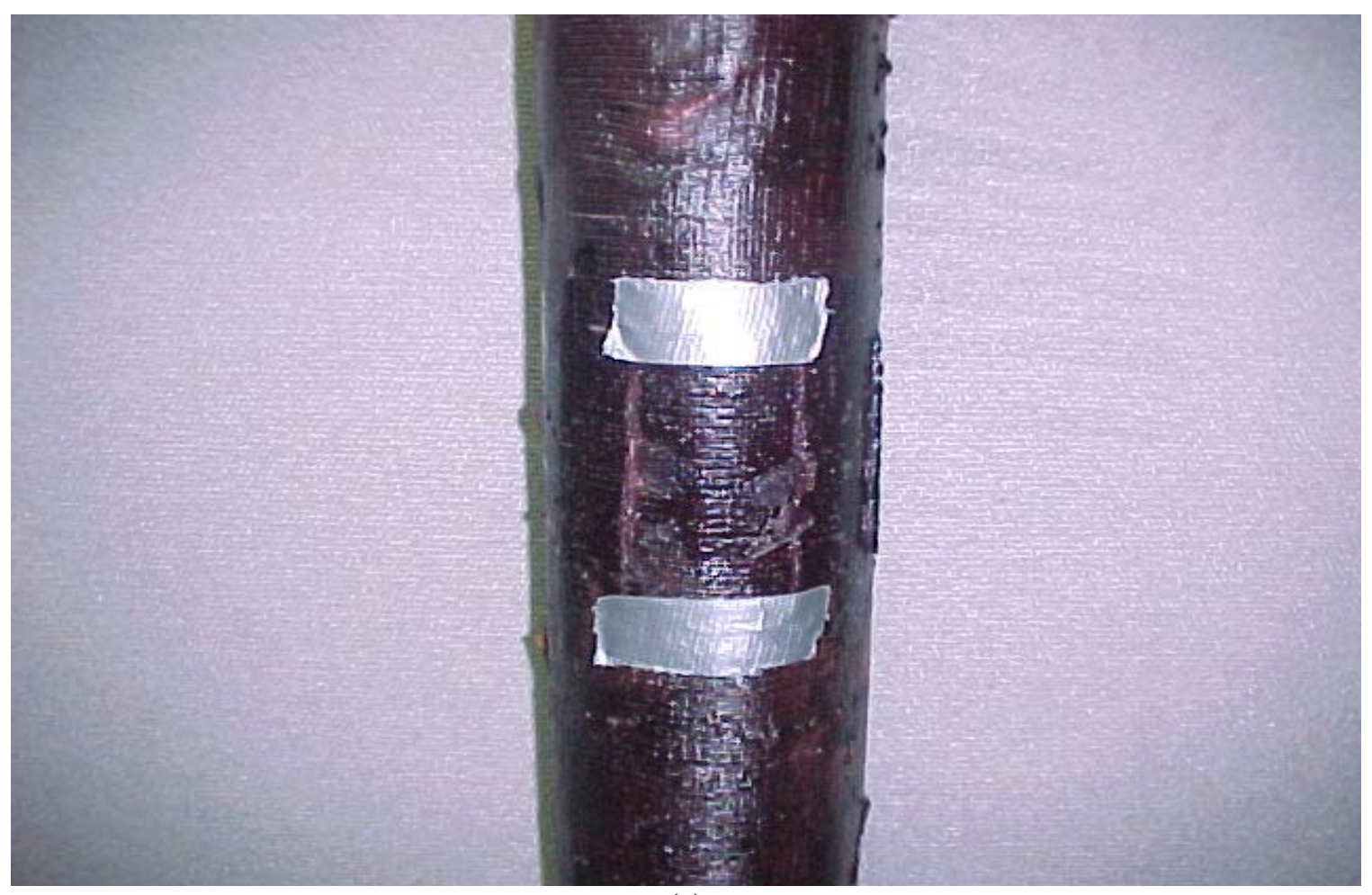

(a)

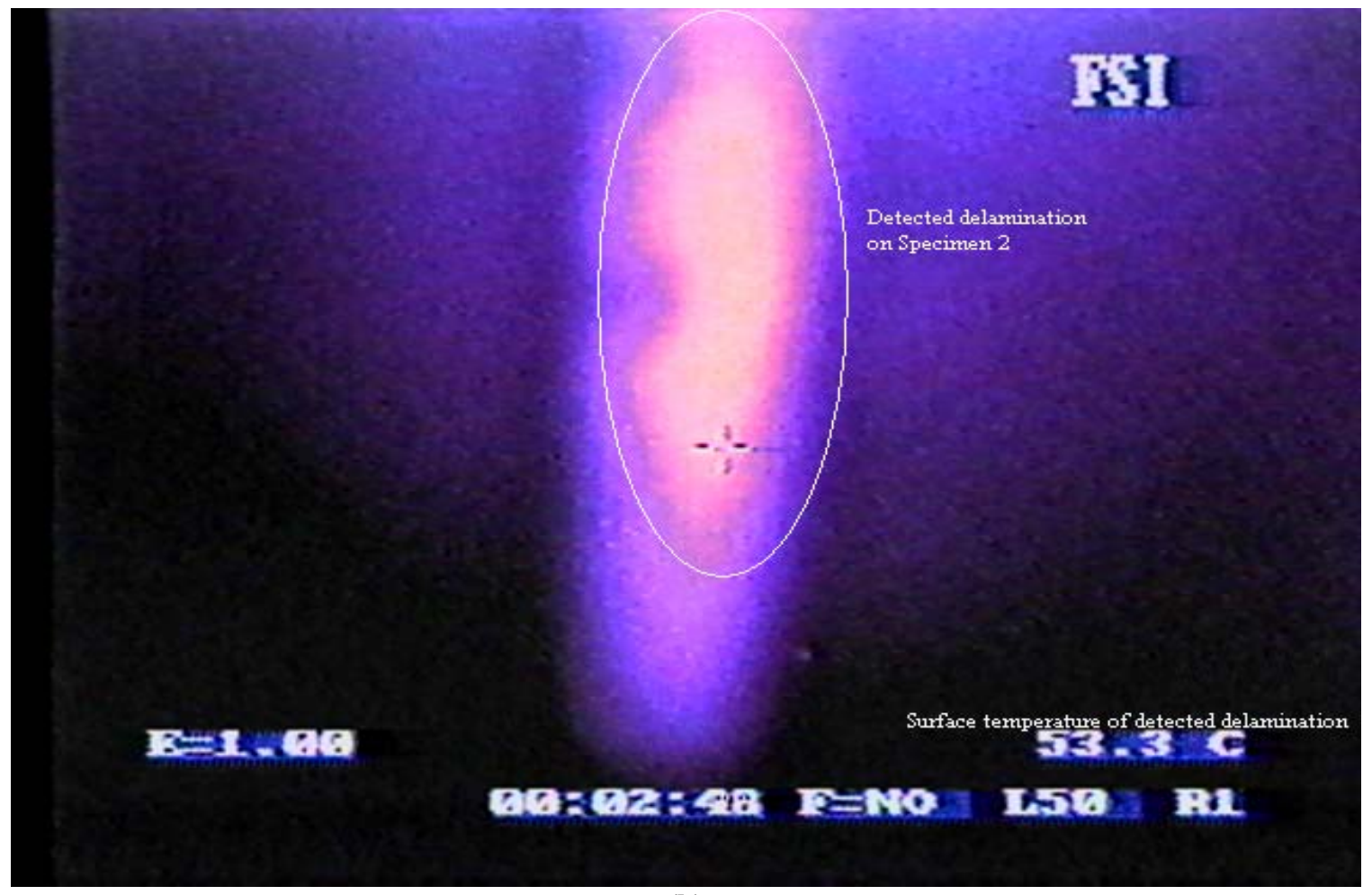

(b)

Figure 4.3.9 (a) Photograph of the surface where the simulated delamination was placed on specimen 2, and (b) thermal image of the simulated delamination detected by the infrared camera 
The last specimen tested (specimen 3) had no known defects or delaminations. This specimen was tested to compare thermal images of defective areas with thermal images of bonded areas. Figure 4.3.10 is the thermal image of a bonded area with no defects or delaminations. The image is relatively uniform. The center section of the thermal image may be slightly brighter than the edges, which indicates the center of the specimen is hotter than the edges. This phenomenon is normal since the center of the specimen absorbs more heat than the edges due to normal heat incidence at the center and heat dissipation to the surrounding air at the edges. One can also observe a difference between the bottom and top section of the thermal image. This is probably due to the fact that heat rises causing the top section of the specimen to be heated more than the bottom section. One needs to be aware of the different factors that can cause temperature differentials so the thermal images are not misinterpreted. 


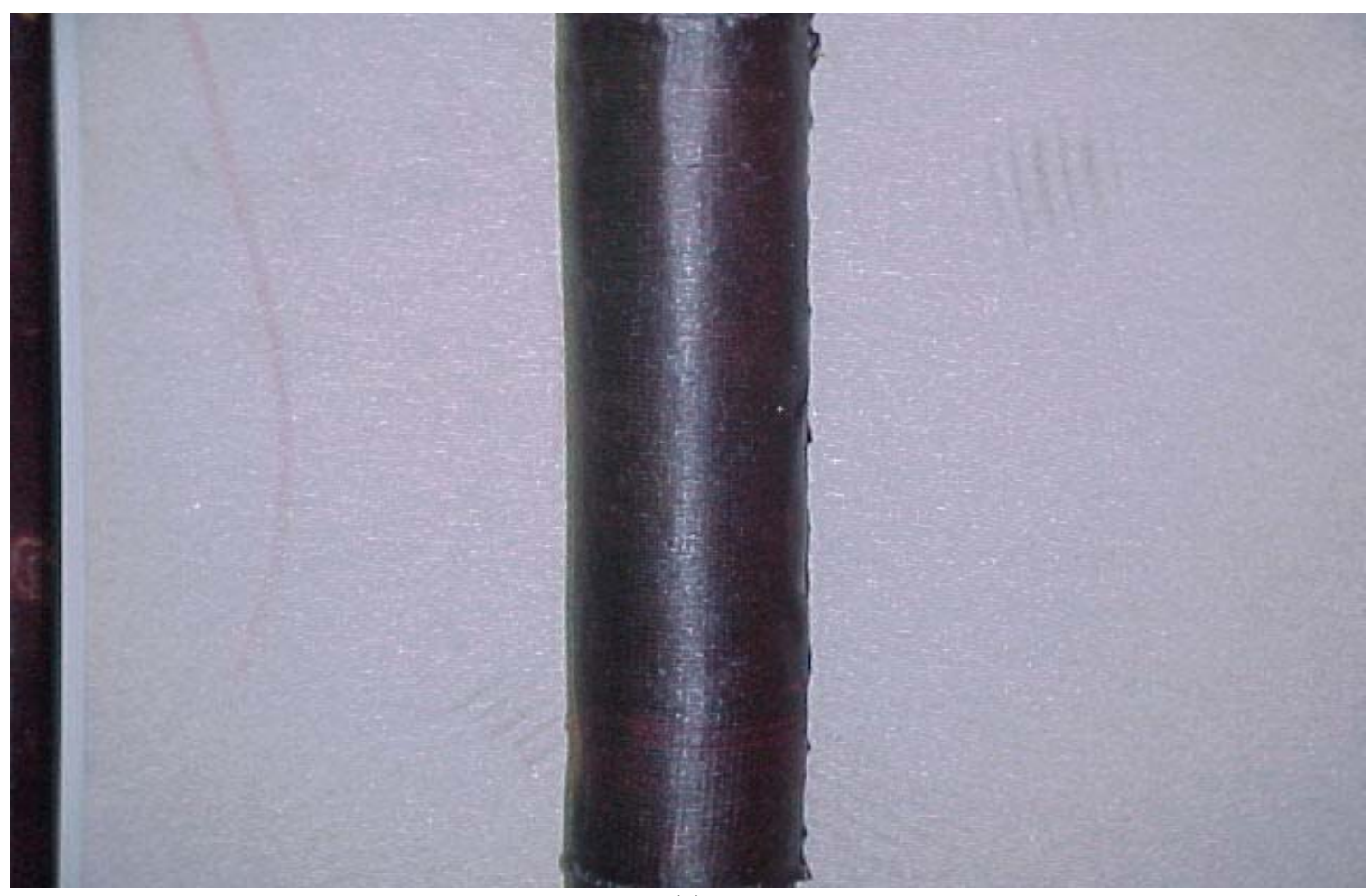

(a)

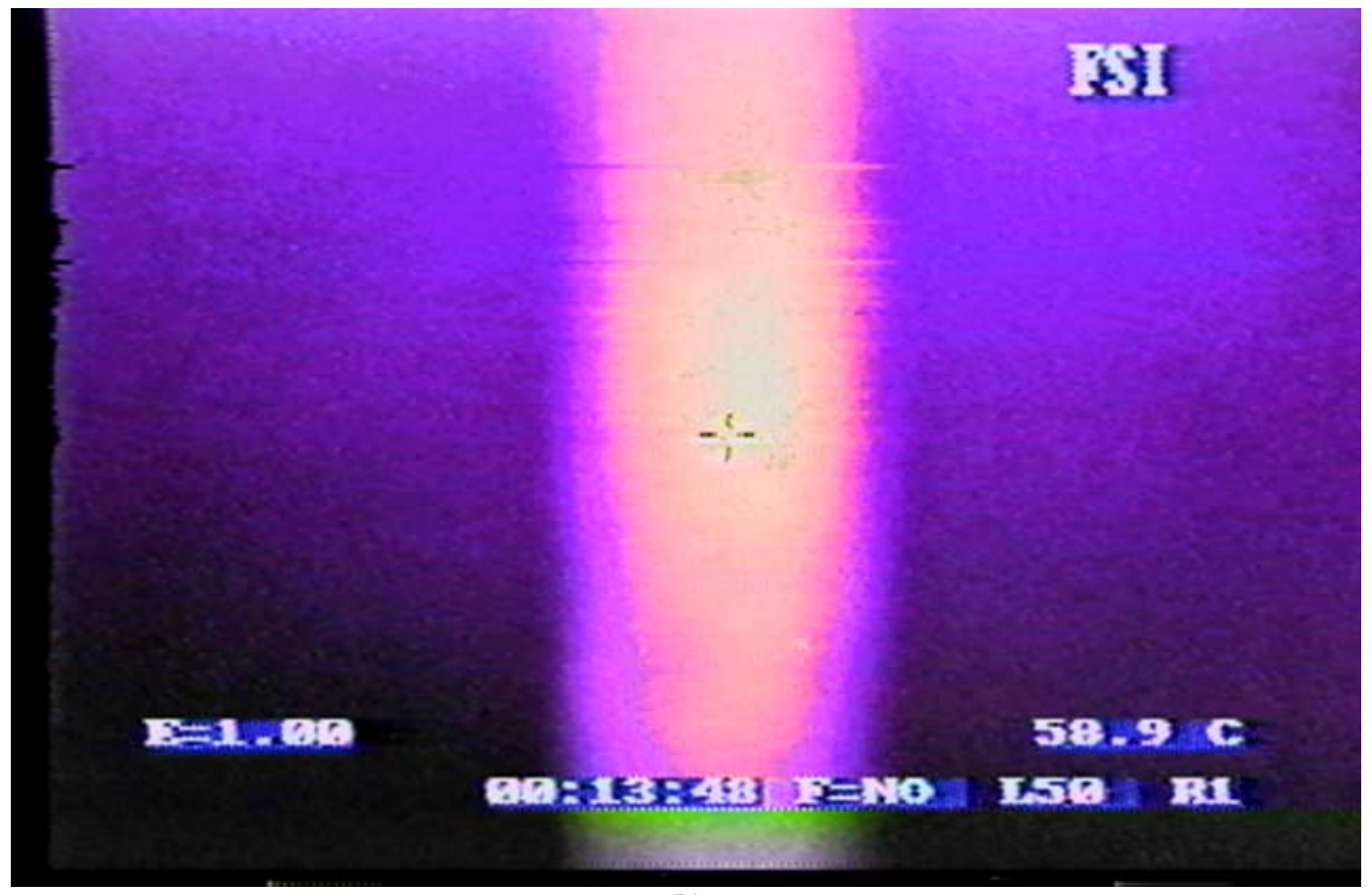

(b)

Figure 4.3.10 (a) Photograph of the surface where no simulated delaminations were detected (specimen 3), and (b) thermal image of the bonded area with no defects or delaminations 


\subsubsection{Solar Radiation}

The next group of tests were conducted using solar radiation as the heat source. This method of heating the specimens did not prove very promising. Although this heating method did increase the temperature of the specimens, it did not produce enough of a thermal gradient to create significant heat transfer through the specimens (front to back). Since there was not a significant amount of heat transfer through the specimens, the delaminations were unable to act as insulators; therefore, were not detected by the infrared camera. Another reason the infrared camera did not show significant surface temperature differentials could be because this camera has a spectral range of $3-5 \mu \mathrm{m}$, which is very close to the visible spectrum, and thus the surrounding environment has a more prominent effect on the infrared measurements.

Figure 4.3.11 shows a thermal image of the two specimens with simulated defects. The infrared camera recorded a temperature of $29.8^{\circ} \mathrm{C}$. On the right side is specimen 1 (with four simulated defects), and specimen 2 is on the left side of the thermal image (with one simulated defect). As one moves from the top of the specimens to the bottom, the colors change from yellow to orange to pink to blue, which indicates that the tops of the specimens are hotter than the bottoms. This is normal since the top of the specimen as well as the edges (but not bottom) are exposed to solar radiation, and at the bottom of the specimen only the edges are exposed to solar radiation. This would cause heat to move from the top of a specimen to the bottom and not through the specimen (front to back). This would be the reason the infrared camera was unable to detect the delaminations, because the defects would not act as insulators with heat 
moving from top to bottom. Heat would need to move through the specimen (front to back) in order for the delaminations to act as insulators.

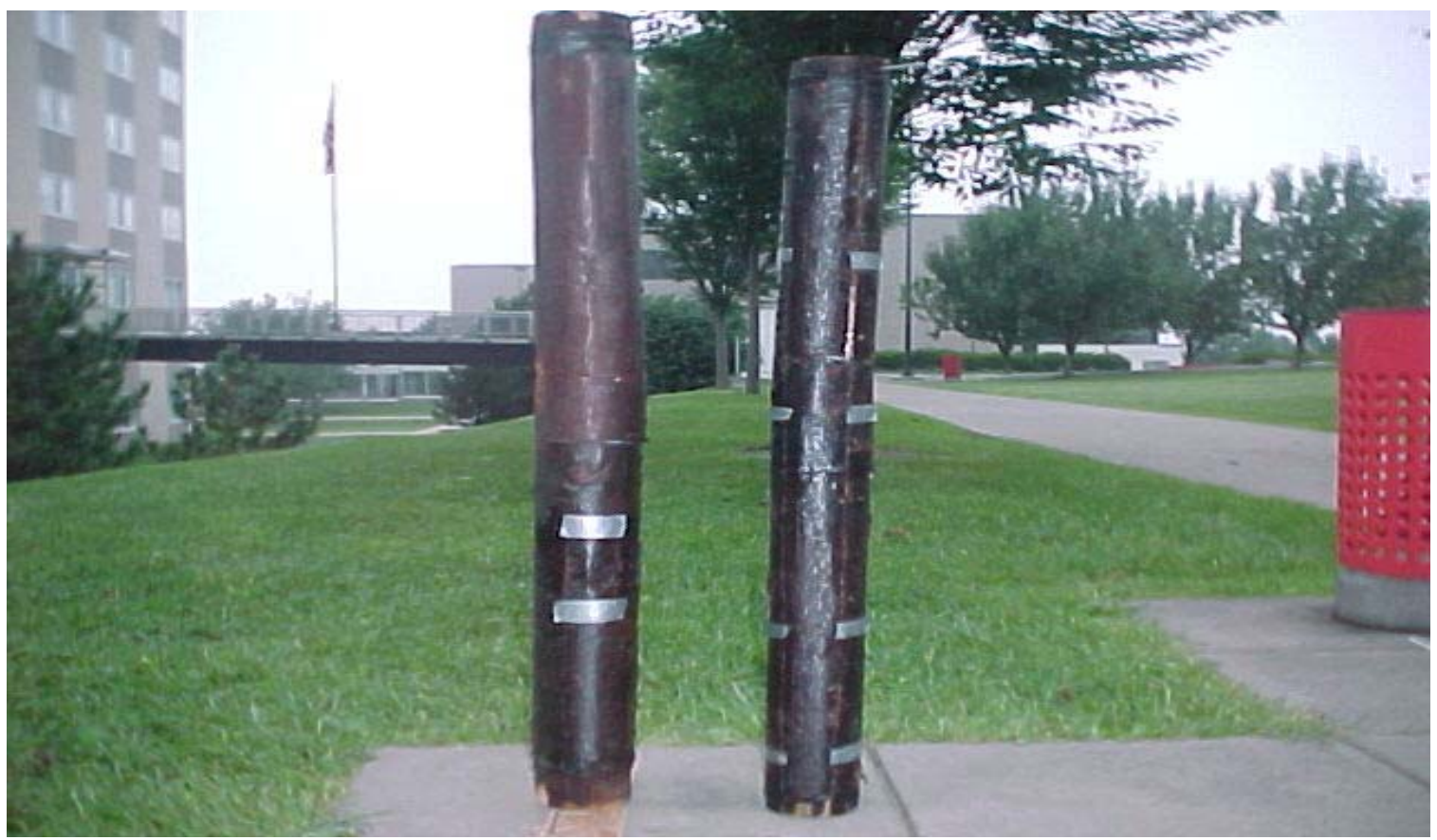

(a)

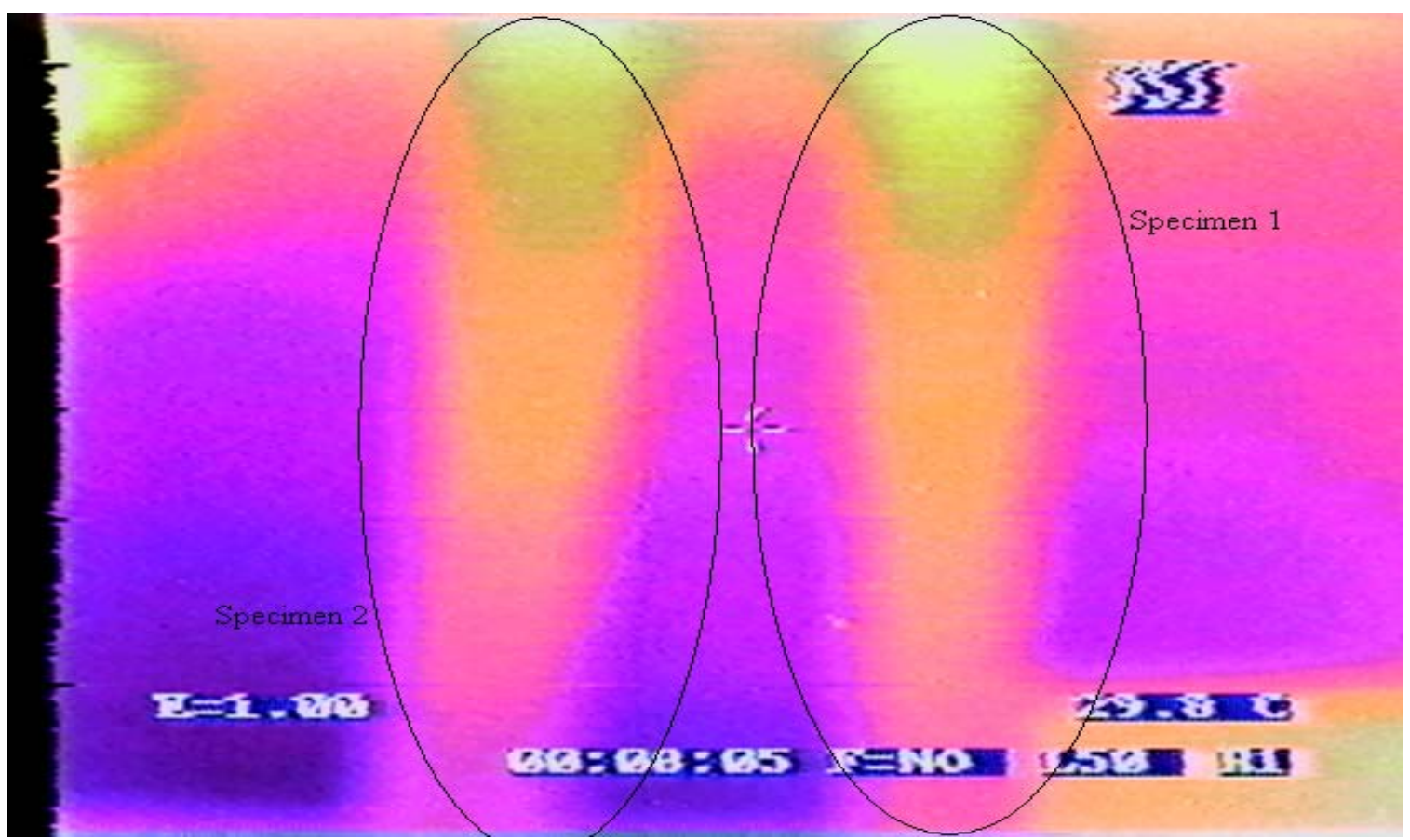

(b)

Figure 4.3.11 (a) Photograph of specimen 1 and 2, and (b) thermal image of specimen 1 and 2 using solar radiation as the heat source 


\subsubsection{Greenhouse Effect}

The last group of tests involved using a simulated greenhouse and solar radiation as the heat source. This thermal image was very similar to the thermal image recorded when sunlight was used as the heat source (Figure 4.3.12). The simulated greenhouse increased the temperature of the specimens to $35.2^{\circ} \mathrm{C}$. The infrared camera was unable to detect the simulated delaminations. Again, the tops of the specimens are hotter than the bottoms causing heat to move from top to bottom and not through the specimen (front to back). It was concluded that the simulated greenhouse did not produce enough of a thermal gradient to create significant heat transfer through the specimens (front to back). Since there was not a significant amount of heat flowing through the specimens, the defects were unable to act as insulators; therefore, were not detected by the infrared camera. The spectral range of the infrared camera $(3-5 \mu \mathrm{m})$ might also have played a prominent role on these infrared measurements since it is very close to the visible spectrum. 


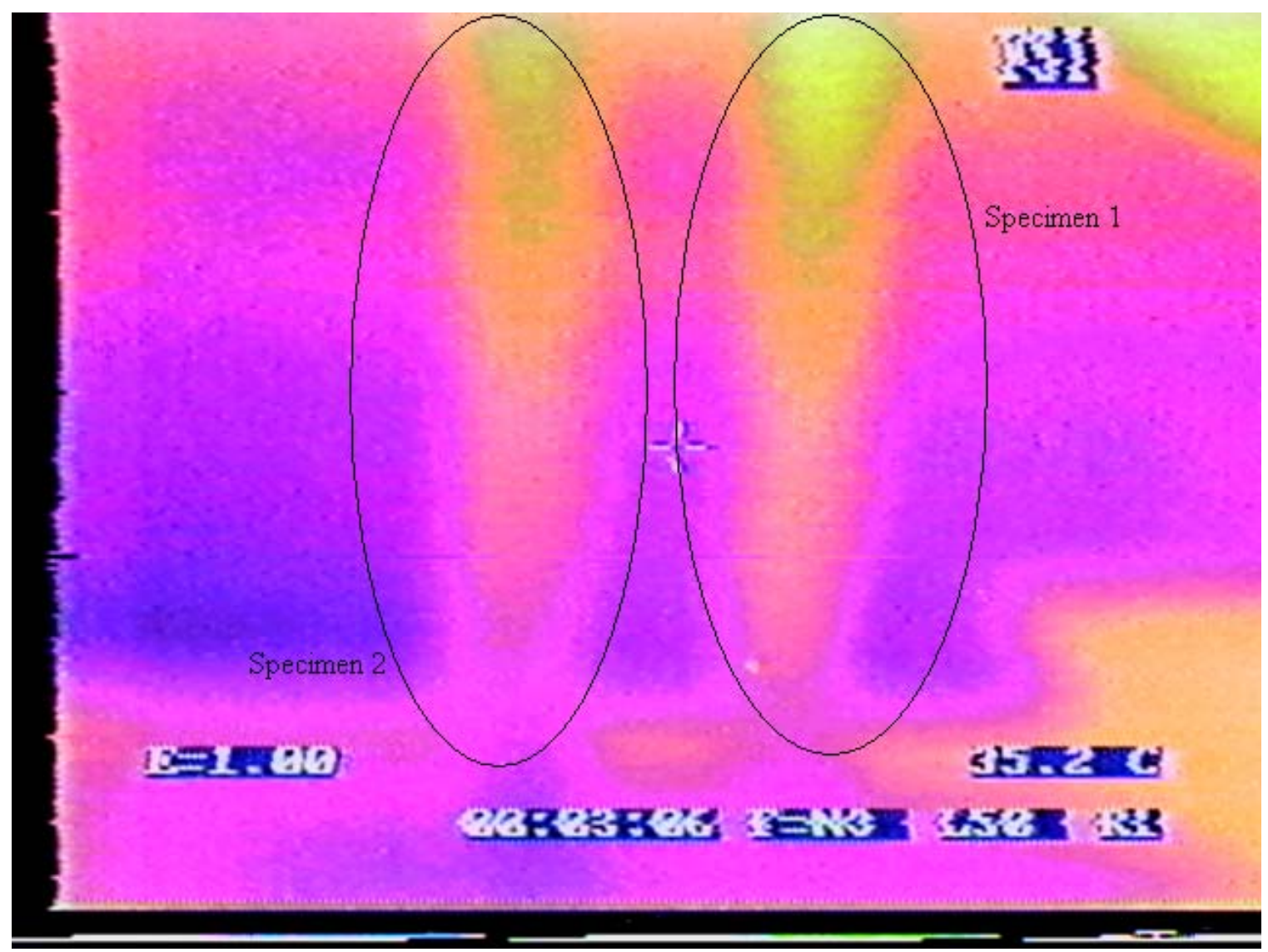

Figure 4.3.12 Thermal image of specimen 1 and specimen 2 using the greenhouse effect and solar radiation as the heat source

\subsection{Conclusions}

The laboratory experiments on the timber pile specimens wrapped with GFRP composite fiber have proven that the infrared thermography technique is able to detect subsurface delaminations at the composite-timber interface. All the delaminations that were detected were approximately 0.125 " beneath the surface. However, the infrared camera detected delaminations as small as $1.5 "$ in diameter and $0.125 "$ thick.

The most promising infrared technique uses a heat source that will produce significant heat transfer through the specimen (front to back). Other heating methods that do not create significant thermal gradients and cause heat transfer from top to bottom 
such as solar radiation could possibly be used to detect delaminations if a more advanced camera is used. In addition, an increase in the delamination thickness or a decrease in its depth will enhance an infrared camera's ability to detect a defect. 


\section{CHAPTER 5}

\section{FIELD STUDY}

\subsection{Introduction}

This chapter presents a description of the nondestructive testing performed on three timber railroad bridges located in Moorefield, West Virginia. These bridges are owned by WVDOT-State Rail Authority. Components of the timber bridges were rehabilitated with composite wrap. Infrared thermography was used to detect any debonding between the timber and composite interface. This chapter will discuss the location of the bridges, the experimental setup, any difficulties that were encountered, and the test results obtained using infrared thermography.

The most promising technique to repair timber bridges to date employs the use of fiber reinforced polymer composite materials. The USDOT-FRA and WVDOT-SRA have placed a group of experimental pile caps, stringers, piles, and crossties in its main track as part of a multiphase project being conducted by the Constructed Facilities Center of West Virginia University. Non-corrosive fiber reinforced plastic (FRP) fabric has been wrapped around and affixed to the timber components to see if FRP wrapping can strengthen and extend the useful life of timber components. The timber structures were hand wrapped with glass reinforced fabrics and phenolic resin composites.

Only the most severely damaged timber components on each of the three bridges were wrapped with the GFRP composite wrap. Figure 5.1.1 shows an example of three 
different timber components wrapped with GFRP composites from the three timber bridges. The timber components were dismantled, wrapped with GFRP, and then reassembled.

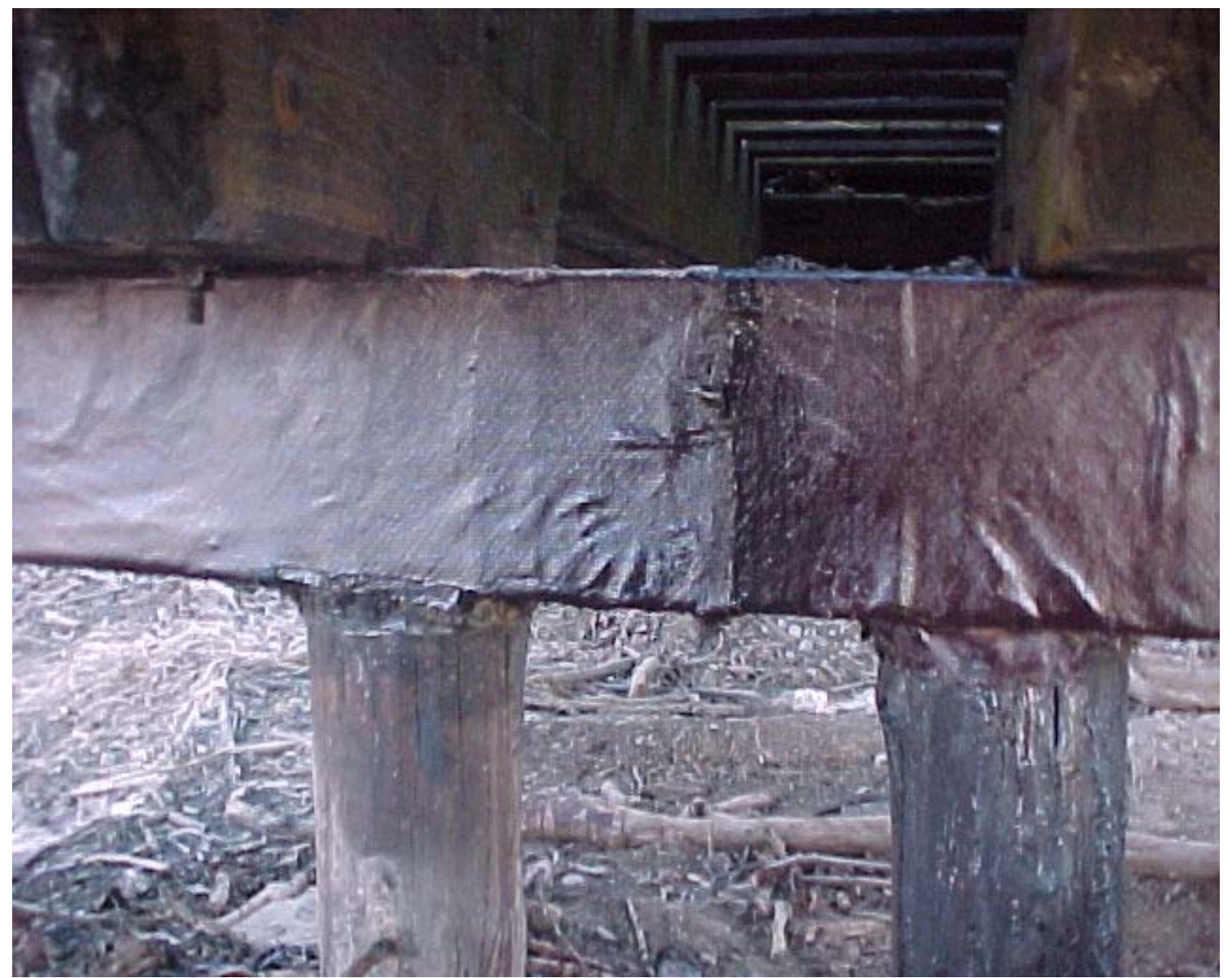

(a) 


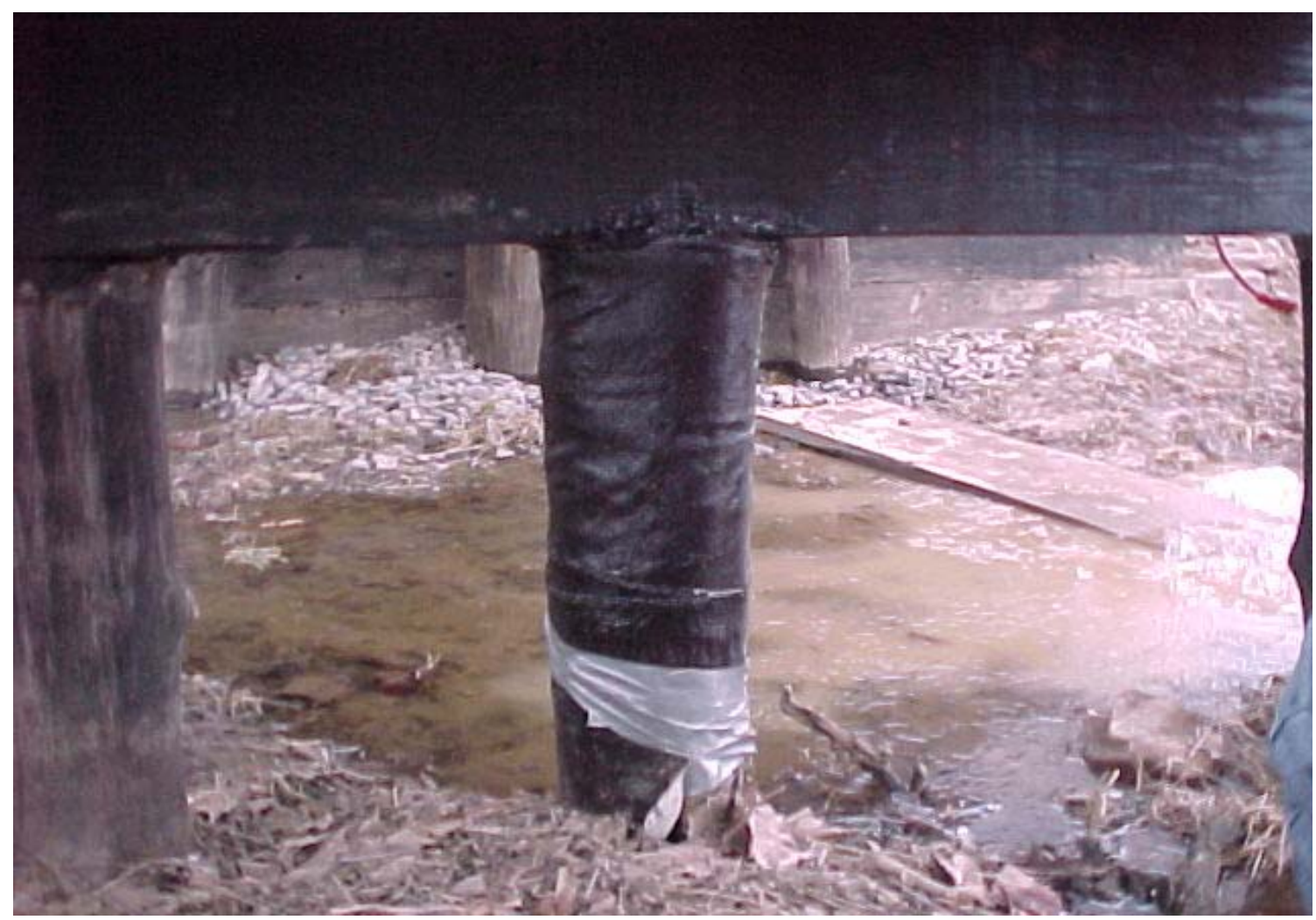

(b)

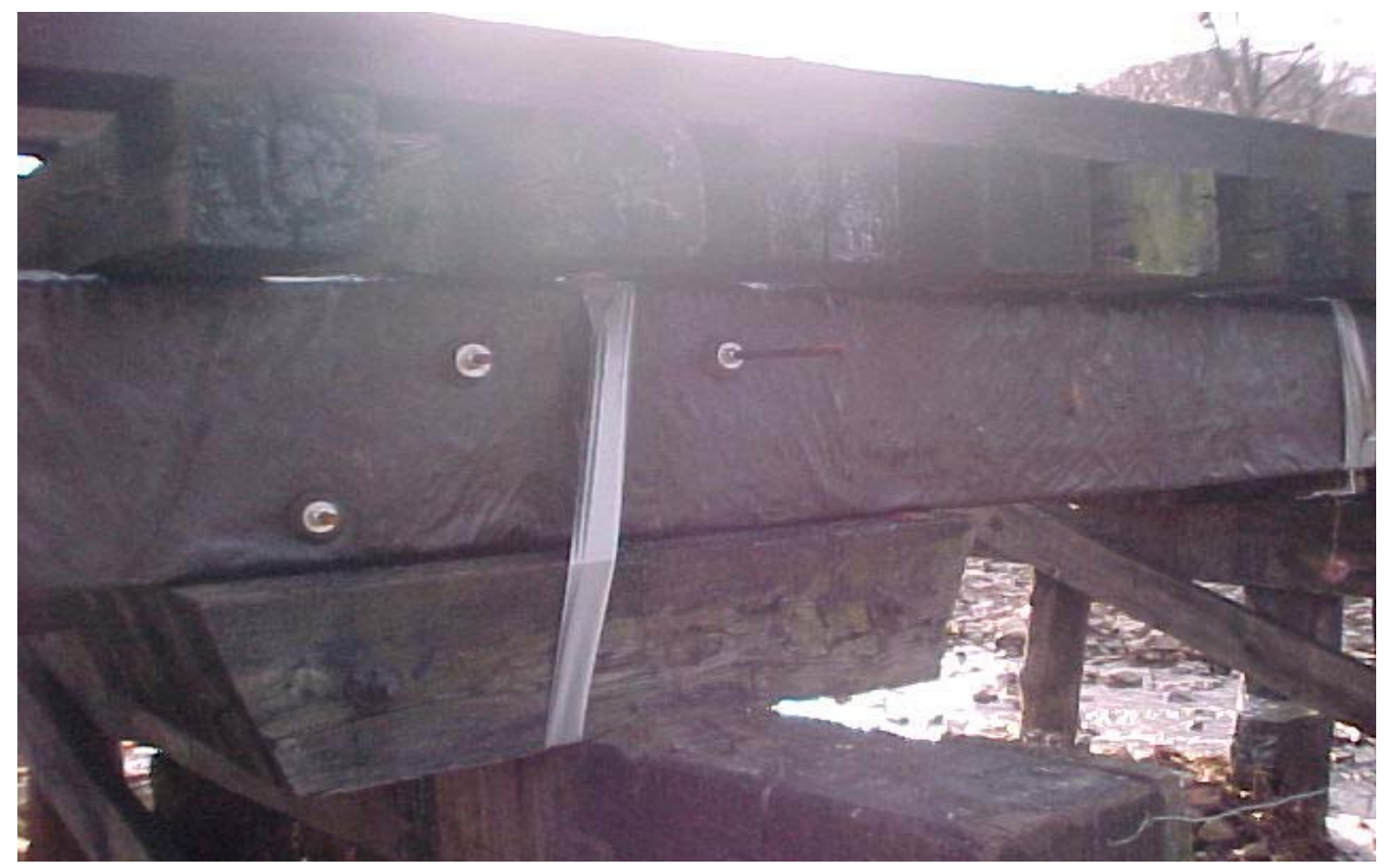

(c)

Figure 5.1.1 (a) A pile cap from bridge \#568 wrapped with GFRP composite wrap, (b) a timber pile from bridge \#570 wrapped with GFRP composite wrap, (c) a stringer from bridge \#583 wrapped with GFRP composite wrap 


\subsection{Description of Bridges}

The 52.4 mile South Branch Valley Railroad (SBVR) is owned and operated by the West Virginia State DOT-Rail Authority (SRA). The line provides freight and passenger service to the state's eastern panhandle. The South Branch line of the Chessie System's Baltimore and Ohio Railroad (now CSXT) had formerly operated the railroad until October 11, 1978 when it was turned over to West Virginia. When Chessie turned over the rail line to the state, West Virginia became the first state in the nation to both own and operate a commercial freight railroad.

There were three timber railroad bridges tested with infrared thermography. The three bridges were part of the WVDOT-South Branch Valley Railroad. The bridges were referred to as Bridge \#568, Bridge \#570, and Bridge \#583. All three bridges were located in Moorefield, West Virginia. The location of Moorefield, West Virginia is shown in Figure 5.2.1. 


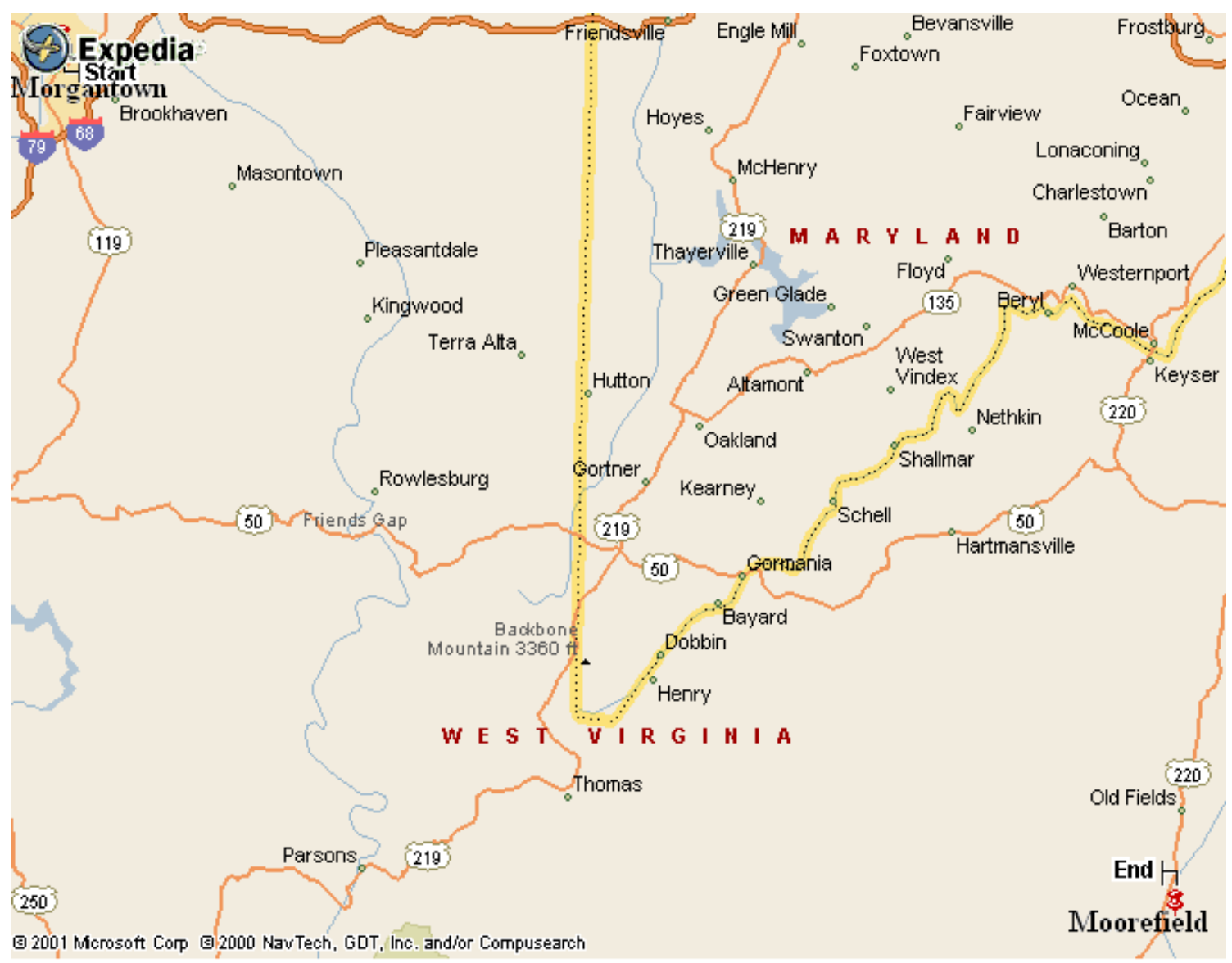

Figure 5.2.1 Location of Moorefield, West Virginia

\subsubsection{Bridge \#568}

Bridge \#568 was the first of the three timber bridges tested using infrared thermography. Figure 5.2.2 shows two photographs of Bridge \#568. One pile cap and one pile wrapped with GFRP composite fabric was tested on this bridge using infrared thermography. 


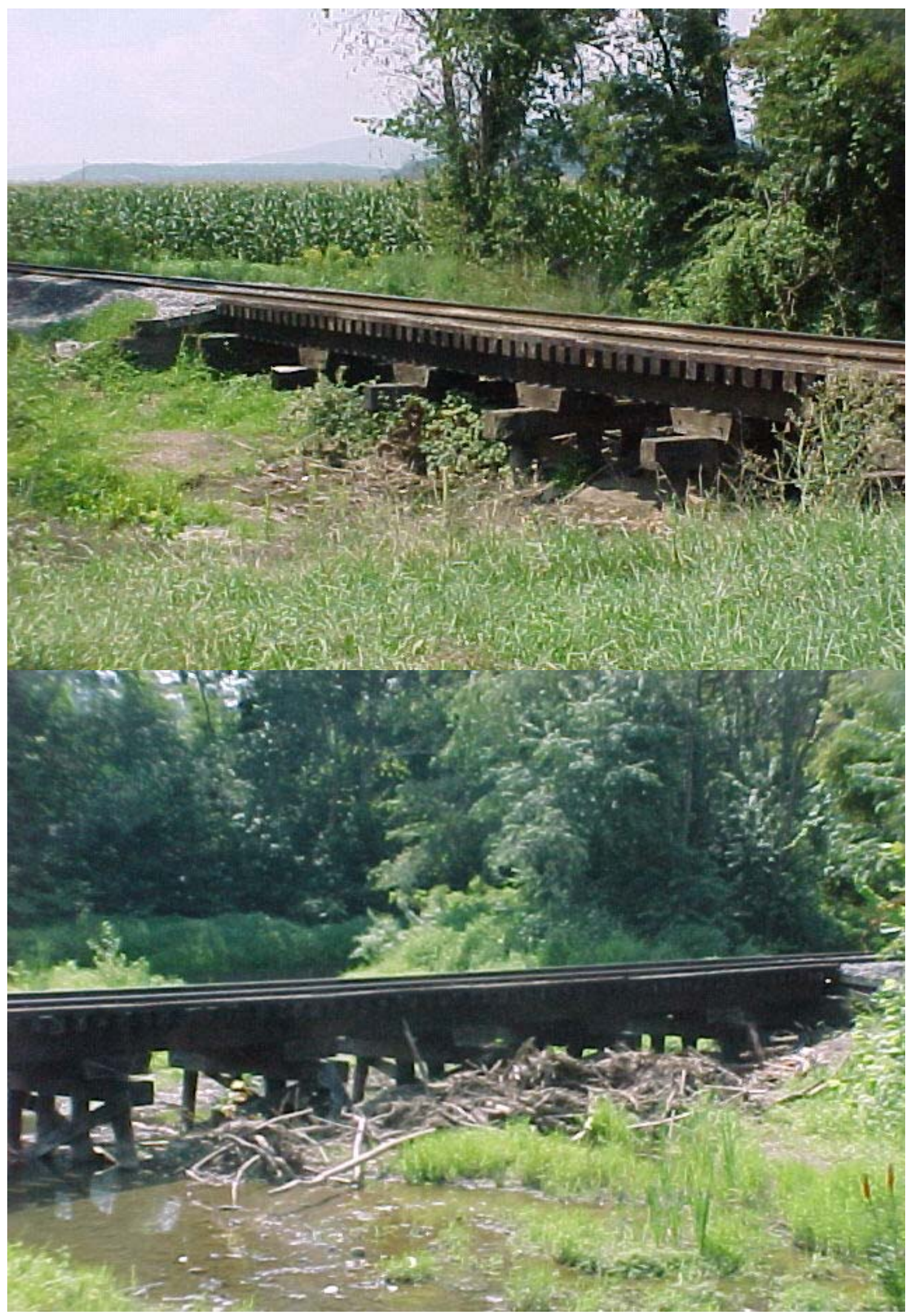

Figure 5.2.2 Two Photographs of Bridge \#568 


\subsubsection{Bridge \#570}

The second bridge tested using infrared thermography was Bridge \#570. Figure 5.2.3 shows a photograph of this bridge. A stringer and one pile wrapped in GFRP composite fabric was tested on Bridge \#570.

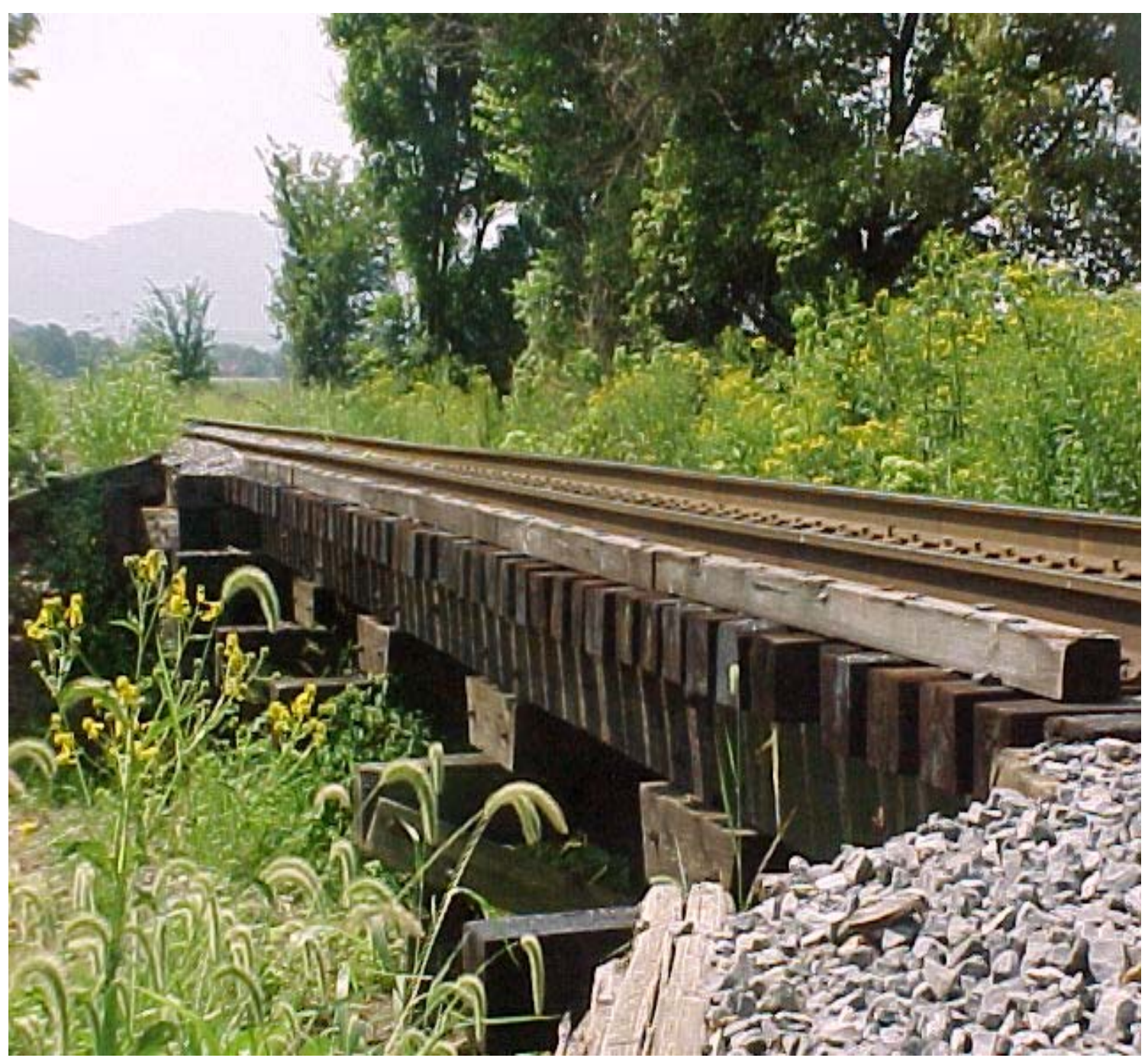

Figure 5.2.3 Photograph of Bridge \#570 


\subsubsection{Bridge \#583}

The last bridge tested with infrared thermography was referred to as Bridge \#583.

Figure 5.2.4 shows two photographs of Bridge \#583. Only one stringer wrapped in GFRP composite fabric was tested on this bridge.

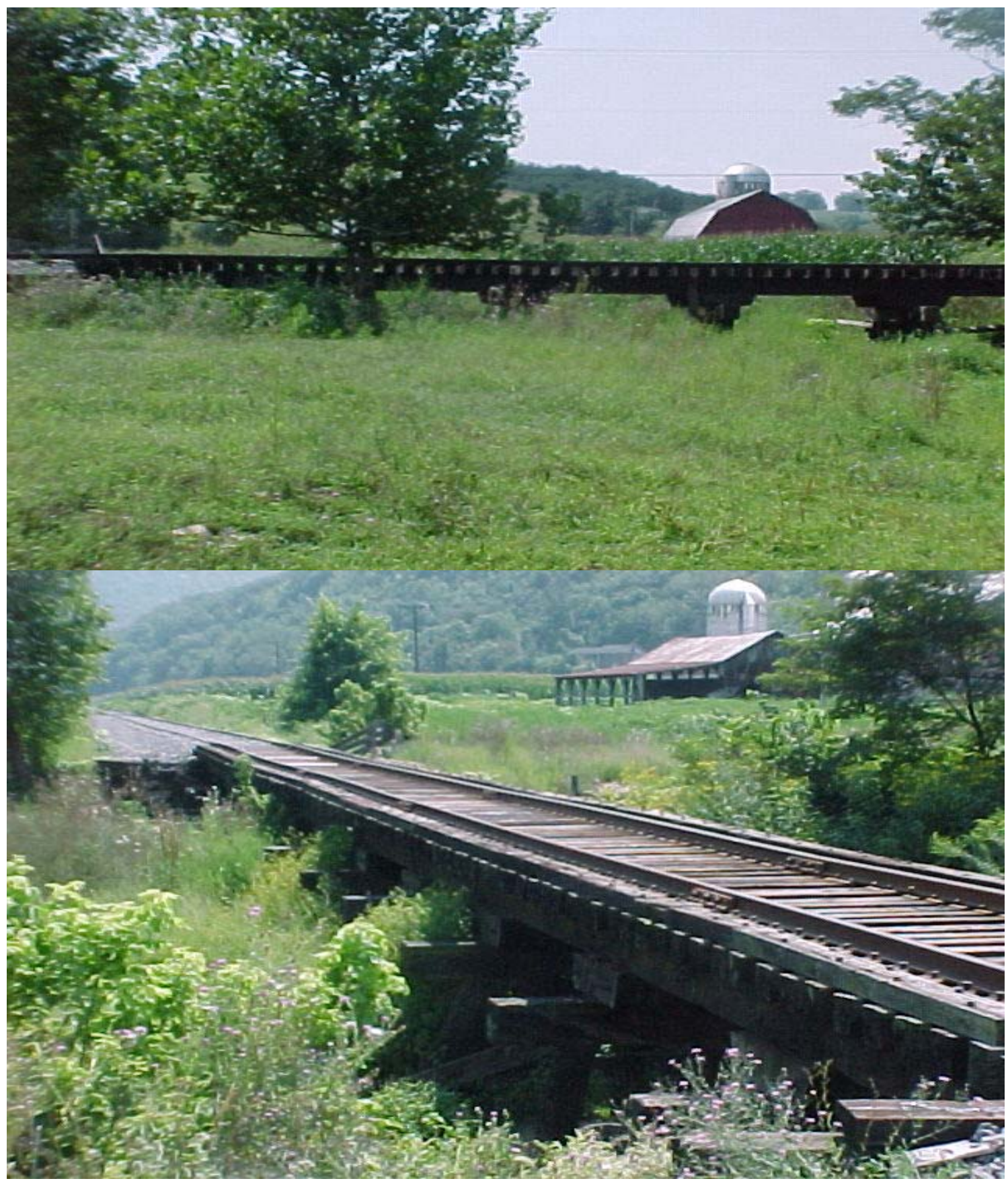

Figure 5.2.4 Two photographs of Bridge \#583 


\subsection{Field Results}

\subsubsection{Equipment Set-up}

The field equipment was the same equipment used in the laboratory. Again, all three heat sources were experimented with in the field (quartz tower heater, solar radiation, and a simulated greenhouse effect). When the quartz tower heater was used as the heat source, different distances between the heater and the timber components, and different time periods of heating were experimented with. Clear plastic wrap was glued around the timber components to create a type of greenhouse effect as a method of heating the components. Solar radiation was also used as a heat source for the GFRP wrapped timber components exposed to sunlight. For the timber components heated by solar radiation, different angles between the infrared camera and the surface of the timber components were experimented with. A previous study (Kadir 1991) has shown that $35^{\circ}$ - $45^{\circ}$ angle between the infrared camera and the surface being tested was the best since it minimizes the effect of the heat reflection from the surrounding environment.

Figure 5.3.1 shows a basic testing situation. Due to the small space under the bridges, the infrared camera could not be mounted on a tripod. A microphone was used to record sound and correlate thermal images recorded by the VCR and the photographs taken with the digital camera. 


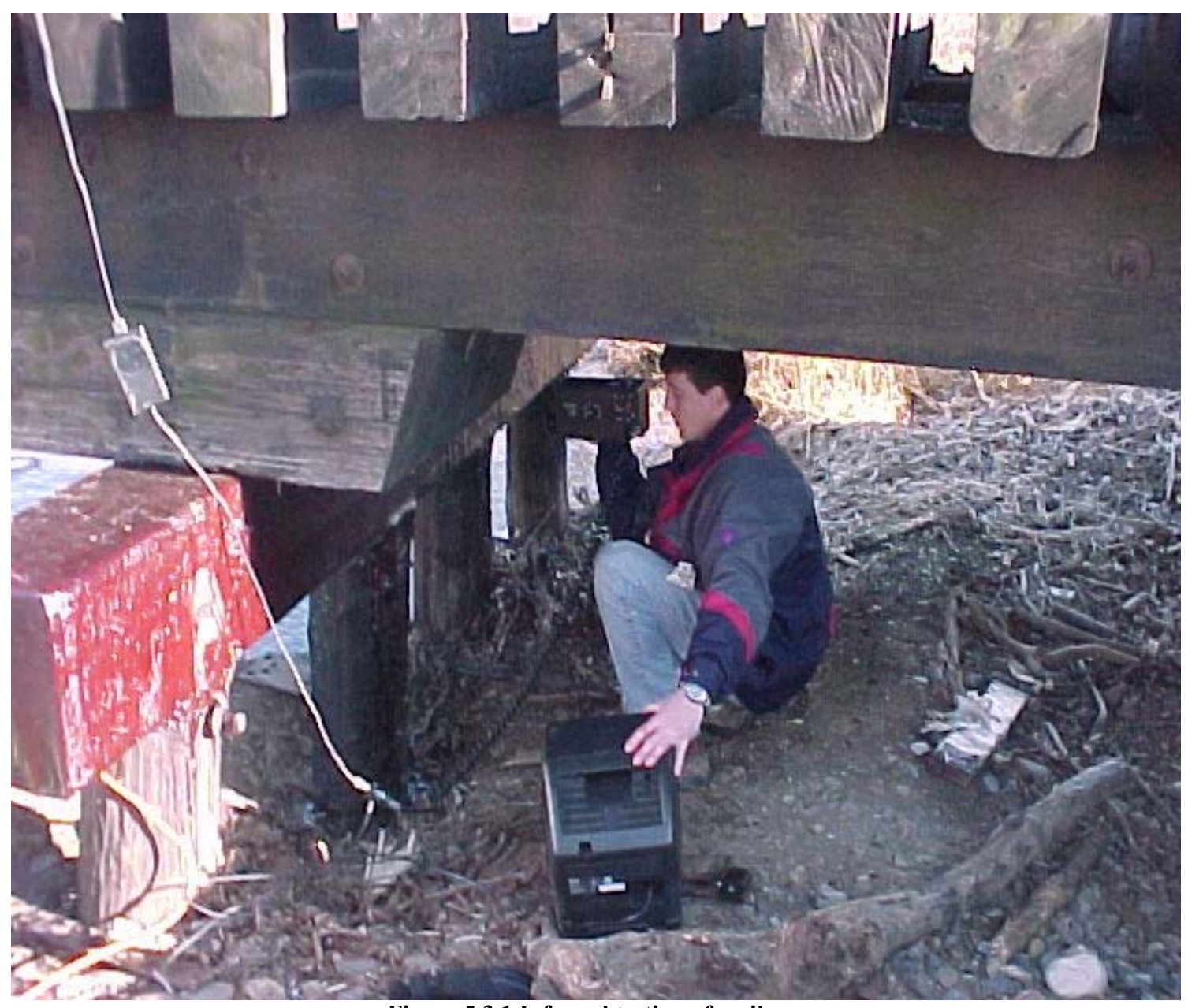

Figure 5.3.1 Infrared testing of a pile cap

\subsubsection{Bridge \#568}

The FLIR Prism Single Point Infrared Camera was used to test a pile cap and pile on Bridge \#568. The field test was conducted on a sunny day with an ambient temperature of approximately $85^{\circ} \mathrm{F}\left(29.4^{\circ} \mathrm{C}\right)$.

The thermal images obtained using solar radiation, as the heat source, did not show any significant surface temperature differentials that would have indicated a delamination. Figure 5.3.2 shows a thermal image of a pile cap section after being wrapped in plastic wrap to create a type of greenhouse effect. The surface temperature 
measured by the infrared camera was $50.3{ }^{\circ} \mathrm{C}$. Figure 5.3 .3 shows a photograph of the pile cap wrapped in plastic wrap. Figure 5.3.4 is another thermal image of a different pile cap section that was also wrapped in plastic wrap. The surface temperature of this section reads $52.2^{\circ} \mathrm{C}$. A black spot was observed in this thermal image, which represents a cooler region than the surrounding area. This could have been caused by a metal nail or bolt under the GFRP composite wrap acting as a heat conductor. Since metal transfers heat faster than timber, the surface temperature of the nail or bolt would be cooler than the surface temperature of the surrounding timber area. 


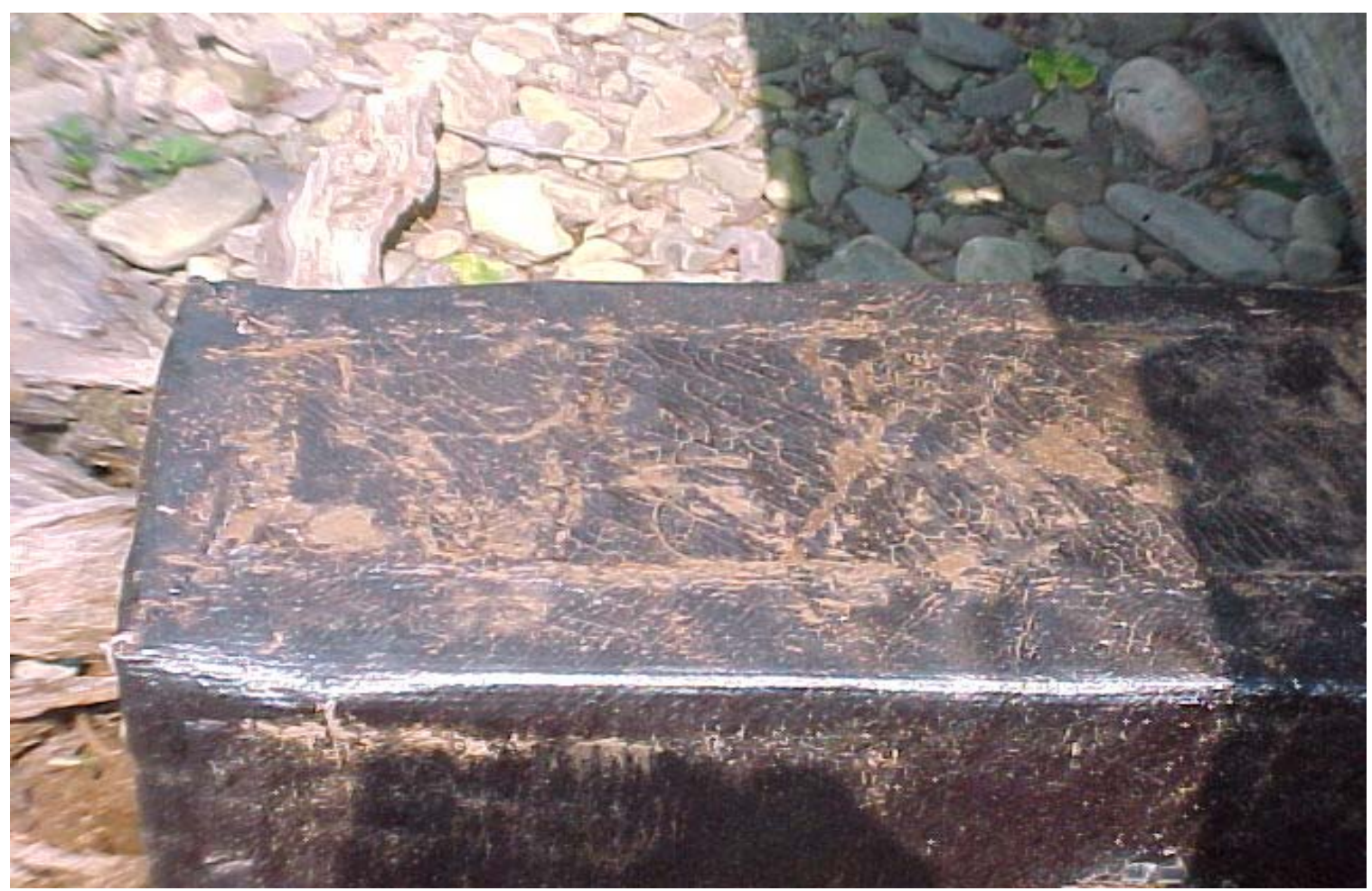

(a)

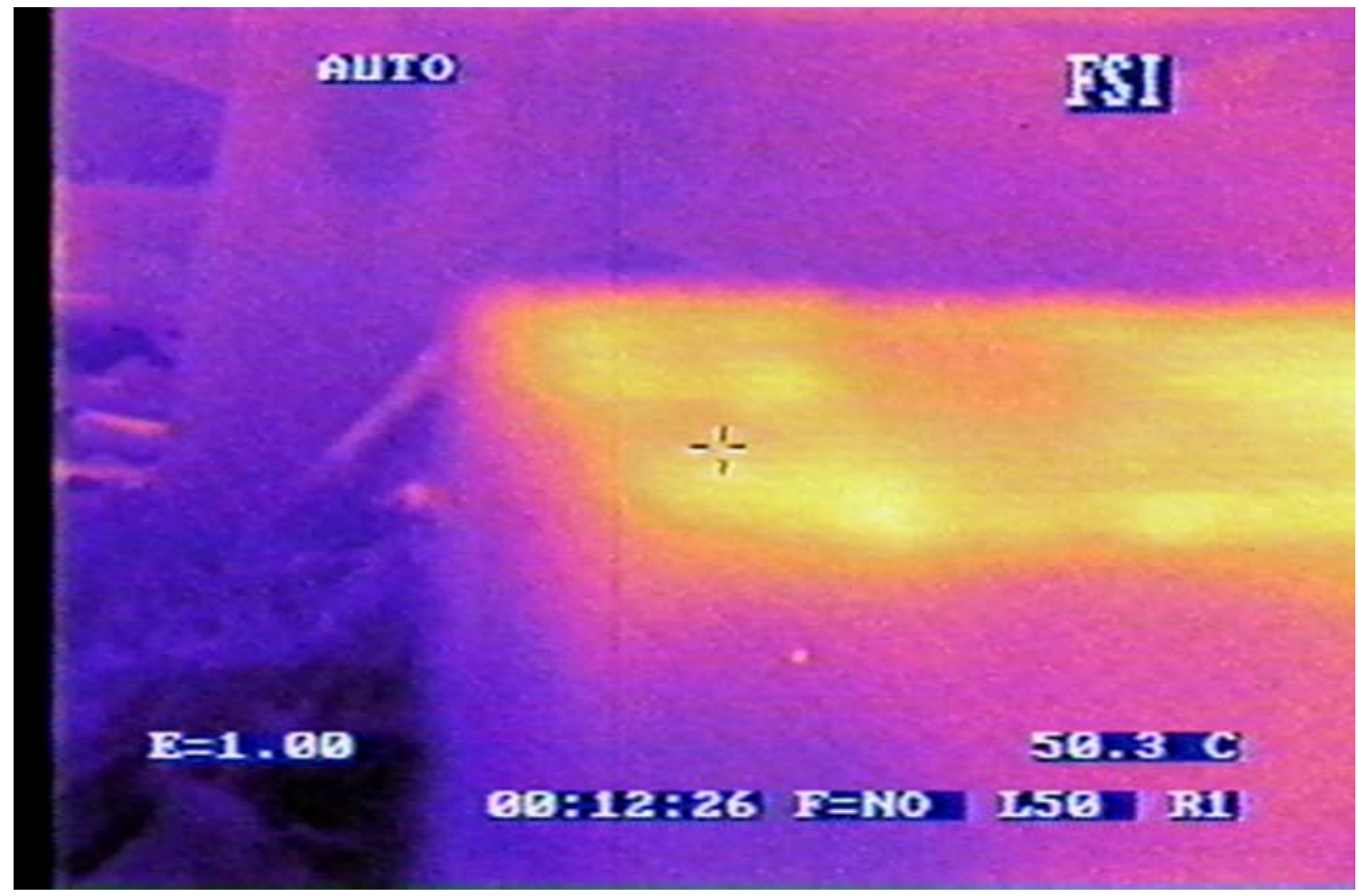

(b)

Figure 5.3.2 (a) Photograph of the pile cap section before being wrapped in plastic wrap, and (b) thermal image of the pile cap section after the plastic wrap was removed 


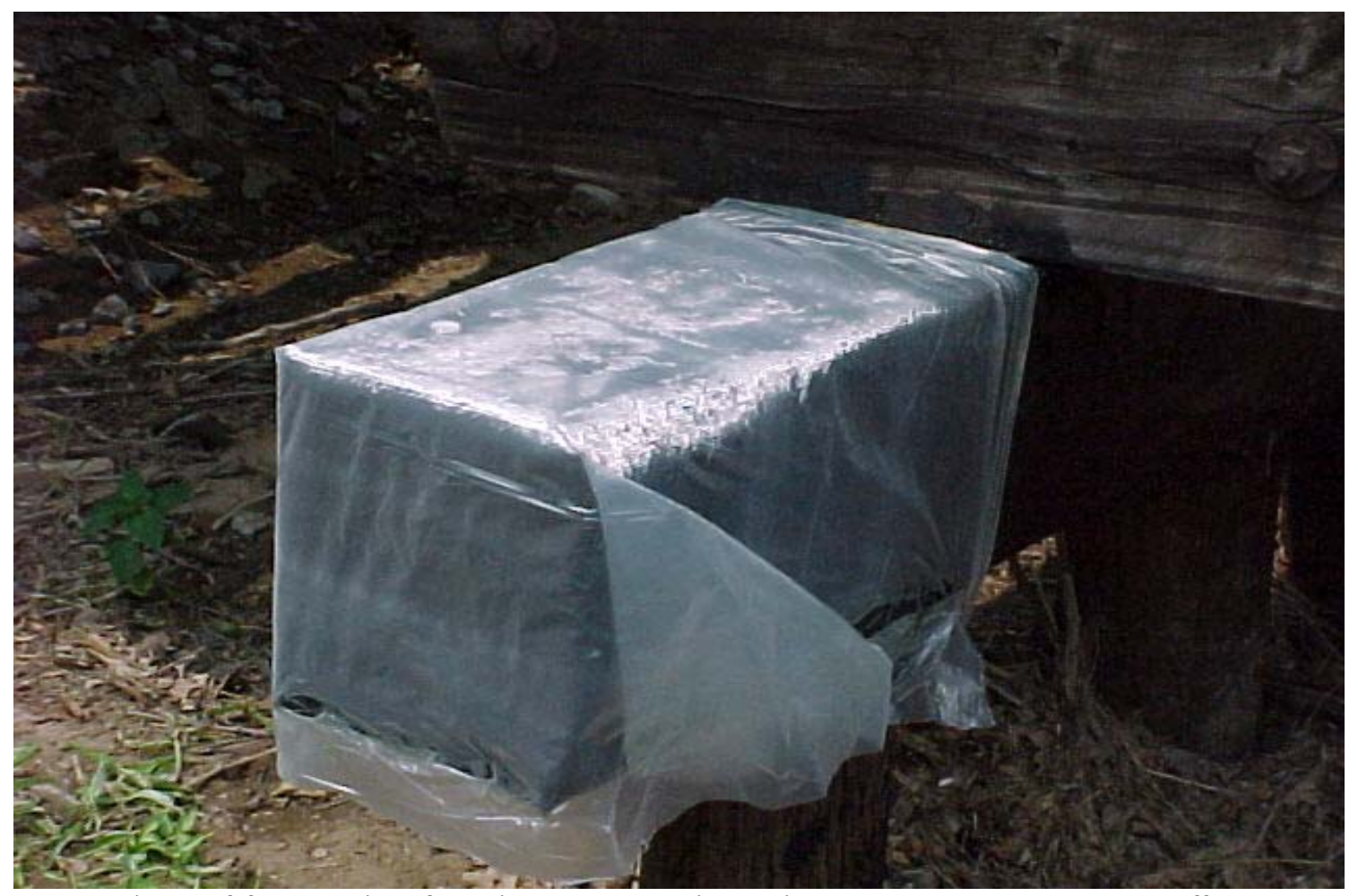

Figure 5.3.3 The section of the pile cap wrapped in plastic wrap to create the greenhouse effect

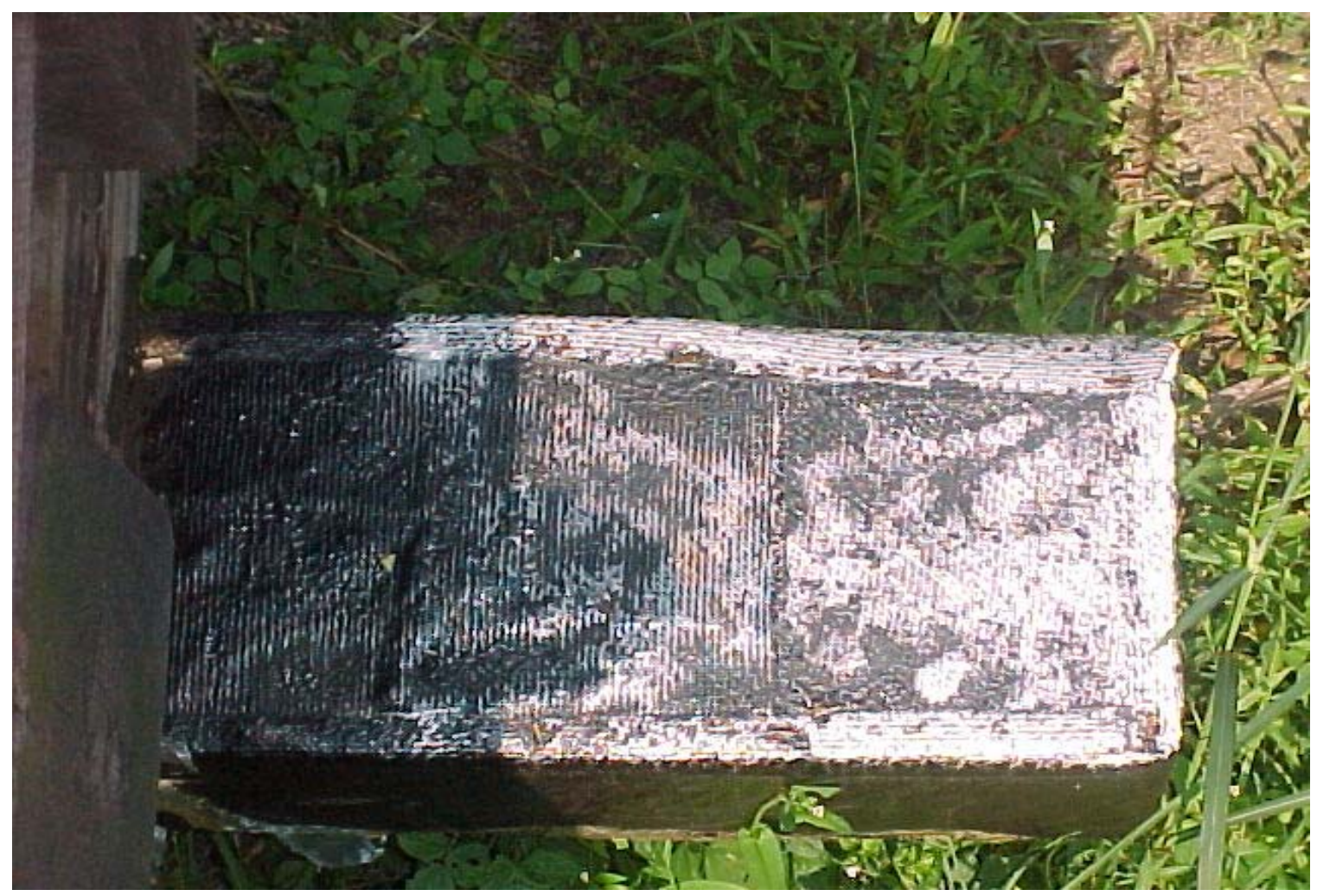

(a) 


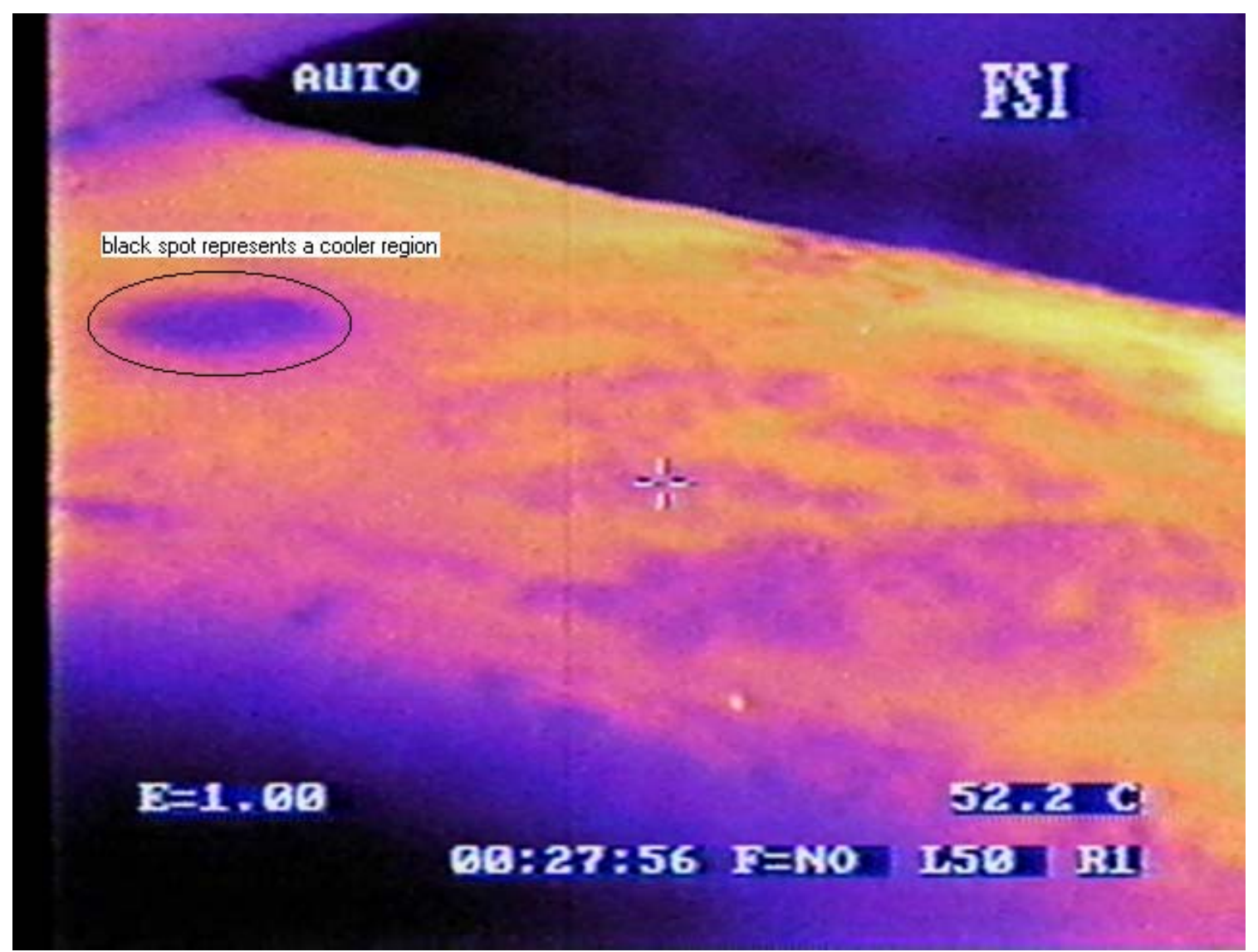

(b)

Figure 5.3.4 (a) Photograph of the pile cap section with the observed black spot, and (b) thermal image of the pile cap section that was wrapped in plastic wrap. The black spot could be caused by a metal nail or bolt under the GFRP wrap

Figure 5.3.5 is a thermal image of a GFRP wrapped timber stringer that has a known bolt going throw the stringer. Again, the darker region represents the bolt, which indicates a cooler region than the surrounding areas. 


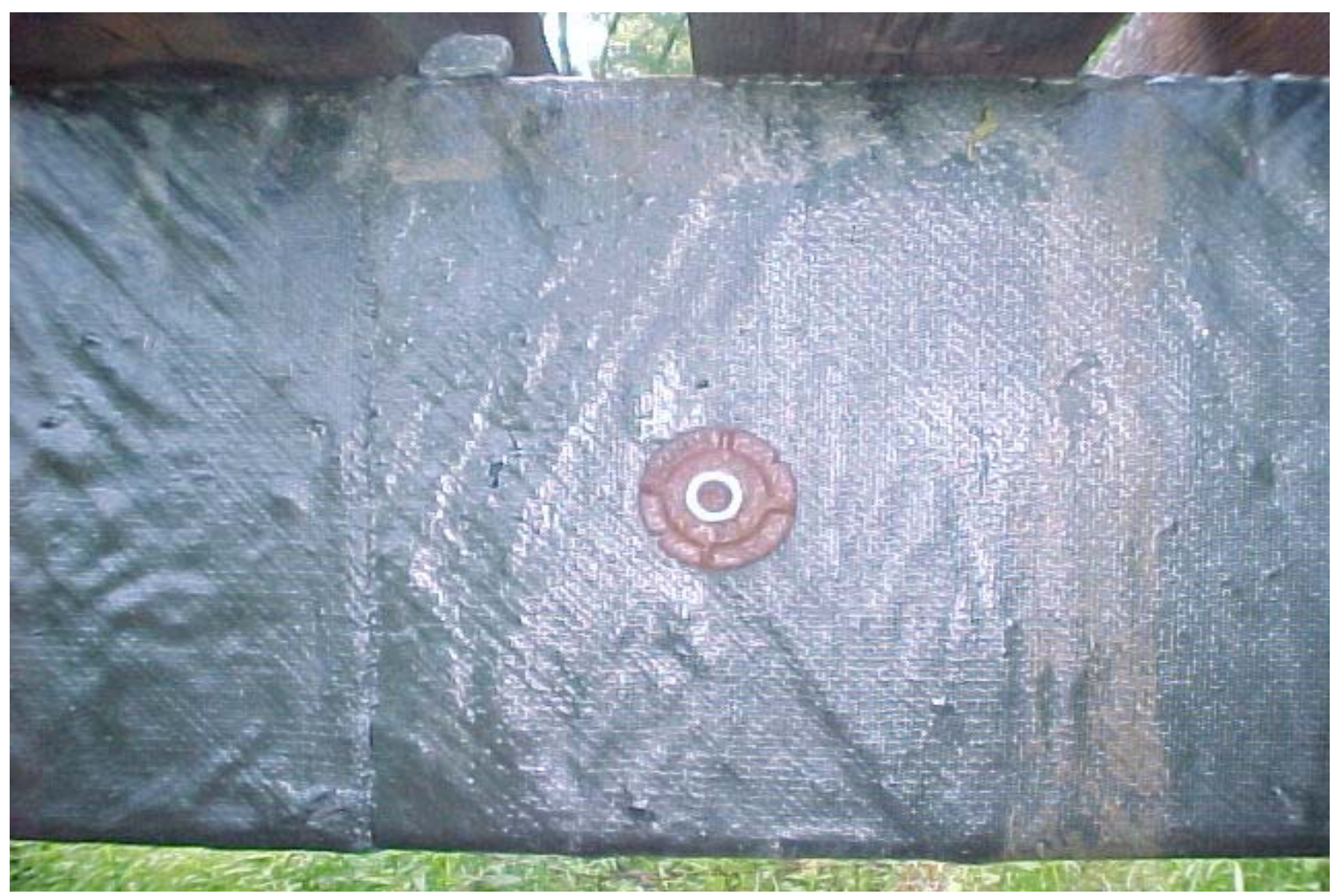

(a)
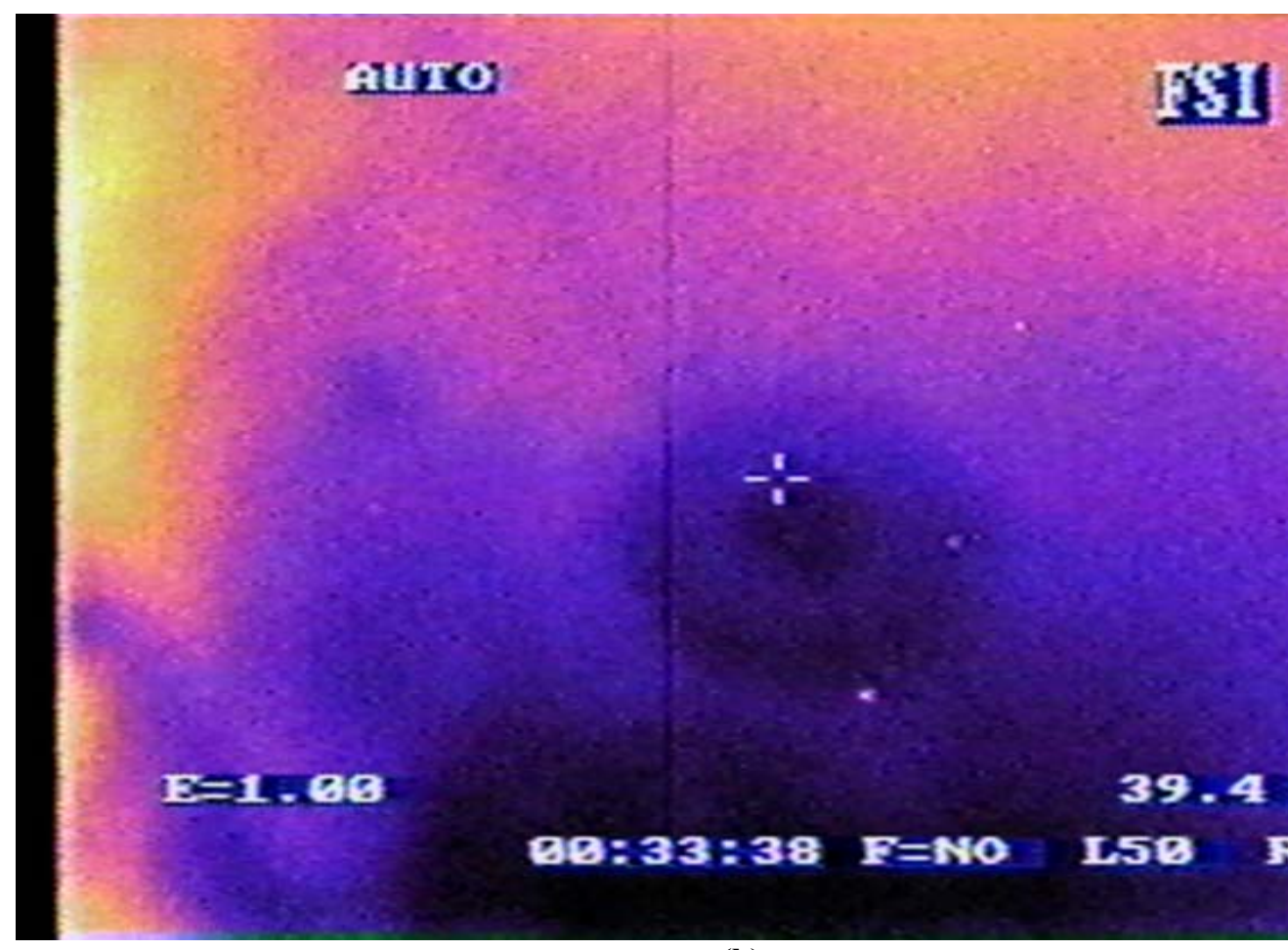

$1 x=1.96$

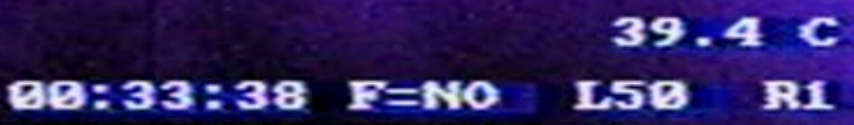

(b)

Figure 5.3.5(a) Photograph of a stringer with a bolt going through it, (b) thermal image of the stringer. The dark area by the cross hairs represents the bolt. 


\subsubsection{Bridge \#570}

The FLIR Prism Single Point Infrared Camera was used to test a stringer and pile on Bridge \#570. The field test was conducted on a sunny day with an ambient temperature of approximately $85^{\circ} \mathrm{F}\left(29.4^{\circ} \mathrm{C}\right)$.

A section of the stringer was wrapped in plastic wrap to create a type of greenhouse effect. However, the thermal images obtained after the plastic wrap was removed did not show significant surface temperature differentials that would have indicated a delamination or defect. A section of the stringer was also tested using the quartz tower heater as the heat source. Figure 5.3.6 shows the stringer being heated with the two different heating methods.

Different distances between the heater and the stringer were experimented with. The most uniform heating occurred when the heater was placed approximately 8 inches away from the surface of the stringer. The surface was heated approximately 60 seconds before the heater was removed. The heater was also placed 4 inches and 2 inches from the surface of the stringer. Figure 5.3.7 shows the thermal images obtained when the heater was placed different distances from the stringer. The plastic wrap and quartz tower heater was also used to heat a pile on Bridge $\# 570$. However, no delaminations or other defects were detected. 


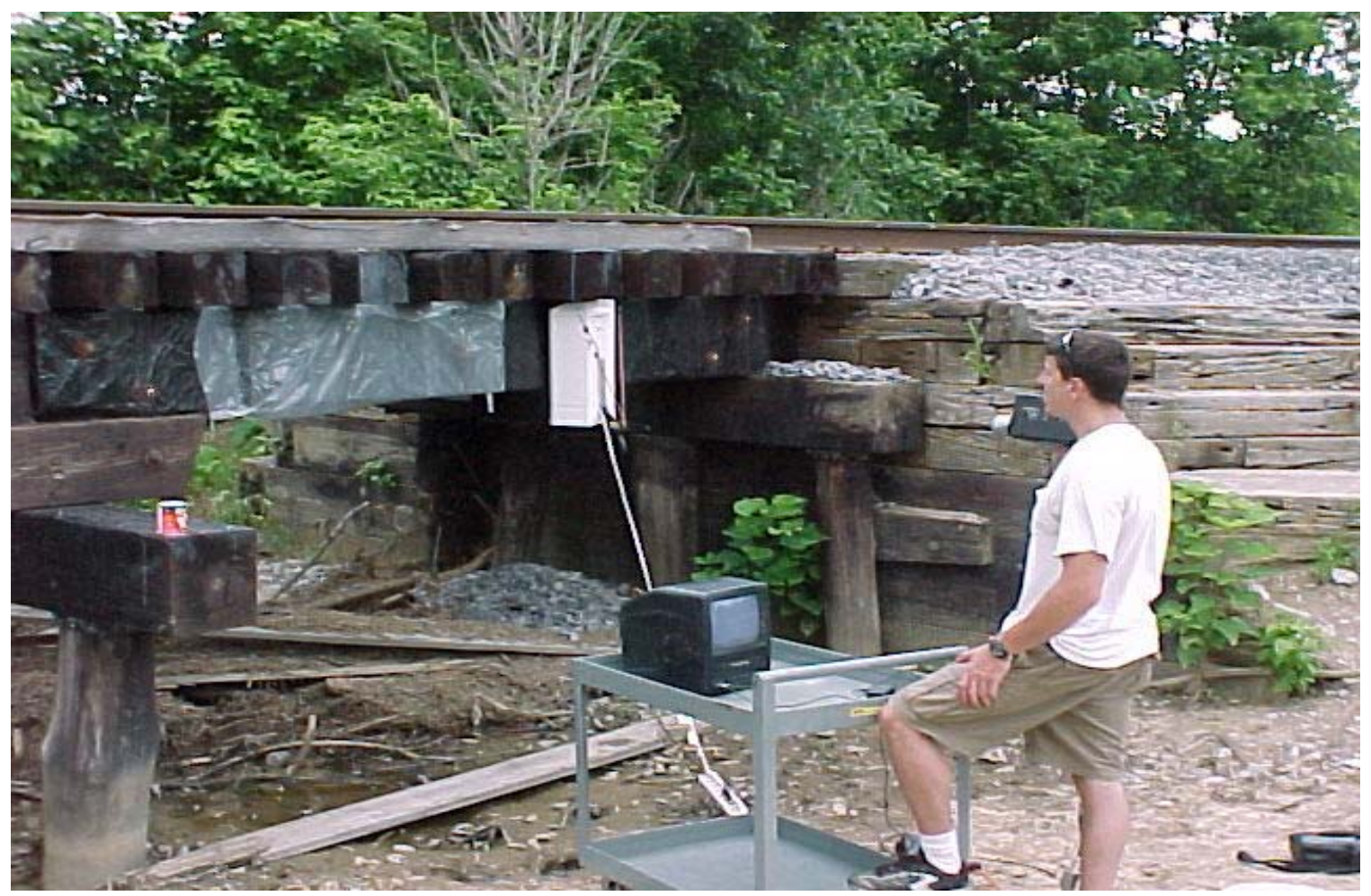

Figure 5.3.6 Different sections of the stringer heated by plastic wrap and a quartz tower heater

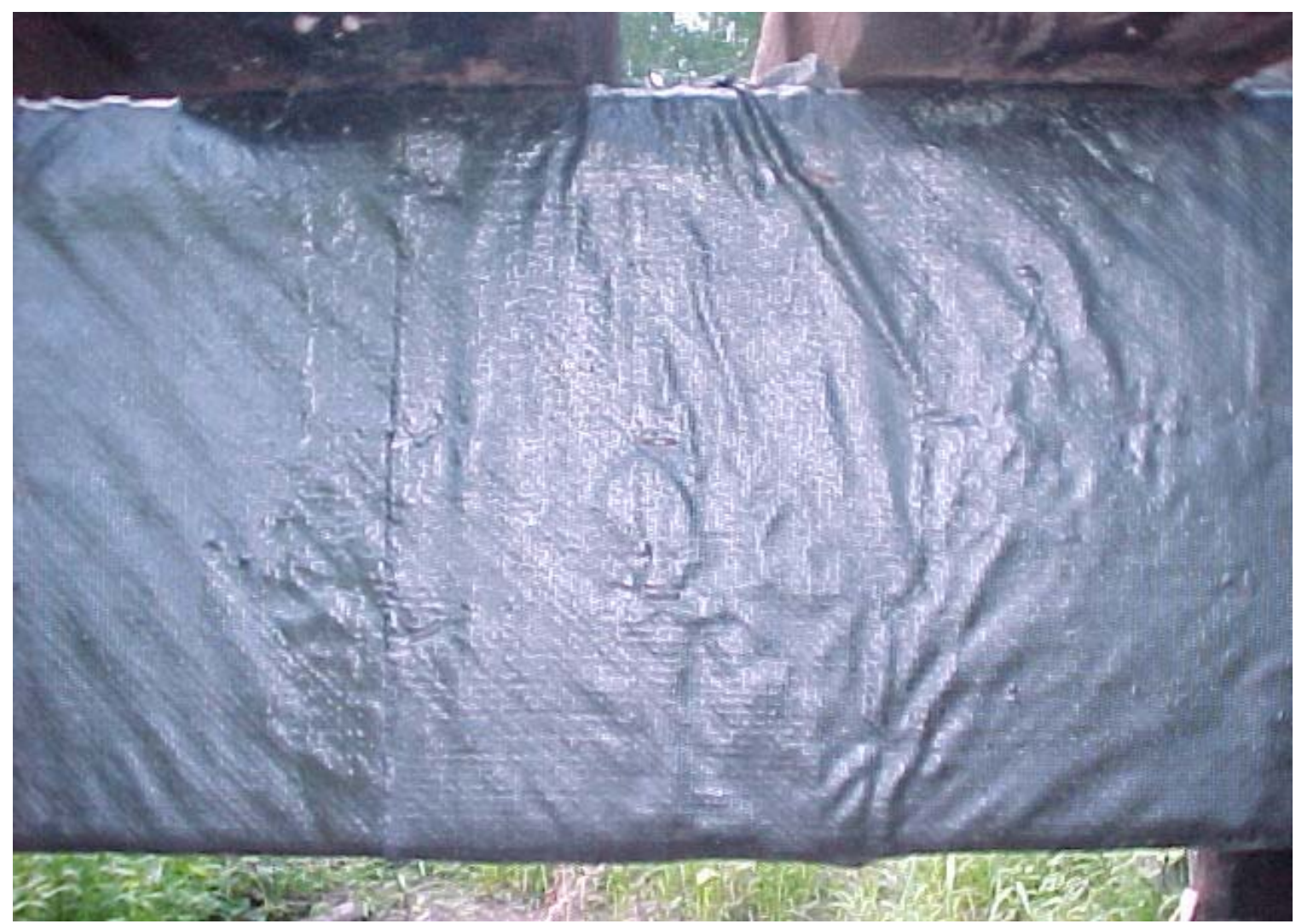

(a) 


\section{คบTo}

\section{ISI}

$-\frac{1}{1}$

$E=1.08$

OUER

05 Jul 10 e4:01:50 F=NO L5e R1

(b)

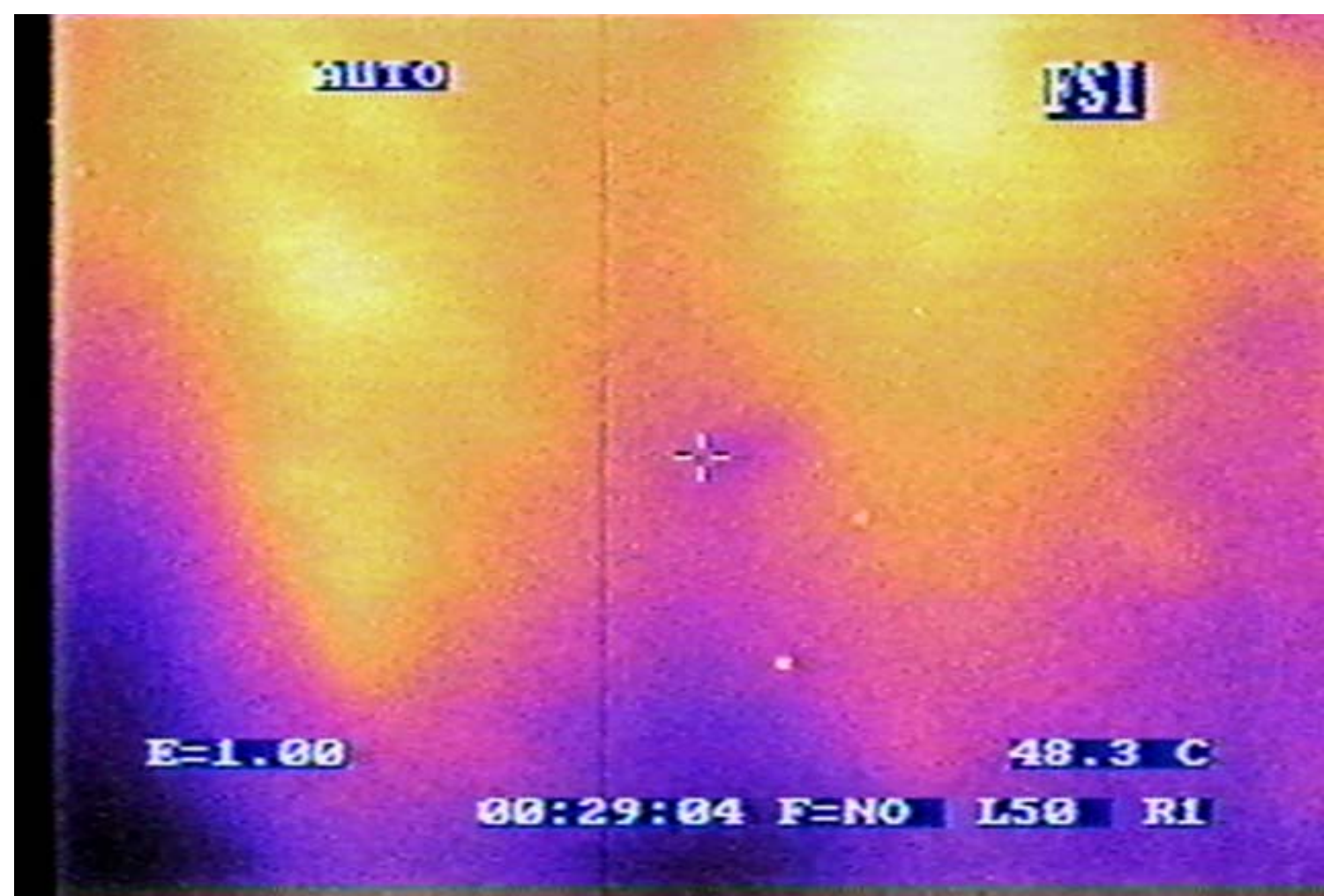

(c) 


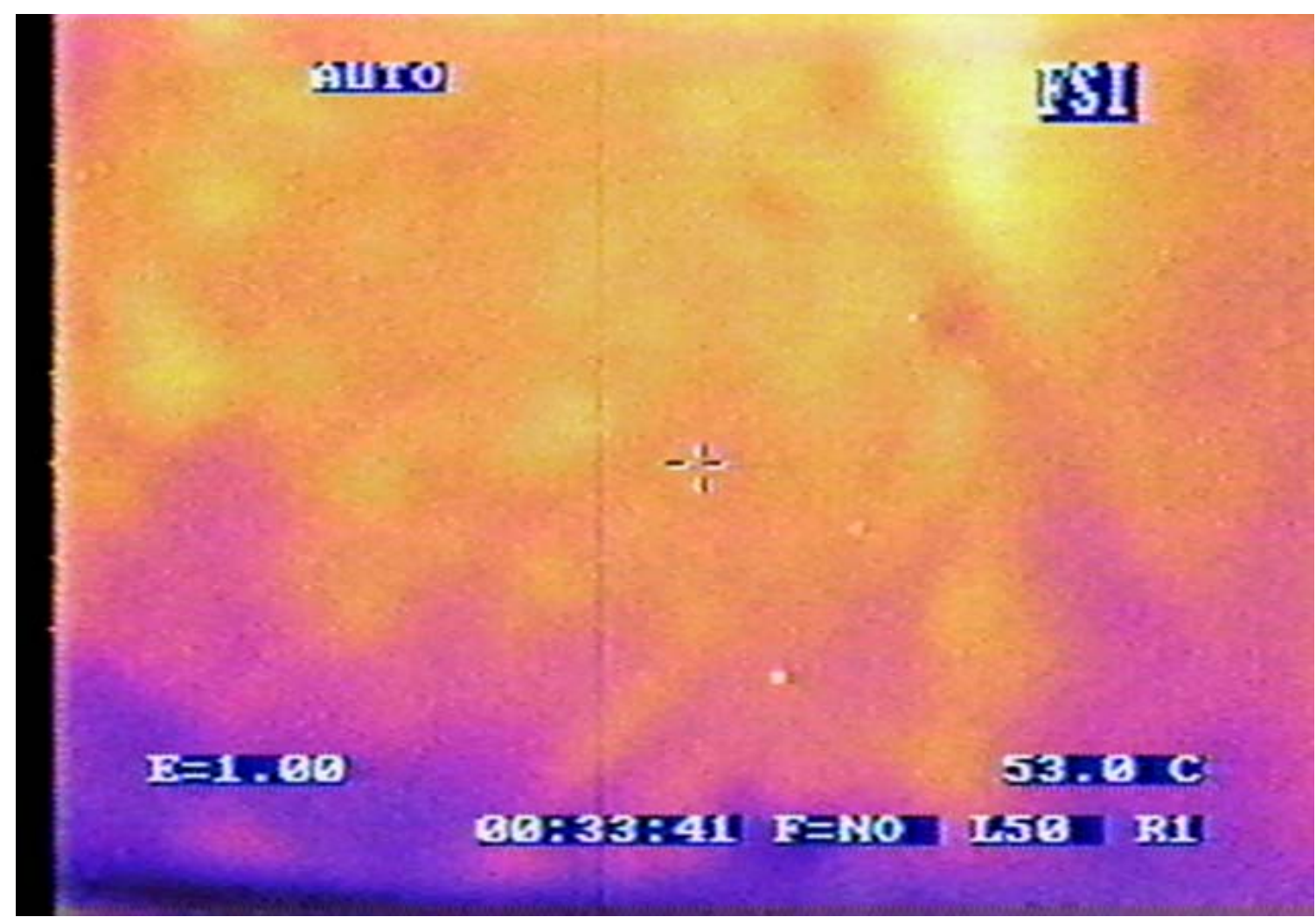

(d)

Figure 5.3.7 (a) Photograph the stringer section heated with the quartz tower heater, (b) thermal image of the stringer with the quartz tower heater placed 2 inches away, (c) 4 inches away, and (d) 8 inches away from the stringer surface

\subsubsection{Bridge \#583}

The FLIR Prism Single Point Infrared Camera was used to test a stringer on Bridge \#583. The field test was conducted on a sunny day with an average ambient temperature of approximately $85^{\circ} \mathrm{F}\left(29.4^{\circ} \mathrm{C}\right)$.

The thermal images obtained after heating the stringer using solar radiation and the plastic wrap to create a type of greenhouse effect did not show any significant surface temperature differentials that would have indicated delaminations or defects. However, significant surface temperature differentials were detected when the quartz tower heater was used to heat a section of the stringer (Figure 5.3.8). 


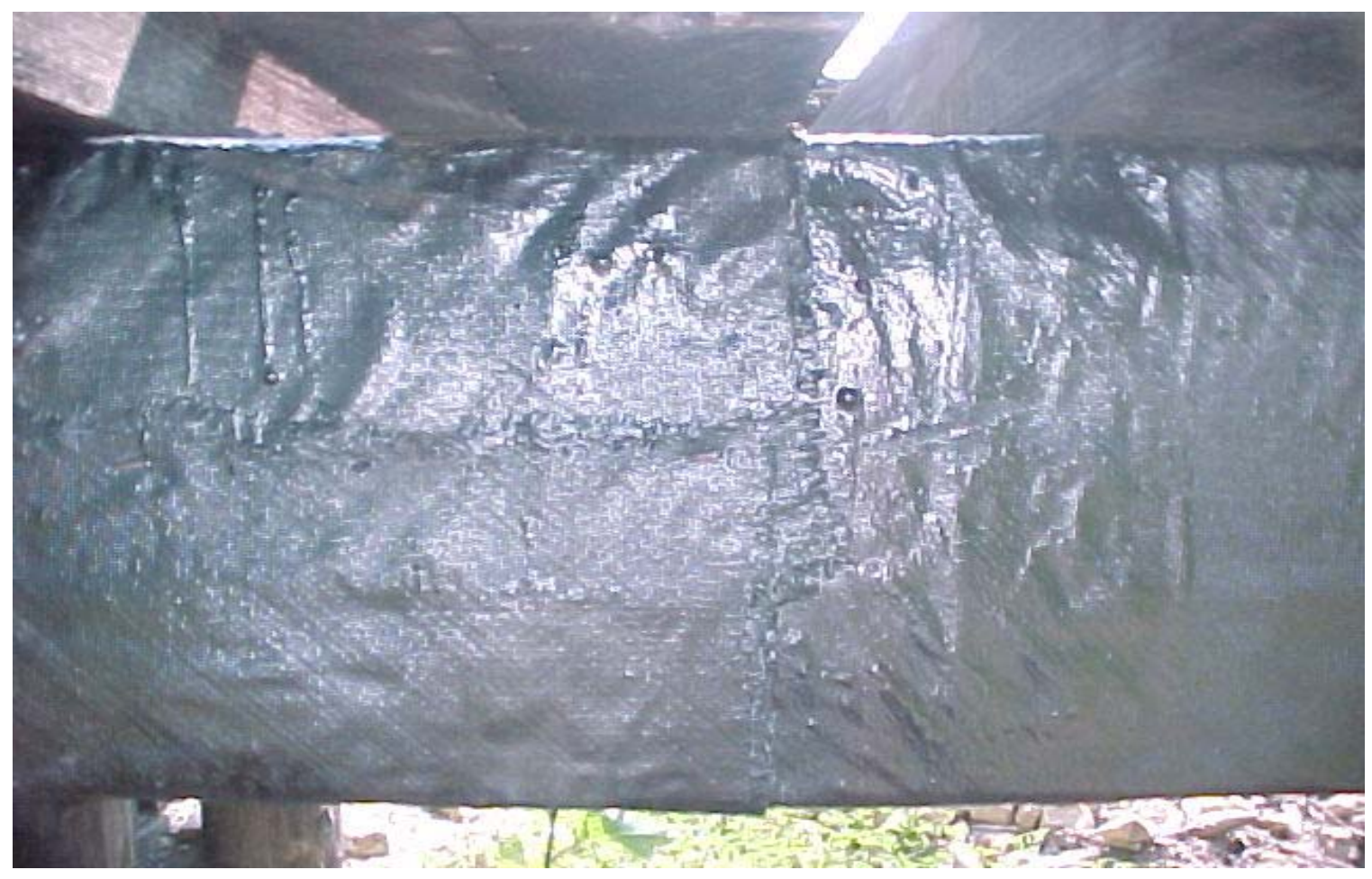

(a)

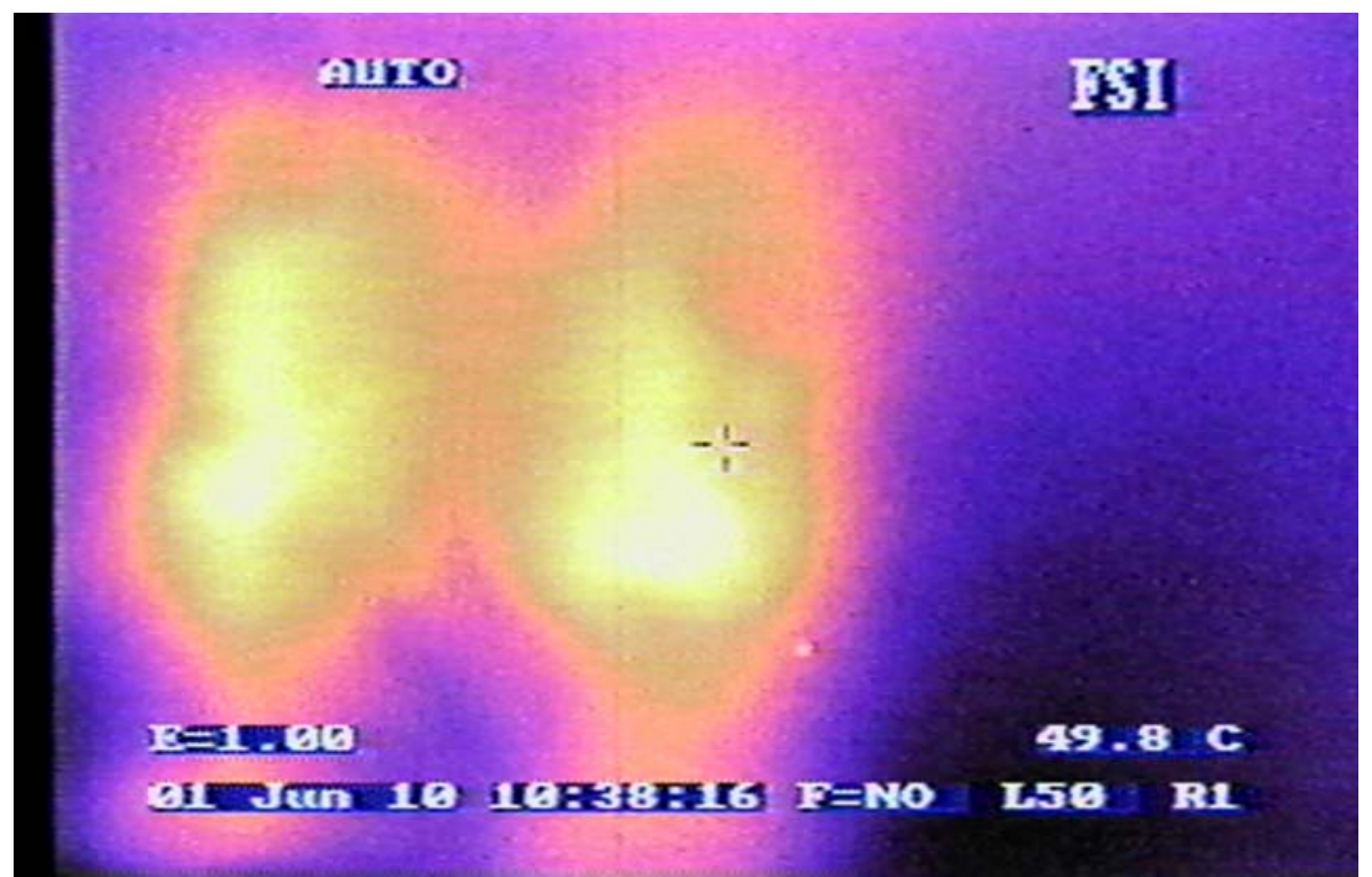

(b)

Figure 5.3.8 (a) A photograph of the stringer's surface, and (b) thermal image of the stringer's surface detecting significant surface temperature differentials 
As one moves from the center of the thermal image in Figure 5.3.8 (b) to the edges, the color changes from yellow to orange to purple to blue, which indicates the center is hotter than the edges. The thermal image in Figure 5.3.8 (b) was taken approximately 5 minutes after being heated. Therefore, this phenomenon is normal since the edges have more heat dissipation to the surrounding and thus return to thermal equilibrium quicker than the center. It was concluded that the temperature differences observed in Figure 5.3.8 were not caused by a delamination.

Figure 5.3.9 is another thermal image that shows surface temperature differentials. However, these surface temperature differentials are a result of inconsistent heating by the quartz tower heater. The heater was placed to close to the timber component being tested and the bottom of the heater was closer to the surface than the top of the heater (the bottom of the thermal image is brighter than the top). Since these temperature differences can be erroneously interpreted as delaminations, an operator must be careful while interpreting thermal images. 


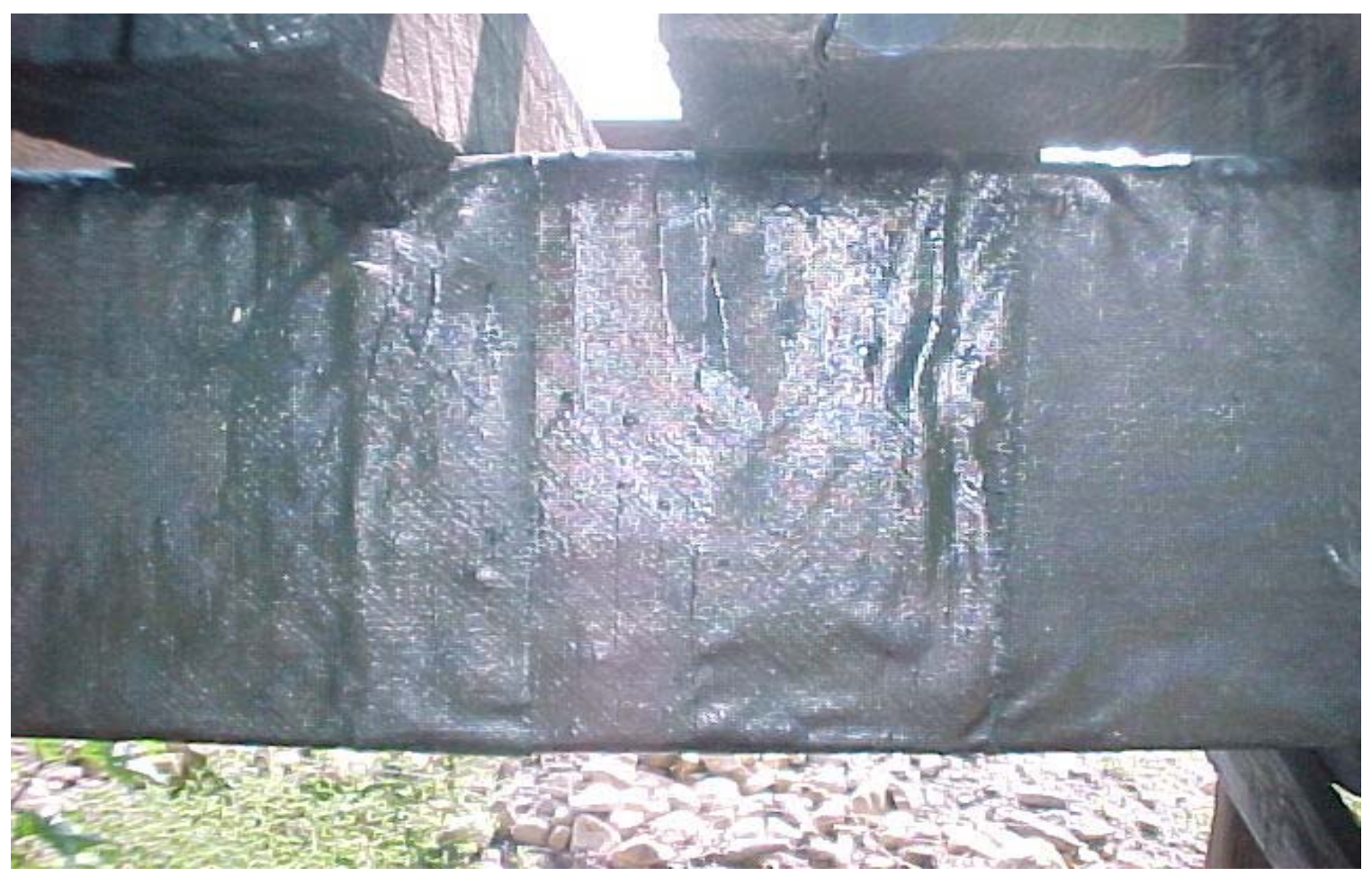

(a)

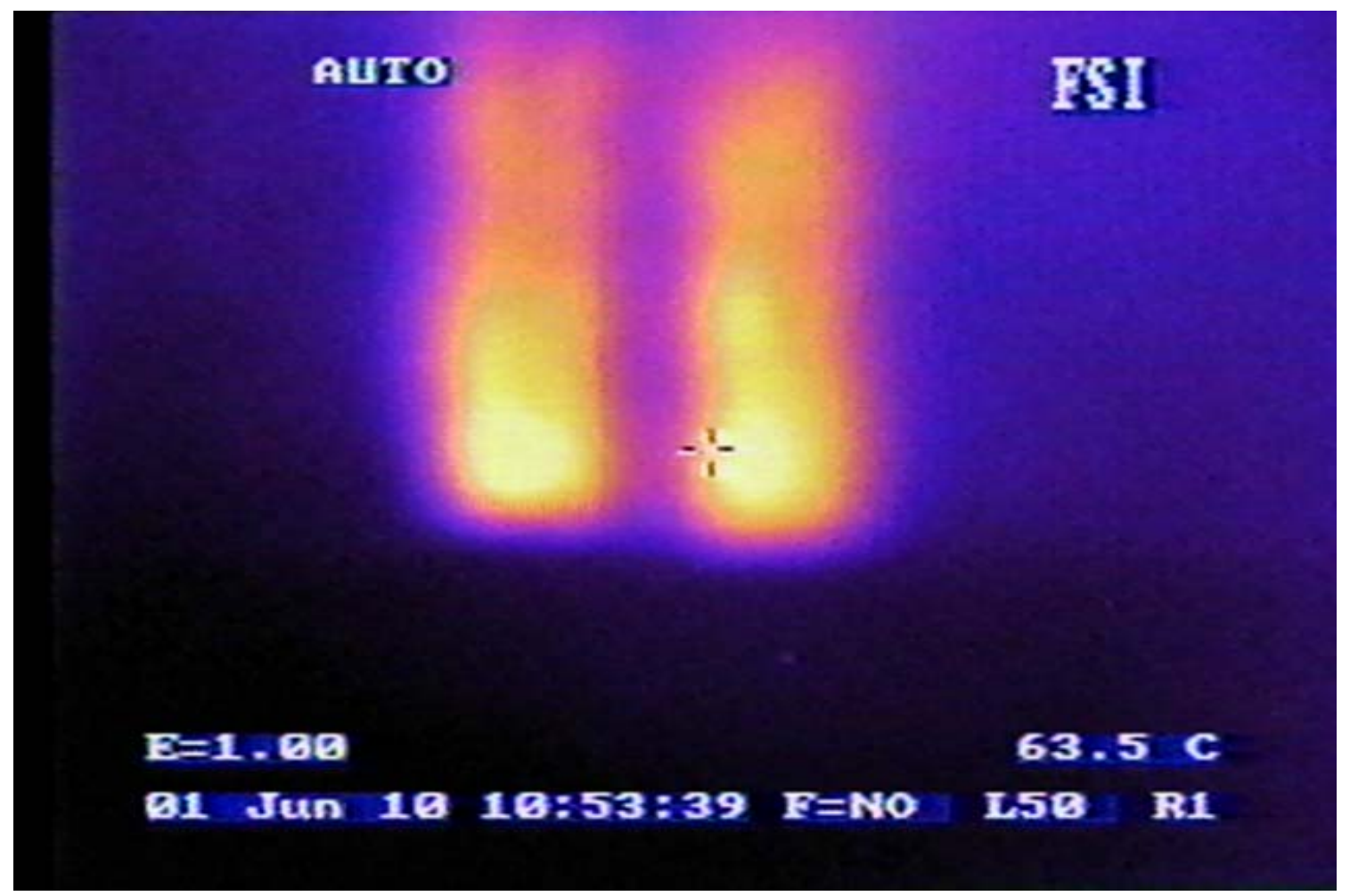

(b)

Figure 5.3.9 (a) Photograph of the stringer's surface, and (b) thermal image of the stringer's surface that was non-uniformly heated 


\subsection{Conclusions}

From the infrared field tests conducted on the three timber railroad bridges belonging to the South Branch Valley Railroad, it was found that the GFRP wraps have sufficiently bonded to the timber components. The GFRP wrapped timber components do not have any subsurface delaminations. However, the infrared camera detected some surface temperature differentials not caused by delaminations.

Three heat sources were used during these field experiments (a quartz tower heater, solar radiation, and a simulated greenhouse effect). The more efficient infrared thermography technique used a quartz tower heater placed approximately 8 inches away from the timber components to heat the surface. The thermal images obtained after using solar radiation to heat the timber components did not show significant surface temperature differentials. This is because solar radiation does not create enough of a thermal gradient to produce significant heat transfer. The plastic wrap used to create a type of greenhouse effect did heat the surface enough to observe some surface temperature differentials. Cooler regions represented by dark spots in the thermal images were also detected. It was concluded that metal nails or bolts beneath the GFRP wrap caused these cooler regions. 


\section{CHAPTER 6}

\section{CONCLUSIONS AND RECOMMENDATIONS}

\subsection{Conclusions}

From the extensive literature review, it was concluded that there are many NDE techniques that have successfully been used for evaluation of composite materials and structures. Each NDE technique has its own strengths and weaknesses. However, it seems the best technique to evaluate FRP wrapped structures is infrared thermography. From the literature review on the application of infrared thermography for the evaluation of structures rehabilitated with composite wraps, the following can be concluded:

1. Infrared thermography has a good potential for application to field monitoring of bridges and other structures rehabilitated with composite wrap.

2. Infrared thermography has distinct advantages over other NDE techniques used to evaluate FRP wrapped structures such as:

- The technique enables rapid, non-contact survey of delaminations between the composite wrap and the underlying structural member.

- The thermal images can be interpreted with relative ease.

- The infrared thermography technique does not require external wire connections or sensors that could get damaged in the field and reduce the quality of the acquired data.

- Infrared thermography allows for the survey of large regions in short periods of time. 
3. Various researchers have used infrared thermography to successfully locate voids and delaminations in structures and components rehabilitated with composite wrap. However, the detection of these defects becomes more difficult as the delaminations become smaller and deeper; therefore, combining other NDE techniques such as ground penetrating radar, ultrasonics, or fiber optics with infrared thermography can be a powerful tool for detecting and assessing various types of defects in FRP wrapped structures.

From the laboratory tests conducted on three timber piles rehabilitated with FRP wrap, the following can be concluded:

1. As the area and the thickness of the subsurface delamination increases, the surface temperature differentials increase, which allows the infrared camera to detect the delaminations easier.

2. The quartz tower heater had distinct advantages over other heat sources for the following reasons:

- The quartz tower heater produced a large thermal gradient between the specimens and the surrounding environment, which caused heat to flow perpendicular to the delaminations (or through the defects).

- The heating period and the distance between the heater and specimen could be easily controlled using a quartz tower heater. 
3. Subsurface delaminations with a thickness as small as 0.125 " buried under a 1/8" FRP wrap were detected by the infrared camera when the quartz tower heater was used to heat the surface.

4. Solar radiation did not prove to be a promising heat source for vertical structural components (e.g. timber piles) for the following reasons:

- Solar radiation did not produce significant surface temperature differentials.

- Solar radiation caused vertical heat flow in the plane of the FRP wrap and delaminations (heat traveled parallel to the delaminations).

- Solar radiation caused the specimens to be heated non-uniformly (the top of the specimens became hotter than the bottom).

5. Creating a type of greenhouse effect did increase the surface temperature of the specimens; however, the infrared camera was unable to detect any surface temperature differentials caused by subsurface delaminations. The disadvantages of using this type of heat source are very similar to the disadvantages associated with solar radiation.

Form the field tests conducted on the three timber railroad bridges rehabilitated with FRP composite wrap, the following can be concluded:

1. The quartz tower heater produced the best results out of the three heat sources considered in this study (quartz tower heater, solar radiation, and a type of greenhouse effect). The best results were obtained when the quartz tower heater was placed approximately 8 inches away from the surface and allowed 
to heat the surface for approximately 60 seconds.

2. It was determined that the surface temperature differentials detected by the infrared camera were not caused by subsurface delaminations. Instead, metal nails and bolts, and greater heat dissipation at the edges caused the surface temperature differentials.

3. No real subsurface delaminations were detected in the field, which led to the conclusion that the wrapping (done one year earlier) are in good condition.

From the literature review, laboratory experiments, and field tests it was concluded that the primary disadvantages associated with infrared thermography include:

1. The infrared technique works well only in the presence of a strong heating source such as the quartz tower heater.

2. Field variables such as shadows, wind velocity, edges or boundaries, and the distance from the object to the infrared camera can influence the readings. Consequently, the inspector/operator must be trained to know when and what adjustments are needed to account for these factors.

3. IR thermography is a multi-disciplinary science that requires a strong understanding of the thermal dynamics of the application.

4. The heat source needs to distribute the heat uniformly since thermal gradients induced by non-uniform heating can be mistaken for defects.

5. Infrared thermography cannot determine depth or thickness of a delamination. 


\subsection{Recommendations}

There are several recommendations that can be made to improve the evaluation of composite bridge decks using infrared thermography. These recommendations are:

1. The use of an infrared camera with a spectral range of $8-12 \mu \mathrm{m}$ or a dualband infrared camera (which acquires infrared images in two different spectra) is highly recommended to minimize the environmental effects.

2. To improve the quality of the images and accentuate the defects, the cold image (image captured prior to heating) can be subtracted from the hot image (image captured during heating).

3. Combining two NDE techniques such as infrared thermography and ground penetrating radar would be a very powerful tool for detecting and assessing various types of defects in FRP wrapped structures. The GPR technique can be used to detect deep defects and/or deterioration of the structure that cannot be detected by IR thermography. The IR thermography can be used to detect very shallow defects, including FRP disbondment, and blisters, which cannot be detected easily with the GPR technique. Therefore, the two techniques complement each other very well.

4. New NDE techniques using lasers and other advanced tools should be explored.

5. Development of finite element models and the use of Fourier domain image processing will be useful analytical tools to establish the magnitude and severity of defects. 


\section{References}

Bar-Cohen, Y., Mal, A., and Chang, Z., "Composite Material Defects Characterization Using Leaky Lamb Wave Dispersion Data," Nondestructive Evaluation of Materials and Composites II, SPIE, Vol. 3396, San Antonio, TX, 31 March - 1 April 1998, pp. 180 186.

Bates, D., Lu, D., Smith, G., and Hewitt, J., "Rapid NDT of composite aircraft components using lock-in ultrasonic and halogen lamp thermography," Nondestructive Evaluation of Aging Materials and Composites IV, SPIE, Vol. 3993, Newport Beach, Ca., 8-9 March 2000, pp. 2 - 13.

Burleigh, D., Bohner, R., and Young, W. P., “ THERMAL NONDESTRUCTIVE TESTING (TNDT) OF ADHESIVELY BONDED COMPOSITE REINFORCEMENTS APPLIED TO CONCRETE CIVIL STRUCTURES," Nondestructive Evaluation of Bridges and Highways III, SPIE, Vol. 3587, Newport Beach, Ca., 3 - 5 March 1999, pp. $105-115$.

Claus, R. O., and Arya, V., “Application of Optical Fiber Sensors to the Intelligent Processing of Materials," Sensing for Materials Characterization, Processing, and Manufacturing, TONE, Vol. 1, ASNT, Columbus, OH, 1998, pp. 363 - 373. 
Cooper, J. D., and Munley, E., "Bridge Research: Leading the Way to the Future," Office of Research and Development, U.S. Federal Highway Administration, 1995, pp. $23-27$.

Duke, Jr., J. C., Lesko, J., and Weyers, R., “ NONDESTRUCTIVE EVALUATION OF CRITICAL PULTRUDED COMPOSITE MATERIAL BRIDGE BEAMS,” Nondestructive Testing and Evaluation of Infrastructure, TONE, Vol. 2, ASNT, Columbus, OH, 1998, pp. $255-260$.

Dunkers, J. P., Zimba, C. G., Flynn, K. M., and Hunston, D. L., "Characterization of Composite Microstructure and Damage Using Optical Coherence Tomography,” Nondestructive Evaluation of Aging Materials and Composites III, SPIE, Vol. 3585, Newport Beach, Ca., 3 - 5 March 1999, pp. 208 - 218.

Edwards, C., Stratoudaki, T., Dixon, S., and Palmer, S., "Non-contact ultrasound studies of composite materials: new developments," Nondestructive Evaluation of Aging Materials and Composites IV, SPIE, Vol. 3993, Newport Beach, Ca., 8- 9 March 2000, pp. $268-275$.

Furrow, P. C., Brown, R. T., and Mott, D. B., 'Fiber optic health monitoring system for composite bridge decks," Smart Systems for Bridges, Structures, and Highways, SPIE, Vol. 3988, Newport Beach, Ca., 6 - 7 March 2000, pp. 380 - 391. 
Georgeson, G. E., "Ultrasonic evaluation of co-cured composite structures," Nondestructive Evaluation of Aging Aircraft, Airports, and Aerospace Hardware IV, SPIE, Vol. 3994, Newport Beach, Ca., 7 - 8 March 2000, pp. 164 - 173.

Hag-Elsafi, O., Kunin, J., and Alampalli, S., "Evaluating Effectiveness of FRP Composites For Bridge Rehabilitation Through Load Testing," Proceedings of Structural Materials Technology IV-An NDT Conference, Atlantic City, NJ, Feb. 28 - March 3, 2000.

Halabe, U. B., Nondestructive Material and Structural Evaluations, CE 364 Course Package, Department of Civil and Environmental Engineering, West Virginia University, Morgantown, WV, 2000, pp. $161-176$.

Hall, D. O., “ Cost Effective Advances in Portable Radioscopic NDT of Composite Materials," Nondestructive Evaluation of Materials and Composites II, SPIE, Vol. 3396, San Antonio, TX, 31 March - 1 April 1998, pp. 96 - 102.

Hawkins, G. F., Johnson, E. C., and Nokes, J. P., “ Detecting manufacturing Flaws in Composite Retrofits," Nondestructive Evaluation of Bridges and Highways III, SPIE, Vol. 3587, Newport Beach, Ca., 3 - 5 March 1999, pp. 97 - 104. 
Jackson, D. R., Islam, M., and Alampalli, S., “Feasibility of evaluating the performance of fiber reinforced plastic (FRP) wrapped reinforced concrete columns using ground penetrating radar (GPR), and infrared (IR) thermography techniques," Proceedings of Structural Materials Technology IV-An NDT Conference, Atlantic City, NJ, Feb. 28 March 3, 2000.

Kadir, R., Detection of Highway Pavement Cracks through Infrared Thermography and Digital Image Processing, Master's thesis, Department of Civil and Environmental Engineering, West Virginia University, Morgantown, WV, August, 1991, pp. 81.

Karabutov, A. A., Murashov, V. V., Podymova, N. B., and Oraevshy, A. A., "Nondestructive characterization of layered composite materials with a laser optoacoustic sensor," Nondestructive Evaluation of Materials and Composites II, SPIE, Vol. 3396, San Antonio, TX, 31 March - 1 April 1998, pp. 103 - 111.

Karabutov, A. A., and Podymova N. B., “ Nondestructive evaluation of fatigue changes of composite structure by laser-excited ultrasonic waves," Nondestructive Evaluation of Materials and Composites II, SPIE, Vol. 3396, San Antonio, TX, 31 March - 1 April 1998, pp. $255-261$.

Liu, J. M., "microwave and ultrasonic NDE of thick glass-fiber-reinforced composites," Nondestructive Evaluation of Materials and Composites II, SPIE, Vol. 3396, San Antonio, TX, 31 March - 1 April 1998, pp. 135 - 146. 
Maldague, X., "NDT by Infrared Thermography: Principles with Applications," Sensing for Material Characterization, Processing, and Manufacturing, TONE, Vol. 1, ASNT, Columbus, OH, 1998, pp. 385-398.

Maldague, X., Nondestructive evaluation of materials by infrared thermography, Springer-Verlag, London, U.K.; NY, 1993.

Mandic, D. G., Martin, R. E., Hemann, J. H., “ Thermal Imaging Technique to Detect Delaminations in CFRP Plated Concrete," Nondestructive Evaluation of Materials and Composites II, SPIE, Vol. 3396, San Antonio, TX, 31 March - 1 April 1998, pp. 22 - 27.

Manning, D. C., and Holt, F. B., “ Detecting delaminations in concrete bridge decks,” Concrete International, 1980, pp. $34-41$.

Morten, F. D., "Infrared detectors and their applications," Applications of Infrared Detectors, Sowan, F. A., ed., Mullard Ltd., London, U.K., 1971, Chapter 1, pp. 1 - 25.

Murukeshan, V. M., Malhotra, S. K., and Asundi, A., “FOPSESPI for Non-Destructive Evaluation (NDE) of Composites," Nondestructive Evaluation of Aging Materials and Composites III, SPIE, Vol. 3585, Newport Beach, Ca., 3 - 5 March 1999, pp. 260 - 268. 
Park, S. W. and Zhou, M., "Nondestructive Assessment of the Post-Impact Properties of FRP Composite Laminates," Proceedings of Structural Materials Technology IV - An NDT Conference, Atlantic City, NJ, Feb. 28 - March 3, 2000.

Ratcliffe, C. P., Gillespie, Jr., J. W., Heider, D., Eckel II, D. A., and Crane, R. M., “ Experimental Investigation into the Use of Vibration Data for Long Term Monitoring of an All Composite Bridge," Nondestructive Evaluation of Highways, Utilities, and Pipelines IV, SPIE, Vol. 3995, Newport Beach, Ca., 7 - 9 March 2000, pp. 64 - 75.

Thevar, T. M., Webster, J. M., Kalshteyn, M., and Mew, J. M., “ Non-contact laser based computation method of NDE of composites and other structures," Nondestructive Evaluation of Aging Materials and Composites III, SPIE, Vol. 3585, Newport Beach, Ca., 3 - 5 March 1999, pp. 219 - 225.

Thomas, D., A Composite Health Monitoring System, Foster-Miller, Inc., Waltham, MA, June 1999.

U.S. Department of Transportation (USDOT), Bureau of Transportation Statistics (BTS). National Transportation Statistics 1998.

U.S. Department of Transportation (USDOT), Bureau of Transportation Statistics (BTS). Transportation Statistics Annual Report 1999. Washington, DC. 
U.S. Department of Transportation (USDOT), Federal Highway Administration (FHWA). 1997. Highway Statistics Summary to 1995. Washington, DC.

U.S. Department of Transportation (USDOT), Federal Highway Administration (FHWA). 1998a. Highway Statistics. Washington, DC.

U.S. Department of Transportation (USDOT), Federal Highway Administration (FHWA). 1998b. National Bridge Inventory Database.

U.S. Department of Transportation (USDOT), Federal Highway Administration (FHWA). 1999 Status of the Nation's Highways, Bridges, and Transit: Conditions and Performance. Washington, DC.

U.S. Department of Transportation (USDOT), Federal Highway Administration (FHWA). 1999. Annual editions. Highway Statistics. Washington, DC.

West Virginia University Constructed Facilities Center, U.S. Department of Transportation Federal Highway Administration, West Virginia Department of Transportation Division of Highways, Fiber Reinforced Polymer Composite Bridges of West Virginia, 2001.

Wyckhuyse, A., Maldague, X., "A study of wood inspection by infrared thermography," Research in NonDestructive Evaluation, submitted Dec 7th, 1999. 
Yannotti, A. P., Alampalli, S., O’Connor, J., Schongar, G., Greenberg, H., and Norfolk, M., "Proof Load Testing And Monitoring of An FRP Composite Bridge," Proceedings of Structural Materials Technology IV-An NDT Conference, Atlantic City, NJ, Feb. 28 March 3, 2000.

Zheng, G., Buckley, M. A., Kister, G., and Fernando, G. F., “ Blind deconvolution of acoustic emission signals for damage identification in composites," Nondestructive Evaluation of Aging Materials and Composites IV, SPIE, Vol. 3993, Newport Beach, Ca., 8- 9 March 2000, pp. $47-57$. 